NIKO SWITEK (HG.)

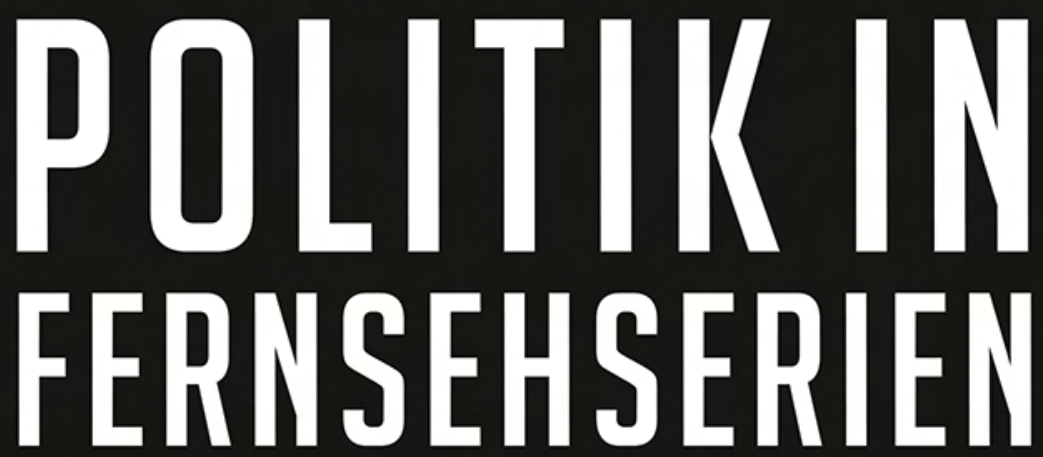

ANALYSEN UND FALLSTUDIEN

ZU HOUSE OF CARDS, BORGEN \& CO.

[transcript] Edition Politik 
Niko Switek (Hg.)

Politik in Fernsehserien

Edition Politik | Band 55 

Niko Switek (Hg.)

\section{Politik in Fernsehserien}

Analysen und Fallstudien zu House of Cards, Borgen \& Co.

[transcript $]$ 
Die Publikation wurde ermöglicht durch Mittel der Fakultät für Gesellschaftswissenschaften (SQVK und Förderung des wissenschaftlichen Nachwuchs) und des Fördervereins der Universität Duisburg-Essen. Die frei zugängliche digitale Publikation wurde ermöglicht mit Mitteln des BMBF-Projektes OGeSoMo der Universitätsbibliothek Duisburg-Essen. In diesem Projekt wird Open Access für geistes- und sozialwissenschaftliche Monografien gefördert und untersucht. Informationen und Ergebnisse finden Sie unter https://www.uni-due.de/ogesomo.

\section{Bibliografische Information der Deutschen Nationalbibliothek}

Die Deutsche Nationalbibliothek verzeichnet diese Publikation in der Deutschen Nationalbibliografie; detaillierte bibliografische Daten sind im Internet über http://dnb.d-nb.de abrufbar.

\section{(c) $\underset{\mathrm{Br}}{\mathrm{i}}$}

Dieses Werk ist lizenziert unter der Creative Commons Attribution 4.0 Lizenz (BY). Diese Lizenz erlaubt unter Voraussetzung der Namensnennung des Urhebers die Bearbeitung, Vervielfältigung und Verbreitung des Materials in jedem Format oder Medium für beliebige Zwecke, auch kommerziell. (Lizenztext:

https://creativecommons.org/licenses/by/4.o/deed.de)

Die Bedingungen der Creative-Commons-Lizenz gelten nur für Originalmaterial. Die Wiederverwendung von Material aus anderen Quellen (gekennzeichnet mit Quellenangabe) wie z.B. Schaubilder, Abbildungen, Fotos und Textauszüge erfordert ggf. weitere Nutzungsgenehmigungen durch den jeweiligen Rechteinhaber.

\section{Erschienen $2018 \mathrm{im}$ transcript Verlag, Bielefeld}

(c) Niko Switek (Hg.), Kapitel: jeweiliger Autor/Autorin

Umschlaggestaltung: Maria Arndt, Bielefeld

Redaktion: Almut Zimmer M.A.

Druck: Majuskel Medienproduktion $\mathrm{GmbH}$, Wetzlar

Print-ISBN 978-3-8376-4200-1

PDF-ISBN 978-3-8394-4200-5

Gedruckt auf alterungsbeständigem Papier mit chlorfrei gebleichtem Zellstoff. Besuchen Sie uns im Internet: https://www.transcript-verlag.de Bitte fordern Sie unser Gesamtverzeichnis und andere Broschüren an unter: info@transcript-verlag.de 


\section{Inhalt}

\section{THEORETISCHE ANSÄTZE \& ANALYTISCHE ZUGÄNGE}

\section{Spiegel, Daten, Narrative}

Politikwissenschaftliche Zugänge zu politischen Fernsehserien | 11

Niko Switek

\section{Polit-Serien im Fernsehen}

Gegenstandsbestimmung, Stand der Forschung

und neue Perspektiven | 33

Andreas Dörner, Stefan Heinrich Simond

\section{Politik in Serie(n)}

Die Politik, das Politische und die Tragödie | 53

Henrik Schillinger

Filme zwischen künstlerischer Freiheit und politischer Erkenntnis Zum Realitätsgehalt fiktionaler Medien | 77

Manfred Mai

\section{FALLSTUDIEN POLITISCHER FERNSEHSERIEN}

\section{Eichwald, MdB}

Überleben im Haifischbecken Berlin-Mitte | 105

Florian Gilberg, Raphael David Moser, Said Rezek, Steffen Schulze

\section{Eurokrise, Sex und Klimawandel}

Krisenmanagement in der britischen Sitcom Yes, Prime Minister | 125

Ann-Kathrin Binot, Ulrike Gansen, Anatoli Kolembach, Martin Miller

The Thick of It

Macht, Medien und Marionetten | 153

Lisa Brose, Julia Linn, Michelle Magaletta

\section{Spiel der Kräfte}

Politik, Medien und Familie in Borgen | 177

Johannes Bongardt, Rieke Gießelmann, Matthias Jüschke, Jan Pfeifer, Christina-Johanne Schröder, Jonas Seyferth 
Sex, Drugs and Politics

Die Polit-Serie Marseille | 201

Jonathan Beierl, Yannick Chougrani, Ilka Rasch, Sören Witt, Almut Zimmer

\title{
Gomorrha
}

Mafia und Staat im Verhältnis | 225

Taylan Yildiz

\section{House of Cards}

The American Machiavelli | 243

Florian Breitweg, Jakob Hager, Vanessa Molter, Cornelius Witt

Fernsehserien und die Wahrnehmung der US-Präsidentschaft

Einstellungen, politischer Zynismus und House of Cards | 271

Zaira Batroff, Lorena Capuozzo, Kai Jardner, Klaus Kamps

\section{Veep}

Das Amt des Vizepräsidenten der USA als institutionalisierte Bedeutungslosigkeit? | 285

Alexander Stock

\section{"Whatever it takes“}

24 und die Normalisierung des Ausnahmezustandes | 305

Frank Gadinger

\section{人民的名义 (Im Namen des Volkes) versus House of Cards}

Polit-Serien als Legitimierungsinstrument aktueller

chinesischer Reformpolitik | 327

Ulrike Gansen, Martin Miller, Nele Noesselt, Jonas Seyferth

Sind Drachen, Zombies und Aliens politisch?!

Das Politische in der Phantastik am Beispiel

der SF-Serie The Expanse | 345

Arne Sönnichsen

\section{REFLEKTION \& DIDAKTISCHER EINSATZ}

\author{
Warum eigentlich nicht? \\ Über die Unmöglichkeit, deutsches Politikmanagement \\ im Fernsehen abzubilden | 363 \\ Karl-Rudolf Korte
}


Polit-Serien im Fachunterricht

Empirische Befunde zum Medieneinsatz

aus Schüler- und Lehrerperspektive | 373

Sabine Manzel

\section{ANHANG}

Autorinnen- und Autorenverzeichnis | 395 

Theoretische Ansätze \& analytische Zugänge 



\title{
Spiegel, Daten, Narrative
}

\author{
Politikwissenschaftliche Zugänge
}

zu politischen Fernsehserien

\section{Niko Switek}

\section{EINLEITUNG: MEHR ALS UNTERHALTUNG}

Politik verkauft sich aktuell gut. Die Fernsehserie House of Cards über den durch eine Zurückstellung gekränkten Francis Underwood, der in einem Rachefeldzug unter Einsatz aller legalen und illegalen Mittel und Strategien das USPräsidentenamt erobert, erzielte weltweite Aufmerksamkeit und Erfolg. Parallel dazu reüssierten weitere Produktionen, wie Veep, Scandal oder Alpha House, die alle explizit unterschiedliche Facetten des Politikbetriebs in der US-Hauptstadt Washington D.C. fokussieren. Aber auch in Europa finden sich in letzter Zeit Beispiele erfolgreicher Polit-Serien: Borgen über eine Premierministerin in Dänemark, Baron Noir über innerparteiliche Machtkämpfe in der sozialistischen Partei und die Präsidentschaftswahl in Frankreich sowie in Deutschland Eichwald, $M d B$ als Satire über einen Hinterbänkler im Bundestag.

Zum Zusammenhang von Popkultur - zu der die als Unterhaltungsformate konzipierten Fernsehserien zählen - und Politik existieren zahlreiche Arbeiten und Studien. Das ist aufgrund des ubiquitären Charakters von Politik nicht überraschend, weswegen auch popkulturelle Produkte ohne direkten Bezug auf die politische Sphäre auf ihren politischen Gehalt oder Aussagen hin inspiziert werden können. Ausgangspunkt für diesen Band ist aber die zunehmende Zahl von Serien, die sich dezidiert dem Politikbetrieb widmen und diesen als Stoff für unterhaltsame, dramaturgisch aufgebaute und fiktive Erzählungen verwenden. Aus zwei Gründen ergibt sich eine besondere Aktualität und Relevanz einer solchen Betrachtung: Das ist erstens eine Aufwertung des Serien-Formats und 
zweitens die zunehmende Zahl von Politikserien, die aus anderen Ländern als den USA als Stammland der Unterhaltungsindustrie stammen.

Anders als Spielfilme sind Serien zeitlich weniger begrenzt und können ihre Geschichten über Folgen und Staffeln hinweg entwickeln (Kelleter/JahnSudmann 2014). Charaktere und Rollen können über längere Zeit aufgebaut sowie Erzählstränge pausiert oder wiederaufgenommen werden. Neu ist eine Verschiebung bei den Produktionsbedingungen: Mit dem Aufkommen der auf Abonnement-Modellen basierenden Fernsehsendern (HBO, Showtime) und in jüngerer Zeit den Streaming-Portalen im Internet (Netflix, Amazon Prime) sinkt die Bedeutung von Werbung und steigt die Relevanz der Bindung von Kunden und Zuschauern. Die Bereitschaft, mehr Ressourcen und finanzielle Mittel für Serien-Produktionen einzusetzen, zieht renommierte Filmregisseure und prominente Schauspieler an, wodurch sich paradoxerweise die Serien in Qualität und Charakter wiederum Spielfilmen annähern. Die Sender oder Plattformen setzen auf Profilierung und Sichtbarkeit, welche sie durch aufwendige und qualitativ hochwertige Eigenproduktionen gewinnen wollen (Kelleter/Jahn-Sudmann 2014). Damit werden einerseits sonst als zu sperrig oder zu komplex eingestufte Themen als Gegenstand interessant. Zugleich ändern sich die Erzählstrukturen, da einzelne Folgen nicht mit Berücksichtigung von Werbeunterbrechungen und Cliffhangern konzipiert werden müssen (Kelleter 2012). Das ermöglicht insgesamt längere und kontinuierliche Erzählungen und speziell für Politikserien eine andere Art des Aufgreifens und Behandelns von Politik.

Neben den USA mit ihrer umfangreichen Unterhaltungsindustrie kommen aus Großbritannien einige bekannte Politikserien - meist beauftragt und ausgestrahlt von der öffentlich-rechtlichen British Broadcasting Corporation (BBC). So ist die viel gerühmte Netflix-Produktion House of Cards eine Adaption einer BBC-Miniserie aus den 1990er Jahren, die Serien Yes, Minister, Yes, Prime Minister und The Thick of It rücken das Amt des britischen Premierministers und seines Kabinetts sowie die Charakteristika der Ministerialbürokratie in den Mittelpunkt. In jüngerer Zeit kommen jedoch zunehmend Produktionen aus weiteren europäischen Ländern hinzu, wodurch sich in der Konsequenz eine originelle Vergleichsebene ergibt. Die verschiedenen Serien stehen für bestimmte politische Kulturen und spiegeln nationale Eigen- und Gegebenheiten (Neumann/ Nexon 2013: 13ff). Über eine vergleichende Betrachtung lassen sich Erkenntnisse zu in ihnen enthaltenen Mustern und Bildern von Politik sowie in diesen sich ausdrückenden kulturellen Spezifika gewinnen.

Das liefert einen Hinweis darauf, inwieweit Serien als unterhaltsame popkulturelle Produkte sinnvoll für eine ernsthafte Bearbeitung durch die Sozial- und Politikwissenschaften aufgegriffen werden können. Dieser konzeptionell ange- 
legte Beitrag skizziert den Rahmen einiger möglicher Forschungsperspektiven auf Polit-Serien und zeigt verschiedene Analysedimensionen auf. Grundsätzlich stehen dabei die Serien und ihre Inhalte als Erzählungen von Politik im Mittelpunkt (und nur am Rande der Produktionsprozess bzw. deren Rezeption und Wirkung). Da die Serien auf ihre eigene Art politische Prozesse abbilden, in denen Akteure in politischen Regelsystemen und Institutionen ihre Ziele verfolgen und um Macht und Einfluss konkurrieren, ergibt sich zunächst eine Verbindung zur politikwissenschaftlichen Systemforschung und (vergleichenden) Regierungslehre, die sich wissenschaftlich ebenfalls einer solchen Rekonstruktion widmen. In einem ersten Schritt ist es daher sinnvoll, deskriptiv das in einer Serie zugrunde gelegte politische System zu erläutern. Unter Rückgriff auf Ansätze, Konzepte und Modelle von Systemforschung und Regierungslehre werden der Kontext dargestellt und die systembedingten Besonderheiten der politischen Entscheidungsfindung aufgezeigt. Durch die Kontrastierung von Serie und Wissen über das politische System werden sowohl die realitätsgetreue Abbildung wie die erzählerischen Abweichungen sichtbar und interpretierbar. Das schärft zugleich den Blick, wonach diese spezifischen Perspektive in Serien sucht.

Allerdings bildet diese Kontrastierung nur einen ersten Schritt der Auseinandersetzung, auf welchem wiederum weitere Analyseperspektiven aufbauen können. Versucht man, eng an den Polit-Serien als Untersuchungsgegenständen zu bleiben, so bieten sich die von Neumann und Nexon (2006) vorgeschlagenen Analysedimensionen Serien als Spiegel und Serien als Daten an. Der Einsatz von Serien als Spiegel stützt sich auf die geschilderten Überschneidungen von politischen Erzählungen und Ausprägungen des politischen Systems. Da audiovisuelle Medien eindrücklicher und direkter wirken, können diese pädagogisch zur Illustration von bekannten Zusammenhängen eingesetzt werden. Aufgrund der teilweise hohen Zuschauerzahlen ganz unabhängig von Lehrkontexten erfüllen die Serien damit unfreiwillig eine Funktion der politischen Bildung. Während die Serien einerseits Interesse an politischen Zusammenhängen wecken können, besteht andererseits die Gefahr, dass negative und zynische Politikbilder entsprechende Einstellungen bei den Zuschauern befödern (z.B. den Einsatz von Folter als legitim erscheinen lassen). Damit ist der umfangreiche Bereich der Wirkungsforschung angesprochen, der hier jedoch nicht im Mittelpunkt steht. Neben dem pädagogischen Einsatz erhält der Forscher in dieser Dimension durch eine analogische Brille möglicherweise neue Ideen oder Impulse zu seinem Untersuchungsgegenstand, den er druch die Serien in einer anderen Anordnung und Rahmung erlebt. Offen und unvoreingenommen gestaltet sich die Auseinandersetzung mit Serien als Daten, wobei gerade oft die identifizierten Abweichungen im Mittelpunkt stehen. Das popkulturelle Aufgreifen von Politik in Serien fügt - 
explizit wie implizit - Normen und Wertvorstellungen hinzu, kodiert zeitgeistliche Effekte und dominierende Weltsichten mit in die Geschichte ein. Popkultur verkörpert immer eine bestimmte Zeit und Gesellschaft. Gerade in einer vergleichenden Betrachtung von Serien lassen sich dabei trefflich länderspezifische und kulturelle Unterschiede herausstellen.

Der vierte und letzte Punkt greift die Entwicklung auf, dass in der Politikwissenschaft verstärkt narrative oder erzähltheoretische Ansätze zum Einsatz kommen (Gadinger/Yildiz 2017). Ihnen ist gemein, dass sie das Erzählende als Kernelement der Politik sehen. Sinngebung vollzieht sich mittels Narrativen aus wiederkehrenden Metaphern, Rollen und Handlungsschemata. Kausalität entsteht in dieser Perspektive erst durch die Anordnung von Deutungsangeboten im Rahmen eine Erzählung. Der Erfolg eines Wahlkampfs oder einer politischen Rede hängt nicht nur an Strategie und Rhetorik, sondern auch daran, inwieweit eine überzeugende und legitimierende Erzählung enthalten ist (Jarzebski 2015). In Anlehnung an die Narrativforschung lässt sich somit in den Serien den Mechanismen der Transformation politischer Systeme, Regeln, Inhalte und Begrifflichkeiten in Erzählungen nachgehen. In diesem Sinne lässt die Betrachtung von Serien, die fiktional Politik unterhaltsam erzählen, zugleich Rückschlüsse über die Mechanismen der realen Politik zu. Auch in dieser Dimension eignet sich ein Vergleich von Serien im Zeitverlauf oder über Ländergrenzen hinweg, um Unterschiede und Ähnlichkeiten von Erzählmustern zu identifizieren. Ähnliches gilt für den Vergleich unterschiedlicher Genres: Wie erzählt oder verarbeitet ein Drama Politik im Gegensatz zu einer Satire?

Der vorliegende Beitrag führt diese vier Punkte im Folgenden mit Beispielen und möglichen Fragestellungen aus, um Impulse für die wissenschaftliche Auseinandersetzung mit Polit-Serien zu liefern. Dabei gilt es den von Heck und Schlag (2015) formulierten Anspruch zu berücksichtigen, dass solche Arbeiten und Studien selbst bei ihrem popkulturellen Gegenstand sorgfältig und systematisch Fragestellung, Analyserahmen und Methode entwickeln. Zugleich ist ihrer Auffassung nach in besonderer Weise eine interdisziplinäre Brille notwendig, welche ergänzend zum politikwissenschaftlichen Ausgangspunkt Erkenntnisse relevanter Fachrichtungen, wie Medien- und Filmwissenschaften oder Narrativforschung, ergänzend einbezieht.

Mit der Ausdifferenzierung der geschilderten vier Dimensionen, die im Folgenden ausführlich beschrieben werden, soll eine häufige Falle bei der politikwissenschaftlichen Auseinandersetzung mit fiktionalen Politikerzählungen vermieden werden. Viele Arbeiten verschreiben sich einem Vergleich von Realität und Fiktion und bleiben bei einer Beschreibung oder Bewertung der gefundenen Abweichungen stehen. Ein solcher Abgleich greift jedoch meist zu kurz, da Se- 
rien letztlich von dieser Abweichung leben und in ihren Erzählungen politische Themen und Inhalte neu arrangieren und ordnen. Sie sind Fiktionen (und keine Dokumentationen), auch wenn sie sich mehr oder weniger auf die Realität beziehen. Dörner (2017) weist darauf hin, dass Teil des Erfolgsrezepts einer Serie eine gewisse Wiedererkennbarkeit ist, bei einem völligen Abweichen droht durch Belanglosigkeit ein Desinteresse der Zuschauer. Das gilt selbst für Fantasy- oder Science-Fiction-Serien, die damit spielen, dass die Zuschauer sie auf sich oder aktuelle gesellschaftliche Probleme rückbeziehen (Whitehall 2003, Graeber 2015: 183ff.). Relevant ist in der hier zugrunde gelegten Perspektive nicht die Frage, ob und wie stark Serien in ihren Erzählungen von der echten Politik abweichen, sondern wofür diese Abweichung in verschiedenen Dimensionen steht, auf was sie verweist und was sich aus ihr ergibt.

\section{KONTEXT: POLITISCHES SYSTEM UND POLITIKMANAGEMENT}

In einem ersten Schritt gilt es zunächst, das tatsächliche politische System, in welches eine Serie gesetzt ist, näher zu betrachten. Es fungiert als eine Art Hintergrundfolie, auf welcher die Drehbuchschreiber und Regisseure ihre Geschichte konstruieren. Einerseits kann man dabei den Bedeutungen von Abweichungen nachgehen, andererseits aber auch fragen, welche Merkmale und Ausprägungen besonders fokussiert und hervorgehoben werden. Dabei sind zwei komplementäre Betrachtungsweisen möglich, die sich wiederum gut ergänzen: Erstens können entlang der Typologien der vergleichenden Regierungslehre (Lauth et al. 2014) verdichtete und zentrale Punkte oder Kriterien herausgestellt werden, anhand derer sich verschiedene Staatsformen gruppieren und kategorisieren lassen (z.B. parlamentarisches vs. präsidentielles Regierungssystem). Zweitens können aber anhand der umfangreichen Literatur zu einem bestimmten nationalen politischen System dessen Spezifika und Strukturmerkmale herausgearbeitet werden (für Deutschland z.B. Korte/Fröhlich 2009), so dass eine detaillierte und tiefergehende Annäherung an den Politikbetrieb eines einzelnen Landes möglich wird (z.B. die deutsche Kanzlerdemokratie).

Bei der Betrachtung von US-amerikanischen Filmen und Serien fällt auf, dass dort häufig der Präsident im Mittelpunkt steht. Die amerikanische Verfassung weist ihm die Rolle als Regierungschef und Staatsoberhaupt zu, er verkörpert mit seiner Person den Staat (Stüwe 2008: 543). Gerade seine Rolle als commander-in-chief (Oberbefehlshaber der Streitkräfte) bildet dabei oft den Ausgangspunkt für Erzählungen, wenn etwa die USA gegen Angriffe von außen 
(feindselige Staaten, Terroristen, Außerirdische) verteidigt werden müssen. ${ }^{1} \mathrm{Zu}$ sätzlich unterfüttert wird das durch die global hervorgehobene Stellung der USA, aufgrund welcher der US-Präsident oft als ,mächtigster Mann der Welt“ bezeichnet wird und seine Handlungen immer Konsequenzen für die gesamte Staatengemeinschaft haben. Die von der Verfassung vorgesehene institutionelle Einbindung und Einhegung des Präsidenten durch ein System der „,checks and balances“ (Stüwe 2008: 543) geht in den Erzählungen oft unter. Der Fokus auf die exekutive Gewalt findet sich auch bei europäischen Serien. Die zentralen Protagonisten sind häufig Regierungschefs (Yes, Prime Minister, Borgen) oder Minister als Teil der Exekutive (Yes, Minister, The Thick of It). Auch die kurzlebige ZDF-Produktion Kanzleramt folgte diesem Muster. ${ }^{2}$ Selbst wenn von der nationalen Politik abgewichen und im föderalen Mehrebenensystem beispielsweise die Kommunalpolitik fokussiert wird, steht oft das Amt des Bürgermeisters als Chef der Verwaltung und Oberhaupt der Gemeinde im Mittelpunkt (Marseille, Baron Noir, Ellerbeck).

Diese Exekutivlastigkeit von Filmen und Serien erklärt sich einerseits mit der Notwendigkeit, Erzählungen auf einige zentrale und wiedererkennbare Personen zuzuspitzen. Das Aufgreifen und Abbilden von Kollektivorganen wie Parlamenten fällt hingegen schwerer. Auf der anderen Seite interessieren sich Serienmacher gerade für Führungspositionen in Demokratien, die mit hoher öffentlicher Sichtbarkeit ausgestattet an der Spitze einer sich nach oben verdichteten Hierarchie stehen. Hier fallen Macht und Einfluss scheinbar am größten aus, hier werden die für alle Bürger weitreichendsten Entscheidungen getroffen. „Dezisionistische Situationen eignen sich vor allem für das serielle Drama.“ (Kelleter/Jahn-Sudmann 2014: 17) Schließlich erklärt diese Macht-Hierarchie, warum Akteure in den Serien wie selbstverständlich ehrgeizig immer weiter nach oben streben.

Ein Grund für den Erfolg von House of Cards resultiert möglicherweise gerade in einer ungewöhnlichen Abweichung von dieser Regel, indem - zumindest in der ersten Staffel - der Protagonist Underwood mit seiner Position weiter unten in der Hierarchie angesiedelt ist. Als Democratic Majority Whip ist er im Repräsentantenhaus für die Organisation von Mehrheiten zuständig und kommt

1 Es existiert auch eine Serie Commander in Chief, die zeitgleich mit dem Ende von The West Wing startete und in welcher nach dem Tod des amtierenden Präsidenten die Vizepräsidentin Mackenzie Spencer Allen das Amt übernimmt.

2 Als ein Grund für den ausgebliebenen Erfolg der Serie wird vorgebracht, dass diese sich gerade ohne ausreichende Reflexion der systemischen Unterschiede zu sehr an dem Vorbild The West Wing orientierte. 
damit in der formalen Rangordnung nach Speaker und Majority Leader (Stüwe 2008: 556). Auch als gewiefter Stratege und Machtmensch ist er stark in vorgegebene Prozesse und Strukturen eingebunden und muss zumindest temporäre Allianzen schließen, um seine Ziele voranzutreiben. Seine Position erlaubt es den Machern der Serie, in der ersten Staffel wiederkehrend Bezug auf die parlamentarische Entscheidungsfindung im Repräsentantenhaus zu nehmen. So wird einerseits deutlich, wie unabhängig im amerikanischen Präsidialsystem die Abgeordneten von ihrer Partei agieren, wenn etwa Underwood an einer großen Magnettafel in seinem Büro für jede Gesetzesinitiative jeweils die Stimmungen einschätzen muss. Andererseits zeigt sich die dem US-Wahlsystem innewohnende starke Rückbindung der gewählten Repräsentanten an den Wahlkreis. Beim Jonglieren der Details einer weitreichenden nationalen Bildungsreform etwa muss Underwood parallel vor Ort in seinem Wahlkreis gegen Missstimmungen bei seinen Wählern ankämpfen, die ansonsten sein Mandat gefährden könnten. Später zeigt sich analog dazu, wie er andere Abgeordnete mit Versprechen für Ressourcen oder Programme, die ihren Wahlkreisen zugutekommen, für sich einnimmt.

Ein ähnliches Bild von Politikern zwischen eigener Autonomie und systemischen Zwängen findet sich in der dänischen Serie Borgen. Der Protagonistin Birgitte Nyborg gelingt es zwar, das Amt der Premierministerin zu erreichen, wie in parlamentarischen Demokratien aber häufig der Fall muss sie dafür eine Koalition mit anderen Parteien eingehen (Switek 2013). Dieses Zweckbündnis auf Zeit setzt ihren Möglichkeiten zum Durchregieren Grenzen, Politik wird als ständiges Suchen und Finden von Kompromissen und Tauschgeschäften gezeigt. Als Idealistin mit hehren politischen Zielen gestartet, wird ihr Aufstieg im politischen System als Entwicklung zu einer kalkulierenden Machtpolitikerin geschildert, die lernt, dass Freundschaften oder Werte hinderlich für das eigene Fortkommen sind. Trotz der unterschiedlichen Hintergründe ähnelt sich damit in beiden Serien die unterschwellige Beschreibung der politischen Sphäre, in der ultimativ die am erfolgreichsten sind, die skrupellos und egoistisch ihre eigenen Interessen und Pläne verfolgen.

Schließlich verfügen die neueren Polit-Serien über eine weitere Gemeinsamkeit, indem sie vermehrt Aspekte des anwendungsorientierten Politikmanagements aufgreifen, bei dem es um die Steuerbarkeit des politischen Systems und die Steuerungsfähigkeit politischer Entscheider geht (Korte/Fröhlich 2009: 173ff.). Filme und Serien blieben lange bei einer oberflächlichen Schilderung politischer Akteure stehen, bei welcher Macht und Einfluss stark an die Personen selbst geknüpft sind. Wenige Produktionen versuchten sich an einer komplexeren Betrachtung und Darstellung politischer Akteure, wenn etwa wie in dem US- 
Spielfilm Wag the Dog die Rolle von Kommunikationsexperten und Spindoktoren hinter den Kulissen ausgeleuchtet wird, die ihren aufgrund der sexuellen Belästigung einer Minderjährigen bedrohten Präsidenten durch einen medial inszenierten Kriegseinsatz schützen wollen. Auch in der Serie The West Wing ist der Präsident Josiah Bartlet stets von seinem Kernteam umgeben, auf das er trotz seines Charismas und seiner intellektuellen Fähigkeiten angewiesen bleibt. Bei neueren Serien ist eine solche Perspektive selbstverständlicher, indem sie stets die Hinterbühne in ihre Erzählungen mit einbeziehen. Underwood hat seinen loyalen Bürochef Doug Stamper, der ihm auch die schmutzigen Aufgaben abnimmt (House of Cards); Nyborg vertraut ihrem Spindoktor und Medienexperten Kasper Juul, der in alle Prozesse eingebunden ist und dabei vor allem auf ihr mediales Bild achtet (Borgen); den Parlamentarier und Bürgermeister Philippe Rickwaert begleitet immer sein Vertrauter Cyril, der letztendlich mit seinem Chef ins Ministerbüro aufrückt (Baron Noir). Die Mitarbeiter sind Schatten ihrer Chefs, kennen ihre Geheimnisse, sind durch Nähe und Erfahrung unverzichtbare Berater und steigen mit ihnen auf wie ab. Die Serien richten ihr Scheinwerferlicht damit auf die informelle Dimension der Politik (Grunden 2009), indem sie Redenschreiber, Berater und Netzwerke thematisieren. Sie räumen mit der Illusion auf, dass Politik und Geschichte ausschließlich von großen Persönlichkeiten gemacht wird. Der Fokus liegt weniger auf Verfassungstheorie und formalen Ämtern, sondern mehr auf der politischen Praxis. Auf diese Perspektiverweiterung stützt sich wohl auch das Lob des Grünen-Politikers Jürgen Trittin, der trotz der zynischen Grundmelodie House of Cards sogar als Material für den Politikunterricht in der Schule empfiehlt: „Frank Underwood führt durch das Unterholz von Max Webers Theorie über das Wesen von Politik im Kompromiss“ (Trittin 2014).

\section{SPIEGEL: WIEDERERKENNUNG VON THEORIEN}

Bei der wohl häufigsten Verwendung von Popkultur in den Sozialwissenschaften wird diese im Sinne eines Spiegels genutzt (Neumann/Nexon 2006: 11ff.): Filme, Serien, Musik oder Romane werden herangezogen, um wissenschaftliche Konzepte oder Theorien zu illustrieren und zu verdeutlichen. Anders als bei dem gerade geschilderten systemischen Kontext, ist dieser illustrative Einsatz nicht auf Institutionen und Regelsysteme beschränkt, sondern kann ein breites Spektrum von empirischen Regelmäßigkeiten, über ad-hoc-Theorien bis zu Theorien hoher Komplexität, umfassen. In der Regel werden einzelne Elemente oder Szenen herausgegriffen, bei denen Wissenschaftler oder Lehrende eine hohe Passgenauigkeit sehen - Abweichungen sind hier weniger von Interesse. Das Ziel ist 
es nicht, offen an popkulturelle Erzeugnisse heranzugehen, um Neues zu entdecken, sondern vielmehr bekannte Zusammenhänge über eine andere Darstellungsform direkter und intuitiver zu erläutern.

Entsprechend viel Literatur liegt in diesem Bereich vor. Oft ist damit die Idee verbunden, den Zugang zur Sozialwissenschaft niedrigschwelliger zu gestalten, da Laien oder Studienanfänger mit dem Konsum popkultureller Erzeugnisse vertrauter sind und diese einfacher erfassen können. Popkultur ist umfassend in unserem Alltag präsent und bestimmt zu großen Teilen unsere Freizeit (Hamenstädt 2017). So liegen beispielsweise Einführungen in die Politikwissenschaft vor, die je nach Themenfelder Bezüge zu Filmen, Serien oder Romanen suchen. Van Belle (2012) stützt sich für sein Lehrbuch zum politischen System der USA vorrangig auf fiktionale Werke, da in diesen seiner Ansicht nach die Charaktere antreibenden Motive deutlicher herausgestellt sowie expliziter politische und soziale Dynamiken von Entscheidung, Handlung und Konsequenz präsentiert werden. Die Studierenden können somit einfacher, eindrücklicher und umfassender die komplexen Strukturen politischer Phänomene erfassen. Darüber hinaus existiert ein breites Repertoire an Bänden, die philosophische oder politik-theoretische Fragen und Ansätze anhand von Serien durchdeklinieren (z.B. Hackett 2015, Irwin et al. 2016). Der nur am Rande politische, aber immens populäre Kult-Film Big Lebowski wird genutzt, um sich der US-amerikanischen Politik und Gesellschaft anzunähern, indem beispielsweise die Charaktere auf ihre Parteiidentifikation und politische Einstellungen hin untersucht werden (Leckrone 2013). Die Webseite The Economics of Seinfeld (www.yadayadayadaecon.com) illustriert zentrale ökonomische Begriffe und Konzepte, wie Anreize, Wettbewerb oder Nullsummen-Spiele, anhand kurzer Ausschnitte der weltweit erfolgreichen Fernsehserie Seinfeld über die Alltagserlebnisse einer Gruppe von Freunden in New York.

Auch das Medium der Musik kann als Aufhänger genutzt werden, um in Lehrveranstaltungen mittels populärer Titel und Lieder aktive politische Diskussionen auszulösen und Lerneffekte zu verbessern - unter anderem, da Musik eine emotionalere Ansprache ermöglicht (Malang 2016: 230f.). Hawn (2013: 527) setzte etwa in einem Einführungskurs den Rock-Klassiker Born in the USA von Bruce Springsteen ein, den viele bei oberflächlicher Betrachtung für ein patriotisches Lied halten und bei dem erst durch die vertiefte Auseinandersetzung die gesellschaftskritische Konnotation auffällt. ${ }^{3}$

3 „Sein Erfolg veranlasste Präsident Ronald Reagan, 1984 in Wahlkampfreden Springsteen als Verkörperung desselben Patriotismus hervorzuheben, den dieser in seinen Songs als scheinheilig kritisierte. Der pathetische Sound von Born in the USA und die 
Gemeinsam ist diesen Verwendungen eine pädagogische Zielrichtung, bei der Popkultur als Gegenstand oder Instrument der Wissensvermittlung eingesetzt wird. In diesem Sinne können politische Fernsehserien in der Lehre als didaktisches Instrument genutzt werden, um theoretische Ansätze leicht verständlich und vergleichend für die Kontexte unterschiedlicher Länder und Staaten aufzuzeigen.

Neumann und Nexon (2006) weisen aber auf eine zweite Möglichkeit hin, wie der popkulturelle Spiegel sinnvoll zum Einsatz kommen kann. Die Kontrastierung zwischen Theorie und Erzählung ermöglicht das Herstellen von Analogien - der Sozialwissenschaftler betrachtet die ihm bekannte Materie in einem anderen Licht. Die Reflektion historischer Ereignisse im Spiegel der Popkultur kann die Plausibilität spezifischer Interpretationen des Geschehens unterstützen und fördern (Neumann/Nexon 2006: 12). Die fiktionale Aufarbeitung konstruiert Zusammenhänge oder Ereignisse neu und ermöglicht dadurch eine Art Zurücktreten oder distanzierte Reflektion. Schwindet durch eine fiktionale Erzählung der Wahrheitsgehalt, kann die Neuordnung zugleich unterbelichtete oder unterschätzte Faktoren sichtbar machen. Diese Art der Erkenntnis bringt der Schriftsteller Eugen Ruge eindrücklich in dem seinem Roman Cabo de Gata vorangestellten und widersprüchlich wirkenden Zitat auf den Punkt: „Die Geschichte habe ich erfunden, um zu erzählen wie es war." (Ruge 2013)

Das schließt wiederum an die Einschätzung von Neumann und Nexon (2006) an, dass die Unterschiede zwischen den Welten von Popkultur und Politik ohnehin nicht grundsätzlicher, sondern gradueller Natur sind. So versucht ein Politiker in einer Rede, politisches Geschehen abzubilden und zu repräsentieren, was letztlich genauso für fiktionale Erzählungen über Politik gilt: „Popular entertainment usually takes the form of second-order representation, in that its narratives re-present elements of social and political life through a layer of fictional representation.“ (Hervorhebung im Original; Neumann/Nexon 2006: 7) Einstellungen, Vorstellungen und Meinungen vieler Bürger sind geprägt von der Popkultur, die in der Regel in höherem Maße konsumiert wird als die Berichterstattung über Politik.

vieldeutige, häufig wiederholte Titelzeile des Refrains übertönten offenbar jegliche Autorintention in den Strophen." (Helms 2005: 33) 


\section{DATEN: AUFDECKEN VON NORMEN UND WERTEN}

Populärkultur bzw. politische Fernsehserien können umgekehrt aber auch unvoreingenommen als Belege für kollektiv geteilte Normen, Ideen, Identitäten oder Überzeugungen in Staaten, Gesellschaften oder Regionen betrachtet werden (Neumann/Nexon 2006: 13). Der Wissenschaftler nähert sich ihnen offen und induktiv. Die Serien stehen in diesem Sinne für Bedeutungszuschreibungen in einer spezifischen Gesellschaft und reflektieren gesellschaftliche Leitmotive oder Annahmen (und das möglicherweise treffender, als es eine Analyse des Eliten-Diskurses leistet). Das mag besonders bei abgeschotteten Staaten, wie es die frühe Sowjetunion war oder wie es für das heutige Nordkorea gilt, eine praktikable Annäherung an die ansonsten schwer zu fassende Sphäre des Politischen sein.

Damit ergibt sich eine gewisse Verbindung zur Rezeptionsforschung, da Reaktionen auf globalisierte popkulturelle Produkte dem Forscher beim vergleichenden Aufdecken oder Beschreiben nationaler Identitäten helfen können. Im Band von Neumann und Nexon (2006) wird das beispielsweise durch die Rezeption der weltweit erfolgreichen Harry Potter Filmreihe betrachtet, anhand dessen sich je nach Land unterschiedliche kollektive Überzeugungen zu Staat, Gesellschaft und politischer Beteiligung aufdecken lassen. In diesem Sinne ist es etwa auch ein interessanter Fingerzeig, dass House of Cards trotz der ansonsten strengen Zensur in China überhaupt zur Ausstrahlung zugelassen wurde. ${ }^{4}$ Flos (2014) verweist darauf, dass die chinesischen Zuschauer vor dem Hintergrund ihrer eigenen Erfahrungen mit Politik die Serie weniger kritisch rezipieren, da sie in vielen Punkten genau ihren Vorstellungen der Mechanismen von Politik entspricht. Über die dargestellten Intrigen, Ränke und illegalen Aktivitäten ergibt sich eine Verbindungslinie zwischen einer eigentlich auf freiem Parteienwettbewerb fußenden US-amerikanischen Demokratie und der kommunistischen Einparteienherrschaft in China.

Bei dieser Dimension gilt es, dass die Analyse einzelner Serien an ihre Grenzen stößt und der Blick daher oft über einzelne Werke hinaus erweitert wird. Man sucht umfassender nach dem Ausdruck eines gesellschaftlichen Wandels, indem beispielsweise die zunehmende Popularität ganzer Genres in den Blick genommen wird. So illustriert Graeber (2015) anhand von Superhelden-Comics, wie sehr sich in diesen eine konservativ-reaktionäre Grundmelodie äußert. Mit ihrer Rolle als Beschützer und Verteidiger der bestehenden Ordnung erhalten

4 „In totalitären Systemen war und ist Filmpolitik immer in den Dienst der Propaganda und Umerziehung im Sinne des Regimes gestellt.“ (Mai 2006: 40) 
Wandel und Neuerungen eine negative Konnotation. „Almost never do superheroes make, create, or build anything. The villains, in contrast, are relentlessly creative." (Graeber 2015: 211) Die Comic-Hefte transportieren somit unterschwellig ein moralisches Argument an ihre Haupt-Zielgruppe von Jugendlichen: Zwar mag jeder eine gewisse Sehnsucht nach Chaos und Zerstörung in sich tragen, aber ultimativ muss dieses Verlangen kontrolliert und eingehegt werden (Graeber 2015: 213). Gerade das Aufkommen und die wachsende Popularität der Superhelden-Geschichten in den USA im frühen 20. Jahrhundert parallel zum Aufstieg des Faschismus sagen etwas über diese Zeit und die Verunsicherung in der amerikanischen Gesellschaft über die richtige staatliche Ordnung und die Rechtfertigung staatlicher Gewalt aus. Es ist nicht ungewöhnlich, dass Superhelden als propagandistische Symbole für Nationalismus, Patriotismus und Ethnizität eingesetzt wurden, etwa um Eigen- und Fremdgruppen zu trennen (Costello/Worcester 2014: 86). Steinitz (2017) erläutert, wie sich in Science-Fiction-Werken als imaginierten Zukunftsentwürfen zugleich Hoffnungen und Ängste manifestieren. Die Industrialisierung und die Entwicklung moderner Technologien lösten ein „Trauma des Kontrollverlusts“ (Steinitz 2017: 13) aus, was vor allem in Verbindung mit der Paranoia während des Kalten Krieges dystopische und düstere Zukunftsvisionen bedingte. Filme über auf die Erde zusteuernde Meteoriten wirken inzwischen vielleicht weniger furchteinflößend, spiegeln aber heute noch die damaligen Ängste und Befürchtungen über eine Invasion oder eine nukleare Katastrophe (Hamenstädt 2016: 20).

Im Kern handelt es sich dabei um einen weiter gefassten soziologischen Blick. Bilder und Muster hinterlassen unterschwellig in Serien und Genres ihre Spuren, die sich in einer bestimmten Zeit und bestimmten Wertordnung bewegen (Neumann/Nexon 2006: 13; Hamenstädt 2016: 13-17). Die in einer Polit-Serie verhandelten Inhalte transportieren somit subtil, wie eine Gesellschaft sich selbst sieht.

Zugleich existieren umgekehrt durchaus intendierte und explizite Aufnahmen politischer Aussagen oder Botschaften. Bei der Konstruktion von Erzählungen zielen Schriftsteller, Drehbuchschreiber und Regisseure darauf, Botschaften oder Kritik als Teil ihrer Geschichte zu formulieren. Die viel gelobte $H B O$-Serie The Wire zeichnet am Beispiel der US-Großstadt Baltimore ein Abbild der Missstände in einer amerikanischen Großstadt im Strukturwandel (Penfold-Mounce et al. 2011). Grundiert werden die fünf Staffeln, die sich mit fast schon wissenschaftlicher Präzision sukzessive den Subsystemen einer Stadt widmen (Drogenbanden und Polizeiarbeit, Hafen und Gewerkschaften, Politik, Schule, Medien), durch ein Bild versagender Institutionen, die durch Aufbau und Zielsetzung die zentralen Probleme nicht lösen können (Mark 2008). In unterschiedlichen Varianten 
wird erzählt, wie der Kampf gegen Drogen, Drogenschmuggler und Dealer letztlich aussichtslos die gleichen Muster wiederholt und nichts an den zugrundeliegenden sozialen Problemen ändert. Generell greifen Filme und Serien häufig sozial- oder gesellschaftskritische Argumente auf, die besonders in Form einer Erzählung eindrücklicher und appellativer wirken können (Hamenstädt 2016: 25).

Damit verbunden rücken die bereits angesprochenen Produktionsbedingungen popkultureller Erzeugnisse in den Blick. In einer global vergleichenden Perspektive stehen der Unterhaltungsindustrie und den großen Studios in den USA andere Mittel zur Verfügung und ihnen liegen andere Logiken als ihren europäischen Wettbewerbern zugrunde. ,Hollywood“ verfügt über eine dominante Rolle in der globalisierten Popkultur. „Jugendliche in aller Welt lernen über das Medium Film Vorbilder, Rollenangebote oder auch nur den Jargon typisch amerikanischer Subkulturen (z.B. den von Jugendgangs oder Polizisten) kennen, an die sie sich ihr Leben lang erinnern.“ (Mai 2006: 30) In Großbritannien und Deutschland ist hingegen die Bedeutung der öffentlich-rechtlichen Akteure zu nennen, von denen viele Produktionsaufträge ausgehen. Aufgrund ihrer öffentlichen Finanzierung sind sie stärker als die privaten Medien angehalten, einen Bildungsauftrag zu verfolgen und eine umfassende politische Berichterstattung sicherzustellen. Das könnte in der Tendenz andere Erzählungen über Politik befördern, als bei den rein auf Profit ausgerichteten privaten Medien. Interessant ist darüber hinaus die Frage, inwieweit sich die Rolle der öffentlich-rechtlichen Sender als Auftraggeber auf die Ausgestaltung von Serien auswirkt. Sie verfügen in ihren Organisationen durch Nachrichtenredaktionen und erfahrene politische Journalisten über umfangreiches Wissen über politische Prozesse und Strukturen, was wiederum die Beratung bei Planung und Konzeption politischer Serien beeinflussen und zugleich prägen könnte.

\section{NARRATIVE: POLITIK ALS STOFF FÜR ERZÄHLUNGEN}

Selbst ohne den in diesem Beitrag verfolgten Schwerpunkt fiktionaler Erzählungen in Form von politischen Fernsehserien hat der Narrativ-Begriff in der Politikwissenschaft Konjunktur. Politiker fordern wiederholt eine „neue Erzählung“ für die Europäische Union, um die Zustimmung zum Integrationsprojekt zu erhöhen, oder sie diagnostizieren einem gescheiterten Wahlkampf, dass ihm das „überzeugende Narrativ“ gefehlt habe. Yildiz, Gadinger und Jarzebski (2015) versuchen, den facettenreichen Begriff systematisch für die Politikwissenschaft zu übersetzen und zu definieren sowie im Sinne einer Methode der NarrativAnalyse politischer Sprache zu fassen. Sie verweisen darauf, wie grundsätzlich unser Denken und Verstehen von Momenten des Erzählens strukturiert wird. Als 
Kernelemente einer Narrativanalyse beschreiben sie Metaphern, Rollen und Handlungsschemata (Plots), aus denen sich die Narrative zusammensetzen (Yildiz et al. 2015: 426ff.).

Den Konstitutionsbedingungen politischer Narrative kann am Beispiel von politischen Fernsehserien besonders gut nachgegangen werden, suchen sich doch Autoren und Drehbuchschreiber aus der politischen Sphäre gerade Elemente heraus, die sie für geeignet für eine interessante und fesselnde Erzählung halten. Wie wird Politik in Erzählungen eingewoben? Welche wiederkehrenden Muster zeigen sich? Die Schwierigkeit einer solchen Dekonstruktion liegt darin, dass die Grenze zu anderen Erzählstrukturen fließend ist. So verbindet die Geschichte eines alleinstehenden Präsidenten, der sich trotz aller Hindernisse in eine Frau verliebt, das Genre der Romanze mit dem des Polit-Dramas. Darüber hinaus variieren Narrative stark im Umfang: Es kann schon im Sinne eines ShakespeareDramas das überspannende Rache-Motiv eines zurückgewiesenen Frank Underwood in House of Cards sein. Zugleich kann es kleinteiliger die Illustration von der nur scheinbaren Objektivität von Zahlen und Statistiken betreffen, wenn wie in The Wire auf den Druck der Polizeispitze zur Reduktion der Kriminalitätsraten mit der Um-Etikettierung von Straffällen reagiert wird, um die gesteckten Ziele zu erreichen (Mark 2008).

Die Webseite tvtropes.org versucht sich mit Hilfe ihrer Nutzer an einer ausführlichen Sammlung aller möglichen tropes, die sie als wiederkehrende Erzählelemente in Filmen, Serien, Romanen, Comics und Spielen definiert: „A trope is a storytelling device or convention, a shortcut for describing situations the storyteller can reasonably assume the audience will recognize. Tropes are the means by which a story is told by anyone who has a story to tell" (tvtropes.org 2017a). Das ist nahe an der Beschreibung von Leitmetaphern, die Yildiz, Gadinger und Jarzebski als zentrales Element politischer Kommunikation erachten: „Durch die bildhafte Übertragung von Sinn werden selbst hochkomplexe Sachverhalt zu einem geringen Kognitionsaufwand wahrnehmbar und können damit in den kommunikativen Beziehungen auch gezielt angesprochen werden." (Yildiz et al. 2015: 426) Auf der Webseite finden sich die political tropes gesammelt auf einer Seite $^{5}$, für diesen Beitrag werden allerdings vergleichend die unter Polit-Serien gefassten Sendungen auf die in ihnen am häufigsten auftauchenden Figuren und Stilmittel hin betrachtet. ${ }^{6}$ Wie zu erwarten, zeigen sich bei diesen Serien ver-

5 http://tvtropes.org/pmwiki/pmwiki.php/Main/PoliticsTropes (zugegriffen am 3.1. 2018).

6 http://tvtropes.org/pmwiki/pmwiki.php/Main/GovernmentProcedural (zugegriffen am 3.1.2018). 
stärkt Bezugnahmen auf tatsächliche Ereignisse (Ripped From The Headlines, Real Life), teilweise mit erstaunlicher Detailtiefe. Frank Underwood lehnt in der selection campaign für den Vizepräsidenten-Platz letztlich alle Kandidaten ab, um selbst gewählt zu werden - das ähnelt dem von Dick Cheney für George W. Bush koordinierten Verfahren, an dessen Ende Cheney selbst als Kandidat feststand. An anderen Stellen werden hingegen direkte Verbindungen gelegentlich maskiert, etwa wenn eine Parteizugehörigkeit nur angedeutet oder unterschlagen wird (No Party Given). Die Identifikation der Zuschauer mit einzelnen Charakteren soll nicht durch abweichende politische Einstellungen erschwert werden oder die Drehbuchschreiber wollen explizit zu direkte politische Aussagen und Bezüge vermeiden. In verschiedenen Serien findet sich das Muster der bereits angesprochenen Wandlung vom idealpolitisch zum machtpolitisch orientierten Politiker (Dörner 2016): Ein an programmatischen Zielen ausgerichteter Charakter mit hohen moralischen Ansprüchen (Naive Newcomer) wandelt sich als Resultat seines politischen Aktivismus zu einem zynischen Machtmenschen (Sliding Scale Of Idealism vs. Cynicism). In diesem Sinne führen die Regeln des politischen Wettbewerbs zwangsläufig dazu, dass Idealisten scheitern und skrupellose Egoisten politische Führungspositionen besetzen. Das liegt wieder nah an dem Stilmittel des überbordenden Ehrgeizes (Ambition Is Evil), der häufig Protagonisten in Polit-Serien zugeordnet wird und bei dem das Motiv für das Handeln aus dem Wunsch nach Geld, Ruhm oder Macht resultiert. Das positive Gegenmodell - der Überzeugte (Determinator) - tritt hingegen seltener auf. Interessant dabei ist das Muster, welches sich auf der anderen Seite in satirisch orientierten Serien zeigt: Hier kämpft der Politiker meist nicht um den Aufstieg, sondern umgekehrt gegen den Abstieg. Als ungeschickte, inkompetente und reaktive Charaktere versuchen Politiker mühsam, ihren erreichten Status zu halten - so etwa in der Mini-Serie Eichwald, MdB des ZDF über den gleichnamigen Bundestagsabgeordneten.

Schließlich zeigt der Vergleich der Serien einige Muster, was die Erzählung über kollektive Akteure oder systemische Bedingungen angeht. Geschichten benötigen immer Charaktere, Rollen oder Protagonisten, welche die Handlung tragen. Die Polit-Serien versuchen erkennbar, hierüber hinauszugehen. The Thick of It, The West Wing, House of Cards und The Wire stützen sich beispielsweise auf Loads and Loads of Characters: „A show that has so many regulars that you can't fit them all into one episode. Therefore, one week some characters will appear, while some different characters will appear in another. You'll rarely get the same combination twice.“ (tvtropes 2017b) Der Einsatz dieses Erzählelements macht Serien lebendiger und vielschichtiger. Die Zuschauer vollziehen mehrere subjektive Blickwinkel parallel empathisch nach, wodurch ein weiter ausdeutba- 
res Geflecht an Perspektiven, Beziehungen, Vernetzungen und Hierarchien entsteht (Urschel 2013). Durch die sich wiederholende Darstellung der Reaktionen der zahlreichen individuellen Charaktere auf spezifische Zwänge und Anreize der Regelsysteme, in denen sie sich bewegen, schafft The Wire indirekt das Nachzeichnen überindividueller Institutionen und Systembedingungen (Mark 2008). Eng damit verbunden findet sich die Erzähltechnik Put On A Bus vermehrt in den Polit-Serien. Charaktere, verreisen ' mit dem Bus, das heißt, sie tauchen einige Zeit nicht auf, um später wieder in Handlungsstränge eingebunden zu werden. In The West Wing verschwindet Sam Seaborn vollständig nach seiner Wahlniederlage und taucht erst wieder in den letzten Episoden der letzten Staffel auf. Auch The Wire nutzt dieses Stilmittel für mehrere Charaktere (Nick Sobotka, The Greek, Judge Daniel Phelan, Steve Earle). Der Haupt-Charakter der Serie ist somit die Stadt Baltimore selbst. „Consequently, the characters, no matter how apparently central to the story they are, are ultimately expendable for the sake of telling Baltimore's story.“ (Penfold-Mounce et. al 2011: 157)

Ein letzter charakteristischer Punkt beim Aufgreifen von Politik durch Serien ist die Betonung von Sonderfällen oder Ausnahmesituationen. Das liegt insofern nahe, da Routinen und eingeübte Prozeduren als Selbstverständlichkeiten weniger unterhaltendes und dramaturgisches Potential bieten. So werden oft Wahlkämpfe nacherzählt, in denen es verdichtet um die Verteilung von Macht für einen längeren Zeitraum geht, so dass Wettbewerb und Intensität noch stärker ausfallen (House of Cards, Baron Noir, Veep). Oft werden tatsächliche gesetzliche oder prozedurale Sonderregelungen thematisiert, die den Zuschauer erstaunen und überraschende Wendungen ermöglichen. Ausgangspunkt der Serie Designated Survivor bildet die wohl nur wenigen bekannte und bisher nicht zum Einsatz gekommene Regel für den Fall eines umfassenden Verlusts der politischen Führung in den USA (Georgi 2017). Bei großen Veranstaltungen (z.B. Rede zur Lage der Nation oder Amtseinführung des neuen Präsidenten) befindet sich eine Person, meist ein Mitglied des Kabinetts, an einem sicheren Ort, um im Notfall als neuer Präsident die Kontinuität der Regierung sicherzustellen. Ein damit verwandtes Muster ist es, wenn Protagonisten in einer Serie sich auf eine existierende, aber in der Praxis nie oder selten genutzte Regel stützen, um sich in einem Konflikt durchzusetzen: so etwa in House of Cards, wenn die USAdministration auf Grundlage der UN General Assembly Resolution 377 ,Uniting for Peace 'versucht, gegen ein russisches Veto im Sicherheitsrat eine Friedensmission für den Nahen Osten durchzusetzen (Odermatt 2015). In Baron Noir droht Philippe Rickwaert seiner Konkurrentin Amélie Dorendeu, ihre Wahl als Parteivorsitzende auf einem Parteitag zu verhindern, da er eine Missachtung von Übergangsfristen herausgefunden hat. 
Eine gegenläufige Besonderheit findet sich wiederum bei der Serie The Wire, wo die Autoren auf die Abbildung von Routinen und Organisationsalltag abzielen. Gerade durch deren wirkungslose Wiederholungen demonstrieren sie eine Aussichtslosigkeit und Fehlausrichtung der Ziele. Zugleich wird geschickt die Darstellung standarisierter Abläufe (als systemischer Zwänge) in Organisationen genutzt, um den verbleibenden Spielraum individueller Akteure aufzuzeigen, denen ultimativ immer eine gewisse Freiheit im Handeln verbleibt (Crozier/Friedberg 1993). In jeder Organisation stehen die Gesamtziele in einem Spannungsverhältnis zu den internen Spielen der Organisationsakteure. „Even well-intentioned cops and teachers are forced to play bureaucratic games in order to survive in their organisations." (Mark 2008)

\section{FAZIT: UNTERHALTSAME POLITIK}

Der vorliegende Beitrag zielte darauf, ausgehend von einer Perspektive der Systemforschung und Regierungslehre unterschiedliche Perspektiven für die Auseinandersetzungen und Analyse fiktional erzählter Politik in Form von Fernsehserien aufzuzeigen. Damit wird ein Forschungsfeld abgesteckt, in welchem sich politikwissenschaftliche Arbeiten und Studien zu Politikserien sinnvoll bewegen können. Eine konkrete Serie kann somit auf das in ihr präsentierte politische System hin inspiziert, auf ihre Tauglichkeit für die Illustration politikwissenschaftlicher Theorien hin abgeklopft, hinsichtlich der in ihr vorhandenen Vorstellungen über Politik und Gesellschaft dekonstruiert sowie bezüglich der in ihr verwendeten Mechanismen der Konstruktion von erzählter Politik betrachtet werden. Die vier Punkte können detailliert Grundlage einer Fallstudie sein, oder eine solche fokussiert nur ein oder zwei dieser Dimensionen angehen. Zugleich ermöglichen die Punkte einen strukturierten Vergleich. Mehrere Serien, etwa im Zeitverlauf oder aus verschiedenen Ländern, können im Hinblick auf eine der Dimensionen kontrastiert werden, so dass ein Wandel über Zeit oder charakteristische Unterschiede zwischen Kulturen sichtbar werden. In jedem Fall gilt, dass solche Arbeiten gegenstandsbezogen jeweils ihre Perspektive, ihren Analyserahmen sowie ihre systematische Vorgehensweise darlegen müssen (Heck/ Schlag 2015). Die in diesem Text aufgeführten Beispiele waren vorrangig sprach- und textorientiert, genauso ließe sich aber durch Rückgriff auf Methoden anderer Disziplinen Elemente der Visualität oder des medialen Formats thematisieren.

Der vorliegende Band versammelt Aufsätze, die sich alle mehr oder weniger den in diesem Beitrag skizzierten Perspektiven und Fragen widmen. Er gliedert sich dabei in drei Blöcke: In einem ersten Teil beschäftigen sich die Autoren 
ähnlich der hier dargelegten Ausführungen damit, wie man sich wissenschaftlich-systematisch mit Polit-Serien als Teil der Popkultur auseinandersetzen kann. Wie gestaltet sich der Forschungsstand? Was macht das Politische in den Serien aus? Welche Perspektiven, Fragestellungen und Methoden existieren, um sich dem Politischen in Fernsehserien anzunähern? Der zweite Teil umfasst Fallstudien unterschiedlicher Polit-Serien. Dabei wurde darauf geachtet, sowohl zeitlich wie geographisch eine gewisse Varianz der behandelten Serien sicherzustellen. Die Mehrzahl der Texte orientiert sich dabei in der Analyse der Serien-Inhalte an den hier präsentierten Dimensionen. Dadurch ergibt sich eine Vergleichsmöglichkeit zwischen den Beiträgen, was zum Beispiel die in ihnen transportierten kulturellen Werte und Normen oder die narrativen Mechanismen der Transformation von Politik in Geschichten angeht. Es finden sich aber auch Abweichungen, wenn etwa für House of Cards die Wirkung der präsentierten Bilder von Politik auf die Zuschauer untersucht wird. Schließlich reflektiert ein dritter Teil die bislang immer noch unterentwickelte deutsche Serienlandschaft und geht gezielt den Möglichkeiten des didaktischen Einsatzes von Filmen und Serien in der universitären Lehre nach.

Grundsätzlich kann es den Politikwissenschaftler nur freuen, dass gerade die Elemente des Politischen, die ihn höchstwahrscheinlich zu seinem Untersuchungsobjekt gebracht haben, nun in Erzählungen in Serien verpackt eine breitere Öffentlichkeit erreichen. Über den unterhaltenden Wert hinaus hat dieser Beitrag versucht aufzuzeigen, inwieweit die Sozial- bzw. Politikwissenschaften den Trend zu politischen Fernsehserien systematisch und vergleichend nutzen können. In jedem Fall bleibt zu hoffen, dass Polit-Serien weiter im Trend bleiben, sodass der Fundus an wissenschaftlichen Studien und Arbeiten weiter wachsen kann. Zuschauer wie Wissenschaftler sind sicher beide gleich dankbar.

\section{LITERATUR}

Costello, Matthew J./Worcester, Kent (2014): The Politics of the Superhero, in: PS 47 (1), S. 85-89.

Crozier, Michel/Friedberg, Erhard (1993): Die Zwänge kollektiven Handelns. Über Macht und Organisation, Frankfurt a.M.

Dörner, Andreas (2016): Politserien: Unterhaltsame Blicke auf die Hinterbühne der Politik, in: Aus Politik und Zeitgeschichte 66 (51), S. 4-11.

Dörner, Andreas (2017): Machthungrige Schurken, in: tv diskurs (2), S. 54-59. Flos, Felix (2014): „Eine Katze, die Mäuse fängt, ist eine gute Katze.“ Warum House of Cards in China erfolgreich ist, in: INDES 3 (4), S. 89-99. 
Gadinger, Frank/Yildiz, Taylan (2017): Politik, in: Martínez, Matías (Hrsg.): Erzählen. Ein interdisziplinäres Handbuch, Stuttgart, S. 158-165.

Georgi, Oliver (2017): Der Notfall-Präsident, in: faz.net 20.1., http://www.faz. net/aktuell/politik/trumps-praesidentschaft/designated-survivor-notfall-praesi denten-gibt-es-tatsaechlich-14696072.html (zugegriffen am 3.1.2018).

Graeber, David (2015): The Utopia of Rules. On Technology, Stupidity, and the Secret Joys of Bureaucracy, Brooklyn/London.

Grunden, Timo (2009): Politikberatung im Innenhof der Macht. Einfluss und Funktion der persönlichen Berater deutscher Ministerpräsidenten, Wiesbaden.

Hackett, J. Edward (Hrsg.) (2015): House of Cards and Philosophy. Underwood's Republic, Chichester.

Hamenstädt, Ulrich (2016): Politik und Film. Ein Überblick, Wiesbaden.

Hamenstädt, Ulrich (2017): Behind the Scenes? Fünf Thesen zum Einsatz von Filmen und Serien in der politikwissenschaftlichen Lehre, in: Zeitschrift für Politikwissenschaft 27 (3), S. 397-407.

Hawn, Heather (2013): Utilising Popular Music to Teach Introductory and General Education Political Science Classes, in: European Political Science 12, S. 522-534.

Heck, Axel/Schlag, Gabi (2015): „And... Cut!“, in: Zeitschrift für Internationale Beziehungen 22 (2), S. 125-148.

Helms, Dietrich (2005): Pop Star Wars, in: Aus Politik und Zeitgeschichte (11), S. 28-34.

Irwin, William/Conard, Mark/Skoble, Aeon (2016): Die Simpsons und die Philosophie: Schlauer werden mit der berühmtesten Fernsehfamilie der Welt, Stuttgart.

Jarzebski, Sebastian (2015): Wahlkampf als Erzählung: Metaphern und Narrative im TV-Duell, in: Korte, Karl-Rudolf (Hrsg.): Die Bundestagswahl 2013. Analysen der Wahl-, Parteien-, Kommunikations- und Regierungsforschung, Wiesbaden, S. 367-385.

Kelleter, Frank (2012): Serien als Stresstest, in: Frankfurter Allgemeine Zeitung vom 4.2., S. 31.

Kelleter, Frank/Jahn-Sudmann, Andreas (2014): „Eine interessante Affinität zwischen dem seriellen Erzählen und dem Thema Politik." Von Soap Operas zum Quality TV, in: INDES 3 (4), S. 5-22.

Korte, Karl-Rudolf/Fröhlich, Manuel (2009): Politik und Regieren in Deutschland: Strukturen, Prozesse, Entscheidungen, Paderborn.

Lauth, Hans-Joachim/Pickel, Gert/Pickel, Susanne (2014): Vergleich politischer Systeme, Paderborn. 
Leckrone, J. Wesley (2013): Hippies, Feminists and Neocons: Using The Big Lebowski to Find the Political in the Nonpolitical, in: PS: Political Science \& Politics 46 (1), S. 129-136.

Mai, Manfred (2006): Künstlerische Autonomie und soziokulturelle Einbindung. Das Verhältnis von Film und Gesellschaft, in: Mai, Manfred/Winter, Rainer (Hrsg.): Das Kino der Gesellschaft - die Gesellschaft des Kinos. Interdisziplinäre Positionen, Analysen und Zugänge, Köln, S. 24-47.

Malang, Thomas (2016): Die politische Dimension von Popmusik: Theoretische Zugänge, empirische Befunde und Potenzial der politikwissenschaftlichen Analyse, in: Zeitschrift für Politikwissenschaft 26 (2), S. 229-240.

Mark, Clif (2008): „All in the Game“: HBO's The Wire, Oxonian Review 7.2, http://www.oxonianreview.org/wp/\%E2\%80\%9Call-in-the-game\%E2\%80\% 9D-hbo\%E2\%80\%99s-the-wire/ (zugegriffen am 30.12.2017).

Neumann, Iver/Nexon, Daniel (2006): Harry Potter and the Study of World Politics, in: Nexon, Daniel/Neumann, Iver (Hrsg.): Harry Potter and International Relations, Lanham, S. 1-25.

Odermatt, Jed (2015): House of Cards (Season 3): International Law and American Power, Centre de Droit International, Université Libre de Bruxelles, 21. Oktober, http://cdi.ulb.ac.be/house-of-cards-season-3-international-law-andamerican-power-a-review-by-jed-odermatt/ (zugegriffen am 3.1.2018).

Penfold-Mounce, Ruth/Beer, David/Burrows, Roger (2011): The Wire as Social Science-Fiction?, in: Sociology 45 (1), S. 152-167.

Ruge, Eugen (2013): Cabo de Gata, Reinbek bei Hamburg.

Steinitz, David (2017): Zurück in die Zukunft, in: Süddeutsche Zeitung vom 30./31.12., S. 13.

Stüwe Klaus (2008): Das politische System der USA, in: Stüwe Klaus/Rinke Stefan (Hrsg.): Die politischen Systeme in Nord-und Lateinamerika, Wiesbaden.

Switek, Niko (2013): Koalitionsregierungen. Kooperation unter Konkurrenten, in: Korte, Karl-Rudolf/Grunden, Timo (Hrsg.): Handbuch Regierungsforschung, Wiesbaden, S. 277-286.

Trittin, Jürgen (2014): Wer das Feuer liebt, in: Der Freitag, Ausgabe 2, online unter: https://www.freitag.de/autoren/der-freitag/wer-das-feuer-liebt (zugegriffen am 28.12.2017).

tvtropes.org (2017a): Tropes, http://tvtropes.org/pmwiki/pmwiki.php/Main/Tropes (zugegriffen am 28.12.2017).

tvtropes.org (2017b): Loads and Loads of Characters, http://tvtropes.org/pmwiki/ pmwiki.php/Main/LoadsAndLoadsOfCharacters (zugegriffen am 28.12.2017). Urschel, Martin (2013): The Wire. Netzwerke der Gewalt, Baden-Baden. 
Van Belle, Douglas A. (2012): A Novel Approach to Politics: Introducing Political Science through Books, Movies and Popular Culture, Los Angeles.

Whitehall, Geoffrey (2003): The Problem of the „World and Beyond“: Encountering ,the Other“ in Science Fiction, in: Weldes, Jutta (Hrsg.): To Seek out New Worlds, New York, S. 169-194.

Yildiz, Taylan/Gadinger, Frank/Jarzebski, Sebastian (2015): Das narrative Element des Politischen: Überlegungen zu einer Poetologie des Wissens in der Politikwissenschaft, in: Zeitschrift für Politikwissenschaft 25 (3), S. 421431. 



\title{
Polit-Serien im Fernsehen
}

\author{
Gegenstandsbestimmung, Stand der Forschung \\ und neue Perspektiven
}

Andreas Dörner, Stefan Heinrich Simond

\section{EINLEITUNG}

Polit-Serien erleben derzeit im internationalen Fernsehen eine ausgeprägte Konjunktur. Vor allem in den USA gibt es kaum einen bedeutsamen TV-Anbieter, der keine eigene politische Serie produziert oder in den letzten Jahren gesendet hat. Dabei werden einerseits zahlreiche US-Produktionen in europäische Medienkulturen exportiert und andererseits dienen europäische Produktionen als Inspirationsquellen für US-amerikanische Formate. Der internationale Erfolg von Polit-Serien wie The West Wing und House of Cards deutet darauf hin, dass Produzenten wie Rezipienten die seriell-fiktionale Konstruktion politischer Welten und Akteure für besonders relevant und/oder erfolgversprechend halten.

Der folgende Beitrag möchte das Forschungsfeld fiktionaler Konstruktionen des Politischen im Format von Fernsehserien knapp umreißen. Dazu wird zunächst eine kurze Gegenstandsbestimmung formuliert, um im zweiten Schritt die einschlägige Forschungsdiskussion im deutsch- und englischsprachigen Raum darzustellen. Der dritte Teil entwirft dann ein interdisziplinäres Forschungsprogramm, das die bisher vorliegenden Perspektiven auf innovative Weise erweitern kann. 


\section{GEGENSTANDSBESTIMMUNG: WAS SIND POLIT-SERIEN?}

Eine Polit-Serie ist eine in Episoden und Staffeln unterteilte, fiktionale und audiovisuelle Erzählung über politische Wirklichkeiten. ${ }^{1}$ Mit ,politischen Wirklichkeiten“ sind dabei zunächst politische Zusammenhänge im engeren Sinne gemeint: Geschehnisse, die mit politischen Institutionen und Akteuren wie Regierungen und Parlamenten, Präsidenten, Parteien und politischen Journalisten, Geheimdiensten und Spionage zu tun haben. Teilweise wird bei dem Begriff ,Polit-Serie“ auch ein weiterer Politikbegriff zugrunde gelegt, sodass Serien, die sich mit Macht und Herrschaft sowie mit Kämpfen um Anerkennung befassen, ebenfalls einbezogen werden. In diesem Sinne können Produktionen wie die Lindenstraße und Game of Thrones ebenfalls als Polit-Serie bezeichnet werden.

Der kommunikative Geltungsanspruch einer Serie ist ein anderer als der eines Berichts oder einer Reportage. Die Erzählung ist gerahmt wie eine Als-obWelt, vergleichbar einem Spiel, bei dem alle wissen, dass es eine zur Alltagswelt differente Wirklichkeit konstruiert (Huizinga 1938/2004). Und dennoch bleibt sie stets auf die Alltagswelt bezogen. Gerade dieser Rückbezug, der ständige Vergleich zwischen fiktionaler Welt und außermedialer Alltagswelt begründet die Relevanz der erfundenen Wirklichkeit einer Serie. ${ }^{2}$

Entscheidend ist, dass Polit-Serien keine Dokumentationen oder Teile der journalistischen Berichterstattung sind, sondern Unterhaltungsformate. Somit sind sie der Verpflichtung auf Faktizität entbunden. Stattdessen können sie sich, Chuck Tryon (2016) zufolge, darauf konzentrieren, kohärente Erzählungen zu vermitteln, deren sinnstiftendes Potenzial in dem Maße bedeutsamer ist, in dem die journalistische Berichterstattung von der Bevölkerung als fragmentiert wahrgenommen und deren Wahrheitsgehalt in Zweifel gezogen wird. Polit-Serien müssen, um den dramaturgischen Anforderungen gerecht zu werden, verkürzen, verdichten, zuspitzen und beschleunigen. Alles passiert schneller, einfacher, eindeutiger als in der alltäglichen Wirklichkeit. Sie wollen mit dramaturgischen Mitteln unterhaltsame Spannungsbögen oder amüsante Situationskomik produzieren - und sie dürfen doch bei aller Zuspitzung den Kontakt zur außermedialen Wirklichkeit nicht verlieren, sonst erscheinen sie belanglos. Die Balance zwischen Unterhaltungswert und Realitätsbezug ist der Drahtseilakt, den politische Serien leisten müssen, um Erfolg zu haben.

1 Zur Definition, Struktur und Funktionsweise der Polit-Serie siehe Dörner (2016).

$2 \mathrm{Zu}$ dieser grundlegenden Logik der fiktionalen Unterhaltungswelten und ihrer Bezüge zur außermedialen Realität siehe Luhmann/Kieserling (2010: 112). 
Warum sind die Serien nicht nur in den USA, sondern auch in vielen europäischen Ländern so erfolgreich? Für das Publikum besteht die große Faszination politischer Serien in dem Versprechen, einen sonst in der medialen Berichterstattung unmöglichen Blick auf die Hinterbühne ${ }^{3}$ des politischen Betriebs erheischen zu können. Die Zuschauer sind dabei, wenn geheime Absprachen getroffen und Intrigen geschmiedet werden. Sie blicken den Protagonisten über die Schulter bei der politischen Arbeit. Sie erfahren sogar die geheimsten Gedanken der Akteure, wenn diese mit sich selbst sprechen, mit der Ehefrau intim eine Zigarette am Fenster teilen oder sich in vertrauensvollem Ton direkt an das Publikum wenden. Mit solchen Direktadressierungen produziert etwa die amerikanische Serie House of Cards eine Komplizenschaft zwischen dem skrupellosen Antihelden Frank Underwood und den Zuschauern, die durch die direkte Ansprache immer wieder ins gemeinsame Boot geholt werden.

Dabei wird den fiktionalen Erzählungen durch Bezüge zur außermedialen Welt eine besondere Authentizität verliehen. Die Serien erhalten auf diese Weise Realitätsindikatoren, wenn etwa reale politische Akteure in Gastrollen auftreten oder reale Geschehnisse thematisiert werden. Oftmals sind die fiktionalen Figuren an Persönlichkeiten des öffentlichen Lebens angelehnt wie beispielsweise die Hauptfigur der Serie Scandal, Olivia Pope, an die echte Kommunikationsberaterin Judy Smith. Smith ist in der Serienproduktion zugleich als beratende Expertin tätig und bringt so gleichsam Hinterbühnenwissen in die fiktionalen Konstruktionen des Politischen ein. Eine solche Einbindung von Fachleuten und Insidern ist in der Serienproduktion durchaus an der Tagesordnung. Dergestalt findet ein Wissenstransfer von der außermedialen Politik in die fiktionalen Serien hinein statt.

\section{EIN BLICK AUF DEN FORSCHUNGSSTAND}

In der deutschen und europäischen Kommunikationsforschung bildeten fiktionale TV-Formate über lange Zeit hinweg keinen relevanten Gegenstand. Die große Ausnahme waren, etwas quer zu den traditionellen Fächergrenzen stehend, die British Cultural Studies, die schon früh die Relevanz der Unterhaltungskultur für gesellschaftliche Kommunikationsprozesse erkannten und dann auch gezielt fiktionale Genres als Formen politischer Kommunikation fokussierten. ${ }^{4}$ In den

3 Zur Begrifflichkeit von Vorder- und Hinterbühne siehe den Klassiker von Erving Goffman (1959/2013).

4 Exemplarisch etwa Fiske (1996) und Kellner (1995). 
USA dagegen hat die Analyse fiktionaler Medienunterhaltung als Form politischer Kommunikation eine längere Tradition, da in den Vereinigten Staaten die Verflechtung von Unterhaltungskultur und Politik schon frühzeitig beobachtbar war. Schon der finanziell erfolgreichste Spielfilm der Stummfilmzeit war kein Liebesmelodram, sondern mit The Birth of a Nation (1915) ein Epos über Bürgerkrieg und nationale Einigung. Bis heute gehören Präsidentenfilme im Kino und politische Satireformate im Fernsehen zur reichweitenstarken kommunikativen Normalität; Wahlkampfmanager greifen folgerichtig immer wieder auf unterhaltungskulturelle Instrumente bei der Gestaltung ihrer Kampagnen zurück. Während diese Entwicklung sich auch in der US-amerikanischen Forschung niederschlägt (siehe dazu ausführlich Dörner 2000), wendete sich die politische Kommunikationsforschung in Europa erst mit der Diskussion über die , amerikanisierten' Formen der Wahlkampfführung in den 1990er Jahren dem Phänomen der politischen Unterhaltungskultur genauer zu. In Deutschland begannen einzelne Publikationen, einen Prozess der „Entertainisierung“ (Holtz-Bacha 2000) bzw. eines zunehmenden „Politainments“ zu diagnostizieren. ${ }^{5}$ Ulrich Saxer (2007) versuchte einzelne Befunde systematisch zu verorten und in den Prozess der Theoriebildung aufzunehmen. Allmählich haben dann auch quantitativempirisch arbeitende Forscher unterhaltende TV-Formate stärker fokussiert (siehe dazu vor allem die Beiträge in Dohle/Vowe 2014 und die weiteren Ausführungen unten).

\section{Politische Serienforschung: quantitative Zugänge}

Um die Forschungsbemühungen unterschiedlicher Disziplinen in Beziehung zueinander zu setzen, wurden verschiedene Ansätze einer Typologisierung des Feldes entwickelt. ${ }^{6}$ Dabei erscheint der Entwurf von Nitsch/Eilders (2014) besonders überzeugend. Sie bestimmen die Dimensionen Politikgehalt (hoch/ niedrig) und Realitätsnähe (hoch/niedrig) als ausschlaggebende Achsen zur Typologisierung fiktionaler Serien. Die Transparenz des Vorgehens erlaubt zudem eine flexible Modifikation der zugrundeliegenden Indikatoren. Jenseits der typologischen Bemühungen beschäftigen sich die inhaltsanalytischen Ansätze etwa mit Rollentypen des amerikanischen Präsidenten (Holbert u.a. 2005), mit negativen Zuschreibungen an die fiktionalen politischen Akteure (Lichter u.a. 2000, van Zoonen 2012) und mit genrespezifischen Clustern, die entlang der Dimen-

5 Siehe Dörner (2001), Diehl (2011) und Stenz (2015).

6 Siehe Holbert (2005), Christensen/Haas (2005), Schwer/Brosius (2008), Friedrich (2011), Kleinen-von Königslöw (2013). 
sionen Politikgehalt und Realitätsnähe aufgespannt sind (Eilders/Nitsch 2015). Auch für komparative, transnationale Untersuchungen kommen quantitative Inhaltsanalysen zum Einsatz. Nitsch/Eilders (2015) stellen etwa im Vergleich von The West Wing und Kanzleramt heraus, dass die Unterschiede der nationalen politischen Strukturen sich kaum in den fiktionalen Politikserien niederschlagen, während Jandura u.a. (2016) die Darstellung parlamentarischer Arbeit im politischen Magazin berlin direkt und der fiktionalen Politserie Borgen vergleichen.

Im Laufe der 2000er-Jahre gewann auch die Rezeptionsdimension fiktionaler Unterhaltungsformate an Relevanz für die quantitative politische Kommunikationsforschung (Holbert 2005: 437). Viele Studien befassen sich mit Wirkungseffekten wie Priming, Framing und Agenda Setting. Vorwiegend arbeiten sie mit einem experimentellen Design samt eigener Stichprobe; Holbrook/Hill (2005) beziehen überdies eine Sekundärauswertung der National Election Study von 1995 mit ein. Zudem liegt den Untersuchungen oftmals die Annahme zugrunde, dass fiktionale Erzählungen ein besonderes persuasives Potenzial entfalten nicht obwohl, sondern gerade weil sie primär zum Zweck der Unterhaltung rezipiert werden (Hastall u.a. 2014: 295).

In einer der ersten Studien zur Wirkung fiktionaler Polit-Serien konnten für The West Wing Priming-Effekte nachgewiesen werden. Zuschauer der Serie bewerteten die Charaktereigenschaften des damals amtierenden US-Präsidenten George W. Bush deutlich positiver als die Kontrollgruppe (Holbert u.a. 2003: 436). Zu einem ähnlichen Ergebnis kommen Moy/Pfau (2010), die eine Verbindung zwischen der Rezeption fiktionaler Polit-Serien und dem Bürgervertrauen in politische Institutionen nachweisen, sowie Nitsch/Wünsch (2016), die in einem Prolonged Exposure-Experiment eine geringere Politikverdrossenheit bei den Zuschauern feststellen. Auch konnten Wünsch u.a. (2012) zeigen, dass Zuschauer der Lindenstraße eine größere Neigung äußerten, die Linkspartei zu wählen. Aufgrund einer Online-Befragung konstatiert Annekatrin Bock (2014: 31) hingegen negative Effekte des Serienkonsums auf das Politik- und Politikerbild bei Zuschauern von House of Cards. Morris und Evans (2014) bestätigen diesen Befund, indem sie zeigen, dass Zuschauer der Serie im Vergleich mit der Kontrollgruppe Rücksichtslosigkeit und Manipulation für politisches Handeln als relevanter betrachten. Andere Studien thematisieren das Agenda Setting, können hier jedoch keine signifikanten Effekte verzeichnen (Wünsch/Czichon 2016).

Auch nationale Sicherheitsdramen wie 24 können einen Einfluss auf die $\mathrm{Zu}$ schauer entfalten, etwa wenn es um deren Meinung zur Legitimität von Folter und Todesstrafe (Mutz/Nir 2010) oder um das Faktenwissen und den Willen zur Kooperation im Falle bioterroristischer Anschläge geht (Arendt/Rössler 2014). 
Unter welchen Bedingungen genau dergleichen Effekte zustande kommen und wie nachhaltig sie sind, ist jedoch nur bedingt geklärt. Auffällig ist in jedem Fall, dass in den Studien zur Medienwirkung die konkreten Inhalte der Sendungen, vor allem aber die Erzählweise und Ästhetik kaum berücksichtigt werden. ${ }^{7}$

\section{Qualitative Analysen}

Anfänge einer qualitativen Serienanalyse sind aus kulturwissenschaftlichen, insbesondere amerikanistischen Kontexten entstanden, wo man sich mit neueren Entwicklungen der TV-Serie beschäftigt (Kelleter 2012, 2014, 2017) und dabei auch Fallstudien zu einzelnen politischen Serienprodukten bietet. ${ }^{8}$ Diese Arbeiten weisen ein starkes Interesse an den ästhetischen Formen der Serien auf und thematisieren darüber hinaus auch Fragen der Konstruktion politischer Bilder. Letzteres wiederum steht im Zentrum der Analysen, die in einer interdisziplinären Arbeitsgruppe um Mark Arenhövel, Anja Besand und Olaf Sanders an der TU Dresden entwickelt wurden. Hier kooperieren sozial- wie kulturwissenschaftliche Forscher, um das gesellschaftliche Potenzial von Serienproduktionen offenzulegen. Interessanterweise hat man sich dabei jedoch bislang auf die Analyse von Serienproduktionen konzentriert, die ,politisch ' nur im weiteren Sinne dimensioniert sind wie Breaking Bad oder True Detective. ${ }^{9}$

Der Großteil qualitativer Inhaltsanalysen ${ }^{10}$ von Polit-Serien befasst sich international mit der Repräsentation politischer Prozesse. Insbesondere die Serie The West Wing erfährt dabei große Aufmerksamkeit als idealistisches Politikbild. ${ }^{11}$ Für House of Cards hingegen liegt der analytische Fokus überwiegend auf der Darstellung machtpolitischen Handelns. ${ }^{12}$ Der Figurenzeichnung des US-

7 Während Rezeptionsstudien mit qualitativem Fokus zu Polit-Serien bislang wenig verbreitet sind (Wolling 2014), finden sich vereinzelt Untersuchungen zur Anschlusskommunikation (Williams 2011) und zur transnationalen Rezeption (Bochner/Thomas 2007, Bacqué/Flamand 2016).

8 Siehe etwa Kaklamanidou/Tally (2017), Däwes u.a. (2015), Nesselhauf/Schleich (2015) und Kiegeland u.a. (2014).

9 Siehe Sanders u.a. (2016), Arenhövel u.a. (2017) sowie Besand u.a. (2017).

10 Die Literaturrecherche ergab eine eindrucksvolle Anzahl und Vielfalt qualitativer Inhaltsanalysen. Aus Platzgründen erschöpfen die nachfolgenden Referenzen daher nicht das Forschungsfeld, sondern stellen lediglich eine Auswahl typischer Zugangsweisen dar.

11 Siehe Lörke (2014), Rothöhler (2012), Wodak (2009), Morsch (2010), Rollins (2003).

12 Siehe Kirchmeier (2016), Schubert (2017), Hackett (2015), Lim (2014). 
amerikanischen Präsidenten, seiner Rolle im politischen Gefüge und seiner Körperlichkeit kommt dabei besondere Aufmerksamkeit $\mathrm{zu} .{ }^{13} \mathrm{Zu}$ The Wire finden sich überdies qualitative Inhaltsanalysen, welche die lokalpolitische Ebene von Baltimore beleuchten. ${ }^{14}$

Ein augenfälliger Schwerpunkt ist überdies die Auseinandersetzung mit dem internationalen Terrorismus. Serien wie 24 und Homeland sind Gegenstand von Inhaltsanalysen, die sich mit der Darstellung von Folter, Ausnahmezuständen, Paranoia und Kriegstraumata beschäftigen. ${ }^{15}$ Dazu gehört ebenfalls die Sonderfolge ,Isaac \& Ishmael ' von The West Wing, ausgestrahlt kurz nach 9/11. ${ }^{16}$

Die Genderdimension bildet einen weiteren analytischen Schwerpunkt. Serien wie Commander in Chief, The Amazing Mrs. Pritchard, Scandal und Veep sind - neben den bereits genannten Produktionen - Gegenstand zahlreicher feministischer und intersektionaler Inhaltsanalysen. Besonderes Augenmerk liegt dabei auf der Reproduktion von Geschlechterstereotypen und dem Potenzial, eine weibliche Präsidentin denkbar zu machen. ${ }^{17}$ Der politische Prozess wird überdies im Kontext anderer institutioneller Bereiche thematisiert. ${ }^{18}$

Immer wieder berührt sich der Diskurs über politische Serien auch mit der internationalen Debatte über , Quality TV‘. Dabei geht es um ein normativ eingefärbtes analytisches Konzept zur Analyse von TV-Serien, das insbesondere an neuere, komplex angelegte und hochwertig produzierte Serienprodukte herangetragen wird (Feuer u.a. 1984). Robert J. Thompson (1997) hat schon vergleichsweise früh einen Katalog von zwölf Kriterien für Quality TV benannt, der unter anderem stilistische Prägnanz, Komplexität, Authentizität und Selbstreflexivität umfasst. Weitere Aspekte wie eine operationale Ästhetik, der selbstbewusste Umgang mit Formen der Episoden- und Fortsetzungsserie sowie ein hoher Anspruch an die Rezeptionsleistung von Zuschauern wurden ebenfalls beschrieben. $^{19}$

13 Siehe Balke (2015), Hermann (2015), Celikates/Rothöhler (2007), Parry-Giles/ParryGiles (2006).

14 Siehe Painter (2017), Ahrens u.a. (2014), Kennedy/Shapiro (2012).

15 Siehe Dunst (2016), Peacock (2007), Morsch (2014), Miniter/Wilson (2008).

16 Siehe Holland (2011), Wodak (2010), Freda (2010).

17 Siehe Loidolt (2016), Sheeler/Anderson (2013), van Zoonen (2003).

18 Für die Schnittstelle zum Journalismus siehe exemplarisch Vint (2013), Eschkötter (2012), Skewes (2009).

19 Siehe Mittell (2012), Nesselhauf/Schleich (2016) und Schlütz (2016); zur Kritik des Begriffs und der Debatte siehe Schneider (2017) und Borsos (2017). 
Überdies sind oftmals Produktionsanalysen mit qualitativen Inhaltsanalysen verknüpft, besonders bei jenen Serien, an deren Produktion renommierte Autoren und Regisseure beteiligt sind. Der biographische Hintergrund von Schlüsselfiguren wie Aaron Sorkin (The West Wing), David Simon (The Wire) und Beau Willimon (House of Cards) ist oftmals als Grund und Zeugnis für die Authentizität der fiktionalen Serien angeführt. Entsprechend findet sich der Zusammenhang zwischen Autorenbiographie, politischer Agenda und Serientext in zahlreichen Analysen betont. ${ }^{20}$ Darüber hinaus werden der Produktions- und Distributionskontext als relevante Faktoren beschrieben. Markt- und Eigentümerstruktur, technische Innovationen und ökonomische Kalküle beeinflussen demnach auch, welche Serien produziert werden.

Insgesamt besteht allerdings eine erhebliche Forschungslücke: Es fehlen vergleichend angelegte qualitative Studien, die Semantik und Ästhetik von Serien untersuchen und dabei die Dimensionen von Produktion, Text und Rezeption im Zusammenhang analysieren. Hier möchte der nachfolgende Entwurf Pionierarbeit leisten. Dabei werden politische Semantik und Ästhetik systematisch aufeinander bezogen, um herauszuarbeiten, inwiefern die Gestaltungsmittel zur Konstruktion von Politikbildern beitragen, ihnen Evidenz verleihen und sie für politisch-kulturell gegebene Wert- und Vorstellungsmuster anschlussfähig machen. Dabei kann an Grundüberlegungen zur politischen Ästhetik angeknüpft werden $^{21}$, aber der spezifische Bezug auf moderne Bewegtbild-Ästhetik ist im Wesentlichen noch zu leisten.

\section{EINE FORSCHUNGSPERSPEKTIVE: POLIT-SERIEN UND POLITISCHE KULTUR IM INTERNATIONALEN VERGLEICH}

Polit-Serien stellen einen komplexen Forschungsgegenstand dar, der semantisch und ästhetisch dimensioniert ist und der enge Bezüge zu den politischkulturellen Kontexten aufweist, in denen die Serien produziert und rezipiert werden. Im Folgenden soll in der gebotenen Kürze ein Forschungszugang skizziert werden, der dem komplexen Gegenstand gerecht wird. Er soll die narrativen Konstruktionen von Politik und politischen Akteuren in neueren TV-Serien und dabei auch das Zusammenspiel von semantischer Dimension und ästhetischen

20 Siehe Pilipets/Winter (2017), McCabe (2013), Crawley (2006), Fahy (2005).

21 Siehe etwa Sartwell (2010), Braungart (2012), Kompridis (2014), Kohns (2016), Virmani (2016) oder Emmerling/Kleesattel (2016). 
Gestaltungsmitteln erfassen. Dabei ist das Ziel zu klären, wie jeweils die fiktionale politische Welt funktioniert, welche kollektiven und individuellen Akteure sie gestalten, welche Institutionen relevant sind, welche politischen Handlungsformen mit Einsatz welcher Ressourcen und Instrumente beobachtet werden können. Auf der ästhetischen Ebene ist zu untersuchen, welche Erzählformen und Dramaturgien zum Einsatz kommen, wie die Figurenkonstruktion erfolgt, welche Formen der Bild- und Tongestaltung zum Einsatz kommen und welchen Einfluss all diese Gestaltungsmittel auf die Konstruktion des Politischen ausüben.

Die rekonstruierten Muster der politischen Semantik und der politischen Ästhetik können dann kontextualisiert werden in der politischen Kultur der jeweiligen Gesellschaft. ${ }^{22}$ Hier wäre das Ziel, zu klären, inwieweit die Serien Vorstellungsmuster, Wahrnehmungsmuster und Werte der politischen Kultur aufgreifen und gegebenenfalls modifiziert verarbeiten, inwieweit die produzierenden Akteure der Serien gezielt deutungskulturelle Praxis betreiben, indem sie den Serien intendiert bestimmte politisch-kulturelle Muster einschreiben (so etwa Aaron Sorkin mit The West Wing), und inwiefern Rezipienten politischer Serien bei der Aneignung der audiovisuellen Texte soziokulturelle Dispositionen der jeweiligen politischen Kultur einbringen. An dieser Stelle wird deutlich, dass vor allem systematisch-vergleichende Analysen reizvoll erscheinen, weil im Kontrast das Spezifische der jeweiligen Medienkulturen und politischen Kulturen hervortritt.

Die Analyse kann dann konkret entlang einer Differenzierung der drei Dimensionen Produktion, Text und Rezeption erfolgen ${ }^{23}$ :

\section{Produktion}

Zunächst sind die Produktionsabläufe zu rekonstruieren, um unter anderem zu klären, inwiefern Wissen über reale politische Prozesse eingeholt wird und welche Akteure an der Konstruktion der fiktionalen politischen Welt beteiligt sind. Werden systematisch politische Experten herangezogen und welche sind dies konkret? Mit welchen Motiven und mit welchem Selbstverständnis agieren die zentralen Akteure des Produktionsprozesses? Verfolgen sie mit ihren Produktionen so etwas wie ein deutungskulturelles Projekt mit entsprechenden politischen

22 Zur Verbindung von qualitativer politischer Kulturforschung und Medienanalyse siehe Dörner (2003).

23 Zur konkreten Methodik einer solchen mehrdimensionalen Analyse im Rahmen einer ethnografisch eingebetteten Medienanalyse siehe Dörner u.a. (2015), Dörner/Vogt (2015) sowie Dörner/Vogt (2017). 
Bildungsabsichten? Inwiefern werden Vorläufer- und Konkurrenzproduktionen als Vorbilder, Anregungsquelle oder Kontrastfolien genutzt? Bilden sich auf diese Weise spezifische Kulturen politischer Serien mit eigenen Traditionslinien heraus? An dieser Stelle ist auch die Spezifik der jeweiligen Rundfunk- und Mediensysteme zu beachten, in deren Kontext produziert wird. Die duale Rundfunkordnung in Deutschland definiert etwa andere Voraussetzungen der Produktion als das fast rein kommerzielle System der USA.

\section{Text}

Die audiovisuellen Texte der Serien können erstens daraufhin analysiert werden, welche Bilder der Politik (politischer Prozess, politische Inhalte und Themen, politische Institutionen, Normen und Werte) und der politischen Akteure (Figurenkonstruktion im Hinblick auf soziale Merkmale, Charakterzüge, Handlungsformen, Bindung des Handelns an Normen und Werte) sie konstruieren. Dabei wäre auch zu untersuchen, welche Bezüge die konstruierten fiktionalen Welten der Serien zur nichtfiktionalen, außermedialen Realität aufweisen: Werden reale Ereignisse und Prozesse verarbeitet, treten ,echte' politische Akteure auf (wie beispielsweise die ehemalige US-amerikanische Außenministerin Madeleine Albright in der Serie Madam Secretary) oder sind klare Bezüge zwischen fiktionalen Figuren und realen Personen erkennbar? Wie werden reale politische Institutionen, beispielsweise Parlamente oder Regierungskabinette, dargestellt? Inwieweit bietet der Text den Zuschauern jeweils Einblicke auf die politischen Hinterbühnen, wie sind diese Hinterbühnen beschaffen, wie ist die innerdiegetische Diskrepanz zwischen politischer Vorder- und Hinterbühne jeweils konstruiert? Agieren die politischen Akteure primär aufrichtig oder agieren sie mit Täuschungen und Zynismus?

Diese semantischen Dimensionen der Konstruktionen des Politischen müssen zweitens jeweils in Relation gesetzt werden zu den Formen der ästhetischen Gestaltung der Serien. Wie wirken sich Besonderheiten der Erzählweise oder der Figurenkonstruktion auf die konstruierten Politik- und Politikerbilder aus? ${ }^{24}$ Finden beispielsweise eher Gestaltungsmittel konventionellen Erzählens Anwendung oder aber Mittel narrativer Komplexität, wie sie im Kontext der Debatte über Quality TV erörtert werden, und führen diese unterschiedlichen Gestaltungsmittel auch zu unterschiedlich angelegten Konstruktionen politischer Abläufe? Im Vergleich verschiedener Serienwelten kann untersucht werden, inwie-

24 Siehe hierzu Nuy (2017) über den Zusammenhang von Dramaturgie und Politikbildkonstruktion im Kinofilm. 
fern es intertextuelle Ähnlichkeiten und Bezüge zwischen den Serien oder sogar so etwas wie gemeinsame Diskursräume gibt.

Drittens können die Konstruktionen des Politischen in den audiovisuellen Serientexten in Beziehung gesetzt werden zu den politischen Kulturen, in denen sie jeweils kontextualisiert sind, um etwa zu fragen: Haben die Texte politischkulturelle Traditionsbestände sowie aktuelle Vorstellungs- und Wertmuster aufgenommen und verarbeitet? Werden vermeintliche Selbstverständlichkeiten bestätigt oder irritiert? So ist beispielsweise bei einer populären und kommerziell erfolgreichen Produktion wie The West Wing unverkennbar, dass hier zentrale Muster der republikanischen Tradition in der US-amerikanischen politischen Kultur zugrunde liegen. Die Figuren agieren altruistisch und gemeinwohlorientiert, sie stellen private Belange konsequent hinter ihre öffentlichen Aufgaben zurück und verstehen die aktive Teilhabe am politischen Prozess als Voraussetzung einer gelingenden menschlichen Existenz. ${ }^{25}$ Demgegenüber lässt sich die politische Welt einer Serie wie House of Cards verstehen als radikale Interpretation des utilitaristischen Individualismus, wenn die Hauptfigur Frank Underwood ihren individuellen Nutzen maximiert, indem sie das politische Handeln vollkommen dem Erwerb und Erhalt persönlicher Macht und Anerkennung unterordnet. Im deutschen Kontext könnte die starke Ausrichtung der politischen Medienkultur auf Kriminalserien und -reihen als Hinweis auf die Wirksamkeit der traditionellen Staatsorientierung in der politischen Kultur gelesen werden, da die Polizei als eines der wichtigsten ordnungsstiftenden Exekutivorgane des Staates fungiert (Rohe 1994: 171ff.).

\section{Rezeption}

In der Rezeptionsdimension kann untersucht werden, auf welche Weise Zuschauer sich das in den audiovisuellen Texten konstruierte Politikbild aneignen. Welche Elemente aus dem vielfältigen Potenzial der Texte werden tatsächlich in der Rezeption ausgewählt, welche Vorstellungs- und Wertmuster aktualisiert und wie positionieren sich real existierende Zuschauer zu dem Gesehenen? Werden die fiktionalen Welten als realistisch, utopisch oder dystopisch bewertet? Werden die Serienfiguren als getreue Abbilder politischer Wirklichkeit, als positive Vorbilder oder als kritisch zugespitzte Gegenbilder wahrgenommen? Realisieren die Rezipienten eher den Unterhaltungswert des populären Formats oder aber eher das politische Potenzial eines deutungskulturellen Beitrags? Welche je

$25 \mathrm{Zu}$ den Traditionslinien der amerikanischen politischen Kultur siehe Bellah u.a. (1987: 52ff.). 
eigenen, politisch-kulturell geprägten Wert- und Vorstellungsmuster bringen die Zuschauer in den Aneignungsprozess ein? Wie unterscheiden sich verschiedene Rezipientengruppen in ihrer Rezeption? Wie wirkt sich ein interkulturell angelegter Rezeptionsprozess aus, wenn beispielsweise deutsche Zuschauer eine britische oder amerikanische Serie rezipieren und dabei einen anderen politischen Systemkontext beobachten können? Wie werden jene politischen Weltkonstruktionen in den eigenen Kontext übersetzt? Letztlich ist die öffentliche Anschlusskommunikation zu berücksichtigen, sowohl im Feuilleton überregionaler Zeitungen als auch in den Blogs, Foren und sozialen Netzwerken.

\section{FAZIT}

Der Beitrag hat den Gegenstand der Polit-Serien beschrieben und den aktuellen Forschungsstand zum Thema dargestellt. Vor diesem Hintergrund wurden Perspektiven einer zukünftigen, qualitativ und interdisziplinär angelegten Forschung aufgezeigt. Eine solche Forschung setzt sich das Ziel, politische TV-Serien nicht nur in ihrem Inhalt, sondern auch in ihrer ästhetischen Gestaltung zu analysieren und beides an den Kontext der politischen Kultur der Länder rückzubinden, in denen die Serien produziert und/oder rezipiert werden. Nationale Besonderheiten und interkulturelle Gemeinsamkeiten können dann vor allem in komparativen Studien erkennbar gemacht werden. Der Komplexität des Gegenstandes und seiner Einbettung in die kulturelle Praxis wird die Forschung dann gerecht, wenn sie die Dimensionen Produktion, Text und Rezeption berücksichtigt und - vermittelt über Triangulationsprozesse - systematisch aufeinander bezieht.

\section{LITERATUR}

Ahrens, Jörn/Cuntz, Michael/Koch, Lars/Krause, Marcus/Schulte, Philipp (2014): The Wire. Analysen zur Kulturdiagnostik populärer Medien, Wiesbaden.

Arendt, Kathleen/Rössler, Patrick (2014): Entertainment-Education. Wirkungsstrategien politischer Kommunikation in Unterhaltungsformaten, in: Dohle, Marco/Vowe, Gerhard (Hrsg.): Politische Unterhaltung - Unterhaltende Politik. Forschung zu Medieninhalten, Medienrezeption und Medienwirkungen, Köln. S. 315 - 343.

Arenhövel, Mark/Besand, Anja/Sanders, Olaf (Hrsg.) (2017): Wissenssümpfe.

Die Fernsehserie True Detective aus sozial- und kulturwissenschaftlichen Blickwinkeln, Wiesbaden. 
Bacqué, Marie-Hélène/Flamand, Amélie (2016): The Wire as seen from France, in: City 20 (6). S. $845-862$.

Balke, Friedrich (2015): Doppelkörper und Korridorbildung. Souveränität und Subversion in The West Wing, in: Klein, Rebekka A./Finkelde, Dominik (Hrsg.): Souveränität und Subversion. Figurationen des Politisch-Imaginären, Freiburg, S. $68-98$.

Bellah, Robert N./Madsen, Richard/Sullivan, William/Swidler, Ann/Tipton, Steven (1987): Gewohnheiten des Herzens. Individualismus und Gemeinsinn in der amerikanischen Gesellschaft, Köln.

Besand, Anja u.a. (Hrsg.) (2017): Väter allerlei Geschlechts. Generationenverhältnisse und Autoritätsfiguren in Fernsehserien, Wiesbaden.

Bochner, Kate/Thomas, Julian (2007): Political Theatre in ,The West Wing'. Swinburne University of Technology. Faculty of Life and Social Sciences. Institute for Social Research.

Bock, Annekatrin (2014): Machtkampf, Intrigen und Manipulation. Die negative Wahrnehmung von Politikgeschehen in aktuellen Politikserien, in: INDES 3 (4), S. $23-31$.

Borsos, Stefan (2017): Nach dem ,Quality TV‘. Anatomie und Kritik eines Diskurses, in: MEDIENwissenschaft Rezensionen (1), S. 8 - 25.

Braungart, Wolfgang (2012): Ästhetik der Politik, Ästhetik des Politischen. Ein Versuch in Thesen, Göttingen.

Celikates, Robin/Rothöhler, Simon (2007): Die Körper der Stellvertreter. Politische Repräsentation zwischen Identität, Simulation und Institution, in: Diehl, Paula/Koch, Gertrud (Hrsg.): Inszenierungen der Politik. Der Körper als Medium, München, S. $58-75$.

Christensen, Terry/Haas, Peter J. (2005): Projecting Politics. Political Messages in American Film, Armonk.

Crawley, Melissa (2006): Mr. Sorkin Goes to Washington. Shaping the President on Television's The West Wing, Jefferson.

Däwes, Birgit/Ganser, Alexandra/Poppenhagen, Nicole (Hrsg.) (2015): Transgressive Television. Politics and Crime in 21st-Century American TV Series, Heidelberg.

Diehl, Paula (2011): Populismus, Antipolitik, Politainment. Eine Auseinandersetzung mit neuen Tendenzen der politischen Kommunikation, in: Berliner Debatte Initial 22 (1), S. 27 - 39.

Dohle, Marco/Vowe, Gerhard (Hrsg.) (2014): Politische Unterhaltung - Unterhaltende Politik. Forschung zu Medieninhalten, Medienrezeption und Medienwirkungen, Köln. 
Dörner, Andreas (2000): Politische Kultur und Medienunterhaltung. Zur Inszenierung politischer Identitäten in der amerikanischen Film- und Fernsehwelt, Konstanz.

Dörner, Andreas (2001): Politainment. Politik in der medialen Erlebnisgesellschaft, Frankfurt am Main.

Dörner, Andreas (2003): Politische Kulturforschung, in: Herfried Münkler (Hrsg.): Politikwissenschaft. Ein Grundkurs, Reinbek bei Hamburg, S. 587 619.

Dörner, Andreas (2016): Politserien. Unterhaltsame Blicke auf die Hinterbühnen der Politik, in: Aus Politik und Zeitgeschichte 66 (51), S. 4 - 11.

Dörner, Andreas/Vogt, Ludgera (2015): Medienwissenschaft und Sozialwissenschaften: Ein Plädoyer für forschungspraktische Kooperation am Beispiel der ethnografisch eingebetteten Medienanalyse, in: Medienwissenschaft (3), S. $325-430$.

Dörner, Andreas/Vogt, Ludgera (Hrsg.) (2017): Wahlkampf mit Humor und Komik. Selbst- und Fremdinszenierung politischer Akteure in Satiretalks des deutschen Fernsehens, Wiesbaden.

Dörner, Andreas/Vogt, Ludgera/Bandtel, Matthias/Porzelt, Benedikt (2015): Riskante Bühnen. Inszenierung und Kontingenz - Politikerauftritte in deutschen Personality-Talkshows, Wiesbaden.

Dunst, Alexander (2016): Unheimliche Heimat. Homeland und die Ästhetik des Imperiums, in: Doll, Martin/Kohns, Oliver (Hrsg.): Figurationen des Politischen, Paderborn, S. $19-42$.

Eilders, Christiane/Nitsch, Cordula (2015): Politics in Fictional Entertainment. An Empirical Classification of Movies and TV Series, in: International Journal of Communication (9), S. 1563 - 1587.

Emmerling, Leonhard/Kleesattel, Ines (Hrsg.) (2016): Politik der Kunst. Über Möglichkeiten, das Ästhetische politisch zu denken, Bielefeld.

Eschkötter, Daniel (2012): The Wire, 2. Aufl., Zürich.

Fahy, Thomas Richard (Hrsg.) (2005): Considering Aaron Sorkin. Essays on the Politics, Poetics, and Sleight of Hand in the Films and Television Series, Jefferson.

Feuer, Jane/Kerr, Paul/Vahimagi, Tise (Hrsg.) (1984): MTM, ,Quality Television', London.

Fiske, John (1996): Media Matters. Race and Gender in U.S. Politics, Minneapolis.

Freda, Isabelle (2010): Survivors in The West Wing. 9/11 and the United States of Emergency, in: Dixon, Wheeler W. (Hrsg.): Film and Television after 9/11, Carbondale, S. 226 - 244. 
Friedrich, Katja (2011): Publikumskonzeptionen und Medienwirkungsmodelle politischer Kommunikationsforschung. Zum Einfluss theoretischer Grundannahmen auf die empirische Forschungspraxis, Wiesbaden.

Goffman, Erving (1959/2013): Wir alle spielen Theater. Die Selbstdarstellung im Alltag, München und New York.

Hackett, J. Edward (Hrsg.) (2015): House of Cards and Philosophy. Underwood's Republic, Chichester, Malden.

Hastall, Matthias R. u.a. (2014): Fiktionale Geschichten und ihre Wirkungen auf die Wahrnehmung der politischen und sozialen Realität, in: Dohle, Marco/Vowe, Gerhard (Hrsg.): Politische Unterhaltung - Unterhaltende Politik. Forschung $\mathrm{zu}$ Medieninhalten, Medienrezeption und Medienwirkungen, Köln, S. 294 - 314.

Hermann, Sebastian M. (2015): „How is it possible that this was kept a secret?“. Representation, Realism and ,Epistemic Panic' in The West Wing, in: Ernst, Christoph/Paul, Heike (Hrsg.): Amerikanische Fernsehserien der Gegenwart. Perspektiven der American Studies und der Media Studies, Bielefeld, S. 225 -248 .

Holbert, R. Lance (2005): A Typology for the Study of Entertainment Television and Politics, in: American Behavioral Scientist 49 (3), S. 436 - 453.

Holbert, R. Lance/Pillion, Owen/Tschida, David/Armfield, Greg/Kinder, Kelly/Cherry, Kristin/Daulton, Amy (2003): The West Wing as Endorsement of the U.S. Presidency. Expanding the Bounds of Priming in Political Communication, in: Journal of Communication, 53 (3), S. 427 - 443.

Holbert, R. Lance/Tschida, David/Dixon, Maria/Cherry, Kristin/Steuber, Keli/ Airne, David (2005): The West Wing and Depictions of the American Presidency. Expanding the Domains of Framing in Political Communication, in: Communication Quarterly 53 (4), S. 505 - 522.

Holbrook, R. Andrew/Hill, Timothy G. (2005): Agenda-Setting and Priming in Prime Time Television. Crime Dramas as Political Cues, in: Political Communication 22 (3), S. $277-295$

Holland, Jack (2011): ,When You Think of the Taliban, Think of the Nazis'. Teaching Americans ,9/11'in NBC's The West Wing, in: Millenium-Journal of International Studies 40 (1), S. 85 - 106.

Holtz-Bacha, Christina (2000): Entertainisierung der Politik, in: Zeitschrift für Parlamentsfragen 31 (1), S. 156 - 166.

Huizinga, Johan (1938/2004): Homo ludens. Vom Ursprung der Kultur im Spiel, Hamburg.

Jandura, Olaf/Gladitz, Peter/Nitsch, Cordula (2016): Parlamente in nonfiktionalen und fiktionalen Angeboten, in: Publizistik 61 (3). S. 287 - 304. 
Kaklamanidou, Betty/Tally, Margaret (Hrsg.) (2017): Politics and Politicians in Contemporary US Television. Washington as Fiction, London/New York.

Kelleter, Frank (Hrsg.) (2012): Populäre Serialität. Narration - Evolution - Distinktion. Zum seriellen Erzählen seit dem 19. Jahrhundert, Bielefeld/Berlin.

Kelleter, Frank (2014): Serial Agencies. The Wire and Its Readers, Lanham.

Kelleter, Frank (2017): Media of Serial Narrative, Columbus.

Kellner, Douglas (1995): Media Culture. Cultural Studies, Identity, and Politics between the Modern and the Postmodern, London.

Kennedy, Liam/Shapiro, Stephen (Hrsg.) (2012): The Wire. Race, Class, and Genre, Ann Arbor.

Kiegeland, Julia/Klatt, Jöran/Rahlf, Katharina (2014): INDES 3 (4).

Kirchmeier, Christian (2016): The President's Address. Zur politischen Parabase in House of Cards, in: Zeitschrift für Literaturwissenschaft und Lingusitik 46 (3), S. $369-385$.

Kleinen-von Königslöw, Katharina (2013): Politische Kommunikation zwischen Information und Unterhaltung. Eine Analysematrix, in: Gonser, Nicole (Hrsg.): Die multimediale Zukunft des Qualitätsjournalismus. Public Value und die Aufgaben von Medien, Wiesbaden.

Kohns, Oliver (Hrsg.) (2016): Perspektiven der politischen Ästhetik, Paderborn. Kompridis, Nikolas (2014): The Aesthetic Turn in Political Thought, New York u.a.

Lichter, S. Robert/Lichter, Linda/Amudson, Daniel (2000): Government Goes Down the Tube. Images of Government in TV Entertainment, 1955-1998, in: The Harvard International Journal of Press/Politics 5 (2), S. 96 - 103.

Lim, Il-Tschung (2014): Mal Freund, mal Feind, mal Konkurrent. Ein soziologischer Blick hinter die Kulissen des Politikbetriebs in House of Cards, in: INDES 3 (4), S. 55 - 61.

Loidolt, Claudia (2016): „It's Handled!“. Empowerment and Feminism in Shonda Rhimes’ TV Show Scandal. Diplomarbeit. Karl-Franzens-Universität Graz. Institut für Amerikanistik.

Lörke, Melanie (2014): What's next? The West Wing als positive Gegenwelt, in: Lillge, Claudia/Breitenwischer, Dustin/Glasenapp, Jörn/Paefgen, Elisabeth (Hrsg.): Die neue amerikanische Fernsehserie. Von Twin Peaks bis Mad Men, Paderborn, S. 229 - 252.

Luhmann, Niklas/Kieserling, André (Hrsg.) (2010): Politische Soziologie, Berlin.

McCabe, Janet (2013): The West Wing, Detroit.

Miniter, Richard/Wilson, Leah (2008): Jack Bauer for President. Terrorism and Politics in 24, Dallas. 
Mittell, Jason (2012): Narrative Komplexität im amerikanischen Gegenwartsfernsehen, in: Kelleter, Frank (Hrsg.): Populäre Serialität. Narration - Evolution - Distinktion. Zum seriellen Erzählen seit dem 19. Jahrhundert, Bielefeld/Berlin, S. $97-122$.

Morris, Joseph M./Evans, Henry T. (2014): Our House of Cards? Political Fiction and Belief Change. Western Political Science Association Conference. Seattle, Washington. 17.04.2014.

Morsch, Thomas (2010): Repräsentation, Allegorie, Ekstase. Phantasien des Politischen in aktuellen Fernsehserien, in: Dreher, Christoph/Akass, Kim (Hrsg.): Autorenserien. Die Neuerfindung des Fernsehens, Stuttgart, S. 199249.

Morsch, Thomas (2014): 24. Der permanente Ausnahmezustand, in: Lillge, Claudia/Breitenwischer, Dustin/Glasenapp, Jörn/Paefgen, Elisabeth (Hrsg.): Die neue amerikanische Fernsehserie. Von Twin Peaks bis Mad Men, Paderborn, S. $105-130$.

Moy, Patricia/Pfau, Michael (2010): With malice toward all? The media and public confidence in democratic institutions, Westport.

Mutz, Diana C./Nir, Lilach (2010): Not Necessarily the News. Does Fictional Television Influence Real-World Policy Preferences?, in: Mass Communication and Society 13 (2), S. $196-217$.

Nesselhauf, Jonas/Schleich, Markus (Hrsg.) (2015): Gegenwart in Serie. Abgründige Milieus im aktuellen Qualitätsfernsehen, Berlin.

Nesselhauf, Jonas/Schleich, Markus (2016): Das andere Fernsehen?! Eine Bestandsaufnahme des „Quality Television“, Bielefeld.

Nitsch, Cordula/Eilders, Christiane (2014): Die Repräsentation von Politik in fiktionaler Unterhaltung. Instrument, Anwendung und Befunde zur Systematisierung von Filmen und Fernsehserien, in: Studies in Communication: Media 3 (1), S. $120-143$.

Nitsch, Cordula/Eilders, Christiane (2015): Fictional Politics on TV. Comparing the Representations of Political Reality in U.S. Series The West Wing and the German Series Kanzleramt, in: Global Media Journal - German Edition 5 (1), S. 1 - 19.

Nitsch, Cordula/Wünsch, Carsten (2016): Fiktionale Videomalaise? Ein Prolonged-Expose-Experiment zur Wirkung von fiktionalen Politikdarstellungen auf die Politikverdrossenheit der Rezipienten, in: Czichon, Miriam/Wünsch, Carsten/Dohle, Marco (Hrsg.): Rezeption und Wirkung fiktionaler Medieninhalte, Baden-Baden, S. 115 - 133.

Nuy, Sandra (2017): Die Politik von Athenes Schild. Zur dramaturgischen Logik des Politischen im fiktionalen Film, Münster. 
Painter, Chad (2017): All in the Game, in: Journalism Studies 18 (1), S. 11 - 27.

Parry-Giles, Trevor/Parry-Giles, Shawn J. (2006): The Prime-Time Presidency.

The West Wing and U.S. Nationalism, Urbana, Ann Arbor, Michigan.

Peacock, Steven (Hrsg.) (2007): Reading 24. TV against the clock, London/New York.

Pilipets, Elena/Winter, Rainer (2017): House of Cards - House of Power. Political narratives and the cult of serial sociopaths in narrative politics in American quality dramas in the digital age, in: Kaklamanidou, Betty/Tally, Margaret (Hrsg.): Politics and politicians in contemporary US television. Washington as Fiction, London, S. $91-104$.

Rohe, Karl (1994): Politik. Begriffe und Wirklichkeiten, 2. Aufl., Stuttgart u.a.

Rollins, Peter C. (Hrsg.) (2003): The West Wing. The American presidency as television drama, Syracuse.

Rothöhler, Simon (2012): The West Wing, Zürich.

Sanders, Olaf/Besand, Anja/Arenhövel, Mark (Hrsg.) (2016): Ambivalenzwucherungen. Breaking Bad aus bildungs-, kultur- und sozialwissenschaftlichen Blickwinkeln, Köln.

Sartwell, Crispin (2010): Political Aesthetics, Ithaca u.a.

Saxer, Ulrich (2007): Politik als Unterhaltung. Zum Wandel politischer Öffentlichkeit in der Mediengesellschaft, Konstanz.

Schlütz, Daniela (2016): Quality-TV als Unterhaltungsphänomen. Entwicklung, Charakteristika, Nutzung und Rezeption von Fernsehserien wie The Sopranos, The Wire oder Breaking Bad, Wiesbaden.

Schneider, Irmela (2017): Quality TV, in: MEDIENwissenschaft Rezensionen 2, S. $287-292$.

Schubert, Christoph (2017): Constructing the antihero. Linguistic characterisation in current American television series, in: Journal of Literary Semantics 46 (1), S. $25-46$.

Schwer, Katja/Brosius, Hans-Bernd (2008): Sphären des (Un-)Politischen. Ein Modell zur Analyse von Politikdarstellung und -rezeption, in: Bonfadelli, Heinz/Imhof, Kurt/Blum, Roger/Jarren, Ottfried (Hrsg.): Seismographische Funktion von Öffentlichkeit im Wandel, Wiesbaden, S. 191 - 209.

Sheeler, Kristina Horn/Anderson, Karrin Vasby (2013): Woman president. Confronting postfeminist political culture, College Station.

Skewes, Elizabeth A. (2009): Presidential Candidates and the Press on The West Wing and in the Real World, in: Quarterly Review of Film and Video 26 (2), S. $131-142$.

Stenz, Christina Anna (2015): Die Politainment-Faktoren. Ein integratives Modell, Saarbrücken. 
Thompson, Robert J. (1997): Television's Second Golden Age. From Hill Street

Blues to ER, New York.

Tryon, Chuck (2016): Political TV, New York/London.

van Zoonen, Liesbet (2003): ,After Dallas and Dynasty we have ... Democracy“.

Articulating Soap, Politics and Gender, in: Pels, Dick/Corner, John (Hrsg.):

Media and the restyling of politics. Consumerism, celebrity and cynicism, London, S. 99 - 116.

van Zoonen, Liesbet (2012): Trends in political television fiction in the UK.

Themes, characters and narratives, 1965-2009, in: Media Culture \& Society 34 (3), S. $263-279$.

Vint, Sherryl (2013): The Wire, Detroit.

Virmani, Arundhati (Hrsg.) (2016): Political Aesthetics. Culture, Critique, and the Everyday, London/New York.

Williams, Rebecca (2011): „This Is the Night TV Died“. Television Post-Object Fandom and the Demise of The West Wing, in: Popular Communication 9 (4), S. $266-279$.

Wodak, Ruth (2009): The Discourse of Politics in Action. Politics as Usual, Basingstoke/New York.

Wodak, Ruth (2010): The Glocalization of Politics in Television. Fiction or Reality?, in: European Journal of Cultural Studies 13 (1), S. 43 - 62.

Wolling, Jens (2014): Politikverdrossenheit als Folge von Unterhaltungsrezeption?, in: Dohle, Marco/Vowe, Gerhard (Hrsg.): Politische Unterhaltung Unterhaltende Politik. Forschung zu Medieninhalten, Medienrezeption und Medienwirkungen, Köln, S. 259 - 293.

Wünsch, Carsten/Czichon, Miriam (2016): Fiktion oder Realität. Agenda-Setting und Medien-Priming durch fiktionale Medieninhalte?, in: Czichon, Miriam/Wünsch, Carsten/Dohle, Marco (Hrsg.): Rezeption und Wirkung fiktionaler Medieninhalte, Baden-Baden, S. 135 - 151.

Wünsch, Carsten u.a. (2012): Politische Kultivierung am Vorabend. Ein prolonged-exposure-Experiment zur Wirkung der Fernsehserie „Lindenstraße“, in: M\&K 60 (2), S. 176 - 196. 



\title{
Politik in Serie(n)
}

\section{Die Politik, das Politische und die Tragödie}

\author{
Henrik Schillinger
}

\section{WARUM POLIT-SERIEN ANSEHEN?}

Können Serien über Politik mehr sein als Unterhaltung? Können Zuschauer etwas über Politik lernen? Leisten Polit-Serien einen Beitrag zur politischen Bildung? Warum sollte man sich bei politischem Interesse fiktive, fiktionale Politik ansehen, statt seine volle Aufmerksamkeit Nachrichten, Reportagen und Dokumentationen über reale Politik zuzuwenden? Polit-Serien sind in erster Linie Unterhaltungsformate und nicht Bildungsfernsehen. Das Weiße Haus (häufig ${ }^{1}$ ) oder Christiansborg $\left(\right.$ selten $\left.^{2}\right)$ sind vor allem ,Setting', eine Kulisse, um einen für moderne Qualitätsserien typischen Genre-Mix aus Kriminalgeschichte, Action, Thriller und Melodram zu erzählen (Dörner 2016: 6).

In diesem Sinne sollen Polit-Serien hier auch verstanden werden: So wie die Arztserie durch das Setting des Krankenhauses als Ort und Institution sowie Ärz$\mathrm{te}^{3}$ als Protagonisten gekennzeichnet sind, definieren sich Polit-Serien über Regierungsgebäude, Ministerien, Parlamente als Orte und Institutionen sowie Politiker, Beamte, Berater und ggf. Journalisten als Haupt- und Nebenrollen. Mit diesem offensichtlichen Politikbezug bilden Polit-Serien einen ,leichten Fall` für die eingangs aufgeworfene Frage. Zwar mögen vor dem Hintergrund eines wei-

1 Z.B. House of Cards, The West Wing, Scandal, Designated Survivor, Veep und viele andere mehr.

2 Borgen. Andere Settings bilden etwa das Rote Rathaus (Die Stadt und die Macht), der Bundestag (Die Lobbyistin, Eichwald, MdB) oder das Rathaus von Marseille (Marseille).

3 Hier wie nachfolgend gilt, dass immer alle Geschlechter gemeint sind. 
teren Politikbegriffs andere Settings oder gar Formate einen Mehrwert im Sinne des Lernens über Politik bilden (vgl. Dörner 2006), aber bei Polit-Serien ist zu erwarten, dass dieser Mehrwert besonders deutlich wird.

Um produziert und im Produktionszyklus verlängert zu werden, müssen sie vor allem unterhalten. Und um zu unterhalten, müssen auch die Polit-Serien dramaturgisch verkürzen und verdichten, zuspitzen und beschleunigen. „Alles passiert schneller, einfacher, eindeutiger als in Wirklichkeit, daher sind die Serien orientierungsfreundlich" (Dörner 2016: 7). Trotzdem wird die realistische Darstellung ,echter' (langsamer und komplexer) Politik in Polit-Serien als wichtiges Argument angeführt, warum diese Serien sehenswert sind - wie im folgenden zweiten Abschnitt ausgeführt werden soll. Explizit oder implizit wird so die Vermittlung politischen Wissens als Mehrwert der Polit-Serie für den Zuschauer (und die kritische, auch politikwissenschaftliche Rezeption) behauptet.

Daran anschließend wird im dritten Abschnitt argumentiert, dass der Realismus der Polit-Serien eine an ,Fakten“ orientierte, konventionelle und instrumentelle Darstellung der Politik als politics reproduziert, dabei die zugrundeliegende Dimension des Politischen ausblendet und so letztlich eine antipolitische Haltung vermittelt. Im vierten Abschnitt wird vorgeschlagen, Polit-Serien eher aus der Perspektive der erzählten ,Story` als Tragödie zu verstehen, statt als Referenzpunkt für ,Fakten“. In Analogie zur politischen Rolle der Tragödie in der klassischen griechischen Polis kann die Polit-Serie so einen Beitrag zur politischen Bildung der Zuschauer als Bürger hin zu Demokratie leisten. Die Zeit vor dem Fernseher wäre dann gut investiert.

\section{REALISMUS UND RELEVANZ}

\section{Das Realismusprinzip: Relevanz durch Realitätsimitation}

Eine mögliche Antwort auf die Frage nach Relevanz von Polit-Serien jenseits der Unterhaltung verweist auf einen Zugewinn an Wissen über Politik.

\footnotetext{
„Die Serien bieten uns komprimierte Seinsbestimmungen der politischen Welt. Sie können im besten Falle zeigen, wie Politik funktioniert und was die beteiligten Akteure antreibt. Sie führen vor, mit welcher Währung im politischen Geschäft bezahlt wird und welche Kooperationen und Konfrontationen sich zwischen Politik und Medien herausbilden können.“ (Dörner 2016: 7)
}

Fiktive Politik in qualitativ hochwertigen Polit-Serien muss dieser Argumentation zufolge realistisch sein, um relevant zu sein. Die Befassung mit Polit-Serien 
lohnt sich dann, weil (und, als Qualitätsargument, wenn) sie Wissen über ,echte Politik vermitteln können. Zwar sind Zuschauer bereit, ein gewisses Maß an dramaturgisch begründeter Abweichung von der Realität zu dulden, vielleicht sogar zu fordern, weil sie unterhalten werden wollen (willing suspension of disbelief), aber die Serie dürfe ,doch bei aller Zuspitzung die Verbindung mit dem, was wir als außermediale Wirklichkeit kennen, nicht völlig verlieren, sonst erscheint sie uns belanglos“ (Dörner 2016: 7).

Auch wenn es in Polit-Serien als Unterhaltungsformaten (im Gegensatz zu journalistischen oder dokumentarischen Formaten) nicht um ,reale“ Politik gehen kann und soll, wird zumindest implizit gesetzt, dass die fiktive Politik als Setting, gemäß dem Realitätsprinzip (Walton 1993), bestenfalls minimal von den bekannten Fakten politischer Institutionen und Ereignisse abweichen soll, um relevant zu sein. Realismus bedeutet so verstanden, dass Polit-Serien die politische Wirklichkeit imitieren, indem sie Geschichten erzählen, ,als ob` sie wahr sein könnten. Polit-Serien gewinnen in dieser Sicht Relevanz, indem sie dem Zuschauer erstens Faktenwissen über die ,reale“ Politik vermitteln - und damit zweitens reale politische Rückschlüsse ermöglichen. So argumentiert etwa ein wissenschaftlicher Text über die Darstellung von Demokratie in House of Cards:

„On this view, House of Cards pose a significant challenge to those of us who want to defend or reform democracy, since it is our responsibility to say how (if at all) our real-world political institutions can be safeguarded against the sort of schemes that threaten the citizens of Frank and Claire's world.“ (Shea 2015: 141-142)

In Anlehnung an das Realitätsprinzip lässt sich in der Rezeption von Polit-Serien von einem Realismusprinzip sprechen, wenn der Mehrwert der Serie auf ihre Leistung in der Vermittlung von Wissen über ,reale Politik` zurückgeführt wird. Dieses Realismusprinzip zieht sich durch journalistische Texte über Polit-Serien: „Willkommen bei ,House of Cards', der Serie, die Politik zeigt, wie sie wirklich ist." (Hanfeld 2013) Dabei wird teilweise durchaus das komplexe Verhältnis von Politik und Fiktion reflektiert und auf unrealistische oder utopische Elemente hingewiesen. Die Einordnung dieser Elemente findet dann aber unter Betonung des grundsätzlich realistischen Charakters der Politikdarstellung statt (z.B. Hardt 2016). Auch wissenschaftliche Diskussionen fiktionaler Inhalte folgen wie im oben angeführten Zitat häufig implizit oder explizit dem Realismusprinzip, wenn im Rahmen interpretativer Vorgehen der Gehalt an realer Politik ,extrahiert` wird (Carpenter 2016: 53) oder die realitätsnähere Politikdarstellung sogar als Auswahlkriterium von Fällen für die sozialwissenschaftliche Analyse von Poli- 
tikfiktionen angeführt wird, wenn etwa Zero Dark Thirty der Serie 24 vorgezogen wird (Heck/Schlag 2015: 138-139).

\section{Realistische Politikfiktion: ,echter als echt'}

Das Realismusprinzip wird dabei auf verschiedene Weise durch die Ausgestaltung des Settings gefördert. Auf der inhaltlichen Ebene der Polit-Serien stehen häufig Entscheidungsprozesse und die vorhergehenden Konflikte im Mittelpunkt, denn ,[d] ezionistische Situationen eigenen sich vor allem für das serielle Drama“ (Kelleter/Jahn-Sudmann, 2014: 17). Die Zuspitzung auf Entscheidungen und Entscheider geht mit einem erzählerischen Fokus auf politisches Spitzenpersonal (Präsidenten, Premierministerinnen, Mehrheitsfüher) und die ihnen unmittelbar zuarbeitenden Stabsstellen (Spindoktoren, Pressesprecher, Stabschefs, Berater) einher (z.B. Switek in diesem Band). Die Hauptfiguren der fiktiven Politik vor der Kamera entsprechen so in der Regel den Hauptfiguren der realen Politik vor der Kamera (oder bilden zumindest deren Referenzpunkt). Das Realismusprinzip wird inhaltlich unterstützt, indem sich die Handlung auf jenes Personal konzentriert, das Zuschauer auch in der Berichterstattung über die echte Politik wiedererkennen können. Dieser Effekt wird durch die Medialisierung vor allem von Wahlkämpfen und die damit einhergehende emotionale Aufladung und Narrativisierung der realen Politik begünstigt (Deacon/Stanyer 2014).

Der realistische Anspruch wird paradoxerweise dadurch verstärkt, dass PolitSerien „unterhaltsame Blicke auf die Hinterbühnen der Politik“ gewähren, die der Berichterstattung über reale Politik unmöglich sind (Dörner 2016). PolitSerien lassen den Zuschauer an der ,unsichtbaren' formellen wie informellen politischen Arbeit der Protagonisten teilnehmen - mit Blick etwa auf die Bildungsreform in der ersten Staffel von House of Cards: von Arbeitsbesprechungen mit dem Präsidenten über Verhandlungen mit verschiedenen Interessensvertretern bis hin zur Überredung einzelner Abgeordneter. Dabei werden im Beispiel alle Mittel ausgeschöpft: politische Gegengeschäfte, argumentative Überzeugung, mediale Enthüllungen und der Druck der Öffentlichkeit, aber auch Erpressung und Mord. Polit-Serien greifen dabei oft erkennbar auf reale Gegebenheiten zurück, können aber in der Fiktionalisierung aus dem oft nur bruchstückhaft vorliegenden Wissen und den unauflösbaren Ambivalenzen des realen Falls eine eindeutige und abgeschlossene Erzählung mit klaren Ursachenzuweisungen entwickeln (vgl. für ein Beispiel Sneed 2014). So entsteht eine fiktionale Darstellung politischer Vorgänge, die realistischer als die Wirklichkeit wirkt, weil sie vollständiger sein kann als die mediale Abbildung realer Politik.

Das Versprechen des Blicks auf die Hinterbühnen wird auch auf der Dialogebene immer wieder bekräftigt: „There's a whole layer of D.C., you know, whe- 
re real politics happen. Where decisions are made. [...] This is about things that go bump in the night. Stuff that regular Americans never hear about. This is the real deal“" (Scandal, S 2/F 10). Oder mit The West Wing: „There are two things in the world you never want to let people see how you make'em: laws and sausages“ (S 1/F 4). Am weitesten treibt diese Perspektive House of Cards, wenn der Protagonist Underwood die , vierte Wand" (den Bildschirm) durchbricht und sich in ,asides ' direkt an das Publikum wendet. Dem Zuschauer wird so vermittelt, dass er einen Einblick in das Sein statt nur den Schein ,echter' politischer Probleme erhält (Gray 2015). Die ,Asides` sind ein wesentliches Element für die Identifikation des Zuschauers mit dem Anithelden Underwood (Kajtár 2015: 235) und damit für eine ,suspension of disbelief ${ }^{*}$, die unrealistische Momente in den Hintergrund treten lässt.

\section{Hyperrealistische Politikfiktion}

Mit dem Blick auf Hinterbühnen wandelt sich der Realismus der Polit-Serie in die Hyperrealität, eines , authentischen Fakes':

„This is the reason for this journey into hyperreality, in search of instances where the American imagination demands the real thing and, to attain it, must fabricate the absolute fake; where the boundaries between game and illusion are blurred, the art museum is contaminated by the freak show, and falsehood is enjoyed in a situation of ,fullness ", of horror vacui.“ (Eco/Weaver 2014: 8)

Die Beschleunigung und Verdichtung, die Zuspitzung und Verkürzung, die notwendig sind, um langsame, mühsame Realität der Politik unterhaltsam zu machen, erzeugen, weil alles schneller, einfacher und eindeutiger sein muss, das Bild einer Politik, die in der Überzeichnung kraft ihrer Vollständigkeit authentischer, ,echter als die echte' Politik scheint.

Vor diesem Hintergrund sind auch die ,Echtheitszertifikate' realer Personen aus der Politik zu verstehen - so etwa die Empfehlung Jürgen Trittins zum Einsatz von House of Cards im Politikunterricht: „Frank Underwood führt durch das Unterholz von Max Webers Theorie über das Wesen der Politik im Kompromiss“ (Trittin 2014). Oder wenn Bill Clinton, dem Hauptdarsteller der gleichen Serie (dessen Erinnerung zufolge) bescheinigt, dass die Serie in ihrer Darstellung von Politik zu 99 Prozent real sei (Hochman 2015).

In diesen Äußerungen realer Politiker über eine fiktive Polit-Serie offenbart sich eine Vermischung von Realität und Fiktion. Die reale verweist auf die fiktive Politik, um sich dem Bürger verständlich zu machen. Entsprechende Äußerungen aus der politischen Praxis werden von Produzenten wie auch journalisti- 
schen Kritikern als Beleg einer authentischen und damit realistischen Darstellung von Politik aufgegriffen. Die Polit-Serie wird so in die reale Politik integriert. Hier deutet sich die Vermischung von Politik und Unterhaltung an, die in der Konsequenz fiktionale und reale Repräsentationen von Politik gleichsetzt.

Der Verweis auf die Einbindung von Fachleuten und Insidern beglaubigt weiter den Anspruch auf eine realistische Darstellung als Relevanzargument und wird nicht in der Vermarktung wie auch in der journalistischen und kritischen Rezeption regelmäßig als wichtige Kontextinformation präsentiert (z.B. Dörner 2016: 4). Indem sich Polit-Serien auf die Beratung oder Vorlage politischer Insider, wie der Krisenmanagerin Judy Smith für die Serie Scandal, berufen, können sie ihren Anspruch auf eine Darstellung von Politik beglaubigen, die ,echter als echt' ist, und so einen sehenswerten Mehrwert besitzen.

In der Vermarktung von Polit-Serien geht die Vermischung aber noch weiter. So bewarb Netflix die vierte Serienstaffel inmitten der realen Vorwahlkämpfe 2016 mit einer fiktiven Präsidentschaftskampagne für den Protagonisten Underwood. Teil dieser Kampagne waren eine Wahlkampfhomepage wie auch Wahlwerbespots, deren fiktiver Charakter nicht weiter gekennzeichnet war - so, , als ob“ (Searle 1997: 156) Underwood ein realer Kandidat wäre. Handwerklich waren echte und fiktive Kampagnen kaum zu unterscheiden (Steinmetz 2015). Mit diesem Nachweis handwerklichen Könnens und politischen Sachverstands unterstrichen die Produzenten ihr Versprechen auf eine realistische Darstellung von Politik als unique selling point der Serie. Der realistischen Politikdarstellung wird offensichtlich verkaufsfördernde Relevanz zugebilligt.

Zusammenfassend lässt sich festhalten, dass Perspektiven, die einen Mehrwert von Polit-Serien in der Vermittlung von Wissen über Politik sehen, diesen Mehrwert implizit oder explizit auf die realistische, faktengetreue Darstellung ,echter' Politik zurückführen: Die Kunst imitiert das Leben. Die realistische Anmutung basiert aber im Kern auf einem Hyperrealismus, der durch Überzeichnung intransparente, komplexe und umstrittene politische Zusammenhänge zu eindeutigen Erzählungen verdichtet. Polit-Serien können so eine ,echtere als echte' Politik zeigen und beglaubigen diesen Anspruch durch die Vermischungen fiktionaler und realer Politik, etwa durch die Einbindung politischer Insider als Experten.

Wie realistisch die fiktive Darstellung von Politik ist, ist auch politisch relevant. Fiktionalisierungen autoritärer Politik können beispielsweise entsprechende politische Einstellungen bei Zuschauern aktivieren (Glas/Taylor 2017). Auch wenn Studien zur politischen Wirkung von Populärkultur aufgrund methodologischer Einschränkungen kritisch zu betrachten sind, beeinflussen populäre Darstellungen zumindest die Konstitution des politischen Diskursraums (Carpenter 
2016). Fiktive Darstellungen können sowohl bestehendes Wissen über Politik bestärken und reproduzieren und legitimieren als auch subversiv unterlaufen oder kritisieren (Erickson 2007). Die Darstellung der Politik in Polit-Serien beinhaltet folglich eine Politik der Serie. Wenn also der Realismus einer Polit-Serie zum Merkmal ihrer Relevanz und Qualität wird, dann rückt die Frage in den Mittelpunkt, welche Form der Politik als ,echte' Politik dargestellt wird - und so eine hyperrealistische Simulation (Baudrillard 2010) von Politik erzeugt, die das Bild des Zuschauers von realer Politik möglicherweise beeinflusst.

\section{POLITIK UND DAS POLITISCHE}

\section{Idealtypen der Serienpolitik}

Wie sehen realistische Darstellungen von Politik in Polit-Serien aus, die uns vermeintlich zeigen können, wie Politik funktioniert? Auf den ersten Blick bieten Polit-Serien dabei ein vielfältiges Angebot. Mit Dörner lassen sich drei Idealtypen der Darstellung von Politik in Polit-Serien unterscheiden (Dörner 2016: 7 10). ${ }^{4}$ In der Idealpolitik der Serie The West Wing verfolgt demnach ein ,guter US-Präsident (gebildet, aufrichtig, prinzipientreu) mit guten Mitteln (legal, legitim, ethisch) gute Zwecke (Gemeinwohl, Gerechtigkeit, Frieden). Die Polit-Serie definiert so einen ,utopischen Raum' der als Maßstab und positiver Gegenentwurf zur politischen Realität dienen soll (Dörner 2016: 8). Borgens Realpolitik zwingt die dänische Ministerpräsidentin zur Einsicht, dass zum Erreichen guter Ziele manchmal Mittel vonnöten sind, die den eigenen hehren Prinzipien zuwiderlaufen. Die Polit-Serie wird zum politischen Bildungsroman, der als zentrale Erkenntnis eines politischen Coming-of-Age die Botschaft vermittelt, dass der Zweck bisweilen die Mittel heiligt. ${ }^{5}$ Die Machtpolitik in House of Cards zeigt Macht und Machtstreben als Mittel und (Selbst-)Zweck allen politischen Han-

4 Die Bildung der Idealtypen erfolgt bei Dörner schon auf der Grundlage einer Voreinschränkung auf politische Prozessdramen, die einerseits sogenannte Sicherheitsdramen (wie bspw. 24) und andererseits fiktionale Inhalte ausschließen, die im Sinne eines erweiterten Politikverständnisses ein anderes Bild von Politik präsentieren könnten. Sie entspricht so der oben getroffenen Definition von Polit-Serien.

5 Siehe hierzu auch die Ausführungen im Beitrag zur Serie Borgen in diesem Band. 
delns. Politik ist frei von äußeren, höheren Werten und Prinzipien. Die PolitSerie wird zur Fernsehfassung von Macchiavellis Der Fürst. ${ }^{6}$

Idealpolitik wie auch Machpolitik bilden in diesem Verständnis die entgegengesetzten Pole eines Kontinuums in der Darstellung von Politik, die das Bild realer Politik jeweils auf ihre Weise verzerren. Dazwischen liegt der Idealtypus der Realpolitik, der so aus der Perspektive realistischer Darstellung wie die ,goldene Mitte“ erscheinen mag: „Realpolitische Szenarien in Politserien scheinen einen besonders realistischen Bezug zur außermedialen Wirklichkeit herzustellen, da sie ein simples Schwarz-Weiß-Schema vermeiden und den Preis für Machterwerb und politische Gestaltung aufzeigen.“ (Dörner 2016: 9)

Die Unterschiede in der fiktionalen Politik sind allerdings eher stilistisch bedeutsam. In den Polit-Serien verschwimmen die Idealtypen immer wieder auf eine Weise, die nahelegt, dass die Unterschiede im (dargestellten) Politikverständnis letztlich geringer sind als die Gemeinsamkeiten. Bezeichnenderweise findet Dörner selbst, dass in Scandal, „,das Abrücken von moralischen Werten um der positiven Ziele willen hier ganz besonders drastisch gezeigt wird und viele der Akteure Entwicklungen durchlaufen, die eher schon dem dritten Muster, der Machtpolitik, entsprechen“ (Dörner 2016: 9), obwohl er die Serie zuvor als Musterbeispiel einer realpolitischen Politikdarstellung anführt.

The West Wing, als ,Left Wing ' utopischer Entwurf eines besseren Amerika aus liberaler Sicht, bleibt grundsätzlich ,realpolitisch“ in seiner Politikdarstellung: „So ideal hier phasenweise das Personaltableau aufgestellt scheint, so entschieden visionär manche State-of-the-Union-Rede klingt: WEST WING ist auch eine große Desillusionierungsmaschine, die zeigt[,] wie weitreichende Reformideen an den Kompromisszwängen überparteilich erreichbarer common grounds scheitern“ (Rothöhler 2012: 36). Zur Verwirklichung des ,guten“ Zwecks lassen sich die eigenen Prinzipien immer wieder nur retten, indem man die Verwendung der weniger edlen Mittel delegiert. „Hinter den Kulissen, das ist das Realitätsprinzip der Serie, reicht der zwanglose Zwang des besseren Arguments oft nicht aus, auch wenn ein idealistischer, idealer Präsident sich nie in die Niederungen der Realpolitik herablassen würde. , The president doesn't hold a grudge. That's what he pays me for“." (Rothöhler 2012: 37)

Auch der Machtpolitiker Underwood aus House of Cards ist fest in der Welt der realpolitischen Zwänge verankert. Der Zuschauer erlebt Underwood als Meister der politics, der zwar letztlich immer die Mehrung seiner Macht im Sinne hat, dafür aber in mühevoller Kleinarbeit Kompromisse und Bündnisse aus-

6 Siehe hierzu auch die Ausführungen im Beitrag „House of Cards: The American Machiavelli" in diesem Band. 
handeln, überzeugen und sich auf politische Gegengeschäfte einlassen muss. Seine politischen Intrigen erzeugen mittelfristig Feindschaften und Widerstände, wie zum Beispiel in den Handlungssträngen um eine Bildungsreform in Staffel 1 (Fallis 2015) oder in seinen Bemühungen um sein America-Works-Programm in Staffel 3 (Byron/Wood 2015) gezeigt. Macchiavelli wäre nicht beeindruckt (Fallis 2015).

\section{Serien-politics und das Fehlen des Politischen}

Die Unterschiede in der Politikdarstellung zwischen verschiedenen Polit-Serien sind somit kleiner, als es die stilistischen Variationen der gezeigten Politiker und ihrer Handlungen vermuten lassen. Politik wird vor allem als politics gezeigt, als dynamischer Prozess, in dem Akteure (vor allem die Protagonisten) in Machtbeziehungen versuchen, ihre Interessen durchzusetzen (Patzelt 2003: 29) und auszuhandeln „who gets what, when, how“ (Lasswell 1936). Die verfolgten Ziele (policies) bleiben dabei in der Regel vage und Vehikel für die dramaturgisch interessanteren politics. Die formalen Strukturen der polity bilden dabei nur das Spielfeld, auf dem die Protagonisten ihre meisterliche Beherrschung der politics bis hin auf die Ebene der Verfahrenstricks vorführen. Ein illustratives Beispiel findet sich wieder in House of Cards in der Handlung um eine drohende Haushaltssperre in der zweiten Staffel (Näheres dazu Sneed 2014).

Der gemeinsame Nenner fiktionaler Politik in Politik-Serien ist damit ihr Fokus auf ,die Politik' im Gegensatz zu ,dem Politischen`.

„Indem ich substantivisch von dem Politischen [ $d u$ politique] spreche, qualifiziere ich damit sowohl eine Modalität der Existenz des gemeinsamen Lebens als auch eine Form kollektiven Handelns, die sich implizit von der Ausübung der Politik unterscheidet. Sich auf das Politische und nicht auf die Politik beziehen, d.h. von Macht und von Gesetz, vom Staat und der Nation, von der Gleichheit und Gerechtigkeit, von der Identität und der Differenz, von der citoyenneté und Ziviltät, kurzum: heißt von allem sprechen, was ein Gemeinwesen jenseits unmittelbarer parteilicher Konkurrenz um die Ausübung von Macht, tagtäglichen Regierungshandelns und des gewöhnlichen Lebens der Institutionen konstituiert." (Rosanvallon 2003: 14 zit. n. Marchart 2010: 13).

Was hier als , die Politik' beschrieben wird, Politik im konventionellen Sinne von politics, policies und polity, erfasst demnach nur die ontische Ebene von Politik - eine historisch bedingte und beschränkte Ausprägung der Gestaltung von Gesellschaft, die ihre Bedingtheit und Beschränktheit aber nicht reflektiert: Politik im ,Normalmodus“ - ,politics as usual' (Wodak 2011). Das Politische bezeichnet im Gegensatz dazu die ontologische Ebene von Politik, die das be- 
gründende Moment von Politik für die Gesellschaft in den Mittelpunkt stellt (Mouffe 2007: 15).

Dieses begründende Moment von Gesellschaft, die spezifische Rationalität des Politischen, kann auf verschiedene Weise gedacht werden. In einem dissoziativen Paradigma ist das Politische durch Antagonismen, also die Konstruktion von Differenzierungen in Freunde und Feinde, Selbst und Anders, gekennzeichnet:

„Unter ,dem Politischen“ verstehe ich die Dimension des Antagonismus, die menschlichen Verhältnissen inhärent ist, viele Formen annehmen kann und in unterschiedlichen Typen sozialer Verhältnisse entsteht. Auf der anderen Seite bezeichnet ,Politik' das Ensemble von Praktiken, Diskursen und Institutionen, die eine bestimmte Ordnung zu etablieren versuchen und menschliche Ko-Existenz unter Bedingungen organisieren, die immer potenziell konfliktorisch sind, da sie von der Dimension ,des Politischen“ affiziert werden. Ich denke, dass wohl die zentrale Frage demokratischer Politik nur gestellt werden kann, wenn wir die Dimension ,des Politischen“ anerkennen und verstehen, dass ,Politik' in der Domestizierung von Feindschaft besteht und im Versuch, den potenziellen Antagonismus, der in menschlichen Verhältnissen herrscht, zu entschärfen.“ (Mouffe 2007: 103)

Die dissoziative Perspektive des Politischen wird durch eine assoziative ergänzt, der zufolge kommunikative und kommunale Momente des acting in concert oder acting together das spezifisch Politische ausmachen (Marchart 2010: 36-37):

„Ich werde das Politische als Ausdruck der Idee verstehen, dass eine freie Gesellschaft, die aus Diversitäten zusammengesetzt ist, sich dennoch Momenten der Gemeinschaftlichkeit erfreuen kann, sobald durch öffentliche Deliberation kollektive Macht eingesetzt wird, um das Wohlergehen der Kollektivität zu fördern oder zu beschützen. Politik bezieht sich auf den legitimierten und öffentlichen Konflikt, vor allem durch organisierte und ungleiche soziale Kräfte, um Zugang zu Ressourcen, die den öffentlichen Autoritäten der Kollektivität zu Verfügung stehen. Politik ist kontinuierlich, ohne Unterbrechungen und endlos. Im Gegensatz dazu ist das Politische episodisch und selten." (Wolin 1966: 32 zit. n. Marchart 2010: 37)

\section{Serienpolitik als Antipolitik}

Was beide Traditionslinien neben ihrer Betonung einer konstitutiven Differenz zwischen dem Politischen und der Politik eint, ist die Neutralisierungs- oder Sublimierungsthese, nach der die Politik das Politische verdrängt (Marchart). Für Mouffe beispielweise sind die politischen Diskurse der Gegenwart durch einen Liberalismus dominiert, der entpolitisierend wirkt, weil er eine post-politische 
Politik des Kompromisses und Konsenses befördert. Aushandlungsprozesse zwischen individuellen Interessensvertretern (methodologischer Individualismus) oder die deliberative Findung eines inklusiven Konzernes ermöglichen diesem Liberalismus zufolge das ,Ende der Politik“ in ihrer antagonistischen Dimension (Mouffe 2007: 20-21). Politische Akteure werden als Konkurrenten (statt als Freunde oder Feinde) gedacht, die auf einem als neutral gedachtem Feld der Politik um Machtanteile und -positionen innerhalb der gegebenen Verhältnisse agieren. „Politik ist für sie nur ein Wettstreit zwischen Eliten.“ (Mouffe 2007: 31)

Wo aber die Frage nach den begründenden Momenten von Gesellschaft in Form von Antagonismen oder von Politik als gemeinschaftsstiftender Lebensform zugunsten einer Post-Politik von Kompromiss und Konsens ,entpolitisiert wird, entstehen antipolitische Bewegungen. Diese umfassen sowohl Politikverdrossenheit als Apathie gegenüber politischen Fragen wie auch radikalisierte Gegenbewegungen. Das Erstarken des islamistischen Terrorismus wie auch der Aufstieg rechter Bewegungen in Europa sind für Mouffe beispielsweise Ausdruck einer antagonistischen Gegenreaktion auf einen entpolitisierten, (neo-) liberalen Diskurs (Mouffe 2007: 85-87).

Polit-Serien zeichnen in ihrem ,Realismus“ diese Neutralisierung des Politischen durch die Politik nach. Der Zuschauer sieht Politik als politics, also als Wettstreit zwischen Individuen, die Teil der politischen Elite sind, um größere Machtanteile mit den Mitteln des Kompromisses oder des Konsenses. Wenn House of Cards' Underwood als „American Macchiavelli“ beschrieben wird (Littmann 2015), dann, weil er den ,Machiavellischen Moment` verkörpert, ab dem gilt: politics is politics (Sartori 1973: 11). Der Umgang mit dem grundlegenden und gründenden Moment des Politischen ist bestenfalls noch strategisch. Underwood fehlt eine gesellschaftliche Gründungsidee (vgl. Meyer 2015), gegenüber der man sich als Freund oder Feind verhalten kann.

Die Dimension des Politischen bricht in Polit-Serien immer nur kurz in die Handlung ein. In der Handlung um Underwoods radikales sozialpolitisches Arbeitsbeschaffungsprogramm America Works in House of Cards wird beispielsweise über Bilder von Gegendemonstrationen auf der einen Seite und über eine Nebenhandlung um den arbeitslosen ehemaligen Besitzer von Underwoods Lieblingsrestaurant auf der anderen Seite die gesellschaftliche Spaltung und die damit einhergehende Mobilisierung von Widerstand angedeutet. Auch die Frage nach Gerechtigkeit und Religion wird angesichts eines militärischen Fehlschlags in einer Episode behandelt - und als irrelevant zurückgewiesen, wenn Underwood dem Christusbild bescheinigt: „Liebe. Das ist Deine Message? Die kannst Du behalten!“ (House of Cards, S 3/F 4) 
Dissoziative wie assoziative Perspektiven setzen auf ein agonistisches Modell zur Wahrung des Politischen. Eine demokratische und liberale Gesellschaft muss demnach Institutionen schaffen, in denen Konflikte in Gegnerschaft ausgedrückt und so Identitäten und Leidenschaften mobilisiert werden können (Mouffe 2007: 42). The West Wing scheint diese Idee aufzugreifen, wenn zeitweise eine oppositionelle, republikanische Abgeordnete in das Ensemble des demokratischen ,Left Wing ' aufgenommen wird (z.B. The West Wing, S 2/F 4). Aber die Auseinandersetzung wird nie zum gleichberechtigten Konflikt, sondern bleibt ein einseitiger Prozess, in dem die Gegenspielerin ,deliberativ“ vom immer schon bekannten besseren Argument überzeugt wird (Rothöhler 2012). Das agonistische Experiment endet mit der Verbannung der Figur in den Keller und aus der Serie.

Die ,realistische“ Darstellung von Politik, die Polit-Serien einen Anspruch auf einen gewissen Mehrwert in der politischen Wissensvermittlung verleihen könnte, klammert also weitgehend ,das Politische‘ zugunsten ,der Politik‘ aus. Politik erscheint als politics, als Wettstreit zwischen Individuen um die Durchsetzung ihrer Interessen, während antagonistische und assoziative Momente unsichtbar bleiben. Damit tragen Polit-Serien zu einem Bild von Politik bei, das Entpolitisierung und antipolitische Haltungen fördert - und somit als Beitrag zur politischen Bildung nur unter Vorbehalt geeignet ist.

\section{DAS POLITISCHE IN DER SERIE ALS TRAGÖDIE}

\section{Stories statt Fakten: Tragödie statt Realismus}

Wenn der ,Mehrwert‘ im Sinne eines Lerngewinns für den Zuschauer über Politik nicht allein in den dargestellten Fakten über die reale Politik liegen kann, weil mit dem Politischen ein wichtiger Aspekt fehlt - und dieses Fehlen das vermittelte Wissen über Politik verzerren kann -, bietet möglicherweise die Geschichte, die vor der Kulisse der konventionellen Politik erzählt wird, einen Wissensgewinn. „If the world is made up of ,facts“ and ,stories“ that organize these ,facts', then there is no more important skill to pass on students than to make them better readers and writers of stories, better interpreters of not just ,facts“ but the organization of ,facts“." (Weber 2013: xxv)

Eine Möglichkeit, moderne fiktionale Formate als ,Story“ ernst zu nehmen und darin eine Möglichkeit der politischen Bildung zu erblicken, besteht darin, sie als Tragödien zu verstehen (Euben 2003, Euben 1994). Entsprechende Über- 
legungen knüpfen in der Regel an Hannah Arendts Betrachtung des Tragischen in der Politik an. ${ }^{7}$ Diese Überlegungen gründen wiederum in ihren Reflexionen zur Rolle des Theaters und der Tragödie für die Demokratie der klassischen griechischen Polis (Athen).

„Greek tragedy is thought by its modern interpreters to have played an important role, fostering critical reflection on community, while at the same time promoting a sense of belonging in community. Central to theorists' understanding of this role is the notion that Greek tragedy, in its novel combination of ritual (choral song and dance) and narrative elements (mythic tales of heroic exertion and doom), could provoke an aesthetic effect that intensified, in a mutually supportive way, theatergoers' dispositions to act for one's self without compromising the capacity of detachment from one's self that is a condition for acting with others in community.“ (Pirro 2001: 177)

Dem Theater und der Tragödie kommt in dieser Vorstellung eine wichtige Rolle in der politischen Bildung der Zuschauer als Bürger zu, die Politik um ihrer selbst willen als Lebenssinn betreiben, als Quelle ihrer Identität, statt als Mittel $\mathrm{zu}$ anderen Zwecken. ,[T]he actual content of political life [is] the joy and the gratification that arises out of being in company with our peers, out of acting together and appearing in public, out of inserting ourselves into the world by word and deed, thus acquiring and sustaining our personal identity and beginning something entirely new.“" (Arendt 1978: 263)

Politik als Selbstzweck ist damit unmittelbar an die Möglichkeit geknüpft, unter Gleichen aufzutreten und sich durch Worte und Taten auszuzeichnen. Die Polis ist als Lebensform deshalb essentiell, weil sie eine Bühne bietet, auf welcher der Einzelne sich auszeichnen kann. „The Greek polis was precisely that ,form of government" which provided men with a space of appearances where they could act, with a kind of theater where freedom could appear" (Arendt 1978: 154). Politik hat somit immer auch einen ,theaterhaften'A Aspekt.

Auch zum politischen Handeln gehört die Möglichkeit des Scheiterns. Nicht zuletzt darin besteht das tragische Element der Politik. Zu der ,threefold frustration of action“ (Arendt 1958: 20) gehören die Unvorhersehbarkeit der Ergebnisse, die Irreversibilität der angestoßenen Prozesse und die Anonymität des Autors (Pirro 2001: 54-55, Euben 2003: 56). Wer immer Initiative ergreift, wird nicht Herr der Ereignisse bleiben, denn das Handeln wird unvermeidlich Folgen nach sich ziehen, die nicht intendiert und nicht vorhersehbar waren und die sich nicht zurücknehmen lassen. In jedem Fall kann man zwar in Anspruch nehmen, eine

7 Für einen Überblick über entsprechende Ansätze siehe (Pirro 2001: 12-26). 
Kette von Ereignissen angestoßen zu haben, aber das Endergebnis ist in der Regel auf viele Ursachen und Verursacher zurückzuführen.

Die eindeutige Zuordnung von Ereignissen als Folge individuellen politischen Handelns - und somit die Möglichkeit für den Einzelnen, in der politischen Gemeinschaft hervorzutreten und sich in Worten und Taten auszuzeichnen - entsteht über das Erzählen von Geschichten. In Geschichten werden ,Fakten“ erst geordnet und als politische Handlung unterscheidbar - und als historische Ereignisse und Taten erinnerbar (Pirro 2001: 77-78). Die Fähigkeit, ,Geschichte zu schreiben', beruht auf der Möglichkeit, Geschichten zu schreiben und zu erzählen - und dazu sind das Theater und das Drama besonders geeignet.

„The specific quality of action and speech [...] is so indissolubly tied to the living flux of acting and speaking that it can be represented or ,reified“ only through a kind of repetition, the imitation or mimesis which according to Aristotle prevails in all arts but is actually appropriate only to the drama, whose very name (from the Greek verb dran, ,to act") indicates that play-acting actually is an imitation of acting." (Arendt 1958: 187)

Als institutionalisierte Form des gemeinschaftlichen Geschichtenerzählens bilden Theater und Drama somit die Voraussetzungen für politisches Handeln, indem sie die politische Handlung und seinen Urheber sichtbar und erinnerbar machen. Das dramatische Geschichtenerzählen bildet so den Kern des politischen Handelns als Quelle von Identität und der Möglichkeit, Neues zu schaffen, weil es eine Möglichkeit zeigt, dieses Handeln gegen die Vergänglichkeit und das Vergessen abzusichern, das allem von Menschen Gemachtem droht (eine weitere tragische Einsicht) (Euben 2003: 45-53).

Indem das Drama, und hier vor allem die Tragödie (dazu unten mehr), über die Identifikation des Zuschauers mit dem Protagonisten die Möglichkeit zeigt, wirksam zu werden und ein Vermächtnis zu hinterlassen, indem man sich im Wettstreit mit Gleichen auszeichnet (also Agonismus), ermutigt es zu politischem Handeln. Damit wirkt es gegen einen politischen Fatalismus wie auch gegen eine Reduktion der politischen Tätigkeit als Mittel zu anderen Zwecken (Euben 2003: 57). 


\section{,Underwood Tyrannos‘: House of Cards als Tragödie}

Dramen, also auch Tragödien, mit populärem Anspruch werden heute im Fernsehen erzählt. Polit-Serien stellen dabei über ihr Setting einen direkten Bezug zum weiteren politischen Kontext her, der die Zuschauer zur Reflexion einlädt. ${ }^{8}$

House of Cards bietet mit dem Protagonisten Francis Underwood ein besonders aussagekräftiges Beispiel, weil Underwood als idealtypischer Macchiavellist geradezu eine Verkörperung des Antipolitischen zu sein scheint. ${ }^{9}$ Aber Underwood geht es nicht um Macht als Selbstzweck, sondern darum, ein Vermächtnis zu hinterlassen:

„Money is the Mc-mansion in Sarasota that starts falling apart after 10 years. Power is the old stone building that stands for centuries. I cannot respect someone who doesn't see the difference." (House of Cards, S 1/F 2)

Der Kern dieses oft (auch auf T-Shirts) zitierten Underwood-Ausspruchs ist nicht ein absolutes Streben nach Macht, sondern der Wunsch, durch (macht-)politisches Handeln der Vergänglichkeit zu entgehen und einen bleibenden Eindruck in der Welt zu hinterlassen: „I will win and I will leave a legacy.“ (House of Cards, S 3/F 12)

In seinem Streben, sich hervorzutun und sich von seinesgleichen abzuheben, zeigt Underwood alle Züge des tragischen Helden, der sich durch seine Kompromisslosigkeit und seine Maßlosigkeit auszeichnet.

,[I]n Sophoclean tragedy the hero faces an issue on which he cannot compromise and still respect himself. [...] They have a profound sense of their own worth as individuals. And this exasperates the anger they feel at the world's denial of respect. In the crisis of their lives, abandoned by friends, ringed by enemies, unsupported by the gods, they have nothing to fall back on for support but this belief in themselves, their conception of their own unique character and destiny.“ (Knox 1964: 36)

8 Euben argumentiert. dass die klassischen Tragödien zwar implizite, aber für die zeitgenössischen Zuschauer im antiken Athen wahrscheinlich deutlich erkennbare Bezüge zu den aktuellen politischen Fragen der Zeit herstellten, wie etwa die Krise der athenischen Hegemonie zu Ende des Peloponnesischen Krieges (Euben 2003: 53-54).

9 Weitere und ausführlichere Beispiele, wie Tragödien politisch interpretiert werden können, zeigt Euben am Beispiel klassischer antiker Dramen (Euben 1994). 
Diese Maß- und Kompromisslosigkeit steht am Anfang der Tragödie House of Cards. Underwood bringt mit allen Mitteln der politischen Intrige und der Täuschung einen Präsidenten zu Fall - und geht dabei nicht nur im übertragenen Sinne, sondern buchstäblich über Leichen, weil ihm dieser Präsident den zugesagten Kabinettsposten als Secretary of State verweigert - ein Posten, der ihn aus seiner eher unsichtbaren und zuarbeitenden Funktion als Mehrheitsführer auf die Vorderbühne der Politik geführt hätte (House of Cards, S 1/F 1). Underwood kann nicht akzeptieren, dass ihm die Möglichkeit genommen wurde, sich auszuzeichnen - und wird zum Antipolitiker, der nur noch die eigene Macht als Ziel verfolgen kann (Meyer 2015). Das maßlose Selbstwertgefühl und die Isolation des tragischen Helden kommen exemplarisch in Underwoods ,Gebet‘ zum Ausdruck: ,There is no solace above or below, only us, small, solitary, striving, battling one another. I pray to myself, for myself." (House of Cards, S 1/F 13) ${ }^{10}$

House of Cards ist aber vor allem deshalb eine Tragödie, weil die Serie Underwoods Scheitern zeigt. Als (aufgerückter) Präsident findet er in Staffel 3 keine Mehrheit für das sozialpolitische Arbeitsprogramm America Works, sondern muss auf Rechtstricks und Dekrete zurückgreifen, um eine Minimalversion dieser policy einführen zu können. Seine Nahostfriedensinitiative zum Jordan-Tal scheitert an den Komplikationen der Außenpolitik - und nicht zuletzt am russischen Präsidenten Petrov (ebenfalls in der dritten Staffel). Petrov ist gewissermaßen das erfolgreiche Spiegelbild Underwoods, insofern er seine Machtpolitik unverhohlen autoritär durchsetzen kann. Im Gegensatz zu Underwood kann er dazu stehen, dass er selbst schon getötet hat (House of Cards, S 3/F 12) - und macht so deutlich, wie , unpolitisch“ Underwoods Morde sind, da sie als private Verzweiflungstat statt als politische Schachzüge aufgefasst/begangen wurden.

Das Scheitern ist kein Zufall. Seine politischen Machenschaften rufen Widerstände und Feindschaften hervor. Underwood wird von Untersuchungsausschüssen, Gerichtsverfahren und der Presse bedrängt. Seine Intrigen, Betrügereien und Morde machen immer weitere ,Verschleierungstaten" notwendig. Die Maßlosigkeit, mit der er sich an die Macht und in das Präsidentenamt befördert hat, drohen durchgängig über die Handlung der Serie hinweg, ihn wieder zu Fall zu bringen. Am Ende muss er angesichts eines drohenden Amtsenthebungsverfahrens in Staffel 5 gar zurücktreten, um sein Vermächtnis zu schützen - ein Zug, der ihn politisch schnell marginalisiert.

10 Die Qualität dieser Serie zeigt sich vielleicht auch darin, dass sie dieses Streben nach einem Vermächtnis mit der Figur des Thomas Yates als ,Hof-Tragiker' der Underwoods thematisiert (z.B. House of Cards, S 3/F 12). 
Der tragische Held handelt also nicht nur, sondern er leidet auch, weil jedes Handeln letztlich unkontrollierbare und nicht intendierte Konsequenzen hat. Es ist die Kehrseite des Drangs nach Auszeichnung.

„In the tragic perspective, acting and being an agent has a double character. On the one side it consists in taking counsel with oneself, weighing for and against and doing the best one can to foresee the order of means and ends. On the other hand, it is to make a bet on the unknown and incomprehensible and to take the risk on a terrain that remains impenetrable. "11

Indem sie uns das Leiden des Helden als Folge seines Handelns in einem überzogenen Drang, sich auszuzeichnen, zeigt, offenbart die Tragödie als Gattung ihre besondere politische Qualität. Die Inspiration zum Handeln könnten auch Heldenerzählungen etwa im Stile der Illias, oder modern in Form der Superheldengeschichten, aber auch ,Sicherheitsdramen“ wie etwa 24 oder Homeland (Dörner 2016: 6), bieten. Auch Action-Storys bieten Formen des Erinnerns und damit Inspiration zum Handeln.

„To understand the promise of Arendt's mode of tragic storytelling to foster kinds of heroism compatible with democratic community, attention should be given to the other dimension of the tragic hero, his or her role as doomed sufferer. This theory of tragedy raises an expectation that identification with the hero in this aspect will serve to train the imagination to achieve a reflective distancing from the press of need, the pull of desire, the partiality of vision characteristic to the claimant self." (Pirro 2001: 182)

In der Figur Underwood zeigt House of Cards einerseits, dass Politik eine lohnende Beschäftigung ist. Der Zuschauer identifiziert sich mit Underwood - und lässt sich möglicherweise inspirieren -, weil er zeigt, dass politisches Handeln möglich ist, dass der Einzelne sich auszeichnen, etwas Neues schaffen und ein Vermächtnis hinterlassen kann. Den Politiker Underwood darf man sich als glücklichen Menschen vorstellen. Es macht aber die Tragik Underwoods aus, dass er das gemeinschaftliche Moment des Politischen vergisst. Mit maßloser politischer Intrige und Betrug verlässt Underwood die agonistische Ebene des Wettstreits unter Gleichen und schafft Antagonismen. Er radikalisiert den politi-

11 Das Zitat wird in der einschlägigen Literatur häufig verwendet und meist ohne Seitenangabe Vernant/Pierre (1988) zugeschrieben. Leider findet sich das Zitat in dieser Form dort jedoch nicht. Möglicherweise handelt es sich um eine ältere Eigenübersetzung aus dem französischen Orginal. 
schen Raum und schafft sich Feinde, deren Widerstand ihn immer wieder zu Fall zu bringen droht (Shea 2015: 144-146).

Im tragischen Scheitern, im Erleiden, liegt paradoxerweise immer noch eine Ermutigung zum Handeln, weil in der Tragödie eine erzählens- und erinnernswerte Geschichte liegt. So betrachtet Arendt beispielsweis den gescheiterten Ungarn-Aufstand von 1956 als Tragödie, die aber zugleich zeigt, dass das Streben nach Demokratie lebendig ist (Pirro 2001: 82).

\section{Polit-Serien und die Politik der Tragödie}

In der Spannung zwischen Handeln und Erleiden, zwischen Machen und Scheitern, liegt der Kern der Tragödie. Die Tragödie beschränkt sich nicht auf eine unqualifizierte Feier des Handelns. Theater funktionierte in der antiken Polis als eine zweite Agora $^{12}$, als öffentliche Institution, die es der Gemeinschaft ermöglichte, ihre politischen Optionen in dramaturgisch gebrochener Version abzuwägen. Das Fehlen des Entscheidungsdrucks, der in der eigentlichen Agora herrschte, erlaubte eine umfassendere Betrachtung von Optionen und Implikationen angesichts der dramatisch präsentierten gesellschaftlichen und politischen Probleme (Euben 2003: 56).

Vor allem aber ermöglicht es das Betrachten der vorliegenden Probleme und Optionen aus der Perspektive anderer. Die Möglichkeit des unvoreingenommenen Blicks, die Möglichkeit, die Welt aus anderen Augen zu sehen, die Fähigkeit, unabhängiges Urteilen zu entwickeln, ist Teil der Erfahrung des Theaters (Euben 2003: 59). Die Tragödie schreibt nicht bestimmte Handlungen vor, sondern versucht, die Bedingungen des Handelns zu erfassen und zu reflektieren. Nicht das Lösen von Problemen steht im Vordergrund, vielmehr soll über die Tragödie soll ein tieferes Verständnis dieser Probleme gewonnen werden. „Arendt expected from story-telling not only the inspiration of glory-seeking publicly individuating heroes but the cultivation of citizens' capacities to see things form the points of view of their fellow citizens." (Pirro 2001: 139)

In Underwoods Streben und tragischem Scheitern können Bürger als $\mathrm{Zu}$ schauer reflektieren, wie eine Politik, die nur der maß- und kompromisslosen Verfolgung von Partikularinteressen dient, ihre eigenen Grundlagen in der Anerkennung durch Gleichgestellte untergräbt. Underwood ermöglicht den Blick auf einen politischen Menschen, der in einem auf Kompromiss und Konsens ausgerichteten politischen System keinen Weg findet, sich als Einzelperson aus-

12 Die Agora ist der Versammlungsplatz des antiken Athen, auf dem Volks-, Gerichtsund Heeresversammlungen stattfanden. 
zuzeichnen. In seiner maßlosen Gegenreaktion entwickelt er letztlich eine antipolitische Haltung, unterläuft oder zerstört die demokratischen Institutionen und versucht, sich zum Tyrannen aufzuschwingen: „Underwood. Underwood. 2016. 2020. 2024. 2028. 2032. 2036. One nation... Underwood.“ (House of Cards, S 5/F 4). In der tragischen Perspektive betrachtet, erweckt Underwood aber nicht einfach Unverständnis und Ablehnung, sondern bietet dem Betrachter eine Identifikationsmöglichkeit - ein Verständnis für den Wunsch nach Anerkennung und die Folgen ihrer Verweigerung (Kajtár 2015).

Als Tragödien können Polit-Serien so mehr als (mehr oder weniger realistisch bewertete) ,Geschichten über Politik' sein. Sie sind selbst Politik. Diese Politik der Tragödie (Euben 1994) fördert die demokratische Bildung der Bürger, indem sie zu politischem Handeln als Selbstzweck inspiriert und das kritische Denken über die gemeinschaftliche Bedingtheit dieses Handelns anregt. Die Polit-Serie als Tragödie schafft in den Begriffen der sozialwissenschaftlichen Literatur zur Frage des sozialen Zusammenhalts so einerseits die Voraussetzungen zum Erwerb sozialen Kapitals (Putnam 2001), indem sie zeigt, dass Anerkennung des eigenen Handelns und der eigenen Besonderheit möglich ist.

Zum anderen trägt sie über die kritische Reflexion zur Bildung von bridgingKapital (Putnam 2001: 22-23) bei, indem sie über die Identifikation mit dem Helden als Leidendem die Entwicklung eines überparteiischen Blicks ermöglicht. Auf diese Weise wirkt sie opportunistischen Tendenzen in der repräsentativen Demokratie entgegen und trägt zur Förderung gesellschaftlichen Zusammenhalts und Gemeinsinns bei (Pirro 2001: 185-186).

Eine ,tragische' Perspektive auf Polit-Serien als Instrument politischer Bildung, wie hier vorgeschlagen, wird durch Befunde der Rezeptionsforschung durchaus gestützt. Die Präsentation politischer Themen resultiert demnach weniger in einer direkten Beeinflussung politischer Einstellungen, sondern eher in der Bildung eines vergleichsweise offenen Raumes diskursiver Auseinandersetzung (Carpenter 2016). Insbesondere die fiktionale Darstellung von Politik in popkulturellen Formaten fördert eher eine kritische Distanzierung (Holland 2016). Der Politikwissenschaft kann hier eine neue Rolle zukommen, wenn sie Polit-Serien nicht länger nur als Untersuchungsgegenstand versteht, der Erkenntnisse über Politik ermöglicht (die dann wieder vermittelt werden), sondern mit Interpretationshilfen die politische Reflexion anhand der erzählten Geschichten unterstützt. Auf diese Weise kann die Befassung mit Polit-Serien (und anderen popkulturellen Erzeugnisse) möglicherweise der Politikwissenschaft wieder mehr Relevanz verleihen, die sie im Zuge einer zunehmend eng verstandenen Verwissenschaftlichung (Probst 2016) in der Konzentration auf Fakten verloren hat. 
Das Versprechen der Politik der Tragödie liegt folglich in einem Beitrag zur demokratischen Bildung der Zuschauer-Bürger. Das Setting der Polit-Serie in der konventionellen Politik bietet dabei einen Hintergrund, der besonders geeignet ist, politisches Denken anzuregen, weil er einen unmittelbaren Bezug zum ,realen' politischen Kontext wie ihn der Zuschauer kennt, schafft. So wird der Hyper-Realismus der Polit-Serie sogar zum Vorteil. Auf diese Weise können Polit-Serien als Tragödien über die Politik als Setting auf den Horizont des Politischen verweisen und mehr sein als Unterhaltung in einer beliebigen Kulisse.

\section{SCHLUSS}

Welchen Mehrwert bieten Polit-Serien dem Zuschauer im Lichte der oben geführten Diskussion? Können sie mehr als Unterhaltung in der Kulisse des Politikbetriebs sein?

Eine mögliche Antwort liegt darin, dass der Zuschauer aus Polit-Serien etwa über Politik lernen kann. Die fiktive Politik weist diesem Argument zufolge zwar aus dramaturgischen Gründen zahlreiche und für den Zuschauer auch erkennbare Unterschiede und Übertreibungen im Vergleich zur realen Politik auf. Zugleich ist sie aber realistisch genug, um Einblicke in die reale Politik zu vermitteln. Realismus ist bei Polit-Serien ein wichtiges Bewertungsmerkmal. Dies gilt sowohl für die öffentliche Rezeption und die Verwendung als popkulturelle Referenz wie auch für die wissenschaftliche Bewertung ihres Mehrwerts. Dieses Bewertungsmuster wird von Produzentenseite gefördert, die durch Einbeziehung von Experten und durch hyper-realistische Werbung die Realitätsnähe der Serien als Verkaufs- und Relevanzmerkmal hervorzuheben versucht.

Polit-Serien können in ihrem Realismus zur Vermittlung von Wissen über politische Prozesse und Institutionen, über Verfahren und im Einzelfall auch Politikfelder nützlich sein. Aber in der realistischen Darstellung, der Politik“ liegt auch eine Verzerrung begründet, indem , das Politische" und damit antagonistische wie auch assoziative Dimensionen ausgeblendet werden. Polit-Serien tragen so zur Reproduktion eines liberalen und instrumentalistischen Bilds von Politik bei, wenn man die Forschung zum Einfluss popkultureller Darstellungen auf politische Einstellungen betrachtet. Damit fügen sich Polit-Serien, wie auch die Politikwissenschaft (Probst 2016), in ein Politikverständnis, das antipolitischen Ressentiments Vorschub leisten kann.

Als Vehikel zur Vermittlung von Faktenwissen über reale Politik sind PolitSerien somit nur bedingt zu empfehlen und gewissermaßen nur ,unter Aufsicht* zu gebrauchen. Betrachtet man Polit-Serien aber nicht so sehr unter dem Aspekt der realistischen Faktenvermittlung, sondern mit Blick auf die Form der Erzäh- 
lung als Politik, können sie einen Beitrag zur politischen Bildung und zur Erziehung der Bürger als Demokraten leisten. Als Tragödien im politischen Setting ermutigen sie zu politischem Engagement, indem sie zeigen, dass das Individuum im politischen Handeln Geschichte schreiben kann, während sie zugleich eine kritische Reflexion der gemeinschaftlichen Bedingungen dieses Handelns anregen und so gesellschaftlichen Zusammenhalt befördern.

\section{LITERATUR}

Arendt, Hannah (1958): The Human Condition, Chicago.

Arendt, Hannah (1978): Between Past and Future, New York.

Baudrillard, Jean (2010): Simulacra and simulation, 17. Aufl., Ann Arbor.

Byron, Chris/Wood, Nathan (2015): „Money Gives Power ... Well, a Run for Its Money“: Marx's Observations on Why Capital and Not Frank Is Really in Charge of the White House, in: Hackett, J. Edward (Hrsg.): House of Cards and Philosophy, Chichester, S. 152-162.

Carpenter, Charli (2016): Rethinking the Political/-Science-/Fiction Nexus: Global Policy Making and the Campaign to Stop Killer Robots, in: Perspectives on Politics 14 (1), S. 53-69.

Deacon, David/Stanyer, James (2014): Mediatization: key concept or conceptual bandwagon?, in: Media, Culture \& Society 36 (7), S. 1032-1044.

Dörner, Andreas (2006): Politik als Fiktion, in: Aus Politik und Zeitgeschichte 56 (7), S. 3-11.

Dörner, Andreas (2016): Politserien: Unterhaltsame Blicke auf die Hinterbühnen der Politik, in: Aus Politik und Zeitgeschichte 66 (51), S. 4-11.

Eco, Umberto (2014): Travels in Hyperreality, San Diego.

Erickson, Christian W. (2007): Counter-terror culture: Ambiguity, subversion, or legitimization?, in: Security Dialogue 38 (2), S. 197-214.

Euben, J. Peter (1994): The Tragedy of Political Theory: the Road not Taken, 2. übera. Aufl., Princeton.

Euben, J. Peter (2003): Platonic Noise, Course Book, Princeton.

Fallis, Don (2015): Machiavelli Would Not Be Impressed, in: Hackett, J. Edward (Hrsg.): House of Cards and Philosophy, Chichester, S. 92-101.

Glas, Jeffrey M./Taylor, J. Benjamin (2017): The Silver Screen and Authoritarianism: How Popular Films Activate Latent Personality Dispositions and Affect American Political Attitudes, in: American Politics Research, Online first, S. 1-30. 
Gray, John Scott (2015): Being versus Seeming: Socrates and the Lessons of Francis Underwood's Asides, in: Hackett, J. Edward (Hrsg.): House of Cards and Philosophy, Chichester, S. 16-27.

Hanfeld, Michael (2013): Der Haifisch liebt das Blut, in: Frankfurter Allgemeine Zeitung vom 9.2., online unter http://www.faz.net/aktuell/feuilleton/ fernsehserie-house-of-cards-der-haifisch-liebt-das-blut-12655049.html (zugefriffen am 07.02.2018).

Hardt, Maria-Xenia (2016): Serienversteher: „Scandal“. Blogseminar, http://blogs.faz.net/blogseminar/serienversteher-scandal/ (zugegriffen am 07.02.2018).

Heck, Axel/Schlag, Gabi (2015): „And... Cut!“‘. In: Zeitschrift für internationale Beziehungen 22 (2), S. 125-148.

Hochman, David (2015): What Bill Clinton told Kevin Spacey about ,House of Cards', in: Gotham vom 20.4.

Holland, Jack (2016): Visual Literacy in International Relations: Teaching Critical Evaluative Skills through Fictional Television, in: International Studies Perspectives 17 (2), S. 173-186.

Kajtár, László (2015): Rooting for the Villain: Frank Underwood and the Lack of Imaginative Resistance, in: Hackett, J. Edward (Hrsg.): House of Cards and Philosophy, Chichester, S. 227-236.

Kelleter, Frank/Jahn-Sudmann, Andreas (2014): „Eine interessante Affinität zwischen dem seriellen Erzählen und dem Thema Politik." Von Soap Operas zum Quality TV, in: INDES, 3 (4), S. 5-22.

Knox, Bernard MacGregor (1964): The Heroic Temper: Studies in Sophoclean Tragedy, Berkley.

Lasswell, Harold D. (1936): Politics: Who Gets What, When, how, New York/London.

Littmann, Greg (2015): American Machiavelli, in: Hackett, J. Edward (Hrsg.): House of Cards and Philosophy, Chichester, S. 81-91.

Marchart, Oliver (2010): Die politische Differenz: zum Denken des Politischen bei Nancy, Lefort, Badiou, Laclau und Agamben, Berlin.

Meyer, Matt (2015): Why Underwood Is Frankly Not an Overman. House of Cards and Philosophy, in: Hackett, J. Edward (Hrsg.): House of Cards and Philosophy, Chichester, S. 68-80.

Mouffe, Chantal (2007): Über das Politische: wider die kosmopolitische Illusion, Frankfurt a.M.

Patzelt, Werner J. (2007): Einführung in die Politikwissenschaft: Grundriß des Faches und studiumbegleitende Orientierung, 6. übera. u. erw. Aufl., Passau. Pirro, Robert (2001): Hannah Arendt and the Politics of Tragedy, DeKalb. 
Probst, Lothar (2016): Was ist Politik, in: Blätter für deutsche und internationale Politik (10), S. 105-114.

Putnam, Robert D. (2000): Bowling Alone: The Collapse and Revival of American Community, New York.

Rothöhler, Simon (2012): The West Wing, Zürich.

Sartori, Giovanni (1973): What is „Politics“, in: Political Theory 1 (1), S. 5-26.

Searle, John R. (1997): Die Konstruktion der gesellschaftlichen Wirklichkeit: zur Ontologie sozialer Tatsachen, Reinbek bei Hamburg.

Shea, Brendan (2015): „Democracy Is So Overrated“: The Shortcomings of Popular Rule. House of Cards and Philosophy, in: Hackett, J. Edward (Hrsg.): House of Cards and Philosophy, Chichester, S. 141-151.

Sneed, Tierney (2014): Explaining That Bonkers ,House of Cards“ Senate Sequence, in: U.S. News am 14.2, online unter https://www.usnews.com/ news/articles/2014/02/13/explaining-frank-underwoods-bonkers-house-ofcards-senate-maneuver (zugegriffen am 07.02.2018).

Steinmetz, Vanessa (2015): Der bessere Trump, in: Spiegel Online am 16.12., online unter http://www.spiegel.de/kultur/tv/a-1068074.html (zugegriffen am 07.02.2018).

Trittin, Jürgen (2014): Wer das Feuer liebt, in: Der Freitag am 9.2., Ausgabe 2, online unter https://www.freitag.de/autoren/der-freitag/wer-das-feuer-liebt (zugegriffen am 07.02.2018).

Vernant, Jean-Pierre/Pierre, Vidal-Naquet. (1988): Tragedy and Myth in Ancient Greece, New York.

Walton, Kendall L. (1993): Mimesis as Make-Believe. In the Foundations of the Representational Arts, Cambridge.

Weber, Cynthia (2013): International Relations Theory: A Critical Introduction, London.

Wodak, Ruth (2011): The Discourse of Politics in Action: Politics as Usual, Basingstoke. 



\title{
Filme zwischen künstlerischer Freiheit und politischer Erkenntnis
}

\author{
Zum Realitätsgehalt fiktionaler Medien
}

\section{Manfred Mai}

\section{EINLEITUNG}

Einer der ältesten Diskurse in der Kunst ist der nach ihrem Verhältnis zur Realität. Sind Kunstwerke Spiegel der Realität oder konstituieren sie eine eigene Realität? Und: Welche Realität bilden sie ab? Die innere (die ,Seele', die Psyche, die Fantasien, die persönlichen Konflikte) des Künstlers oder die äußere (die Gesellschaft, die politische Struktur, die Ökonomie), in der der Künstler lebt?

Künstler leben in ihrer Zeit. Sie sind eingebunden in Netzwerke mit anderen Künstlern, wo sie z.B. einen bestimmten Stil entwickeln, sie haben Beziehungen zu Freunden, Mäzenen, Akademien, Kunsthändlern, Auftraggebern und nicht zuletzt auch mit der Politik. Nicht wenige sind politisch engagiert und verstehen ihr Werk als politisch. Die Surrealisten um André Breton, Luis Buñuel, Salvador Dalí u.a. sahen sich in den 1920er Jahren im Lager der Kritiker gegen die bürgerliche Gesellschaft. Mit ihren Filmen Ein andalusischer Hund (1929) und Das goldene Zeitalter (L'age d'or) (1930) schrieben sie Filmgeschichte. Aus ihrer Sicht sagten sie die Wahrheit über die bürgerliche Gesellschaft und die Kirche als eine ihrer Stützen. Viele Filme Buñuels kritisieren immer wieder das katholische Spanien und die reaktionäre Gesellschaft unter der Diktatur Francos. „In a world as badly made as ours there is only one road - rebellion. " So sah Luis Buñuel die Welt und so waren seine (frühen) Filme: Rebellion gegen die bürgerliche Gesellschaft.

Buñuel zeigt in L'age d'or alles, was er hasst: heuchlerisches und bigottes Großbürgertum, verlogenen Nationalismus und den ,,institutionalisierte[n] Katholizismus als ideologische[n] Überbau“ (König o.J.). Erfahren wir durch die- 
sen und andere Filme Buñuels mehr über die ,Wahrheit' der spanischen Gesellschaft in den 1920er Jahren oder sehen wir nur seine subjektive Sicht auf diese Welt? Sagen die zahlreichen Filme, Dokumentationen und Romane ${ }^{1}$ aus dem spanischen Bürgerkrieg mehr über den Faschismus aus als die empirischen Studien des Instituts für Sozialforschung (Horkheimer/Adorno)? Immerhin haben diese Studien Standards in der empirischen Sozialforschung gesetzt.

Die Frage nach dem Verhältnis zwischen Film und Realität wurde bereits zu Beginn des Filmzeitalters gestellt ist auch heute in Bezug auf Serien, die wie der Film audiovisuelle Produkte sind, aktuell. Diese Debatte geht wiederum auf die viel ältere Debatte innerhalb der Literaturwissenschaft zurück, wie sich Dichtung und Wahrheit zueinander verhalten. Können Methoden der qualitativen und quantitativen Sozialforschung die ,Wahrheit' oder wenigsten die Realität der Politik erfassen? Oder ist es die Freiheit künstlerischen Schaffens, die mit einem gelungenen Werk die ,Wahrheit' und Realität der Politik auf den Punkt bringt ganz ohne Methodenkritik, valide Indikatoren und statistische Vertrauensmaße? Ist das Bild, das z.B. die Serie House of Cards und Filme wie Mr. Smith geht nach Washington, Staatsfeind Nr. 1 oder Thirteen Days über die amerikanische Politik zeigen, näher an der Realität als Studien über Entscheidungsstrukturen, Handlungsoptionen und Nutzenkalküle von Regierungsakteuren? Im Folgenden soll diese Frage genauer erörtert werden.

Bei dem Vergleich zwischen der empirischen Realität der Politik einerseits und der im Film gezeigten ,Realität“ der Politik andererseits muss der grundlegende Unterschied zwischen diesen beiden Realitäten immer bedacht werden: Filme sind als fiktionale Produkte ,nur im Ansatz der Realität verpflichtet“, ansonsten ,einzig und allein der Fantasie, die das Konkret-Politische/Soziale eines Landes zu einer bestimmten Zeit in existenzielle Befindlichkeiten generalisiert. Das heißt, die Filme sind stets sozial verankert, gleichzeitig aber schicksalhaft verdichtet." (Grob 2008: 9) Empirische historische, biografische und sozialwissenschaftliche Studien werden daher immer zu anderen Ergebnissen kommen als Regisseure, Drehbuchautoren oder Romanschreiber, die über den gleichen Gegenstand wie etwa ein bestimmtes politisches Regime schreiben. „Aber von großer Literatur ist [...] zu erfahren, wie die feinen psychologischen, sozialen, persönlichen und nationalen Prozesse ablaufen - Prozesse, die den Mechanismus diktatorischer Regime und den Charakter brutaler und manchmal auch verrückter Despoten geformt und hervorgebracht haben." (Grossmann 2017) Das gilt auch für Filme.

1 Die bekanntesten sind Max Aubs Romanzyklus Das magische Labyrinth, George Orwells Roman Mein Katalonien und Ernest Hemingways Wem die Stunde schlägt. 


\section{KUNST UND FILME ALS SPIEGEL DER REALITÄT}

Die Frage, ob Filme die Realität wiedergeben oder sogar einen Erkenntnischarakter haben und damit mehr sind als ein bloßes Unterhaltungsmedium, betrifft auch andere Künste - insbesondere die Literatur mit ihren Gattungen Lyrik, Epik und Dramatik. Vor allem dem Roman wird in der Literaturwissenschaft ein Erkenntnischarakter zugesprochen. Der Essay, sofern man ihn als vierte Gattung neben den drei klassischen akzeptiert, kommt einer wissenschaftlichen Abhandlung am ehesten recht nahe. Essayisten wie Michel de Montaigne haben diese vierte Literaturgattung - neben den drei klassischen - zu einer eigenständigen Kunstform entwickelt. Was ist an einem Roman, an einem Gedicht oder an einem Drama Realität und was heißt das für den Film, der zu keiner dieser Gattungen gehört?

Bereits in der Antike wurde über das Verhältnis zwischen Kunst und ,Wahrheit' reflektiert und darüber, ob Künstler eine höhere Wahrheit verkünden, da sie offenbar von rauschhaften, ,dionysischen “ und göttlichen Eingebungen inspiriert sind. Auf keinen Fall wurden von den antiken Philosophen die zeitgenössischen Tragödien und Skulpturen als Widerspiegelung der Realität gesehen. Erst im 19. Jahrhundert wurde die Frage, ob Kunst die Realität der ökonomischen und gesellschaftlichen Realität wiedergebe, u.a. von Karl Marx, der ein Kenner der antiken Kunst war, aufgeworfen und von marxistischen Kunst- und Literaturphilosophen aufgegriffen. Für sie stand fest, dass die Künste den ideologischen Überbau über die materielle Basis bildeten. In den Romanen von Honoré de Balzac und denen von Charles Dickens sah man das hässliche Bild des Kapitalismus mit seiner zerstörerischen Kraft für die menschlichen Beziehungen (Demetz 1969).

Die materialistische Widerspiegelungstheorie war bis zum Ende des Sowjetregimes die offizielle Linie der Kunst- und Literaturtheorie, die auch an westlichen Universitäten viele Vertreter hatte. Alle anderen Theorien galten aus dieser Sicht als ,bürgerlich', da sie den kreativen Künstler mit seinen Phantasien und seiner Individualität in den Mittelpunkt stellte und nicht den Grundwiderspruch der bürgerlichen Gesellschaft, der sich im Klassenkampf zeige und der den Künstler zwinge sich zu positionieren. Mit den Werken von Baudelaire, Rimbaud, Rilke oder Kafka, die von einer radikalen Subjektivität ausgehen und jede Verbindung zur äußeren Welt zugunsten der Erschaffung einer eigenen Welt abbrechen, kann die Widerspiegelungstheorie nichts anfangen, zumal sich deren Werke nicht für den politischen Kampf eignen.

Es war daher nur konsequent, dass die sowjetische Kulturpolitik von ihren Filmemachern Filme verlangte, die ,realistisch“ sind und einen klaren Propagandazweck erfüllen. Diese Forderung erfüllten Sergei Eisenstein mit seinen Filmen 
Panzerkreuzer Potemkin und Streik, und Dziga Vertov mit seinen Dokumentationen und Wochenschauen. Beide Regisseure wurden damit zu Paradigmen des ,sozialistischen Realismus` im Bereich des Films. Die Frage ist, was an diesen und vielen anderen Filmen des sozialistischen Realismus eigentlich realistisch ist. Schon zu Lebzeiten musste Eisenstein erleben, dass Stalin mit seinen Filmen Oktober (weil er Lenin mehr als Stalin würdigt) und Iwan der Schreckliche unzufrieden war (diesmal, weil er zu viel an Stalin erinnert). Die Folgen waren Zensur und Aufführungsverbot. Diese Filme aus den 1920er Jahren sind das Ergebnis der Vorgaben und Erwartungen seiner Auftraggeber, denen jedoch viele Filmemacher und Autoren gerne nachkamen, da sie sich freiwillig in den Dienst der Revolution stellten. Auch für viele Künstler aus dem Westen war die russische Revolution 1917 die Hoffnung auf eine bessere Welt. Für sie war die Welt so, wie sie sie in ihren Werken glaubten objektiv widerzugeben. Dabei stehen die ästhetische Qualität und die an den Expressionismus erinnernde Form sowie die innovative Schnitttechnik der Filme Eisensteins auch aus heutiger Sicht außer Frage. ${ }^{2}$

Die zur gleichen Zeit entstandenen deutschen Filme waren das genaue Gegenteil des sozialistischen Realismus. Ihre Revolution beschränkte sich weitgehend auf die ästhetische Form: In diesem Sinne revolutionär waren die Filme von Friedrich Murnau (Nosferatu), Fritz Lang (Dr. Mabuse, der Spieler) oder von Robert Wiene (Das Cabinet des Dr. Caligari). Die Inhalte dieser Filme waren reine Fantasie ohne politische Botschaft. Der Dokumentarfilm von Walther Ruttmann (Berlin - Die Sinfonie der Großstadt) fand wegen seiner avantgardistischen Form - und trotz seines unpolitischen Inhalts - in der zeitgenössischen Kritik ein geteiltes Echo: Die eine Seite (u.a. Sigfried Kracauer) „monierte, dass der Film sich an Formen berausche und nur die Oberfläche zeige. [...] Begeistert hingegen zeigt sich Rudolf Kurtz: ,Das ist die Großstadt, wie sie ein Künstler er-

2 Wie in der damaligen Sowjetunion unter Stalin wird auch im heutigen Russland Eisensteins Homosexualität verschwiegen. In dem Film Eisenstein in Guanajuato von Peter Greenaway wird das Coming-Out Eisensteins sehr explizit dargestellt. Die russische Filmförderung, der Gosfilmofond, weigerte sich, an der Finanzierung dieses Films teilzunehmen, ,wenn - wie dessen Direktor es formulierte - ,Details über Eisensteins nicht traditionelle sexuelle Orientierung ( Homosexualität wurde 1936 in der Sowjetunion zu einem Verbrechen erklärt, und vor zwei Jahren [2013] verabschiedete die Duma ein Gesetz, das ,homosexuelle Propaganda ' in den Medien unter Strafe stellt.“ (Rodek 2015) Dieses Beispiel zeigt, dass nicht nur Filme, sondern auch die Biografien ihrer Regisseure dem sozialistischen Realismus angepasst wurden, um als Vorbild für die Massen zu gelten. 
fühlt, eine Gestaltung aus Eisen, Blut und Licht - erfüllt von dem mächtigen Brausen des Lebens, das von diesem Film in das Parkett überspringt und es überwältigt."،3

In dieser zeitgenössischen Kritik an Ruttmanns Film werden die beiden verschiedenen Positionen auf die Frage nach dem Realitätsgehalt von Filmen sichtbar, die sich unversöhnlich gegenüberstehen: auf der einen Seite die Kritik an der vermeintlichen ,Oberfläche', auf der anderen Seite das Lob für die Darstellung, wie sie der Künstler erfühlt‘. Eine vermittelnde Position scheint kaum möglich. Entweder man sieht in dem Film das Abbild einer hinter der ästhetischen und verschleiernden Oberfläche verborgenen gesellschaftlichen Realität, oder man erkennt an, dass Filme ihre eigene Realität haben, die eher zufällig auf die Gesellschaft verweist, womit der Versuch, etwas durch diesen Film über die Gesellschaft zu erfahren, sinnlos ist. Zudem wird unterstellt, dass ,Realität ' im Sinne des sozialistischen Realismus beim Zuschauer eine eindeutige Wirkung hat, nämlich die, dass die Korruptheit der bürgerlichen Gesellschaft (des Kapitalismus, des Imperialismus, der herrschenden Klasse) entlarvt und dass nur durch eine klare und aktive Positionierung jedes Einzelnen gegen diese Realität eine bessere - sozialistische - Welt möglich wird.

Filme wie Frank Beyers Spur der Steine verschwanden daher in der DDR kurz nach ihrer Premiere, da sie nicht nur Widersprüche im Alltag der DDR zeigten (moralische Verfehlungen eines Funktionärs, Mängel in der Organisation auf dem Bau, menschliche Schwächen), sondern auch keine ,Perspektive‘ für den Zuschauer lieferte. Willkommen waren der SED-Führung dagegen Filme wie Professor Mamlock, Fünf Patronenhülsen oder Ich war neunzehn, die die DDR als antifaschistischen Staat inszenieren und die damit die DDR gegenüber der Bundesrepublik abgrenzen. Dennoch waren nicht alle Produktionen der DEFA $^{4}$ im Sinne der Partei. So wurde der Film Berlin - Ecke Schönhauser als Antwort der DEFA auf Jugendfilme aus den USA (... denn sie wissen nicht, was sie tun) und der Bundesrepublik (Die Halbstarken) verstanden:

„Lakonisch im Gestus, genau in der Beschreibung des Lebensgefühls einer Generation zwischen den Fronten, kritisch gegenüber gesellschaftlichen Fehlentwicklungen in der

3 Berlin. Die Sinfonie der Großstadt, in: Stummfilmkonzert.de - Glossar, http://www. stummfilmkonzerte.de/glossar/stummfilme/berlinsinfoniedergrossstadtlang.html (zugegriffen am 6.12.2017).

41946 erhielt die Deutsche Film AG (DEFA) die Lizenz für die Herstellung von Filmen in der damaligen Sowjetischen Besatzungszone, der späteren DDR. Insgesamt hat die DEFA 700 Kinofilme produziert. 
DDR. Die staatliche Filmkritik [der DDR] reagierte prompt: Sie bemängelte eine ,zu große Konzession an den italienischen Neorealismus‘. Dieser brächte die Menschen in Opposition zum Staat, sei also unbrauchbar im Sozialismus, der nur , lösbare Widersprüche vorübergehender Art' kenne. Gerade die ,Konzession“ an den Neorealismus macht diesen Film und die anderen sogenannten Berlin-Filme von Gerhard Klein und Wolfgang Kohlhaase auch heute noch zu einem filmischen Ereignis und zeigt auf, welchen Weg die DEFA hätte gehen können. “5

Interessant ist die parteioffizielle Feststellung, dass dieser Film für den Sozialismus unbrauchbar ist, der nur ,lösbare Widersprüche vorübergehender Art“ kennt. Nicht nur aus heutiger Sicht ist die These plausibel, dass die Filme des italienischen Neorealismus (Rom - offene Stadt, Bitterer Reis, Fahrraddiebe) vermutlich mehr Menschen die Gedanken des Sozialismus nähergebracht haben als die Filme der DEFA.

Dass auch unpolitische Filme eine politische Funktion haben können und damit auch politisch sind, nutzte vor allem die Filmpolitik des nationalsozialistischen Deutschland. Gaben nach der ,Machtergreifung' der Nazis zunächst noch Propagandafilme wie Hitlerjunge Quex, Ohm Krüger oder Carl Peters den Ton an, folgten später vor allem Unterhaltungsfilme und Komödien. Ihr Zweck war, Ablenkung und Unterhaltung zu bieten. Nichts sollte die Zuschauer an den Kriegsalltag erinnern. Dass diese Filme zum Teil gut gemacht waren - der Film Leni Riefenstahls über die Olympischen Spiele 1936 setzte darüber hinaus auch filmtechnische Maßstäbe ${ }^{6}$ - ändert nichts am eigentlichen Zweck dieser Filme für den Erhalt des ,Dritten Reichs‘. Je unpolitischer und je lustiger es in diesen Filmen (Die Feuerzangenbowle, Glückskinder) zuging, umso leichter schien es, den Alltag während des Krieges zu ertragen. ${ }^{7}$ Goebbels wollte, so Quentin Tarantino in einem Interview „Entertainment, Comedies, Operetten, Musicals“ (Rodek 2009).

5 Berlin - Ecke Schönhauser, http://www.mdr.de/home/sendung655362_ipgctx-true_zc6569ce81_zs-48c98c3d.html (zugegriffen am 6.12.2017).

6 Hitlers Lieblingsregisseurin ,setzt filmische Maßstäbe für Aufnahme und Schnitt, erfindet neue Kameratechniken. Sie bugsiert die Kamera auf Schienen, in Aufzüge und Gräben. [...] Keine Parfumwerbung kommt mehr ohne ihren Stil aus, kein Dokumentarfilm mehr ohne ihre Technik, keine Sportfotografie ohne ihren Einfluss.“ (Brauer 2008).

7 Unterhaltung und Ideologie im NS-Film, Filmportal.de, http://www.filmportal.de /thema/unterhaltung-und-ideologie-im-ns-film (zugegriffen am 6.12.2017). 


\section{FILME ZWISCHEN KUNST UND UNTERHALTUNG}

Der Film wurde 1893 in Frankreich erfunden. Bereits in seiner Geburtsstunde war er von den Paradigmen Kunst und Unterhaltung geprägt und zwischen Dokumentation und Inszenierung angesiedelt. Während die Gebrüder Lumière - die eigentlichen Erfinder - den Film als verbesserte Fotografie sahen ${ }^{8}$ und vor allem technisch weiterentwickelten, entdeckte George Méliès das Prinzip der Inszenierung durch fantasievolle Arrangements: Fiktion statt Dokumentation. Obwohl Méliès auch Dokumentationen produzierte, gilt er als Erfinder des narrativen Films. Er „hatte bewiesen, dass die Kamera mehr kann als gleichgültige Reportage fotografieren" (Waldekrenz/Arpe 1956: 56).

Filme sind mehr als andere Kunstwerke arbeitsteilig hergestellte Produkte. Eine besondere Bedeutung für den Inhalt haben Produzenten, Regisseure und Drehbuchautoren. Aber auch alle anderen an der Herstellung beteiligten Akteure haben Einfluss auf die Qualität eines Films. Kameraleute, Beleuchter, Filmarchitekten, Maskenbildner, Filmkomponisten, Cutter, Toningenieure u.a. verstehen sich ebenfalls als künstlerisch ambitionierte Professionals mit hohem Anspruch an ihre Tätigkeit. ${ }^{9}$ Ohne die Mitwirkung von Studios, Casting-Agenturen, Filmversicherungen und -verleihern, Technikern, Marketing und nicht zuletzt ohne staatliche Filmförderung käme kein Film ins Kino. Durch die Digitalisierung des Films erhalten Softwarespezialisten und Techniker eine zunehmende Bedeutung. Über den Erfolg eines Films entscheidet dennoch oft weniger die Qualität aller dieser Beteiligten, sondern die Prominenz der Hauptdarsteller. So wie sich Cineasten jeden Film eines Kultregisseurs anschauen, wollen viele Zuschauer jeden Film ihres Stars sehen. Dennoch spielt bei der Beurteilung von Filmen fast nur der Regisseur eine Rolle, obwohl die Regisseure selbst immer wieder ihre Abhängigkeit von Produzenten, Drehbuchautoren und Schauspielern betonen (Schnakenberg 2010).

In den 1920er Jahren wurde die Eigenart dieser neuen Kunst definiert. Der Lyriker und Regisseur René Clair gibt einen Überblick über die damalige Diskussion (Clair 1995: 16). Jean Cocteau - ebenfalls Dichter und Regisseur meinte, dass der Film in einem Engpass stecke: „Schon am ersten Tag, als noch die Erfindung blendete, griff der Irrtum um sich: man fotografierte Theater. Mit der Zeit ist daraus verfilmtes Theater geworden, aber nie echter Film." (Clair

8 Allein die nur wenige Minuten dauernden gefilmten Alltagsszenen der Gebrüder Lumière begeisterten die zeitgenössischen Zuschauer.

9 So wird etwa der der bedeutendste Filmpreis, der Oscar, in verschiedene Kategorien (Schnitt, Drehbuch, Musik, Spezialeffekte u.a.) verliehen. 
1995: 17). Paul Valéry war der Ansicht, dass man zum „reinen“ Film gelangen müsste, „nämlich zu einer von eigenen Mitteln ausgehenden Kunstform; und diese Kunstform sollte zu jenen anderen, die es mit dem Wort halten, Theater oder Roman, einen bewussten Gegensatz bilden.“(Clair 1995: 19) René Clair resümierte die Ansichten seiner zeitgenössischen Künstlerkollegen: „Der Film ist ein autonomes Ausdrucksmittel, das seine Zukunft in sich selbst trägt" (Clair 1995: 19). Und noch etwas anderes erkannte Clair:

„Dichtung, Musik und die bildenden Künste schienen auf dem Weg, esoterische Bereiche zu werden: Dichtung für Literaten, Musik für Musiker, Malerei für Maler; uns es sah so aus, als sei die Öffentlichkeit von einem Spiel ausgeschlossen, dessen Regeln nur noch die Spezialisten beherrschten. [...] Der Film hingegen war für die Masse, er brauchte sie, um zu bestehen, und es gab Streifen, die sowohl anspruchsvolle wie breite Kreise beeindruckten. [...] Aber der schlimmste Irrtum, dem wir in unserem Taumel verfielen, war der, dass wir für alle Zukunft eine bestimmte Filmkunst im Auge hatten und nicht darauf gefasst waren, dass technische Neuerungen ihre Züge verändern können.“(Clair 1995: 27)

Dabei hatte Clair nur an die Erfindung des Tonfilms (1927) gedacht.

Die Frage, ob Film auch eine Kunst ist, wurde schließlich positiv beantwortet. Viele Kunstkritiker und -philosophen sahen im Film eine Nähe zum Theater und nicht wenige Stummfilme erinnerten an Theaterinszenierungen, bevor die kreative Anwendung von Perspektivwechseln, Schnitten, bewegter Kamera u.a. Techniken dem Film eine eigene Identität gaben. Susan Sontag stellte noch 1965 die Frage, ob ,der Film Nachfolger, Rivale oder Erneuerer des Theaters“ ist, und konstatierte, dass ,diejenigen, die den Tod des Theaters voraussagen und meinen, dass der Film seine Funktion übernommen hat, dazu neigen, eine Beziehung zwischen Film und Theater als gegeben zu betrachten, die an jene Beziehung erinnert, die man einst zwischen Fotografie und Malerei sehen wollte" (Sontag 2015: 228).

Schon zu Beginn des Films zeichnete sich ab, dass der größte Teil der Filmproduktion der Massenunterhaltung dient. Wurden Filme um 1900 noch als Pausenfüller in Varietés und auf Jahrmärkten gezeigt (Klooss/Reuter 1980, Engell 1992: 32), entstanden bald darauf eigenständige Abspielstätten (Kinos) und groBe Produktionsstätten (Studios). Anfang des 20. Jahrhunderts gab es in fast allen europäischen Hauptstädten und vor allem in Hollywood eine Filmindustrie, die die gesamte Wertschöpfungskette von der Herstellung bis zum Verleih umfasste (Engell 1992: 75, Waldekrenz/Arpe 1956: 54). Bereits in der Stummfilmzeit war die Dominanz Hollywoods bei der Produktion von Filmen erkennbar, was auch eine Folge der Sehgewohnheiten war: „Der Stil des englischen Spielfilms war 
und verblieb theatralisch und steif. Wie der französische Film, so verlor auch der englische nach Schluss des Krieges sein Publikum mehr und mehr. Auf breiter Front rückte der amerikanische Film vor, und es wurde unmodern, sich englische Filme anzusehen.“ (Waldekrenz/Arpe 1956: 218) Trotz dieser eindeutig am Massenmarkt und -geschmack orientierten Filmproduktion haben sich Filme als eigenständige Kunstform entwickelt. Vor allem in der EU werden künstlerisch ambitionierte Filme gefördert, um sie gegen die Dominanz US-amerikanischer Produktionen zu schützen.

Es waren vor allem die Erfahrungen mit den industriellen Dimensionen der amerikanischen Filmindustrie, die Günther Anders (2002) ${ }^{10}$, Theodor W. Adorno und Max Horkheimer (1971) zu ihrem Urteil veranlassten, dass Filme als Produkte der Unterhaltungs- und Kulturindustrie nur den Zweck der Ablenkung und damit der Stabilisierung des bürgerlich-kapitalistischen Systems dienen. Dieses Verdikt der Kritischen Theorie gegenüber allen Produkten der populären Künste hat Generationen von Kulturwissenschaftlern und Filmkritikern geprägt. Eine Folge dieser Sichtweise ist die Trennung der Filmproduktion in kommerzielle Massenware einerseits und in Filmkunst andererseits. Abgesehen davon, dass diese idealtypische Trennung bei der Masse der Filmproduktionen kaum Sinn macht, können massenkompatible Kommerzfilme mehr über Gesellschaft und Politik aussagen als elaborierte Filmexperimente, die die Filmkritik als avantgardistisch feiert und die die Filmwissenschaft als Paradigma einer Theorievariante des Konstruktivismus entdeckt (Sanders 2006). Es ist fast schon die Regel, dass Filme, die auf Festivals ausgezeichnet wurden, in den Kinos keine $\mathrm{Zu}$ schauer finden - was die staatlichen Filmfördereinrichtungen dieser Filme wiederum zum Anlass nehmen, ihre Förderung vom erwarteten Erfolg (d.h. Zuschauerzahlen) abhängig zu machen.

Diese kulturkritische Sicht und Verachtung des Populären seitens der ,Frankfurter Schule' wurde durch die cultural studies aufgebrochen. Dieser sehr heterogene Forschungsansatz sieht sich zwar wie die Autoren der Kritischen Theorie auch im linken, antikapitalistischen Lager. Aber er entdeckt gerade in der Popu-

10 Günther Anders hat mit seinem Verdikt über das Fernsehen die radikalste Medienkritik geliefert: „Durch sein Kleinformat verwandelt TV jedes Ereignis in eine synchrone Nippesszene [...] Die Absicht der Bildlieferung, ja die Lieferung des ganzen Weltbildes besteht eben [...] darin, das Wirkliche abzudecken, und zwar mit Hilfe des angeblich Wirklichen selbst; also die Welt unter ihrem Bild zum Verschwinden zu bringen.“ Weil das Fernsehbild übersichtlich ist, verfälscht es das Unabsehbare, „und weil es uns überhaupt ins Bild setzt“ (Anders 2002: 151), betrügt es uns. 
lärkultur wie Fernsehserien u.a. Medien ein Widerstandspotenzial gegen die herrschende Lesart (Winter 2006). Gerade daher sei Popkultur subversiv:

„Wie die Arbeiten von Henry Giroux, Douglas Kellner, Peter McLaren und anderen zeigen, sollten Medien- und Kulturanalyse im Rahmen von Cultural Studies immer auch verknüpft sein mit einer kritischen Pädagogik, die der impliziten Pädagogik medialer Texte opponiert und eine produktive Auseinandersetzung intensivieren oder erst ermöglichen möchte. Dabei wird der Alltag als ,contested terrain“ bestimmt, der auf einen kollektiven Dialog hin geöffnet werden soll, damit viele unterschiedliche Stimmen sich artikulieren können, um eine demokratischere und gerechtere Gesellschaft zu schaffen.“ (Winter 2004: 13)

Der Einfluss auf das Verständnis von Politik ist in populären Filmen und Fernsehserien größer als bei den Kunstfilmen, die ein weitaus geringeres und eher elitäres Publikum erreichen. Schon im 18. Jahrhundert waren die populären ,Moralischen Wochenschriften“ wegen ihren ,Hausmacher-Weisheiten“ zu Themen wie Moral, Erziehung, gesitteter Umgang, Verschrobenheit der Gelehrten, Geschmacklosigkeit, Ehe u.a. einem größeren Publikum näher als die Texte der Aufklärer. Bereits damals gab es Kritik an der „,minderwertigen Massenware“ an ,Weitschweifigkeit, Banalität und Schulmeisterei“" (Klawitter 2017: 110) dieser Journale. Dennoch hatten sie nicht trotz, sondern wegen ihrer niedrigschwelligen Ansprache ihrer Leser einen maßgeblichen Anteil an der Selbstaufklärung der damaligen Öffentlichkeit. „Das Publikum hält sich mit dem Tatler, dem Spectator, dem Guardian den Spiegel vor; es versteht sich noch nicht auf dem Umweg einer Reflexion über Werke der Philosophie und Literatur, der Kunst und der Wissenschaft, sondern dadurch, dass es selbst als Gegenstand in die ,Literatur" eingeht." (Habermas 1976: 60)

Ähnlich verläuft die Debatte über die politische Relevanz populärer Filme oder Fernsehserien in der Gegenwart. Dörner (2001) hat in seiner Studie Politik in der Erlebnisgesellschaft untersucht, wie das „Politische im Unterhaltungsformat konstruiert wird“ und kommt zu dem Ergebnis, dass die „Gefühlsqualität unterhaltender Politik und politischer Unterhaltung als Integrationsfaktor einer modernen Massengesellschaft keineswegs von geringem Wert ist“" (Dörner 2001: 241). Dieser Befund widerspricht der Mediokratie-These, dass „,vor allem der exzessive Fernsehkonsum [...] von mehreren Seiten zugleich die soziokulturellen Grundlagen der Demokratie [beeinträchtigt]“" (Meyer 2001: 203). Meyer sieht eine Überformung des Politischen durch die Medien und konstatiert, dass „die Macht des Mediensystems zur Prägung der politischen Kultur [...] offen- 
kundig bei weitem den Einfluss der politischen Kultur auf die Praxis des Mediensystems [übertrifft]“ (Meyer 2001: 205).

Diesen beiden sich widersprechenden Thesen über die Funktion und Wirkung von Fernsehserien liegen unterschiedliche Vorstellungen von Politik und von der Rolle der Medien für den Zuschauer zugrunde. Die Mediokratie-These geht von einem idealtypischen Verständnis von Politik und der politisch Handelnden aus, während Dörner Unterhaltung als legitimes Bedürfnis anerkennt. Die Unterhaltungsgesellschaft ist keine Abweichung eines Idealstaates im Sinne Rousseaus, sondern eine typische Form der modernen Gesellschaft. Es gab und gibt in jeder Gesellschaft Bürger, die sich - aus welchen Gründen auch immer einem politischen Engagement entziehen und die mit politischen Fragen nichts zu tun haben wollen. Diese aus demokratietheoretischer Sicht nicht zu akzeptierende Haltung wirft die Frage auf, wie denn die immer größer werden Teile ,unpolitischer' Bürger zumindest für das Problem des Populismus sensibilisiert werden können. Eine Möglichkeit besteht z.B. darin, sie mit populären Serienformaten zu erreichen. Eine Verpflichtung zum Besuch politischer Filme (wie bei den Nazis) verbietet sich in einer Demokratie auch dann, wenn die Filme ,korrekt' sind. Sinnvoll ist dagegen, Filme als Teil der Allgemeinbildung etwa im Schulunterricht zu verwenden und mit ihnen gesellschaftliche, politische oder philosophische Fragen zu erörtern.

Der Befund der Autoren der Kritischen Theorie u.a., wonach populäre Medien nur Auswüchse der Freizeitindustrie sind, die die entfremdeten Individuen mit dem Kapitalismus versöhnen, geht an der Realität der modernen Gesellschaft vorbei: Diese ist wesentlich eine Mediengesellschaft, in der eine weitaus größere soziale Vielfalt an Lebensstilen und soziokulturellen Milieus existiert, wie sie Theodor Adorno, Max Horkheimer, Günther Anders, Herbert Marcuse u.a. kannten, und die die Medien produktiv in ihre Lebenspraxis integrieren. Statt Opfer einer alles überformenden Mediokratie zu sein, wissen die Bürger als Mediennutzer meist genau, was sie von welchem Medium erwarten. So werden z.B. Filme danach ausgesucht, wo die Zuschauer für sich persönlich den größten Nutzen ziehen können, sei es Information, Unterhaltung, Thrill oder intellektuelle Anregung. Eine Folge davon ist, dass diese zersplitterten Öffentlichkeiten in ihren jeweiligen Subkulturen und soziokulturellen Nischen fast nur noch von solchen Inhalten erreicht werden, die sie selbst aussuchen. Wer z.B. politische Filme nicht mag, findet unzählige Alternativen, und umgekehrt, wer nur ARTE schaut und in Programmkinos geht, wird nie von seichter Unterhaltung behelligt. Damit schwindet für die Gesamtgesellschaft der Vorrat gemeinsam geteilter Erinnerungen, Inhalten und Themen. Diese kulturellen ,Filterblasen ‘ werden durch die Algorithmen der sozialen Netzwerke verstärkt, die dem Nutzer vornehmlich 
diejenigen Themen, Meinungen und Personen vorschlagen, die aufgrund des Suchverhaltens scheinbar naheliegen.

Die Zeit, in der bestimmte Filme oder einzelne Fernsehendungen von nur zwei TV-Programmen (ARD und ZDF) ausgestrahlt wurden, von fast allen Bürgern gesehen und am nächsten Tag zum Gesprächsthema wurden, wird es im multimedialen Zeitalter nie wieder geben. Es ist fraglich, ob mit diesen, lediglich zwei Fernsehprogrammen damals wirklich alle Bedürfnisse der Zuschauer befriedigt wurden oder ob es sich nur um eine ideologische Rechtfertigung des öffentlich-rechtlichen Rundfunks handelte, der den Zerfall der Gesellschaft durch die Einführung des Privatfernsehens (1984) befürchtete. Der damalige Intendant des Zweiten Deutschen Fernsehens, Dieter Stolte, sah eben dies mit seiner Feststellung, dass das Fernsehen vor einem grundlegenden Wandel stehe, weil es „in seiner Funktion für den Zuschauer vom Angebots- zum Nachfragemedium“ geworden sei (Stolte 1992: 13).

Die Vielfalt der Medienangebote ist eine Reaktion auf die Vielfalt der Gesellschaft und nicht umgekehrt: Die Einführung eines vielfältigen Medienangebots hat nicht die die Vielfalt der Gesellschaft verursacht, sondern die Medien haben auf die Vielfalt der Interessen der Zuschauer, der aktiven und der passiven, reagiert.

Die Frage ist also nicht mehr nur: Was machen die Medien mit uns? Sondern auch: Was machen wir mit den Medien? Auch fiktionale Fernsehunterhaltung bewirkt unterschiedliche Interpretationen und Wirkungen. Es kann daher keine „verbindliche Antwort auf die Frage geben, was die Menschen an bestimmten Medienangeboten fasziniert und welchen Gewinn sie aus der Nutzung ziehen“ (Jäckel 2001: 42). Was der Schriftsteller David Grossman über Literatur feststellt, gilt auch für Filme: „Selbst wenn zehntausend Menschen ein bestimmtes Buch zur gleichen Zeit lesen, ergreift es jeden auf andere Art und hilft einem jeden, sein Wesen auf individuelle Weise auszubuchstabieren. Verschiedene Partikel unseres inneren und äußeren Lebens, unserer Erinnerungen und unserer Identität streben dem starken Magneten Buch entgegen.“ (Grossmann 2017)

\section{DER POLITISCHE FILM}

Explizit politische Filme wollen eine Botschaft vermitteln und die Gesellschaft und ihre Herrscher (mit jeweils unterschiedlicher Akzentuierung) als bürgerlich, kapitalistisch, patriarchalisch, ungerecht und/oder totalitär entlarven. Die Frage bleibt, was das Politische an Filmen ist: Ist es der Inhalt, ist es die Wirkung auf die Zuschauer, oder kommt es bei der Wirkung der Filme auf die Lesart und Decodierung seitens der Rezipienten an? 
Abgesehen von Propagandafilmen, bei denen eine bestimmte Wirkung (z.B. die Denunziation der westdeutschen Gesellschaft als Erbe des Faschismus seitens der DDR-Propaganda) auf die Zuschauer von der Politik gefordert und beabsichtigt ist, haben Filme oft auch eine eher unbeabsichtigte politische Wirkung. Das ist z.B. dann der Fall, wenn dem Film eine bestimmte Ideologie zugrunde liegt. So stehen Filme, in denen einzelne Helden (Dirty Harry, Stirb langsam, Rambo) gegen korrupte Behörden, unfähige Politiker und/oder gegen übermächtige Verbrechersyndikate kämpfen, immer unter dem Verdacht, konservative Weltbilder zu transportieren und damit entsprechende Parteien zu stärken. Im Prinzip zeigt jeder Kriminal- oder Mafiafilm das Versagen der Politik, die die Menschen offenbar nicht vor Verbrechen schützen kann. Politische Institutionen und die in ihnen handelnden Polizisten, Richter und Politiker kommen in diesen Filmen immer schlecht weg: Entweder sind sie unfähig oder korrupt. Die Helden - und damit der Zuschauer, der sich mit ihnen identifiziert - bleiben meistens einsam. Sogar die persönlichen Beziehungen der Helden zerbrechen an ihrer Obsession, die allgegenwärtigen Feinde zu besiegen und der Gerechtigkeit zum Sieg zu verhelfen.

Aber nicht alle Filme, in denen einsame Helden mit Law and Order-Denken gegen ,das System“ kämpfen, sind konservativ. Im Gegenteil: In der „ParanoiaTrilogie“ (Klute, Zeuge einer Verschwörung und Die Unbestechlichen) von Alan J. Pakula wird vor dem Hintergrund der Watergate-Affäre und der VietnamProteste in den USA der legitime Widerstand gegen die Auswüchse einer allmächtigen Regierung in Szene gesetzt. Anders als bei den Law and OrderHelden und Selbstjustiz-Filmen werden Politik und Behörden nicht durch lästige Vorschriften wie Gewaltenteilung, Rechte der Beschuldigten oder liberale Medien behindert, sondern umgekehrt: Eine diffuse Verbindung zwischen Geheimdiensten, Konzernen, rechten Politikern, Justiz und Regierung ist verantwortlich für staatliche Repression, gegen die der Einzelne aufstehen muss und die seinen Widerstand legitimiert (z.B. in den Filmen Staatsfeind Nr. 1 oder Erin Brockovic). Sowohl in der konservativen als auch in der linksliberalen Sicht werden in diesen o.g. Filmen Politik und ihre Institutionen als im Kern korrumpiert dargestellt. Alle diese als Beispiele genannten Filme waren weltweit auch kommerziell erfolgreich.

Es gibt jedoch noch andere politische Filme von Regisseuren, die weniger erfolgreich waren als die Blockbuster der amerikanischen Regisseure: Bernardo Bertolucci, Pier Paolo Pasolini, Costa Gavras, Jean Luc Godard und Ken Loach gehören zu denen, die nicht nur das Kino, sondern auch die Welt revolutionieren wollen. Ihr politisches Engagement wird in ihren Filmen unmittelbar sichtbar: Die Filme von Gavras z.B. (Der unsichtbare Aufstand, Z, Das Geständnis) las- 
sen auch ohne Dechiffrierung von Bildern und Metaphern keinen Zweifel daran, wer Täter und wer Opfer ist. Hier gibt es keine vagen Verschwörungen oder komplizierten Beziehungsnetze, sondern eindeutige Verbindungen, gegen die auch Widerstand mit Gewalt legitim ist. Weil die USA ihre Interessen in den Militärdiktaturen Südamerikas durchsetzen wollen, ist in Der unsichtbare Aufstand die Gewalt der Guerillas gerechtfertigt. „Weitgehend differenziert in der Darstellung der Unrechtsprobleme, rechtfertigt er letztlich allzu parteiisch Gewalt als Mittel des Widerstands. Als ambitionierter Beitrag zur politischen Meinungsbildung für eine gerechtere Welt diskussionswert. “11

Ein anderes Beispiel für einen Film mit einer eindeutigen Botschaft ist der argentinische Film Aufstand in Patagonien (Patagonia rebelde) von Osvaldo Bayer, der besonders in anarchistischen Kreisen zum Kultfilm wurde. Er war während der argentinischen Militärdiktatur verboten und erhielt bei der Berlinale 1974 den Silbernen Bären. Inhalt des Films ist der Aufstand von Landarbeitern gegen die Großgrundbesitzer 1921/22 in Patagonien im Süden Argentiniens. Auch in diesem Film gibt es wie bei Der unsichtbare Aufstand kaum Zwischentöne: Er zeigt die Ausbeutung rechtloser Landarbeiter und legitimiert ihren Widerstand, der von der argentinischen Armee blutig niedergeschlagen wird. $\mathrm{Ob}$ man auf diese gewaltsame Niederschlagung ebenfalls mit Gewalt reagieren müsse, wurde lange bei den Anarchosyndikalisten in Südamerika und in Europa diskutiert. Einige der südamerikanischen Linken, die sich dem bewaffneten Widerstand gegen die damaligen Militärdiktaturen anschlossen, wurden später - wie José Mujica (2010 bis 2015 Präsident von Uruguay) oder Dilma Rousseff (2011 bis 2016 Präsidentin von Brasilien) - eher pragmatische Politiker. Die Erinnerung an diese Geschichte des bewaffneten Widerstandes spielt auch in der aktuellen Kritik an einigen linken Parteien in Südamerika eine Rolle, die „oft als soziale Bewegungen [begannen], die sich entschieden gegen politische und wirtschaftliche Eliten wandten und auf Entscheidungen von unten pochten“ (Hübener 2014). Aufstand in Patagonien ist auch ein Beispiel dafür, wie Filme die Erinnerung an Ereignisse, die vom Mainstream der Politikwissenschaft sowie vom „kollektiven Gedächtnis“ (Assmann/Assmann 1994, Reinhardt/Jäckel 2005) vernachlässigt werden, lebendig halten.

Die Filme des bekennenden Trotzkisten, Ken Loach (Bread and Roses, $\mathrm{Na}$ vigators, Angels' Share), sind weniger radikal als die o.g. Costa Gavras, aber nicht weniger engagiert. „Immer wieder ist Loach, vor allem im ThatcherEngland, das sich nach Kräften um seine Kaltstellung bemühte, als unbeugsamer

11 Der unsichtbare Aufstand, in: Zweitausendeins Filmlexikon, https://www.zweitausend eins.de/filmlexikon/?sucheNach=titel\&wert=47679 (zugegriffen am 9.12.2017). 
Sozialist oder auch als linker Utopist angeeckt - und hält doch bis heute am Glauben an die Widerständigkeit derer fest, die in weltpolitischen oder auch mikrokosmischeren Hackordnungen niedergehalten werden." (Schulz-Ojala 2006)

Andere, ebenfalls politisch links engagierte Regisseure wie Bernardo Bertolucci, Pier Paolo Pasolini oder Jean Luc Godard machten dagegen Filme, die zu den wertvollsten der Filmkunst zählen: Accatone - Wer nie sein Brot mit Tränen aß, Decamerone, Der große Irrtum, 1900, Vor der Revolution, Außer Atem, Zwei oder drei Dinge, die ich von ihr weiß. Eine unmissverständliche politische Botschaft wie die Filme von Gavras oder Loach haben diese ästhetisch anspruchsvolleren Filme nicht. Es ist kein Zufall, dass die Filme dieser Regisseure eher ein linksliberales Publikum ansprechen, da in allen diesen Filmen totalitäre Strukturen und bürgerliche Konventionen entlarvt und - wie in Bertoluccis Der große Irrtum - in die Nähe des Faschismus gerückt werden. Allein die sexuelle Freizügigkeit in den Filmen von Pasolini und Bertolucci ${ }^{12}$ hatte eine gesellschaftskritische und damit politische Dimension. Auch die anderen als die o.g. Filme von Godard, Bertolucci und Pasolini haben eine starke erotische und sexuelle Dimension. In weiten Teilen der ,Neuen Linken“ im Umkreis der ,Frankfurter Schule', die den Mainstream des kritischen Denkens seit 1968 bildete, bedingten sich sexuelle und politische Befreiung gegenseitig. Für Herbert Marcuse z.B. war Sexualität ein Vehikel zur Emanzipation. „In einer Welt der Entfremdung würde sich die Befreiung des Eros notwendig als zerstörerische, verderbliche Kraft auswirken - als die vollständige Verneinung jenes Prinzips, das die repressive Wirklichkeit beherrscht.“ (Marcuse 1973: 96)

Die meist sehr anspruchsvollen Filme vor allem Pasolinis und Godards treffen auf ein Publikum, das die gesellschaftskritische Haltung seiner Regisseure im Wesentlichen teilt. Ein Massenpublikum erreichen diese Filme nicht - zu elaboriert sind ihre Ästhetik, Bildsprache und Anspielungen. ${ }^{13}$ Selbst die Helden - Zuhälter, Landarbeiter, Außenseiter - laden nicht gerade zur Identifikation ein.

12 Vor allem die Filme 1900 von Bertolucci und Salò oder die 120 Tage von Sodom von Pasolini wurden wegen expliziter Szenen zensiert.

13 „Wenn in Godards absurder Dystopie (Weekend) ein schicker Sportwagen mit einem schwerfälligen Traktor kollidiert, ist das zugleich ein Bild für den Kampf der Klassen. Mobilität ist auch nur Lüge und Illusion. Die Wahrheit offenbart sich nur im Stillstand [...]: Die Bourgeoisie ist allzeit mobil, aber intellektuelle und emotional hoffnungslos steckengeblieben.“ (SAW 2018: 18) Diese Erkenntnis erschließt sich dem unbefangenen Zuschauer nicht unmittelbar und offenbar nur denen, die das sehen, was sie sehen wollen indem sie ihr politisches Bewusstsein in die vieldeutigen Bilder hineinprojizieren. 
Ganz anders dagegen die Helden in Filmen wie Stirb langsam, Dirty Harry und Rambo. Ihre Darsteller sind Weltstars (Bruce Willis, Clint Eastwood, Sylvester Stallone) und wurden zu Markenzeichen. Die Botschaft dieser Filme ist durch Verzicht ihrer Regisseure auf ästhetische Innovationen, filmtheoretische Experimente und radikale Erzählstrukturen auch einem breiten Publikum in der ganzen Welt verständlich. Gerade wegen ihrer eher konventionellen Machart und leichten Verständlichkeit sind dies die eigentlich politisch relevanten Filme. „Wer nur Filmkunst gelten lässt, die sich per definitionem kritisch mit herrschenden Verhältnissen auseinandersetzt [...], der verfehlt den Möglichkeitsraum der Massenkünste.“ (Kaspar Maase zit. n. Zywietz 2012: 29)

\section{POLITIK IM FILM}

Filme können auch dann politisch sein, wenn Politik scheinbar nur am Rande vorkommt. So waren viele Filme der Nazis gerade deshalb auch politisch, weil sie eine heile Welt - gelegentlich mit doppeldeutigen Anspielungen auf die Politik - vorgaukelten. Viele Western und Polizeifilme sind auch deshalb politisch, weil in ihnen eine populistische Werthaltung gegenüber dem Stellenwert von Recht und Gesetz zum Ausdruck kommt. ${ }^{14}$ Politische Institutionen und die in ihrem Namen handelnden Akteure sind in diesen Filmen entweder schwach, korrupt oder unfähig. Und genau deshalb - so die Botschaft dieser Filme - bleibt nur die Hoffnung auf einen ,starken Mann', der , aufräumt'.

Es gibt aber auch Filme, in denen Politiker nicht nur Randfiguren sind, sondern im Kontext politischer Institutionen und Strukturen gezeigt werden: $M r$. Smith geht nach Washington (1939) von Frank Capra, Wag the Dog (1997) von Larry Beinhart, Thirteen Days (2000) von Roger Donaldson oder die Fernsehserie House of Cards (2013). In allen diesen Filmen liegt der Fokus auf dem Politikbetrieb zwischen dem Weißen Haus, dem Kongress, der Lobby - dem Iron Triangle - und den Medien. Ihnen ist gemeinsam, dass sie den Politikbetrieb anschaulicher und realistischer vermitteln als jede politikwissenschaftliche Analyse. Filme verleihen den handelnden Akteuren ein Gesicht, eine persönliche Iden-

14 Eine der wenigen Ausnahmen ist der ungewöhnliche Western von William A. Wellmann Der Ritt zum Ox-Bow. Dieser 1943 veröffentlichte Film prangert Lynchjustiz und faschistisches Denken in den USA an. Bemerkenswert ist, dass der Autor der Romanvorlage, Walter Van Tilburg Clark, darauf bestand, dass er den Faschismus in den USA meint und nicht den in Japan und Deutschland, mit denen sich die USA damals im Krieg befanden. 
tität und Motivationen, die jenseits der Rationalitätsunterstellungen von politikwissenschaftlichen Theorien und von Handlungstheorien liegen. Indem diese Filme die Akteure als Menschen in einem Geflecht menschlicher Beziehungen zeigen und nicht als idealtypische Repräsentanten gesellschaftlicher Funktionssysteme oder von Verfassungsorganen, liegen sie näher an der Realität als sozialwissenschaftliche Theorien. Das ,Gesicht', das Filme Funktionsträgern und ihrer Entourage geben, zeigt zugleich die Problematik des Films als Erkenntnisquelle auf. Die Filmgesichter etwa von Präsidenten der Vereinigten Staaten oder anderen hohen Politikern gehören meist bekannten Schauspielern (Kevin Kostner, Matt Damon, Gene Hackman u.v.a.) und prägen damit auch die Wahrnehmung der von ihnen dargestellten historischen Figuren, da diese Schauspieler durch andere Rollen bekannt und z. T. zu einer Marke für bestimmte Charaktere wurden.

„Literarische Interpretationen der Gesellschaft sind weder - wie die Massenmedien - an Aktualität, noch an wissenschaftliche Wahrheit gebunden. Und doch schaffen es literarische Texte immer wieder, Aktualität und Wahrheit nicht nur zusammenzuführen, sondern sogar präziser auf den Punkt zu bringen.“ (Kron/Schimank 2004: 11) Dies gilt auch für die o.g. Filme: Auch sie bringen bestimmte Dinge , auf den Punkt': so z.B. die Art, wie der Held von House of Cards (Frank Underwood) vorgeht, um sein Ziel, Präsident der Vereinigten Staaten zu werden, zu erreichen. Der rein instrumentelle und jeder Moral spottende Umgang mit Kollegen, Parteifreunden, Lebenspartnern und Weggefährten, wie er in dieser Serie dargestellt wird, wird oft von der Realität bestätigt oder überholt. Der Film Wag the Dog bildet nicht eine Realität ab, sondern erscheint vor dem Hintergrund der realen Ereignisse wie eine Blaupause für politisches Handeln: Um von einem persönlichen Skandal des US-Präsidenten abzulenken, inszeniert er einen Krieg auf dem Balkan. „Man mag es schier nicht glauben, dass Barry Levinsons neuer Film Wag the Dog lange vor der Lewinsky-Affäre fertiggestellt wurde. Es drängen sich so viele Parallelen zwischen filmischer Fiktion und Bill Clintons Sex-Nöten auf, dass man zu dem Schluss gelangt, der Drehbuchautor David Mamet habe vorab Insider-Informationen bekommen oder besitze hellseherische Fähigkeiten." (Deutscher Depeschendienst vom 24. März 1998) In House of Cards erhalten die Zuschauer bessere Einblicke in die Mechanismen der Macht als durch die einschlägigen Theorien der Sozialwissenschaft. Hinzu kommt, dass es in der Wissenschaft Tabus gibt, die im Film ohne weiteres angesprochen werden können. So bedient sich etwa die Frau des Serienhelden (Claire Underwood), die für eine NGO Unterstützung sammelt, der gleichen Methoden wie alle anderen Politiker: Sie schmiedet Intrigen und nutzt 
persönliche Schwächen von Personen aus, um ihr Projekt in Afrika zu finanzieren. $^{15}$

Filme und andere Kunstwerke dürfen mit der Realität, die sie vorfinden, so umgehen. Shakespeare hat z.B. mit seinem Königsdrama Richard III den Prototyp eines machiavellistischen und über Leichen gehenden Herrschers geschaffen und sich dabei von Raphael Holinshed's Chronicles (1577) inspirieren lassen. Damit die Handlung des Dramas - der Aufstieg und Fall Richard III (1452 bis 1485) - schlüssig erzählt werden konnte, wurden einige Fakten von ihm kreativ angepasst, z.B. wurden Lebensdaten von zeitgenössischen Akteuren geändert und ungeklärte Todesfälle Richard zur Last gelegt. Nur so gelang es Shakespeare, mit seinem Richard III den größten Bösewicht der Literaturgeschichte zu schaffen. In dem nach Hamlet (,Sein oder Nichtsein“) berühmtesten Monolog des Theaters nennt Richard III das Motiv für sein politisches Handeln: „Ich bin so dermaßen hässlich, dass ich nie den Liebhaber geben kann und mir keine andere Wahl bleibt, als Böses zu tun“ (, Since I cannot prove a lover...I am determined to prove a villain. "). ${ }^{16}$ Im Unterschied $\mathrm{zu}$ Shakespeare stellt die Geschichtswissenschaft quellenkritisch fest, dass das Bild Richard III von der zeitgenössischen Geschichtsschreibung verzerrt wurde.

„Our research has found that such inaccuracies are caused by the complexity of Holinshed's Chronicles, which stems from multiple authorship, religious tensions among the contributors, and fraught circumstances when it was published. The authors and revisers came from diverse backgrounds and they used an extraordinary variety of conflicting sources. “17

Künstler können mit historischen oder anderen Quellen, die sie gern zur Inspiration verwenden, anders umgehen als Wissenschaftler. Was Historiker als religiös und politisch verzerrte Propaganda des mit Richard III verfeindeten Königshauses (es war die Zeit der Rosenkriege zwischen den englischen Adelshäusern

15 Moralisch stehen sich Mrs. und Mr. Underwood in nichts nach, obwohl sie zunächst unterschiedliche Ziele verfolgen. Dass Claire schließlich ihren Mann als Präsident der Vereinigten Staaten ablöst, wird als unstimmig kritisiert, weil es in der Serie so dargestellt wird, als würde nach wie vor ihr Mann die Fäden ziehen (Klode 2017).

16 „Weil ich den Liebhaber nicht geben kann ... heißt der Beschluss: Ich geb den Bösewicht.“ (Übersetzung: Rainer Iwersen)

17 Paulina Kewes, Ian W. Archer und Felicity Heal zit. nach http://www.ox.ac.uk/news /2013-02-07-source-shakespeares-inaccurate-richard-iii-portrayal-explored (zugegriffen am 29.12.2017) 
York und Lancaster) werten (wie die Holinshed's Chronicles), kommt Shakespeare gerade recht, weil es in sein konstruiertes Bild von Richard III passt. Am wirklichen König Richard III hatte Shakespeare kaum Interesse. Ihm ging es darum, einen Typus zu schaffen, der gerade deshalb einen eigenen Wahrheitscharakter hat. Es ist der Typus eines machtversessenen Herrschers, der alle Mittel nutzt, um sein Ziel - König von England zu werden - zu erreichen. Dieser Typus des machiavellistischen Herrschers ist bis heute aktuell. Auch dem Helden von House of Cards ist jedes Mittel recht: Er ist ein moderner Machiavellist, bei dem Shakespeares Richard III Pate gestanden hat. Im Übrigen hat Shakespeares Lady Macbeth bei der Figur der Mrs. Underwood zumindest teilweise Pate gestanden. Die Frau von Macbeth stachelt ihn an (,wenn Du ein Mann bist“), den König von England zu ermorden, damit er - Macbeth - zum nächsten König gekrönt werden kann. ${ }^{18}$

Durch die künstlerische Freiheit, von den realen Vorbildern zu abstrahieren, entsteht eine neue ,Wahrheit', die sich empirischen Belegen entzieht und ihnen sogar widerspricht. „Literatur und Film haben - anders als Politik, Soziologie und Geschichtswissenschaft - von Natur aus eine ausgeprägte Lizenz zur freien Aufnahme, Behandlung und Ausdeutung historischer Stoffe.“ (Braun 2010: 8) Dadurch können fiktionale Charaktere mehr über das Wesen der Politik aussagen als empirische Studien über Machtstrukturen und Machtkämpfe. Fiktionale Charaktere können auf ein größeres Bündel an Motiven zurückgreifen als die Theorien der Rational Choice und das Netzwerk relevanter Akteure im Film umfasst weit mehr als nur Funktionsträger von Verfassungsorganen.

Im realen Leben wie in Filmen spielen auch Ehepartner, heimliche Liebhaber, enttäuschte Praktikanten, vergessene Partner aus früheren Zeiten, falsche Freunde, labile Geschäftspartner, sich zurückgesetzt fühlende Konkurrenten u.v.a. eine entscheidende Rolle. Jede dieser Figuren kann im Film zum Stolperstein für die Karriere werden oder ein unverhoffter Türöffner. Ein Politikwissenschaftler, der z.B. die Büroleiterin eines Stabschefs als relevante Akteurin einstuft, wird in der scientific community kaum ernst genommen. Im Film ist sie es aber, die dem Helden einen - nicht erlaubten - Einblick in die Termine des Regierungschefs gibt und ihm damit Handlungsoptionen eröffnet. Auch die Erpressung eines alkoholkranken Konkurrenten eröffnet im House of Cards einen interessanten Handlungsstrang, der für den Helden letztlich zum Erfolg führt. In soziologischen und politikwissenschaftlichen Theorien spielen derartige Motive im

18 „Bist du zu feige, derselbe Mann zu sein in Tat und Mut, der du in Wünschen bist? Möchtst du erlangen, was du den Schmuck des Lebens schätzen musst, und Memme sein in deiner eignen Schätzung?“ (Macbeth I./VII; Übersetzung: Dorothea Tieck). 
Unterschied zu biografischen und historischen Darstellungen keine Rolle. Immerhin werden Korruption und Filz durchaus als deviantes Verhalten thematisiert. Aber auch bei diesen Studien geht man von einem überschaubaren Netzwerk von Akteuren aus, die mehr oder weniger rational handeln. Auch im Verhältnis zu nicht-fiktionalen Nachrichtensendungen zeichnen Fernsehserien „ein ganzheitlicheres Bild der Politiker“(Jandura/Gladitz/Nitsch 2016: 292).

\section{FAZIT}

Unabhängig von der Frage, was genau an Filmen politisch ist und nach welcher Medienwirkungstheorie (Brose 1997) die Folgen für politisches Handeln erklärbar sind, bleibt festzustellen, dass Filme und Fernsehserien politisch relevant sind. Sie können politische Einstellungen verfestigen, prägen oder hinterfragen. Allein dadurch, dass Filme und Serien ein gesellschaftsweites Echo erzeugen können und von vielen Blogs, Fangemeinden und Fachmagazinen ${ }^{19}$ teilweise noch nach Jahren begleitet werden, sind sie gesellschaftlich und politisch relevant. Sie sind damit Teil der gesamtgesellschaftlichen Kommunikation und dienen als Kitt für den sozialen Zusammenhalt. Blockbuster sind wie viele andere Produkte der Populärkultur fast überall verbreitet und prägen als, soft power ' die Einstellungen gegenüber dem in diesen meist amerikanischen Filmen gezeigten Lebensstil.

Fernsehserien können zu politischen Konflikten zwischen zwei Staaten führen oder im Gegenteil bestehende Stereotype korrigieren. Die türkische Fernsehserie Aryilik (Abschied/Trennung) (2009) sei nach Ansicht des israelischen Außenministers Avigdor Lieberman „staatlich geförderte Hetze“. Aus Protest über diese Serie bestellte er den Vertreter der türkischen Botschaft in Tel Aviv ein (Günther 2009). Auch die türkische Serie Tal der Wölfe (2007) war in Israel als ,antisemitische[s] Machwerk" heftig kritisiert worden und führte zu einem diplomatischen Eklat. „Viele Türken hatten das blutig-nationalistische SöldnerDrama Tal der Wölfe frenetisch im Kino gefeiert, der Held des Films wurde zur Identifikationsfigur für die Jugend. Die gleichnamige TV-Serie wurde jetzt nach nur einer Folge abgesetzt. 2006 hatte die Wölfe-Serie Zuschauerrekorde gebrochen $[\ldots .$. . Doch schon nach der ersten Folge wurde die Reihe jetzt abgesetzt, auf Druck der türkischen Rundfunkaufsicht RTÜK. Die Kritik: Die Serie sei gewalt-

19 Einen Überblick mit Episodenführer, Cast, Hintergründen, Sendeterminen, Hinweisen auf Communities u.a.M. über alle im deutschen TV gesendeten Serien bietet www.fernsehserien.de. 
verherrlichend und schüre Spannungen in der Gesellschaft.“ (Großbongardt 2007)

Die 2013 vom ZDF produzierte dreiteilige Fernsehserie Unsere Mütter, unsere Väter führte ebenfalls zu einer Verstimmung zwischen Deutschland und Polen: „Ein 92 Jahre alter Veteran der polnischen Heimatarmee, die im Zweiten Weltkrieg gegen die deutsche Besatzung kämpfte, sieht durch die Fernsehfilme seine Persönlichkeitsrechte und die Würde der polnischen Nation verunglimpft. Zusammen mit dem ,Weltverband der Soldaten der polnischen Heimatarmee“ fordert er eine Entschuldigung des ZDF im polnischen Fernsehen und einen Schadenersatz in Höhe von umgerechnet 5700 Euro. [...] Die Produktionsfirma weist die Anschuldigung [...] zurück und verweist auf die Kunstfreiheit.“ (Zaboji 2016)

Eine positive Wirkung hatte dagegen die in Japan laufende koreanische Fernsehserie Wintersonate (fuyu no sonata). Das Verhältnis beider Staaten ist durch die Besetzung Koreas durch Japan im Zweiten Weltkrieg bis heute getrübt und von wechselseitigen Vorurteilen geprägt. Das änderte sich mit der Ausstrahlung dieser Serie im japanischen TV. In Japan spricht man vom „Korea-Wind“ (Hanryu), den diese Serie ausgelöst hat. Die Folgen zeigen ein eindrückliches Beispiel für die Wirkung von soft power:

„Der Tourismus nach Korea nahm stark zu, koreanische Sprachkurse in Japan boomten wie noch nie, und die Fotoalben von Yong Joon Bae [der Hauptdarsteller] wurden in unzähligen Exemplaren verkauft. In Japan hat sich als Folge ein vielversprechender Markt für koreanische Kulturgüter wie Filme, TV-Dramen und Populärmusik aufgetan. Über das Phänomen ,Hanryu ' kam in Japan ein Diskurs in Gang, ein Diskurs, der die Einschätzung koreanischer Kultur grundlegend verändert hat. Diese Entwicklung ist besonders bemerkenswert, rückt man die durch die koloniale Vergangenheit belasteten schwierigen Beziehungen beider Länder in den Blick.“ (Seelmann 2005)

Besonders die Reaktion von Japanerinnen auf das in dieser Serie gezeigte Frauenbild ist bemerkenswert: „Bei den Ergebnissen der Umfrage zeigten sich u.a. die Auswirkungen der Ausstrahlung des koreanischen Fernsehdramas Wintersonate im persönlichen Umfeld der Fans. So äußerten 37,2 \% der Befragten, aufgrund dieser Serie hätten sie ein neues Gesprächsthema in der Familie und unter Freunden gefunden, 31,2 \% schlossen neue Freundschaften unter den Fans, 10,5\% gaben an, ein neues Lebensziel gewonnen zu haben, und 7,7 \%, dass die Beziehung zum (Ehe-)Partner besser geworden sei. Diese Ergebnisse decken sich mit den Aussagen von zahlreichen Fanbriefen an den Fernsehsender. Wie aus ihnen hervorgeht, hat sich durch das Fernsehdrama den Frauen häufig eine neue Welt eröffnet. Sie 
stießen auf etwas, das ihre Begeisterung hervorrief.“ (Gössmann mit Bezug auf Kaori Hayashi 2008: 90)

Angesichts dieser Beispiele ist es müßig, darüber zu spekulieren, ob Filme und Fernsehserien gesellschaftliche und politische Wirkungen haben. Selbst wenn sie nicht so drastisch ausfallen wie in den o.g. Beispielen, sondern weniger dramatisch sind wie bei der Lindenstraße oder den Simpsons (Dörner 1998 und 2001), so ist ihre Wirkung selbst nicht zu bestreiten. Sie sind integraler Bestandteil der modernen Gesellschaft, in der Medien und Unterhaltung einen anderen Stellenwert haben als in totalitären Gesellschaften, die der radikalen Medienund Kulturkritik zugrunde liegen.

Diesen Belegen für die Wirksamkeit von Filmen und Serien stehen auf der anderen Seite Erfahrungen entgegen, dass das seit einigen Jahren verstärkte Bemühen um politische Korrektheit ${ }^{20}$ - z.B. die Vermeidung von ethnischen und Geschlechterklischees - negative gesellschaftliche Entwicklungen wie die $\mathrm{Zu}$ nahme von Rassismus und Gewalt gegen Minderheiten hingegen scheinbar kaum beeinflusst hat. Gerade Fernsehserien wie Tatort und Lindenstraße gelten als Musterbeispiele politisch korrekter Filme. Auch die weitgehende mediale Abschottung der DDR hat ihren Zusammenbruch nicht verhindert, da ihren Medien doch keiner glaubte. Dies bestätigt und ergänzt den Befund bezüglich nichtfiktionaler und journalistischer Medien, dass sie in der Realität nicht so einflussreich sind, ,sondern nur, solange Politiker glauben, dass sie es sind, und dann dementsprechend handeln“" (Newton/Merz 2015: 462).

20 In dem Actionthriller Unstoppable (Außer Kontrolle) von Tony Scott aus dem Jahr 2010 ist der Held - ein erfahrener Lokführer, der einen außer Kontrolle geratenen Güterzug retten will - schwarz (Denzel Washington) und die Fahrdienstleiterin ebenfalls (Rosario Dawson). Die negativste Figur - der um den Aktienkurs der ihm gehörenden Eisenbahngesellschaft besorgte Eigentümer - ist ein alter weißer Mann und wird beim Golfen gezeigt, während der Held und die Heldin verzweifelt eine Katastrophe verhindern wollen. Auch der weiße Chef der schwarzen Fahrdienstleiterin kommt schlecht weg, da er den Helden zuvor bereits entlassen hatte. „Der Beharrlichkeit des Eisenkolosses steht nur diejenige des rechtschaffenen amerikanischen Arbeiters entgegen. Um Geld zu sparen, würden die Eisenbahnunternehmer - Prototypen amerikanischer Kapitalisten - den sicheren Tod von Hunderten in Kauf nehmen. Gegen den Willen der Bosse treffen die Helden die richtige Entscheidung - und begleichen damit ausstehende Rechnungen.“ (Thumfart 2010) Dieser und andere populäre Filme, die nicht gerade Meilensteine der Filmkunst sind, haben das gegenwärtige gesellschaftspolitische Klima in den USA offenbar kaum beeinflusst. 
Filme können zweifellos Fakten (z.B. diplomatische Konflikte) und Realitäten (für viele Zuschauer) schaffen. Aber sind sie auch ein Abbild der konkreten politischen Realität mit ihren Konflikten, Akteuren, Institutionen und Strukturen? Wenn von Publizisten, Historikern und Politikern nach historischen Filmen „eine Debatte darüber losgetreten wird, ob sich die Macher auch in allem an die Fakten gehalten haben“ (Everschor 2002: H27), dann verkennen sie das Verhältnis von Film und Realität. Als fiktionale Produkte können sie nicht mehr als bestimmte Aspekte - Korruption, Machtgier, Systemversagen - auf den Punkt bringen und ins Bild setzten. Das kann erhellender und vor allem unterhaltsamer sein als eine nüchterne politikwissenschaftliche Analyse. Die doppelte Subjektivität von Filmen - bei der Herstellung und bei der Rezeption - macht sie der Wissenschaft suspekt. Und gerade deshalb können Filme der Politikwissenschaft in weiten Teilen überlegen sein.

\section{LITERATUR}

Adorno, Theodor W./Horkheimer, Max (1971): Kulturindustrie, in: Dialektik der Aufklärung, Frankfurt/Main, S. 108-150.

Albersmeier, Franz-Josef (2001): Filmtheorien im historischen Wandel, in: ders. (Hrsg.): Texte zur Theorie des Films, Stuttgart, S. 3-29.

Anders, Günther (2002): Die Antiquiertheit des Menschen. Über die Seele im Zeitalter der zweiten industriellen Revolution, 2. Aufl., München.

Assmann, Aleida/Assmann, Jan (1994): Das Gestern im Heute. Medien und soziales Gedächtnis, in: Merten, Klaus/Schmidt, Siegfried/Weischenberg, Siegfrid (Hrsg.): Die Wirklichkeit der Medien. Eine Einführung in die Kommunikationswissenschaft, Opladen, S. 114-140.

Brauer, Wiebke (2008): Die Frau, die den perfekten Nazi-Körper schuf, in Spiegel Online, http://www.spiegel.de/einestages/riefenstahl-filme-a-946887.html (zugegriffen am 4.12.2017).

Braun, Michael (2010): Erinnerungskultur 2010, in: Wem gehört die Geschichte? Erinnerungskultur in Literatur und Film, St. Augustin.

Brose, Hans-Bernd (1997): Modelle und Ansätze der Medienwirkungsforschung. Überblick über ein dynamisches Forschungsfeld. Düsseldorfer medienwissenschaftliche Vorträge. Düsseldorf.

Clair, René (1995): Kino. Vom Stummfilm zum Tonfilm, Zürich.

Demetz, Peter (1969): Marx, Engels und die Dichter. Ein Kapitel deutscher Literaturgeschichte, Frankfurt/Main und Berlin. 
Dörner, Andreas (1998): Medien als politische Identitätsgeneratoren. Zur Inszenierung des Republikanismus in der amerikanischen Medienkultur, in: Politische Vierteljahresschrift 39, S. 3-27.

Dörner, Andreas (2001): Politainment. Politik in der medialen Erlebnisgesellschaft, Frankfurt/Main.

Engell, Lorenz (1992): Sinn und Industrie. Einführung in die Filmgeschichte, Frankfurt/Main und New York.

Everschor, Franz (2002): Kino als Geschichtskurs? Wachsende Polemik gegen politische und historische Hollywoodfilme, in: 2001 Lexikon des internationalen Films, Band 2, Frankfurt/Main, H. 27-H 28.

Gössmann, Hilaria (2008): Die Macht der Fiktion: Zum Einflusspotenzial von Fernsehdramen in Japan, in: Jäckel, Michael/Mai, Manfred (Hrsg.): Medienmacht und Gesellschaft. Zum Wandel öffentlicher Kommunikation, Frankfurt/Main und New York, S. 83-98.

Grob, Norbert (2008): Kino der Verdammnis, in: ders. (Hrsg.): Filmgenres Film noir, Stuttgart, S. 9-53.

Großbongardt, Annette (2007): Sendeverbot für "Tal der Wölfe"- Türkischer Rambo fällt in Ungnade, in: Spiegel Online, http://www.spiegel.de/kultur/ gesellschaft/sendeverbot-fuer-tal-der-woelfe-tuerkischer-rambo-faellt-inungnade-a-466766.html (zugegriffen am 5.1.2018)

Grossmann, David (2017): Gegen die Masse, in: Die ZEIT Nr. 11, S. 43.

Günther, Inge (2009): TV-Serie empört Israel, in: Frankfurter Rundschau vom 16.10., http://www.fr.de/politik/tuerkei-tv-serie-empoert-israel-a-1074543 (zugegriffen am 5.1.2018).

Habermas, Jürgen (1976): Strukturwandel der Öffentlichkeit, 8. Aufl., Neuwied/Berlin.

Hübener, Karl-Ludolf (2014): Anarchisten am Rio de la Plata, in: SWR2 Wissen, Manuskript unter https://www.swr.de/-/id=12437876/property=down load/nid=660374/1avl8ug/swr2-wissen-20140103.pdf (zugegriffen am 3.1.20 18).

Jäckel, Michael (2001): Ein endloses Thema. Zur Akzeptanz von Unterhaltungsangeboten und Serien, in: Cippitelli, Claudia/Schwanebeck, Axel (Hrsg.): Pickel, Küsse und Kulissen. Soap Operas im Fernsehen, München, S. 39-47.

Jandura, Olaf/Gladitz, Peter/Nitsch, Cordula (2016): Parlamente in nonfiktionalen und fiktionalen Angeboten. Was man in Berlin direkt und Borgen über parlamentarische Abläufe erfährt, in: Publizistik 61, S. 287-304.

Klawitter, Nils (2017): Schnörkelreiche Sittenlehre, in: Saltzwedel, Johannes (Hrsg.): Die Aufklärung. Das Drama der Vernunft vom 18. Jahrhundert bis heute, München, S. 110-115. 
Klode, Ulrike (2017): Das lange Warten auf Claire Underwood, in: dwdl.de https://www.dwdl.de/wocheinserie/61891/das_lange_warten_auf_claire_und erwood/ (zugegriffen am 5.1.2018).

Klooss, Reinhard/Reuter, Thomas (1980): Körperbilder. Menschenornamente in Revuetheater und Revuefilm, Frankfurt a.M.

Köck, Nicole (2004): Romane als Datenmaterial soziologischer Analysen, in: Kron, Thomas/Schimank, Uwe (Hrsg.): Die Gesellschaft der Literatur, Opladen, S. 243-263.

König, Siegfried (o.J.): Das goldene Zeitalter, in: filmzentrale, http://www.film zentrale.com/rezis/goldenezeitaltersk.htm (zugegriffen am 7.2.2018).

Kron, Thomas/Schimank, Uwe (2004): Die Gesellschaft der Literatur. Vorwort, Opladen, S. 7-18.

Mai, Manfred/Winter, Rainer (Hrsg.) (2006): Das Kino der Gesellschaft - die Gesellschaft des Kinos, Köln.

Marcuse, Herbert (1973): Triebstruktur und Gesellschaft, Frankfurt/Main.

Meyer, Thomas (2001): Mediokratie. Die Kolonisierung der Politik durch die Medien, Frankfurt/Main.

Newton, Kenneth/Merz, Nikolas (2015): Regieren die Medien?, in: Merkel, Wolfgang (Hrsg.): Demokratie und Krise. Zum schwierigen Verhältnis von Theorie und Empirie, Wiesbaden, S. 439-471.

Reinhardt, Jan/Jäckel, Michael (2005): Massenmedien als Gedächtnis- und Erinnerungsgeneratoren - Mythos und Realität einer ,Mediengesellschaft', in: Rössler, Patrick/Krotz, Friedrich (Hrsg.): Mythen der Mediengesellschaft, Konstanz, S. 93-112.

Rodek, Hanns-Georg (2009): Nur zehn Prozent der Filme waren NaziPropaganda, in: Welt, http://www.welt.de/kultur/article4409638/Nur-zehnProzent-der-Filme-waren-Nazi-Propaganda.html (zugegriffen am 5.12.2017).

Rodek, Hanns-Georg (2015): Jungfrau Eisenstein, 33, sucht schwule Erweckung, in: Welt, https://www.welt.de/kultur/article137395100/Jungfrau-Eisenstein-33sucht-schwule-Erweckung.html (zugegriffen am 6.12.2017).

Sanders, Olaf (2006): Cronenbergs Pädagogik, Wachowskis Pädagogik - Deleuzes ermächtigende Filmtheorie, in: Mai, Manfred/Rainer Winter (Hrsg.): Das Kino der Gesellschaft - die Gesellschaft des Kinos. Interdisziplinäre Positionen, Analysen und Zugänge, Köln, S. 259-275.

SAW (2018): Mobilität ist Kampf. Jean Luc Godards bürgerliche FilmApokalypse Weekend, in: k.west - Magazin für Kunst, Kultur, Gesellschaft 2, S. 18.

Schnakenberg, Robert (2010): Die großen Filmregisseure und ihre Geheimnisse, Zürich. 
Schulz-Ojala, Jan (2006): Drehen heißt Kämpfen. Zum 70. Geburtstag des britischen Filmemachers Ken Loach, in: Der Tagesspiegel vom 17.06.

Seelmann, Hoo Nam (2005): Wenn der Wind weht aus Korea, in: NZZ vom 7.2., https://www.nzz.ch/articleCJP6P-1.90482 (zugegriffen am 5.1.2018).

Sontag, Susan (2015): Kunst und Antikunst. 24 literarische Analysen, 11. Aufl., Frankfurt/Main.

Stolte, Dieter (1992): Fernsehen am Wendepunkt. Meinungsforum oder Supermarkt? München.

Thumfart, Johannes (2010): Mit Rechtschaffenheit gegen Godzilla, in: Die ZEIT vom 10.11., http://www.zeit.de/kultur/film/2010-11/film-unstoppable (zugegriffen am 10.1.2018).

Waldekrenz, Rune/Arpe, Verner (1956): Das Buch vom Film. Berlin/Darmstadt.

Winter, Rainer (2004): Cultural Studies und kritische Pädagogik, in: Hipf, Brigitte/Ernst, Katharina (Hrsg.): Medienpädagogik, Heft 8: Der Beitrag der Cultural Studies für die Medeinpädagogik, S. 1-16.

Winter, Rainer (2006): Die Filmtheorie und die Herausforderung durch den ,perversen Zuschauer‘. Kontexte, Dekonstruktionen und Interpretationen, in: Mai, Manfred/Winter, Rainer (Hrsg.): Das Kino der Gesellschaft - die Gesellschaft des Kinos. Interdisziplinäre Positionen, Analysen und Zugänge, Köln, S. 79-94.

Zaboji, Niklas (2016): Polnische Veteranen ziehen vor Gericht, in: FAZ vom 21.7., http://www.faz.net/aktuell/feuilleton/medien/klage-gegen-zdf-wegen-unseremuetter-unsere-vaeter-14349561.html (zugegriffen am 5.1.2018).

Zywietz, Bernd (2012): Grenzen des seismografischen Films. Zum konzeptionellen Verhältnis von Film und Gesellschaft am Beispiel des Stereotyps zwischen Funktionalität und Angemessenheit, in: Rabbit Eye - Zeitschrift für Filmforschung 4, S. 16-34. 
Fallstudien politischer Fernsehserien 



\section{Eichwald, MdB}

\section{Überleben im Haifischbecken Berlin-Mitte}

Florian Gilberg, Raphael David Moser, Said Rezek, Steffen Schulze

\section{EICHWALD, MDB: EIN HINTERBÄNKLER IM POLITISCHEN ÜBERLEBENSKAMPF}

Eine Polit-Satire, die auf dem deutschen Markt große Beachtung gefunden hat, ist die ZDF-Produktion Eichwald, MdB. Gedreht und produziert wurden die vier halbstündigen Folgen Anfang 2014 in Kooperation mit ZDFneo. Zunächst nur in der Mediathek verfügbar, liefen die einzelnen Folgen ab Mai 2015 im wöchentlichen Turnus im ZDF-Hauptprogramm. Zwei Jahre nach der Erstaustrahlung wurde die geplante Veröffentlichung einer zweiten Serienstaffel für voraussichtlich 2018 bekannt (Niemeier 2017).

Satirisch erzählt wird die Geschichte des gleichnamigen Protagonisten HansJosef Eichwald, Spitzname Hajo, Abgeordneter im Deutschen Bundestag für den Wahlkreis Bochum II, und seines Büro-Teams. Dazu zählen Berndt Engemann, ein erfahrener Routinier des Berliner Politikbetriebs, Sebastian Grube, der als Social Media affiner Politik-Neuling das Pendant dazu verkörpert, sowie die junge Büroleiterin Julia Schleicher, bei der die Fäden zusammenlaufen. Eichwalds Hauptmotivation nach über 20 Jahren als Bundestagsabgeordneter beschränkt sich im Wesentlichen darauf, wiedergewählt zu werden und möglichst ohne Komplikationen das Alter seiner Pensionierung zu erreichen. In seinem Alltag manövriert er sich jedoch immer wieder von einer Krise in die nächste. Der permanente Ausnahmezustand wird hierbei zur gefühlten Normalität. So überspitzt sich die Serie auch dem Arbeitsalltag eines Abgeordneten nähert, so sehr ist sie doch am Puls der Zeit.

Um ein besseres Verständnis für den Serienkontext zu vermitteln, geht es im ersten Abschnitt der Analyse zunächst darum, das allgemeine Setting von Eichwald, $M d B$ aus einem organisationssoziologischen Blickwinkel kurz zu umrei- 
Ben. Daran anknüpfend geht es im zweiten Kapitel schwerpunktmäßig mit dem politikwissenschaftlich interessanten Verhältnis von Medien und Politik weiter. Medien und insbesondere soziale Netzwerke spielen schließlich eine zentrale Rolle in der Serie, illustrieren sie doch idealtypisch die sich im Laufe der Jahre veränderten Mechanismen der politischen Kommunikation.

Im dritten Kapitel wird ein detaillierter Blick auf Eichwalds Nutzung von sozialen Medien geworfen und die damit transportieren Bilder analysiert. Im vierten und letzten Kapitel erfolgt schließlich eine Betrachtung der narrativen Elemente von Eichwald, MdB. Der Protagonist als ein von äußeren Umständen Getriebener und sein Umgang mit dem Dauerzustand der Krise sind hierbei ebenso Thema wie die Machtverhältnisse und die Beziehung zur Wählerschaft.

\section{PARTEILICHE SPIELREGELN UND INSTITUTIONELLES SETTING}

Der Humor und die satirischen Elemente von Eichwald, $M d B$ sind ohne Kenntnisse der institutionellen Besonderheiten des deutschen politischen Systems kaum zu verstehen. Der erste Abschnitt der Analyse bietet daher eine kurze Einführung in den organisationalen Kontext. Der Abschnitt konzentriert sich vor allem auf die politikwissenschaftlich interessanten Prozesse innerhalb einer Partei und einer Fraktion. Wie lässt sich beispielsweise eine Partei unter Rückgriff auf Erkenntnisse der benachbarten Soziologie konzeptionell beschreiben? Und welche Prozesse laufen im Inneren einer Fraktion ab?

Der Hauptteil der satirischen Erzählung von Eichwald, $M d B$ konzentriert sich auf die Tätigkeit des fiktiven Abgeordneten Hajo Eichwald. Er ist hin- und hergerissen zwischen den Anforderungen, die die Partei, die Fraktion und seine Wähler an ihn stellen. Eichwalds stärkste politische Motivation beschränkt sich in erster Linie darauf, das eigene politische Überleben zu sichern: Office-Seeking wird zum elementaren Treibstoff seiner politischen Ambitionen. Die Figur des Hajo Eichwald verkörpert einen Politikertypus, dessen oberste Maxime der Machterhalt ist. Politik wird hier porträtiert als permanenter Kampf um Deutungshoheit und Einflusssicherung in Partei und Öffentlichkeit.

\section{Abgeordnete und ihre Teams}

Da die Serie fast ausschließlich im Setting des Abgeordnetenbüros spielt, stellt sich aus wissenschaftlicher Sicht die Frage, was von Seiten der empirischen Politikwissenschaft über die klassischen Abgeordnetentätigkeiten zu erfahren ist. Patzelt (2014) erklärt in dem Zusammenhang, dass deutsche Abgeordnete unge- 
fähr fünfzig Prozent ihrer Arbeit am Sitz des Parlaments und etwa dreißig Prozent im Wahlkreis verbringen; der Rest entfällt auf sonstige Verpflichtungen und Veranstaltungen. Darüber hinaus haben Abgeordnete eine Arbeitswoche von im Schnitt fünfundsechzig Stunden, die gestückelt auf Sitzungen von Arbeitskreisen und Arbeitsgruppen sowie auf Fraktionsversammlungen, Plenarzeiten und Ausschusssitzungen entfallen (Patzelt 2014: 3-5).

Wie auch in der Serie immer wieder deutlich wird, konzentriert sich der zweite wichtige Arbeitsbereich eines Abgeordneten auf dessen Wahlkreis. Hier geht es, zugespitzt, weniger um profunde Fachexpertise als vielmehr um ostentative Bodenhaftung und die Sicherung der eigenen Wiederwahl (Patzelt 2014: 5). Dafür muss ein Abgeordneter die Balance zwischen Parlaments- und Basisarbeit meistern und neben dem regulären politischen Betrieb weiterhin in engem Kontakt zur örtlichen Parteibasis stehen. Wenngleich dieser Aspekt zwar nicht besonders prominent in der Serie thematisiert wird, fällt bei Hajo Eichwald doch sofort auf, dass ihn die Geschehnisse in seinem Wahlkreis keinesfalls kalt lassen. Das, was auf dem Spiel steht, ist die Organisation des vorpolitischen Raums, die den Grundstein für die politische Karriere eines jeden Abgeordneten legt (Patzelt 2014: 5).

Patzelt zieht aus dieser empirischen Beobachtung die Schlussfolgerung, dass unter den jetzigen Abgeordneten mehrheitlich Netzwerker seien, die es gelernt hätten, professionelles Beziehungsmanagement zu betreiben und sich an die Spielregeln des politischen Settings anzupassen (Patzelt 2014: 11). Letztgenannter Punkt schlägt erneut die Brücke zum Protagonisten Hajo Eichwald: Sein politisches Kapital besteht gerade darin, dass er sich innerhalb der eigenen Partei Allianzen und Rückhalt aufgebaut hat - eine Fähigkeit, die er noch in Zeiten der Bonner Republik kultiviert hat. Im Übrigen spielt der Clash of Cultures zwischen der analogen politischen Kultur der Bonner Republik, in der Eichwald politisch sozialisiert wurde, und der gegenwärtigen, oft digitalen politischen Realität in Berlin-Mitte eine ganz zentrale Rolle für den Humor der Serie.

\section{Handlungsrahmen: Partei und Parlamentsfraktion}

Neben der politikwissenschaftlichen Definition, wonach Parteien eine Interessenbündelungs- und Artikulationsaufgabe erfüllen (Korte 2009), sticht unter soziologischen Gesichtspunkten ein anderer Aspekt hervor: Die Rede ist von der sogenannten Omnibustendenz (Eldersveld 1964: 6-7). Dahinter verbirgt sich die Beobachtung, dass Parteien immer mit organisatorischen Spannungen und Richtungsentscheidungen zu kämpfen haben (Hoebel 2012: 70). Der Grund: Parteien sind auf die Akquise neuer Mitglieder angewiesen, um in der parlamentarischen und öffentlich-medialen Auseinandersetzung ihr Stimmgewicht weiter ausbauen 
zu können. Gleichzeitig konfrontiert sie diese Situation mit dem Problem, heterogene Interessenslagen und unterschiedliche Motive miteinander in Einklang bringen zu müssen, ohne dabei allerdings das eigene programmatische Profil aus den Augen zu verlieren (Hoebel 2012: 70, Donges 2008: 75). Dieser Balanceakt kommt in der Serie immer wieder zum Ausdruck, etwa wenn der Protagonist versucht, einen eigenen inhaltlichen Vorstoß zu einem bestimmten Thema in der Partei zu wagen. Hajo Eichwalds eigenes politisches Überleben ist immer an seine Fähigkeit geknüpft, die unterschiedlichen Interessensströmungen in seiner Partei auszutarieren oder in seinem Sinn gegeneinander auszuspielen.

Inspiriert von der Denkschule des Neo-Institutionalismus charakterisiert Elmar Wiesendahl (1998: 242) Parteien als offene und lose verkoppelte Handlungssysteme. Aus einer organisationstheoretischen Perspektive bleibt zu resümieren, dass Parteien unter den politischen Organisationen ausgesprochen komplexe und vielschichtige Systeme sind. Der Parteivorsitzende muss den Balanceakt vollbringen, die verschiedenen Logiken der Parteimitglieder - das Vote-, Policy-, Office-, und Democracy-Seeking - zu bündeln und zusammenzuführen (Donges 2008: 80).

Das Besondere an Parteien, was Jun (2010) noch einmal aufgreift, ist, dass sie Zusammenschlüsse von individuell handelnden Personen sind, die unterschiedlich gelagerte Interessen verfolgen (Donges 2008: 13f.). Diese Handlungsund Wirkungslogiken miteinander in Einklang zu bringen, ist die vorrangige Aufgabe von zentralen Führungsfiguren und Politikmanagern, wie den Parteivorsitzenden oder den Generalsekretären. Wenngleich der Parteivorsitzende in der Serie nicht zu Wort kommt, so spielen doch Hierarchie und Führung insbesondere in all jenen Szenen eine große Rolle, in denen die Fraktionsvorsitzende Birgit Hanke die Kulisse betritt.

In Analogie zu Parteien stehen Fraktionen, die „den sozialen Kontext für das faktische Abgeordnetenverhalten in Gesetzgebungsverfahren bilden“ (Hoebel 2012: 72), vor der Herausforderung, konfligierende Interessen und Positionen und die daraus möglicherweise entstehenden innerfraktionellen Spannungen auszuhalten. Vor diesem Hintergrund kommt den Fraktionsvorständen eine ganz essenzielle Bedeutung zu (Rudzio 2015: 228). Sie sichern in der parlamentarischen und in der medialen Arena als zentrale Akteure des Politikmanagements die Geschlossenheit ihrer Fraktion nach außen. Außerdem entwickeln die Fraktionsvorstände die Marschroute bei anstehenden Entscheidungen. Neben diesen planerisch-strategischen Fragen verteilen sie die Arbeit der Fraktion, indem sie zur Ausarbeitung gemeinsamer Programme fraktionsinterne Arbeitskreise und -gruppen bilden; außerdem erarbeiten die Vorstände Vorschläge für die Besetzung von Gremien und der Ausschüsse des Bundestages (Rudzio 2015: 228). 
Fasst man die ersten konzeptionellen Überlegungen zu Organisationsproblemen von Parteien und Parlamentsfraktionen zusammen, dann wird unter Rückgriff auf Elmar Wiesendahl deutlich, dass die Fähigkeit, widersprüchliche Handlungs- und Motivationslogiken auszubalancieren, maßgeblich über die Arbeitseffektivität von Organisationen entscheidet (Hoebel 2012: 78). Sowohl Parteien als auch Fraktionen stehen als Organisationen beide vor der Herausforderung, Interessen zu bündeln und kompakt zu artikulieren und dabei gleichzeitig das ihnen innewohnende Organisationsdilemma aufzulösen (Hoebel 2012: 78). Schließlich kristallisiert sich die Tendenz heraus, dass sich Organisationen - und politische insbesondere - mit steigender Zahl ihrer Mitglieder zu „fragmentierten und lose verkoppelten Anarchien“ entwickeln (Wiesendahl 1998: 242). Dieser Aspekt wird in der Serie vor allem dann deutlich, wenn Hajo Eichwald zwar auf die Zustimmung seiner Fraktionsvorsitzenden angewiesen ist, er jedoch mit List und Intrigen seinen Vorteil sucht, indem er die verschiedenen Interessen der Akteure gegeneinander ausspielt.

Wie lassen sich nun die vorangegangenen Überlegungen auf die Serie rückbeziehen? Hajo Eichwald ist sowohl in seiner Partei als auch in seiner Fraktion in verschiedene, bisweilen auch nur schwer in Einklang zu bringende Erwartungsstrukturen eingebunden. Von ihm wird der permanente Balanceakt erwartet, die unterschiedlichen Funktionslogiken in den einzelnen Arenen zu kennen und sich darin zu behaupten. Eine der Herausforderungen, mit denen sich Eichwald konfrontiert sieht, ist die Fähigkeit, eine gewisse Ambiguitätstoleranz zu entwickeln und sich die Funktionsmechanismen der unterschiedlichen Arenen zu vergegenwärtigen. Der Zuschauer begleitet Eichwald dabei und wird auf diesem Weg mit den Stilmitteln der Satire dazu angeregt, seine eigene Erwartungshaltung zu hinterfragen, die er den gewählten Abgeordneten entgegenbringt.

\section{SERIEN ALS SPIEGEL: DAS VERHÄLTNIS VON MEDIEN UND POLITIK}

Zur Beschreibung der Beziehung von Medien und Politik haben sich drei Paradigmen in der Forschung etabliert. Selbst in Serien, welche primär eine Unterhaltungsfunktion zu haben scheinen, spiegeln sich diese Paradigmen wider. Im Folgenden werden am Beispiel einer ausgewählten Sequenz die unterschiedlichen Konstellationen zwischen Medien und Politik dargestellt. Als Beziehungsmuster zwischen diesen beiden Akteuren können hierbei das Instrumentalisierungs-, Symbiose- und Gewaltenteilungsparadigma identifiziert werden, welche in Tabelle 1 näher erläutert werden. 
Tabelle 1: Zum Verhältnis von Politik und Medien

\begin{tabular}{|l|l|l|l|}
\hline Paradigmen & $\begin{array}{l}\text { Dominanter } \\
\text { Beziehungs- } \\
\text { modus }\end{array}$ & $\begin{array}{l}\text { Medien im politischen } \\
\text { Prozess }\end{array}$ & Analyseebenen \\
\hline Gewaltenteilung & Autonomie & $\begin{array}{l}\text { Neutralität, } \\
\text { Kritik und Kontrolle, } \\
\text { Gegenmacht }\end{array}$ & $\begin{array}{l}\text { vorwiegend } \\
\text { makroanalytisch }\end{array}$ \\
\hline Instrumentalisierung & Steuerung & $\begin{array}{l}\text { a) Politik als Steuerungsob- } \\
\text { jekt der Medien } \\
\text { b) Medien als Steuerungs- } \\
\text { objekt der Politik }\end{array}$ & makro- und \\
mikroanalytisch
\end{tabular}

Quelle: Sarcinelli 2011: 125.

\section{Das Instrumentalisierungsparadigma: Übermacht der Medien}

Eichwald und sein Team planen in der ersten Folge der Serie eine Anti-FastfoodKampagne. Die Inhalte dieser Initiative sind höchstens sekundär. Aus Eichwalds Sicht geht es vor allem darum, mediale Aufmerksamkeit zu erzielen. Das soll dadurch geschehen, dass übergewichtige Personen auf Burger-Schachteln zur Abschreckung abgedruckt werden. Das Medienecho fällt auf unterschiedlichen Kanälen katastrophal aus, darunter im Radio, in sozialen Netzwerken und in Printmedien, hierbei auch in der reellen Bild-Kolumne, Post von Wagner, die hier Bestandteil der fiktiven Serie ist. ${ }^{1}$ Der veröffentlichte Kommentar wird von Eichwalds Referenten während der Morgenlage vorgelesen.

Szenen-Beispiel:

Im Abgeordnetenbüro (S 1/F 1/09.25 - 09.36)

Grube: Lieber Hajo Eichwald, ich würde Sie mal gerne zum Essen einladen. Und dann, wenn wir gemeinsam vor zwei großen Cheeseburgern säßen, würde ich Ihren Kopf greifen und so lange mit voller Wucht auf den Pappteller...

Eichwald und sein Team reagieren entsetzt auf die außerordentlich negative Berichterstattung und ziehen die Gesetzesinitiative in der Konsequenz zurück. Die

1 Die täglich in der Bild erscheinende Kolumne des Boulevard-Journalisten Franz Josef Wagner ist für ihre oft polemische Art bekannt. 
„Rolle rückwärts“ wird als Überzeugungstat öffentlich kommuniziert. Die Maßnahme sollte als Weckruf an die Bevölkerung dienen, um sie vor möglichen zukünftigen und unverhältnismäßigen Vorschlägen dieser Art zu warnen.

Eichwalds Handeln infolge der negativen medialen Resonanz verdeutlicht in zweierlei Hinsicht die Übermacht der Medien (Sarcinelli 2011: 123): Zum einen orientiert sich Eichwalds Team bei der Politikdarstellung an der Medienlogik, indem die Gesetzesinitiative plakativ inszeniert wird. Dadurch geraten die Inhalte der Initiative in den Schatten und die oberflächliche Bebilderung der BurgerSchachteln in den Vordergrund. Zum anderen wird die geplante Gesetzesinitiative aufgrund der negativen Berichterstattung unmittelbar zurückgenommen. Insofern steht und fällt die Implementierung der Maßnahme mit der medialen Resonanz.

\section{Instrumentalisierungsparadigma: Übermacht der Politik}

Eichwald und sein Team ziehen sich, wie bereits erwähnt, aus der Affäre, indem sie die Gesetzesinitiative als bewusste Provokation inszenieren. Um dies zu verdeutlichen, zerreißt Eichwald vor laufender Kamera eine Burger-Schachtel mit dem Bild einer übergewichtigen Frau darauf und appelliert mittels einer direkten Videobotschaft an die Bürger.

\section{$\underline{\text { Szenen-Beispiel: }}$}

Im Abgeordneten Büro vor laufender Kamera (S 1/F 1/15.57 - 16.01)

Eichwald: Hallo, mein Name ist Hajo Eichwald. Und ich glaube an Sie, den mündigen Verbraucher.

In dieser Szene instrumentalisieren Eichwald und sein Team die Medien als puren Verlautbarer (Sarcinelli 2011: 123). Die politischen Akteure haben aufgrund des Wissensvorsprungs eindeutig die Oberhand und die Medien werden über Eichwalds Motive völlig im Unklaren gelassen. Sie können höchstens erahnen, dass Eichwalds ,Rolle rückwärts ' auf das negative Medienecho zurückzuführen ist - Belege gibt es für dieses Handeln jedoch keine. Dementsprechend erfüllen die Medien aus Eichwalds Sicht lediglich den Zweck, die Botschaft mit einer möglichst hohen Reichweite zu verbreiten. Unfreiwillig und ohne Absprache betreiben die Medien als verlängerter Arm die Schadensbegrenzung der Politik. Das nächste Problem lässt in Eichwald, $M d B$ jedoch nie lange auf sich warten.

Eichwalds Referenten Engemann und Grube realisieren während eines Bürogesprächs, dass die übergewichtige Dame namens Heinemann, deren Bild auf der Burger-Schachtel aufgeklebt war, die Eichwald vor laufender Kamera zerris- 
sen hatte, eine Prominente ist. Unmittelbar danach laufen die Telefone am Arbeitsplatz heiß und der nächste Skandal ist vorprogrammiert.

\section{Symbioseparadigma: Politik und Medien als Partner}

Eichwald und seine beiden Referenten stellen sich die Frage, wie sie den durch das Foto ausgelösten Skandal lösen können. Zunächst zieht Eichwald in Erwägung, sich zu entschuldigen, indem er Frau Heinemann einen Blumenstrauß mit einem Brief schickt. Engemann ist bereits auf dem Weg, um die Idee umzusetzen, als Grube einen alternativen Vorschlag macht.

$\underline{\text { Szenen-Beispiel: }}$

Im Abgeordnetenbüro (S 1/F 1/20.57 - 21.33)

Grube: Oder wieder fahren da jetzt hin.

Eichwald: Zu der Heinemann?

Grube: Ja.

Eichwald: Hast du das Foto gesehen? Spitzname Venus Fugidor.

Grube: Nein. Jetzt mal im Ernst. Ich mein, das ist doch wahre Größe. Hajo Eichwald macht einen Fehler, vielleicht auch zwei. Aber dann. Er fährt in den Grunewald, mit dem besten Blumenstrauß, den man für Geld kaufen kann, schüttelt der fetten Alten die Hand. Und ein Fotograf von der BILD ist auch noch dabei.

Engemann: Können wir nicht einmal was machen, weil es richtig ist, und nicht, weil es gut in der Zeitung aussieht?

Grube: Ja, Berndt. Aber wenn im Wald ein Baum umkippt und keiner hört zu. Macht es dann trotzdem Krach?

Engemann: Ach was. Der Baum in deinem Bild. Soll das Hajo sein?

Die Bild-Zeitung, die das Gesetzesvorhaben noch zuvor polemisch kommentierte, soll nun in einer partnerschaftlichen Beziehung als Mittler fungieren und bei der Inszenierung behilflich sein. Die Bild-Zeitung ist demnach exklusiv bei der geplanten Entschuldigung dabei und Eichwald erhält einen öffentlichkeitswirksamen Auftritt in einem reichweitenstarken Medium. Die Kooperation zwischen Medien- und Politikvertretern verläuft in dieser Episode schlussendlich zum Vorteil beider Akteure.

Im Rückgriff auf die zuvor definierten Beziehungsmuster handelt es sich bei dieser Konstellation um das Interdependenz- und Symbioseparadigma. Politiker sind auf Journalisten angewiesen, um mit den Wählern zu kommunizieren, wohingegen Medien wiederum an Meldungen aus der Politik interessiert sind. Diese Zusammenarbeit findet zum größten Teil auf der Hinterbühne statt, also jen- 
seits der Öffentlichkeit. Die politischen Eliten sind in dem Modell tendenziell die Agenda-Setter, wohingegen Medien die Rahmung der Themen besser beeinflussen können (Sarcinelli 2011: 123).

\section{Gewaltenteilungsparadigma: Medien als ,Vierte Gewalt‘}

In der zweiten Folge von Eichwald, $M d B$ soll in Hajo Eichwalds Wahlkreis ein Unternehmen mit 80 Angestellten geschlossen werden. Nun gilt es, einen Ausweg zu finden, um die Schließung bestenfalls zu verhindern oder sich elegant aus der Affäre zu ziehen. Im Zuge einer Krisensitzung des ,Team Eichwald“ macht Engemann seinen Vorgesetzten darauf aufmerksam, dass sein ehemaliger Studienfreund, Dominik Wohlfahrt, in der Wirtschaft tätig ist und die Schließung des bedrohten Unternehmens möglicherweise verhindern könnte. Letztlich vereinbaren Eichwald und Wohlfahrt ein Treffen auf Sylt, um die Angelegenheit persönlich zu besprechen.

Ein Bild-Journalist namens Hofmann erfährt durch ein öffentliches Facebook-Foto von der Begegnung auf der nordfriesischen Insel und vermutet informelle Vereinbarungen. Hajo gerät wegen einer zweifelhaften Verbindung zwischen Wirtschaft und Politik in das Visier des Journalisten. Kurze Zeit später ereignet sich eines der raren direkten Aufeinandertreffen zwischen einem Journalisten und einem Volksvertreter in der Serie. Die Rolle der Medien als treibende Kräfte, die den Protagonisten keine Verschnaufpause gönnen, wird hier erstmals deutlich.

Der Bild-Journalist Hofmann gibt sich im Interview investigativ und lässt sich weder von Eichwalds Büroleiterin noch von der Fraktionsvorsitzenden ablenken, die versuchen, Eichwald aus der Schusslinie zu manövrieren. Der Medienvertreter verkörpert zunächst das Idealbild eines investigativen Journalisten, welcher einzig der Wahrheit verpflichtet ist. Er tritt als unabhängiger Vertreter der vierten Gewalt und Kontrolleur staatlichen Handelns auf. Voraussetzung für die Erfüllung dieser Funktion ist eine weitestgehende Autonomie und Distanz zwischen Medien und Politik (Sarcinelli 2011: 122). Diesem Anspruch wird Hofmann im ersten Teil des Gesprächs mehr als gerecht. Im darauffolgenden Gesprächsverlauf beginnt diese Fassade jedoch zu bröckeln.

\section{Medienkritik: fließende Grenze zwischen Politik- und Boulevardberichterstattung}

Durch eine unbedachte Äußerung Eichwalds erfährt der Bild-Journalist von einer Geliebten des Unternehmers Wohlfahrt, die letzterer während seines SyltAufenthaltes im Beisein von Eichwald getroffen hat. 
Szenen-Beispiel:

Im Abgeordnetenbüro während des Interviews (S 1/F 2/18.36 - 19.02)

Eichwald: $\quad$ Ich hab' Dominik eingeladen, ob er mal wieder persönlich... Mit seiner Frau - die eine hervorragende Volleyballspielerin ist!

Hofmann: Ach Frau Wohlfahrt war mit auf Sylt?

Eichwald: $\quad$ Selbstverständlich. Und ich habe mit beiden bei langen Strandspaziergängen darüber geredet, was die Brenotek [Unternehmen] ganz konkret machen kann, um den Standort Deutschland auch in Zukunft zu stärken.

Hofmann: Entschuldigung. Ich muss eine Sache mal eben [sucht ein Bild auf seinem Tablet]. Ist das Frau Wohlfahrt?

Eichwald: Ja, ach das ist...

Hofmann: $\quad$...eine ganz angenehme Erscheinung. Oder?

Es folgen keine weiteren Fragen zu vermeintlichen Interessenkonflikten zwischen Eichwald und seinem Unternehmerfreund Wohlfahrt. Der Bild-Journalist blickt Eichwald mit einem verschmitzten Lächeln und ertappendem Blick an so als hätte er gefunden, wonach er gesucht hat. In dieser Szene gelingt den Drehbuchautoren und Regisseuren die Kür, indem sie ein und dieselbe Person, namentlich den Bild-Journalisten Hofmann, von einem zum nächsten Moment von der Rolle des investigativen Journalisten hin zum Boulevard-orientierten Medienmacher wechseln lassen.

Diese Szene kann als Medienkritik verstanden werden, welche den Trend zur Personalisierung und Skandalisierung der Politikberichterstattung anprangert. Kritisiert wird die hierdurch vermutete Entpolitisierung der Berichterstattung, welche unter anderem auf den Quoten- und Reichweitendruck von Medien zurückzuführen ist (Steg 2012: 68).

\section{SERIEN ALS DATEN: EICHWALD IM NETZ}

Wenngleich die Serie Eichwald, $M d B$ als Satire naturgemäß überspitzt und sich das Dargestellte nur sehr begrenzt auf die Realität übertragen lässt, variiert der Grad dieser Übertreibung jedoch stark. Die äußerst derbe Ausdrucksweise der Protagonisten von Eichwald, MdB untereinander stellt im Alltag von Parlamentariern und deren Mitarbeitern vermutlich eine Ausnahme dar - dies lässt sich jedoch schwerlich überprüfen. Anders steht es um die triste Einrichtung im Abgeordnetenbüro von Hajo Eichwald, die sich in der Realität nicht ganz so deprimierend vorfinden lässt (Heissler 2015). Eine andere in der Serie dargestellte Thematik erscheint jedoch nicht übertrieben oder verzerrt, sondern recht plausi- 
bel: die Nutzung von Social Media durch Eichwald und sein Team. Welche Ziele verfolgt der fiktive MdB Eichwald in den sozialen Netzwerken und was für ein Bild von Politikern transportiert die Serie damit? Bei der Beantwortung dieser Fragen lohnt sich zunächst ein Blick auf die tatsächlichen Parlamentarier aus Bund und Ländern hinsichtlich ihrer Social Media-Nutzung anhand der Studie Politiker im Netz, um anschließend die Erkenntnisse aus der Studie als Vergleichswerte für die Social Media-Nutzung von Hajo Eichwald zu verwenden.

\section{Abgeordnete in sozialen Medien}

In der Studie Politiker im Netz - Treiber und Hürden der Social Media-Nutzung bei Bundes- und Landtagsabgeordneten (Meckel/Hoffmann 2013) wurde das Verhalten von deutschen Parlamentariern in sozialen Netzwerken analysiert.

Erfragt wurde unter anderem, welche Funktion die Social Media-Nutzung für die Politiker hat. Dabei trat deutlich zutage, dass das Polit-Marketing hier eine dominierende Rolle einnimmt. Das zentrale Motiv für Parlamentarier ist das Erreichen ihrer potenziellen Wähler, Parteifreunde und Unterstützer (Meckel/Hoffmann2013: 3). Der Kontakt zu den genannten Gruppen wird jedoch meist als Einbahnstraße verstanden, ein Austausch wird selten als Ziel genannt.

Die Authentizität des Internetauftritts ist nur bei knapp 40 Prozent der Befragten durch ein ausschließlich durch sie selbst gepflegtes Social Media-Profil gewährleistet. Jeder zehnte lässt seine Kanäle gar überwiegend oder vollständig von Dritten betreuen, beispielsweise durch Mitarbeiter oder Agenturen (Meckel/Hoffmann 2013: 4).

Im ,Neuland Internet“ zeigt sich - wenig überraschend - eine deutliche Spaltung der Generationen, was die Intensität der Social Media-Nutzung angeht. Jüngere Abgeordnete sind eigenständiger, aktiver und mehr im Austausch. Neu ist zudem die Möglichkeit, an den klassischen Medien vorbei direkt eigene Zielgruppen anzusprechen. Journalisten sind selten Teil dieser Gruppe, es ist ohnehin schwierig für einen einzelnen Abgeordneten, in der breiten medialen Öffentlichkeit durchzudringen (Meckel/Hoffmann 2013: 5).

In Betrachtung der Gesamtheit der Parlamentarier lassen sich drei Typ-Profile bilden, die in der Studie ,der Profi ‘, , der Begeisterte ‘ und ,der Skeptiker' getauft werden. Der überwiegende Anteil (62 Prozent) macht der Profi aus, der meist aus einer der beiden Volksparteien CDU und SPD stammt und seinen Kanal in geübter Weise für Eigenwerbung nutzt. Es folgt der Begeisterte (24 Prozent), der durchaus auch in Kontakt mit seiner Anhängerschaft tritt und oft den Piraten, aber auch der Linken oder der CSU angehört. Der Skeptiker ist oftmals älter und eher die Ausnahme (14 Prozent). Wenig überraschend zeichnet er sich durch 
eine geringe Nutzung sozialer Medien aus und entstammt überproportional häufig den Grünen und der Linken (Meckel/Hoffmann 2013: 6).

\section{Eichwald in sozialen Medien}

Der Protagonist Hajo Eichwald nutzt die sozialen Medien regelmäßig, jedoch nicht immer zu seinem Vorteil. Kommunikative Schnellschüsse sind ebenso häufig wie versuchte Befreiungsschläge über Social Media, die kläglich scheitern. In der Hektik der Serie, vor allem verursacht durch Eichwalds ständige Fehltritte, sind persönliche Stellungnahmen in den sozialen Medien oft das erste Mittel, das dem Abgeordneten und seinen Mitarbeitern in den Sinn kommt.

Für Eichwald als Direktkandidat des Wahlkreises Bochum II und Hinterbänkler, der um jedes Fünkchen Aufmerksamkeit mühsam kämpfen muss, ist die Möglichkeit, die Öffentlichkeit und potenzielle Wähler an den klassischen Medien vorbei zu erreichen, von großer Wichtigkeit. Ein konstruktiver Dialog mit der Wählerschaft im Internet ist jedoch bei Eichwald - wie auch bei den meisten seiner realen Kollegen (Meckel/Hoffmann 2013: 3) - nicht zu erkennen. Eichwald erreichen stattdessen E-Mails oder erboste Sprachnachrichten auf seinem Anrufbeantworter, die dieser jedoch nicht als Anreiz empfindet, mit den Bürgern in einen Austausch zu treten Vielmehr stellen die Nachrichten für ihn einen Seismographen dafür dar, wie tief er sich mit seinen verfehlten politischen Kniffen in die Misere manövriert hat. In den sozialen Medien ist Eichwald auf ,Senden ' und nicht auf ,Empfangen' ausgerichtet.

\section{Szenen-Beispiel:}

Eichwald kommt mit seinem Tablet in der Hand aus einem Sitzungssaal des Bundestags, seine drei Mitarbeiter erwarten ihn bereits vor der Tür (S 1/F 1/9.29 - 9.38).

Eichwald: Habe im Ausschuss gerade euer Statement gelesen und geantwortet und gerade bei Facebook schon auf ,Gefällt mir!‘ geklickt. So. Wie mache ich Apps?

Grube: Ja, Hajo, Folgendes...

Seine Social Media-Aktivitäten delegiert Eichwald - aus Mangel an eigenen Fähigkeiten - fast vollständig. Sein Mitarbeiter Grube kann hier durchaus Kompetenzen vorweisen und versucht auch, diese als seine Existenzberechtigung im Abgeordnetenbüro in den Vordergrund zu rücken. In der Serie wird allerdings deutlich, dass soziale Medien nur ein Mittel zum Zweck sein können und eine Nachricht nur so gut ist wie der Inhalt, den sie transportiert. Entsprechend selten sind Eichwalds Versuche von Erfolg gekrönt, sich im Netz als erfolgreicher Politiker zu präsentieren. So floppt in Folge 3 der ersten Staffel seine unausgegorene 
Idee, eine Lebensmittelampel einzuführen, schon aufgrund der hastigen, unprofessionellen Vorbereitung seiner Videobotschaft und sie endet in einem PRDesaster, wie bereits im dritten Kapitel beschrieben.

Mit seinen in der Serie thematisierten Tweets und Facebook-Beiträgen gelingt es Eichwald durchaus, Journalisten zu erreichen und seine Statements so an die Öffentlichkeit zu bringen. Diese stehen in aller Regel unter dem Zeichen der Krisenkommunikation, da er immer wieder versucht, von ihm selbst verursachte Probleme mit medialen Ausweichmanövern in den Griff zu bekommen. Die Krise als Dauerzustand ist jedoch nichts, was den Alltag der deutschen Bundes- und Landtagsabgeordneten kennzeichnet. Entsprechend ist der genannte Bruch von Eichwalds Social Media-Nutzung mit den Studienergebnissen an dieser Stelle dem satirischen Unterhaltungsformat geschuldet.

Eichwald entstammt einer Volkspartei und nutzt soziale Medien in der Regel zur gezielten Eigenwerbung. Er weist auf eigene Aktivitäten und Erfolge hin und versucht damit, Wähler, Parteifreunde und Unterstützer zu erreichen, aber auch in die breite mediale Öffentlichkeit durchzudringen. Am ehesten lässt sich Eichwald dem beschriebenen Nutzerprofil des "Profis` zuordnen, wenngleich dies eine Bezeichnung ist, mit der man den vom einen in das nächste Fettnäpfchen Tretenden ansonsten kaum assoziieren würde.

\section{Soziale Medien als Mittel zum Zweck}

Eichwald vermittelt das Bild eines Abgeordneten, dessen politischer Gestaltungswille längst erloschen ist. Die Selbstdarstellung über soziale Medien wird als Substitut für tatsächliche politische Erfolge genutzt. Das fatale Alleinstellungsmerkmal von Eichwalds Social Media-Nutzung ist die Tatsache, dass seine abgesetzten Nachrichten meist verzweifelte Versuche sind, irgendwie noch in der öffentlichen Debatte vorzukommen, um so seine Daseinsberechtigung als Volksvertreter zu demonstrieren. Das Zu-Wort-Melden in sozialen Netzwerken wird so zum Selbstzweck und die Serie zelebriert diese Tatsache mit den absurdesten Ideen, mit denen Eichwald und sein Team Aufmerksamkeit im Netz generieren wollen - beispielsweise mit den erwähnten Abschreckbildern auf Burger-Schachteln gegen ungesunde Ernährung, wie man sie von Zigarettenpackungen her kennt.

Andreas Jungherr erkennt in den sozialen Medien die Möglichkeit, dass Hinterbänkler und Bürger an den etablierten Medien vorbei an die Öffentlichkeit gelangen können und damit ermächtigt sind, in gewissem Umfang Agenda-Setting zu betreiben. In diesem Zusammenhang fragt er auch: „Wie kontrollieren Parteien die öffentliche Themensetzung, wenn sich Hinterbänkler durch intensive Nutzung von Social Media-Kanälen zu inoffiziellen Sprechern der Partei entwi- 
ckeln?“ (Jungherr 2009: 23). Diese weitere Problematik bilden in der Serie die Szenen mit der Fraktionsvorsitzenden Hanke ab. Vor ihr muss sich Eichwald regelmäßig rechtfertigen, wenn er erneut ein kommunikatives Chaos angerichtet hat.

Der Protagonist beweist ein bemerkenswertes Talent, zu demonstrieren, warum soziale Medien ein zweischneidiges Schwert sind. Mit einfachen Mitteln kann er wesentlich mehr Aufmerksamkeit generieren als über klassische Wege im Kontakt mit Journalisten. Zugleich besteht die Gefahr, sich selbst bloßzustellen oder zu blamieren. Eichwald, $M d B$ zeichnet das Bild eines überforderten, älteren, verzweifelten Hinterbänklers, der zu sozialen Medien als einem rettenden Strohhalm greift, um irgendwie an die Öffentlichkeit durchzudringen. Die Serie verdeutlicht durch die Social Media-Thematik, wie sich die neu gewonnene Macht des Zugangs zur Öffentlichkeit auch gegen den Veröffentlichenden wenden kann.

\section{NARRATIVE ELEMENTE: HAUTNAH IM BERLINER HAIFISCHBECKEN}

Eine der Hauptfragen des Berufspolitikers beschäftigt sich mit der Inszenierung seiner Tätigkeit und Persönlichkeit sowohl nach innen als auch nach außen. Hajo Eichwald stellt hierbei keine Ausnahme dar, was seine Ambitionen zur Selbstvermarktung in den sozialen Medien unter Beweis stellen. Die Form und die Möglichkeiten seiner Inszenierung sind hierbei durch äußerliche Einflüsse bestimmt. Er reagiert mit seinem Handeln meist auf diese Einflüsse und passt seine Inszenierung hierbei an - ähnlich wie ein Schauspieler, welcher versucht, den unterschiedlichen Ansprüchen des Publikums bei einer Aufführung gerecht zu werden (Horn/Warstat 2003). Dem Versuch einer solchen Inszenierung des Protagonisten Hajo Eichwald in der Serie Eichwald, $M d B$ wohnt der Zuschauer ähnlich wie bei einer Theateraufführung bei - mit dem Unterschied, dass sich die Charaktere in der Serie der Anwesenheit eines unbeteiligten Beobachters unbewusst sind. Hierbei treten drei narrative Elemente immer wieder in den Vordergrund: die Allgegenwärtigkeit einer akuten Krise, die Machtverhältnisse im Politikbetrieb sowie das Verhältnis und die Interaktion beziehungsweise Nichtinteraktion zwischen Wählern und Gewählten.

\section{Krise als Dauerzustand}

Durch das zentrale, wiederkehrende narrative Element eines Skandals, in den Hajo Eichwald verwickelt ist, werden die Inszenierungs- und Reaktionsversuche 
Eichwalds im Verlauf der Serie immer wieder auf die Probe gestellt. Die Krise ist in Eichwald, $M d B$ allgegenwärtig und fordert stets die vollständige Konzentration aller Mittel und Ressourcen seines Mitarbeiterstabs, um ein Ende Eichwalds politischer Karriere zu verhindern und seinen Kopf noch einmal aus der Schlinge zu ziehen.

Die politische Krise und der Umgang mit einem Skandal dienen bei Eichwald, $M d B$ als zentrale Qualitätsmerkmale für die Bewertung der Fähigkeit eines Politikers - andere wichtige Bereiche des Berufsalltags eines Bundestagsabgeordneten, wie etwa die Bedeutung der innerfraktionellen Koordination oder der Austausch mit dem Bürger und dem Wahlkreis, finden kaum oder gar keine Erwähnung. Die Fähigkeit eines durchdachten Krisenmanagements ist bei Eichwald bestenfalls schwach ausgeprägt: Von verschiedenen Vorschlägen seines Teams geleitet, übernimmt er meist einen der präsentierten Lösungsvorschläge, ohne über etwaig nachhaltigere Lösungskonzepte oder mögliche Konsequenzen seiner kurzsichtigen Handlungen nachzudenken.

Vor allem diese Verhaltensweisen zeigen seine Überforderung mit den von ihm als unfair empfundenen Forderungen der Öffentlichkeit an einen Politiker. Er entspricht keinem klassischen Typus eines Skandalpolitikers ${ }^{2}$, sondern stellt eine unberechenbare Mischform verschiedenster Arten des Umgangs mit einem politisch-medialen Skandal dar (Bude 1989).

Bezeichnend für diese Unfähigkeit eines professionellen Umgangs mit der Krise ist die jeweilige Auflösung der Krisensituation in den einzelnen Episoden, welche unter dem Begriff , glückliche Umstände‘ zusammengefasst werden können. Der Ablauf eines Skandals folgt in Eichwald, MdB stets dem gleichen Muster: Nach dem medialen Bekanntwerden des Skandals verschlimmert Eichwald seine Lage durch eigene Bewältigungsversuche immer weiter, bis etwa im bereits genannten Beispiel der zweiten Folge ein Bild-Journalist von einer Affäre des Unternehmers Wohlfahrt auf Sylt erfährt und sich die mediale Berichterstattung danach auf diese Thematik verschiebt - Eichwald entwischt dem Skandal um seine Person durch Zufälle und glückliche Fügungen.

Trotzdem erfüllt die durchlittene Krise einen regulatorischen Effekt: Die Angst vor einem erneuten Losbrechen einer Empörungswelle wirkt als soziale Selbstkontrolle (Neckel 1990) und beeinflusst Eichwald in seinem Handeln auch wenn ihm kein wirklicher Lernprozess attestiert werden kann.

2 In der Literatur wird hier zwischen dem Unbeirrbaren, dem taktierenden Skandalierer und dem vom Skandal überwältigten unterschieden (vgl. Bude 1989) 


\section{Macht und Machterhalt}

Ein ebenfalls in jeder Folge wieder auftretendes Element ist die Bedeutung von Macht und Hierarchie im politischen Berlin. Hierbei sind vor allem die Rollen Hajo Eichwalds und der komplett gegensätzlich porträtierten Fraktionsvorsitzenden Hanke zu erwähnen. Während Hanke als kühl, kalkuliert und grundsätzlichen Prinzipien folgend dargestellt wird, erscheint Eichwald als unterwürfiger, durchaus sympathischer Hitzkopf, der in der Zeit stehen geblieben ist und keine grundlegenden Ideale mehr vertritt. Beide Schlüsselfiguren verfolgen zudem unterschiedliche Ziele: Hanke arbeitet als Fraktionsvorsitzende vor allem auf ihren Machtausbau hin und würde zum Erreichen ihrer Ziele in der Radikalität sicherlich auch nicht vor Methoden eines Frank Underwood aus House Of Cards zurückschrecken. Verdeutlicht wird dies sehr gut durch das Rollenprofil der Fraktionsvorsitzenden im (realen) Presseportal des ZDF, in welchem ihr eine Sicht auf die Welt als „,ein ewiges Fressen und Gefressen werden“ attestiert wird (ZDF 2015a). Eichwald wiederum kämpft nur ums Überleben im Berliner Politikbetrieb und um seinen Machterhalt - auch wenn er außerhalb seines Stimmrechts in Form des Bundestagsmandates über keine andere nennenswerte Form von Einfluss zu verfügen scheint.

Besonders stark wird dieses Machtungleichgewicht bereits in einer Szene der ersten Folge verdeutlicht, in welcher die Fraktionsvorsitzende Hanke zum ersten Mal auftritt. Das sich kurzfristig ankündigende Erscheinen Hankes versetzt Eichwald in helle Aufregung und er versucht noch im letzten Moment, kleinstmögliche Anpassungen wie das Öffnen eines Fensters oder das Reichen eines Obsttellers zu veranlassen, um den ihm drohenden Ärger abzumildern - ganz wie ein kleiner Junge, der weiß, dass ihm unvermeidlich Ärger für seine Taten droht. Der Symbolik des Essens kommt als zentrales Besänftigungselement für heikle Situationen in der Serie generell eine besondere Bedeutung zu: Beim gemeinsamen Wackelpuddingessen wird das Fehlen von Vanillesauce zum Streitthema und Lebensmittelfunktionäre sollen mit Kuchen milde gestimmt werden.

Auch ein hohes Stresslevel Eichwalds und seine Überforderung mit Stresssituationen sind wiederkehrende Motive. Untermalt wird dieses zusätzlich von einem minimalistischen Soundtrack aus hektischen Schlagzeugsoli, die immer dann eingespielt werden, wenn zwischen zwei Handlungsszenen Berliner Parlamentsgebäude als Übergangsbilder gezeigt werden. Diese unterstreichen die besondere Form der Rastlosigkeit des Politikbetriebes und verstärken das Gefühl, getrieben zu sein. 


\section{Wahlen und Gewählte}

Ein Besonderer Blick lohnt sich bei Eichwald, $M d B$ darauf, was explizit nicht gezeigt und erwähnt wird, aber trotzdem über allem schwebt: der direkte Einfluss des Wählers und die starke Abhängigkeit Eichwalds von der Gunst der Wähler seines Wahlkreises.

Eichwalds Versuchen der Krisenbewältigung liegt die Intention zugrunde, dem potentiellen Wähler ein Bild von sich als dessen erfolgreicher Repräsentant $\mathrm{zu}$ vermitteln und somit auch künftig seinen Machtanspruch zu legitimieren (Hitzler 1994: 290). Für eine erfolgreiche Ausrichtung seiner Tätigkeit anhand der Vorstellungen seiner Wähler ist er jedoch auf Feedback angewiesen, welches im realen Politikbetrieb beispielsweise mit dem Instrument der Meinungsforschung einen ganzen Wirtschaftszweig beschäftigt. In Eichwald, MdB erfährt der Protagonist jedoch ausschließlich über die mediale Berichterstattung vom Misserfolg seiner Aktionen.

Die einzige Ausnahme hierzu bildet eine Szene am Ende der vierten Episode, in welcher einige Sprachnachrichten von Bürgern auf dem Anrufbeantworter Eichwalds vorgespielt werden. Eine direkte Interaktion mit dem Wähler findet aber auch hier nicht statt - Hajo Eichwalds Handeln und der Politikbetrieb Berlins erscheinen so als losgelöst und fern von den Interessen des Volkes.

\section{FAZIT}

Eichwald, $M d B$ ist eine Satire, die im Umfeld eines Berliner Abgeordnetenbüros spielt und den gleichnamigen tragikomischen Protagonisten porträtiert. Eichwald wird stets von unterschiedlichen Interessen, Zwängen und Akteuren begleitet, die zuweilen offensichtlich oder nur angedeutet zutage treten. Die klassischen Beziehungsverhältnisse zwischen Medien und Politik, namentlich das Instrumentalisierungs-, Symbiose- und Gewaltenteilungsparadigma, werden in der Serie geradezu idealtypisch inszeniert. Eine eindeutige Antwort darauf, welche Konstellation zur Beschreibung der Beziehung von Medien und Politik zutreffend ist, lässt die Serie aber offen. Der situative Kontext und die rationalen Interessen der Akteure in den jeweiligen Arenen entscheiden letztlich, welches Verhalten sie an den Tag legen, die Bewertung dieses Verhaltens bleibt dem $\mathrm{Zu}$ schauer überlassen.

Eichwald, $M d B$ begnügt sich nicht mit der Rolle der traditionellen Medien wie Print und Fernsehen, sondern zeichnet auch ein Bild vom Umgang des politischen Betriebes mit sozialen Netzwerken wie Twitter oder Facebook. Auch in diesem Feld gibt es deutliche Parallelen zwischen dem durchschnittlichen Nut- 
zungsverhalten eines realen Bundestagsabgeordneten und dem fiktiven Protagonisten Eichwald. Der Fokus auf das Veröffentlichen eigener Erfolge und die Delegation der Profilpflege an Mitarbeiter spiegelt wider, was vielfach Realität in Berliner Abgeordnetenbüros ist. Gleichwohl führt die Krise als wiederkehrendes narratives Element in Eichwalds Team zu Stilblüten, die den Satirecharakter der Serie verdeutlichen. Es ist kein Zufall, dass die Krise als Dauerzustand ebenfalls in der BBC-Serie The Thick of it ein Charakteristikum darstellt, wie im Kapitel "Narrativ“ der diesbezüglichen Serienanalyse dargestellt wird. Das ZDF bezeichnet Eichwald, $M d B$ als ,inspiriert“ von der derben Polit-Comedy aus Großbritannien (2015b).

Eichwald hat es im Laufe seiner Zeit als Abgeordneter perfektioniert, zwischen den unterschiedlichen Erwartungslogiken innerhalb seiner Partei und seiner Fraktion hin und her zu changieren. Eine der Herausforderungen, mit denen sich Eichwald permanent konfrontiert sieht, ist die Fähigkeit, eine gewisse Ambiguitätstoleranz zu entwickeln und sich die Funktionsmechanismen der unterschiedlichen Arenen immer wieder aufs Neue zu vergegenwärtigen.

Aus erzählerischer Sicht konzentriert sich die Serie Eichwald, $M d B$ auf zwei Kriterien zur Bewertung der Kompetenz von Berufspolitkern: auf den Umgang mit übergroß erscheinenden Herausforderungen in Form von Krisen und auf die Frage nach der politischen Macht. Durch den Anspruch an einen Berufspolitiker, sich stets unverrückbar als Fels im medialen Sturm zu behaupten, vermittelt die Serie die Fähigkeiten zum Krisenmanagement und zur Selbstinszenierung als zentrale Elemente zur Bewertung der Kompetenz eines Politikers. Wenngleich humoristisch überzeichnet inszeniert, zeigt dieser Anspruch den Wunsch nach politisch starken Leitfiguren und Vorbildern, die vorangehen und agieren, anstatt bloß zu reagieren.

In Bezug auf die persönliche Stellung und informelle Macht eines Abgeordneten zeigt die Serie unterschiedliche Ansprüche und Selbstverständnisse der eigenen Rolle. Die Existenz von Macht wird mit dem Begriff der Stärke assoziiert - eine vergleichsweise machtlose Person wie der Hinterbänkler Hajo Eichwald wird als schwach und unsicher porträtiert.

Eichwald, $M d B$ schafft den Spagat, die wechselseitigen Zwänge in der administrativen, parlamentarischen und medialen Sphäre zugespitzt anzudeuten, ohne den Humor auf der Strecke zu lassen. Eichwald ist als Getriebener stets unter Druck, versucht einem Skandal nach dem anderen auszuweichen und Schadenbegrenzung zu betreiben. Der Kampf um das politische Überleben als kleiner Fisch im großen Haifischbecken des Berliner Politikbetriebes ist hierbei allgegenwärtig. 


\section{LITERATUR}

Alemann, Ulrich von (1995): Parteien, Hamburg.

Bude, Heinz (1989): Typen von Skandalpolitikern, in: Ebbighausen, Rolf/ Neckel, Sighard (Hrsg.): Anatomie des politischen Skandals, Frankfurt a.M., S. 396-414.

Deutscher Bundestag (1967): Gesetz über die politischen Parteien (Parteiengesetz), in: https://www.bundestag.de/blob/189336/c8a22a376ea9165443a4771 01f2352dd/pg_pdf-data.pdf (zugegriffen am 1.3.2017).

Donges, Patrick (2008): Medialisierung politischer Organisationen, Wiesbaden.

Eldersveld, Samuel J. (1964): Political Parties - A Behavioural Analysis, Chicago.

Heissler, Julian (2009): Nachbau made in Germany, in: Der Freitag, https:// www.freitag.de/autoren/julian-heissler/nachbau-made-in-germany (zugegriffen am 4.3.2017).

Hesse, Jens (2012): Das Regierungssystem der Bundesrepublik Deutschland, 10. Aufl., Baden-Baden.

Hitzler, Ronald (1994): Die Banale Seite der Macht. Politik als Beruf heute und morgen, in: Berking, Helmuth/Hitzler, Ronald/Neckel, Sighard (Hrsg.): Politikertypen in Europa, Frankfurt a.M., S. 280-295.

Hoebel, Thomas (2012): Politische Organisationen, in: Apelt, Marion/Tacke, Veronika (Hrsg.): Handbuch Organisationstypen, Wiesbaden, S. 63-91.

Jun, Uwe (2010): Parteien als fragmentierte Organisationen: Erfolgsbedingungen und Veränderungsprozesse, Opladen.

Jungherr, Andreas (2009). Twitternde Politiker: Zwischen buntem Rauschen und Bürgernähe 2.0, in: Bieber, Christoph/Eifert, Martin/Groß, Thomas/Lamla, Jörn (Hrsg.): Soziale Netze in der digitalen Welt: Das Internet zwischen egalitärer Beteiligung und ökonomischer Macht, Frankfurt a.M., S. 99-127.

Marcinkowski, Frank/Pfetsch, Barbara (Hrsg.): Politik in der Mediendemokratie, Wiesbaden.

Meckel, Miriam/Hoffmann, Christian Pieter (2013). Politiker im Netz: Treiber und Hürden der Social Media-Nutzung unter Bundes- und Landtagsabgeordneten, in: http://www.isprat.net/fileadmin/downloads/projekte/2011/Politiker\%20im\% 20Netz.\%20Verständnis\%20für\%20das\%20Web\%202.0\%20und\%20eLiteracy \%20unter\%20politischen\%20Entscheidungsträgern/ISPRAT_Abschlussbericht _Politiker_im_Netz.pdf (zugegriffen am 3.12.2017).

Neckel, Sighard (1990): Die Wirkungen politischer Skandale, in: Aus Politik und Zeitgeschichte (7), S. 3-10. 
Niemeier, Timo (2017): Überraschung: ZDF setzt „Eichwald, MdB“ doch noch fort, DWDL vom 21.5., in: https://www.dwdl.de/nachrichten/61577/berra schung_zdf_setzt_eichwald_mdb_doch_noch_fort/ (zugegriffen am 3.12.20 17).

Patzelt, Werner (2014): Abgeordnete und ihr Beruf, Wiesbaden.

Rudzio, Wolfgang (2015): Das politische System der Bundesrepublik Deutschland, 9. Aufl., Wiesbaden.

Sarcinelli, Ulrich (2011): Politische Kommunikation in Deutschland: Medien und Politikvermittlung im demokratischen System, 3. Aufl., Wiesbaden.

Steg, Thomas (2012): Formwandel. Personalisierung und Privatisierung von Politik, in: Hombach, Bodo (Hrsg.): Politik und Medien, Bonn, S. 67-82.

Warstat, Matthias/Horn, Christian (2003): Politik als Aufführung. Zur Performität politischer Ereignisse, in: Fischer-Lichte, Erika/Horn, Christian/Uma thum, Sandra/Warstat, Matthias (Hrsg.): Performativität und Ereignis, Tübingen und Basel, S. 395-417.

Wiesendahl, Elmar (1998): Parteien in Perspektive: Theoretische Ansichten der Organisationswirklichkeit politischer Parteien, Opladen.

ZDF (2015a): Rollenprofile - Eichwald, MdB - Vierteilige Polit-Satire, in: https://presseportal.zdf.de/pm/eichwald-mdb/ (zugegriffen am 29.3.2017).

ZDF (2015b): Vorwort - Eichwald, MdB - Vierteilige Polit-Satire, in: https://presseportal.zdf.de/pm/eichwald-mdb/ (zugegriffen am 29.10.2017). 


\title{
Eurokrise, Sex und Klimawandel
}

\author{
Krisenmanagement in der britischen Sitcom
}

Yes, Prime Minister

Ann-Kathrin Binot, Ulrike Gansen, Anatoli Kolembach, Martin Miller

\section{NEUAUFLAGE VON YES, PRIME MINISTER}

Nach über 30 Jahren wurde 2012 mit Yes, Prime Minister eine der erfolgreichsten und beliebtesten Sitcoms des Vereinigten Königreichs neu aufgelegt. Erstmalig zwischen 1980 und 1988 auf $B B C$ ausgestrahlt, gewannen die Vorgänger Yes, Minister (drei Staffeln) und Yes, Prime Minister (zwei Staffeln) zahlreiche Preise und Auszeichnungen und wurden 2004 in einer Zuschauerwahl zu Britain's Best Sitcom auf Rang sechs gewählt (BBC 2004). Selbst Margaret Thatcher erklärte sie zu ihrer Lieblingsserie und spielte bei einer Preisverleihung im Jahr 1984 in einem Sketch mit (Day 2013). Im Zuge der Neufassung wurde nun eine weitere Staffel mit insgesamt sechs Episoden für BBC Gold produziert.

Die Handlung spannt sich dabei nach wie vor um James ,Jim“ Hacker, der sich im Serienverlauf vom Minister zum Premierminister des Vereinigten Königreichs hochgearbeitet hat, dennoch aber oftmals hilflos, naiv und übermütig wirkt. Als sein Konterpart tritt Sir Humphrey Appleby auf, der als beamteter Cabinet Permanent Secretary den traditionsbewussten civil service verkörpert und stets versucht, seine Privilegien zu schützen. Bernard Woolley steht dazwischen - faktisch Teil des civil service, als Parliamentary Private Secretary des Premierministers jedoch gleichzeitig zur Loyalität gegenüber Hacker verpflichtet, versucht er die Interessen seiner beiden ,Herren“ auszubalancieren. Als weiterer Charakter wurde mit der Neuauflage von 2012 Claire Sutton eingeführt, welche den modernen Typus des politischen Beraters repräsentiert.

In der neuen Staffel sieht sich Hacker als Veranstalter einer Konferenz des Ministerrats der Europäischen Union (EU) unter der aktuellen britischen Rats- 
präsidentschaft mit den Folgen der Finanzkrise konfrontiert, die sich zunehmend zu einer Krise des Euroraums zuspitzt. Eine schnelle Lösung der Geldsorgen verspricht ein Kreditangebot von Kumranistan, das an den Bau einer Ölpipeline in die EU geknüpft ist. Dass dabei ein Beitritt des Vereinigten Königreichs zum Euroraum zwingend notwendig ist, verschweigt Appleby dem Premierminister zunächst, bis Hacker und Sutton die Intrige aufdecken und auf eine Änderung der Vertragsbedingungen hinwirken. Nach einem gemeinsamen Abendessen mit der diplomatischen Delegation aus Kumranistan scheint der Deal in greifbarer Nähe, bis der Außenminister von Kumranistan eine pikante Bedingung aufstellt: Er verlangt noch am selben Abend die Dienste mehrerer Prostituierten, ansonsten käme keine Einigung zustande. Hacker und seine Berater sehen sich in einem Dilemma: Sollen sie ihre moralischen Vorbehalte übergehen und das Risiko, dass die Sexaffäre an die Öffentlichkeit kommt, in Kauf nehmen, oder die Zukunft der EU aufs Spiel setzen? Zusätzlich bezichtigen Hacker und Appleby sich gegenseitig der Steuerhinterziehung beziehungsweise Veruntreuung und streiten sich über die Reformierung des civil service. Dann erscheint auch noch der schottische Vize-Premierminister und kündigt ein Referendum zur Unabhängigkeit Schottlands an. Den Medien werden immer wieder Informationen über Vorgänge im Landhaus des Premierministers zugespielt, sodass das Team um Hacker sich auch mit der Öffentlichkeit auseinandersetzen muss. Aufgelöst wird die Problemsituation - nachdem der Kumranistan-Deal aufgrund des Zögerns von Hacker geplatzt ist und die Medien fälschlicherweise über einen Prostitutionsskandal berichten - durch ein Fernsehinterview, in dem Hacker verkündet, dass nicht die Eurokrise, sondern der Klimawandel das eigentliche Problem sei und bearbeitet werden müsse.

Bemerkenswert ist die weitestgehend realitätsgetreue Abbildung des politischen Systems, für die die Drehbuchautoren Anton Jay und Jonathan Lynn Informationen aus dem direkten Umfeld des Premierministers beziehungsweise der britischen Regierung bezogen haben (Brew 2007, Jay 2013). Zu beachten gilt jedoch, dass die Serie als politische Satire das Denken und Handeln von Politikern und Regierungsangestellten karikiert. Durch die Verwendung von Ironie, Sarkasmus, Übertreibung, Vergleiche und Analogien wird die Realität verzerrt. Dennoch muss eine Satire auf anerkannten und geteilten Einstellungen, Stereotypen oder Denkmustern fußen, um Wirkungskraft zu entfalten und nicht Abwehrreaktionen beim Rezipienten zu evozieren. Entscheidend ist also, dass Satire sich nicht zu sehr von der Wirklichkeit entfernt - was sie zu einem idealen Analysegegenstand macht (Behrmann 2002: 35-36, 352-353).

Im Folgenden wird die 2013 ausgestrahlte Neuauflage von Yes, Prime Minister anhand der von Switek im einleitenden konzeptionellen Beitrag vorgeschla- 
genen vier Forschungsperspektiven analysiert, wobei zu einem vollständigen Verständnis teilweise Rückbezüge zu den Vorgänger-Serien notwendig sind.

\section{KONTEXT: REGIEREN IM VEREINIGTEN KÖNIGREICH - STRUKTUREN, AKTEURE UND ENTWICKLUNGEN}

Yes, Prime Minister gewährt den Zuschauern Einblicke in die Strukturen, Funktionsweisen und Regierungsorganisation des politischen Systems des Vereinigten Königreichs von Großbritannien und Nordirland. Ein vollständiges Verständnis der Handlungen und Machtstrukturen ist ohne Kenntnisse der Charakteristika britischer Regierungsführung kaum möglich. Aus diesem Grund werden in diesem Kapitel wichtige Kontextinformationen skizziert. Das erste Unterkapitel nimmt das Westminster-Modell, allgemeine Systemstrukturen, die herausgehobene Machtposition des Premierministers sowie seine Beziehung zum civil service in den Blick, das zweite Unterkapitel erläutert die Bedeutung des britischen Imperialismus und der fortschreitenden Devolution, und zuletzt wird die Struktur des britischen Mediensystems komprimiert dargestellt.

\section{Politisches System der Westminster-Demokratie}

Das britische Regierungssystem beschrieb und beschreibt mit seiner Absenz einer kodifizierten Verfassung, seiner Konkurrenzdemokratie und seinem Zweiparteiensystem, der festgeschriebenen Parlamentssouveränität und seiner konstitutionellen Monarchie einen ,Klassiker'vergleichender Regierungslehre. In diesem Zusammenhang wird oftmals der Begriff des Westminster-Modells verwendet, um die spezifischen Charakteristika der britischen parlamentarischen Demokratie zu bündeln. ${ }^{1}$ Dieses politische System steht vor allem für die Durchsetzung der Bill of Rights. Zusammen mit der Rule of Law, der Bindung staatlichen Handels an Gesetze, bildet diese Parlamentssouveränität einen der Grundpfeiler des britischen Politiksystems (Sturm 2009c: 37-46). Im 19. und 20. Jahrhundert wurde ein System von checks and balances implementiert, welches erst Anfang des neuen Jahrtausends teilweise entzerrt und durch zusätzliche Kontrollmechanismen ergänzt wurde (Sturm 2009c: 1-9, 37-52). Noch heute stehen ,sich nicht Legislative und Exekutive, sondern Parlamentsmehrheit und Regierung auf der

1 Welche Merkmale tatsächlich das Modell einer Westminister-Demokratie charakterisieren, wird in der wissenschaftlichen Literatur oftmals nicht abschließend geklärt (Rhodes/Weller 2005: 4-6). 
einen und die Opposition [...] auf der anderen Seite gegenseitig kontrollierend“ (Kuhlmann/Wollmann 2013: 84) gegenüber, wobei die Exekutive eine privilegierte Stellung einnimmt. Das Vereinigte Königreich gilt damit als Beispiel einer majoritarian democracy, die politische Macht in der Hand weniger bündelt (Lijphart 1999: 9). Zwar ist es grundsätzlich eine konstitutionelle Erbmonarchie, das politisch wichtigste Amt übernimmt jedoch der Premierminister. Er bildet zusammen mit dem Kabinett die exekutive Spitze. Im britischen System nimmt er eine ausgesprochen dominante Rolle ein; seine Machtposition wird nur durch wenige institutionelle Schranken begrenzt (Sturm 2009c: 111-122, Kuhlmann/Wollmann 2013: 84). Diese „Präsidentialisierung“ (Sturm 2009c: 111) spiegelt sich auch in der wachsenden Bedeutung der öffentlichen Arena für den Regierungschef. Das administrative Rückgrat der Regierung bildet der civil service oder Whitehall, welcher oftmals als Synonym für die Londoner Ministerialbürokratie verwendet wird (Hartmann 2011: 75). Der civil service umfasst den Verwaltungsstab auf zentralstaatlicher Ebene in Ministerien und Agenturen (Kuhlmann/Wollmann 2013: 90). Seine Aufgabe ist die Unterstützung der amtierenden Regierung ,in developing and implementing its policies, and in delivering public services" (GOV UK 2015). Der damit einhergehende Einsatz von political advisors bildet sukzessive ein Äquivalent für den bis dahin unbekannten politischen Beamten (Krumm/Noetzel 2006: 222). In der Serie Yes, Prime Minister gelingt es, zentrale Charakteristika des britischen Westminister-Systems abzubilden. Der Fokus auf Hacker und seine Darstellungspolitik begründet sich in der Machtzentralisierung im Amt des Premierministers; das Parlament oder die Parteien bleiben außen vor. Die bedeutende Position des civil service wird insbesondere verkörpert durch Appleby und Woolley.

\section{Vereinigtes Königreich zwischen Kolonialmacht und Devolution}

Mit der Kontrolle über das britische Weltreich (British Empire) avancierte Großbritannien von der zweiten Hälfte des 19. Jahrhunderts an zur führenden Weltmacht (Sturm 2009b). Noch heute ist die Auseinandersetzung mit der kolonialen Vergangenheit von gesellschaftlicher Relevanz und wird auch in der Serie Yes, Prime Minister aufgegriffen.

Das Vereinigte Königreich ist ein Einheitsstaat mit einer „quasi-föderativen“ (Hartmann 2011: 78) staatsrechtlichen Konstruktion. Es unterteilt sich in die Gebietskörperschaften England, Schottland, Wales und Nordirland. 1999 wurden im Zuge der durchgeführten Devolution Regionalparlamente und -regierungen in Nordirland, Schottland und Wales eingeführt (GOV UK 2016). Die Rückbin- 
dung von Wales, Schottland und Nordirland in der „Ever Looser Union“² (Paun/Munro 2015) scheint mit der Zeit brüchiger zu werden. So hat das schottische Parlament - nach einem gescheiterten Unabhängigkeitsreferendum 2014 im März 2017 als Reaktion des geplanten Austritts des Vereinigten Königreiches aus der EU ein weiteres Referendum angekündigt. Auf eine ähnliche Situation muss auch Hacker in Yes, Prime Minister reagieren, was den aktuellen zeitgeschichtlichen Hintergrund der Serie verdeutlicht.

\section{Die Macht der Medien im Vereinigten Königreich}

Medien nehmen in Demokratien traditionell eine Vermittlungsinstanz zwischen Politik und Bevölkerung ein. Das britische Mediensystem steht dabei für eine kritische Öffentlichkeit, die tief in der Gesellschaft verwurzelt ist (Krumm/ Noetzel 2006: 86). In Yes, Prime Minister sind es die Medien, die Skandale offenbaren, Handlungszwänge hervorrufen und politische Entscheidungen maßgeblich beeinflussen. Die reale britische Medienlandschaft ist hoch konzentriert, so kontrollieren die vier Unternehmen News UK, Daily Mail \& General Trust und Trinity Mirror 71 Prozent des britischen Zeitungsmarktes. Eine ähnliche Konzentration lässt sich auch in den Bereichen Fernsehen und Radio feststellen. Gleichzeitig wird der britischen Medienlandschaft mit seiner traditionellen Unterscheidung zwischen Massenblättern (tabloids) und seriösen Tageszeitungen (quality papers) eine große Vielfalt nachgesagt, die verschiedene Zielgruppen bedienen kann (IfM 2015, Media Reform Coalition 2015: 4-5). Auf der einen Seite ist das

,journalistische Selbstverständnis [...] traditionell geprägt von der gesellschaftlichen Verantwortung, die Öffentlichkeit vollständig und rückhaltlos über alle politischen Entscheidungsprozesse zu informieren, [andererseits] aber auch zugleich aktiv gestaltend in die Politik einzugreifen“ (Lenz/Kamm, zit. n. Sturm 2009a).

Im Bereich Rundfunk herrscht ein duales System aus der BBC (British Broadcasting Corporation) als gebührenfinanziertes Sender-Konglomerat und den

2 Die Autoren beschreiben die Staatskonstruktion des Vereinigten Königreiches wie folgt: „What is clear is that there are now multiple centres of power, divergent party political systems, and a complex division of responsibilities and resources between the four nations of the UK“ (Paun/Muno 2015: 81). 
werbefinanzierten Privatsendern (IBA, Independent Broadcasting Authority) $)^{3}$ (IfM 2015, Sturm 2009a). Diese Struktur ist immer wieder Mittelpunkt kontroverser Diskussionen. Insbesondere die hohen Kosten für die Allgemeinheit und eine mögliche Verzerrung des Wettbewerbs werden als Argumente vorgetragen, um den Etat für die $B B C$ zu kürzen (Martinson 2016). Dies kann - so die Einschätzung eines deutschen Auslandskorrespondenten in London - jedoch nur gegen den Willen der britischen Bevölkerung gehen:

„Was die BBC einstweilen rettet, ist ihre enorme Popularität in der Bevölkerung. Ihr Spitzname ist ,Tantchen', sie gehört zur Familie. Sie hat eine emotionale Bedeutung für Großbritannien, eine Bindung zum Land, und selbst die konservativsten unter den Tories wissen, dass sie sich mit der Abschaffung der BBC wenig Freunde machen würden.“ (Zaschke 2016)

Auch in Yes, Prime Minister verkörpert die $B B C$ ihre herausragende Rolle der Medienlandschaft des Vereinigten Königreichs, : So beeinflusst die Rundfunkanstalt durch Telefonanrufe, Interviewanfragen und Fernsehsendungen immer wieder den Verlauf der Geschehnisse auf dem Landsitz des Premierministers.

\section{SPIEGEL: DARSTELLUNG DES POLITIKBETRIEBS IN YES, PRIME MINISTER}

Europäische Finanzkrise, Öl-Abkommen, schottische Unabhängigkeit, globale Erderwärmung - dazu (Beinahe-)Skandale, Sex und krumme Geschäfte. Dies sind i die Themen, mit denen sich Yes, Prime Minister auseinandersetzt. Es gehört dabei zu den Charakteristika einer politischen Satire, Kritik an den herrschenden politischen Zuständen auszuüben und humorvoll zu überzeichnen. Die Aufgabe dieses Kapitels besteht demnach darin, unter Berücksichtigung aktueller politikwissenschaftlicher Konzepte und Diskussionen die Persiflage des politischen Alltags nachzuzeichnen. Die hier vorgestellte Analyse fokussiert im Wesentlichen das Verhältnis von Politik und Medien, die politische Entscheidungsfindung, wobei zunächst die wichtigen Akteure und ihre Beziehungen unterei-

3 Die IBA war von 1955 bis 1990 ein werbefinanzierter Privatsender. 1990 wurde aus der IBA die Independent Television Commission (ITC), die nicht mehr als Sendeveranstalter agierte, sondern nur noch für die Lizenzierung der Privatsender zuständig war (Sturm 2009a). 
nander dargestellt werden und schließlich eine konkrete politische Dilemmasituation als Anwendungsfeld für Politik in Yes, Prime Minister.

\section{Politik versus Medien: Wer instrumentalisiert eigentlich wen?}

Regieren findet immer auch in der medialen Arena statt. Politische Akteure müssen öffentliche Zustimmung generieren, mobilisieren und ihre Entscheidungen legitimieren. Mit dem Aufkommen der Massenmedien hat auch die Mediatisierung der Politik und die Bedeutung von politischer Kommunikation zugenommen (Korte/Fröhlich 2009: 272-283). Auch Yes, Prime Minister geht auf die Bedeutung von Medien für politische Prozesse ein ${ }^{4}$, wie bereits an der ersten und letzten Szene der Staffel ersichtlich wird, die jeweils mit einem Interview des Premierministers gefüllt werden. Im ersten Fall gibt Hacker ein Interview zur laufenden Konferenz des Rats der EU, deren ungünstiger Verlauf in einer Nachbesprechung mit Appleby und Woolley nochmals den Handlungsdruck und den Bedarf an öffentlichkeitswirksamen Reaktionen verdeutlicht. Dagegen schafft er es mit dem letzten Interview, seine eigene Agenda zu setzen und von der Eurokrise abzulenken. Der Vergleich beider Interviewsituationen lässt die Funktion von Medien als ambivalent erscheinen: Einerseits sind sie kontrollierender Akteur, der im Krisenfall Antworten seitens der Politik fordert, andererseits Dienstleister der Politik.

Auch an anderen Stellen wird deutlich, dass die Medienlogik bei der Entscheidungsfindung stets berücksichtigt wird. Als Aushängeschild der Regierung und damit „democratically elected scapegoat“ (S 6/F 3/3.53 - 3.57) hofft Hacker oftmals darauf, dass ein bestimmter Sachverhalt nicht an die Öffentlichkeit gerät: „I hate the BBC. It's like having two oppositions. One at Westminster and the other at Broadcasting House“" (S 6/F 2/18.39 - 18.48). In dieser Hinsicht stellen Medien abermals einen Akteur mit Kontrollfunktion dar, der bei schwerwiegenden wie vermeintlich einfachen Entscheidung berücksichtigt wird. Diametral entgegengesetzt ist die Instrumentalisierung der Medien durch die Regierungsakteure einzuordnen; sie dient dazu, ein bestimmtes Dilemma aufzuheben oder den Charakteren eine positive Ausgangslage zu verschaffen. Hier drohen gerade Hacker und Appleby gegenseitig damit, ihre nicht ganz sauberen Geschäfte an die Öffentlichkeit zu bringen, um so auf den jeweils anderen Druck aufzubauen. Auch die Regierungskrise lösen Appleby und Hacker dadurch, dass sie eigenmächtig den Fokus auf globale Erderwärmung setzen und damit die Medien nut-

4 Unterschiedliche Konzeptionen zum Verhältnis von Medien und Politik werden im Kapitel „Spiegel“ des Beitrags zu Eichwald, MdB ausführlich erläutert. 
zen, um die eigene Agenda in die Öffentlichkeit zu bringen und negative Berichterstattung bezüglich der EU-Krise zu vermeiden.

Der mediale Akteur an sich wird eher als neutrale Kontrollinstanz, denn als eigenständig handelnder und bewusst beeinflussender Akteur dargestellt.

Szenen-Beispiel: S 6/F 2/23.09-23.20

Director-General: Obviously, we don't want to destabilize Britain as you put it. But we are not the government information service either. We have a duty to reflect all shades of opinion. We are journalists. Our job is to keep the public fully informed.

Hacker bemüht sich darum, negative Berichterstattung über ihn zu verhindern oder zumindest auf einen für ihn günstigeren Zeitpunkt zu verschieben. Der Generaldirektor der $B B C$ repräsentiert hier den medialen Akteur und wirkt zunächst unnahbar und unbeeindruckt von Hackers Bestrebungen. Gegen die anstehende Reform und damit Beschneidung der $B B C$ kann er jedoch nichts ausrichten und auch Gegenmaßnahmen erfolgen keine.

Yes, Prime Minister ermöglicht nur scheinbar einen Einblick in das schwierige Verhältnis von Vertrauen und Transparenz, mit dem politische Akteure bei Entscheidungen konfrontiert werden. Der Vorwurf: Hinter verschlossenen Türen werden Lösungen diskutiert, die jenseits moralischer und politischer Vernunft sind. Der Zuschauer bekommt den Eindruck, dass Medien als vierte Gewalt und kritische Öffentlichkeit dringend benötigte Kontrollmechanismen sind, die den Politikbetrieb, wenn auch indirekt, positiv beeinflussen können. Ob nun überspitzt oder nicht - Yes, Prime Minister präsentiert Medien zwar nicht als übermächtigen und eigeninitiativ handelnden, wohl aber als einflussreichen Akteur, der durchaus auf das politische Handeln und die Entscheidungsfindung einwirkt.

\section{Politische Entscheidungsfindung I: „Wer trifft auf welche Weise die wichtigen politischen Entscheidungen tatsächlich?“}

Mit der hier verwendeten Titelfrage eröffnet Rudzio (2005: 10) die Debatte über politische Entscheidungsfindung, also mit der Frage, wer eigentlich Macht besitzt, um Entscheidungen treffen zu können. Der Politikbetrieb ist dabei durchdrungen von Informalität, sprich Verfahren und Institutionen, die nicht auf formal festgelegten Verfahren beruhen (Grunden 2013: 219-220). Aus politikwissenschaftlicher Sicht ist dies aufgrund einer immer komplexer werdenden Umwelt notwendig. Gerade Spitzenakteure sind daher auf Personen in ihrem Umfeld angewiesen, die als Machtmakler für ein ausgewogenes Informationsmanage- 
ment sorgen (Korte/Fröhlich 2009: 214-215). Auch bei Yes, Prime Minister spielen insbesondere die Hauptakteure Appleby, Hacker, Woolley und Sutton und ihre Beziehungen untereinander eine gesonderte Rolle. Darüber hinaus treten nur wenige andere Charaktere in Erscheinung, noch dazu meistens ohne Eigeninitiative oder gewichtigen Einfluss auf die Entscheidungsfindung. Erwähnenswert ist zudem, dass die Serie überwiegend in Hackers Büro im Chequers, dem offiziellen Landsitz des Premierministers, stattfindet. Damit entfernt sich die Darstellung des Politikbetriebs von gängigen Konzepten wie einer Unterteilung in öffentliche, parlamentarische und administrative Arena (Korte/Fröhlich 2009: 230-233). Während die Öffentlichkeit in Ansätzen durch relativ einflussreiche Medien repräsentiert wird und die Administration durch Appleby als starken Akteur verkörpert wird, findet das Parlament gar keine Erwähnung, ebenso wenig wie Parteien. Drehbuchautor Jay begründet dies mit dem Anspruch, die Regierungsführung realitätsgetreu abzubilden:

„There was not a single scene set in the House of Commons because the series is about the government. Government does not take place in the House of Commons; some politics takes place there, and much theatre takes place there. Government happens in private. As in all public performances, the real work is done in rehearsal, behind closed doors. Then the public, and the House, are shown what the government wishes them to see." (Jay 2013)

Die Unterbelichtung formal-demokratischer Prozesse und des gesetzgebenden Parlaments vermittelt dem Zuschauer ein Bild von Politik, das vor allem durch starke Persönlichkeiten geprägt wird. Zwischen ihnen und ihren Interessen finden die politischen Aushandlungsprozesse statt. Eines der markantesten Elemente der Serie besteht dabei in einem fortlaufenden Schlagabtausch zwischen Premierminister Hacker auf der einen und Appleby als höchstem Vertreter der britischen Bürokratie auf der anderen Seite. Appleby beschreibt sich und den civil service als die eigentliche Machtzentrale des Landes. Gerade die Dauerhaftigkeit seiner Anstellung ist dafür ausschlaggebend (,permanence is power" $S$ 6/F 3/3.00 - 3.05), auch wenn er dabei aus dem Hintergrund agiert. Hacker wiederum ist als Premierminister davon überzeugt, dass im civil service nur politische Amateure - Generalisten ohne Expertise in den einzelnen Feldern - arbeiten. Auch Korruption wirft er der Bürokratie vor: „Claire, this latest outrage by Humphrey is just one more example of that corruption of those who think they are above the law" (S 6/F 2/20.25 - 20.32). Über geplante Gesetze und Regierungsvorhaben ist er trotz seiner Position nicht immer informiert. So weiß er zunächst nichts über das geplante Öl-Abkommen mit Kumranistan. Erst kurz vor 
der Möglichkeit zur Unterzeichnung wird er informiert, jedoch ohne Erwähnung der zentralen Details. Auf die Frage, ob es noch mehr gibt, dass er über das Abkommen wissen müsse, antwortet Appleby: „I hardly know where to begin“ (S 6/F 2/3.20 - 3.27). Nachdem klar wird, dass dieser sich durch das Abkommen zudem einen persönlichen Vorteil verschafft hat, versucht Hacker die Machtposition des „Außer Kontrolle“-Geratenen (S 6/F 2/19.27 - 19.29) Appleby dauerhaft einzuschränken. Die Drohung einer Reform des civil service kontert Appleby jedoch mit Enthüllungen über Steuerhinterziehung des prime ministers. Dass auch der civil service nicht immer saubere Geschäfte betreibt, kann Hacker wiederum zum Gegenschlag nutzen; eine Pattsituation entsteht. Ob dies noch Informalität oder schon Erpressung ist, wird an anderen Stellen zumindest gefragt:

\section{Szenen-Beispiel: S 6/F 4/9.49 - 10.02}

Appleby: We do not approve a blackmail as an instrument of government policy.

Hacker: Since when?

Appleby: Prime Minister, blackmail is criminal. We use leverage.

Auch Woolley und Sutton sind in diesen Schlagabtausch involviert. Als persönliche Berater sorgen sie für die notwendige Beschaffung von Informationen. Woolley selbst bezeichnet sich als „servant of two masters“. Er sieht den Premierminister, dem er eigentlich untersteht, als seinen „temporary boss“ und Appleby, der ihn in seine geheimen Pläne einweiht, als „permanent boss“. Gleichzeitig erläutert er, dass beide oft verschiedene, teilweise antagonistische Interessen verfolgen und sich gegenseitig die Schuld für etwaiges Versagen geben ( $\mathrm{S}$ 6/F 2/5.15 - 6.55). Sutton dagegen ist eine starke und informative Akteurin, die Hacker als Beraterin zugeordnet ist. Sie präsentiert sich als Machtmaklerin. Während sowohl Appleby als auch Woolley nicht alle Informationen an Hacker preisgeben, besteht zwischen Sutton und Hacker ein absolutes Vertrauensverhältnis.

Die Serie zielt damit auf eine akteurszentrierte Politikdarstellung, die Wissen im Sinne von Information als die größte Machtressource sieht - denn mit brisanten Informationen schafft man sich eine günstige Verhandlungsposition (Korte/Fröhlich 2009: 207). Während der Premierminister nicht immer im Zentrum der Entscheidungsfindung steht (und dies aus Angst vor negativer Berichterstattung auch teils gar nicht möchte), sind es vor allem die im realen Politikbetrieb eher im Hintergrund agierenden Akteure wie die persönliche Beraterin oder die Verwaltung, die aufgrund von Informationsvorsprüngen ein hohes Maß an Einfluss besitzen. 


\section{Politische Entscheidungsfindung II: Prostitutions-Dilemma}

Der Eindruck, dass Entscheidungen oft auf informeller Basis unter Ausschluss der Öffentlichkeit und vor allem schnell getroffen werden, wird durch Dilemmasituationen, in denen sich die Hauptcharaktere befinden, weiter verstärkt. Hier wird behauptet, dass im politischen Prozess Situationen entstehen, die kurz vor der Eskalation stehen und nur mit Mühe verhindert werden können, wobei die Öffentlichkeit außen vor bleibt. Die Unterzeichnung des Öl-Abkommens wird in Episode 4 geknüpft an die Forderung des Außenministers von Kumranistan nach „,sexual entertainment“. Nach anfänglicher Ratlosigkeit beschließen Hacker, Appleby, Sutton und Woolley den Botschafter Kumranistans dazu zu holen, der zunächst die moralischen Bedenken aller Anwesenden relativiert und eine neue Sichtweise auf das Problem eröffnet:

\section{Szenen-Beispiel: S 6/F 4/17.59-18.28}

Aitikeev: Prime Minister, I urge you to be practical. [...] These girls are doing a job. They will be well paid. And as a result, unemployment will drop, oil prices will drop, repossession will stop, Greece, Italy and Spain will be bailed out and Europe will be saved from disaster.

So einfach die Argumentationskette erscheint, in der drei Prostituierte letztendlich die EU-Krise verhindern könnten - „the public wouldn't understand“ (Hacker S 6/F 4/19.47 - 19.49). Die Hauptcharaktere bemühen sich daher um Handlungsalternativen, die letztendlich auch Woolley - als letzte moralische Bastion - seine Grundprinzipien verlieren lassen. Die Entscheidung fällt zugunsten der Prostitution, allerdings weiß keiner genau, wie dies zu arrangieren ist. Die Unfähigkeit, das Dilemma zu lösen, sorgt letztendlich dafür, dass das Abkommen nicht unterzeichnet wird. Nur ein Ablenkungsmanöver kann jetzt noch Abhilfe schaffen: Das bereits geplante Fernsehinterview Hackers über die Lösung der EU-Krise wird instrumentalisiert, um die globale Erderwärmung auf die Agenda zu setzen. Das riskante Manöver scheint zu funktionieren und Hacker hat das Interview dieses Mal fest in der Hand. Dieses Ersetzen komplizierter Aushandlungsprozesse durch publikumswirksame Maßnahmen in der Öffentlichkeit wird in der Politikwissenschaft auch als Telepolitik bezeichnet (Korte/Fröhlich 2009: 263).

Moralische Verrohung, Skandalthemen, Unfähigkeit, inhaltliche Lösungen für gesellschaftlich relevante Probleme zu finden - diesen Eindruck erhält der Zuschauer vom Politikbetrieb im Vereinigten Königreich. Wer als politisch Interessierter nun empört aufschreit, ist dennoch im Unrecht. Die Serie spiegelt im 
Grunde das wider, was aus dem für viele Menschen wenig attraktiven Politikgeschäft an die Oberfläche gespült wird und für Politik(er)verdrossenheit sorgt: Sex-Skandale, Steuerhinterziehung, Durchsetzung persönlicher Interessen, ,Klüngelpolitik' hinter verschlossenen Türen. Wer jedoch den satirischen Charakter erkennt, darf mitlachen und dann zeigen, wie wahre gestaltende, zielgerichtete, am Gemeinwohl orientierte politische Entscheidungsfindung stattfindet - wenn dem denn so ist...

\section{DATEN: YES, PRIME MINISTER ALS AUSDRUCK POLITISCHER KULTUR}

Yes, Prime Minister eröffnet nicht nur eine (satirische) Perspektive auf den Politikbetrieb, sondern auch einen umfassenden Einblick in die vorherrschenden gesellschaftlichen Normen und Werte im Vereinigten Königreich. So spiegelt der Umgang mit den sexuellen Wünschen des Außenministers von Kumranistan die restriktive Einstellung der britischen Bevölkerung gegenüber Prostitution (IPSOS Mori 2008, Wintour 2008). Die artikulierte moralische und kulturelle Überlegenheit des Vereinigten Königreichs kontrastierend zu Kumranistan sowie die häufigen Anspielungen auf die rückwärtsgewandte Sehnsucht nach einem starken britischen Königreich lassen sich als Verweise auf die Postkolonialismus-Debatte und die postcolonial melancholia (Gilroy 2005) verstehen. Aus politikwissenschaftlicher Perspektive sind jedoch vor allem jene Erkenntnisse zentral, die sich über die politische Kultur - also über die Einstellungen, Perzeptionen und Werte der Bevölkerung gegenüber Institutionen, Policies und dem politischen System als Ganzem - ableiten lassen (Schubert/Klein 2016: 233). Das Vereinigte Königreich bewegt sich dabei in einem Spannungsfeld aus Liberalismus, Multikulturalismus und Exzeptionalismus, zwischen historischer Kontinuität und gesellschaftlichem Wandel:

„Die britische Gesellschaft ist sich - ebenso wie die britische Politik - ihrer Sonderstellung bewusst, die nicht nur die Geographie (Insellage), sondern auch die jüngere Geschichte, insbesondere die immer wieder ins kollektive Bewusstsein gehobene Rolle des Vereinigten Königreichs im II. Weltkrieg, zu bestätigen scheint, und der durch den Glücksfall, Mutterland der heute unumstrittenen Weltsprache zu sein, heute neue Bedeutung zukommt.“ (Sturm 2009: 12)

Wie ein vertiefter Blick auf die filmische Darstellung der politischen Orientierungen und Einstellungen in Yes, Prime Minister helfen kann, das Wesen der 
politischen Kultur im Vereinigten Königreich zu verstehen, wird exemplarisch an den beiden Themen Euroskeptizismus und Verwaltungskultur dargestellt.

\section{Eine verzwickte Beziehung: Vereinigtes Königreich und EU}

Betrachtet man aus heutiger Sicht die Darstellung des Verhältnisses des Vereinigten Königreichs zur EU in Yes, Prime Minister und den Vorgängern, so könnte man meinen, die Drehbuchschreiber hätten das Ergebnis des BrexitReferendums im Sommer 2016 vorhersagen können. In den auf das Referendum folgenden Wochen erschienen zahlreiche Zeitungsartikel und Blog-Artikel, die auf Yes, Minister rekurrieren, um das spezifische Verhältnis der Briten zur EU zu charakterisieren (Dallison 2016, Westbrook 2016, Payne 2016, York 2016). Im Guardian erschien sogar ein Yes, Minister Brexit special, in dem Appleby erklärt, was der Austritt für das Vereinigte Königreich bedeutet (Lynn/Jay 2016).

Dabei ist die britische Europapolitik seit jeher geprägt durch ein entemotionalisiertes und rationales innenpolitisches Kalkül und durch das Misstrauen gegenüber der fortschreitenden europäischen Integration sowie gegenüber jeglichen Visionen europäischer Staatlichkeit. Mit dem Referendum zum EU-Austritt setzt sich ein „deprimierender Zyklus enttäuschter Erwartungen“ (Sturm 2009: 206) fort: Vom verspäteten Beitritt zur Europäischen Gemeinschaft, Margaret Thatchers berühmter Rede in Brügge 1998, welche die Vision einer europäischen Gemeinschaft souveräner, unabhängiger Staaten beförderte ${ }^{5}$, bis hin zur Skepsis gegenüber einer Gemeinschaftswährung oder den Verträgen von Maastricht und Lissabon (Leconte 2010: 3-4, Smith 2016: 12-16, Sturm 2009: 208, 214). Deutlich sichtbar werden die verschiedenen Facetten des Euroskeptizismus - gemeint ist eine kritische bis aversive Haltung gegenüber den europäischen Institutionen, bestimmten Politiken und Integrationsbestrebungen (Niedermeier/Ridder: 3, Weßels 2009: 51-55) - in den zynischen Dialogen der Akteure in Yes, Prime Minister. Mit Applebys Intrige, durch die Inanspruchnahme des Kredites aus Kumranistan das Vereinigte Königreich zum Beitritt in die Eurozone zu zwingen, wird eine rote Linie überschritten, wie der Premierminister in einem Gespräch mit Sutton betont:

5 Im Wortlaut „My first guiding principle is this: willing and active cooperation between independent sovereign states is the best way to build a successful European Community“ (Thatcher 1988). 
Szenen-Beispiel: S 6/F 1/24.27-24.58

Hacker: We can't join the Euro, it would be a catastrophe! Look what's happened to Greece, Spain, Italy, Ireland, Portugal! Hand over control of interest rates, control of exchange rates, control of the money supply? What if I need to inflate to get re-elected? If the Germans are worried about rising prices, the ECB could deflate and I could get kicked out.

Die Ablehnung richtet sich jedoch nicht nur gegen die Gemeinschaftswährung, sondern gegen die EU insgesamt. So werden Defizite in der institutionellen Konstruktion angeprangert. Eine effiziente und effektive Entscheidungsfindung wäre fast unmöglich, wie Premierminister Hacker Sutton erklärt: „Dealing with the Europeans is like herding cats" (S 6/F 1/6.57 - 6.59). Es ginge nicht darum, Erfolge zu erziehen, sondern ,it's about concealing failure“ (S 6/F 1/7.52 7.57). Die zähen Aushandlungs- und Kompromissfindungsprozesse zwischen den europäischen Mitgliedsstaaten sind nicht nur langwierig, sondern enden auch in suboptimalen Ergebnissen, wie anhand der geplanten Route der ÖlPipeline von Kumranistan in die EU illustriert wird. Diese führt in einem ZickZack-Kurs durch alle 28 Mitgliedsstaaten. An anderer Stelle finden sich Anspielungen auf das Demokratiedefizit - so bemerkt Hacker kritisch, dass der Präsident des Europäischen Rates nicht gewählt, sondern im Geheimen benannt wird (S 6/F 1/10.23 - 10.27). Auf Applebys Nachfrage hin, ob es noch weitere Beratungen benötige, um die Ausgabe des Kredites von Kumranistan in Britische Pfund zu ermöglichen, antwortet der Präsident der Europäischen Zentralbank Müller: „No, that would be anarchy. Brussels decides, then everyone does what we say“ (S 6/F 3/28.19 - 28.22). Brüssel sei eine Supermacht, die einer Autokratie ähnlich die Entscheidungsprozesse steuere und Macht akkumuliere.

Es ist ein potenzieller Widerspruch, wenn auf der einen Seite langwierige Entscheidungsprozesse in der EU kritisiert und auf der anderen Seite autokratische Züge konstatiert werden. Dies lässt sich zumindest teilweise mit der Komplexität und Intransparenz institutioneller Strukturen und Entscheidungsprozesse sowie mit der generellen Unwissenheit über die Funktionsweise der EU erklären. So ist selbst der Premierminister nicht in der Lage, das derzeit von ihm innegehaltene Amt in der EU richtig zu benennen oder seine Machtposition davon abzuleiten:

\section{Szenen-Beispiel: S 6/F 1/10.38-11.24}

Hacker: I don't understand this. I'm sure I'm President of the Council. I remember being in the chair. 
Woolley: Well, it is a little confusing, Prime Minister. You see, we, the British, have the presidency of the Council of the European Union.

Hacker: That's what I said!

Woolley: Uh, no. The European Council and the Council of the European Union are not the same thing. The President of the European Council is there for 30 months and is in charge, well, preparing the agenda and chairing their meetings of the European Council. Whereas the presidency of the Council of the European Union is held for six months each by rotating states and not by individual leaders and not by you.

Hacker: So which of them really runs Europe?

Des Weiteren eröffnet Yes, Prime Minister einen Einblick in die Angst vor Souveränitätsverlust und politischer Marginalisierung des Vereinigten Königreichs innerhalb einer starken EU. So antwortet Appleby, nachdem Woolley die Sorge des Premierministers teilt, dass Brüssel danach strebt, alle nationalen Machtbefugnisse an sich zu reißen, ungerührt: „They already have“ (S 6/F 1/9.11 - 9.13). Angesichts der wirtschaftlichen, finanziellen und politischen Probleme der EU und insbesondere des Euroraums zeigt man sich im Vereinigten Königreich besorgt, durch die Krise selbst Schaden zu nehmen und mit in der Bedeutungslosigkeit zu versinken. Für die Argumentationsweise charakteristisch sind die häufigen Rückbezüge zur britischen Kolonialgeschichte, die die Sehnsucht nach dem alten Glanz des Britischen Königreiches sichtbar machen:

\section{Szenen-Beispiel:}

Appleby erklärt dem Prime Minister, welche Machtposition er durch den Vorsitz des Rates der EU innehat (S 6/F 1/12.35 - 12.43).

Appleby: Prime Minister, what it all boils down to, as far as you're concerned, is the difference between years of being head of an empire of nearly 500 million people and chairing the Council of Ministers for six months.

Ob Identitätsfragen, Institutionenkritik, Unwissenheit oder Nostalgie - es sind sowohl kognitive als auch affektive Einflussfaktoren, die das spezifische Verhältnis des Vereinigten Königreichs zur EU als Ganzer, ihren Politiken und den Integrationsbestrebungen in Yes, Prime Minister begründen. Damit wird eine Perspektive auf den britischen Euroskeptizismus eingenommen, dessen zentrales Charakteristikum in einem spezifischen Nationalismus liegt:

„Ein wesentliches Element des britischen Europaskeptizismus liegt in einem stark auf das Englische fokussierten Nationalismus, welcher auf eine an Permanenz orientierte Anglo- 
Souveränität hin ausgerichtet ist. [...] Auch im Kontext des Brexit wurden traditionelle Vorbehalte und lange gehegte Feindseligkeiten gegenüber europäischen Mächten und Einstellungen, die seit jeher als bedrohlich für die Insel empfunden wurden, auf die EU übertragen und dem kontinentaleuropäisch-institutionalisierten Hort der Undemokratie die einzigartige Tradition des britischen Parlamentarismus gegenübergestellt." (Niedermeier/Ridder 2017: 4)

Interessant ist nun, dass viele von den dargestellten Einflussfaktoren in der politischen und wissenschaftlichen Debatte um die Erklärung des BrexitReferendums wiederzufinden sind. Parallelen lassen sich zum Beispiel mit Blick auf die Angst der Bürger vor Souveränitätsverlust und vor der Untergrabung der eigenständigen britischen Identität (Hobolt 2017: 1270-1271, Niedermeier/Ridder 2017: 4, Tombs 2016) finden. Außerdem zeigen sich auch in der Konzentration auf ökonomische Vor- und Nachteile einer EU-Mitgliedschaft (Hobolt 2017: 1270-1271, Niedermeier/Ridder 2017: 15-16), in der Bedeutung des Commonwealth-Narrativs und in der postkolonialen Nostalgie der BrexitKampagne (Ashe 2016, Namusoke 2016: 471-473) viele Ähnlichkeiten. Ein systematischer Vergleich von Fiktion und Realität könnte hier zu interessanten Erkenntnissen führen.

\section{Civil service: Verwaltungskultur zwischen Tradition und Reformen}

Die Verwaltungskultur beschreibt einen eher institutionenspezifischen Ausschnitt der politischen Kultur und nimmt die Einstellungen, Perzeptionen und Erwartungen sowohl der Gesamtbevölkerung als auch der civil servants als Teilpopulation auf den Gegenstandsbereich Verwaltung in den Blick (Prätorius 2002: 625). Yes, Prime Minister zeichnet in diesem Zusammenhang das Selbstverständnis eines traditionell ausgerichteten und durch das Gentleman-Ideal geprägten britischen civil service, welches auch mit dem Begriff , Geist von Whitehal' umschrieben wird:

„Dieser Geist äußert sich in einer Art müheloser Überlegenheit, in Behutsamkeit, Diskretion und nicht zuletzt makellosen Umgangsformen. Er beruht auf einer sicheren und breiten Allgemeinbildung, Menschenkenntnis und einem in den Public Schools geübten intensiven Charaktertraining.“ (Händel 1979: 117)

Soziale Exklusivität und Homogenität, die Vormachtstellung der Generalisten, der Zusammenhalt unter den Beamten, eine strenge Geheimhaltungspflicht so- 
wie parteipolitische Neutralität gehörten bis Ende der 1970er Jahre zu den wesentlichen Charakteristika des civil service (Hartmann 2001: 75-78, Kingdom 2014: 18, Kuhlmann/Wollmann 2013: 90-92, Sturm 2009: 135-138). Appleby und Woolley portraitieren diese nahezu perfekt: Beide sind Oxford-Absolventen und Generalisten, statusbewusst, haben eine gehobene Ausdrucksweise, ein makelloses Erscheinungsbild, eine profunde Allgemeinbildung sowie genaue Kenntnisse über die Funktionsweise der politischen Institutionen. Des Weiteren helfen sie einander und zeigen sich loyal gegenüber dem civil service. Dagegen wird die Geheimhaltungspflicht durch Appleby konterkariert, indem er zum eigenen Profit sensible Regierungsinformationen an eine Bank weitergibt. Appelby ist parteipolitisch neutral und versteht sich als aktiver Gestalter, der von politischen Wechseln unabhängig als permanente Machtinstanz das Regierungshandeln steuert:

\section{$\underline{\text { Szenen-Beispiel: }}$}

Appleby und Woolley reden über das Selbstverständnis des Premierministers und die Rolle des civil service (S 6/F 3/2.19-3.34).

Woolley: He seems to think he's in charge and we're just paid officials. Functionaries.

Appleby: Good god! It's not his business to interfere in the way government is run. As the Head of the Home Civil Service, I am the true head of government.

Woolley: But what's the Prime Minister then?

Appleby: Well, he's our marketing consultant. The party leaders pitch for our business every four or five years, Woolley. And the electorate simply chooses the mouthpiece of the moment.

Woolley: But politicians do have their uses though, don't they?

Appleby: Yes, they make the cases for our policies on television better than you and I would. And when we write speeches, we need someone to read them out for us in the House of Commons. But they come and go, Woolley, whereas we stay. Permanence is power. [...] Minister average 15 months in their jobs, Woolley. And they're only in their departments part-time. Their real job is getting re-elected. Their attention and skill, well, such as it is, is focused on the House of Commons, their constituencies, the media, and try to so down their opponents. Whereas we are here for 30 or 40 years. And we run a budget of 600 pounds, Woolley. It's quite obvious who's actually running the country.

Eine solche Selbstwahrnehmung als policy maker war und ist immer noch prägend für den britischen civil service. Es scheint ohnehin so, dass die Verwal- 
tungskultur im Vereinigten Königreich von der Dualität von Kontinuität und Wandel geprägt ist. Auf der einen Seite gab es seit Ende der 1970er Jahre im Rahmen der New Public Management-Reformen Maßnahmen zur Effektivitätssteigerung, stärkeren Politisierung, sozialen Öffnung sowie Verkleinerung der Verwaltungsstruktur. ${ }^{6}$ Obwohl ambitionierte Reformentwürfe oftmals nur teilweise beschlossen und unzureichend umgesetzt wurden, scheinen sich allmählich Veränderungen in Bezug auf das Selbstverständnis und die Arbeitsweise der civil servants bemerkbar zu machen. Man sehe sich zunehmend verantwortlich für die erfolgreiche Umsetzung und Durchführung von Policies, akzeptiere vereinbarte Zielvorgaben, öffne sich für externe Einflüsse und lege mehr Wert auf Führungs- sowie Managementqualitäten (Greer/Jarman 2011: 19-29, Panchamia/Thomas 2014: 6-9, 22-81). Auf der anderen Seite halten sich gefestigte Denkmuster und Arbeitsweisen oftmals hartnäckig, wie einer der Drehbuchautoren bei der Recherche festgestellt hat:

And our research made it clear that there had been a lot of changes in the 23 years since our last script. [...] And the people were different, too. The civil servants had lost some of Sir Humphrey's effortless superiority, though underneath the facade there was still the same patronising attitude to the amateurs who were their political masters. (Jay 2013)

Frauen, Migranten und Menschen mit Behinderung sind nach wie vor insbesondere in gehobenen Positionen unterrepräsentiert, die Wahrnehmung von Diskriminierung durch verschiedene Gruppen eröffnet nicht das Bild eines offenen, inklusiven civil service (Krumm/Noetzel 2006: 223, National Audit Office 2015: 15-28, Sturm 2009: 137-141). Auch Appleby verwehrt sich ausdrücklich einer Reform des civil service, die vorsieht, Generalisten in der Verwaltung durch Spezialisten für die jeweiligen Politikbereiche zu ersetzen. Sutton aber, die eine moderne politische Beraterin repräsentiert, unterstützt die Reform.

Korrupt, intrigant, arrogant, elitär - es ist nicht unbedingt ein positives Bild, das Yes, Prime Minister und auch der Vorgänger Yes, Minister vom civil service zeichnet. Nicht umsonst titelt The Independent (2012) „Britain’s new top civil servant says he wants to kill off the Sir Humphrey stereotype“ (Merrick 2012) ${ }^{7}$. Fest steht jedoch, dass das Vertrauen der Bevölkerung in den civil service in den letzten Jahrzehnten massiv gestiegen ist: Während 1983 nur 25 Prozent der Be-

6 Für einen Überblick über die Reformmaßnahmen siehe Parry 2011: 354-365, Panchamia/Thomas 2014: 22-75, Sturm 2009: 137-146.

7 In vielen Artikeln britischer Zeitungen finden sich Bezüge zu Yes, Minister oder Yes, Prime Minister. Siehe zum Beispiel McCartney 2016 und Slawson 2015. 
fragten der Meinung waren, dass ein civil servant die Wahrheit sagt, sind es 2013 mit 55 Prozent mehr als doppelt so viele (Freeguard 2015). Inwiefern die Serienproduktionen das Selbstverständnis, den Ethos und die Rolle des civil service im Vereinigten Königreich tatsächlich prägten, ist also ungewiss.

\section{DIE NARRATIVE: KONZENTRATION AUF POLITISCHE INHALTE}

Wie in Filmproduktionen üblich, bedienen sich die Macher von Yes, Prime Minister einer Reihe von Gestaltungsmitteln und narrativen Elementen, um eine weitere Erzählebene zu ergänzen, Charakterzüge der Figuren zu verdeutlichen und der Handlung mehr Tiefe zu verleihen.

\section{Das Setting und der Inhalt: Reduktion versus Komplexität}

Zeitraum, Setting und Cast der Serie sind äußert reduziert. So geben die sechs Folgen der Staffel den Verlauf eines Abends wieder. Der Schauplatz ist überwiegend Chequers, der offizielle Landsitz des britischen Premierministers außerhalb Londons. Bis auf wenige Ausnahmen findet die Handlung im dortigen Büro des Premiers statt. Dieses ist im britischen Stil eingerichtet - mit dunklen Wandvertäfelungen, Chesterfield-Möbeln und einer Bücherwand mit antiken Büchern. Vorherrschende Farben sind samtrot, dunkelgrün und braun. Dieser Stil ist typisch für viele reale politische Schauplätze des Vereinigten Königsreichs und spiegelt gleichzeitig die Konservativität des britischen Systems wider. Hinsichtlich des Cats stellen dem Premierminister Hacker und dem Cabinet Permanent Secretary Appleby als Hauptpersonen lediglich noch die politische Beraterin Sutton, und der Parliamentary Private Secretary Woolley übergeordnete Rollen dar. Vereinzelt treten auch weitere Personen auf: der Botschafter von Kumranistan, der Chef der Europäischen Zentralbank, der schottische Außenminister, der Vizepräsident der $B B C$ und ein Interviewer der $B B C$. Diese Nebencharaktere stehen allerdings nicht im Vordergrund der Handlung, sondern leisten lediglich ihren Beitrag, wenn es darum geht, die politischen Themen zu diversifizieren sowie Gegenspieler und deren Meinungen und Einstellungen darzustellen.

Im Kontrast zur Reduktion des Schauplatzes, des Zeitraums und des Casts ist die Politikdarstellung relativ komplex, wodurch deutlich wird, dass darauf der Fokus der Serie liegt. Neben Europapolitik und Eurokrise werden auch der Klimawandel, die Außenpolitik des Vereinigten Königsreichs, die Beziehung zu 
Schottland und moralisch aufgeladene Themen wie Immigration und Prostitution aufgegriffen. dem Krisenmanagement kommt dabei eine übergeordnete Rolle.

Auch wenn das Chequers als eine Art ,Backstage-Bereich“ in der Politik gesehen werden kann, ist es immer noch ein politischer (wenn auch informeller) Raum und kein privater Bereich. Dies verdeutlicht auch die Kleidung der Hauptpersonen: Hacker, Appleby und Woolley tragen jeweils einen Anzug und Sutton ein schwarzes Kleid.

\section{Kleidung, Sprache \& Bildung: Charaktere als Stereotypen}

Mit Einstecktuch und Anzugweste ist Appleby am formellsten gekleidet. Dies verdeutlicht seinen Platz im politischen System. Im Gegensatz zu Hacker und Sutton ist er nicht von den Wahlen abhängig und hat einen permanenten Sitz in der Exekutive. Er ist sozusagen ,Teil des Mobiliars'. Dieser Eindruck wird durch seine sehr gewählte Sprache vertieft. Er repräsentiert damit gemeinsam mit Woolley den Beamtentypus des studierten Generalisten, der seine Sprachgewandtheit und Bildung strategisch nutzt, um z.B. eindeutige Festlegungen zu vermeiden oder andere Akteure - insbesondere Hacker - zu verwirren oder zu manipulieren. Dieses klassisch-konservative Auftreten von Woolley und Appleby steht im Gegensatz zu dem Suttons und Hackers. Der Bildungshintergrund von Hacker ist nicht bekannt, wobei auch Appleby bemerkt, dass der Job des Premierministers „the only top job [is] that requires no experience“ (S 6/F 1/4.50 - 4.53) und Hacker persönlich in dieser Funktion als ,,intellectually challenged“ (S 6/F 4/23.00 - 23.04) abgestempelt wird. Hacker spricht eine einfache, direkte Sprache, achtet nicht auf politische Korrektheit und benutzt häufiger Ethnophaulismen ${ }^{8}$. Er versteht Appleby häufig nicht und lässt sich dessen Antworten von Woolley, übersetzen'. Des Weiteren ist er nicht besonders vertraut mit dem System der EU, und politisch-strategisch verlässt er sich auf seine Berater. James D. Barber (Barber 1972: 90-96) würde ihn als passiv-positives Regierungsoberhaupt bezeichnen, da Hacker hauptsächlich damit beschäftigt ist, ein möglichst positives oder heroisches Außenbild abzugeben, und sich dabei so wenig wie möglich in umstrittene politische Diskussionen einmischen möchte.

Er wird von seiner politischen Beraterin Sutton unterstützt. Allerdings stellt sie im Kontrast zu Appleby und Woolley einen völlig neuen - auch in der Neuauflage der traditionsreichen Sitcom neu eingeführten - politischen Charakter dar. Sie ist jung und gebildet. Sie ist keine Generalistin, sondern hat Politik studiert und ihre Doktorarbeit über den civil service geschrieben. Auch ihre Klei-

8 Abwertende, verunglimpfende Bezeichnungen für ethnische Bevölkerungsgruppen. 
dung ist modern und gleichzeitig professionell. Sie benutzt eine einfachere und prägnantere Sprache als Woolley und Appleby, wobei sie diese mit ihrer Redegewandtheit ohne Probleme versteht. Auch dies zeigt, dass sie eine neue Generation der politischen Berater darstellt, welche sich Mühe geben, Politik weniger wissenschaftlich und volksnaher erscheinen zu lassen.

Bezüglich der Charaktere lässt sich zusammenfassend sagen, dass Kleidung, Sprache und Bildungshintergrund die Akteurskonstellation symbolisch unterstützen. Sie stellen verschiedene politische Stereotypen dar: Appleby verkörpert den konservativen civil service, Hacker einen eher ungebildeten, oftmals hilflosen Politiker, der sich leicht manipulieren lässt und nur um seine Außenwirkung bemüht ist, Woolley verkörpert den hin- und hergerissenen Beamten, der gerne an seinen Moralvorstellungen festhalten möchte, und Sutton spiegelt eine neue Generation von politischen, jungen, spezialisierten Politikberatern wider.

\section{Krisenmanagement: Panik versus Gelassenheit}

Diese politischen Stereotype treten auch in Problemsituationen hervor, in denen Krisenmanagement angesagt ist. Während der Premierminister sich überschätzt und die Lage zu optimistisch einschätzt, haben die anderen drei Charaktere einen klareren Blick auf die Probleme - wenn auch aus verschiedenen Perspektiven. Appleby und Sutton bleiben bei der Konfrontation mit schlechten Neuigkeiten gelassen, während Woolley eher überfordert wirkt. Wenn die Dilemmata ausführlich diskutiert werden, nehmen Appleby und Sutton rationale, machtstrategische Positionen ein, welche aufgrund der Verschiedenheit ihrer Interessen allerdings nicht immer übereinstimmen, und Woolley spielt eine Art Moralapostel. Hacker wirkt meist hilflos gegenüber der Komplexität der Situationen und fordert mehrfach von seinen Beratern, einen Ausweg zu finden.

Szenen-Beispiel: S 6/F 1/15.40 - 15.44

Hacker: So it is time for me to get hands-on and give some leadership... [Zu Appleby] So, tell me what I should do!

Als seine Berater keine Lösung des Prostitutionsdilemmas liefern können, ist er bereit, sich an jedem Strohhalm festzuhalten: Er betet zu Gott um seine Hilfe und deutet ein Gewitter als dessen Antwort. Mit Zuspitzung der Krise verhalten die Charaktere sich unterschiedlich: Sutton ist bereit, jede Möglichkeit - inklusive der Ermordung des Außenministers - in Erwägung zu ziehen. In ihrem Eifer, das Problem zu lösen, wird sie unvorsichtig und verschlimmert die Lage. Auf dem Wendepunkt der Krise wird auch sie leicht panisch, aber versucht weiter, Lösungsansätze zu finden. Woolley wird durch seine moralischen Vorstellungen 
davon abgehalten, sich aktiv zu beteiligen, und versucht weiter erfolglos, die anderen zu überzeugen. Hacker gerät völlig in Panik. Er will sich nicht aktiv mit dem Problem konfrontieren, lässt sich verleugnen und fordert weiterhin von seinen Beratern eine Lösung. Er verkriecht sich unter seinem Schreibtisch, weinerlich wie ein Kind. Schließlich ist er sogar bereit, sein Amt als Premierminister aufzugeben.

Appleby erscheint zweimal als Retter in der Not. Besonders inszeniert wird dies nach der völligen Resignation des Premierministers, als es donnert, das Licht ausgeht und er in einer hell erleuchteten Tür erscheint. Während die übrigen Charaktere über mögliche Lösungen diskutiert haben, hat er im Hintergrund seine Kontakte und sein Wissen genutzt, um Lösungen zu finden.

Diese verschiedenen Verhaltensweisen verstärken die Stereotype der Charaktere und lassen den alteingesessenen civil servant Appleby als überlegen erscheinen. Auch Sutton, die zwischenzeitlich seine Konkurrentin zu sein schien, ist ihm aufgrund ihres Mangels an Erfahrung und Beziehungen schließlich unterlegen. Woolley ist schlussendlich davon überzeugt, seinen „,moralischen Kompass" verloren zu haben, was eine Andeutung darauf ist, dass man mit einem ausgeprägten moralischen Gewissen in der Politik nicht weit kommen kann.

\section{Politikdarstellung: Lösung der politischen Krise versus Lösung der realen Krise}

Das drohende Scheitern der EU-Konferenz kann im Endeffekt abgewendet werden. Dies bedeutet jedoch nicht, dass eine Lösung für die Eurokrise gefunden wird. Die Idee Applebys, ein Klimaabkommen, wird als Lösung der politischen Krise angenommen, auch wenn dadurch nicht zur Lösung der realen Krisen - der Eurokrise und des drohenden Klimawandels - beigetragen wird. Dies wird bemerkt, dennoch billigend in Kauf genommen.

\section{Szenen-Beispiel: S 6/F 6/22.36-22.49}

Woolley: There is one problem: Nothing will have actually been achieved.

Appleby: It will sound as though it has. So people will think it has.

Hacker: That's all that matters.

Diese ,Lösung ' verstärkt die über die Serie aufgebaute negative Darstellung der Politik, in welcher die Akteure nur in ihrem eigenen Interesse handeln und im Endeffekt keine realen Probleme lösen, sondern Fehler und Scheitern mit schön klingenden, aber wenig effektiven Policies verschleiern. 


\section{Die fehlende Haarpracht des Premierministers und weitere narrative Elemente}

Der Name der Serie, Yes, Prime Minister, ist auch das Ende jeder Episode. Der Tonfall, in dem diese Zustimmung dem Premierminister entweder von Appleby oder Woolley ausgedrückt wird, zeigt jeweils auf, wer diese Folge ,gewonnen“ hat beziehungsweise wer im Spannungsfeld zwischen dem Premierminister auf der einen sowie Appleby und Woolley auf der anderen Seite in der endenden Folge seine Interessen erfolgreich durchbringen konnte.

Ein Kommentar Woolleys über die fehlende Haarpracht des Premierministers (in Episode 1), kann als Anspielung auf die Realität gesehen werden, da in den britischen Medien 2010 und 2011 mehrfach über eine kahle Stelle an David Cameroons Hinterkopf berichtet wurde (Hoggart 2010, O. V. 2010).

Interessant ist auch, dass der Premierminister das Buch Ghost von Robert Harris ${ }^{9}$ zu lesen scheint. Dies könnte man so deuten, als wolle der Premierminister sich über seinen Vorgänger und die gewöhnlichen Vorgänge innerhalb der Regierung informieren.

Es lassen sich noch viele weitere narrative Elemente und Gestaltungsmittel in der Sitcom finden, deren nähere Beschreibung und Deutung allerdings den Rahmen dieses Beitrags sprengen würde.

\section{FAZIT}

Mit der Neuauflage von Yes, Prime Minister lassen die Produzenten einen britischen Sitcom-Klassiker aufleben, der bereits im vorigen Jahrhundert - weit vor dem Boom von politischen Fernsehserien - seine eigene Perspektive auf die britische Regierungsführung vermittelte. Zwar immer noch an der Tradition verhaftet, wie die Kleidung, die Sprache oder die konservative Darstellung des civil service deutlich zeigen, erscheinen die Themen und Politikinhalte doch hochaktuell. Dreh- und Angelpunkt der Politik ist - so die Auffassung des Drehbuchautors Jonathan Lynn - nach wie vor das individuelle Verhältnis der Politiker zur Macht: „Nothing has really changed. Nothing much will. It's about people and their relationship to being in power. That doesn't change.“ (Day 2013) Die Stärke von Yes, Prime Minister liegt darin, auf satirisch-humoristische Art die Hand-

9 Ghost ist ein Politthriller über einen Ghostwriter, der die Memoiren eines Premierministers (welcher als Tony Blair zu identifizieren ist) verfassen soll und schließlich aufdeckt, dass letzterer sich mit der CIA verschworen hat. 
lungsimperative des Politikbetriebes zu karikieren, Machtstrukturen zu enthüllen und den Fokus von der parlamentarischen Arena auf die Medien und die oftmals im Verborgenen agierende Administration zu verschieben. Dabei wird Politik nie eindimensional dargestellt; vielmehr zeigen sich die Komplexität, die Ungewissheit und Widersprüche von politischen Entscheidungen - wie zum Beispiel durch die Illustration der Dilemmata. Dadurch eröffnet sich ein idealer Anknüpfungspunkt, anhand von Szenen aus Yes, Prime Minister Elemente des Politikmanagements anschaulich zu exemplifizieren.

\section{LITERATUR}

Ashe, Stephen (2016): UKIP, Brexit and postcolonial melancholy, in: http://discoversociety.org/2016/06/01/ukip-brexit-and-postcolonial-melancholy (zugegriffen am 21.3.2017).

Barber, James David (1972): The Presidential Character - Predicting Performance in the White House, Englewood Cliffs.

British Broadcasting Corporation (BBC 2004): The Final Top 10 Sitcoms, in: http://web.archive.org/web/20130430222338/http://www.bbc.co.uk/sitcom/w inner.shtml (zugegriffen am 26.3.2017).

Behrmann, Sven (2003): Politische Satire im deutschen und französischen Rundfunk, Wiesbaden.

Brew, Simon (2007): The Den of Geek interview: Jonathan Lynn, in: Den of Geek vom 6.12., http://www.denofgeek.com/tv/thor/19336/the-den-of-geekinterview-jonathan-lynn (zugegriffen am 21.3.2017).

Dallison, Paul (2016): What Yes, Minister taught us about Europe, in: Politico Online vom 24.8., http://www.politico.eu/article/sir-antony-jay-what-yesminister-satire-tv-show-taught-us-about-europe (zugegriffen am 22.3.2017).

Day, Patrick Kevin (2013): ,Yes, Prime Minister's‘ Jonathan Lynn remembers Margaret Thatcher, in: Los Angeles Times Online vom 9.4., http://articles. latimes.com/2013/apr/09/entertainment/la-et-st-yes-prime-ministers-jonathan -lynn-remembers-margaret-thatcher-20130409 (zugegriffen am 26.3.2017).

Freeguard, Gavin (2015): Public trust in public servants, in: https://www.insti tuteforgovernment.org.uk/blog/public-trust-public-servants-\%E2\%80\%93six-graphs (zugegriffen am 22.3.2017).

Gilroy, Paul (2005): Postcolonial melancholia, New York.

Government of the United Kingdom (GOV UK 2016): Guidance on devolution, in: https://www.gov.uk/guidance/guidance-on-devolution (zugegriffen am 22.3.2017). 
Government of the United Kingdom (GOV UK 2015): Statutory guidance. The Civil Service Code, in: https://www.gov.uk/government/publications/civilservice-code/the-civil-service-code (zugegriffen am 22.3.2017).

Greer, Scott L./Jarman, Holly (2011): The British Civil Service System, in: van der Meer, Frits M. (Hrsg.): Civil Service Systems in Western Europe, Cheltenham/Northampton.

Grunden, Timo (2013): Formales und informelles Regieren in rechtsstaatlichen Demokratien. Analysezugänge und Untersuchungsgegenstände, in: Korte, Karl-Rudolf/Grunden, Timo (Hrsg.): Handbuch Regierungsforschung, Wiesbaden, S. 219-228.

Händel, Heinrich (1979): Großbritannien. Band 1: Staat und Verwaltung, neubearb. Ausg. 1991, München.

Hartmann, Jürgen (2011): Westliche Regierungssysteme. Parlamentarismus, präsidentielles und semi-präsidentielles Regierungssystem, 3. Aufl., Wiesbaden.

Hobolt, Sarah B. (2017): The Brexit vote: a divided nation, a divided continent, in: Journal of European Public Policy 9, S. 1259-1277.

Hoggart, Simon (2010): Sticky patch headed off: Cameron goes for hair apparent, in: The Guardian Online vom 17.12., https://www.theguardian.com/ politics/2010/dec/17/sticky-patch-head-cameron-hair (zugegriffen am 24.9. 2017).

Institut für Medien- und Kommunikationspolitik (IfM) (2015): Länderporträt Großbritannien, in: http://www.mediadb.eu/europa/grossbritannien.html (zugegriffen am 22.3.2017).

IPSOS Mori (2008): Public's views on prostitution, in: https://www.ipsosmori.com/researchpublications/researcharchive/2308/Publics-views-onprostitution.aspx (zugegriffen am 22.3.2017).

Jay, Antony (2013): Antony Jay on the new Yes, Prime Minister, in: The Telegraph Online vom 9.4., http://www.telegraph.co.uk/comment/9774984/ Antony-Jay-on-the-new-Yes-Prime-Minister.html (zugegriffen am 26.3.20 17).

Kingdom, John (2014): The United Kingdom, in: Chandler, J. A. (Hrsg.): Comparative Public Administration, 2. Aufl., London/New York, S. 7-34.

Korte, Karl-Rudolf/Fröhlich, Manuel (2009): Politik und Regieren in Deutschland, 3. Aufl., Paderborn.

Krumm, Thomas/Noetzel, Thomas (2006): Das Regierungssystem Großbritanniens. Eine Einführung, München.

Kuhlmann, Sabine/Wollmann, Helmut (2013): Verwaltung und Verwaltungsreformen in Europa. Einführung in die vergleichende Verwaltungswissenschaft, Wiesbaden. 
Leconte, Cécile (2010): Understanding Euroscepticism, London/New York.

Lijphart, Arend (1999): Patterns of democracy: government forms and performance in thirty-six countries, New Haven.

Lynn, Jonathan/Jay, Antony (2016): Yes Minister Brexit special - Sir Humphrey explains all, in: The Guardian Online vom 7.8., https://www.theguardian. com/tv-and-radio/2016/aug/07/yes-minister-brexit-eu-jonathan-lynn-sirhumphrey (zugegriffen am 22.3.2017).

Martinson, Jane (2016): BBC News faces $£ 80$ m cuts over the next four years, in: The Guardian Online vom 29.2., https://www.theguardian.com/media/2016/ feb/29/bbc-news-cuts-news-channel (zugegriffen am 30.3.2017).

McCartney, Jenny (2016): Yes, Minister: We need Sir Humphrey now more than ever, in: The Telegraph Online vom 24.8., http://www.telegraph.co.uk/opinion /2016/08/24/yes-minister-we-need-sir-humphrey-now-more-than-ever (zugegriffen am 21.3.2017).

Media Reform Coalition (2015): Who owns the UK media?, in: http://www. mediareform.org.uk/wp-content/uploads/2015/10/Who_owns_the_UK_media-re port_plus_appendix1.pdf (zugegriffen am 21.3.2017).

Merrick, Jane (2012): Britain's new top civil servant says he wants to kill off the Sir Humphrey stereotype, in: Independent Online vom 14.4., http://www. independent.co.uk/news/uk/politics/britains-new-top-civil-servant-says-he-wan ts-to-kill-off-the-sir-humphrey-stereotype-7645962.html (zugegriffen am 21.3. 2017).

Namusoke, Eva (2016): A Divided Family: Race, the Commonwealth and Brexit, in: The Round Table 5/2016, S. 463-476.

Niedermeier, Alexander/Ridder, Wolfram (2017): Das Brexit-Referendum. Hintergründe, Streitthemen, Perspektiven, Wiesbaden.

National Audit Office (2015): Equality, diversity and inclusion in the civil service. Report by the Comptroller and Auditor General, London.

O.V. (2010): Is David Cameron going bald? In: Sunday Express Online vom 9.8., http://www.express.co.uk/news/uk/192028/Is-David-Cameron-going-bald (zugegriffen am 24.9.2017).

Panchamia, Nehal/Thomas, Peter (2015): Civil Service Reform in the Real World. Patterns of success in UK civil service reform. Institute for Government, London.

Paun, Akash/Munro, Robyn (2015): Governing in an Ever Looser Union. How the four governments of the UK co-operate, negotiate and compete, in: https://www.instituteforgovernment.org.uk/sites/default/files/publications/Go verning\%20in\%20an\%20ever\%20looser\%20union\%20-\%20final.pdf (zugegriffen am 22.3.2017). 
Payne, Sebastian (2016): ,Yes Minister' remains an unrivalled guide to British politics, in: Financial Times Online vom 27.8., https://www.ft.com/content/ fe329b28-69fa-11e6-a0b1-d87a9fea034f (zugegriffen am 22.3.2017).

Prätorius, Rainer (2002): Verwaltungskultur, in: Greiffenhagen, Martin/Greiffenhagen, Sylvia/Neller, Katja (Hrsg): Handwörterbuch zur politischen Kultur der Bundesrepublik Deutschland, Wiesbaden, S. 626-628.

Rhodes, R. A. W./Weller, Patrick (2005): Westminster transplanted and Westminster implanted: Exploring political change, in: Patapan, Haig/Wanna, Regina/Weller, Patrick (Hrsg.): Westminster Legacies: Democracy and Responsible Government in Asia and the Pacific, Sidney, S. 1-13.

Rudzio, Wolfgang (2005): Informelles Regieren. Zum Koalitionsmanagement in deutschen und österreichischen Regierungen, Wiesbaden.

Sarcinelli, Ulrich (2011): Politische Kommunikation in Deutschland. Medien und Politikvermittlung im demokratischen System, 3. Aufl., Wiesbaden.

Schubert, Klaus/Klein, Martina (2016): Das Politiklexikon, Bonn.

Slawson, Nicola (2015): Whitehall still has a diversity problem - here's is what it's doing about it, in: The Guardian Online vom 2.9., https://www.the guardian.com/public-leaders-network/2015/sep/02/whitehall-diversityproblem-civil-service-fast-stream (zugegriffen am 21.3.2017).

Sturm, Roland (2009a): Bildung und Kultur, in: Informationen zur politischen Bildung, Heft 262, http://www.bpb.de/izpb/10563/bildung-und-kultur?p=all (zugegriffen am 30.03.2017).

Sturm, Roland (2009b): Grundlinien britischer Außenpolitik, in: Informationen zur politischen Bildung, Heft 262, https://www.bpb.de/izpb/10548/grund linien-britischer-aussenpolitik (zugegriffen am 30.3.2017).

Sturm, Roland (2009c): Politik in Großbritannien, Wiesbaden.

Smith, Julie (2016): Europa und das Vereinigte Königreich. Kleine Geschichte der Beziehungen seit 1945, in: Aus Politik und Zeitgeschichte 49-50/2016, S. 11-16.

Thatcher, Margaret (1988): Speech to the College of Europe (,The Bruges Speech"), in: http://www.margaretthatcher.org/document/107332 (zugegriffen am 22.3.2017).

Tombs, Robert (2016): The English Revolt, in: New Statesman Online vom 24.7., http://www.newstatesman.com/politics/uk/2016/07/english-revolt (zugegriffen am 22.3.2017).

Weßels, Bernhard (2009): Spielarten des Euroskeptizismus, in: Decker, Frank/Höreth, Marcus (Hrsg.): Die Verfassung Europas. Perspektiven des Integrationsprojekts, Wiesbaden, S. 50-68. 
Westbrook, Caroline (2016): This 1980 clip from classic comedy Yes Minister pretty much sums up Brexit..., in: Metro Online vom 25.6., http://metro.co.uk/ 2016/06/25/this-1980-clip-from-classic-comedy-yes-minister-pretty-much-su ms-up-brexit-5966657 (zugegriffen am 22.3.2017).

Wintour, Patrick (2008): Harman: poll shows public support for ban on buying sex, in: The Guardian Online vom 4.9., https://www.theguardian.com/ politics/2008/sep/04/harrietharman.socialcare?gusrc=rss\&feed=networkfront (zugegriffen am 22.3.2017).

York, Chris (2016): ,Yes Minister' Clip Gains New Found Fame After EU Referendum Brexit Vote, in: Huffington Post UK vom 25.6., http://www. huffingtonpost.co.uk/entry/yes-minister-explains-brexit_uk_576e92a8e4b0232 d331e0fa8 (zugegriffen am 22.3.2017).

Zaschke, Christian (2016): Die BBC - so hochgelobt wie umstritten, in: Süddeutsche Online vom 13.10., http://www.sueddeutsche.de/medien/britischesfernsehen-was-die-bbc-fuer-grossbritannien-bedeutet-1.3205270 (zugegriffen am 30.3.2017). 


\title{
The Thick of It
}

\author{
Macht, Medien und Marionetten
}

Lisa Brose, Julia Linn, Michelle Magaletta

\section{WILLKOMMEN IM MINISTERIUM FÜR ALLES UND NICHTS}

Anfang der ersten Staffel

Lawton: Thank you. Morning.

Coverley: Morning, Cliff. Let me take that. [nimmt die Tasche des Ministers]

Lawton: Up all night with these bloody things.

Stets überforderte Politiker im Dauerstress, Konflikte zwischen ihnen, Spindoktoren, Beratern, Beamten und den Medien - darum dreht sich The Thick of It. Die britische Polit-Satire wirft einen Blick auf das Innenleben der britischen Regierung. Die von der $B B C$ produzierte Serie wurde zwischen 2002 und 2015 in unregelmäßigen Abständen ausgestrahlt. Die dieser Analyse zugrundeliegende erste Staffel umfasst drei Episoden. Regie führte der schottische Comedian Armando Iannucci (BBC Online 2017).

Im Deutschen wird The Thick of It treffenderweise mit Der Intrigantenstadl übersetzt. Zentrales Setting der Serie ist das fiktive Department of Social Affairs. Wofür dies genau steht und welche Zuständigkeiten das Ministerium hat, erfährt man in der ersten Staffel nur unzureichend. Es scheint, als würde dieses Ministerium alle Themenfelder bearbeiten, die in den anderen Ministerien keinen Platz finden. Auch der Minister selbst scheint die Abgrenzung nicht genau zu kennen, aber dazu später mehr. Entlang des Settings im Department of Social Affairs wird nach einer kurzen Serieneinführung die Ministerialdemokratie im Vereinten Königreich näher beleuchtet. Danach fokussiert die Analyse die sich in der Serie widerspiegelnden Theorien. Im darauffolgenden Kapitel wird die Serie als Datengrundlage betrachtet. Auf Basis dessen werden Besonderheiten der in der 
Serie dargestellten politischen Kultur herausgearbeitet, bevor der Aspekt der Erzählweise der Serie, also der Narration, thematisiert wird. Abschließend werden die Ergebnisse in einem Fazit noch einmal zusammengefasst.

\section{SERIENEINFÜHRUNG: DER MARIONETTEN-MINISTER UND SEIN GEFOLGE}

Im Fokus der Serie stehen das fiktive Department of Social Affairs and Citizenship, der amtierende Sozialminister Hugh Abbot sowie sein Antagonist und Vertreter des Premierministers Malcom Tucker. Der formelle Zuständigkeitsbereich des Ministeriums ist weitreichend und umfasst interdisziplinäre Themen, wie beispielsweise Wohnungsbau, Sozialleistungen und Bildung. Aufgrund seiner thematischen Weitläufigkeit und ungenauen Abgrenzung genießt das Ministerium allerdings keine große Beliebtheit, wie eine Aussage des Ministers selbst unterstreicht: „Social Affairs. What the fuck does that actually mean? It's so vague“" (S 1/F 3/26.30 - 26.34).

Die Parteizugehörigkeit der Regierungsmitarbeiter bleibt im gesamten Serienverlauf unerwähnt. In einem Serienkommentar wird jedoch eine Parallele zur britischen New Labour unter Tony Blair und seiner Image- und Medienfokussiertheit angedeutet (The Independent 2014). Die Serie ist keine rein fiktive, sondern orientiert sich an britischer Realpolitik. Dazu wurde inhaltlich mit politischen Westminster-Insidern zusammengearbeitet (The Guardian Online 2012a). Das Hauptmerkmal der Serie bildet der klassische britische Humor trockenster Sarkasmus und Kraftausdrücke weit unterhalb der Gürtellinie.

Abbildung 1: Beziehungsgeflecht der Akteure

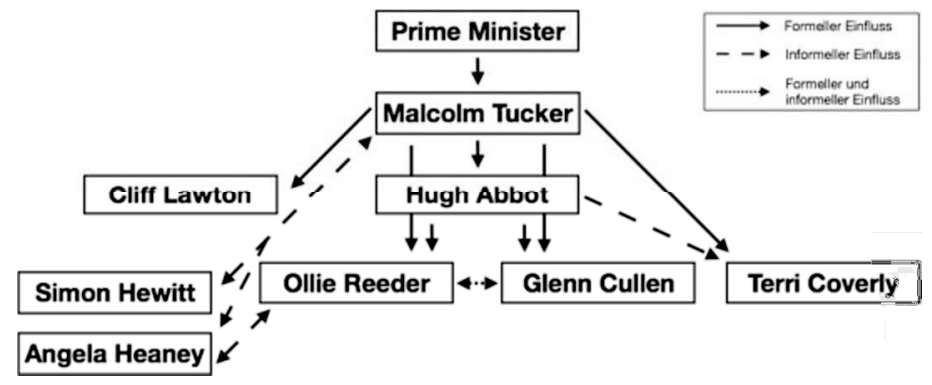

Eigene Darstellung. 
Abbildung 1 stellt die wichtigsten Akteure und ihre Beziehungen untereinander dar. Die prominenteste Beziehung ist wohl die zwischen Politik und Medien. Allmorgendlich wird im Ministerium die aktuelle Presse diskutiert und der Tagesablauf danach ausgerichtet. Den Medien wird insgesamt eine ambivalente Rolle beigemessen: Je nach Tonalität der Berichterstattung ist sie Heilbringer oder Sensenmann für die politischen Akteure.

Die zweite herausragende Beziehung besteht zwischen dem Spindoktor des Premierministers, Malcom Tucker, sowie dem Sozialminister Hugh Abbot und dessen Mitarbeiterteam. Dieses wird einseitig dominiert von Tucker, der mit seinen autoritären Anweisungen das Ministerium leitet.

\section{Spitzenakteure: Premierminister und Malcolm Tucker}

Der Premierminister ist der große Unbekannte in der Serie. Der Zuschauer bekommt ihn im gesamten Verlauf nicht zu Gesicht. Für die Abwicklung des alltäglichen Politikgeschäfts setzt er Malcolm Tucker ein, der in seinem Namen handelt und die Leitung des Ministeriums durch seine autoritären Anweisungen an sich reißt. Letztlich bleibt der Zuschauer im Unklaren darüber, ob Tucker wirklich dem Willen des Premiers oder seiner eigenen Agenda folgt.

Malcolm Tucker ist der director of communications oder Spindoktor bzw. Meinungsmacher des Premierministers in der ersten Staffel und ist somit für das PR- und Imagemanagement zuständig. Seine Aufgabe ist es, ein positives Image und wohlwollende Berichterstattung über seinen Vorgesetzten zu erzeugen, was ihm nicht immer gelingt. Tuckers Job ist dadurch automatisch an den Premierministerposten gekoppelt und von dessen politischer Machtstellung abhängig. Seine Ranghöhe erlaubt ihm ein cholerisches und erbarmungsloses Auftreten.

\section{Machtlose Minister}

Der sehr kurze Auftritt von Cliff Lawton, dem Vorgänger Abbots im Amt des Sozialministers, macht bereits zu Beginn deutlich, dass die Medien und Tucker sehr einflussreiche Akteure sind. Der Grund für Lawtons Rücktritt in den ersten fünf Serienminuten ist die schlechte Presse über seine Arbeit und sein Ministerium. Das Risiko, dass die Negativberichterstattung auf die Regierung und somit auf den Premierminister abfärbt, wird als zu bedrohlich eingeschätzt. Gegen seinen Willen muss Lawton seinen Posten räumen.

Anschließend wird Hugh Abbot als Nachfolger Lawtons eingesetzt. Abbot ist verheiratet und hat zwei Kinder. Er wirkt motiviert und legt ein hohes Arbeitspensum an den Tag. Jedoch kommt seine Ehe dadurch regelmäßig zu kurz. Für den Zuschauer unterscheidet er sich dennoch nur wenig von seinem 
Vorgänger - er ist zwar durchaus engagiert, inhaltlich voranzukommen, jedoch tragen seine Vorhaben selten Früchte. Darüber hinaus scheint sein allgemeines, gesellschaftliches Wissen begrenzt zu sein, was ihn wenig qualifiziert erscheinen lässt. Da ihm selten Positives gelingt, schwankt der Zuschauer bei seiner Betrachtung zwischen Ablehnung aufgrund seiner Amateurhaftigkeit und empathischer Zuneigung für den scheinbar dauerhaft Unglücklichen.

\section{Politische Berater versus Verwaltungsangestellte}

Der politische Seniorberater des Ministers ist Glenn Cullen. Abbot und Cullen sind über die berufliche Zusammenarbeit hinaus eng miteinander befreundet. Geschieden und resigniert widmet Cullen den Großteil seines Lebens der Politikberatung. Trotz seiner langjährigen Erfahrung erscheint Cullen dem Zuschauer wenig kompetent oder sympathisch. In seinem Auftreten wirkt er tendenziell überheblich und unmodern, besonders im Hinblick auf sein Team und seine politischen Positionen.

Politischer Juniorberater Abbots ist Ollie Reeder. Er spielt die Rolle des gebildeten und karriereorientierten Aufsteigers. In der ersten Staffel ist Reeder von konstanter beruflicher Professionalität jedoch noch weit entfernt und bewegt sich schwankend zwischen angriffslustigem Gegenspieler zu Cullen und amateurhaftem Tollpatsch hin und her.

Als dritte im Bunde zählt Terri Coverly zum erweiterten Kreis des Sozialministers Abbot. Coverly ist Verwaltungsangestellte und für die Öffentlichkeitsund Pressearbeit des Ministers zuständig. Obwohl von politischen Entscheidungen meist exkludiert, muss sie diese dennoch nach außen gewinnbringend kommunizieren, was ihr aufgrund der inkonstanten Politikgestaltung nicht selten Kopfschmerzen bereitet. Sie ist an verwaltungsinterne Prozeduren und Abläufe gebunden, die die politischen Akteure in der Regel nicht so genau nehmen. Daher steht sie häufig mit Abbots politischem Team in Konflikt, was das gegenseitige Verhältnis zunehmend belastet. Dennoch weiß sie ihre Position gesichert und ist daher gegen jegliche politische Krise immun.

\section{Kritische Medien}

Die Medien werden stets als Antagonisten der Politik inszeniert. Die beiden wichtigsten Medienvertreter der ersten Staffel sind Angela Heaney vom Standard, später Daily Mail, und Simon Hewitt ebenfalls vom Standard. Aufgrund der ehemaligen persönlichen Beziehung zwischen Reeder und Heaney wird letztere vom Minister als kontrollierbar eingeschätzt und des Öfteren für die Streuung interner Informationen verwendet. Im Serienverlauf wird Heaney jedoch zu- 
nehmend kritischer und investigativer. Hewitt gilt von Anfang an als medienpolitischer Gegner - seine Medienberichterstattung wird zumeist von den politischen Akteuren zu verhindern versucht.

\section{POLITISCHER KONTEXT: DIE BRITISCHE EXEKUTIVE}

Im Folgenden wird nun zunächst genauer auf das politische System Großbritanniens eingegangen. Um möglichst nah an den Gegebenheiten der Serie zu bleiben, wird hierbei ein Fokus auf die britische Exekutive und somit die Regierung sowie die öffentliche Verwaltung gesetzt.

\section{(Premierminister-)Regierung}

Die britische Regierung lässt sich auf zwei Weisen erfassen. Zum einen als ,Regierung im engeren Sinne', die sich aus dem Premierminister und seinem aus circa 20 Ministern bestehenden Kabinett zusammensetzt. Bei einem breiteren Verständnis zählt man zu ihr ,,im weiteren Sinne auch Minister ohne Kabinettsrang, stellvertretende oder niederrangige Minister [...], parlamentarische Staatssekretäre [...] und vor allem private parlamentarische Staatssekretäre“ (Hartmann 2011: 68). Gemäß Roland Sturm (2009: 26) haben sich die Machverhältnisse innerhalb der britischen Regierung im Laufe der Zeit deutlich verändert. So hat die nun bestehende Premierministerregierung den vormals präsenten Typ der Kabinettsregierung ersetzt. Die Bedeutung dieser Feststellung für das politische Alltagsgeschäft wird bei der Betrachtung der beiden Komponenten der ,Regierung im engeren Sinn', ergo Premierminister und Kabinett, deutlich.

\section{Premierminister}

Der Premierminister residiert in der Downing Street No. 10 im Londoner Stadtteil Whitehall. Aufgrund des dortigen Konglomerats von Ministerien wird ,Whitehall' auch als Synonym für die britische Ministerialbürokratie verwendet (Hartmann 2011: 72, Sturm 2009: 133). Die vielzähligen und wichtigen Befugnisse des Premierministers lassen sich in erster Linie auf Verfassungskonventionen zurückführen (Hartmann 2011: 72). Diese sind „dann wirksam, wenn sie von allen betroffenen Personen und Institutionen akzeptiert werden und wenn einflussreiche Verfassungskommentatoren bestätigen, dass dies Regeln sind, an die man sich halten soll“" (Sturm 2009: 39-40). Grundlegend lassen sich drei zentrale Kompetenzen des Premierministers herausstellen. So ist er einerseits der Parteiführer der Regierungspartei im Parlament. Gleichzeitig übernimmt er als Re- 
gierungschef die Führung der Regierung. Die Minister werden zudem nicht nur durch den Premierminister ernannt, sondern können auch durch ihn entlassen werden (Hartmann 2011: 72). Dies ist die sogenannte Ernennungs- und Entlassungskompetenz des Premierministers (Korte 2001: 6). In dem Regelwerk Questions of Procedure for Ministers, das erst 1992 der Öffentlichkeit zugänglich gemacht wurde und heute unter dem Namen Ministerial Code bekannt ist, hält der Premierminister Vorschriften fest, die für seine Minister während seiner Amtszeit gelten (Sturm 2009: 111). Doch der Premierminister wirkt nicht nur nach innen, sondern ebenso nach außen. Da er als Einzelperson im Gegensatz zum Kollektiv des Kabinetts oder der Komplexität der gesamten Regierung einen hohen Wiedererkennungswert besitzt, fokussiert sich die öffentliche Aufmerksamkeit hauptsächlich auf ihn (Heffernan 2006, nach Hartmann 2011: 75). Es lässt sich festhalten, dass der Premierminister eine „dominierende politischstrategische Rolle im politischen Institutionengeflecht" ausübt (Korte 2001: 7). Hieran anknüpfend wird die Macht des Premierministers in Großbritannien durch vergleichsweise wenige institutionelle Schranken begrenzt, solange er denn die entsprechende parlamentarische Mehrheit innehat (Sturm 2009: 111). Die Regierungszentrale des Premierministers setzt sich aus dem Cabinet Office und dem Prime Minister's Office zusammen, die ihn in jeweils unterschiedlichen Tätigkeitsfeldern unterstützen. Das Cabinet Office steht dem Premierminister bei der Lenkung der Regierungsgeschäfte durch die Vorbereitung von Sitzungen des Kabinetts, die Pflege der Beziehungen zu den verschiedenen Ressorts sowie die Anfertigung einer Übersicht zu den Tätigkeiten der Ministerien zur Seite. Zudem ist es an der Bildung von Kabinettsausschüssen beteiligt, die eine zentrale Rolle im Falle „ressortübergreifender Probleme“ spielen (Hartmann 2011: 74). Das Prime Minister's Office ist für die Organisation der persönlichen politischen Umgebung des Premierministers sowie der Beziehungen zur Regierungspartei zuständig. Zum einen wirkt es an der inhaltlichen Erarbeitung von Lösungsansätzen für politische Probleme mit. Zum anderen betreibt es ein permanentes Monitoring der Medien und überprüft die dortige Darstellung der Regierungsarbeit (Sturm 2009: 116).

\section{Kabinett}

Das Kabinett ist aus circa zwanzig durch den Premierminister bestimmten Ministern zusammengesetzt. Nur die wichtigsten Ministerialverwaltungen sind im Kabinett repräsentiert. Die zentrale Aufgabe des Kabinetts liegt im Beschluss von Gesetzesvorlagen der Regierung an das Parlament. Da sich hierbei alle Minister an den Diskussionen beteiligen, wird das Kabinett auch als Kollegialorgan charakterisiert. Jedoch handelt es sich bei einer entsprechenden Kabinettsbera- 
tung nicht um eine formelle Abstimmung. Stattdessen wird diese durch den Premierminister geleitet, beendet und im Anschluss zusammengefasst. Durch eben jene Zusammenfassung der Ergebnisse der Beratung ergibt sich eine umfassende Richtlinienkompetenz des Premierministers. Denn sie stellt in erster Linie die Sichtweise des Premierministers dar, die den Konsens des Ministerkollektivs widerspiegeln kann, aber nicht muss. Darüber hinaus legt die Zusammenfassung verpflichtend fest, wie die Minister die Entscheidungen in ihren jeweiligen Ressorts realisieren und in der Öffentlichkeit erklären müssen. Sollte ein Minister eine solche Entscheidung nicht unterstützen können, bleibt ihm nur die Option des Rücktritts. Denn die Nichtbeachtung einer Richtlinie entspricht einem Verstoß gegen die Kabinettsdisziplin (Hartmann 2011: 72-73). Es wird deutlich, dass der Premierminister nicht seinem Kabinett verantwortlich ist, sondern seine Entscheidungen eher von den Kabinettsmitgliedern ohne Debatten akzeptiert werden. Somit wurde das Kabinettsprinzip, also das gemeinsame Entscheiden des Kabinetts, aufgegeben (Sturm 2009: 111, 113). Des Weiteren gilt für die Minister das Prinzip der Ministerverantwortlichkeit, denn sie haben die vollständige Verantwortung für ihr Ressort. Sollte sich ihre Führung als nicht sonderlich erfolgreich herausstellen oder eine durchweg negative Resonanz bekommen, kann der Premierminister auf das Instrument der Kabinettsumbildung zurückgreifen. Der jeweilige Minister wird dann ausgewechselt und entweder vollständig entlassen oder einem anderen Ressort zugeteilt (Hartmann 2011: 75). Interessanterweise sind Kabinettsumbildungen jedoch nicht mit Krisen der amtierenden Regierung gleichzusetzen, sondern vielmehr ein regelmäßig eingesetztes Mittel, um beispielsweise die Effizienz der Regierung zu erhöhen (Sturm 2009: 112). Anhand dieser kurzen Darstellung ist deutlich geworden, warum, wie eingangs erwähnt, die britische Regierung auch als Premierministerregierung bezeichnet wird. Während der Premierminister der zentrale und dominante Akteur innerhalb der britischen Regierung ist, fungiert das Kabinett hauptsächlich als sein Instrument (Sturm 2009: 114).

\section{Ministerialverwaltung und civil service}

Den britischen Ministerien werden keine institutionellen Eigeninteressen beigemessen, da die Beamten keine Parteizugehörigkeit innehaben. Der Typus des politischen Beamten ist in der britischen Ministerialverwaltung nicht existent. Ausgenommen hiervon sind die parlamentarischen Staatsekretäre, die Mitglieder des Unterhauses sind. Das britische Beamtentum wird unter dem Begriff civil service zusammengefasst, der nun im Folgenden hierfür verwendet wird. Zwei entscheidende Ereignisse formten das Selbstverständnis des civil service. Der Northcote-Trevelyan Act 1854 legte die grundlegende Struktur des civil service 
fest. Im Zuge der Professionalisierung kristallisierten sich zwei Zuständigkeitsbereiche heraus. Auf der einen Seite war der civil service für die inhaltliche Beratung der Politik und Gestaltung von Entscheidungen zuständig. Auf der anderen Seite kümmerte er sich um die Umsetzung und Durchführung von Gesetzen. Durch den Haldane Report im Jahr 1918 wurde dann der Schwerpunkt auf die erste der beiden Zuständigkeiten gelegt. Es entwickelte sich ein Rollenverständnis, das, wenn auch in abgeschwächter Form, teilweise bis heute besteht. So konnten sich die Minister bei der Gestaltung von Lösungsansätzen auf das Fachwissen ihrer Beamten verlassen und ihren Fokus ganz auf die eigene Rolle, nämlich die Präsentation eben jener Lösungen in Parlament und Öffentlichkeit richten (Hartmann 2011: 75-76). Fünf Merkmale charakterisierten den traditionellen civil service. Bedingt durch die Dominanz von Absolventen der Universitäten Oxford und Cambridge, zeichnete er sich durch eine starke Homogenität und soziale Exklusivität aus. Innerhalb der Beamtenschaft überwiegten Generalisten, die durch ein geisteswissenschaftliches Studium dazu befähigt waren, sich in eine Vielzahl unterschiedlicher Probleme einzuarbeiten. Der civil service war zudem zur parteipolitischen Neutralität verpflichtet. Durch das Verbot einer parteipolitischen Tätigkeit wurde gewährleistet, dass die Beamten wechselnde Regierungen und politische Positionen gleichermaßen loyal unterstützten. Darüber hinaus bestand die Tradition, das Handeln der Regierung geheim zu halten und nicht an die Öffentlichkeit dringen zu lassen. Der civil service bildete eine eingeschworene Gemeinschaft, die einen großen Einfluss auf die politischen Amtsträger und die Art und Weise des Regierens ausüben konnte. In den Regierungszeiten Margareth Thatchers, John Majors und Tony Blairs wurden jedoch zentrale Reformen eingeleitet, die den traditionellen civil service erheblich veränderten und zum heutigen, ,modernen' civil service umgestalteten. Wandel fand hierbei in allen der erwähnten fünf Charakteristika statt. (Sturm 2009: 135-137). Der Auslöser für die Veränderung war ein Grundmisstrauen gegenüber der sozial homogenen und politisch neutralen Beamtenschaft. Entlang der neuen Leitbilder Effizienz und Wirtschaftlichkeit wurden die Aufgaben der Verwaltung reduziert und an privatwirtschaftliche Akteure übertragen. Die Neuerungen reichten bis in die Kernkompetenzen des civil service hinein. In dem Bereich der politischen Beratung, Gestaltung und Gesetzesvorbereitung hat die Beamtenschaft ihre Monopolstellung verloren. Stattdessen konkurriert sie hier nun mit einer Vielzahl weiterer Berater aus Wissenschaft und Wirtschaft (Hartmann 2011: 77-78). 


\section{SERIE ALS SPIEGEL DER THEORIE}

\section{Arenenmodell: Dominanz der medialen Sphäre}

Das Arenenmodell (Korte 2009) kann als grober Rahmen verstanden werden, in dem sich die Serie wiederfindet. ${ }^{1}$ Malcolm Tucker repräsentiert in The Thick of It die parlamentarische Arena, die auch als Parteiendemokratie bezeichnet wird. Er handelt im Namen des Premierministers und gibt für diesen die Parteilinie vor, was an mehreren Stellen in der Serie zum Ausdruck kommt und dann auch im Rahmen dessen in die Analyse einfließen wird. Die öffentliche Arena beziehungsweise Mediendemokratie spielt in The Thick of It eine besonders tragende Rolle. In diese Arena fällt beispielsweise die Orientierung bei Entscheidungen an Stimmungen. Auch das Feld Darstellungspolitik und der kommunikative Austausch zwischen Bürgern und politischen Akteuren finden sich in der öffentlichen Arena wieder (Korte 2009: 232-233). Dies lässt sich anhand folgender Szene beobachten:

\section{Szenen-Beispiel: S 1/F 2/08.48-11.18}

Reeder: [...] This is a tape of this morning's focus group. This is Mary, she is a focus group legend. [...] Everything that she's said in the last twelve months in every focus group has chimed exactly with the core voter. And she loved my arts for hearts and minds policy. [...] And she said, Glenn, that it chimed brilliantly with stay-at-home mums like her.

[Coverly, Abbot, Reeder und Cullen sitzen zusammen im Besprechungsraum. Sie schauen sich ein Video einer Fokusgruppe an. Im Video ist unter anderem Mary zu sehen.]

Abbot: And she's really core Middle England, is she?

Reeder: Totally, totally core Middle England.

In dieser Szene kommt zum Ausdruck, dass sich bei einer Entscheidung an der Stimmung orientiert wird. Die Entscheidung über eine Policy wird von einer Fokusgruppe abhängig gemacht bzw. von einer einzelnen Frau, die als „Fokusgruppen-Star" bezeichnet wird, da sie den perfekten Querschnitt darstelle und somit andere Mitglieder einer Fokusgruppe überflüssig mache. Zudem treten im Anschluss an diese Szene die politischen Akteure in den kommunikativen Austausch mit einer Bürgerin.

1 Eine ausführliche Erläuterung des Arenenmodells findet sich im Kapitel „Spiegel“ des Beitrags zur Serie House of Cards, weshalb an dieser Stelle von einer Wiederholung dessen abgesehen wird. 


\section{Verschränkung zwischen Medien und Politik}

Medien und Politik sind untrennbar miteinander verbunden, was auch in der Serie deutlich wird. Die Medien sind stets präsent und in Entscheidungsprozessen ausschlaggebend.

Hierzu lassen sich zwei theoretische Konzepte hinzuziehen - das Interdependenzmodell sowie die Mediendemokratie. Nach dem Interdependenzmodell stehen Medien und Politik in einem symbiotischen Verhältnis. Sie sind wechselseitig voneinander abhängig, tauschen sich aus und verschmelzen so miteinander (Donges/Jarren 2001: 231-232). Der Begriff Mediendemokratie kann als Nachfolgekonzept zum Interdependenzmodell, insbesondere in der Abgrenzung zur Parteiendemokratie, verstanden werden. Sowohl das Interdependenzmodell als auch das Konzept der Mediendemokratie fokussieren Akteurskonstellation und die Funktionen von Medien. Diese sind die Herstellung von Öffentlichkeit, Information und Kontrolle. Damit haben Medien im politischen System eine unverzichtbare Funktion (Donges/Jarren 2001, Marcinkowski/Pfetsch 2009). Marcinkowski und Pfetsch verstehen mit ihrem Konzept der Mediendemokratie die Medien als Bestandteil des politischen Systems. Demnach beeinflussen die Mechanismen der Medien politische Prozesse und kontextualisieren diese (Marcinkowski/Pfetsch 2009: 13). Damit ist die Verschmelzung von Politik und Medien nicht wieder rückgängig zu machen.

Diese durch die Konzepte beschriebene Verschränkung von Medien und Politik kommt in mehreren Szenen der ersten Staffel von The Thick of It zum Ausdruck. Exemplarisch wird die enge Beziehung zwischen Medien und politischem System in einem Ausschnitt der ersten Folge deutlich. Der press digest, also die Presseschau, ist das erste, was am Tag passiert. Seit einigen Tagen wird in der Presse spekuliert, ob Minister Cliff Lawton zurücktritt oder nicht. Aufgrund der negativen Berichterstattung erklärt Malcolm Tucker Cliff Lawton, dass dieser zurücktreten müsse. Der Premierminister und Tucker selbst seien zwar der Meinung, dass der Minister seine Arbeit gut mache, aber die Regierung wirke durch die negative Presse schwach. Hieran wird die starke Beeinflussung der Politik durch die Medien deutlich. Die Politik orientiert sich in dieser Szene an den Medien (S 1/F 1/01.41 - 03.10).

Das sowohl im Interdepenzmodell als auch im Konzept der Mediendemokratie beschriebene Wechselverhältnis zwischen Medien und Politik zeigt sich in einer weiteren Szene. Zunächst versucht die Journalistin Angela Heaney, die Politik zu beeinflussen. Sie droht Malcolm Tucker mit einer Enthüllungsstory über das Chaos im Ministerium. Doch dann dreht Malcolm Tucker den Spieß um und droht der Journalistin, dass sie keine Hinweise aus dem Ministerium mehr 
bekäme und er dafür sorgen würde, dass sie keine Arbeit mehr fände. Hier wird klar, dass sowohl die Medien einen starken Einfluss auf die Politik haben, die Politik aber auch die Medien beeinflusst - sich beide nahezu erpressen ( $\mathrm{S}$ 1/F $1 / 24.19-26.32$ ).

\section{Spin doctoring}

Malcolm Tucker agiert in The Thick of It als Spindoktor des Premierministers beziehungsweise der Regierung. Spin doctoring ist ein Begriff aus den USA und wird dort vor allem im Zusammenhang mit Nachrichtenmanagement verwendet. Der Spindoktor ist für die Imagepflege zuständig. Bei ihm ist, wie bei Tucker als Kommunikationsdirektor der Regierung, die Kommunikation zentralisiert. $\mathrm{Zu}$ den Merkmalen des Spindoktors zählen außerdem die Perfektion der Medienbeobachtung und die professionelle Sammlung von Informationen, wie auch bei Tucker zu beobachten ist. Zudem beeinflussen Spindoktoren Journalisten und werden als manipulativ beschrieben (Donges/Jarren 2011: 177-178). All diese Punkte treffen auch auf Tucker zu, was im Folgenden verdeutlicht werden soll.

Szenen-Beispiel: S 1/F 1/05.00-05.35

Tucker: One more thing. The daily mail. David Topham has got it into his ear that we are going to sack you because of press pressure.

Lawton: I wonder why.

Tucker: Look, you're in no position to dish out fucking sarcasm. That's over. You no longer have purchase in the sarcasm world. Get on the phone. Tell him that you're jumping before you're pushed, although we were going to push you, but not because of press pressure, but because of your deeply held fucking personal issues, whatever they were.

Tucker versucht in dieser Szene, eine Geschichte zu konstruieren, damit es nicht so wirkt, als würde die Regierung dem Druck der Presse nachgeben und Lawton wegen der negativen Berichterstattung der Presse zurücktreten. Tucker betreibt in dieser Szene also aktiv Imagepflege. Außerdem zeigt sich, dass er die Medienbeobachtung perfektioniert hat. So handelt er aufgrund der Berichterstattung, die er ständig im Blick hat. Eine weitere Szene aus der zweiten Folge behandelt gleichermaßen den Umgang mit negativer Berichterstattung. Abbot, Reeder und Cullen arbeiten an einer Strategie, um auf diese zu reagieren. Sie wollen sich rächen, allerdings fällt ihnen nichts ein. Tucker kommt hinzu und hat schließlich eine Strategie, die umgesetzt werden soll: „This is how we do it!“ Hieran werden die Beeinflussung der Medien durch den Spindoktor Tucker und seine manipulative Herangehensweise deutlich, mit der er auf negative Bericht- 
erstattung reagiert. In dieser Szene zeigt sich zudem der Zusammenhang des spin doctoring mit dem Nachrichtenmanagement. Zudem verordnet Tucker Abbot eine zusätzliche Presseschau, was einmal mehr die perfektionierte Medienbeobachtung und die professionelle Sammlung von Informationen durch den Spindoktor beweist (S 1/F 2/04.03-06.51).

Das spin doctoring kommt neben den USA vor allem in Großbritannien vor. Als Beispiel aus der Realität sei hier Tony Blairs Spindoktor Peter D. Mandelson genannt. Der Wahlkampfmanager und Vertraute Blairs war auch noch als Minister im Kabinett für die Imagepflege des Premierministers verantwortlich. Diese Kombination aus Mandelsons Tätigkeit als Spindoktor einerseits und seiner Zugehörigkeit zur Regierung andererseits sorgte immer wieder für Debatten (Donges/Jarren 2011: 177-178). Gegenüber der englischen Zeitung The Guardian zog Peter Capaldi - alias Malcolm Tucker - Parallelen zwischen den Figuren Mandelson und Tucker. Im Interview bestätigte Capaldi zudem, dass die Rolle des Kommunikationsdirektors und Spindoktors Tucker ursprünglich an die Person Alastair Campell, Tony Blairs Kommunikationsdirektor, angelehnt wurde, was zuvor öffentlich vielfach vermutet wurde (The Guardian Online 2009).

\section{Machtmakler}

Glenn Cullen erfüllt in The Thick of It unterschiedliche Funktionen eines Machtmaklers, die im Folgenden kurz skizzieren werden ${ }^{2}$. Zunächst sei hier die Reziprozität genannt. Cullen und Abbot verbindet ein langjähriges Vertrauensverhältnis. Sie besprechen alles miteinander. Ein wechselseitiger Informationsaustausch, wie er zwischen Machtmakler und Spitzenakteur zentral ist, ist gegeben. Basierend auf dem Vertrauen, das Abbot Cullen entgegenbringt, genießt dieser einen Sonderstatus. Während die anderen Mitarbeiter immer wieder in ihre Schranken verwiesen werden, hört Abbot stets auf Cullen und befolgt dessen Rat. Zudem ist er Abbots Sparringpartner und sein Sprachrohr (S 1/F 3/07.24 - 08.24). Cullen koordiniert alles für seinen Minister: Termine, Policies, aber auch Persönliches. So ruft er beispielsweise Abbots Frau an, um ihr mitzuteilen, dass dieser im Büro übernachtet. Gleichzeitig ist er auch Seelentröster, bei dem der Minister seinen Frust ablassen kann (Korte 2003: 23-26).

Cullen ist Abbots Vertrauter und ältester Freund. Das langjährige Vertrauensverhältnis zwischen dem Spitzenakteur und seinem Machtmakler wird in einer Szene besonders deutlich (S 1/F 3/22.46 - 23.06). Alle Mitarbeiter werden

2 Vgl. hierzu das Kapitel „Spiegel“ der Analyse zur Serie House of Cards, in dem das Konzept des Machtmaklers auf Doug Stamper übertragen wird. 
zu Tucker gerufen, um das weitere Vorgehen zu besprechen. Abbots Rücktritt steht im Raum. Allerdings darf er als Einziger nicht an dem Treffen teilnehmen. Als alle zu Tucker aufbrechen, bleibt Cullen einen Moment länger beim Minister. Er betont, dass er zu ihm stehe und sie ein Team bleiben würden, egal was komme.

\section{Informalität als Strategie}

Informalität kann als Mechanismus zur Problembewältigung beschrieben werden. „Informelle Entscheidungsverfahren gewährleisten die Entscheidungsfähigkeit in formalen Entscheidungsinstitutionen.“ (Grunden 2009: 62) Ziele dessen sind die Reduktion von Unsicherheit, die Gewinnung von Informationen sowie die Sicherung von Legitimation. Charakteristisch für Informalität sind die Vermeidung formaler Verfahren sowie der Ausschluss von Akteuren, die eigentlich ein Partizipationsrecht haben, um so Blockaden zu vermeiden (Grunden 2009: 62-63). Eine weitere informelle Praktik ist die Drohung als ,spezifische Form der konflikthaften Interaktion bzw. Kommunikation zwischen (Gruppen von) Akteuren“ (Rüb 2014: 16).

Die Arbeit des Ministers Abbot und seines Teams ist von informellen Strukturen geprägt. In der Serie gibt es immer wieder informelle Gesprächsrunden die Beamtin Terri Coverly wird mehrmals von Gesprächen ausgeschlossen. Es bildet sich ein informeller enger Beraterzirkel um Abbot, bestehend aus Cullen und Reeder. Zudem wird mit Drohungen gearbeitet - vor allem gegenüber der Journalistin Angela Heaney, wie bereits im Abschnitt zur Verschränkung zwischen Medien und Politik beschrieben. Ein weiteres Anzeichen für informelles Handeln ist das Missachten von Vorgaben. Der Minister und sein Team halten sich nicht an Regeln und Abläufe, wie die folgende Szene zeigt.

\section{Szenen-Beispiel: S 1/F 1/07.42-08.38}

Coverly: I can see that you've all got big, stiff hard-ons for this. I'm not saying that's not nice. But there is no way we're gonna clear it by this afternoon.

Reeder: Why not?

Coverly: So cool it, and I'll ring Paul at the treasury.

Alle: No, no, no!

Abbot: No phone calls to the treasury!

Reeder: If you call the treasury, to get near the announcement, he's going to have to dress up as catering with a big tray of drinks and a pot of sliced lemons.

Abbot: I'm not doing that.

Coverly. I'm just going by procedure. 
Abbot: Terri. I love doing things the right way, that ethical stuff. I love it, we all do. But it's difficult when you're the first person to put your gun down because people jump on your head as if it's a ripe watermelon. We don't want that.

Reeder: The Prime Minister said he wants to do it. He's above the treasury in the hierarchy. I can write it down on a chart.

\section{Macht als zentrale Motivation}

Auffällig in The Thick of It ist die ausbleibende politische Gestaltung. Eine mögliche Erklärung hierfür bietet der mangelnde Wille der Protagonisten, gestaltend tätig zu werden. Ihr zentraler Antrieb lässt sich vielmehr im Streben nach Macht verorten. Nutzt man die Termini der Parteienforschung nach Kaare Strøm und Wolfgang Müller (1999: 5, 7), so ist der zentrale Antrieb der Akteure das officeseeking. Sie wollen in ein Amt kommen, im Amt bleiben und ihr Amt vor allem nicht verlieren. Das policy-seeking also der Wunsch der inhaltlichen Gestaltung beziehungsweise der Realisierung von bestimmten politischen Zielen steht dahinter zurück. Deutlich wird dies zum Beispiel dann, wenn Abbot als Reaktion auf negative Presseberichterstattung eine neue policy implementieren möchte. Seine Motivation gründet sich hierbei nicht in der Umsetzung eines bestimmten, gut ausgearbeiteten Inhaltes. Vielmehr ist die policy selbst nur Mittel zum Zweck der guten Publicity und letztendlich des Machterhalts (S 1/F 2/01.44 $03.46)^{3}$

\section{DATEN: KNOW YOUR PLACE!}

Im Folgenden wird nun die Serie selbst als Gegenstand betrachtet, der Auskunft über Politik, ihre Funktionsweisen und Arbeitsweisen geben kann. Es ist nicht das Ziel der Analyse, Aussagen über die Realitätstreue der Serie zu treffen.

\section{Organisationskultur des Departments of Social Affairs}

Das Department of Social Affairs ist der zentrale Schauplatz in The Thick of It. Hier beginnen und beenden die Protagonisten ihren Arbeitstag. Hier nehmen die meisten Verstrickungen ihren Anfang. Somit liegt es nahe, dass die Serie dem

3 An dieser Stelle sei auf die Analyse der Serie Borgen verwiesen, die den Machtaspekt in Kapitel „Narrative“ näher betrachtet. 
Zuschauer einen Blick hinter die Kulissen des Ministeriums ermöglicht. Es wird zu einem lebendigen und dynamischen Ort mit Abläufen, Routinen und Eigenheiten sowie spezifischen Beschäftigungsverhältnissen und Rollen der Akteure. Typisch satirisch und stereotypisch wird die Arbeitsweise der Verwaltung dargestellt.

Szenen-Beispiel: S 1/F1/22.49-23.02

Abbot betritt ein offensichtlich leeres Büro.

Abbot: Where are all the people? Where are my people?

Coverly: They've gone home, Secretary of State, it`s 5:40.

Diese Szene verweist auf die - vermeintlichen - Vorteile des Beamtentums. Die Beamten üben einen klassischen nine-to-five-job aus und kommen in den Genuss geregelter Arbeitszeiten. Zum anderen lässt sie sich jedoch auch als Kritik an eben jenen geregelten Abläufen verstehen. Mit einem Augenzwinkern bestätigt sie traditionelle Vorurteile gegenüber der öffentlichen Verwaltung: eine geringe Arbeitsmoral sowie eine von Langsamkeit geprägte Arbeitsweise. ${ }^{4}$ Des Weiteren finden auch die unterschiedlichen Befugnisse und das jeweilige Selbstverständnis der Beamten im Vergleich zu den politischen Beratern besondere Beachtung. Vor dem Hintergrund eines sich zuspitzenden Skandals in der dritten Folge und der Tatsache, dass Minister Abbot höchstwahrscheinlich zurücktreten wird, telefoniert Coverly mit einer dem Zuschauer unbekannten Person.

Szenen-Beispiel: S 1/F 3/16.30 - 16.43

Coverly: It 11 be fine in the end, we'll just get another doomed middle-aged man in on Monday morning, come through the door, stride about a bit, spunk off and I'll have to mop up the mess. It'll be business as usual.

An dieser Aussage wird sowohl die Rolle der Beamten als auch die Austauschbarkeit des Ministers deutlich. Als civil servant bleibt Coverly auch dann, wenn der Minister zurücktritt und durch einen neuen Minister ausgewechselt wird. Ihre

4 Weiterführend sind hierzu die Forschungsergebnisse von Dieter Grunow und Daniela Strüngmann von Interesse, die sich mit Bürokratiekritik in der öffentlichen und veröffentlichten Meinung beschäftigen. Allgemein bestehe in der Bürgermeinung eine Diskrepanz zwischen auf Stereotypen basierender negativer Bürokratiekritik und positiv bewerteten konkreten Erfahrungen. Die mediale Berichterstattung sei hierfür ein Erklärungsfaktor. Durch die Beförderung negativer Stereotype leiste sie einen Beitrag zu ihrer Verstetigung in der gesellschaftlichen Meinung (Grunow/Strüngmann 2008). 
Loyalität gilt dann dem nächsten „middle-aged man“. Nur die Vertrauten des Ministers, seine politischen Berater, werden mit ihm gehen müssen. Die Gleichgültigkeit, mit der Coverly über den bevorstehenden Rücktritt des Ministers spricht, verdeutlicht, dass es sich hierbei um ein übliches Vorgehen handelt. Immerhin beginnt auch für den Zuschauer die Serie mit der Abdankung des ehemaligen Sozialministers Lawton. Dass nicht alle Mitarbeiter mit derselben Gelassenheit der drohenden Entlassung Abbots entgegensehen können, wird anhand einer weiteren Szene deutlich.

Szenen-Beispiel: S 1/F 3/25.10 - 25.50

Die politischen Berater Glenn Cullen und Ollie Reeder unterhalten sich mit der Beamtin Terri Coverly. Sie erörtern die Möglichkeit, dass an Stelle des Ministers eine andere Person innerhalb des Ministeriums gehen muss.

Coverly: One of you got to decide. [...] Which of you will do the decent thing?

Cullen: We've been through this. Apparently, I'm Ronald fucking McDonald.

Reeder: Hang on, why are you not included in this? Why is it one of us two?

Coverly: If I've got to resign, then you've got to resign. [... I I answer to you on matters of policy.

Reeder: In which case, he's got to go, because I answer to him.

Cullen: So we've all got to go now, eh?

Coverly: Well, I am going nowhere. I didn't do anything, I did nothing. I am as clean as a whistle.

Cullen: Oh Terri, grow up! Don't give me that pile of fucking bollocks. Just because you're a civil servant, don't think that your shit doesn't smell. I know things about you.

So wird hieran neben der Ordnung innerhalb des Ministeriums auch das unterschiedliche Selbstverständnis Coverlys als civil servant im Vergleich zu Cullen und Reeder deutlich. Sie fühlt sich für den aktuellen Skandal nicht mitverantwortlich, da sie eben nicht für die Gestaltung der Politik, sondern nur für ihre Umsetzung und Vermarktung zuständig sei. Sie führe im Gegensatz zu Cullen und Reeder nur loyal aus, was von ihr verlangt werde, und sei deshalb für eventuelle Fehltritte nicht zur Verantwortung zu ziehen. Im Verlauf der Serie wird zudem die Inkompetenz des Ministers im Sinne eines mangelnden Fachwissens deutlich. Der folgende Dialog soll hierfür als Beispiel dienen.

Szenen-Beispiel: S 1/F 2/16.44-18.00

Abbot: How fucked am I? [...] In terms of negative publicity. On the fuckometer, where am I? 
Cullen: Oh, 12.

Reeder: Yeah. 12 say.

Abbot: Out of what?

Cullen: Er...50.

Reeder: Oh. Mine was out of ten. [...]

Cullen: Terri, have you got anything?

Coverly: I can't ask them to drop the piece, it would make us look pathetic.

Abbot: I don't mean to come across all Mr Gradgrind but this is your job isn't it, sorting out the press. This is what you do for a living.

Coverly: This is Malcolm's problem, anyway, it's him that spun that...

[Malcolm Tucker betritt das Büro]

Tucker: All right, this what we're gonna do. I'm bringing forward Hugh's interview with Heaney this afternoon.

Abbot weiß selbst nicht, was zu tun ist. Zum wiederholten Mal benötigt er die Hilfe und Ratschläge Tuckers. Diese Szene verdeutlicht die politische Unfähigkeit des Ministers sowie sein mangelndes inhaltliches Fachwissen. Er ist auf Berater angewiesen, um handlungsfähig zu bleiben. Auch wenn Abbots Inkompetenz deutlich überspitzt dargestellt wird, so knüpft sie doch an der Tatsache an, dass Minister generell ohne jegliche fachliche Qualifikation die Führung eines Ministeriums übernehmen (Hartmann 2011: 76).

\section{Hierarchie}

Neben den vorgestellten Einblicken in die Organisationskultur des Ministeriums ist Hierarchie das zweite Moment dieser Analyse. Die gesonderte Herausstellung erfolgt, da sich dieser Gesichtspunkt nicht nur auf die Organisation des Ministeriums beschränkt. Eine spezifische hierarchische Ordnung beziehungsweise ein hierarchisches Rollenbild ist erkennbar. So wird deutlich, dass der Premierminister der zentrale Entscheidungsträger ist. Er steht an der Spitze und mit seiner Zustimmung oder Ablehnung steht oder fällt eine Entscheidung. Während Abbot und Cullen noch selbstgefällig eine Radio-Berichterstattung über ihre neue Poli$c y$ hören, werden sie von einem aufgebrachten Tucker angerufen und zurechtgewiesen.

\section{Szenen-Beispiel: S 1/F 1/09.20 - 11.09}

Tucker: What the fuck was that? Was this Snooper Force thing from you?

Abbot: Malcolm, I talked to the PM, this is completely kosher as far as he's concerned. He gave the go-ahead and he said, you know, bounce the Treasury. 
Tucker: Don't you realise. We have got 17 different issues we are fighting with the Treasury about.

Abbot: I can hear that you are, as usual, upset...

Tucker: I'll tell you why I'm upset. I'm upset because these fucking morons at the Treasury, these people, they are so paranoid. If you don't tell them about this stuff, cc them on email, they think you started a palace coup. [...] You don't seem to understand that I $\mathrm{m}$ going to have to mop up a fucking hurricane of piss from all these neurotics. What did the Prime Minister actually say to you?

Abbot: He actually said: ,This is exactly the kind of thing we should be doing“.

Tucker: What did he actually say?

Abbot: He said: „This is exactly the sort of thing we should be doing“.

Tucker: Should be doing. Should does not mean yes. Now, there`s only one thing to do here and it's what I'm going to tell you to do. Kill it.

Als Folge der nichtexistenten Zustimmung des Premierministers muss sich der Minister somit von der policy distanzieren. Wie vorangehend beschrieben, hat der Premierminister in vielen Entscheidungen das letzte Wort. Tucker agiert hierbei stellvertretend in seinem Namen und steht in der Hierarchie somit direkt unter dem Premierminister und noch über Abbot. Das Wohlwollen des Premierministers scheint eine wichtige Währung im politischen Alltagsgeschäft zu sein. Doch auch innerhalb des Ministeriums gibt es entlang der unterschiedlichen Beschäftigungsverhältnisse eine klare, hierarchische Rollenverteilung. Sichtbar werden die Rollen besonders daran, wer wem formell untersteht und wer an welchen Treffen beteiligt sein darf. So wird in einer anderen Szene zunächst Reeder von einem Gespräch zwischen Cullen und Abbot ausgeschlossen, im nächsten Schritt dann wiederum Coverly von Cullen und letztendlich darf auch Cullen dem Gespräch zwischen Abbot und Tucker nicht mehr beiwohnen (S 1/F 1/19.14 - 20.05). Im Kontrast zu dem sonst vorherrschenden rauen und vulgären Ton gelten starre Hierarchiestrukturen und es ist ein deutliches Machtgefälle sichtbar, das das Handeln der Protagonisten bestimmt.

\section{NARRATION: POLITIK ALS SKANDAL}

Die Narration der Serie, also ihre stilistische Konstruktion und Erzählstruktur, ist minimalistisch gehalten. Das klassische Stilmittel der Wiederholung steht im Vordergrund. Ob Szeneninhalt, -ort oder -ablauf, vieles ist repetitiv, und das nicht ohne Grund. Der Zuschauer sieht die Akteure kontinuierlich in derselben 
Umgebung dieselben Fehler machen. Dadurch wird die inhaltliche Botschaft narrativ unterstützt: Die politischen Akteure sind unkreativ und unfähig.

\section{Skandalös: ein immer gleicher Folgenablauf}

Die einzelnen Folgen der ersten Staffel sind strukturell sehr ähnlich aufgebaut und erinnern an die politische Skandaluhr nach Christoph Bieber, Constantin Härthe und Caja Thimm (2015) in Anlehnung an Steffen Burkhardt (2006). Diese Uhr bildet verschiedene Phasen eines Skandals ab, die der Reihe nach durchlaufen werden. Inhaltlich bedeutet dies, dass in jeder Folge ein mehr oder weniger hochkarätiger politischer Skandal im Fokus steht. Ein dilettantisch erarbeiteter Vorschlag oder ein brisantes Regierungsdetail wird durch die Medien zum Skandal aufgewertet, der, wenn er nicht gleich personelle Konsequenzen fordert, zumindest für den Zuschauer höchste Unterhaltung bietet.

Exemplarisch ist der sogenannte flatgate scandal der dritten Folge der ersten Staffel zu nennen, der geradlinig der Skandaluhrstruktur folgt. Der Skandal beginnt mit der sogenannten Latenzphase, die eine inhaltliche Einführung in das Problem umfasst: Der Sozialminister Abbot besitzt neben seinem Familienwohnhaus noch eine städtische Zweitwohnung. Sie ist faktisch unbewohnt, weil Abbot berufsbedingt selten dazu kommt, hier zu übernachten. Dies jedoch steht im Widerspruch zum Housing Bill der Regierung, einem Wohnraumgesetz zur Entlastung des Londoner Wohnungsmarktes, welches das Leerstehen von Wohnraum unterbinden soll. Um kein Aufsehen zu erregen, einigen sich Abbot und sein engster Berater Cullen darauf, dass die Wohnung offiziell zum Verkauf ausgeschrieben wird, tatsächlich jedoch niemals verkauft werden soll.

In der nachfolgenden Aufschwungphase nimmt das Problem an Fahrt auf. Abbots Vorgesetzter Tucker erfährt, dass die Medien einen Bericht über das Thema planen. Ungünstigerweise befindet sich der Sozialminister zeitgleich bereits in einem Interview mit Heaney vom Standard. Er eilt also zum Interviewort und weist den Minister im indirekten Beisein der Medienvertreterin sprachlich unverhohlen auf sein Fehlverhalten hin. Dies ist der Übergang in die Etablierungsphase, da der Skandal nun die Interna der Politikarena verlässt und als Faktum in das Wissen der Presse übergeht.

Die Skandalklimax lässt nicht lange auf sich warten: Während sich alle Beteiligten noch gegenseitig die Schuld in die Schuhe schieben, arrangiert Tucker den Verkauf der Wohnung deutlich unter Marktwert. Leider kommt sein Schachzug zu spät, denn die Presse hat das Thema bereits flächendeckend aufgegriffen. Der Skandal befindet sich auf seinem Höhepunkt, als die Entlassung Abbots im Raum steht. Kurz darauf wird allerdings auch schon die Abschwungphase des Skandals eingeläutet, als klar wird, dass Abbots direkte Entlassung 
durch Tucker verhindert werden konnte. Jedoch muss ein Kopf über den Skandal rollen. Der vorher hochgelobte und am Skandal unbeteiligte Kollege Dan Miller muss seinen Rücktritt bekannt geben. Damit beginnt die Rehabilitationsphase des Skandals, der politische Alltag beginnt von Neuem.

Die Skandaluhr wird einerseits als repetitives Stilmittel genutzt, um die Politik in der Serie eintönig, kreativ- und erfolglos erscheinen zu lassen. Die Skandaluhr kann aber andererseits auch als ein dramaturgisches Instrument verstanden werden, das den Inhalt auf einen Höhepunkt hin ausrichtet und dadurch einen Spannungsbogen für den Zuschauer erzeugt (Hickethier 1996: 92). Das Element der Wiederholung zeigt sich auch zu Beginn und zum Schluss einer jeden Folge - zumindest in der ersten Staffel. Die Folgen beginnen immer ähnlich, in etwa mit dem Minister, der morgens das Ministerium betritt und auf dem Weg in sein Büro von seinen Mitarbeitern gebrieft wird. Und sie enden auch stets im Ministerium, das am Tagesende verlassen wird, weil dort spät noch gearbeitet oder gar übernachtet wird. Somit wird der inhaltliche Fokus auf das Ministerium auch mit szenischen Elementen unterstützt.

\section{Thema Überarbeitung erregt Spott und Empathie}

Häufig thematisiert wird der Faktor ,Stress und Arbeitsbelastung'. Wie der beschriebene Skandal aufzeigt, befinden sich die Akteure unter dauerhafter öffentlicher Beobachtung. Dies resultiert in mangelnder Erholung und erhöhter Fehleranfälligkeit, wodurch die Handelnden immer weniger intelligente Entscheidungen treffen. Einerseits kann dies spöttisch bewertet werden, da die politischen Akteure nicht dazu befähigt sind, einfach ihren Job zu machen. Andererseits wird der Zuschauer zum Nachdenken angeregt, ob die Akteure nicht doch wahrhaftig überarbeitet sind und hier ein Missverhältnis im Politiksystem vorliegt.

Zum Thema Stress gesellt sich der Faktor Zeitdruck. Kaum ein politischer Inhalt kann in Ruhe erarbeitet werden. Was die Serie aufzeigt, beschreibt Korte (2012) verkürzt gesagt als das Paradox der digitalen Beschleunigung in Zeiten einer entschleunigten Politiklogik. Gemeint ist der Zwist zwischen den Medien, die stets unmittelbare Reaktionen verlangen, und der Politik, die Zeit für Absprache und Abstimmung benötigt. Szenisch wird der Zeitdruck dadurch aufgegriffen, dass politische Inhalte zum Beispiel im Auto erarbeitet werden. Dennoch lassen die Macher im Unklaren, ob tatsächliche Überforderung oder reines Unvermögen der Akteure hier der ausschlaggebende Faktor ist. 


\section{Humor durch Kraftausdrücke}

Wer britische Unterhaltungssendungen schätzt, kennt das gern genutzte Mittel des Sarkasmus und beißenden Spotts. The Thick of It treibt es mit der Verwendung von Kraftausdrücken und persönlichen Beleidigungen auf die Spitze. Nicht umsonst gilt für die Serie eine Altersfreigabe ab 18. Der schroffe Ton fügt sich nahtlos in das raue politische Tagesgeschäft und sorgt an vielen Stellen für allgemeine Belustigung oder Entrüstung. Das Stilmittel Humor wird neben dem offenkundigen Unterhaltungsfaktor im Sinne von Michael Hellenthal (1989: 33) zusätzlich aber auch dazu verwendet, um auf existierende Missstände hinzuweisen und Kritik an der britischen Politikpraxis zu üben.

\section{Sick because of it: Ton, Bild und Kameraführung so realitätsgetreu wie möglich}

Auffälligstes technisches Merkmal der Serie ist die spezielle ,mitten drin“Kameraführung. Die Aufnahmen werden hauptsächlich ohne Stativ gemacht und sind daher bewusst wackelig, mit vielen Bewegungen und Richtungswechseln. Einem Guardian-Artikel zufolge soll Zuschauern von der hektischen Kameraführung sogar schlecht geworden sein (The Guardian Online 2012b). Gegen die flüchtige Kameraführung kontrastiert eine fast gänzlich gleiche Perspektive, nämlich die einer beobachtenden dritten Person. Als Zuschauer fühlt man sich so immer als Teil der Szene, da die Kameraperspektive die eigene Augenhöhe reflektiert. ${ }^{5}$ Die Materialqualität hat passend dazu den Anschein einer Dokumentation: teilweise unscharfe, unter- beziehungsweise überbelichtete Bilder, unbearbeitete Farben und nicht standardisierter Ton. Es soll real wirken, und das gelingt den Machern auch.

\section{Bei der Szenerie ist weniger mehr}

Zur puristischen Bildgewinnung gesellt sich das Stilmittel der begrenzten Szenerie. Die Folgen der ersten Staffel spielen mit wenigen Ausnahmen im Ministerium, im Auto des Ministers oder in Tuckers Büro. Das jeweilige Mobiliar passt sich dem szenischen Inhalt an. Das unbeliebte Ministerium ist sporadisch und veraltet eingerichtet, das Büro von Tucker hingegen elegant und edel ausgestattet. Auch die Anzahl der Schauspieler ist überschaubar. Neben dem Hauptkon-

5 Auf das Stilmittel der Zuschauereinbindung und dessen direkte Ansprache wird ebenfalls in Kapitel „Narrativ“ der Analyse der Serie House of Cards eingegangen. 
flikt Politik versus Medien existieren nur wenige Nebenschauplätze, wie die Rivalität der zwei politischen Berater Reeder und Cullen. Alle narrativen Serienelemente sind also darauf ausgerichtet, den Hauptkonflikt auf simple, kritischhumoristische Weise nachzuzeichnen.

\section{FAZIT: WENN HANDLUNGSFÄHIGKEIT DEM MACHTSTREBEN ZUM OPFER FÄLLT}

Wie ausführlich erörtert, finden Theorien, die sich mit der Verschränkung von Politik und Medien beschäftigen, ihre Bestätigung in der Serie. Trotz der bestehenden wechselseitigen Abhängigkeit nehmen die Medien in The Thick of It hauptsächlich die Rolle des Antagonisten ein. Sie beschränken oder verhindern die Handlungsmöglichkeiten der Politik und begrenzen in diesem Sinne deren Gestaltungsfähigkeit. Diese Feststellung fügt sich in das negative und pessimistische Bild der Politik ein, das sich beim Zuschauer einprägt, wenn er am Ende der letzten Folge gemeinsam mit den Protagonisten das Department of Social Affairs verlässt. Gestaltet wurde wenig, gescheitert dafür umso mehr. Wenn eins bewiesen wurde, dann ist es die Unfähigkeit der politisch Handelnden. Auch im Ansatz sinnvolle Policies missglücken letztendlich in der Phase der Implementation und fallen internen Machtkämpfen oder medialen Skandalen zum Opfer. Im Spiel der Medien und wirklich mächtigen Akteure ist Minister Hugh Abbot nicht viel mehr als eine Marionette. Ebenso wie sein Vorgänger Cliff Lawton hängt er am Faden des omnipräsenten Puppenspielers in Gestalt des Premierministers. Bei Bedarf werden diese Fäden durch den Spindoktor und Vertrauten des Premierministers Malcolm Tucker durchgeschnitten. Inhalte und Wertvorstellungen sind fluide Gebilde und bloß Mittel zum Zweck des Machterwerbs oder Machterhalts. Im Einklang mit Andreas Dörner (2006), der drei mögliche Bilder des Politischen identifiziert, die von politischen Serien vermittelt werden können, wird Politik in The Thick of It somit als Machtpolitik dargestellt. Macht wird eben nicht als Mittel zur Umsetzung von politischen Inhalten verstanden, sondern ist vielmehr selbst Zweck des politischen Handelns. Die politischen Akteure streben nach der Festigung und Verbesserung der eigenen Position (Dörner 2016: 9).

\section{Ende der ersten Staffel The Thick of It}

Abbot: Actually, can I stay on your sofa tonight?

Cullen: Yep.

Abbot Thanks. Take your time. I'll wait for you, then. 


\section{LITERATUR}

BBC Online (2007): The Thick of It, in: http://www.bbc.co.uk/programmes/ b006qgrd (zugegriffen am 26.2.2017).

Bieber, Christoph/Härthe, Constantin/Thimm, Caja (2015): Erregungs-kampagnen in Politik und Wirtschaft. Digitale Öffentlichkeit zwischen Candy- und Shitstorms, in: Bonner Akademie für Forschung und Lehre Praktischer Politik, http://www.bapp-bonn.de/medien/ downloads/publikation_forschungsergebnis se/erregungskampagnen_in_politik_und_wirtschaft-final.pdf (zugegriffen am 10.03.2017).

Burkhardt, Steffen (2006): Medienskandale. Zur moralischen Sprengkraft öffentlicher Diskurse, Köln.

Donges, Patrick/Jarren, Ottfried (2011): Politische Kommunikation in der Mediengesellschaft. Eine Einführung, 3. Aufl., Wiesbaden.

Dörner, Andreas (2016): Politserien: Unterhaltsame Blicke auf die Hinterbühne der Politik, in: Aus Politik und Zeitgeschichte (51), S. 4-11.

Grunden, Timo (2009): Politikberatung im Innenhof der Macht. Zu Einfluss und Funktion der persönlichen Berater deutscher Ministerpräsidenten, Wiesbaden.

Grunow, Dieter/Strüngmann, Daniela (2008): Bürokratiekritik in der öffentlichen und veröffentlichten Meinung: Impulse für Verwaltungsreformen?, in: Der moderne Staat (1), S. 121-140.

Hartmann, Jürgen (2011): Westliche Regierungssysteme. Parlamentarismus, präsidentielles und semi-präsidentielles Regierungssystem, 3. Aufl., Wiesbaden.

Hellenthal, Michael (1989): Schwarzer Humor. Theorie und Definition, Essen.

Hickethier, Knut (1996): Film- und Fernsehanalyse, 2. Aufl., Stuttgart/Weimar, S. 42-155.

Korte, Karl-Rudolf (2001): Was kennzeichnet modernes Regieren? Regierungshandeln von Staats- und Regierungschefs im Vergleich, in: Aus Politik und Zeitgeschichte (5), S. 3-13.

Korte, Karl-Rudolf (2003): Maklermacht. Der personelle Faktor im Entscheidungsprozess von Spitzenakteuren, in: Hirscher, Gerhard (Hrsg.): Information und Entscheidung: Kommunikationsmanagement der politischen Führung, Wiesbaden, S. 15-28.

Korte, Karl-Rudof (2009): Handeln im Vermittlungsdreieck: Steuerung, Kommunikation, Macht, in: Korte, Karl-Rudolf/Fröhlich, Manuel: Politik und Regieren in Deutschland: Strukturen, Prozesse, Entscheidungen, 3. Aufl., Paderborn, S. 173-350. 
Korte, Karl-Rudolf (2012): Beschleunigte Demokratie: Entscheidungsstress als Regelfall, in: Aus Politik und Zeitgeschichte (7), S. 21-26.

Marcinkowski, Frank/Pfetsch, Barbara (2009): Politik in der Mediendemokratie, Wiesbaden.

Rüb, Friedbert W. (2014): Informelles Regieren - oder: Vergeblicher Versuch, die Farbe eines Chamäleons zu bestimmen, in: Bröchler, Stephan/Grunden, Timo (Hrsg.): Informelle Politik. Konzepte, Akteure und Prozesse, Wiesbaden, S. 51-80.

Sturm, Roland (2009): Politik in Großbritannien, Wiesbaden.

Strøm, Kaare/Müller, Wolfgang C. (1999): Political Parties and Hard Choices, in: Müller Wolfgang C./Strøm, Kaare (Hrsg.): Policy, Office, Or Votes? How Political Parties in Western Europe Make Hard Decisions, Cambridge, S. 1-35.

The Guardian Online (2009): Peter Capaldi: Malcolm Tucker is Alastair Campbell. But Mandelson is in there, too, in: https://www.theguardian.com/tv-andradio/2009/oct/17/peter-capaldi-malcolm-tucker-tv (zugegriffen am 26.2.2017).

The Guardian Online (2012a): The Thick of It: ,it feels more like politicians copy us', in: https://www.theguardian.com/media/2012/aug/22/the-thick-ofit-politicians (zugegriffen am 27.2.2017).

The Guardian Online (2012b): The Thick of It: good news, minister, the show is over, in: https://www.theguardian.com/tv-and-radio/2012/oct/25/thick-of-itbbc-political-satire-ends (zugegriffen am 6.3.2017).

The Independent (2014): Don't let Malcolm Tucker be your Big Brother, in: http://www.independent.co.uk/voices/comment/dont-let-malcolm-tucker-beyour-big-brother-9039083.html (zugegriffen am 26.1.2017). 


\section{Spiel der Kräfte}

\section{Politik, Medien und Familie in Borgen}

Johannes Bongardt, Rieke Gießelmann, Matthias Jüschke, Jan Pfeifer, Christina-Johanne Schröder, Jonas Seyferth

\section{BORGEN: ,THE BEST TELEVISION SHOW ABOUT POLITICS EVER“1}

Der Reiz der Serien Borgen für die Politikwissenschaft findet sich nicht nur in der Betrachtung unterschiedlicher politischer Arenen, sondern auch darin, dass die Serie, beispielsweise mit dem Thema Minderheitsregierungen, präzise eine Besonderheit des politischen Systems aufgreift. Seit 1909 konnte keine einzelne Partei im Folketing, dem dänischen Parlament, eine absolute Mehrheit erringen. Es hat sich eine Tradition von Minderheitsregierungen etabliert, die seit 1982 ausschließlich aus Koalitionen bestehen. Daraus hat sich eine politische Kultur entwickelt, die auf Verhandlungen, Konsens und Kompromissen zwischen politischen Akteuren basiert. Das Schloss Christiansborg, im Volksmund Borgen genannt, ist nicht nur Sitz des Folketing, sondern vereint die Spitzen von Exekutive, Legislative und Judikative unter einem Dach; auch die Königin hat Empfangsräume in Christiansborg. Dieses Zentrum der Macht ist nicht nur namensgebend für die Serie, sondern auch zentraler Schauplatz des Zusammenspiels zwischen Akteuren unterschiedlicher Parteien, des Parlaments und der Regierung. Dabei thematisiert Borgen die politischen Abläufe auf der Hinterbühne der Politik.

„Borgen ist ein Lehrstück in politischer und journalistischer Rhetorik. Die Serie vermittelt, was bei Raab, Jauch oder Maischberger zwischen den Zeilen der Moderationskarten steht

1 Dyson 2014. 
und Steinbrück, Merkel oder Özdemir nur vor ausgeschalteten Kameras sagen würden. Sie schärft den Blick für das, was dem zwar wachen, aber doch nur beobachtenden Bürger vorenthalten werden soll.“ (Weihser 2012)

Die Serie beginnt in der heißen Phase des dänischen Wahlkampfes. Birgitte Nyborg, die Hauptfigur der Serie, ist Parteivorsitzende der Moderaten und wird überraschend zur ersten dänischen Ministerpräsidentin ${ }^{2}$ gewählt. In ihrer neuen Rolle als Ministerpräsidentin muss Nyborg ihre Macht behaupten und wird dabei mit Intrigen und der Härte von Realpolitik konfrontiert. Gleichzeitig macht die Serie deutlich, wie schwierig es ist, Familie und Beruf zu vereinen. Quasi jeder der handelnden Akteure muss sich zwischen Karriere und Privatleben entscheiden. Darüber hinaus kommt dem Einfluss der Medien als vierte Gewalt eine zentrale Rolle in Borgen zu. Die Serie beleuchtet das Verhältnis zwischen Medien und Politik und die diesem zugrunde liegenden Mechanismen (Riepler 2013).

Borgen - Gefährliche Seilschaften (Originaltitel: Borgen) wurde von Denmarks Radio $(D R)$, der öffentlich-rechtlichen Sendeanstalt Dänemarks, produziert und von 2010 bis 2013 auf DRl ausgestrahlt (Wirries 2014: 63). Die deutsche Erstausstrahlung erfolgte 2012 auf ARTE. Die Serie wurde mittlerweile in über 70 Ländern gesendet und gilt als eine der erfolgreichsten dänischen Fernsehproduktionen (Teuwsen 2013). Als ein Grund für den großen Erfolg wird unter anderem genannt, dass die Serie dramaturgische Parallelen zum skandinavischen Exportschlager, dem Krimi-Genre, aufweist (Hurard 2015).

\section{KONTEXT UND RAHMENBEDINGUNGEN: BORGEN UND SEINE VORBILDER}

In der Serie werden aktuelle Themen wie beispielsweise in der ersten Staffel die Bedeutung von Pressefreiheit, die Einführung einer Frauenquote oder Beziehungen zu autokratischen Ländern behandelt (Jessen 2015). Neben dem zeithistorischen Kontext haben die thematisierten Politikinhalte auch die Eigenschaft, dass sie theoretisch auf viele Länder übertragbar sind. Diese Allgemeingültigkeit ist neben der Aktualität der Themen ein wesentlicher Faktor für den weltweiten Erfolg der Serie (Hurard 2015). Während der Produktion der Serie gab es eine Zu-

2 Die Amtsbezeichnung des Regierungschefs ist in der deutschen Übersetzung nicht einheitlich. In der Serie wird der Begriff des Premierministers verwendet und entsprechend in den Szenen-Beispielen zitiert. Da die Bezeichnung des Ministerpräsidenten jedoch in der Fachliteratur geläufiger ist, wird im Fließtext dieser Begriff verwendet. 
sammenarbeit zwischen Produzenten und verschiedenen Experten aus der Politik, um die Realität so gut wie möglich abzubilden (Helmes 2014).

Auch für die Figur von Birgitte Nyborg ist eine Inspiration aus der realen dänischen Politik naheliegend. Mit Helle Thorning-Schmidt und Margrethe Vestager gibt es gleich zwei Politikerinnen, die Ähnlichkeiten zu der fiktiven Ministerpräsidentin aufweisen. Thorning-Schmidt war nicht nur von 2005 bis 2015 Vorsitzende der dänischen Sozialdemokraten, sondern auch von 2011 bis 2015 die erste Ministerpräsidentin Dänemarks. Die zweite Politikerin, Vestager, hatte von 2007 bis 2014 den Vorsitz der sozialliberalen Partei Det Radikale Venstre inne, war unter anderem Wirtschaftsministerin, Innenministerin und Bildungsministerin und ist seit 2014 EU-Kommissarin für Wettbewerb.

Neben der Inspiration aus der Realität gibt es ebenso Beispiele dafür, wie die Serie die Politik Dänemarks beeinflusst hat. So wurde die Serie von verschiedenen Akteuren zum Agenda-Setting benutzt (Jessen 2015). Die Opposition startete beispielsweise eine Initiative zu dem Thema Prostitutionsverbot nach genau dem Sonntag, an dem eine Serienfolge ausgestrahlt worden war, in der die fiktive Ministerpräsidentin Birgitte Nyborg dieses Thema aufgriffen hatte. Die heutige Sozialministerin von Det Konservative Folkeparti, Mai Mercardo, gab damals den politikstrategischen Schachzug unverblümt zu. Eine populäre TVMinisterpräsidentin, die dieselbe Meinung wie eine konservative Oppositionsführerin vertrete, helfe bei der Durchsetzung der eigenen Agenda (Borchert 2013). Generell können Agenda-Setting-Effekte erzielt werden, indem Serien Aufmerksamkeit für ein Thema erzeugen (Dörner 2016: 11, Holbrook/Hill 2005). Der hier geschilderte Vorgang spitzt dies sogar noch zu, indem reale politische Akteure diesen Effekt der Serie antizipieren und instrumentalisieren.

\section{Dänemark: eine konstitutionelle Monarchie}

Die heute noch geltenden Prinzipien des parlamentarischen Systems Dänemarks entstammen der Verfassung von 1849. Das Ende des Absolutismus wurde mit der Implementierung des freien Wahlrechts, grundlegenden Menschenrechten und einem parlamentarischen Zweikammersystem vollzogen. Grundsätzlich kann die Verfassung von Dänemark nur durch Volksabstimmung geändert werden. Obligatorische und fakultative Referenden wurden mit der Verfassungsreform von 1953 eingeführt (Jochem 2012: 69-70, Nannestad 1999: 88-90).

Borgen greift zu Beginn der Serie die Rolle der Monarchie auf. Königin Margarethe lässt die sichtlich nervöse Birgitte Nyborg eine Zigarettenlänge warten, bis sie den Auftrag zur Regierungsbildung erteilt (S 1/F 2/1.06 - 3.00). Üblicherweise äußert sich die Königin nicht zum politischen Alltagsgeschehen und bewahrt politische Neutralität. Jedoch unterschreibt sie sämtliche Gesetze, was 
in der Praxis zu häufigen Treffen zwischen ihr und den Ministern führt. Ferner verfügt die Königin über ein gesetzliches Initiativrecht, das sie sich mit den Parlamentariern teilt. Für den Fall, dass sich die Parlamentarier nicht auf eine Regierungsbildung einigen können, schlägt die Königin eine Koalition vor, die regierungsfähig ist, ohne sofort mit einem Misstrauensvotum konfrontiert zu werden. Die Königin ist gleichzeitig Oberbefehlshaberin über das Militär, auch wenn dies in der Praxis keine Anwendung findet (Förster/Schmid/Trick 2014: 29, Ismayr 1999: 18).

Die Verfassung des Landes gilt auch in Grönland und auf den Färöern - beides sind dänische Autonomiegebiete. Seit 2009 verfügt Grönland über einen umfassenden Autonomiestatus. Vier Abgeordnete repräsentieren die Autonomiegebiete im Folketing (Findeisen 1999: 233, Lykketoft 2014: 5). Borgen thematisiert das Verhältnis zwischen der dänischen Regierung und dem Autonomiegebiet in der vierten Folge der ersten Staffel. Soziale Probleme, geringe Bildung und Suizide werden von Birgitte Nyborg anlässlich eines offiziellen Besuchs beim grönländischen Premierminister angesprochen. Dieser beklagt seinerseits, bei militärischen Entscheidungen auf der grönländischen Thule Airbase nicht beteiligt zu sein. Denn Angelegenheiten der Außen- und Sicherheitspolitik, Staatsbürgerschaft und Fragen der Währungsangelegenheiten obliegen nicht der grönländischen autonomen Regierung (Jochem 2012: 20). In Dänemark leben außerdem eine deutsche Minderheit in Nordschleswig und eine dänische Minderheit in Südschleswig, die beide in den jeweiligen Ländern Minderheitenschutzrechte genießen und dadurch beispielsweise von der Sperrklausel bei Parlamentswahlen befreit sind (Frandsen 1994: 191-194).

\section{Regieren in Dänemark: Wer kann (nicht) bis 90 zählen?}

Über das aktive und das passive Wahlrecht verfügen alle Dänen ab dem 18. Lebensjahr. Auf kommunaler Eben gilt dieses Recht auch für Drittstaatsangehörige, die sich seit drei Jahren legal im Land aufhalten. Das Folketing konstituiert sich aus 179 Parlamentariern. 175 von ihnen werden über das Verhältniswahlrecht gewählt, während je zwei Sitze den Autonomiegebieten vorbehalten sind (Förster/Schmid/Trick 2014: 27-30, Jochem 2012: 69-74, Lykketoft 2014: 5).

Seit der Einführung des parlamentarischen Systems ist die dänische Politik durch den negativen Parlamentarismus geprägt (Bergman 1993). Die Regierung besteht solange, bis ihr das Misstrauen durch eine Gegenmehrheit im Parlament ausgesprochen wird oder reguläre Neuwahlen stattfinden. Enthaltungen oder Abwesenheiten einzelner Parlamentarier genügen, solange keine Mehrheit gegen die Regierung stimmt. Zur Regierungsbildung muss demnach keine aktive Mehrheit vorliegen. Diese Regelung ermöglicht die lange Tradition von Minder- 
heitsregierungen mit der Aufgabe, Verhandlungen, Konsens und Kompromisse zu erzielen, da die Regierung Dänemarks gezwungen ist, mit Oppositionsparteien zusammenzuarbeiten, um Gesetzesvorschläge durchzubringen und Ämter zu erhalten (Förster/Schmid/Trick 2014: 30-32, Lykketoft 2014: 7). Es ist demnach weniger wichtig, dass die Regierung selbst die absolute Mehrheit der Parlamentarier hinter der eigenen Position versammeln kann. Viel wichtiger ist, dass sich keine Mehrheit von 90 Abgeordneten des Folketings gegen die Regierung formiert. In der Tradition der Minderheitsregierung verfolgen insbesondere die tolerierenden Oppositionsparteien keine office-seeking-Strategien bei der Regierungsbildung, sondern verhandeln rein auf der policy-Ebene um Inhalte ihres Parteiprogramms oder Ziele einzelner Wahlkreise. Auf diesen Normen fußen die vielschichtigen narrativen Linien in der Serie Borgen, die sich dem Rezipienten in Verhandlungen innerhalb der Regierung, aber auch mit einzelnen Parlamentariern offenbaren. In Minderheitsregierungen ist die Fraktionsdisziplin von großer Bedeutung, hat doch der einzelne Abgeordnete die Macht, eine Regierungsmehrheit zu gefährden (Jensen 1991: 114-115). In der Serie wird dieses Strukturmerkmal anhand der Abstimmung über den Staatshaushalt demonstriert (S 1/F 3/19.04 - 22.45).

Die Regierung konstituiert sich aus dem Regierungschef und seinen Ministern. Die Ressorts und ihr Zuschnitt werden vom Regierungschef bestimmt, sind faktisch aber Teil der Verhandlungsmasse bei der Regierungsbildung. Üblich sind zusätzlich zur Staatskanzlei um die 20 Ministerien. In der Regel verbleiben Mitglieder des Kabinettes zugleich im Parlament (Förster/Schmid/Trick 2014: 32). Die Ministerien sind in flacher Hierarchie in verschiedene Fachdezernate organisiert. Die in den Ministerien beschäftigten Beamten sind politisch neutral und verbleiben nach einem Regierungswechsel in ihrer Position (Nannestad 1999: 79-80). Borgen greift die Rolle des neutralen Beamten mit der Figur von Niels Erik Lund auf, der unter mehreren Ministerpräsidenten als Staatssekretär in der Staatskanzlei arbeitet.

\section{Parteien und politische Entwicklung}

In der Nachkriegszeit begannen sich die Milieus der dänischen Gesellschaft verstärkt auszudifferenzieren, was sich auch im politischen Parteiensystem widerspiegelte. Aus einem Vierparteiensystem aus Det Konservative Folkeparti, Socialdemokraterne, Venstre und Det Radikale Venstre wurde 1960 durch die Gründung der grün-linken Socialistisk Folkeparti ein Fünfparteiensystem. Studentenbewegung, Proteste gegen innenpolitische Entscheidungen und der Beitritt zur EG führten 1973 zur Neuordnung der Parteienstruktur. Bei den sogenannten Erdrutschwahlen konnten die fünf etablierten Parteien nur Zweidrittel des Parla- 
ments besetzen. Die Sperrklausel von zwei Prozent begünstigt den Einzug neuer politischer Parteien ins Parlament. Diese fortschreitende Fragmentierung des Parlaments brachte vergleichsweise kurzlebige Koalitionsregierungen in den 1970er Jahren hervor (Jochem 2012: 72, Nannestad 1999: 70). Die zunehmende Etablierung von Minderheitsregierungen in Mehrparteienkoalitionen stärkt das Parlament gegenüber der Regierung (Förster/Schmid/Trick 2014: 66). Das dänische Parlament ist ein Arbeitsparlament mit umfassenden Mitwirkungsrechten in den Ausschüssen. Das macht Verhandlungen ,vielschichtiger, unkalkulierbarer und zahlreicher" (Jochem 2012: 74). Neben den fünf Parteien mit längerer Historie sind seit 2015 die Dansk Folkeparti, die Enhedslisten, die Liberal Alliance und die Alternativet im Parlament vertreten.

Die Parteien in Borgen sind stark an den realen Vorbildern orientiert. Venstre wurde 1870 gegründet und ist damit die älteste Partei Dänemarks. Ihre Grundwerte sind konservativ liberal ausgerichtet: Freiheit, Verantwortung und freier Wettbewerb. In der Serie heißen sie De Liberale. Die Socialdemokraterne, Serienname Arbejderpartiet, ist die zweite Traditionspartei. Sie ist eng mit den Gewerkschaften verbunden und treibende Kraft bei der Gestaltung des dänischen Wohlfahrtstaates. Vorbild für Birgitte Nyborgs Moderate ist Det Radikale Venstre. Die zu Beginn des 20. Jahrhunderts gegründete Partei übt den Balanceakt zwischen sozialer Gerechtigkeit und freiem Handel und tritt für den Umweltschutz ein. Det Konservative Folkeparti, in der Serie Neue Konservative, ist konservativ liberal ausgerichtet und stützt seit 2015 die Regierung gemeinsam mit der rechtspopulistischen Dansk Folkeparti und der Liberal Alliance. Eine weitere Partei ist die seit 1960 im dänischen Parlament vertretene Socialistisk Folkeparti. Mehrfach hat sie sozialdemokratische Regierungen gestützt und war von 2011 bis 2014 erstmals an der Regierung beteiligt. Sie steht Pate für die Milieupartei und ist dem grünen Parteienspektrum zuzurechnen. Die Enhedslisten sind seit 1994 im dänischen Parlament vertreten und entsprechen der Solidarischen Sammlung (Wirries 2014: 65).

\section{SPIEGEL: POLITIK ZWISCHEN ÖFFENTLICHER, PARLAMENTARISCHER UND ADMINISTRATIVER ARENA}

Borgen weist nicht nur Parallelen zur Wirklichkeit auf, sondern liefert auch zahllose Inhalte, in denen sich politikwissenschaftliche Theorien und Erkenntnisse widerspiegeln lassen. Im Sinne eines Spiegels lässt sich in der Serie anschaulich nachvollziehen, wie die Akteure in der öffentlichen, der administrativen sowie der parlamentarischen Arena des Politikmanagements agieren (Korte/Fröhlich 
2009: 230). Wie in den theoretischen Annahmen ${ }^{3}$ formuliert, sind sie dabei nur erfolgreich, wenn sie den Anforderungen aller drei Arenen gerecht werden. Dieses Kapitel beschränkt sich auf die Analyse der Darstellung folgender Aspekte: die Funktion der Staatskanzlei als Regierungszentrale und Ort der administrativen Arena, die Strukturmerkmale der Mediendemokratie sowie die Koalitionsverhandlungen als Beispiel für strategisches Regierungshandeln.

\section{Politikberatung durch die Regierungszentrale}

Die Staatskanzlei als Regierungszentrale hat die Funktion, die Arbeit der Regierung zu koordinieren und den Spitzenakteur zu beraten (Frohn 2011: 226). Den Beraterkreis um Birgitte Nyborg bilden Medienberater Kasper Juul, Finanzminister und Mentor Bent Sejrø sowie Staatssekretär Niels Erik Lund. Alle drei pflegen Netzwerke und Kanäle zu maßgeblichen Akteuren: Partei, Koalitionspartner, Ministerialbürokratie und Medien. Die Berater haben drei Hauptaufgaben: die policy-Akzentuierung, die stille Konfliktregulierung sowie die Selektion und Interpretation von Informationen (Grunden 2011: 265). Dabei ist letztere Funktion sowohl in der Realität als auch in Borgen besonders wichtig. Häufig kommen die Berater zusammen, um Informationen auszutauschen, und erst auf Grundlage dieser Informationen werden Entscheidungen getroffen.

Wenn das Informationsmanagementsystem eines Spitzenakteurs versagt, geraten die Politiker unter Druck, etwa der Verteidigungsminister in der vierten Folge: Er hat nicht alle Informationen über geheime Flüge der CIA über Grönland und kann deshalb keine erfolgreiche Kommunikationsstrategie aufstellen. In Folge 9 geht es um den Kauf von neuen Kampfflugzeugen. Nachdem Birgitte Nyborg das Vertrauen in die Informationsweitergabe des Verteidigungsministeriums und ihrer Mitarbeiter verloren hat, liest sie selbst die Akten. „Hast du ernsthaft vor, das alles zu lesen“, fragt ihr Ehemann Phillip Christensen. „Kann das kein anderer für dich lesen und dir das Wichtigste berichten?“ (S 1/F 9/9.57 - 11.30). Birgitte Nyborg lernt im Zuge dieser Episode, dass sie auf das Informationsmanagement ihrer Mitarbeiter vertrauen muss, da sie die Regierungsgeschäfte sonst nicht führen kann.

Die Staatskanzlei ist ein Ort der administrativen Arena, denn hier finden informelle Prozesse statt. Die Akteure treffen unter Ausschluss der Öffentlichkeit Entscheidungen (Szenen-Beispiele in S 1/F 5). Der Staatssekretär Niels Erik Lund ist ein besonders guter Kenner dieses informellen Politikmanagements,

3 Nähere Ausführungen zum Arenenmodell finden sich im Beitrag zu House of Cards im Kapitel „Spiegel“. 
überdauert er als politischer Beamter doch wechselnde Ministerpräsidenten. Folgende Zitate definieren sein Rollenbild:

Szenen-Beispiele:

Birgitte Nyborg über Lund, der ihren Vorschlag einer Frauenquote skeptisch betrachtet (S 1/F 5/2.25)

Nyborg: Staatssekretäre können sich nie für Veränderungen begeistern, es sind Beamte.

Niels Erik Lund nach Birgitte Nyborgs Rede zur Parlamentseröffnung (S 1/F 10/57.20)

Lund: Ich muss zugeben, ich hätte nicht damit gerechnet, dass Sie sich ein Jahr auf diesem Posten halten können. Aber wenn Sie so weitermachen, werden wir noch einige Legislaturperioden zusammenarbeiten.

Besonders letzteres Zitat offenbart das Selbst- und Standesbewusstsein des Beamten, das er aus seiner dauerhaften Anstellung bezieht. Das formale Hierarchiegefälle zwischen Ministerpräsidentin und Staatssekretär verflacht, wenn Lund gegenüber Nyborg als Berater und Organisator der Regierungsgeschäfte auftritt. Seine Figur offeriert damit beispielhaftes Anschauungsmaterial zu internen sowie informellen Prozessen der Politikberatung.

\section{Mediendemokratie}

Die Beziehung von Medien und Politik ist in Borgen von wechselseitiger Abhängigkeit geprägt. Die Medien stehen unter Druck, gute Quoten oder Verkaufszahlen zu erzielen. Dafür ist es entscheidend, als Erster politische Skandale aufzudecken oder die Politiker als Interviewpartner für das eigene Medium zu gewinnen. Den Politikern wiederum ist bewusst, dass Kommunikation für die Legitimation von demokratischer Politik konstitutiv ist (Sarcinelli 2013: 93). Diese Kommunikation vollzieht sich vor allem durch das Leitmedium Fernsehen. Der Fokus der Mediendarstellung liegt auf dem öffentlich-rechtlichen Sender TVI. Die Darstellung von Medien und Politik in Borgen spiegelt dabei veraltete Rollenmuster. Weder das Internet noch soziale Medien spielen eine Rolle. Dadurch kommunizieren Politiker nicht direkt mit der Öffentlichkeit, sondern nur über die Medien. Dazu werden klassische Kanäle wie Pressekonferenzen und Interviews genutzt oder Informationen informell weitergegeben. Sarcinelli (2009: 119) beschreibt das Verhältnis von Medien und Politik als eine Tauschbeziehung: „In- 
formation gegen Publizität - und umgekehrt “4 . Diese Beschreibung wird in Borgen bestätigt. „Der Informationsaustausch wie der Informationsbedarf sowohl in als auch zwischen diesen beiden Bereichen scheinen unermesslich zu sein", so Wirries (2014: 62). Zwar werden Medien und Politik als getrennte Arenen dargestellt. Doch enge persönliche Verbindungen zwischen den Akteuren machen die geradezu ,symbiotische Beziehung beider Arenen“ (Wirries 2014: 62) deutlich. Auch reibungslose Wechsel von Akteuren aus der Politik in den Journalismus oder aus den Medien in die Politik, wie Michael Laugesen, Kaper Juul oder Katrine Fønsmark sie vollziehen, verdeutlichen dieses Prinzip.

Das Verhältnis von Medien und Politik ist von Professionalisierung und Kooperation gekennzeichnet. Verstoßen die Medien gegen die Regel, sich an Absprachen zu halten, geraten Politiker unter Druck, wie eine andere Szene aus der ersten Folge zeigt. Es ist Wahlkampf und die Moderatorin Katrine Fønsmark führt ein Interview mit Birgitte Nyborg (S 1/F 1/3.10 - 6.05). Unangekündigt schaltet die Redaktion Michael Laugesen von der Arbeiterpartei hinzu, der einen Kurswechsel in der Asylpolitik verkündet. Diese nicht abgesprochene Aktion setzt Birgitte Nyborg unter Druck. Nach der Sendung fährt sie Katrine Fønsmark an, dass deren Verhalten nicht in Ordnung gewesen sei. „Man verletzt die Vereinbarungen nicht drei Tage vor der Wahl“ (S 1/F 1/5.55). Kasper Juul kündigt an, sie werde nicht mehr von der Moderatorin interviewt. Es wird deutlich: Aufgrund des interdependenten Verhältnisses sind beide Bereiche auf die Kooperation des anderen angewiesen. Wird gegen unausgesprochene Gesetze verstoßen, kann dies mit Entzug der Kooperationsbereitschaft sanktioniert werden.

Politische Entscheidungen orientieren sich in Borgen an den Erfolgsbedingungen der medialen Öffentlichkeit. Kasper Juul als Spindoktor (S 1/F 1/43.50) von Birgitte Nyborg ist die Personifikation dieses Prinzips. Der Begriff Spindoktor - in etwa als Meinungsmacher zu übersetzen - stammt ursprünglich aus dem amerikanischen Wahlkampf und bezeichnet einen ,professionalisierten Politikvermittlungsexperten“ oder „PR-Krisenmanager einer Partei“ (Hofer 2005: 8-9). Beide Begriffe treffen auf das Aufgabenprofil von Kasper Juul zu. Er prüft jede Entscheidung hinsichtlich ihrer Medienrezeption. Befürchtet er schlechte Presse oder Umfrageergebnisse, dann wirbt er für eine Kurskorrektur. Nicht immer wird dem nachgegeben. Nyborg entgegnet in einer Szene: „Wir berücksichtigen die Meinungsumfragen, aber wir richten die Politik nicht daran aus" (S 1/F

4 Näheres zu Theorien über das Verhältnis von Medien und Politik findet sich im Beitrag zu Eichwald, MdB. 
1/15.10 - 15.25). Trotzdem hat er Zugang zum engsten Beraterkreis, in Entscheidungssituationen scheint er beinahe immer anwesend zu sein.

Bezugnehmend auf die Funktion von Beratern ist Kasper Juul für die PolicyAkzentuierung zuständig. Er identifiziert „Gewinnerthemen, mit deren Hilfe die Identifikation von Partei, Fraktion und Wählern mit den Regierungen gefestigt oder unpopuläre Entscheidungen kompensiert werden“ (Grunden 2011: 264). Kasper Juul betreibt auch aktive Pressearbeit und Agenda-Setting mit dem Ziel, den öffentlichen Diskurs nach seinen Vorstellungen zu formen (Hofer 2005: 18).

\section{Koalitionsverhandlungen als rationale Entscheidung}

Die Regierungsbildung in Borgen wird anhand einiger Erklärungsansätze aus der Koalitionstheorie in aller Kürze analysiert (S 1/F 2/50.16 - 55.05).

Tabelle 1: Faktoren von Koalitionsverhandlungen mit Szenen-Beispielen

\begin{tabular}{|c|c|c|}
\hline Faktoren & Beispiel & $\begin{array}{l}\text { Verweis } \\
(\text { je S 1/F 2) }\end{array}$ \\
\hline office-seeking & $\begin{array}{l}\text { Nyborg sucht die effektivste Machtoption, also je- } \\
\text { ne mit den meisten Ministerposten. } \\
\text { Ein neues Ministerium wird geschaffen, um Ko- } \\
\text { operation der Milieupartei zu gewinnen. } \\
\text { Um des Machterhalts willen würde Hesselboe ein } \\
\text { bisher unbekanntes Bündnis eingehen. }\end{array}$ & $\begin{array}{l}23.00-26.00 \\
50.37-51.08 \\
54.40 \\
48.25-50.31\end{array}$ \\
\hline policy-seeking & $\begin{array}{l}\text { Sejrø lehnt eine Koalition mit den Liberalen ab, da } \\
\text { er keine inhaltliche Übereinstimmung sieht. }\end{array}$ & $51.10-51.50$ \\
\hline $\begin{array}{l}\text { Mehrheiten und } \\
\text { Normen }\end{array}$ & $\begin{array}{l}\text { Zwar sind Minderheitsregierungen typisch für Dä- } \\
\text { nemark, gegen die Norm spricht es jedoch, dass } \\
\text { mit den Moderaten nicht die größte Fraktion den } \\
\text { Ministerpräsidenten stellt. }\end{array}$ & \\
\hline $\begin{array}{l}\text { Innerparteiliche Pro- } \\
\text { zesse }\end{array}$ & $\begin{array}{l}\text { Die Arbeiterpartei ringt als heterogene Partei um } \\
\text { Führung und Eigeninteressen von Spitzenakteuren } \\
\text { stehen im Vordergrund. }\end{array}$ & $23.00-25.30$ \\
\hline $\begin{array}{l}\text { Vertrauen als Grund- } \\
\text { lage von Verhand- } \\
\text { lungen }\end{array}$ & $\begin{array}{l}\text { Hesselboe schafft einen vertrauensvollen Rahmen } \\
\text { für sein Gespräch mit Nyborg. Laugesen verliert } \\
\text { das Vertrauen der Bündnispartner, da er gegen } \\
\text { Abmachungen verstößt. }\end{array}$ & $48.25-50.31$ \\
\hline $\begin{array}{l}\text { Begründungspflicht } \\
\text { gegenüber Partei und } \\
\text { Wählern }\end{array}$ & $\begin{array}{l}\text { Die Milieupartei argumentiert, sie könne die vor- } \\
\text { geschlagene Ressortverteilung nicht gegenüber } \\
\text { den Wählern vertreten, und erhält ein Ministerium } \\
\text { mehr. }\end{array}$ & $54.35-55.00$ \\
\hline Personalfragen & $\begin{array}{l}\text { Nyborg lehnt den Kandidaten der Arbeiterpartei } \\
\text { für das Amt des Ministerpräsidenten ab, da sie } \\
\text { ihm Führungskompetenzen abspricht. }\end{array}$ & $43.30-44.40$ \\
\hline
\end{tabular}

Eigene Darstellung. Quelle: Kropp/Sturm 1999: 40-59, Switek 2011. 
Für die Ausgestaltung dieser Phase ist die Ausgangslage in Form von Wahlergebnis, Vorwahlallianz und offener Situation entscheidend (Switek 2013: 279 280). Hierfür sei auf die entsprechende zweite Folge verwiesen. Für die Koalitionsverhandlungen spielen verschiedene Faktoren eine Rolle (Kropp/Sturm 1999, Switek 2011), welche in der Tabelle 1 jeweils mit Beispielen aus Borgen versehen sind.

Die Akteure haben Ziele und Präferenzen, was in dieser Szene gemäß dem office-seeking-Theorem das Streben nach Regierungsämtern ist. Dem wird vieles untergeordnet, etwa der Faktor, dass normalerweise eine Trägheit bei neuen Bündnisoptionen besteht. Doch Hesselboe erwägt es - des Machterhalts wegen. Für alle Akteure bieten sich Handlungsoptionen, die im Hinblick auf dieses Ziel bewertet werden. Gewählt wird die Option, die die Werte maximiert. Für Birgitte Nyborg stellt sich die Situation nach dem Gespräch mit Lars Hesselboe wie folgt dar:

\section{$\underline{\text { Szenen-Beispiel: }}$}

Birgitte Nyborg kommt aus Verhandlungen mit Lars Hesselboe und berichtet ihrer Fraktion von den Ergebnissen (S 1/F 1/50.37 - 51.08).

Nyborg: Sein Vorschlag ist eine Regierungsteilnahme mit fünf Ministerien. Und wie ich es sehe, wäre das das effektivste Angebot. Wir nehmen der Freiheitspartei ihren Einfluss und nutzen unsere Mandate für effektive politische Arbeit, statt mit ihnen verheizt zu werden.

Erst durch ein Gespräch mit Bent Sejrø erkennt sie weitere Handlungsoptionen. Das spiegelt die Erweiterung des Konzepts des office-seeking (Kropp/Sturm 1999: 53). So entstehen Spielräume, wenn die Anzahl der Ressorts aufgestockt wird und die Gewinne aller Koalitionsparteien erhöht werden können (Kropp/Sturm 1999: 52). Zudem wird das office-seeking als Wert um den Faktor policy-seeking ergänzt. Denn Politiker müssen über Ressorts verfügen, mit denen sie ihre ,politischen Präferenzen in gestalterische Politik umsetzen“ (Kropp/ Sturm 1999: 52) können. Darauf weist Sejrø hin, als er einwendet, dass ein angedachtes Ministerium für Internationale Entwicklung der Partei nicht helfen wird, ihre policies umzusetzen. Unter Abwägung aller Optionen gelingt es Nyborg schließlich, eine Regierung zu bilden, die ihren Nutzen maximiert. 


\section{DATEN: DIE KULTURELLE UND NORMATIVE DIMENSION IN BORGEN}

In der ersten Staffel von Borgen manifestieren sich in Form von impliziten Normen, Werten und kulturellen Spezifika verschiedene Elemente einer skandinavischen und europäischen Kultur, die Einfluss auf das fiktive politische Geschehen nehmen. Konkret geht es dabei um folgende Themen: das Staatsverständnis am Beispiel des Verhältnisses von Wirtschaft und Politik, die parlamentarische Kultur und liberale Grundwerte einer europäischen Demokratie.

\section{Borgen als Beispiel für skandinavisches Staatsverständnis}

Das Zusammenspiel von Politik und Wirtschaft wird in einem Szenen-Beispiel aus der fünften Episode thematisiert: Die Regierung plant die Einführung einer Frauenquote für Aufsichtsräte und die Industrie, verkörpert durch Joachim Chrone, versucht mit massivem Lobbydruck, das Gesetz zu verhindern beziehungsweise abzuschwächen. Im Sinne der Datenanalyse seziert Borgen hier sehr kleinteilig die Abhängigkeiten zwischen Politik und Wirtschaft. Nyborg tritt Chrone zunächst entschieden entgegen und artikuliert damit das Ideal eines Primats der Politik und der staatlichen Steuerung.

\section{Szenen-Beispiel:}

Nyborg stellt gegenüber Chrone das Verhältnis von Wirtschaft und Politik dar (S 1/F $5 / 48.42-48.48)$.

Nyborg: In diesem Land leiten Sie das größte Unternehmen, doch die Gesetze für dieses Land machen wir und das wollen wir nicht vermischen.

Borgen zeigt jedoch im Folgenden, dass die informellen Verflechtungen zwischen Politik und Wirtschaft enger sind, als es auf den ersten Blick ersichtlich ist. Auch wenn die Norm politischer Steuerungsautarkie postuliert wird, bestehen auf einer funktionalen Ebene wechselseitige Abhängigkeiten. Die Politik ist auf gute Wirtschaftsdaten angewiesen, um Wahlen zu gewinnen und Legitimität zu behaupten. Joachim Chrone weiß als enger Vertrauter des ehemaligen Ministerpräsidenten Hesselboe um diese politischen Gesetzmäßigkeiten und macht sich dies zunutze: Er stellt Nyborg gegenüber die Forderung auf, das Gesetz zur Frauenquote fallen zu lassen, da er sonst sein gesamtes Unternehmen ins Ausland verlagern würde (S 1/F 5/21.05 - 23.50). Nyborg fühlt sich in die Ecke gedrängt und entwickelt einen Plan als Antwort auf diese Drohung. Sie verkörpert damit an dieser Stelle einen handlungsfähigen Staat, der sich durch ein hohes Maß an Steuerungsfähigkeit auszeichnet. Das Ausbalancieren der konkurrie- 
renden Politikpräferenzen erfolgt in diesem Zusammenhang, indem sie Informationen über Chrone einholt und so aufdeckt, dass es sich bei seiner Drohung mit der Verlagerung von Arbeitsplätzen höchstwahrscheinlich lediglich um einen Bluff handelt (S 1/F 5/44.30 - 46.14). Gleichwohl Nyborg alle Trümpfe in der Hand hält, verständigt sie sich letztlich mit Chrone darauf, dass dieser den Vorschlag einer Frauenquote als erster Unternehmer unterstützt und sich somit die besten weiblichen Führungskräfte für sein Unternehmen sichern kann. Im Gegenzug gewährt ihm Nyborg einen Aufschub bei bestimmten Umweltauflagen (S 1/F 5/48.20 - 50.30). Neben der Einlösung des politischen Steuerungsprimats eines starken Staates treten damit Elemente einer Kultur im Verhältnis von Politik und Wirtschaft zu Tage, die auf Konsens- und Kooperationsorientierung verweisen.

\section{Parlamentarische Kultur von Konsens und Kooperation}

Die zehnte Folge der ersten Staffel thematisiert ein Kernelement demokratischer Regierungsführung, und zwar die parlamentarische Rede als politisches Gestaltungsmittel. In Dänemark eröffnet der Ministerpräsident traditionell jedes Jahr das Parlament mit einer Rede am ersten Dienstag im Oktober. Diese Situation wird in Borgen dargestellt. Nyborg steht politisch unter Druck, ihre Macht bröckelt, in ihrer Minderheitsregierung gärt es. In dieser Situation nutzt sie die parlamentarische Rede, um sich aus der Defensive $\mathrm{zu}$ befreien und politischen Handlungsspielraum zurückzugewinnen. In ihrer Rede beschwört Nyborg die Einheit der Nation: Alle im Parlament vertretenen Parteien müssten jetzt zusammenarbeiten und damit der Verantwortung gegenüber dem Volk gerecht werden. Gleichzeitig beschwört sie den gesellschaftlichen Zusammenhalt und bedient sich des Sports als eines verbindenden Elements (S 1/F 10/52.22 54.50). Nyborgs Rede ist damit Ausdruck einer parlamentarischen Kultur, die Konsens und Zusammenarbeit über die Grenzen von Regierung und Opposition hinaus betont. Diese normative Orientierung ist in Zusammenhang mit den politisch institutionellen Rahmenbedingungen zu betrachten, die Minderheitsregierungen zum Regelfall in Dänemark machen und so intensive Kooperation von Regierung und Parlament erfordern. In einem solchen kulturellen Kontext kann ein eher weiches Instrument der Machtausübung wie die parlamentarische Rede besondere Wirkmächtigkeit entfalten, da sie ein auf Verständigung ausgerichtetes Kommunikationsinstrument darstellt und ihr allgemein eine hohe Bedeutung beigemessen wird. Birgitte Nyborg gelingt es, dieses Instrument zielgerichtet einzusetzen, so dass sie wieder in eine Position der Stärke zurückfindet und ihre Regierung stabilisiert. 


\section{Kulturelle Grundwerte liberaler Demokratien}

Zwei Episoden der ersten Staffel behandeln ausführlich kulturelle Eckpfeiler der Werteordnung europäischer Demokratien. Die Darstellung von Borgen zeigt dabei, wie einerseits im innerstaatlichen Rahmen Spannungsverhältnisse in solchen Werteordnungen zwischen Presse- und Meinungsfreiheit, Regierungshandeln und staatlichen Geheimnissen verhandelt werden und sich andererseits nach außen kulturelle Normen im Umgang mit autokratisch verfassten Staaten ausdrücken.

In Episode 4 (S 1/F 4) veröffentlicht TV1 Bilder und Listen von geheimen CIA-Flügen mit gefangenen Terrorverdächtigen über Grönland. Der Informant, ein Major der Streitkräfte, sowie TVI sehen sich anschließend staatlichen Repressionen ausgesetzt, denn die Veröffentlichung gefährdet das Verhältnis zum wichtigen Bündnispartner USA und damit die nationale Sicherheit. Agenten des Geheimdienstes brechen bei der verantwortlichen Redakteurin ein, TVI wird untersagt, die Bilder und Listen der Flüge weiterhin für die Berichterstattung zu benutzen, und dem Informanten wird gedroht, sein Privatleben zu ruinieren. Diese drastischen Methoden verdeutlichen die Bedeutung des Schutzes staatlicher Geheimnisse und des Verhältnisses zu wichtigen Bündnispartnern. Im Prinzip geht es somit um die Grenzen der Pressefreiheit und Borgen zeigt klar auf, dass auch eine demokratische Regierung die Pressefreiheit in bestimmten Fällen einschränken muss. Es ist ein weiteres Merkmal eines demokratischen Rechtsstaats, dass die Presse als sogenannte vierte Gewalt zwar eingeschränkt werden kann, die Regierung sich dafür aber öffentlich rechtfertigen muss und somit weiterhin einer gewissen Kontrolle durch die Medienöffentlichkeit unterworfen ist. Damit wird sowohl der Wert der Pressefreiheit verdeutlicht, als auch, in welchem normativen Rahmen sie sich bewegen kann, bis der Staat aufgrund widerstreitender Werte eingreift. Die Grenzen der Pressefreiheit, die Frage, wie weit das berechtige Informationsinteresse der Öffentlichkeit reicht, und die Bewertung, welche Informationen die nationale Sicherheit gefährden, sind ständigen Aushandlungsprozessen unterworfen. Dieser Grundkonflikt wird in der vierten Episode beispielhaft aufgearbeitet.

Die zweite Episode (S 1/F 6) behandelt den Umgang einer westlichen Demokratie mit einem illiberalen Regime und wie in dieser Konstellation spezifische Wertevorstellungen aufeinandertreffen. Während eines Staatsbesuchs in Dänemark fordert Alexander Grozin, Präsident des fiktiven Turgisiens, Nyborg auf, den bekannten turgisischen Regimekritiker Vladimir Bayanov zu verhaften, der sich ebenfalls in Dänemark aufhält. Grozin beschuldigt ihn des Terrorismus. Problematisch wird diese Forderung, da Grozin die Verhaftung zur Bedingung 
für einen lukrativen Windkraftdeal mit der dänischen Industrie macht. Gleichzeitig gibt Bayanov Interviews in den dänischen Medien und bezichtigt Grozin der Verletzung von Menschenrechten und der Unterdrückung von Minderheiten.

Schematisch betrachtet kommt es damit zwischen Nyborg und Grozin zur Konfrontation unterschiedlicher Werteordnungen, in denen sich Repression zwecks Aufrechterhaltung von Sicherheit und Ordnung auf der einen sowie freiheitliche Grundordnung und individuelle Schutzrechte auf der anderen Seite gegenüberstehen. Borgen verwischt diese strikte schematische Differenzierung jedoch, da Birgitte Nyborg sich in dieser Konstellation einem Dilemma ausgesetzt sieht: Sie hat als Repräsentantin einer westlichen Demokratie die moralische Verpflichtung, den Opfern von Unterdrückung beizustehen und sie muss gleichzeitig verhindern, dass Bayanov für öffentliche Unruhe sorgt, damit der Windkraftdeal mit Grozin zustande kommt. Im Folgenden lässt Nyborg den Regimekritiker Bayanov schließlich mithilfe eines Anti-Terror-Gesetzes verhaften, obwohl sie das Gesetz für fragwürdig hält und sich bei dessen Beschluss klar dagegen positioniert hat. Die Verhaftung steht somit nicht im Einklang mit ihren persönlichen Werten, ist aber aus realpolitischen Gründen unvermeidlich. Die Pressefreiheit ermöglicht den Medien, die Verhaftung live zu dokumentieren ( $S$ 1/F 6/32.32 - 34.01) und Nyborg damit öffentlich unter Druck zu setzen, denn es sieht so aus, als würde sie die wirtschaftlichen Interessen über ein Menschenleben stellen und dem Druck Grozins nachgeben. Daraufhin setzt sich Nyborg nochmal mit der Menschenrechtslage in Turgisien auseinander und entscheidet sich schließlich auch aufgrund des öffentlichen Drucks gegen die Auslieferung. Stattdessen greift sie zu einem Trick: Sie lässt Grozin im Glauben, sie würde Bayanov ausliefern und lässt ihn öffentlich den Windkraftdeal ohne andere Bedingungen zusagen. Erst danach offenbart sie ihre wahren Motive (S 1/F 6/52.17 $-54.30)$.

\section{NARRATIVE: DER MENSCH UND DIE MACHT - ERZÄHLUNGEN VON POLITIK IN BORGEN}

Für die Vielfalt aktuell konsumierbarer Fernsehserien mit politischem Bezug und der in ihnen enthaltenen Erzählungen von Politik bietet Andreas Dörner ein kategorisierendes Schema, welches das „Spektrum der fiktionalen Konstruktion des Politischen“ (Dörner 2016: 7) auf drei Idealtypen eingrenzt. In der Darstellung von Idealpolitik werden die handelnden Figuren von einem idealistischen Impetus getragen, ihr Streben gilt einem höheren Gut, das sie mit anständigen Mitteln zu erreichen suchen. Dieser Konzeption stehen als Gegenpol des narrati- 
ven Spektrums Erzählungen von Machtpolitik gegenüber, denen ein verabsolutierter Ich-Bezug in der Handlungsorientierung politischer Akteure gemein ist. Befriedigung erfährt dieser Egoismus in der bedingungslosen Akkumulation von Macht, bei der Moral als relevante Kategorie negiert oder allenfalls als camouflierendes Element akzeptiert wird. In der Realpolitik hingegen finden die beiden zuvor benannten Narrative von Politik zu einer Synthese, nach der im Zweifel der hehre Zweck die Mittel heiligt. Dramaturgisch beziehen derartige Erzählungen ihr Spannungsmoment bevorzugt aus Porträts gebrochener Gesinnungsethiker oder aus der Prozessdokumentation einer Korrumpierung politischer Ideale (Dörner 2016: 8-10). Da Nyborgs Aufstieg zur Ministerpräsidentin in der ersten Staffel von Borgen einen solchen Prozess illustriert, stuft Dörner die Serie als Beispiel für ein realpolitisches Narrativ ein. Dieser erzählerische Archetyp stellt in Form der persönlichen Entwicklungsgeschichte Nyborgs den dominanten Handlungsstrang der ersten Staffel dar.

\section{Politik als Triebkraft einer persönlichen Entwicklungsgeschichte}

Borgen beleuchtet nicht nur politisches Regierungsgeschehen im Umfeld der dänischen Ministerpräsidentin und thematisiert dabei unterschiedliche aktuelle Politikfelder, die Serie begleitet insbesondere auch das Hineinwachsen einer Newcomerin in das höchste dänische Regierungsamt sowie ein stufenartiges Hineinwachsen der Hauptfigur Birgitte Nyborg in diese Rolle.

Die Zuschauer lernen die Herausforderin Birgitte Nyborg mit ihren ursprünglichen Prinzipien und Vorstellungen des Politikbetriebs zu Beginn der Serie besonders im TV-Duell mit den Spitzenkandidaten am Ende der ersten Episode kennen (S 1/F 1/41.30 - 45.31). Sie spricht als Vorletzte und vor ihr haben alle Kandidaten gewöhnliche, vorbereitete Abschlussstatements vorgetragen. Nyborg hingegen spricht plötzlich frei und setzt sich somit bewusst von ihren Konkurrenten ab. Zudem gibt sie zu, nicht das vorgesehene Kleid zu tragen, da sie zugenommen habe und nicht hineinpasse. Nyborg spricht damit öffentlich ein persönliches Problem an, wodurch sie sich als persönlich nahbar und authentisch darstellt. Sie verbindet diese Inszenierung mit dem Bekenntnis, mittels eines Regierungswechsels nicht nur eine andere Politikausrichtung, sondern einen neuen, prinzipienverpflichteten Politikstil anzustreben. Insbesondere wirbt sie für mehr Ehrlichkeit und Mut, Schwächen zuzugeben.

Nach der für die Moderaten unerwartet erfolgreichen Wahl erweist sich Nyborg nach anfänglichen Schwierigkeiten bei den Koalitionsverhandlungen, wie bereits beschrieben, als sehr adaptionsfähig. Während ihr zunächst angeboten 
wurde, die Moderaten könnten als Teil einer Regierungskoalition ein neues Ministerium für Internationale Entwicklung erhalten, nutzt sie diesen Vorschlag ihrerseits, um die Milieupartei von einer Koalition unter ihrer Leitung zu überzeugen (S 1/F 2/53.39 - 54.56). Indem sie die Unerfahrenheit des jungen Fraktionsvorsitzenden der Milieupartei zu ihrem Vorteil nutzt, beweist sie mit Unterstützung ihres Mentors Sejrø politisches Geschick.

Im Amt der Ministerpräsidentin muss sie bald beweisen, inwieweit sie den angekündigten Stilwechsel zu einem wertegeleiteten Ansatz in die Tat umsetzen kann. Nach zweieinhalb Monaten Amtszeit ist absehbar, dass die Regierungskoalition aufgrund interner Abweichler keine Mehrheit für ihren ersten Haushaltsentwurf hat. Nyborg versucht im Angesicht der ersten Krise, auf ihrem eingeschlagenen Pfad zu verharren, und lehnt ein Angebot der Opposition, bei Einarbeitung von Änderungswünschen den Haushalt mitzutragen, ab. Nyborg weigert sich an dieser Stelle, die mit ihrem Budgetentwurf verbundenen Politikziele zu opfern, braucht allerdings eine Alternative, um eine Regierungskrise in Folge eines gescheiterten Haushalts abzuwenden. In der dritten Folge tritt das realpolitische Narrativ gleich in drei Szenen besonders deutlich hervor.

\section{Szenen-Beispiel a:}

Haushaltsverhandlung mit Lars Hesselboe und Yvonne Kjær (S 1/F 3/21.49 - 22.13)

Nyborg: Wir machen keine bürgerlich-liberale Politik.

Hesselboe: Eine Regierung macht die Politik, die nötig ist, um zu überleben. Denken Sie darüber nach.

Nyborg: Lars, die Antwort ist „Nein“! Ich will als Premierministerin nicht als Ersatz für die Liberalen in Erinnerung bleiben.

Hesselboe: Die Alternative ist, gar nicht in Erinnerung zu bleiben.

\section{$\underline{\text { Szenen-Beispiel b: }}$}

Die Opposition droht daraufhin mit einem Misstrauensvotum, sodass Nyborg mit Finanzminister Sejrø und dem temporären Spindoktor Tore Gudme über eine alternative Strategie bezüglich der Forderungen der Abweichler berät (S 1/F 3/25.53 - 26.03).

Sejrø: Vertrauliche Verhandlungen durchsickern zu lassen, ist nicht fair.

Gudme: Dann würde Peterson ${ }^{5}$ bestimmt umgänglicher werden.

Nyborg: Wir sind dabei, die Mehrheit zu verlieren. Das war's mit Fairplay. So sollten wir vorgehen.

5 Peterson ist einer der Abweichler aus den Reihen der Arbeiterpartei. 


\section{Szenen-Beispiel c:}

Nyborg, Gudme und Sejrø kommen zu dem Schluss, dass Verhandlungen nicht mehr aussichtsreich sind (S 1/F 3/28.20 - 28.25).

Gudme: Wie überleben wir morgen die Abstimmung?

Nyborg: Durch Erpressung! ${ }^{6}$

Nyborg handelt hier gemäß dem Prinzip, dass der Zweck die Mittel heiligt, bedient sich instrumentell jedoch ihr ursprünglich suspekter Winkelzüge, die mit der im TV-Duell formulierten Vision einer moralischen Erneuerung der Politik kaum in Einklang zu bringen sind. Im Ergebnis erringt Nyborg einen taktischen Sieg, der ihr mehr Zeit für Verhandlungen eröffnet.

Auch langjährige politische Wegbegleiter von Birgitte Nyborg, und letzten Endes sie selbst, nehmen ihr verändertes Handeln wahr. Dies zeigt ein Beispiel aus der sechsten Folge, in welcher der turgisische Präsident Grozin zum Staatsbesuch in Dänemark empfangen wird.

\section{Szenen-Beispiel:}

Anne Sophie Lindenkrone, Vorsitzende der Solidarischen Sammlung, thematisiert den Staatsbesuch (S 1/F 5/6.54-7.20).

Nyborg: Ich übergebe ihm den Vorsitz [der Organisation für Sicherheit und demokratische Entwicklung], weil Dänemark [...] zufällig den Vorsitz führt.

Lindenkrone: Du empfängst ihn. Offiziell. Früher hättest du dich den Protesten angeschlossen, bevor du Pragmatikerin wurdest.

Nyborg: Es ist dein Privileg, nicht pragmatisch sein zu müssen, Anne Sophie, weil deine Partei nur ein Prozent über der Sperrklausel ist. Auch für die, Anne Sophie, die mich nicht gewählt haben, bin ich Premierministerin.

Nyborg offenbart hier unter Einwirkung ihres Amtes Handlungsstandards, die sich nicht mehr maßgeblich aus ihrer eigenen moralischen Überzeugung ableiten. Die darin angelegte Entwicklung zu einer immer stärker mit ihrem Amt verschmelzenden und die Notwendigkeit von Machterhalt betonenden Politikerin erfährt im weiteren Staffelverlauf eine Beschleunigung. Als Nyborg zum Staffelende hin schließlich Bent Sejrø der Koalitionsräson zum Opfer fallen lässt, indem sie ihn zum Rücktritt drängt, hat sie nahezu alle Verbindungen zu ihrem

6 Die Erpressung ist ein Deal mit Ex-Ministerpräsident Hesselboe (S 1/F 3/28.35 29.50), dessen Partei letztlich einem Aufschub der Haushaltsabstimmung um eine Woche zustimmt. Im Gegenzug versichert Nyborg, dass ein gegen Hesselboe gerichteter Untersuchungsausschuss nicht eingesetzt wird. 
früheren politischen Selbst gekappt. Die Metamorphose, bisweilen erzählt in Form eines nuancierten Gegenentwurfs der Menschwerdung, ist vollendet.

\section{Politik als Spiel der Kräfte mit eigenen Gesetzen}

Ein wesentlicher Bestandteil von Politik ist in der Darstellung von Borgen das stete Ringen um die Deutung von Ereignissen und die Konstruktion der Wirklichkeit. Die Ministerpräsidentin ist dabei in ein komplexes Geflecht von unterschiedlichen Handlungsarenen, Interessen, Praktiken und informellen Institutionen eingebunden, dessen feine Verästelungen in der Serie über die Interaktionen der verschiedenen Akteure abgebildet werden. Daraus ergibt sich ein vielschichtiges Set von Handlungsanforderungen, das die Figuren in Form von Spielanalogien selbst thematisieren. Ein Beispiel hierfür findet sich in der letzten Episode der ersten Staffel.

\section{$\underline{\text { Szenen-Beispiel: }}$}

TV1 berichtet von einer Umfrage, die Mandatsverluste für Nyborgs Partei bei Zugewinnen für einen Koalitionspartner, die Arbeiterpartei, ausweist. Diese möchte die Gunst der Stunde nutzen und die Umfragewerte in politisches Kapital umwandeln, indem sie ein zusätzliches Ministerium für sich beansprucht. Nyborg bittet in der Folge Finanzminister Sejrø zum Gespräch (S 1/F 10/48.40 - 50.35).

Nyborg: Die Arbeiterpartei mach mir Druck. [...] Bent, die wollen das Finanzministerium.

Sejrø: [Lacht] Aber Birgitte, das wirst du ihnen nicht geben, das musst du nicht. [Schweigen und Blickwechsel zwischen Nyborg und Sejrø] Ich bin noch nicht am Ende, Birgitte. Es stimmt schon, dass ich nicht ganz jung bin, aber ich habe Erfahrung, ich kenne das Spiel. Politik ist mein Leben.

Nyborg: Ja, und das hier ist Politik, Bent. Du musst zurücktreten.

Im Nachgang der Kabinettsumbildung kommentiert TV1 wohlwollend, dass die Ministerrochade Veränderungsbereitschaft und Handlungsfähigkeit der Ministerpräsidentin demonstriere.

Die Szene verweist auf die Bedeutung der Spielregeln sozialer Aushandlungsprozesse in der Politik, deren Wirkmächtigkeit hier daraus erwächst, dass Arbeiterpartei und Medien auf Basis von Umfragewerten als hypothetischem politischem Szenario die Deutung etablieren konnten, die Regierung stehe unter Handlungsdruck. Den Medien fällt in diesem Zusammenhang, auch unter erzähltechnischen Gesichtspunkten, eine Rolle als Taktgeber für die politische Auseinandersetzung und als Wächter über die Spielregeln zu, wobei sie im oben zitier- 
ten Beispiel das regelkonforme Verhalten Nyborgs durch den Ministertausch mit einem belobigenden Kommentar bedenken.

Dass die Einhaltung der Spielregeln konstitutiv für die politische Arbeit der Akteure in Borgen ist, illustriert das Schicksal von Tore Gudme, Kasper Juuls temporärem Nachfolger als Spindoktor, in der dritten Episode. Akademisch hochqualifiziert und von Nyborg aufgrund seiner Nähe zu Positionen der Moderaten ausgewählt, scheitert er als Systemaußenseiter am Umgang mit den Provokationen einer Boulevardzeitung und wird kurz nach Amtsantritt bereits wieder entlassen. Die narrative Implikation dieses Serienstoffes weist in die Richtung, dass politisches Handeln unter den Bedingungen einer komplexen Regelanleitung eines eigenständigen Professionalismus bedarf. Das große Spiel ist den Profis vorbehalten, deren besondere Expertise weniger in einer herausragenden Fachqualifikation begründet liegt als in intimer Kenntnis politischer Handlungsanforderungen und der Klaviatur praktischer Regierungsarbeit. Politik nimmt in dieser Erzählvariante die Konturen einer Kunstform sui generis an, deren Materie und Beherrschung sich nicht exklusiv über akademische Qualifikationen und Bildungsabschlüsse erschließt.

Eine Sonderstellung kommt unter den Spielarten des Politischen dem Wahlkampf zu, da ihm in der ersten Staffelfolge der Charakter einer Ausnahmesituation zugewiesen wird.

\section{$\underline{\text { Szenen-Beispiel: }}$}

Kasper Juul hat Kenntnis von kompromittierenden Informationen über den zu Staffelbeginn noch amtierenden Ministerpräsidenten Hesselboe erhalten. Er versucht, Nyborg davon zu überzeugen, die Information für ihren Wahlkampf einzusetzen, um Hesselboe zu schaden (S 1/F 1/28.20 - 28.45).

Nyborg: Hältst du mich wirklich für so unehrenhaft?

Juul: Verstehst du nicht, Birgitte? Wir sind im Hintertreffen! Wir sind im Krieg! So eine Chance dürfen wir uns nicht einfach entgehen lassen.

Nyborg: Ich könnte mir nie wieder in die Augen sehen, wenn ich so an die Macht käme.

Juul: Dann bezweifele ich, dass du jemals an die Macht kommst.

Der Kriegszustand im Kampf um das politische Überleben rechtfertigt in Juuls Augen die Annullierung der Anstandsregeln. Paradoxerweise ist die Verweigerung Nyborgs an dieser Stelle Ausgangspunkt dafür, dass ihr die spätere öffentliche Ausbreitung der Information durch einen politischen Kontrahenten den Weg in das Amt der Ministerpräsidentin ebnet. Da Juul die Information gegen ihren Willen weiterreicht, kann sich Nyborg schlussendlich erfolgreich als Antithese zu dieser Art von Wahlkampfführung inszenieren (abschließende TV-Runde im 
Wahlkampf, S 1/F 1/42.10 - 48.15). Die Darstellung von Moral ist in diesem Kontext ambivalent: Mögen die handelnden Figuren in Borgen Moral in ihren Überlegungen wie im geschilderten Beispiel Nyborgs auch einen intrinsischen Wert zuweisen, so stellt die Episodendramaturgie die Handlungen stets in einen Zusammenhang mit der politischen Wirkung. Moral ist damit nicht ausschließlich ein abstraktes Normsystem, sondern potentiell auch eine Handlungsressource im Kampf um politische Macht.

\section{FAZIT}

Die weitreichende internationale Rezeption von Borgen macht die Serie zu einem lohnenswerten Analysegegenstand in Hinblick darauf, wie Politik einem breiten Publikum gegenüber dargestellt wird. Indem die Kamera eng der Hauptfigur Birgitte Nyborg folgt, öffnet sie Türen zu den vermeintlichen Hinterzimmern der Macht und dem Privatleben einer Spitzenpolitikerin. Dabei zeigen sich elementare Konfliktlinien politischen Handelns zwischen Moral und Macht, Konfrontationen politischer Grundwerte und die Auswirkungen der Kräfte im politischen Betrieb auf die Entscheidungsträger. Der Serie gelingt es, komplexe politische Entscheidungszusammenhänge und -konflikte in die Interaktion ihrer handelnden Figuren hinein zu spiegeln, wodurch abstrakte Prozesse stets greifbar bleiben. Im Zusammenwirken mit dem dynamischen Wechselspiel der unterschiedlichen Politikarenen bildet Borgen so die vielfältigen Handlungsanforderungen an politische Spitzenakteure ab.

Unter politikwissenschaftlichen Gesichtspunkten zeigt sich eine Vielfalt von Verknüpfungsmöglichkeiten zwischen Serieninhalten und Theorien des Politikmanagements. So bieten Elemente der fiktionalen Koalitionsverhandlungen gleichermaßen Anschluss für eine Betrachtung aus office- oder policy-seekingPerspektive. Unterschiedliche wissenschaftliche Analysezugänge finden sich ebenso für Prozesse des Wahlkampfes und der politischen Beratung sowie für das interdependente Verhältnis von Politik und Medien. Borgen ist damit als Anschauungsmaterial geeignet, um ein vertieftes Verständnis von Politik zu vermitteln, wozu auch die hohe Bandbreite von Themen- und Politikfeldern über den Staffelverlauf beiträgt. In der Serie manifestieren sich zudem Elemente der spezifischen politischen Kultur eines skandinavischen und europäischen Staates, die Auskunft über die Rolle des Staates, die Parlamentskultur und die Rolle liberaler Grundwerte geben. Die in der ersten Staffel enthaltenen Politiknarrative offerieren verschiedene Erzählvarianten, den episodenübergreifenden Bogen spannt jedoch die Entwicklungsgeschichte Birgitte Nyborgs im Amt der Ministerpräsidentin. An ihr lässt sich präzise studieren, wie ein politisches Amt den Menschen 
formt. Versucht man die Motive der verschiedenen Narrative zu verdichten, zeigt sich, dass in der Serie häufig ein Wesen von Politik konstruiert wird, das einen Kosmos von eigener Gesetzmäßigkeit in sich birgt und gegenüber den Protagonisten Kräfte einer verzehrenden Vereinnahmung entfaltet. In der analytischen Gesamtschau vermittelt Borgen so die Botschaft, dass eine komplexe Realität ebenso anspruchsvoll wie unterhaltsam in einer vielschichtigen Fiktion abgebildet werden kann.

\section{LITERATUR}

Bergman, Torbjorn (1993): Formation rules and minority governments, in: European Journal of Political Research (1), S. 55-66.

Borchert, Thomas (2013): „Borgen“ in der ARD - Dänische TV-Serie beeinflusst Politik, in: Stern Online vom 4.4., http://www.stern.de/kultur/tv/borgen--in-der-ard-daenische-tv-serie-beeinflusst-politik-3025704.html (zugegriffen am 19.2.2017).

Bundeszentrale für politische Bildung (2015): Parlamentswahlen in Dänemark, in: Hintergrund aktuell vom 17.6., http://www.bpb.de/politik/hintergrundaktuel1/208377/wahlen-in-daenemark (zugegriffen am 19.2.2017).

Dörner, Andreas (2016): Politserien: Unterhaltsame Blicke auf die Hinterbühnen der Politik, in: Aus Politik und Zeitgeschichte 66 (51), S. 4-11.

Dyson, Stephen Benedict (2014): The best television show about politics ever, in: Washinton Post Online vom 2.2., http://wapo.st/MO9dtk (zugegriffen am 19.2.2017).

Findeisen, Jörg-Peter (1999): Dänemark. Von den Anfängen bis zur Gegenwart, Regensburg.

Förster, Christian/Schmid, Josef/Trick, Nicolas (2014): Die nordischen Länder, Wiesbaden.

Frandsen, Steen Bo (1994): Dänemark - der kleine Nachbar im Norden. Aspekte der deutsch-dänischen Beziehungen im 19. und 20. Jahrhundert, Darmstadt.

Frohn, Rüdiger (2011): Staatskanzleien als Regierungszentralen. Erfahrungen und Erkenntnisse eines Akteurs, in: Bröchler, Stephan/von Blumenthal, Julia (Hrsg.): Regierungskanzleien im politischen Prozess, Wiesbaden, S. 225234.

Grunden, Timo (2011): Das informelle Politikmanagement der Regierungszentrale, in: Florack, Martin/Grunden, Timo (Hrsg.): Regierungszentralen, Wiesbaden, S. 249-283. 
Helmes, Irene (2014): TV-Serie „Borgen“ - Wettlauf mit der Wirklichkeit, in: Süddeutsche Zeitung Online vom 31.1., http://sz.de/1.1875680 (zugegriffen am 19.2.2017).

Hofer, Thomas (2005): Spin Doktoren in Österreich, 2. Aufl., Wien.

Holbrook, Andrew R./Hill, Timothy G. (2005): Prime-Time Agenda-Setting and Priming: Television Crime Dramas as Political Cues, in: Political Communication (3), S. 277-296.

Hurard, Oriane (2015): Interview mit Drehbuchautor Adam Price, in: arte.de, http://sites.arte.tv/borgen/de/interview-mit-drehbuchautor-adam-price-borgen (zugegriffen am 20.2.2017).

Ismayr, Wolfgang (1999): Die politisches Syteme Westeuropas im Vergleich, in: Ders. (Hrsg.): Die politischen Systeme Westeuropas, Wiesbaden, S. 9-64.

Jensen, Olaf (1991): Königreich Dänemark, in: Steffani, Winfried (Hrsg.): Regierungsmehrheit und Opposition in den Staaten der EG, Wiesbaden, S. 93125.

Jessen, Lisbeth (2015): Dänische TV-Serie - Wie „Borgen“ die Politik Dänemarks verändert hat, in: Deutschlandradio Kultur vom 2.2., http://www.deutschland radiokultur.de/daenische-tv-serie-wie-borgen-die-politik-daenemarks.976.de.ht ml?dram:article_id=310463 (zugegriffen am 19.2.2017).

Jochem, Sven (2012): Die politischen Systeme Skandinaviens, Wiesbaden.

Korte, Karl-Rudolf/Fröhlich, Manuel (2009): Politik und Regieren in Deutschland: Strukturen, Prozesse, Entscheidungen, Paderborn.

Kropp, Sabine/Sturm, Roland (1999): Koalitionen und Koalitionsvereinbarungen, Wiesbaden.

Lykketoft, Mogens (2014): The parliamentary system of Denmark. Introduction to Danish democracy, in: thedanishparliament.dk http://www.thedanishparliament. $\mathrm{dk} /$ Publications/ /media/PDF/publikationer/English/The\%20Parliamentary\%20S ystem\%20of\%20Denmark_2014.ashx (zugegriffen am 15.3.2017).

Nannestad, Peter (1999): Das politische System Dänemarks, in: Ismayr, Wolfgang (Hrsg.): Die politischen Systeme Westeuropas, Wiesbaden, S. 55-91.

Riepler, Lukas (2013): Was an „Borgen“ und Birgitte Nyborg begeistert, in: Blogeintrag vom 24.10., http://lukasriepler.blogspot.de/2013/10/was-borgenund-birgitte-nyborg.html (zugegriffen am 19.2.2017).

Sarcinelli, Ulrich (2009): Politische Kommunikation in Deutschland. Zur Politikvermittlung im demokratischen System, 2. Aufl., Wiesbaden.

Sarcinelli, Ulrich (2013): Legitimation durch Kommunikation? in: Korte, KarlRudolf/Grunden, Timo (Hrsg.): Handbuch Regierungsforschung, Wiesbaden, S. 93-103. 
Switek, Niko (2011): Wieder einmal Trendsetter? Koalitionstheoretische Annäherung an die Minderheitsregierung in Nordrhein-Westfalen, in: regierungsforschung.de, http://regierungsforschung.de/wieder-einmal-trendsetter-koalitionsth eoretische-annaeherung-an-die-minderheitsregierung-in-nordrhein-westfalen (zugegriffen am 20.3.2017).

Switek, Niko (2013): Koalitionsregierungen: Kooperation unter Konkurrenten, in: Korte, Karl-Rudolf/Grunden, Timo (Hrsg.): Handbuch Regierungsforschung, Wiesbaden, S. 277-287.

Teuwsen, Peer (2013): Die Suche nach dem guten Stoff, in: ZEIT Online vom 20.6., http://www.zeit.de/2013/26/schweiz-fernsehserien-vorbild-daenemark (zugegriffen am 19.2.2017).

Weihser, Rabea (2012): Macht festhalten, Prinzipien loslassen, in: ZEIT Online vom 19.11., http://www.zeit.de/kultur/film/2012-11/borgen-gefaehrliche-seilschaften (zugegriffen am 19.2.2017).

Wirries, Clemens (2014): Borgen in der Wirklichkeit - Historische Vorbilder und aktuelle Rezeptionen einer populären Fernsehserie, in: INDES 3 (4), S. $62-68$. 


\section{Sex, Drugs and Politics}

\section{Die Polit-Serie Marseille}

Jonathan Beierl, Yannick Chougrani, Ilka Rasch, Sören Witt, Almut Zimmer

\section{MARSEILLE - EIN ÜBERBLICK ZUR SERIE}

\section{Hintergrund: ein französisches House of Cards?}

Die Serie Marseille ist die erste Netflix Europa-Produktion und wurde am 5. Mai 2016 zum ersten Mal ausgestrahlt. Das Drehbuch wurde vom französischen Autor Dan Franck geschrieben, als Regisseur fungierten mit Florent Emilio Siri und Thomas Gilou ebenfalls zwei Franzosen. Laut Netflix handelt es sich bei Marseille weniger um eine Politikserie als vielmehr um eine Familiengeschichte im politischen Umfeld (Krekeler 2016). Dennoch eignet sich die Serie zur Analyse mit dem Fokus Politik, da sie die politischen und gesellschaftlichen Machtverhältnisse auf kommunaler Ebene in Frankreich darstellt und diese in den Kontext eines (Wahl-)Kampfes zwischen zwei Politikern einbettet.

\section{Synopsis: Familiendrama und der Kampf um die wahre Macht}

„Wahre Macht bekommt man nicht geschenkt, wahre Macht muss man sich erobern“ (S 1/F 5/36.00). Diese zentrale Aussage verdeutlicht, worum es in der Serie Marseille geht: den Kampf um die politische Vorherrschaft in der Hafenstadt Marseille. Ausgetragen wird dieser Machtkampf zwischen Robert Taro (Gérard Depardieu), dem langjährigen Bürgermeister und seinem politischen Ziehsohn Lucas Barrès (Benoît Magimel). Beide Kontrahenten versuchen, mit teils illegalen Mitteln den Posten des Bürgermeisters zu halten beziehungsweise zu erobern. Ausgangspunkt der Auseinandersetzung ist die Abstimmung über ein Casino auf dem Gelände des ehemaligen Hafens. Initiiert von Taro soll dieses 
Großbauprojekt sein politisches Erbe an die Stadt darstellen, da er sich nach 20 Jahren Amtstätigkeit nicht mehr zur Wahl aufstellen lassen möchte. An seine Stelle soll Barrès treten, dem er auch die Verantwortung für die entscheidende Abstimmung über das Casino im Hafenaufsichtsrat überlässt. Doch Barrès verfolgt eigene Pläne und kündigt im entscheidenden Moment seine Gefolgschaft mit dem Bürgermeister auf. Irritiert von diesem scheinbar ziellosen Schachzug entschließt sich Taro, ein weiteres Mal für das Amt des Bürgermeisters zu kandidieren. Erschwert wird ihm dieser Schritt durch die Krankheit seiner Frau Rachel (Géraldine Pailhas), die ihrem bisherigen Beruf als Cellistin nicht mehr nachgehen kann und daraufhin in Depressionen verfällt.

Währenddessen baut Barrès sein Netzwerk zum Sturz des Bürgermeisters bei der Wahl aus. Ein enges Verhältnis geht er dabei mit der Regionalpräsidentin Vanessa D'Abrantes (Nadia Farès) ein. Diese stellt den Kontakt zwischen Barrès und Cosini (Jean-René Privat), einem einflussreichen Mafiaboss, her. Die Hilfe der beiden ist jedoch nicht uneigennützig: D'Abrantes möchte den kommunalen Machtkampf nutzen, um die Führung der Partei $U P M$ zu übernehmen, während Cosini den Bau des Casinos aus wirtschaftlichen Interessen unter allen Umständen verhindern möchte: Für die Mafia, die in Marseille Herr über das illegale Glücksspiel ist, wäre der Bau eines städtischen Casinos eine Katastrophe. Mithilfe seiner hinzugewonnenen Kontakte schafft es Barrès nicht nur, seine Gegner einzuschüchtern, sondern er kann auch die Einwohner der ärmeren cités von seiner Kandidatur überzeugen und sich ihre Unterstützung im Straßenwahlkampf sichern. Angeführt wird seine Einsatztruppe aus Kleinkriminellen und Drogendealern von Farid (Hedi Bouchenafa) und Julia Taros Jugendfreund Eric (Guillaume Arnault). Erics Freund Sélim (Nassim Si Ahmed), der wie er im Sozialbauviertel Felix-Pyat groß wurde und sich als Drogendealer durchschlägt, wird der Freund von Julia Taro (Stéphanie Caillard), der Tochter des Bürgermeisters. Die Freundschaft der beiden jungen Männer zerbricht durch die Beziehung, noch dazu schlägt sich Sélim im Straßenwahlkampf auf die Seite Taros. Die Drogendealer der cités bekämpfen sich nun auch wegen ihrer politischen Gefolgschaft.

Einen weiteren Spannungsbogen stellt die Suche nach der wahren Identität Lucas Barrès' dar. Angestachelt durch verschiedene anonyme Hinweise, versucht Julia Taro herauszufinden, woher Barrès in Wirklichkeit kommt. Als Journalistin der regionalen Tageszeitung La Provence verfügt sie dabei über mehrere Möglichkeiten der investigativen Recherche. So stellt sie im weiteren Verlauf der Serie fest, dass - anders als von Barrès behauptet - seine beiden Elternteile leben und er in Wahrheit der uneheliche Sohn von Robert Taro ist. Diese Tatsache bringt auch Klarheit bezüglich des wahren Motivs von Lucas Barrès: In seiner Jugend war Robert Taro Teil einer militanten Bande, welche an mehreren 
Raubüberfällen beteiligt war. Ein weiteres Mitglied der Gruppierung war Pharamond (Eric Savin), der heute Chefredakteur bei La Provence ist. Bei einem Überfall wurde ein Mitglied der Gruppe schwer verletzt. Es handelte sich dabei um die Freundin von Robert Taro, welche zu dem Zeitpunkt schon von ihm schwanger war, ihn darüber jedoch im Ungewissen ließ. Die Verletzung war so gravierend, dass Taro sich dafür entscheiden musste, seine Freundin in ein nahegelegenes Krankenhaus zu bringen, was jedoch auch bedeutete, dass sie nach ihrer Genesung eine lange Haftstrafe antreten musste. Durch diesen Vorfall verloren sich die beiden aus den Augen, sodass Robert Taro die Geburt seines Sohnes, Lucas Barrès, nicht mitbekommen konnte. Die scheinbare Vernachlässigung seiner Mutter durch Taro und die fehlende Anerkennung als Sohn werden für Barrès zum Ausgangspunkt seines politischen Rachefeldzugs und begründen seinen Kampf um das Amt des Bürgermeisters.

In der letzten Szene kommt es zum emotionalen Showdown: Während des Besuchs eines Fußballspiels bricht Taro aufgrund eines Herzinfarkts zusammen. In diesem Zustand wird er von Barrès entdeckt, der unmittelbar mit den lebensrettenden Belebungsmaßnahmen beginnt. Mittlerweile hat er nämlich die Intrige von D'Abrantes und ihren Hintermännern durchschaut und möchte nun geläutert zu seinem politischen Ziehvater zurückkehren. Im Hintergrund wird in diesem Moment das Ergebnis der Wahl verkündet: Taro konnte mit wenigen Stimmen Vorsprung die Wahl zum Bürgermeister für sich entscheiden.

\section{KONTEXT: EINE STADT ZWISCHEN KRIMINALITÄT UND GROSSBAUPROJEKTEN}

\section{Geschichte, Wirtschaft und Soziales - zum Hintergrund der Stadt Marseille}

Marseille ist sowohl die älteste als auch mit etwa 900.000 Einwohnern nach Paris die zweitgrößte Stadt Frankreichs. Sie wurde im siebten Jahrhundert vor Christus als antiker griechischer Handelsstützpunkt gegründet (dies und Folgendes Simon 2014: 8-12). Die Bedeutung als Hafenstadt hat sich bis heute gehalten: Der Port autonom de Marseille umfasst circa 10.000 Hektar und ist damit der bedeutendste Frachthafen in Frankreich. Darüber hinaus wurde die Stadt als Afrikas Tor nach Europa bekannt (Fishmann o.J.). Durch mehrere Einwanderungswellen im Verlauf des 19. und 20. Jahrhunderts ließen sich immer wieder neue Bevölkerungsgruppen in Marseille nieder (Liehr 2013: 8). Der hohe Ausländeranteil prägt auch heute noch die Stadt - so haben beispielsweise rund 
80.000 Algerier und 10.000 bis 15.000 Senegalesen in Marseille ihre neue Heimat gefunden (dies und Folgendes Simon 2014: 12).

Die Arbeitslosenquote von Marseille liegt mit 13,5 Prozent weit über dem Landesdurchschnitt von 10,4 Prozent ${ }^{1}$. Jedoch muss hierbei stark differenziert werden, denn in der Stadt gibt es ein starkes soziales Gefälle. Während diejenigen der 16 Bezirke Marseilles, die im Süden liegen, vergleichsweise reich sind, ist der Norden von Armut geprägt. Dies ist einer der Gegensätze, die in der Serie immer wieder aufgegriffen werden.

\section{Schmiere stehen für die Drogenbosse - jugendlicher Alltag in den Sozialbauvierteln}

Aufgrund großer sozialer Schwierigkeiten ist das Alltagsleben der citésBewohner in hohem Maß durch Kriminalität geprägt (dies und Folgendes Wiegel 2012). Die Einwanderer, die vorwiegend in der Industrie, in Fabriken und Raffinerien gearbeitet hatten, verloren im Zuge der Deindustrialisierung massenweise ihren Arbeitsplatz. Heute herrscht dadurch besonders unter den Jugendlichen mit Migrationshintergrund Arbeitslosigkeit, Armut und Orientierungslosigkeit (Wiegel 2011). Das größte Problem stellt der Rauschgifthandel dar, gerade weil Marseille als Hafenstadt dafür einen idealen Handelsplatz bietet (Wiegel 2011, Duplantier 2013). Die Sozialbauviertel werden ,,von vielen kleinen Banden beherrscht, die sich den Rauschgiftmarkt streitig machen“; Bandenkriege fordern immer wieder zahlreiche Menschenleben (dies und Folgendes Ulrich 2012). Gerade Jugendliche sind durch die Anreize des Drogenhandels mindestens 100 Euro gibt es am Tag allein fürs Wacheschieben - besonders gefährdet. In der Amtszeit des amtierenden Bürgermeisters Jean-Claude Gaudin hat sich die Kriminalitätsstatistik der Stadt noch einmal massiv verschlimmert. Einige Wohnviertel gelten inzwischen als verloren, Lokalpolitiker forderten hier schon die Präsenz der Armee (Ulrich 2012).

Die Allgegenwart der Kriminalität prägt nicht nur die Stadt Marseille, sondern spielt auch in der gleichnamigen Serie eine grundlegende Rolle. Die Serie greift die Machtlosigkeit der Politik ebenso auf wie die Hilfslosigkeit der Jugendlichen, die dem kriminellen Milieu und der sozialen Lage der cités entkommen wollen (S 1/F 5/12.50 - 14.00, S 1/F 7/19.15 - 20.45).

1 Die Zahlen zur Arbeitslosigkeit stammen aus dem Jahr 2013. 


\section{Politik und Verwaltung: Frankreichs Kommunalebene}

Die zentralstaatliche Ebene des französischen politischen Systems spielt in der Serie Marseille keine Rolle. Hauptbezugspunkt ist - der Name der Serie lässt es vermuten - die Stadt Marseille, also die kommunale Ebene. Erwähnung findet über die Figur der Regionalpräsidentin Vanessa D'Abrantes aber auch die Ebene der Region.

Marseille ist die Hauptstadt des Départements Bouches-du-Rhône; gleichzeitig sitzt hier der Regionalrat der Region Provence-Alpes-Côte d'Azur (dies und Folgendes Simon 2014: 12). Seit 1995 amtiert der 1939 in Marseille geborene Jean-Claude Gaudin als Bürgermeister im Rathaus der Stadt. Er war zunächst Mitglied der bürgerlich-liberalen Partei Union pour la Democratie Française $(U D F)$, die 2002 in Teilen Mitglied der konservativen Union pour un Mouvement Populaire (UMP, seit 2015 Les Républicains [LR]) wurde (Schild/ Uterwedde 2006: 60, Kempf 2017: 200). Die Parallelen zu Bürgermeister Robert Taro in der Netflix-Serie sind deutlich: Taro regiert seit zwanzig Jahren in Marseille, als Parteimitglied der UPM, deren Mitbegründer er ist. Ebenso wird eine Parallele zum real existierenden Phänomen der ,Erbmonarchie‘ wichtiger kommunaler Ämter in der Serie angesprochen: Anfang der neunziger Jahre war noch die Hälfte der Bürgermeister Söhne von früheren Gemeindevorstehern, und zwar in der gleichen Gemeinde (dies und Folgendes Kempf 2017: 296). Mittlerweile ist es auch durchaus üblich, dass die Nachfolge wichtiger Ämter an die Witwen der bisherigen Amtsinhaber übergeht. In der Serie versucht Taro zu Beginn, Barrès als seinen Nachfolger zu installieren. Auch wenn er zu diesem Zeitpunkt noch nichts von seiner Vaterschaft weiß, wird daran deutlich, dass er es als wichtig erachtet, seinen politischen Ziehsohn als zukünftigen Bürgermeister zu haben. Im Fall der Regionalpräsidentin Vanessa D’Abrantes lässt sich ein ähnliches Phänomen beobachten: Sie ist die Frau des ehemaligen Regionalpräsidenten und hat das Amt direkt von ihrem Mann übernommen.

Berichte über den Zustand von Politik und Verwaltung in Marseille sehen die Lage sehr kritisch. Die Rede ist von klientelistischen Strukturen, einer ,aufgeblähten Verwaltung“ und der „Unreformierbarkeit des Gemeinwesens“ (Lehnartz 2014):

„Allianzen bilden sich fast immer nur um des kurzfristigen Vorteils willen. Das führt dann nicht selten zu einer für Außenstehende nur noch schwer durchschaubaren Bündnispolitik, deren oberstes Prinzip lautet: ,Der Feind meines Feindes ist mein Freund.“ (Lehnartz 2014) 
Als Parteien stehen in der Serie besonders zwei im Vordergrund, die als UPM und Zentrumspartei bezeichnet werden. Zur $U P M$ gehören Bürgermeister Robert Taro und sein politischer Ziehsohn Lucas Barrès. Im Hinblick auf diese Partei liegt die Vermutung einer Parallele zur real existierenden, konservativen Partei Les Républicains nahe, die bis im Mai 2015 noch Union pour un Mouvement Populaire hieß und mit UMP abgekürzt wurde (Kempf 2017: 200). Die in der Serie erwähnte Zentrumspartei, zu der Robert Taro im Verlauf der Serie wechselt, gibt es im französischen System als solche nicht. Vielmehr handelt es sich um ein ganzes Lager, innerhalb dessen sich immer wieder Splittergruppen aufgrund ideologischer Richtungskämpfe voneinander absetzen oder neue Bündnisse schließen (Kempf 2017: 2011). Eine kurze Erwähnung finden auch eine sozialistische Partei, wohl in Anspielung auf den französischen Parti socialiste, und eine rechte Partei namens Parti Français $(P F)$. Aus dem bereits erwähnten Parteiwechsel von Robert Taro lässt sich ableiten, dass es auf kommunaler Ebene eine starke Personalisierung der lokalen Macht gibt, die Parteizugehörigkeit spielt eine untergeordnete Rolle (Kempf 2017: 295). Großstadtbürgermeister wie Taro werden somit zu „Provinzfürsten“, welche eine Schlüsselstellung zwischen der Hauptstadt und der Peripherie einnehmen, in der Literatur wird dieses Phänomen als „lokaler Präsidentialismus“ bezeichnet (Kempf 2017: 296).

\section{Das Bild Marseilles wandelt sich: Euroméditerranée und Kulturhauptstadt 2013}

1995 beschloss man, dem düsteren Gesicht der Stadt durch Modernisierungsmaßnahmen entgegenzutreten: Das Milliardenbauprojekt Euroméditerranée wurde ins Leben gerufen, bis 2013 wollte man die Stadt zukunftsfähig machen ${ }^{2}$ (EPA Euroméditerranée 2017, Simon 2014: 60). Auf 480 Hektar werden nun Büroanlagen, Gebäude für internationale Events, smarte Wohnbauten und Luxushotels hochgezogen. Die Attraktivität des Marseiller Zentrums soll auf diese Weise erhöht und die Stadt selbst zum „,wirtschaftlichen Mittepunkt der Region“ umgestaltet werden. Als Anerkennung für ihre großen Bemühungen wurde der Stadt der Titel der Kulturhauptstadt Europas 2013 verliehen. Die Verwandlung der Stadt ist auch das zentrale Anliegen des Serien-Bürgermeisters Robert Taro. Sein Plan eines Casino-Neubaus, an dessen Ablehnung das Verhältnis zwischen Taro und Lucas Barrès zerbricht, ist nur ein Teil eines größeren Vorhabens, die

2 Das Projekt befindet sich mittlerweile in einer zweiten Phase, die bis 2030 andauern soll (Simon 2014: 15). 
Stadt in ein neues Zeitalter zu führen: Marseille soll Hauptstadt Südeuropas werden (S 1/F 1/16.35 - 17.15).

\section{SERIE ALS SPIEGEL: PERSONENKULT AUS DEM HINTERZIMMER}

In Marseille lassen sich im Hinblick auf politikwissenschaftliche Theorien und Wirkungszusammenhänge vier Themenbereiche ausmachen, deren nähere $\mathrm{Be}$ trachtung sich lohnt. Im Vordergrund steht der Wahlkampf um das Amt des Bürgermeisters. Hier geht es insbesondere um die Art der Wahlkampfführung sowie die dabei angewandten Strategien, respektive die inhaltliche Schwerpunktsetzung. Des Weiteren nimmt das Verhältnis zwischen Medien und Politik ebenfalls eine wichtige Rolle in der Serie ein. Die omnipräsente Informalität, in der sich ein Großteil des politischen Handels abspielt, sowie das Akteursverhalten von Vetospielern schließen die theoriegeleitete Einordnung ab.

\section{Wahlkampf: Marktplätze und Schlammschlachten}

Betrachtet man die Darstellung des Wahlkampfes in Marseille, fällt schnell auf, dass der Fokus eindeutig ausschließlich auf den Kandidaten und ihren Eigenschaften liegt ist. Es geht nicht um relevante Themen, inhaltliche Positionen oder konkrete policies. Auch der zum Anfang der Serie eingeführte Themenkomplex um die Renovierung des Hafens mitsamt Bau eines Casinos wird in der weiteren Darstellung des Wahlkampfes weitestgehend ignoriert. Dafür wird umso detaillierter die Planung und Ausführung der werbewirksamen Auftritte der beiden Kandidaten gezeigt. Wahlen gewinnt man in Marseille durch vermeintlich bürgernahes Auftreten auf dem Marktplatz und das möglichst weitläufige Verbreiten von Plakaten und Flyern mit dem Konterfei der Kandidaten. Dabei ist das volksnahe Auftreten hauptsächlich inszeniert und bis hin zur richtigen Wortwahl für den richtigen Wahlbezirk abgestimmt.

Argumente oder Pläne über die politischen Vorhaben im Falle eines Wahlgewinns spielen keine Rolle. Die Tatsache, dass Taro und Barrès bei den Plänen hinsichtlich des Hafens gänzlich gegensätzliche Positionen einnehmen, ist dem Zuschauer zwar bewusst, wird jedoch in den Interaktionen mit den Wählern sowie den Medien nicht weiter aufgegriffen.

Stattdessen gibt es reichlich Einblicke in die Arbeit der Wahlkampfteams (S 1/F 4/23.00 - 24.00). Mangels Inhalten geht es in diesen um die aktuellsten Umfragewerte, mögliche Methoden, um dem Gegenkandidaten zu schaden, und wie 
man welchen Marktplatz am besten durch Auftritte und Plakate bespielt (S 1/F 4/07.30-08.50).

Das Ziel beider Lager ist es, mediale Öffentlichkeit und Aufmerksamkeit zu erlangen. Personalisierte Botschaften in Form von Attacken und Verleumdungen des politischen Kontrahenten machen den Großteil der Kommunikationsstrategie aus. Sinn und Zweck dieses Vorgehens ist es, Stimmungen in der Bevölkerung zu wecken und diese dadurch für die Wahl zu mobilisieren. Dieser Fokus auf das Attackieren des politischen Kontrahenten macht sich in einer Szene besonders bemerkbar:

\section{$\underline{\text { Szenen Beispiel: }}$}

Barrès und sein Team befinden sich in seinem Büro. Gerade kam die Meldung herein, dass laut den ersten Umfragen Barrès einen deutlichen Vorsprung vor seinen Kontrahenten hat, insbesondere vor Taro (S 1/F 4/08.00 - 08.10).

Barbara: Taro hat nur elf Prozent.

Adrien: Da lohnt es sich fast gar nicht mehr, Taro frontal anzugreifen, also jetzt nur noch halbe Kraft voraus, so wie er mit dir.

Barrès: Ja gut, aber auf wen schlage ich jetzt ein? Die Sozialisten mit ihren 15 Prozent?

Den Vorschlag, aufgrund der guten eigenen Position in den Umfragen keine weiteren offensichtlichen Attacken auf die politische Konkurrenz auszuführen, lässt sich als Anregung zur asymmetrischen Demobilisierung verstehen. Die Tatsache, dass Barrès hier lediglich damit antwortet, dass er schließlich irgendjemanden zum ,Darauf-Einschlagen“ brauche, zeigt deutlich, welches Verständnis von Wahlkampf in der Serie vorherrscht.

Eine weitere große Rolle im Wettstreit um das Amt des Bürgermeisters spielen die cités. Diesbezüglich lässt sich ein interessanter Widerspruch feststellen: Geht es in der generellen Darstellung des Wahlkampfes, wie eingangs erwähnt, hauptsächlich um das öffentlichkeitswirksame Auftreten der Kandidaten, so wird die Unterstützung der cités eher durch informelle Deals gewonnen als durch die Wahlkampfauftritte der Politiker vor Ort. So trifft sich Barrès mit Cosini und Farid, um sich die Unterstützung der cités zu sichern (S 1/F 4/25.00 - 28.00) oder geht gar mit ihnen zusammen zur Wahlwerbung in die Sozialbauviertel (S 1/F $5 / 29.10$ - 30.00). Barrès' Wahlkämpfer aus der cité erkaufen für ihn Stimmen im Tausch gegen Wertgegenstände (S 1/F 6/22.38 - 23.46).

Die Seilschaft von Barrès über Cosini zu Farid bis hin zum Stimmenkauf durch die Gangs der cités ist symptomatisch für die stete Verknüpfung von Politik, Informalität und Kriminalität, die sich durch die gesamte Serie zieht. Diese 
konstante Verbindung zwischen Politik und Illegalität führt auch dazu, dass so ziemlich jeder politische Akteur in der Serie schon eine illegale Tat begangen hat oder begeht. Das Wissen um eben diese Aktivitäten ist entscheidend, um im Wahlkampf den Kontrahenten zu schaden. Es entsteht ein wechselseitiger Wettkampf um Informationen. So will Barrès, als er von der vermeintlichen Krankheit Taros hört, herausfinden, woran dieser leidet und ob er dieses Wissen womöglich gegen ihn einsetzen kann. Gleichzeitig muss er jedoch seinen Kontakt zu Cosini und der Mafia geheim halten. Taro wiederum versucht mehr über die Vergangenheit von Barrès zu erfahren, aber gleichzeitig ist er auch sorgsam darauf bedacht, seinen eigenen Kokainkonsum zu verheimlichen. Diese Ambivalenz von Informationen wurde bereits Anfang der neunziger Jahre von Meyrowitz als eine Nebenwirkung der zunehmenden Medialisierung unserer Gesellschaft auf den politischen Prozess beschrieben (Meyrowitz 1990). Marseille zeigt diesen Kampf um die Kontrolle von Informationen als essentiellen Bestandteil des politischen Prozesses. Dabei spielen die Medien eine besondere Rolle, da sie für die Aufnahme und Verbreitung der Inhalte genutzt werden.

\section{Die Medien als vierte Gewalt? Nicht in Marseille!}

Die Serie zeichnet ein Bild von Politik und Medien, welches zuerst den Eindruck einer Mediendemokratie erweckt. Kennzeichen hierfür ist die Tatsache, dass die Medien und deren Akteure omnipräsent wirken und die primären Adressaten für politische Botschaften sind. Jedoch werden letztere hierbei nicht, wie zu erwarten, einer Mediatisierung unterzogen (Schulz 1997: 14). Konkret bedeutet das, dass die Medien zwar das wichtigste Instrument im Wahlkampf sind, um die hauptsächlich verleumdenden Inhalte zu kommunizieren. Sie zwingen die Politik jedoch nicht dazu, ihre Aussagen auf eine Medienlogik hin anzupassen.

Vielmehr lassen sich hier bei genauerer Betrachtung Merkmale der Instrumentalisierungsthese wiedererkennen (Schulz 1997: 26). ${ }^{3}$ Dieser Theorie folgend instrumentalisiert die Politik in Marseille die Medien, um ihre Botschaften ungefiltert kommunizieren zu können. Einzige Ausnahme stellt ein Radiointerview dar, in Zuge dessen auch kritische Fragen gestellt werden, die sich um die Verwicklungen Taros in den Geldwäscheskandal seiner Partei UPM drehen. Jedoch lässt man ihn, mit Beweisen für seine Unschuld ausgestattet, auch hier souverän auftreten, wohingegen der ihn interviewende Reporter schnell zum Statisten degradiert wird. Im weiteren Verlauf werden Journalisten als willfährige Masse

3 Näheres zu den Theorien über das Verhältnis von Politik und Medien finden sich im Beitrag zur Serie Eichwald, MdB im Kapitel „Spiegel“. 
dargestellt, welche mit ihren Mikrofonen und Kameras den Politikern eine Bühne bieten, die diese ohne Angst vor kritischen Interaktionen mit den Medien nach ihrem Belieben nutzen können.

Zwar wird auch kurzzeitig das Innenleben einer Redaktion gezeigt, jedoch keine wirkliche redaktionelle Arbeit. In den kurzen Momenten, in denen Medienakteure im Fokus stehen, werden eher private Hintergrundgeschichten erzählt, als dass es um Politik oder investigativen Journalismus geht. Betrachtet man beispielsweise Julia Taro, so beginnt ihre Erzähllinie als Reporterin, welche Nachforschungen hinsichtlich Barrès' Vergangenheit betreibt. Mit ihrem Wechsel in das Wahlkampfteam ihres Vaters endet dieser Erzählstrang jedoch, obgleich sie die Recherche fortführt. Sie wird nun nicht länger als Journalistin dargestellt, sondern als Assistentin ihres Vaters.

Vergleicht man Marseille hinsichtlich der Mediendarstellung beispielsweise mit der Serie House of Cards, werden diese dort in einer Rolle als tatsächliche Vetospieler präsentiert, wohingegen sie in Marseille lediglich wie ein filterloses Sprachrohr für politische Botschaften wirken; ihre Vetospielerposition wird außer Acht gelassen.

Beispielhaft für dieses Verhältnis zwischen Politik und Medien ist eine Szene zwischen Barrès, D'Abrantes und dem Redakteur Pharamond.

\section{Szenen-Beispiel:}

Barrès, D'Abrantes und Pharamond sitzen in einem Café. Sie sprechen über die bevorstehenden Wahlen und den finanziellen Zustand von Pharamonds Zeitung. Zum Ende des Gesprächs übernimmt Pharamond die Rechnung und sagt (S 1/F 3/22.30 - 24.40):

Pharamond: Wir sind schon ein seltsames Paar, wir Journalisten und ihr Politiker. Unsere Beziehung ist doch in Wahrheit eine rein wirtschaftliche. Wir sind die, die Papier verkaufen wollen und Sie wollen Stimmen kaufen.

Über diese Beschreibung des Verhältnisses zwischen Politik und Medien geht die Darstellung der Serie bis zum Schluss an keiner Stelle hinaus.

\section{Informalität: Hinterzimmer von Marseille}

Durch die zuvor beschriebene enge Verknüpfung von Politik und Kriminalität ist es nicht verwunderlich, dass ein Großteil des politischen Prozesses in Marseille 
in einem informellen Rahmen stattfindet. ${ }^{4}$ Insbesondere die Darstellung verhandlungsdemokratischer Elemente spielt sich im ,Schatten der Informalität“ ab. Dabei ist die Grenze zur Illegalität oftmals fließend. Auch wenn diese BeinaheGleichsetzung von informellen Verhandlungen und Kriminalität sicherlich überzogen ist, so sind die demokratiegefährdenden Elemente einer zunehmenden Entscheidungsfindung im Hinterzimmer in der Politikwissenschaft durchaus bekannt. Privilegierte Partizipation und zunehmende Verhandlung im nichtöffentlichen Rahmen sind nur zwei von vielen Kritikpunkten an der fortlaufenden Entwicklung (Grimm 2003: 203).

Die Serie selbst lässt nahezu ausnahmslos jegliche Verhandlungen im Hintergrund der politischen Bühne stattfinden - unabhängig davon, ob es um die Zustimmung für den Hafenumbau geht oder darum, Allianzen für die Bürgermeisterwahl zu schmieden. Sämtliche Gespräche finden in verlassenen Restaurants, dem Fußballstadion oder bei privaten Treffen im Garten statt. Taro und Barrès etwa treffen Richterin Marek im Foyer der Sinfonie, um sich ihre Unterstützung hinsichtlich des Hafenprojektes zu sichern (S 1/F 1/37.56 - 38.29), und der Bürgermeister erpresst Chasseron in dessen Privathaus, um dessen Platz als Spitzenkandidat der Zentrumspartei zu erhalten (S 1/F 3/26.47 - 32.20).

Auch das Verhalten der Akteure untereinander spielt sich im Rahmen dieser konstanten Informalität des politischen Handelns ab: Es ist geprägt von Erpressungen und innerparteilichen Ränkespielen.

\section{Vetospieler: Freund oder Feind}

Das Verhalten der politischen Akteure in Marseille ist eindimensional. Es geht ihnen ausschließlich um die Maximierung persönlicher Nutzenkalküle. Diese überzogene Logik des ausschließlich nutzenmaximierenden Handelns fokussiert sich hauptsächlich auf das office- und das damit erhoffte power-seeking. In dem dadurch entstehenden Kampf um Ämter und Positionen wird weder vor Erpressung noch vor sich wiederholenden Parteiwechseln Halt gemacht. Ideologien und Überzeugungen der Akteure spielen keine Rolle, solange durch neue Allianzen der eigene Machtgewinn gesichert wird.

Der Fokus liegt dabei stets auf der öffentlichen Wirkung des politischen Handelns. Dass die Aktionen, die in der öffentlichen Arena Erfolg bringend sind, negative Konsequenzen in der parlamentarischen oder administrativen Arena

4 Das Thema Informalität ist auch ein Element der Serien The Thick of It und Yes, Prime Minister und wird in den entsprechenden Analysen im Kapitel „Spiegel“ besprochen. 
haben können (Korte/Fröhlich 2009: 225), wird nicht dargestellt. Ein gutes Beispiel dafür ist die Figur des Vorsitzenden der Zentrumspartei, Pierre Chasseron (Pascal Elso). Dieser wechselt, soweit für den Zuschauer ersichtlich, dreimal die Partei - zweimal davon mitten im Wahlkampf. Selbst wenn diese Wechsel öffentlichkeitswirksam kommuniziert werden können, so werden die Konsequenzen, die solch ein Verhalten in den anderen Arenen hat, ignoriert. So ist beispielsweise mit einem massiven Vertrauens- und Glaubwürdigkeitsverlust bei zukünftigen politischen Partnern zu rechnen.

Der Umgang mit politischen Gegnern hingegen richtet sich schon mehr an bekannten Akteursstrategien und politischer Rationalität aus. Zuerst wird versucht, die betreffenden Personen einzubinden; schlägt der Versuch fehl, werden sie isoliert. Auch wenn es durchaus weitere Strategien im Umgang mit politischen Gegnern gibt (Korte/Fröhlich 2009: 220), so passt es doch zu der Machart der Serie, dass sie sich auch hier in den zwei Extremen bewegt.

\section{Szenen-Beispiele:}

S 1/F 2/09.40 - 12.00 (Barrès trifft sich mit Chasseron, um ihn davon zu überzeugen, Barrès bei der anstehenden Bürgermeisterwahl zu unterstützen.)

S 1/F 3/26.47 - 32.20 (Taro trifft sich mit Chasseron und erpresst ihn, um dessen Platz als Spitzenkandidat der Zentrumspartei zu erhalten. Dieser schließt sich gezwungenermaßen Taro an.)

S 1/F 4/20.40 - 31.50 (Barrès gibt ein Fernsehinterview, in dem er beweist, dass Chasseron als Schatzmeister der UPM Gelder veruntreut hat.)

Wie an den Beispielen ersichtlich, werden die Vetospieler jeweils von beiden Seiten aufgesucht. Dieses Muster lässt sich über die gesamte Serie hinweg feststellen: Sowohl Taro als auch Barrès suchen die relevanten Vetospieler auf und versuchen, sie auf ihre Seite zu ziehen. Gelingt dies nicht, so probieren sie, diese $\mathrm{zu}$ isolieren oder zu kompromittieren.

\section{SERIE ALS DATEN - DIE POLITISCHE KULTUR IN DER SERIE MARSEILLE}

\section{Machtkämpfe und Rachegelüste}

Die Haupthandlung der Netflix-Serie Marseille beschreibt wie erwähnt den fiktiven politischen Machtkampf zwischen dem amtierenden Bürgermeister von Marseille, Robert Taro, und seinem Widersacher Lucas Barrès. Der Verrat von Barrès im Hafenaufsichtsrat ist allerdings nicht allein politisch motiviert, son- 
dern politische und familiäre Beweggründe können als gleichrangig identifiziert werden. Dieser Umstand macht eine Analyse der politischen Kultur jedoch nicht überflüssig, da sich der Konflikt der beiden Kontrahenten um die Herrschaft über die Hafenstadt Marseille dreht. Während es zu Beginn der Serie so scheint, als würden sich die beiden Hauptcharaktere blind vertrauen, wird der Zuschauer schnell eines Besseren belehrt. Alle Akteure der Serie sind von einem extremen Misstrauen zueinander geprägt, das sie dazu veranlasst, jegliche Werte oder Moralvorstellung zu missachten. Handlungen in der politischen Arena werden keiner normativen Prüfung unterzogen und eine kritische Reflexion durch die Akteure findet nicht statt. Beispielhaft für das stark ausgeprägte Misstrauen ist die Figur des Robert Taro. Sein engster Vertrauter über den Zeitraum der ersten Staffel ist in Folge des allgemeinen Misstrauens im politischen Umfeld sein Chauffeur, dem er seine persönlichen Gedanken, Geheimnisse und Strategien während verschiedener Autofahrten anvertraut. Darüber hinaus beauftragt er seinen Fahrer damit, Koks für ihn zu besorgen, das Taro offensichtlich benötigt, um den stressigen Job als Bürgermeister von Marseille zu bewältigen.

Wie auch in House of Cards ist in Marseille Rache ein zentrales Motiv, das die Serie und die Handlungen der verschiedenen Akteure stark prägt. Es spielt in nahezu allen Akteursbeziehungen eine wesentliche Rolle und dominiert deren Handlungsprämissen. Dies gilt insbesondere für Lucas Barrès, der seinem politischen Ziehvater die Herrschaft über die Stadt Marseille abnehmen möchte und sich dafür rächen will, was Taro seiner Mutter angetan hat. Politische und private Rachefeldzüge spielen aber nicht nur im Konflikt um den Bürgermeisterposten der französischen Hafenstadt die zentrale Rolle, sondern viele weitere Nebenhandlungen weisen explizit auf das Motiv der Rache hin. Taros Frau Rachel will sich an ihrem Ehemann rächen, da dieser als Bürgermeister keine Zeit für die Familie und ihre Ehe hat. Darüber hinaus gibt sie ihm die Schuld an ihrer degenerativen Krankheit. Der enttäuschte Eric sucht Rache an Sélim, da dieser eine romantische Beziehung mit Julia beginnt. Am Wahltag kommt es zu einer Schlägerei zwischen den beiden, in deren Verlauf Eric Sélim mit einer Schere ersticht.

\section{Französischer Machiavelli}

Die handelnden Personen nehmen quasi nie ein Dilemma zwischen Moral und Macht wahr. Herrschaftsansprüche und absolute Machtorientierung stehen in einem übertriebenen Maße über jeglichen Moralvorstellungen. Der politische Kontrahent soll bekämpft und besiegt werden - ohne Rücksicht auf Verluste. Man kann den politischen Akteuren daher durchaus unterstellen, sich an den politischen Ideen von Niccolò Machiavelli orientiert zu haben (siehe hierzu auch 
das Kapitel „Spiegel“ in der Serienanalyse zu House of Cards). Doch selbst Machiavelli würde ein solch eindimensionales Streben nach Macht nicht erklären können - hier wird eine besonders zynische Sicht auf die Politik in der französischen Kommune zum Ausdruck gebracht. Diese gnadenlose Fokussierung auf den Machterwerb macht auch nicht Halt davor, die Familien und Freunde des Kontrahenten in die politische Auseinandersetzung um das höchste Amt der Stadt mit einzubeziehen. Im Gegenteil, Barrès taktiert bewusst mit der mentalen Situation von Taros Frau, um die Eheleute gegeneinander auszuspielen und so die Wahl für sich zu entscheiden. Eine zentrale Szene der ersten Staffel ist das Aufeinandertreffen der beiden Kontrahenten, Barrès und Taro, die beispielhaft darstellt, welche Rollen Familie und Macht in der Serie spielen:

\section{$\underline{\text { Szenen-Beispiel: }}$}

Barrès und Taro stehen auf dem Balkon von Barrès' Penthouse. Taro hat zuvor erfahren, dass er der leibliche Vater von Barrès ist. Im Hintergrund erkennt man den Hafen von Marseille (S 1/F 5/37.30 - 37.55).

Barrès: Inzwischen habe ich es verstanden. Für dich gibt's nur eins, das ist Marseille. Das zählt mehr als deine Familie.

Taro: Warst du deswegen gegen den Verkauf des Piers?

Barrès: Einmal musste ich anfangen, dein Gegner zu sein.

Taro: Das war aber zu früh. Einen Monat danach wäre dir Marseille in den Schoß gefallen.

Barrès: Das wäre zu leicht gewesen.

Taro: Ich gebe dir die Macht und du nimmst nicht an?

Barrès: Die wahre Macht bekommt man nicht geschenkt, wahre Macht muss man sich erobern. Ich werde dir Marseille entreißen und am Ende bist du einsam und allein.

\section{Aura der Männermacht}

Die politische Elite in der Serie Marseille wird von Männern dominiert. Die Bürgermeisterwahl ist zugespitzt auf den Zweikampf zwischen Barrès und Taro. Frauen spielen in der Politik der französischen Hafenstadt nur eine untergeordnete Nebenrolle. Taros Frau Rachel verfällt in eine starke Depression und die Rolle seiner Tochter Julia entwickelt sich nur sehr schwach von einer spätpubertären jungen Frau zu einer politischen Beraterin des amtierenden Bürgermeisters. Wie selbstverständlich bekommt sie diese Tätigkeit allein aufgrund ihrer Rolle als Tochter. Als einzige Ausnahme in diesem patriarchalen Geflecht kann die Regionalpräsidentin Vanessa D'Abrantes angesehen werden. Sie hat ein bedeutsames politisches Amt und versucht im Laufe der Serie, die Macht der Partei 
$U P M$ auf zentralstaatlicher Ebene an sich zu reißen. Hervorzuheben ist an dieser Stelle jedoch, dass nicht nur einmal herausgestellt wird, wie D'Abrantes an ihr Amt gekommen ist. Mehrfach wird betont, dass sie dieses allein ihrem Status als Ehefrau des vorherigen Regionalpräsidenten zu verdanken und es auch direkt von ihm übernommen hat. Darüber hinaus verweist ihre sexuelle Beziehung zu Barrès darauf, auf welche Art und Weise eine Frau in Marseille politisch erfolgreich sein kann. Insgesamt werden Frauen in der Serie als Begleiterinnen der männlichen Machthaber und Strippenzieher dargestellt. Eigene Kompetenzen können Frauen im besten Falle unterstellt werden. Die Männer beherrschen nicht nur Marseille, sondern auch die Frauenwelt. Beispielhaft wird dies durch Barrès' Verhalten in der ersten Folge der Staffel gezeigt. Er streicht uneingeschränkt jeder Frau, der er in der ersten Folge begegnet, zur Begrüßung durch das Haar und bewundert mit übertriebenem Staunen ihre Schönheit. Außerdem wird der Mafiaboss Cosini immer von einer blonden Frau begleitet, die in den entscheidenden Dialogen jedoch von den Gesprächen ausgeschlossen wird.

Darüber hinaus muss erwähnt werden, dass keiner der machtvollen Männer in Marseille einen Migrationshintergrund hat und alle eine weiße Hautfarbe haben. Diese Stereotypisierung könnte unerwähnt bleiben, allerdings haben alle Menschen mit Migrationshintergrund in der Serie Verbindung ins kriminelle Milieu oder sind selbst kriminell. Dieser inszenierte ,Kampf der Kulturen“ prägt dabei nicht nur die Politik in Marseille, sondern das gesamte Stadtleben.

Weiße Machos regieren die zweitgrößte Stadt Frankreichs und Frauen kommen nicht über ihre Rolle als sexualisierte Statistinnen hinaus. Ob dies auf eine spezifisch-französische Form der politischen Kultur hindeuten soll oder als realistische Darstellung der Kommunalpolitik in Marseille zu deuten ist, bleibt ungewiss. Für das Modell der männlichen Herrschaft, welche durch die Serie Marseille dargestellt wird, passt sehr treffend die Analyse des französischen Soziologen Pierre Bourdieu: „Sie ist gleichermaßen [...] in der ganzen sozialen Welt und [...] in den Körpern, in dem Habitus der Akteure präsent, die als systematische Schemata der Wahrnehmung, des Denkens und Handelns fungieren“ (Bourdieu 2005: 19-20).

\section{Kriminalität und Politik}

Marseille soll nach dem Willen der Hauptcharaktere das Tor zum Mittelmeer werden und sich zu einer internationalen Finanzmetropole, ähnlich wie Shanghai, entwickeln. Ein neues Casino und Luxushotels werden gebaut, um die Stadt in neuem Glanz erstrahlen zu lassen. Das sind zumindest die Pläne des amtierenden Bürgermeisters Taro. Er hat diese Rechnung allerdings ohne den Verrat seines Vertrauten Barrès gemacht. Dieser verbündet sich mit dem Mafiaboss Cosi- 
ni, der verhindern möchte, dass die bisherigen Einnahmen der Mafia aus den Spielhallen gefährdet werden. Das Verhältnis zwischen politischen Akteuren und der organisierten Kriminalität wird in der Serie als sehr eng dargestellt. Entweder haben Politiker direkte Beziehungen zur Mafia oder sind auf die eine oder andere Art selbst kriminell. Insbesondere Barrès unterhält nicht nur zweifelhafte Verbindungen zu kriminellen Strukturen, sondern nutzt diese auch in hohem Maße im Bürgermeisterwahlkampf.

\section{$\underline{\text { Szenen-Beispiel: }}$}

Cosini beobachtet das Fußballtraining seines Neffen. Barrès erscheint und fordert mehr Unterstützung durch Cosini im Wahlkampf (S 1/F 4/37.30 - 37.55).

Cosini: Hast du das jetzt gesehen? Da, mein Neffe, der wie ein Volltrottel spielt. Er sitzt bei Olympique nur auf der Ersatzbank, und ich hab' den Kerl einkaufen lassen, unglaublich teuer. Ich bin mit seinem Spielerberater befreundet, da wurde gemauschelt, ich hab' meine Provision. Ich will damit sagen, ich möchte nicht auf jemanden setzen, der mit seinem Arsch im Rathaus die Ersatzbank plattdrückt, nein, ich hoffe du schießt Tore.

Barrès: Du bist gut, ich hab' meinen Teil doch erfüllt. Ab jetzt musst du für mich spielen. Wenn ich nicht gewinne, wird das Casino gebaut und ein anderer Bürgermeister drückt dich platt.

Cosini: Okay, was brauchst du?

Barrès: Das hatten wir doch schon, Geld und Wahlhelfer.

Cosini: [...] Und dann hab' ich noch ein Geschenk für dich. Ich hab' doch gerade telefoniert, der Typ wird dir die Türen zu den nördlichen Vierteln öffnen.

Barrès beendet seine Beziehungen zur Unterwelt erst, als er bemerkt, dass er zum politischen Spielball zwischen D'Abrantes und Cosini geworden ist. Dies geschieht also weniger aus moralischen Beweggründen als aus dem Bewusstsein heraus, außer seinem Vater und letztendlichen Sieger der Wahl, Taro, keine Verbündeten mehr in der städtischen Politik zu haben. Dieser hat zwar weniger direkte Verbindungen zur Mafia, allerdings lässt sein bereits erwähnter Kokainkonsum ihn auch nicht als Schaf unter den Wölfen dastehen.

Des Weiteren ist Korruption ein großes Thema in der gesamten ersten Staffel. Nahezu jeder Politiker ist entweder in eine Korruptionsaffäre verwickelt oder wird dieser bezichtigt. Unterstellte Korruption stellt für die Akteure dabei ein wahltaktisches Element dar: „Im Wahlkampf wiegt ein Verdacht so schwer wie ein Beweis“" (S 1/F 6/15.20). 


\section{SERIE ALS NARRATIV - WAS ERZÄHLT MARSEILLE ÜBER POLITIK?}

In der Politik lässt sich in vermehrter Form eine Hinwendung zu erzählerischen Formen erkennen. Die Begrifflichkeit Narrativ hat dabei in den vergangenen Jahren einen deutlichen Bedeutungszuwachs erfahren. „Der gemeinsame Nenner liegt in der Annahme, dass Sprache nicht nur im Prozess der Politikvermittlung bedeutend ist, sondern viel grundsätzlicher als elementares Medium des Weltverstehens und Weltveränderns funktioniert." (Gadinger/Jarzebski/Yildiz 2014: 3) Die Narrativforschung spielt bei der Serienbetrachtung demnach eine zentrale Rolle:

\section{Politik befindet sich allzu oft im Spannungsfeld zwischen Arm und Reich}

Während die Politiker, als Sinnbild der Oberschicht, die reichen Stadtteile Marseilles bewohnen, verkommen die cités immer mehr. Heruntergekommene Häuser, Bandenkriminalität und allgemeine Gewalt dominieren den Alltag der ärmeren Bevölkerung in diesen Teilen der Stadt. Ein vermeintliches Bindeglied zwischen Arm und Reich schafft dabei der Wahlkampf zwischen Lucas Barrès und Robert Taro. Die Bürger der cités sind zwar, laut eigenen Aussagen, während der Legislaturperiode nur begrenz relevant, für den Wahlkampf beziehungsweise den Wahlsieg aber unverzichtbar. Beide Kontrahenten bemühen sich um Stimmenfang. Dabei agiert die Politik nicht immer im rechtlichen Rahmen. Lucas Barrès setzt auf die Unterstützung Farids. Bandenmitglieder sollen die Bewohner der cités überzeugen, für Barrès zu votieren:

\section{Szenen-Beispiel:}

Farids Bandenmitglieder betreiben Wahlkampf in diversen heruntergekommenen Häusern der cité. Sie klingeln an einer Wohnungstür, eine Frau öffnet (S 1/F 6/22.38 - 22.46).

Bandenmitglieder: Sie müssen Lucas Barrès wählen. Wir schicken Ihnen ein Taxi zum Wahllokal am Sonntag, und im Taxi sagt man Ihnen, wie man wählt. Brauchen Sie irgendetwas?

Da viele cité-Bewohner von der Politik und insbesondere den Politikern enttäuscht sind, scheinen sachliche Argumente unangebracht, weshalb mit neuen Elektrogeräten oder Geld gelockt werden soll.

Beide Gruppierungen, Arm und Reich, kommen nur selten in Kontakt miteinander. Politiker scheinen abgehoben von den unteren Gesellschafsschichten zu 
agieren. Die Beziehung zwischen Julia Taro und Sélim stellt dabei eine Seltenheit dar. Diese personifizierte Verbindung von Arm und Reich ist scheinbar die einzige, die nicht auf einen speziellen Nutzen abzielt:

\section{Szenen-Beispiel:}

Sélim wurde durch einen Schuss von Eric verletzt. Zu Hause wartet Julia auf ihn. Sie verarztet seine Wunde (S 1/F 5/13.00 - 13.06).

Julia: Was bleibst du auch in der cité? In dieser scheiß Vorstadt?

Sélim: Wo soll ich denn hin? Sag's doch, wo soll ich denn hin?

Ihre Unterschiede sind den beiden Protagonisten dabei durchaus bewusst, dennoch verschweigt Julia Sélim ihre Herkunft und klammert ihr wohlsituiertes Dasein zu Beginn wissentlich aus. Gegenüber ihrem Vater erwähnt sie die Beziehung erst zu einem späten Zeitpunkt.

Als stilistische Untermalung dieses Gegensatzes dienen die Musikstile Hip Hop und Klassik. Rachel Taro ist professionelle Cellistin. Der Alltag der Familie des Bürgermeisters wird somit zwangsläufig oft durch klassische Musik begleitet. Das Leben in den cités hingegen wird vom Musik- und Lebensstil Hip Hop dominiert (Beispiel: S 1/F 1/15.40 - 16.33).

\section{Die politische Ebene ist durch wenig Rotation, viel Stagnation geprägt}

Seit mehreren Jahren gab es keinen Bürgermeisterwechsel in Marseille. Barrès und Taro verkörpern dabei zwei unterschiedliche Generationen. Während der ,alte“ Taro schon seit vielen Jahren Bürgermeister Marseilles ist, eine Familie hat und ein Haus besitzt, stellt der ,junge‘ Lucas Barrès vielmehr das freie, ungebundene Pendant zu ihm dar. Die Gegensätzlichkeit alt gegen jung zieht sich durch die gesamte Serienstaffel. Sowohl die Ablehnung des Casino-Projekts als auch der Antritt Barrès' als Gegenkandidat Taros bei der Bürgermeisterwahl wird hiermit, zumindest in Barrès' offizieller Ankündigung, begründet:

\section{Szenen-Beispiel:}

Lucas Barrès steht vor einem Spiegel in seinem Büro, hält einen Monolog (S 1/F 8/2.57 3.15).

Lucas: Ich weiß, Herr Bürgermeister, Sie haben mich unter Ihre Fittiche genommen, aber irgendwann muss der Vogel das Nest verlassen. Natürlich ist mir bewusst, was Sie für unsere Stadt getan haben, aber auch was Marseille 
noch fehlt und was Sie nicht erreichen werden. Ein Mann Ihrer Generation kann nicht begreifen, wie die sozialen Netzwerke die Welt verändert haben.

Der Tenor ,die Politik ist zu alt" wird von Barrès genutzt, um effektiven Wahlkampf zu betreiben.

\section{Politik bedeutet einen schmalen Grat zwischen Freund- und Feindschaft}

Nahezu jeder Hauptcharakter der Serie durchlebt die Entwicklung Freund versus Feind am eigenen Leib. Barrès ist dabei der einzige, der sich scheinbar bewusst für den Seitenwechsel entscheidet. Während er zu Beginn der Serie ein Freund der Familie Taro ist, kapselt er sich frühzeitig ab und wird zu ihrem politischen Feind. Dabei betont er immerzu, dass sich sein Handeln lediglich gegen Robert Taro, nicht gegen dessen Familie richte. Dass sein Plan aufgeht, zeigt sich daran, dass Rachel Taro Lucas' Handeln an vielen Stellen der Serie in Schutz nimmt. Darüber hinaus sucht Rachel Lucas' - auch körperliche - Nähe und besucht ihn sogar privat.

Auch Julia Taro macht derartige Erfahrungen. Für Eric ist die Freundschaft zu Julia nicht rein platonischer Art. Im Verlauf der Serie unternimmt er immer wieder Versuche, Julia für sich zu gewinnen, die sie aber immerzu unterbindet. Als er letztlich von der Beziehung zwischen ihr und Sélim erfährt, bringt Eric seinen ehemals besten Freund im Affekt um.

\section{Traue niemandem in der Politik}

Der Vertrauensbruch zwischen Barrès und Taro verdeutlicht die Notwendigkeit ehrlicher Beziehungen. Während Taro sich zu Beginn der Serie als Bürgermeister in der Machtposition befindet, sein Amt wissentlich und freiwillig abzulegen, manövriert Barrès' Intrige ihn in eine Art Ohnmachtssituation. Taro fühlt sich dazu genötigt, erneut um das Amt des Bürgermeisters zu kämpfen, um dieses nicht, wie einst angedacht, an seinen Ziehsohn abzugeben. Derartige Ohnmachtsgefälle lassen sich auf die gesamte Familie Taro übertragen. Rachel Taro wird im Laufe der Serie mit ihrer Krankheit konfrontiert, die sie dazu zwingt, ihre größte Leidenschaft, das Cellospielen, zu beenden. Obwohl ihr Mann die Diagnose bereits kennt, verschweigt er sie zu Beginn. Julia Taro muss ,ohnmächtig' zusehen, wie Eric Sélim ermordet. 


\section{Personifizierung: Politik als Vollzeitjob, ohne Zeit für Familie und Freunde}

\section{Szenen-Beispiel:}

Julia und Robert betreten ihr Haus. Sie streiten sich im Wohnzimmer. Rachel hört aus dem Nebenzimmer zu (S 1/F 3/12.25 - 12.35).

Julia: Du hast Marseille immer vorgeschoben, um dich nicht um uns kümmern zu müssen.

Robert: Nein, ich brauche euch doch und Marseille braucht mich. Ich kann mich weder von dem einen noch von dem anderen trennen.

Diese Personifizierung der Stadt Marseille führt dazu, dass der Zuschauer den Eindruck erhält, die Stadt würde aktiv in soziale Konstrukte wie auch in das politische Geschehen eingreifen. Taro wird in diesem Zusammenhang von seiner Familie beschuldigt, sich mehr um Marseille als um alles andere zu sorgen. Marseille wird dabei hauptsächlich durch Machtbeziehungen dominiert. Rein zwischenmenschliche Beziehungen und Handlungen stellen eine Seltenheit dar. ,Ehrliche Verbindungen“, wie die zwischen Taro und seiner Familie oder die zwischen Julia und Sélim, werden durch den Handlungsverlauf entweder zerstört oder aber zerbrechen im Verlauf der Serie zumindest teilweise. Dieser ,Schatten der Macht' lässt sich vor allem auf Barrès übertragen. Er befreit sich zu Beginn der Serie von seiner Ziehfamilie, um offiziell seine politische Karriere voranzutreiben, inoffiziell seinen Racheakt zu vollziehen.

\section{Sexualisierung: Männer dominieren die politische Landschaft}

Wie bereits dargestellt, porträtiert die Serie Marseille Politik als eine klare Männerdomäne. Frauen spielen in der Serie generell eine deutlich untergeordnete Rolle. Außer der Regionalpräsidentin Vanessa D’Abrantes bekleidet keine Frau eines der höheren Ämter, die in der Serie von Bedeutung sind. Selbst in diesem Zusammenhang gilt es zu betonen, dass ihr Aufstieg sowohl durch sexuelle Handlungen als auch ihren erfolgreichen Mann begründet wird. Frauen sind in der Politik in erster Linie eine Art Spielball der mächtigen Männer. Sie dienen sowohl der Befriedigung der männlichen Lust als auch möglicher Informationsbeschaffung. Insbesondere der junge Barrès fasst Frauen, an denen er interessiert zu sein scheint, als wiederholendes Muster in die Haare. Nachdem er sich die Informationen beschafft hat, schläft er mit ihnen. Dabei geht es um den reinen Machtgewinn: Sex wird als Mittel zum Zweck verstanden, was auch an den homoerotischen Annäherungsversuchen Barrès' deutlich wird: 


\section{Szenen-Beispiel:}

Lucas Barrès und Pharamond treffen sich in der Dusche eines Schwimmbads, nachdem Lucas ihn abgepasst hat (S 1/F 4/6.08 - 6.46).

Lucas: Ich denke immer noch an Ihre Ansicht über Orson Wells, die Weiblichkeit in den Künsten. Entdecken Sie Weiblichkeit bei Picasso?

Pharamond: Aber natürlich, ja. [...]

Lucas: Die Frauen, die Frauen, die sind nicht das, was mich am meisten anzieht.

Pharamond: Ich spreche von der Kunst.

Lucas: Finden Sie es nicht abartig, dass wir so Schwanz an Schwanz über Picasso reden? [Barrès streicht über den ganzen Körper seines Gegenübers]

Pharamond: Nein, nein. [verschwindet schnell]

\section{ZUSAMMENFASSUNG}

Der Kontext der Serie Marseille greift überaus detailgetreu die realen Zustände der Stadt auf. Eine so genaue Darstellung der politischen und sozialen Lage, des kulturellen und gesellschaftlichen Rahmens bis hin zur genauen Übernahme des Namens der Marseiller Tageszeitung La Provence legt den Verdacht nahe, dass mittels der Serie an genau diesen Zuständen und der Verantwortung der Politik daran Kritik geübt werden soll.

Die Serie bleibt, was politikwissenschaftliche Theorien und Wirkungszusammenhänge angeht, in ihrer Darstellung relativ simpel. Ein Steuerungstrilemma zwischen verschiedenen Arenen wird nicht aufgegriffen. Der Großteil des politischen Prozesses findet im Sinne der Mediendemokratie in der öffentlichen Arena statt, dabei werden die Medien jedoch instrumentalisiert und als filterloses Sprachrohr für politische Botschaften genutzt. Der Wahlkampf bezieht sich hauptsächlich auf das Verteilen von Flyern und Plakaten sowie auf das bürgernahe Auftreten der Kandidaten. All das wird eingebettet in eine allgegenwärtige Atmosphäre der Informalität und Intransparenz, welche fließend ins Kriminelle übergleiten. Inhalte, Policies oder reguläre politische Prozesse, wie das Erlangen von Mehrheiten in der parlamentarischen Arena, werden kaum angedeutet. Was bleibt, ist ein für die heutige Zeit übersimplifiziertes Bild von Politik, welche ohne die komplexen Strukturen einer pluralistischen Gesellschaft oder von Governance-Netzwerken auskommt. Dafür beschränkt Marseille sich auf den Personenkult um die beiden Kandidaten und deren illegale Machenschaften.

Die politische Kultur in der französischen Hafenstadt ist geprägt durch machtbesessene und nach Rache dürstenden Machos. Die politischen Akteure versuchen in einem übertriebenen, machiavellistischen Stil, die Macht über das Rathaus an sich zu reißen, und haben dabei vor allem im Sinn, ihre Kontrahenten 
zu besiegen. Die Verbindungen zur organisierten Kriminalität sind eng, werden insbesondere im Wahlkampf ausgiebig genutzt und stellen aus Sicht der politischen Klasse ein legitimes Mittel zum Machterwerb dar. Normen und Werte werden in politischen Entscheidungen gänzlich ausgeklammert. Frauen kommen über eine Nebenrolle als sexualisierte Statistinnen nicht hinaus.

Das politische Narrativ in Marseille ist durch eine überwiegend negativ konnotierte Erzählart geprägt: Politiker regieren aus ihrem ,Elfenbeinturm ' heraus über die Köpfe der einfachen Bürger hinweg. Schenkt man dieser Darstellung von Politik Glauben, so ist der politische Alltag vor allem durch Informalität, Einsamkeit und Rache geprägt. Vorwiegend alte Männer, die seit Jahrzehnten Teil des politischen Geschäfts sind, bestimmen über Sachverhalte, die sie selbst nur wenig tangieren. Frauen werden vor allem , genutzt ${ }^{\circ}$, reale zwischenmenschliche Beziehungen existieren nur marginal. Auch wenn einige der aufgeführten Darstellungen auf die reale politische Landschaft zutreffen mögen, skizziert die Serie Marseille eine durchweg negative, an vielen Stellen deutlich überzogene Erzählart von Politik.

\section{LITERATUR}

Bourdieu, Pierre (2005): Die männliche Herrschaft, Suhrkamp, Frankfurt am Main.

Duplantier, Ursula (2013): Marseilles radikales Facelifting, in: Deutsche Welle Online vom 8.1., http://www.dw.com/de/marseilles-radikales-facelifting/a16426804 (zugegriffen am 1.3.2017).

EPA Euromeditérannée (2017): Introduction, in: http://www.euromediterranee. fr/districts/introduction.html?L=1 (zugegriffen am 2.3.2017).

Fishman, Robert B. (o.J.): Marseille ist Kulturhauptstadt 2013, in: http:// www.merian.de/europa/frankreich/marseille/artikel/marseille-ist-kulturhaupt stadt-2013 (zugegriffen am 1.3.2017).

Gadinger, Frank/Jarzebski, Sebastian/Yildiz, Taylan (2014): Politische Narrative. Konturen einer politikwissenschaftlichen Erzähltheorie, in: dies. (Hrsg.): Politische Narrative. Konzepte - Analysen - Forschungspraxis, Wiesbaden, 3-38.

Grimm, Dieter (2003): Lässt sich die Verhandlungsdemokratie konstitutionalisieren?, in: Claus Offe (Hrsg.): Demokratisierung der Demokratie. Diagnosen und Reformvorschläge, Frankfurt.

Kempf, Udo (2017): Das politische System Frankreichs, 5. Aufl., Wiesbaden.

Korte, Karl-Rudolf/Fröhlich, Manuel (2009): Politik und Regieren in Deutschland. Strukturen, Prozesse, Entscheidungen, Paderborn. 
Krekeler, Elmar (2016): Vor dieser Serie muss die ARD keine Angst haben, in:

Welt Online vom 13.5., https://www.welt.de/kultur/article155307617/Vordieser-Serie-muss-die-ARD-keine-Angst-haben.html (zugegriffen am 3.3. 2017).

Lehnartz, Sascha (2014): Der kuriose Kampf um das Rathaus in Marseille, in: Welt Online vom 28.3., https://www.welt.de/politik/ausland/article126 333429/Der-kuriose-Kampf-um-das-Rathaus-von-Marseille.html (zugegriffen am 2.3.2017).

Liehr, Günter (2013): Marseille. Portrait einer widerspenstigen Stadt, Zürich.

Meyrowitz, Joshua (1990): Überall und nirgends dabei. Die Fernsehgesellschaft, Weinheim.

Münkler, Herfried (2006): Niccolò Machiavelli, der Fürst, in: Brocker, Manfred (Hrsg.): Geschichte des politischen Denkens, Frankfurt am Main, S. 108122.

Schild, Joachim/Uterwedde, Henrik (2006): Frankreich. Politik, Wirtschaft, Gesellschaft, 2., aktual. Aufl., Wiesbaden.

Schulz, Winfried (1997): Politische Kommunikation. Theoretische Ansätze und Ergebnisse empirischer Forschung zur Rolle der Massenmedien in der Politik, Opladen.

Simon, Klaus (2014): Marseille, 2., aktual. Aufl., Ostfildern.

Ulrich, Stefan (2012): Favela am Mittelmeer, in: Süddeutsche Zeitung Online vom 7.9., http://www.sueddeutsche.de/panorama/drogenkrieg-in-marseille-favelaam-mittelmeer-1.1461454-2 (zugegriffen am 1.3.2017).

Wiegel, Michaela (2011): Spielend in die Kriminalität, in: Frankfurter Allgemeine Zeitung Online vom 6.10., http://www.faz.net/aktuell/marseille-spielendin-die-kriminalitaet-11484496.html (zugegriffen am 1.3.2017).

Wiegel, Michaela (2012): Stadt des Verbrechens, in: Frankfurter Allgemeine Zeitung Online vom 27.9., http://www.faz.net/aktuell/politik/europaeischeunion/marseille-stadt-des-verbrechens-11904925.html\#lesermeinungen (zugegriffen am 1.3.2017). 



\title{
Gomorrha
}

\section{Mafia und Staat im Verhältnis ${ }^{1}$}

\author{
Taylan Yildiz
}

\section{EINLEITUNG}

Der Begriff ,Mafia‘ bezeichnet einen Ordnungstypus, der sich durch zwei Kernprinzipien auszeichnet. Das sind a) die totale Hingabe zur Familie, die zur Sippe ausgedehnt ist und feudal regiert wird und b) das unerbittliche Konkurrenzverhältnis, das deren Mitglieder zu den Repräsentanten und Vollzugsorganen staatlicher Gewaltmonopole unterhalten. Erst beide Prinzipien machen die Mafia zu dem, was wir kennen und allgemeinhin zu fürchten gelernt haben.

Im Zentrum unserer Kenntnis über die Mafia steht damit eine Unerbittlichkeit, die auf zwei Weisen wirkt. Zunächst fungiert sie als Distinktionsmerkmal. Im Gegensatz zu allen anderen Formen menschlicher Vergemeinschaftungen zeichnen sich mafiöse Ordnungen vor allem dadurch aus, dass Regelverstöße rücksichtslos verfolgt werden und unweigerlich Eigenjustiz hervorbringen. Unerbittlichkeit dient aber nicht nur der Kohäsion. Sie prägt darüber hinaus auch das Konkurrenzverhältnis zum Staat, der - wenngleich mit unterschiedlichen Mitteln und Idealen - doch auf derselben ontologischen Ebene den Anspruch über die letztinstanzliche Sozialbindung des Individuums erhebt und sich durch dieselbe paternale Ikonologie konstituiert. Zwar fällt nicht jedes Gesellschaftsmitglied gleichermaßen in die sich gegenseitig ausschließenden Hoheitsbereiche von Staat und Mafia. Aber jedes Familien- und Clanmitglied ist immer zugleich auch ein Mitglied staatlicher Ordnungen, mögen diese noch so prekär oder fragil sein.

1 Für hilfreiche Hinweise danke ich Carlo Latilla, Paolo Rastiello, Paolo Staiano, Niko Switek und Frank Gadinger. 
Dieser Beitrag behandelt durch die Perspektive von Gomorrha - Die Serie (Sky Italia) einige der wichtigsten Überlappungen von Staat und Mafia, sowohl in prinzipieller als auch in praktischer Hinsicht. Dem liegt die These zugrunde, dass in der Mafia Prinzipien und Praktiken hochgetrieben werden und damit in geradezu idealtypischer Weise vorliegen, die in abgeschwächter Form auch den Staat anleiten. Damit soll der Staat keineswegs delegitimiert werden. Es soll nur durch das Medium einer Fernsehserie jene Seite seiner ,janusköpfigen` Existenz belichtet werden, in der sein dunkles Machtpotenzial ruht. Nach einer kurzen Skizze meines theoretischen Verständnisses der Fakt-Fiktion-Relation werde ich mich mit zwei ${ }^{2}$ Prinzipien und ihren Praktiken befassen, durch die sich eine solche Überlappung begründet und eine limitische Zone zwischen Staat und Mafia schafft: Das ist erstens das Leitbild der Familie als elementarer Sozialcode und zweitens das Oberhaupt als höchste Entscheidungsmacht.

\section{SERIEN ALS MITTLER ZWISCHEN FAKT UND FIKTION}

Das Denken über das Verhältnis von Fakt und Fiktion unterliegt in weiten Teilen der Sozialwissenschaften den Prämissen des kartesischen Dualismus. Demnach gründet sich das Faktische in der leiblichen Seinsweise des Menschen, während das Fiktive als jenes Produkt seiner seelischen Bemühungen begriffen wird, das den größtmöglichen Entfremdungsgrad von seiner leiblichen Existenz erreicht. Fakten sind demnach Gegenstand exakter Wissenschaften, werden ,erhoben' oder ,vermessen', wohingegen Fiktionen als Resultate rein schöpferischer Tätigkeiten gelten, die nicht $g e-$, sondern erfunden werden und damit eine von den Fakten differente Seinsweise aufweisen. Aus dieser Spaltung der Episteme wurde lange der Vorrang des Faktischen abgeleitet und als eine der wichtigsten Errungenschaften des neuzeitlichen Weltverständnisses ausgewiesen. Der Irrsinn, der sich durch die großen Utopien im 20. Jahrhundert ausbreitete, hatte zur Stabilisierung dieser Einschätzung nicht unwesentlich beigetragen. Heute aber wird das Verhältnis von Fakt und Fiktion differenzierter eingeschätzt und es wird nicht zuletzt durch die verfassungsrechtlichen Zusammenlegungen beider Episteme (in Deutschland in Art. 5 GG etwa) auch politisch eingestanden, dass sie in der Substanz zwar unterschiedlich, in ihrer ordnungspolitischen Relevanz aber als gleichwertig zu behandeln sind. Wenn das Fiktionale im Dienst der Des-

2 Es lassen sich natürlich eine Reihe weiterer wichtiger Bezüge diskutieren, wie etwa die Tötung als die radikalste Form der Vermännlichung, Abschreckung und Bestrafung. 
truktivität stehen kann, ist es schließlich imstande, in das Faktische zu intervenieren, und es und besteht kein Grund zur Annahme, dass es auf diese Negativrolle festgelegt ist und nicht auch progressive Wirkungen entfalten kann. Seinen Ruhepol hat die Kontroverse über das Verhältnis von Fakt und Fiktion in dieser arbeitsteiligen Umdeutung des kartesischen Dualismus gefunden.

Aber auch dieses Verständnis ist trotz seiner unbestreitbaren Vorteile unstimmig geblieben. Besonders im Anschluss an jene wissenschaftstheoretischen Studien, die sich der Genealogie der neuzeitlichen Aufteilung der Welt in Leibund Seelenphänomene widmeten, ist man immer mehr dazu übergegangen, sich der gegenseitigen Bedingtheit von Fakt und Fiktion zuzuwenden. Offenbar ist die Erhebung oder Vermessung von Fakten ein weitaus schöpferischer Vorgang, als es der kartesische Dualismus zu erkennen erlaubt (Daston/Galison 2007), ebenso wie das Fiktive viel stärker im Realen verankert ist, als unter seinen Prämissen angenommen werden kann (Iser 1993). Die Plausibilität dieses Einwandes ist mit der Verbreitung konstruktivistischer Theorien gestiegen. Heute wird nur noch selten bestritten, dass das Verhältnis von Fakt und Fiktion (wie übrigens das zwischen Empirie und Theorie oder genereller noch: zwischen Zeichen und Bedeutung) nicht arbeitsteilig fixiert werden kann, sondern viel offener gedacht werden muss. Denn ebenso gut wie dem Faktischen nicht zu entkommen ist und selbst in der radikalsten Übersteigerung der Fiktion (Science-Fiction) Bestände des Realen erhalten bleiben, ist der Zugang zum Faktischen immer auch von den fiktiven Fähigkeiten abhängig, die der Mensch in sich trägt, um überhaupt erkennen und über das Erkannte kommunizieren zu können. ${ }^{3}$ Damit etwas als ,wirklich ‘ erfahrbar wird, muss es zuvor als ,möglich“ gedacht worden sein, ebenso wie das Wirkliche in seiner Aktualität immer wieder mit offenen Spielräumen zurechtkommen muss. Deshalb fordert Waldenfels (2002: 236) in seinen Überlegungen zu ,Fiktionalität‘ und ,Realität‘ einleitend:

„Genauso wie die Wirklichkeit ist auch die Möglichkeit als Implikat der Erfahrung zu bestimmen und nicht als Attribut, das wir einem erfahrbaren oder benennbaren Ding zusprechen."

Werden die Ko-Konstitution von Fakt/Fiktion und ihre ontologische Gleichwertigkeit akzeptiert, ist nach den Mittlern zu suchen, durch die sich ihre wechsel-

3 So etwa der Ost-West-Konflikt in Star Trek oder das NS-Regime in Charly Chaplins Der große Diktator. Deshalb werden neuerdings die Möglichkeiten des Einsatzes von Filmen und Serien in der Lehre erörtert, vor allem in den Internationalen Beziehungen. Siehe hierzu Heck/Schlag 2015. 
seitige Durchdringung vollzieht. Ein ,Mittler', so Latour (2010: 70), ist eine Entität, die mit transformativer Kraft ausgestattet ist, also etwas (Bedeutungen oder Elemente), das übermittelt werden soll, zu ,übersetzen, entstellen oder auch (zu) modifizieren“" vermag und so auf den ,Lauf der Dinge" einwirkt. Serien als Mittler zu betrachten, bedeutet also, ihnen eine generative Kraft zuzuschreiben. Sie führen damit zwar nicht unmittelbar an die Realität heran, wenn überhaupt sinnvollerweise von einer Realität, an sich' gesprochen werden kann. Aber sie bestärken doch eine Betrachtungsweise, wonach im Medium der Serie zumindest der Zugang zu den Realitätsdeutungen, die sozial bedeutsam sind, möglich ist. Dabei ist allerdings darauf zu achten, dass dies nicht auf alle Serien gleichermaßen zutrifft. Es kommt vielmehr auf die konkreten Produktionsbedingungen an, ob und wie genau nun das Faktische und Fiktionale aufeinander einwirken.

Als narrative Medien verknüpfen Serien Bilder zu Bilderfolgen und stimulieren durch Einstellungs- und Schnitttechniken ein audiovisuelles Erzählen, das sowohl das ,Reale' fiktional zu transzendieren vermag und dadurch idealtypische Zugänge in unbekannte Wirklichkeitsbereiche ermöglicht (ganz gleich, ob diese Zugänge nun aufklärerisch motiviert sind oder in Verblendungszusammenhangen stehen), als auch imstande ist, das Fiktionale vom Grund des Realen her aufzuspannen. Selbst, Traumwelten' sind über die Abgrenzung zur ,Realwelt" stets konstitutiv mit ihr verbunden und lassen sich in ihrer narrativen Entfaltung auch nie vollständig von ihr lösen. Das Reale kann wie in Alice im Wunderland (Lewis Carroll) etwa nicht nur als Ausgangspunkt einer Reise ins Unbekannte dienen, sondern auch als ein internes Differenzierungsmerkmal fungieren, durch das sich das Fiktionale als Fiktionales erkennbar macht. Und mehr noch: Die narrative Entfaltung von Traumwelten schafft Anlässe dafür, um umgekehrt auch über das Mögliche oder Unerkannte im Realen kommunizieren zu können. Denn die Einbildungskraft, so auch Flusser (1990: 116) ist „eine komplexe, absichtsvolle (,intentionale') Geste, mit welcher sich der Mensch zu seiner Lebenswelt einstellt".

Gomorrha - Die Serie beruht auf einem preisgekrönten Reportage-Roman ${ }^{4}$ von Roberto Saviano und folgt dem Typus der dokumentarischen Methode. Sie ist damit auch in ihren fiktionalen Momenten als realitätsverwandt einzustufen und wird deshalb gelegentlich als ,hyperreal' bezeichnet und dem Genre des

4 Gomorra: Viaggio nell'impero economico e nel sogno di dominio della camorra, 2006 bei Mondadori erschienen, 2007 von Mario Gelardi als Theaterstück uraufgeführt und 2008 unter der Regie von Matteo Garrone verfilmt. Gegenstand des vorliegenden Beitrages ist die erste Staffel der Serie. 
neuartigen global neo-noir (Cilento 2011) zugerechnet. ${ }^{5}$ Das hat vor allem damit zu tun, dass Saviano in der Produktion ,die Funktion des Auteurs“ (Weber 2015: 198) übernimmt und die Serie von seinem detektivischen Blick ${ }^{6}$ auf das opake Innenleben des organisierten Verbrechens profitieren lässt. Da dieser Blick mit Bilderfolgen im Stile des neorealistischen Noir-Stils ausgeführt wird, kann eine ungewöhnliche Realitätsnähe und narrative Stimmigkeit in der Darstellung der Handlungsabläufe und in der Figurenzeichnung entstehen. Hyperrealität wird allerdings nicht nur durch die Verankerung der Skripte in den ethnografischen Aufzeichnungen des Auteurs und durch die bevorzugte Bilderästhetik erzeugt. Auch die Rückwirkungen der Serie auf das Leben Savianos, der seit dem transmedialen Erfolg von Gomorrah bedroht wird und unter Personenschutz steht, und der Imageschaden, den die neapolitanische Vorstadt Casal di Principe als zentraler Handlungsort der Serie erlitten hat (Tzermias 2008), rechtfertigen die Bezeichnung.

Die Serie schafft es damit, wie in The Wire eine Storyworld zu entfalten, die zwischen dem Faktum und der Fiktion des organisierten Verbrechens vermittelnde Momente einschiebt, den Zuschauern dabei aber nicht die Art von Identifikationsmöglichkeiten bietet, die sie üblicherweise an den Erzählverlauf zu binden verspricht. Der Zuschauer wird selbst zum Schnittstellenphänomen und in seiner moralischen Beweglichkeit herausgefordert. Während Hauptfiguren wie Vito Corleone (Marlon Brando) in Der Pate stets von einer Aura der Coolness und Erhabenheit umgeben sind, die selbst in Krisensituationen nicht zu bröckeln vermag, wird in Gomorrha - Die Serie ,die Empathie der Zuschauer mit den Figuren gezielt unterlaufen“ (Weber 2015: 199) und das Bild einer brüchigen Welt gezeichnet, die moralisch ungreifbar ist und allein von existenziellen Funktions-

5 So hat Saviano jüngst in einem Interview auf die Frage, ob die Serie nicht auch Jugendliche inspirieren würde, geantwortet: „Bei den Vorarbeiten zur Serie haben wir in Neapel genau beobachtet, wie junge Camorra-Mitglieder sich kleiden, frisieren, wie sie sprechen. Wir bilden in ,Gomorrha“ nur die Wirklichkeit ab“ (FAS vom 28. Januar 2018). Da es unmöglich ist, die Frage auf einer Seite der Kontroverse aufzulösen - es ist bekannt, dass junge Gang-Mitglieder in Italien Figuren aus der Serie nachahmen (Cilento 2011) -, bringt sich in dieser Interview-Stelle die Mittler-Funktion der Serie gut zum Ausdruck.

6 Über Jahre hinweg hatte sich Saviano als Hafenarbeiter getarnt, so wichtiges Expertenwissen angeeignet und die Romanvorlage an ,realen ' Vorgängen ausgerichtet. Die erste Szene der Staffel spielt sich deshalb auch im Hafen von Neapel ab, einem Ort, in dem die Globalität und Lokalität des organisierten Verbrechens zusammenfällt. Zum detektivischen Blick siehe Boltanski 2015. 
erfordernissen vorangetrieben wird. In den ersten beiden Folgen ist es noch die Figur Ciro Di Marzio (Marco D'Amore), die inmitten einer unmoralischen Welt eine besonnene Perspektive auf das organisierte Verbrechen eröffnet und integer erscheint. Bevor der Zuschauer jedoch eine parasoziale Beziehung zu ihm aufbauen kann, zeichnet sich schon ein moralischer Degenerationsprozess ab, der nicht radikaler ausfallen könnte. Tony Montana beispielsweise wird in Scarface (Brian de Palma) in der moralisch prekärsten Situation seiner Verbrecherkarriere noch von Gewissensgründen überrumpelt und unterbricht das Attentat auf eine Zielperson, die überraschend von ihren Kindern begleitet wird, obwohl der Abbruch - und auch die spezielle Art des Abbruchs (das Töten des Attentäters) sein Leben kosten wird. Ciro aber wird das junge Mädchen ${ }^{7}$ brutal foltern, erschießen und anschließend verbrennen, nur um den Aufenthaltsort ihres Freundes Daniele zu erfahren, mit dem er ein gefährliches Geheimwissen teilt. Seinen Höhepunkt erreicht die ungebremste Gewaltbereitschaft in der letzten Folge der ersten Staffel, in der Ciro seine Familie als Lockvogel benutzt, nur um den zum Widersacher herangereiften Sohn des Bosses in eine Falle zu locken. Während in klassischen Mafiafilmen also noch so etwas wie eine universelle Moral oder Ehre bebildert wird, die dem organisierten Verbrechen vorausgeht und ihr Ausufern von innen begrenzen kann, wird eine solche Moral hier allenfalls angedeutet, fällt aber stets hinter die Frage nach der Effektivität jener Mittel, die einen strategischen Vorteil im Handlungsgeschehen versprechen. Diese Vorgehensweise dient nicht etwa dazu, den Zuschauer zum Innehalten zu bewegen, sondern einen irreversiblen Bruch zwischen den Figuren und den Zuschauern herbeizuführen (Weber 2015: 206). Die Serie bricht also mit den Outlaw-Helden insgesamt und kennt keine ,auf charmante Art gebrochenen Publikumslieblinge wie den HoodRobin-Hood Omar Little oder den netten Junkie Bubbles“ (Weber 2015: 206) aus The Wire, wenngleich die Serie immer wieder scharfe Übergänge zwischen die allgegenwärtigen Gewaltausbrüche und die moralisch entlastenden Alltagsszenen schiebt. Sie schafft vielmehr gerade durch diesen stetigen Perspektivenwechsel den ,Helden als solchen ' ab und kreiert ein neues Mafia-Genre, das von Gerhard Meier (2014) folgendermaßen beschrieben wird:

„Gegen Ende der ersten Staffel von Gomorrha ist jedoch endgültig klar, dass die Serie sich konsequent von den gängigen Mechanismen der Gangster- und Crime-Serie abwendet. Während das Publikum von den verschiedenen Facetten der Camorra-Aktivitäten in

7 Angelehnt an den Fall der 22-Jährigen Gelsomina Verde, deren verkohlter Leichnam in einem Fiat 600 in einem neapolitanischen Vorort am 21. November 2004 aufgefunden wurde. 
einem von moralischen Richtwerten befreiten Taumel überrollt wird, fächert die Serie die undurchdringbare Verflechtung von krimineller Parallelgesellschaft und ,Normalität ‘ auf, die weit über die Stadtgrenzen Neapels hinaus wuchert. Doch weniger durch die faktische Anerkennung dieses Zustandes, sondern durch den erzählerischen, gegen die Gepflogenheiten des Crime-Kinos gebürsteten Umgang mit dieser Erkenntnis entledigt sich Gomorrha der Regeln des Genres.“

\section{DIE FAMILIE}

Es gehört zu den großen Inszenierungen des Liberalismus, dass ausgerechnet im modernen Staat der mächtige Widersacher der individuellen Freiheit ausgemacht wird. Die politische Relevanz dieser Gegenüberstellung mag nachvollziehbar sein. Aber entwicklungsgeschichtlich trifft sie in dieser Eindeutigkeit keinesfalls zu, weil es der moderne Staat war, der erstmals die individuelle Freiheit zur kollektiven Geltung bringen konnte und sich, lange bevor er dieses Recht durch Normierungen zu formen (und auch zu entstellen) begann, eher am Prinzip der Dynastie und der Sippe als am Individualismus gerieben hatte (Tilly 1986).

Diese Reibung äußert sich heute noch auf vielfache Weise. Immer dort, wo starke ,familien- oder sippenähnliche Strukturen' dominieren, stößt der Staat nicht selten auf massive Handlungsgrenzen; sei es, weil ihm reiche Familien

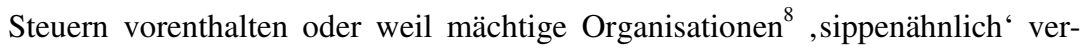
fasst sind und ihre Verstrickungen in illegale Vorgänge effektiv verbergen können. Das Loyalitätengeflecht der Familie wird aus der Perspektive des Staates deshalb auch abfällig, Vetternwirtschaft' genannt:

„Und die Verpflichtung zu unverbrüchlicher Treue gegenüber der eigenen Familie, einer Treue, die höher gilt als staatliche Gesetze und gegen die niemand verstoßen kann, ohne sich der Eigenjustiz dieser Familien zu überliefern, trägt umgangssprachlich einen italienischen Namen: Mafia.“ (Koschorke 2000: 119)

Das organisierte Verbrechen sticht in seiner Reibung mit staatlichen Autoritäten also besonders heraus (Collins 2011), eben weil es dem legitimen Blick des Staa-

8 Ein Beispiel unter vielen ist die FIFA, die stets mit Korruptionsvorwürfen konfrontiert wird, die nur schwer zu überprüfen sind, und es ist hier bezeichnend, dass sich Sepp Blatter in einem Interview mit der $B B C$ als ,the godfather of women's football“ bezeichnet hat: http://www.bbc.co.uk/programmes/p02qw29r (zugegriffen am: 1.2. 2018). 
tes nicht nur ethisch problematische Bereiche entzieht, sondern weil es den Staat in seiner Existenz negiert und ihm dadurch auf seltsame Weise ähnlich wird. Dass Staat und organisiertes Verbrechen funktional äquivalent sind und ein Verhältnis der Isomorphie aufweisen, hatte Tilly (1985) historisch belegt. Demnach war der moderne Staat in seinem frühen Stadium noch eine patrimonial geführte Organisation, die ihren Machtzuwachs durch Verbrechen garantierte, bevor er sich als bürokratische Herrschaftsform im Weber'schen Sinne legitimieren konnte. Gomorrha - Die Serie macht diesen Zusammenhang nun mikroanalytisch fassbar. Das lässt sich an zwei Aspekten verdeutlichen, die in der Serie immer wieder szenisch aufgegriffen und mit den ästhetischen Mitteln des global neonoir verarbeitet werden: (a) an der familiären Organisation der Mafia, die sich aus Kernfamilien speist, aber dennoch ein grundsätzlich ambivalentes Verhältnis zur Familie aufweist, und (b) an der korporalen Metaphorik, durch die sich die Mafia zu einem komplexen (staatsähnlichen) Herrschaftsgebilde ausdifferenziert.

(a) Die Familie ist ein Beziehungsgeflecht, das sich in unterschiedliche Ebenen mit jeweils eigenen Verweisungszusammenhängen und Handlungslogiken gliedert. Da ist zunächst die Ebene der ,Kernfamilie', die in der Mafia - ähnlich wie im Staat - ein durch repressive Maßnahmen herunterformatiertes Verwandtschaftssystem darstellt (Mutter-Kind-Einheit), in dem der männliche Versorger eine „schwankende Stellung“ (Koschorke 2000: 121) einnimmt. Die Serie greift diese liminale Position des Versorgervaters durch eine sehr gewählte Bebilderung der Mutter-Kind-Einheit auf. Denn sie wird nur dann szenisch aufgegriffen, wenn entweder die Alltäglichkeit des Verbrecherlebens dargestellt werden soll oder der Versorgervater es vermeiden will, dass sich die Gewalthandlungen seines Berufslebens auf seine Familie übertragen. Erst in der letzten Folge der ersten Staffel etwa wird Ciros Familie (Mutter-Tochter-Einheit) sichtbar, verbleibt aber in passiver Haltung und wirkt auf seltsame Weise subjektlos, weil Mutter und Tochter als Lockvögel vom Vater missbraucht werden und ihn dann gemeinsam verlassen. Eine etwas umfangreichere Schilderung der Kernfamilie wird in Folge 5 der ersten Staffel (Kleingeld) vorgenommen. Franco Musi (Antonio Zavaterri), der mailändische Finanzverwalter der Savastanos, gerät durch fehlgeschlagene Investitionen in eine verzweifelte Lage. Die intensivere Behandlung Musis Familienleben verdeutlicht nicht nur die mafiösen Verstrickungen des Finanzkapitalismus und deren lebensweltliche Folgen, sondern thematisiert grundlegender die abhängige Rolle der bürgerlichen Kernfamilie vom Berufsleben des Vaters. Musi begeht bezeichnenderweise auf der häuslichen Feier des 18. Geburtstages seiner Tochter Selbstmord, weil er weiß, dass die Savastanos ihren Geldverlust sonst durch das Töten seiner Familie begleichen werden (Le- 
ben als Währung). Auf dieser Ebene wird die ambivalente Rolle der Familie in Staat und Mafia erkennbar. Während sie einerseits immer wieder moralisch aufgeladen wird und Gegenstand tiefster Bekenntnis ist - auch die Mafia kennt die Witwenrente, der Familie darf es schließlich an nichts mangeln -, wird sie andererseits auf der Sollseite der Machtasymmetrie verbucht. Sie stiftet ,Nestwärme“, erlegt den Protagonisten aber damit Pflichten auf, bindet sie und ist nicht mehr als der wunde Punkt, der sie jederzeit angreifbar macht.

(b) Eine zweite familiäre Instanz besteht in der Organisation des Clans. Er ist familienähnlich strukturiert und unternimmt vom Ideal der Kernfamilie ausgehend die Ausdifferenzierung seiner Rollenkonstrukte. Diese Erweiterung vollzieht sich durch eine korporale Metaphorik, die es bereits dem modernen Staat erlaubt hatte, die einzigartige Bindungskraft familiärer Loyalitäten auf größere soziale Einheiten zu übertragen und so aus disparaten Einzelwillen eine Herrscherpersönlichkeit zu schaffen. So steht an der Spitze des Clans ein Oberhaupt, der als souveräner Hausvater über das Gesamtgeschehen wacht und als Kopf des Mafiakörpers mit der schwierigen Aufgabe konfrontiert ist, widerstrebende Teile durch das Familienband zusammenzufassen und die Handlungsprogramme der ökonomischen Akteure mit denen des Soldatentums zu verbinden. So muss sich Don Pietro Savastano (Fortunato Cerlino) stets in zwei Kreisen bewegen, die sich jeweils durch eigene Handlungslogiken auszeichnen und deshalb auch kaum berühren, wenngleich sie in einem ausgeprägten Verhältnis der Interdependenz zueinander stehen. Auf der einen Seite sind die , affektregulierenden` Geld- und Investitionsgeschäfte des Clans und seine Rechtsfragen, die im häuslichen Empfangsraum Don Pietros verhandelt werden und bis in die italienische Finanzmetropole Mailand reichen. Die Inneneinrichtung ist den Repräsentationsräumen barocker Paläste nachempfunden. Da der Raum aber auf die Größenordnung der umliegenden Wohnungen im Vele di Scampia reduziert ist, entsteht eine kitschige und „geschmacklose Opulenz“ (Meier 2014), die den Unterredungen eine ausgeprägte Schwere verleiht. Auf der anderen Seite stehen die ,affektmobilisierenden“ Drogengeschäfte des Clans. Sie werden von Soldaten überwacht und verwickeln den Clan immer wieder in kriegerische Auseinandersetzungen, die sich in den Bildern als Entladungen höchster Emotionalität präsentieren. Die Unterredungen hier finden fast ausschließlich in leerstehenden Lagerhallen statt und werden so von der beklemmenden Ästhetik einer gescheiterten Wirtschaftspolitik begleitet, die dem Clan nicht nur logistische Möglichkeiten verschafft, sondern mehr noch seine Bedeutung in der Region überhaupt erst mitbegründet. Der Zusammenhang der ökonomischen und soldatischen Handlungsprogramme, die auf jeweils unterschiedliche Weisen in den Geldkreislauf des organisierten 
Verbrechens involviert sind, lässt sich in drei aufeinanderfolgenden Szenen nachvollziehen:

\section{Szenen-Beispiel a:}

Don Pietro spricht mit seiner Frau Donna Imma (Maria Pia Calzone) und dem gemeinsamen Sohn Gennaro, kurz Genny (Salvatore Esposito), im Besucherraum des Gefängnisses. Es geht um die Haftbedingungen Don Pietros, um die Erschließung neuer Geschäftskontakte und um die Kapitalanlage des Clans in Mailand (S 1/F 3/ab 22.45).

Don Pietro: Mit Geld lässt sich alles regeln. [Donna Imma schwenkt die Haare.]

Don Pietro: Was hast du mit deinen Haaren gemacht?

Donna Imma: Nichts. Was meinst du?

Don Pietro: Du siehst noch wilder aus, wie eine Löwin.

[Donna Imma leise und lächelnd]

Donna Imma: Hör auf damit.

[Don Pietro konzentriert seine Blickrichtung auf Donna Imma.]

Don Pietro: Nein im Ernst.

[Der Gefängnisaufseher kündigt das Ende der Besuchszeit durch dreimaliges Klopfen an und ein Kuss zwischen Don Pietro und Donna Imma besiegelt das Gespräch.]

[Don Pietro wendet sich abschließend an Genny.]

Don Pietro: Du bist jetzt der Mann im Haus.

\section{Szenen-Beispiel b:}

Don Pietro in der Gemeinschaftszelle im Gespräch mit dem jungen Pasquale, der sich in der Essenszeit auf seine Verhandlung vorbereitet und um seine Erscheinung bangt (S 1/F 3/ab 23:58):

Don Pietro: Pasquali, was ist los?

Pasquali: Verzeihung Don Pie, aber heute ist meine Verhandlung und ich sehe aus wie ein Penner. [Er schaut in den Spiegel.] Mir steht die Strafe schon ins Gesicht geschrieben. Der Richter wird mich nicht mal hinsetzen lassen.

[Don Pietro reicht ihm ein frisches weißes Hemd, das ihm soeben mitgebracht wurde.]

Don Pietro: Hier. Zieh das hier an.

Pasquali: Nein, lassen Sie. Es wird schon gehen.

Don Pietro: Na los, zieh es an.

[Pasquali zieht das Hemd an.]

Don Pietro: Haaah, gut. So sieht ein ehrlicher Mann aus.

Pasquali: Danke, Don Pietro. Das Hemd sieht so verdammt teuer aus.

Don Pietro: Geld, macht ehrliche Männer aus uns, Pasquali. 


\section{Szenen-Beispiel c:}

Genny und Ciro überwachen das Auszählen von Drogengeldern in einem verdunkelten Raum und unterhalten sich (S 1/F 3/ab 25:23).

Genny: Was stinkt hier so?

Ciro: Die Junkies packen ihr Geld in die Unterhose. Glaubst du denn so was riecht nach Parfum? Aber wenn das Geld später in deiner Tasche steckt, stinkt's auf einmal nicht mehr so. Ist Tatsache.

[Das Geld wird in einem Fahrzeug versteckt, um unbehelligt nach Mailand zu gelangen.]

Die Szenenfolge lässt eine Reihe von Interpretationen zu, von denen ich hier nur eine aufgreifen möchte. Sie bezieht sich auf die beiden ,Geld-Aussagen“, denen zufolge Geld ,alles regelt' und , aus uns ehrliche Männer macht‘. Die Aussagen sind nicht etwa in ihrem faktischen Wert interessant, insofern neben dem Geld auch dem Töten ${ }^{9}$ die Fähigkeit beigemessen wird, , alles regeln` zu können, und Ehrlichkeit auch nicht das ist, wonach das organisierte Verbrechen primär bestrebt ist. Geld fungiert hier vielmehr als ein Symbol, durch das sich bestimmte Wahrheits- und Bedeutungseffekte herstellen lassen - etwa den, dass es als ,Verkleidungsmittel' dem organisierten Verbrechen erlaubt, in illegalen wie legalen Umgebungen gleichermaßen diskurs- und handlungsfähig zu sein. Wenn aus Drogengeld Finanzkapital wird und sich das Handlungsprogramm der Ökonomie mit dem des Soldatentums verbunden hat und sich gegenseitig verstärkt, hört das Geld im doppelten Sinne des Wortes auf zu stinken und wird universell einsetzbar (u.a. Korruption). Damit ist weder die Ökonomie eine notwendige Folge des Soldatentums, noch kann der ökonomische Erfolg auf das Handlungsprogramm des Soldatentums reduziert werden. Erst die Verknüpfung beider Logiken im Familienbild macht die Mafia zu einer souveränen und ordnungsbildenden Größe, also staatsähnlich.

\section{DAS OBERHAUPT}

Die Familie ist nicht nur Widersacher, sondern auch Leitbild des Staates. Die Nation wurde schließlich in dem Moment zum gemeinschaftsstiftenden Band moderner Staaten, als es sich der metaphorischen Bindungskraft der Familie bediente und so die sich territorial ausweitende Bevölkerung als Ganzheit vorstell-

9 Genny etwa wird durch das Töten zum ,Mann“ gemacht. Zunächst scheitert der Versuch in Folge 2 (Das erste Mal, ab 26.30) kläglich, geht aber dann mit noch größerer Wirkung auf (siehe unten). 
bar machte (Koschorke 2000). Diese Bindungskraft ist nach wie vor ungebrochen und markiert noch immer einen zentralen Teil der politischen Sprache (Lakoff 1996), was vor allem am juristischen Vokabular des Staates und seinen alltagssprachlichen Abwandlungen erkennbar wird, wo nicht zufällig von Gründungsvätern, Staatsoberhäuptern, Muttersprachen und Vaterländern gesprochen wird.

Zentral für die metaphorische Übertragung des Familienbildes auf den Nationalstaat ist eine paternale Konzeption von politischer Macht. Sie bevorzugt hierarchische Ordnungsgebilde, an deren Spitze das Oberhaupt als ein Hausvater steht, der ein umfassendes Wahrheitsprivileg über die Nation beansprucht und eine autoritäre Führung begründet. Koschorke fasst diesen Zusammenhang folgendermaßen zusammen:

„Während die vormoderne Welt ein Flickenteppich von Mächten war, die miteinander rivalisierten und nur zu häufig in offenen Streit miteinander gerieten, bahnt die bruchlose Übertragbarkeit von Vaterattributen durch alle sozialen Abstufungen hindurch einer Vereinheitlichung des Machtkörpers den Weg, die ihr immanentes Ziel im ,autoritären, alles umfassenden, inquisitorischen, alles fordernden Nationalstaat" finden wird.“ (in Anlehnung an Lawrence Stone, Korschorke 2000: 155)

Diese durch die Übertragung von Vaterattributen ermöglichte Vereinheitlichung des Machtkörpers bleibt jedoch stets prekär. Man kann die Machttechniken des Oberhauptes, seinen autoritären Führungsstil, etwa daran bemessen, wie es ihm gelingt, diesen Zustand unsichtbar zu halten. Weil er ihn nicht grundsätzlich überwinden kann, muss er die Brüchigkeit seiner Macht durch symbolische Investitionen und Gesten überschreiben. Aus dieser Tätigkeit ${ }^{10}$ bezieht er seine Macht und kann sie nur so aufrechterhalten. Das lässt sich an zwei Szenenfolgen diskutieren: erstens an jenem Szenenzusammenhang, in dem die Macht Don Pietros als kommunikative Macht dargestellt wird, die sich mechanischer Formeln bedient und unnachgiebig ist. Die kompromisslose Ansprache suggeriert Willenskraft und steigert damit aber auch Selbstzensur, womit zwar die Machtposition des Oberhauptes gefestigt wird, aber auch zugleich seine Führungskapazitäten erodieren. Seine Macht wird bestätigt, in den zentralen Machtfragen aber bleibt er auf sich selbst beschränkt und von den Möglichkeiten der Beratung ab-

10 Es handelt sich hier um die Übersteigerung oder Radikalisierung einer exekutiven Form der Legitimitätsgenerierung, wie sie in der postdemokratischen Konstellation (Crouch 2008, Körösényi 2015) in abgeschwächter Form durchaus zu beobachten ist (Yildiz et al. 2018). 
geschnitten. Zweitens ist hier auch jene Erzählfolge zu nennen, in der die Frage der Machtdelegation virulent wird, nachdem Don Pietro durch die Inhaftierung in seinen kommunikativen Kapazitäten beschnitten wird. Das zentrale Problem der Delegation liegt, wie die Szenenfolge verdeutlicht, vor allem darin, dass Machtübertragungen im Kontext autoritärer Führungskulturen meist nur sehr schwer zu kontrollieren sind (Prinzipal-Agent-Problematik) und jeder Versuch, einen Kronprinzen zur Macht zu befähigen, zugleich auch die Stärkung eines Rivalen befördert, dem der Vatermord irgendwann zur Option wird.

\section{Erste Szenenfolge}

Szenen-Beispiel a:

Ciro in einem Gespräch in Vele di Scampia mit seinem Mentor Attilio (Antonio Milo) nach einem Bombenanschlag auf ihn, den Ciro in einer Bar unverletzt überlebt hat (S 1/F $1 / \mathrm{ab} 17.57)$

Ciro: Drei Tote, und vielleicht sogar noch mehr, und das war's noch nicht. Da lagen noch Verletzte auf dem Boden. Diese Hurensöhne, zwei Granaten haben sie noch reingeworfen.

Attilio: Und was ist mit dir?

Ciro: Nicht einen scheiß Kratzer, siehst du doch. Du, wenn du da gewesen wärst, wärst du jetzt tot. Wir haben einen riesen Fehler gemacht, Ati. Ich habe gewusst, dass sich Conte für die Brandstiftung rächt.

Attilio: Ciro, Das darfst du Pietro so auf keinen Fall sagen, verstanden!

Szenen-Beispiel b:

Don Pietro in einer Lagerhalle über das weitere Vorgehen nach dem Bombenanschlag auf Ciro (S 1/F 1/ab 18.26)

Don Pietro: [...] Sie haben sich in unser Revier gewagt und haben geschossen. An einem Sonntag. Es hätten Kinder da sein können. So was kann man nicht einfach ignorieren. Er wird dafür büßen. Wir werden dieser Schlange mit Freude den Kopf zertreten.

L'Africano: Er soll sich in so 'nem Gebäude verstecken, gleich neben der Hutwerkstatt.

Don Pietro: Ihr müsst ihn einfach nur schnappen. Wo ist mir egal.

Bolleta: Ist klar Don Pie, nur wir brauchen einen Plan. Wir sollten ihn dazu bringen, raus zu kommen. Das ist kein passender Ort, um... [wird von Don Pietro unterbrochen]

Don Pietro: Kein passender Ort?

Bolleta: Don Pie, man kann da nicht einfach so rein. Da drin wird's auch für uns heiß. 
Don Pietro: Ich war wohl nicht deutlich genug.

L'Africano: Wir sollten uns gut überlegen, wie wir vorgehen. Er hat seine Wachen. Seine Leute sind überall und wir... [wird von Don Pietro unterbrochen]

Don Pietro: Was willst du, Africano. Stell dir vor, dein Sohn wäre in der Bar gewesen. Würdest du dann auch überlegen? Und würdest du lange überlegen [schwenkt seinen Blick auf jemanden, der eingeschüchtert nickt, schwenkt ihn dann weiter auf Zecchinetta] Und du?

Zecchinetta: Schon wahr, Don Pietro, aber wir müssen doch darüber nachdenken... [wird von Don Pietro unterbrochen]

Don Pietro: Halt den Mund [richtet seinen erhobenen Zeigefinger unter die Nase]! Conte hätte ein wenig überlegen sollen, und zwar vorher. Aber dafür ist es zu spät.

An dieser Szenenfolge lässt sich gut nachvollziehen, wie die Kompromisslosigkeit als zentrale Machtressource ${ }^{11}$ des Clanoberhauptes durch mehrfaches Unterbrechen und beharrliches Wiederholen des eigenen Willens zur Geltung kommt. Gleichzeitig zeigt sich hier die Ambivalenz solcher Machtsteigerungen, weil sie Kritiken evoziert, die entweder durch Selbstzensur (Attilio mit gehobenem Zei-

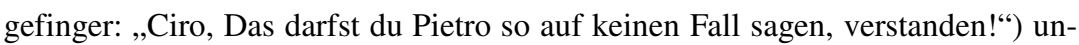
artikuliert bleiben oder durch das Oberhaupt gezielt niedergeschwiegen werden

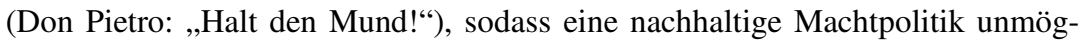
lich wird. Stattdessen reißt der Flickenteppich der Mächte auf und die Gewalt eskaliert, woraufhin Conte sich nach Spanien absetzt und Don Pietro verhaftet wird.

\section{Zweite Szenenfolge}

Don Pietro wurde verlegt, die Besuchsregeln wurden eingeschränkt, was die Gesprächsatmosphäre intimer und klinischer macht.

\section{Szenen-Beispiel c:}

Vorgespräch zwischen Ciro und Genny in Strandnähe. Im Hintergrund Gennys Freunde am Pool (S 1/F 5/ab 40.35)

11 Wichtige Szenen hierfür finden sich in der dritten Folge (Poggioreale), in dem der Machtkampf zwischen dem Gefängnisdirektor Comandante Carcere (Lello Serao) und Don Pietro eskaliert. Diese Szenen bleiben aus Platzgründen unberücksichtigt, müssten in einer breiteren Abhandlung aber stärker ins Zentrum gestellt werden, weil sie das Kräfteverhältnis zwischen staatlicher Macht und Mafia zentral thematisieren. 
Ciro: Warum gehst du nicht zum Besuchstermin, erklär's mir. Du musst dahin. Du. Es war schon falsch, deine Mutter den ganzen Mist regeln zu lassen. Aber mit deinem Vater sprichst natürlich du. Nicht sie. Weil du auch die Befehle geben musst. Kapierst du das?

Genny: Ich soll die Befehle geben?

Ciro: Ja.

Genny: Ich soll befehlen, ja? Wenn ich der bin, der befiehlt, warum sagt mir dann jeder, was ich zu tun hab', mh? Genny hier, Genny da, Genny mach dies, Genny mach das [Genny nimmt die Sonnenbrille ab, rückt näher an Ciro und wird lauter]. Weißt du was. Ich hab' die Schnauze voll davon, kapiert. Ich hab' das Sagen, ja. Also befehl' ich dir jetzt, dass du meine Mama zum Besuchstermin ins Gefängnis fährst, klar. Du fährst sie. Na geh' schon.

[Genny wendet sich seinen Freunden zu. Sie reichen ihm einen Joint und schubsen sich gegenseitig in den Pool.]

\section{Szenen-Beispiel d:}

Besuchergespräch zwischen Donna Imma und Don Pietro im Gefängnis (S 1/F 5/ab 42:24)

Donna Imma: Es ist jetzt alles geregelt. Du kannst beruhigt sein, alles gut.

Don Pietro: Ich hab' mit dem Anwalt geredet. Ich weiß Bescheid. [Sein Blick wendet sich kurzzeitig ab.] Imma, ich bin so erschöpft. Vielleicht ist der Moment gekommen, um mich auszuruhen. Hat Genny denn noch diese Freundin? [Donna Imma nickt verhalten.] Ich bin stolz auf ihn. Mein Sohn ist zum Mann geworden.

Donna Imma: Ich glaube nicht, dass dein Sohn schon ernsthaft beurteilen kann, wer die richtige Frau für ihn ist. Und die jetzt ist es für mein Gefühl noch nicht. Ich als Mutter spüre so etwas. Ich weiß es.

Don Pietro: Imma. Er ist es, der entscheidet. Außerdem hat er Ciro an seiner Seite. Der weiß Bescheid mit den Frauen. Verstehst du mich? [Donna Imma schaut eisern.] Verstehst du mich? [Donna Imma senkt den Blick und nickt verhalten.] Sehr gut. Dann kann ich mich jetzt ausruhen

Im Gefängnis bröckelt die Macht des Oberhauptes und ein eingeschmuggeltes Handy kann den Verfallsprozess nur kurzzeitig aufhalten. In dem Maße, wie die Macht des Oberhauptes schwindet, greifen Konflikte um sich, die nun auch im Inneren der Organisation durch einen angeheizten Generationenkonflikt zu wuchern beginnen. In dieser verschärften Krisensituation vollzieht sich eine Machtdelegation, die sich in Form einer triangulären Figurentransformation ausdrückt. Da ist zum einen das Clanoberhaupt Don Pietro, der das Machtvakuum hinterlässt und im Gefängnis zum gebrochenen Mann wird. Als zweites vollzieht seine 
Frau Donna Imma eine Wandlung von der konservativen Hausmutter zur ,Löwin': Sie widersetzt sich den Anordnungen Don Pietros führt Genny und Ciro in ausweglose Situationen. Ciro wird zum Erzfeind Salvatore Conte (Mario Palvetti) geschickt, um zu verhandeln, Genny nach Honduras, um neue Kontakte zu erschließen. Die weitaus beachtlichste Transformation erlebt allerdings Genny in Honduras. Während Vater und Mutter eher charakterliche Akkumulationen oder Vertiefungen erleben (Weber 2015: 201), erweist sich die Transformation des Sohnes Genny als irreversible Verwandlung (Metamorphose), an deren Ende er nicht mehr der dickliche und machtunfähige Kronprinz ist, der zum Müßiggang neigt, sondern entschieden Befehlstöne erklingen lässt und durch eine Irokesenfrisur und eine ringförmige Tätowierung unterhalb des Halses mit dem Schriftzug „No Confio ni en Dios“ (,Vertraue auch Gott nicht“) seine kampfbetonte und rücksichtslose Führungsbereitschaft ankündigt. Offenbar ist die Drohgebärde seines honduranischen Peinigers zu einer Selbsterkenntnis gereift, die ihn zum Boss werden lässt.

Das stetige Bemühen um Erhalt und Widerherstellung der monolithischen Machtposition ist also von Widerständen und Interferenzen durchzogen, die sich in den Subjekten selbst abspielen und als Schmerzerfahrungen artikulieren. Genny kehrt heim, gewachsen durch einen Schmerz, den er im karibischen Kerker seelisch und körperlich in unübertrefflicher Weise erfahren musste, weil er gezwungen war, einen Mithäftling bestialisch zu zerstückeln, zu dem er Sympathien aufgebaut hatte. Das raubt ihm jegliche emphatischen Reflexe und lässt ihn robust und machtfähig werden. Sein Schmerzempfinden ist nach der Figurentransformation nicht bloß gedämpft, sondern im Grunde überwunden, so dass nicht jedes Geschrei und Gejammer seine Entscheidungsfähigkeit zu beeinträchtigen vermag. Allerdings geht diese Erfahrung weit darüber hinaus, insofern sie neben der Machtreifung des Kronprinzen auch den Vatermord symbolisiert, den Mord am Souverän, der über seinen Leib hinaus zu vernichten ist, wenn er so umfassend ersetzt werden soll, wie es Genny auf dem Schauplatz des sich ausweitenden Generationenkonfliktes vorhat.

\section{FAZIT}

Fiktionale Phänomene als politikwissenschaftliche Untersuchungsgegenstände zu begreifen, gehört nicht zu den Gepflogenheiten der Disziplin. Allerdings schaffen zwei Entwicklungen begründete Anlässe dafür, dass sich momentan eine noch bescheidene Perspektivenerweiterung in diesem Sinne vollzieht. Das ist erstens die sich ausbreitende Überzeugung, dass (pop-)kulturelle Gegenstände wie Filme und Serien niedrigschwellige Zugänge zu komplexen Theorien und 
Modellen bieten und damit einen beachtlichen didaktischen Wert aufweisen. Darüber hinaus artikuliert sich zweitens eine Vorstellung, wonach mittels fiktionaler Strategien ein differenzierteres Realitätsverständnis erzeugt werden kann, als es mittels klassisch-wissenschaftlicher Methoden möglich ist. Beide Entwicklungen greifen gewollt oder ungewollt eine methodologische Position auf, in deren Zentrum die grenzüberschreitende Zirkulation von Zeichen und Symbolen steht. Wenn Weltdeutung eine grundlegende menschliche Aufgabe ist, rücken alle deutenden Praktiken gleichermaßen in den Fokus der Sozialwissenschaften. Bestärkt wird diese Position dadurch, dass sich in den letzten Jahren ein Wandel insbesondere in der Produktion von Serien vollzogen hat, die explizit Realitätsnähe suchen und Methoden einsetzen, die auch in der Wissenschaft genutzt werden, wie etwa die ethnografische Methode oder auch die klassischen Verfahren der Dokumenten- und Berichtsanalyse (Saviano hatte sich auch mit Polizeiberichten über die Camorristi befasst). Der vorliegende Text hat sich vor diesem Hintergrund mit der italienischen Serie Gomorrha auseinandergesetzt, die einige Grundfragen der Politikwissenschaft mit Blick auf Ordnungsbildung und Machtausübung auf innovative Weise zu adressieren erlaubt. Wenngleich das hier eher in demonstrierender Weise geschehen ist und auf ausgewählte Topoi (Familie \& Oberhaupt) beschränkt blieb, so habe ich doch zumindest im Ansatz zeigen können, dass eine Beschäftigung mit Serien die Politikwissenschaft nicht von ihren grundliegenden Anliegen wegführt, wie oftmals befürchtet wird, sondern ganz im Gegenteil neue Möglichkeit für politiktheoretische Reflexionen erlaubt.

\section{LITERATUR}

Boltanski, Luc (2015): Rätsel und Komplotte - Kriminalliteratur, Paranoia, moderne Gesellschaft, Berlin.

Cilento, Fabrizio (2011): Savione, Garrone, Gomorrah: Neorealism and Noir in the Land of Camorra, in: Fast Capitalism 8: 1.

Collins, Randall (2011): Patrimonial Alliances and the Failure of State Penetration: A Historical Dynamic of Crime, Corruption, Gangs, and Mafias, in: The Annals of the American Academy of Political and Social Science 636, S. 1631.

Daston, Loraine/Galison, Peter (2007): Objektivität, Frankfurt a.M.

Flusser, Vilém (2008): Eine neue Einbildungskraft, in: Bohn, Volker (Hrsg.): Bildlichkeit. Internationale Beiträge zur Poetik, Frankfurt a.M., S. 115-126.

Heck, Axel/Schlag, Gabi (2015): „And... Cut!“ Theoretische und methodische Überlegungen zur Analyse von Filmen in Lehre und Forschung, in: Zeitschrift für Internationale Beziehungen 22 (2), S. 125-148. 
Iser, Wolfgang (1993): Das Fiktive und das Imaginäre - Perspektiven literarischer Anthropologie, Frankfurt a.M.

Koschorke, Albrecht (2000): Die Heilige Familie und ihre Folgen, Frankfurt a.M.

Lakoff, George (1996): Moral Politics. How Liberals and Conservatives Think, Chicago.

Latour, Bruno (2010): Eine neue Soziologie für eine neue Gesellschaft, Frankfurt a.M.

Meier, Gerhard (2014): „Höchste Zeit für keine Helden“, spex vom 19.12., http://www.spex.de/hoechste-zeit-fuer-keine-helden-gomorrha/ (zugegriffen am 11.1.2018).

Tilly, Charles (1985): War Making and State Making as Organized Crime, in: Evans, Peter/Rueschemeyer, Dietrich/Skocpol, Theda (Hrsg.): Bringing the State Back in, Cambridge, S. 169-187.

Tzermias, Nikos (2008): „Ghetto Stimmung in Casal di Principe“, Neue Zürcher Zeitung vom 3.12., https://www.nzz.ch/ghetto-stimmung_in_casal_di_principe1.1346790 (zugegriffen am 1.2.2018).

Waldenfels, Bernhard (2002): Virtualität, Fiktionalität, Realität, in: Helmes, Günter/Köster, Werner (Hrsg.): Texte zur Medientheorie, Stuttgart, S. 235240.

Weber, Tanja (2015): „Perché sono tutti cattivi“: Strategien der Anziehung und Abstoßung in Gomorra - La serie, in: Romanische Studien 2, S. 199-232.

Yildiz, Taylan/Gadinger, Frank/Smith, Christopher (2018): Narrative Legitimierung: Exekutive, repräsentative und subversive Erzählstrategien in der Überwachungskontroverse, in: Leviathan 46: 1, S. 135-162. 


\section{House of Cards}

The American Machiavelli

Florian Breitweg, Jakob Hager, Vanessa Molter, Cornelius Witt

\section{EINLEITUNG: DAS FUNDAMENT DES KARTENHAUSES}

Underwood: Okay. This is the memo I've drafted on our middle east policy we've been developing. Now I want to borrow from Reagan. I'd like to coin the phrase ,trickle-down diplomacy“. That way.

Vasquez: Frank, I'm going to stop you there. We are not nominating you for secretary of state. I know he made you a promise, but circumstances have changed (S 1/F $1 / 9.23-9.43)$.

Mit dieser Feststellung beginnt die derzeit erfolgreichste Polit-Serie House of Cards (HoC), die seit 2013 vom Streamingdienst Netflix, basierend auf einer $B B C$-Produktion aus dem Jahr 1990, ausgestrahlt wird. HoC ist, wie der vorangegangene Ausschnitt zeigt, die Geschichte einer Kränkung. Frank Underwood, Whip der Demokraten im Abgeordnetenhaus, wird nach erfolgreichem Wahlkampf vom neuen US-Präsidenten Walker politischen Erwägungen geopfert und wird nicht, wie von Underwood fest angenommen, US-Außenminister im neuen Kabinett. Im Gespräch scheint es kurzzeitig so, als ob Underwood die Fassung verlieren könnte, doch ihm gelingt es - ganz Politprofi -, seine Fassade aufrechtzuerhalten. Professionell und scheinbar zustimmend akzeptiert er die $\mathrm{Zu}$ rückweisung. Allerdings ist dieser Moment Ausgangspunkt der gesamten Erzählung in House of Cards, denn Underwood gibt sich keineswegs geschlagen, im Gegenteil: In ihm reift der Plan, zum ganz großen Coup anzusetzen und die demokratischen Mechanismen durch Macht und Intrigen von innen heraus auszuhebeln, um sich so selbst an die Spitze der Nahrungskette zu katapultieren. Underwood wird zur Spinne im Netz, rücksichtslos und perfide bahnt er sich seinen Weg ins Oval Office - und geht dabei wortwörtlich über Leichen. 
Bei seinen Intrigen nutzt Underwood nicht nur die Führungsschwäche des Präsidenten aus, sondern kann sich auch ganz auf seinen engsten Vertrauten, den loyalen Douglas Stamper, verlassen. Stamper fungiert als Underwoods Büroleiter und tritt vor allem in Erscheinung, wenn es für Underwood selbst zu riskant ist - beispielsweise, wenn es um die Einschüchterung von unliebsamen Zeugen geht. Außerdem sehr bedeutend für Underwood ist seine Ehefrau Claire. Sie ist nicht weniger ambitioniert und skrupellos als er und leitet ihre eigene NGO, die Clean Water Initiative. Diese richtet sie auf internationale Projekte aus, um dem Profil ihres Ehemannes zu entsprechen und seinen erhofften Einfluss im Außenministerium nutzen zu können. Doch als dieser ausbleibt, geht Claire ihren eigenen Weg und trotzt ihrem Ehemann.

Die junge und aufstrebende Journalistin Zoe Barnes entwickelt sich in der ersten Staffel zur Antagonistin Underwoods. Sie hadert mit den konservativen Strukturen ihres Arbeitgebers, des Washington Herald. Um sich dort mehr Einfluss zu verschaffen, geht sie ein Verhältnis mit Underwood ein und erhält von ihm einige Insiderinformationen, was ihr zur Bekanntheit verhilft. Nichtsdestotrotz verlässt sie den Herald und wechselt zum neuartigen Blog Slugline. Nach der Beendigung ihres Verhältnisses mit Underwood beginnt sie damit, den Tod des vormals von Underwood protegierten, inkompetenten Kongressabgeordneten Peter Russo genauer unter die Lupe zu nehmen, und wird somit zur Gefahr für Underwoods Pläne. Ein weiterer Gegenspieler des Protagonisten ist Remy Danton, welcher früher Pressesprecher von Underwood war. Dadurch kennt er auch einige von Underwoods Machtspielen, welche er nun als Lobbyist in einer $\mathrm{Pu}-$ blic Affairs-Agentur zu beeinflussen sucht. Persönlich kann er Underwood nicht ausstehen, was ein Grund dafür war, dass er dessen Team verlies. Durch seinen Einfluss, insbesondere auf Claire, scheitert auch der Water Shed Bill, da dieser den Interessen seiner Klienten widerspricht.

Zwei weitere Schachfiguren in Underwoods Spiel sind der Vizepräsident Jim Matthews und die chief of staff des White House Office, Linda Vasquez. Sie ist loyal und zielstrebig und versucht, den Rücken des Präsidenten freizuhalten. Als einer der wenigen Akteure des Washingtoner Politikbetriebes wird sie auch von Underwood geschätzt, aber nichtsdestotrotz gefügig gemacht. Vom Präsidenten unbeachtet und selten ernst genommen, wachsen Matthews' Unzufriedenheit mit seinem Amt und sein Hass auf den Präsidenten, sodass er ein leichtes Ziel für Underwoods Intrigen wird. Durch geschicktes Manipulieren erreicht Frank Underwood, dass Matthews seinen Posten räumt und der Präsident Underwood zum Vizepräsidenten ernennt, was zum ersten Baustein seines Kartenhauses wird.

Diese Arbeit analysiert die erste Staffel von House of Cards. Da bereits die erste Staffel komplexe und parallele Handlungsstränge aufweist, konzentriert 
sich dieser Teil auf einzelne, zentrale Elemente der Serie, die wiederum auf die Gesamtanlage von $\mathrm{HoC}$ schließen lassen. Ein Überblick über das politische System der USA bereitet zunächst den realen Kontext der Serie auf. Im Anschluss daran erfolgt eine theoretische Analyse, um die Politikdarstellung in $\mathrm{HoC}$ wissenschaftlich zu verorten. Darauf folgt ein Blick auf die Daten, die House of Cards bereitstellt: Welche Rückschlüsse lässt die Serie auf die politische Kultur der USA zu und welche Veränderungen spiegeln sich im Serienformat wieder? Abschließend sollen die Sprache, die eines der wichtigsten Machtinstrumente Underwoods ist, und die zentralen Narrative der Serie näher beleuchtet sowie die Rezeption der Serie aufgegriffen werden.

\section{KONTEXT: VON AUFGABEN UND GESETZEN}

\section{US-amerikanisches Regierungssystem}

Das föderale US-amerikanische System mit Gewaltenteilung und checks and balances wurde von den Gründervätern, basierend auf Charles de Montesquieu (Oldopp 2005) und den zeitgenössischen britischen Verfassungsverhältnissen (Hartmann 2011), eingeführt, um eine Machtkonzentration auf eine Person beziehungsweise eine Gewalt, und damit Machtmissbrauch, zu verhindern. Die starke Ausprägung des präsidialen Systems in Exekutive, Legislative und Judikative mit gleichzeitigen partiellen Verschränkungen hielten die Verfassungsväter für am besten geeignet, obwohl sie nicht zwingend die effizienteste Form des Regierens sein muss (Oldopp 2005: 10).

Trotz dieser Gewaltenteilung kristallisierte sich die heutige große Bedeutung des Präsidenten heraus. Zwar steht ihm verfassungsrechtlich keine Richtlinienkompetenz zu und er darf auch keine Gesetze in den Kongress einbringen, sondern kann diese nur durch ein Veto verhindern. Aber seit dem Beginn des 20. Jahrhunderts konnten sich die Präsidenten - vor allem aufgrund neuer Telekommunikationstechnik - als Anführer darstellen und direkt mit dem Volk kommunizieren. Auch durch die stärkere Fokussierung auf die Außenpolitik kann der Präsident als commander-in-chief und chief diplomat eigene Akzente setzen, da er hier verfassungsrechtlich nur noch der Zustimmung und nicht der Beratung des Kongresses bedarf (Stüwe 2008: 550-551). Doch diese starke symbolische Macht des Präsidenten hat auch ihre Schattenseiten, da er für Ereignisse verantwortlich gemacht wird, für die er selbst nichts kann (Stephenson et al. 1988: 424). Dies passiert vor allem im Falle eines divided government, bei welcher die Oppositionspartei in mindestens einer der beiden Kammern des Kongresses die Mehrheit hat. 
Der Kongress wurde von den Verfassungsvätern als wichtigere Institution angesehen, weswegen er in der Verfassung auch vor dem Präsidenten behandelt wird. Er ist in zwei gleichberechtigte Kammern, Repräsentantenhaus und Senat, geteilt. Im Abgeordnetenhaus repräsentieren direkt gewählte Volksvertreter die Bürgerinnen und Bürger. Ihre Anzahl (derzeit 435) wird auf Grundlage der Bevölkerungszahl und anhand einer festen Berechnungsformel bestimmt; die Wahlkreise müssen hierfür gleich groß sein. Underwood hat im Repräsentantenhaus die Position des Whip inne, welche ein Teil der Leadership ist, die aus dem Speaker, dem majority leader und dem Whip besteht. Die Aufgabe des Whips ist vergleichbar mit der des parlamentarischen Geschäftsführers im Bundestag. Er erkundet die Stimmung in der Fraktion, bringt gegebenenfalls abtrünnige Abgeordnete auf Kurs der Fraktionsführung und meldet auch an diese zurück, wie die Chancen eines Gesetzes sind. Dadurch fungiert er als Scharnier zwischen der Leadership und den anderen Abgeordneten (Hartmann 2010: 109f). Der Speaker of the House ist gleichzeitig Fraktionsvorsitzender der Mehrheitspartei und Präsident des Repräsentantenhauses und hat damit eine Fülle formaler Befugnisse. Er kann „die Geschäftsordnung und die Tagesordnung sowie die Arbeitsanweisungen der Ausschüsse [...] kontrollieren [und] Debatten und Abstimmungen im Plenum [...] beeinflussen“ (Stüwe 2008: 556). Seine stärkste Kompetenz ist aber, dass er die Vorsitze der committees an die Abgeordneten seiner Mehrheitspartei vergeben kann - dadurch ist er die potentiell mächtigste Figur des gesamten Kongresses.

Durch das Organ des Senats werden den Staaten Mitspracherechte beim Beschluss von Bundesgesetzen gegeben. Der Senat besteht dauerhaft aus 100 Senatoren, nämlich zwei pro Staat. Die Mitglieder des Hauses werden alle zwei Jahre neu gewählt, weswegen sie sich kontinuierlich im Wahlkampf befinden, während im Senat ein Drittel alle zwei Jahre gewählt wird, eine Amtszeit also sechs Jahre dauert. Da Gesetzesvorschläge von Mitgliedern des Kongresses eingebracht werden können, hat sich ein starkes Gegengewicht zum Präsidenten entwickelt. Der Präsident muss sich auf sponsors für ein Gesetz verlassen können, also Abgeordnete und Senatoren, die den Vorschlag verfassen oder unterstützen und diesen dann an die jeweiligen committees (Fachausschüsse) übergeben (Oldopp 2005: 29-41).

Der Gesetzgebungsprozess als solcher ist nicht weniger kompliziert. Bei House of Cards werden zwei solcher Gesetze (bills) behandelt. Der Education Reform and Achievement Act wird auf Anregung des Präsidenten und nach Formulierung von Frank Underwood verabschiedet, während der Delaware River Watershed Act, eingebracht von Peter Russo, an zwei Abgeordneten scheitert. 
Kongressmitglieder lassen sich in ihrem Abstimmungsverhalten vor allem von den Interessen ihres Wahlkreises leiten, Wünsche des Präsidenten oder die Linie der Partei treten hier in den Hintergrund. Aus diesem Grund gibt es im Vergleich zu Deutschland auch keine strenge Fraktionsdisziplin, was zu schwierigen Gesetzgebungsverfahren führt (Stüwe 2008: 556-560).

Zur Nachkontrolle der Gesetzgebung gibt es vor allem den Supreme Court als weiteren Vetospieler, welcher „,mit dem Instrument der richterlichen Normenkontrolle (judicial review) legislative Entscheidungen ganz oder teilweise für verfassungswidrig erklären kann“ (Stüwe 2008: 560).

\section{SPIEGEL: UNDERWOOD, DER MODERNE MACHIVELLI}

Nachdem im ersten Teil dieser Arbeit die zentralen Akteure sowie die Grundzüge des politischen Systems der Vereinigten Staaten herausgearbeitet wurden, befasst sich das vorliegende Kapitel mit den politikwissenschaftlichen Bezügen und relevanten Theorien der Serie. Denn neben dem breiten MainstreamPublikum kommen bei House of Cards auch Politik-Nerds und Sozialwissenschaftler auf ihre Kosten.

\section{Streben nach Macht: moderner Machiavelli}

„Der, welcher einem anderen zur Macht verhilft, geht selbst zugrunde; denn er macht ihn stark mit Geschick oder durch Gewalt, und beides ist dem, der zur Macht gelangt ist, verdächtig." (Machiavelli 2001: 30) Diese Überlegungen Machiavellis, niedergeschrieben und veröffentlicht unter dem Titel Der Fürst, sind zwischenzeitlich zu einem Klassiker der Weltliteratur geworden. Und es liegt nahe, dass die Schreiber von House of Cards mit dem knapp 500 Jahre alten Werk vertraut sind: $\mathrm{Zu}$ deutlich sind die Parallelen im Handeln von Hauptcharakter Frank Underwood und den Zeilen Machiavellis. Das oben aufgeführte Zitat kann gleichermaßen als Underwoods Leitmotiv sowie auch Motivation verstanden werden. Der Fürst bietet viele Anknüpfungspunkte an Underwoods Handlungen. Die allgemeine These, wonach der Zweck die Mittel des politischen Akteurs heiligt, lässt sich auch bei Underwood erkennen. Hierfür lässt sich Machiavellis 18. Kapitel heranziehen, in dem es um die Aufrichtigkeit und Ehrlichkeit des Fürsten geht:

Man muss nur sein Gemüt so gebildet haben, daß man, wenn es nötig ist, auch das Gegenteil vermag. Und dies ist so zu verstehen, daß ein Fürst, insbesondere ein neuer Fürst, nicht all das beachten kann, was bei anderen Menschen für gut gilt; denn oft muß er, um 
seine Stellung zu behaupten, gegen Treu und Glauben, gegen Barmherzigkeit, Menschlichkeit und Religion verstoßen. Daher muß er ein Gemüt besitzen, das sich nach den Winden und nach dem wechselnden Glück zu drehen vermag (Machiavelli 2001: 88).

Dieses Verständnis von Politik hat Underwood verinnerlicht, beispielsweise bei seiner hinterlistigen Kampagne gegen Peter Russo: Er baut den zunächst gescheiterten Politiker zum erfolgreichen Wahlkämpfer auf, bis er ihn - in dem für Underwood besten Moment - fallen lässt und diskreditiert, und verstößt damit, um bei den Worten Machiavellis zu bleiben, gegen Menschlichkeit und Barmherzigkeit, um seine eigene „Stellung zu behaupten“ (Machiavelli 2001: 88). Außerdem stellt der Machterwerb, der durch unterschiedliche Methoden erreicht werden kann, für Machiavelli eine zentrale Handlungsmaxime politischer Akteure dar (Zimmer 2004: 44). Entsprechend ist auch Underwood, vor allem in den Staffeln 1 und 2, getrieben vom Streben nach Macht. Dies wird beispielsweise mit Blick auf seinen Rachefeldzug gegen die Regierung des amtierenden USPräsidenten Garrett Walker ersichtlich. Erstes Opfer dieser Machtgier wird Michael Kern, der anstelle Underwoods zum neuen Außenminister ernannt wird. Mit einer orchestrierten Medienkampagne, basierend auf falschen Aussagen eines ehemaligen Studienkollegen Kerns, leitet Underwood den Rücktritt Kerns ein - ohne Rücksicht auf Reputationsverluste seiner Partei oder der neuen demokratischen Regierung. Ebenso findet sich Machiavellis Prinzip der „Nützlichkeit“" als zentrales Mittel zum Machterhalt in Underwoods Karriere wieder. Es lassen sich zahlreiche Belege finden, die zeigen, wie Underwood seine Kollegen und Rivalen ausnutzt und eigensinnig einsetzt - beispielsweise bei seiner Abmachung mit der Journalistin Zoe Barnes, die er nur aus Eigennutz und politischem Kalkül mit Informationen versorgt. Oder bei seinem Schachzug, nach Russos Scheitern den Vizepräsidenten Jim Matthews ins Rennen um den Gouverneursposten in Pennsylvania zu schicken - nur um selbst dessen Position im Weißen Haus einzunehmen. Eine machiavellistische Handlungsmaxime, die dabei zum Tragen kommt, ist die Verschleierung der eigenen Absichten. Diese Handlungslogik wird bei Underwood vor allem deutlich, wenn er direkt mit dem Zuschauer kommuniziert und dabei seine wahren Absichten äußert (siehe dazu „Die vierte Wand“ im Kapitel „Narrativ“").

Frank Underwoods Handeln im Geiste Machiavellis lässt nur einen Schluss $\mathrm{zu}$ : Er ist ein reiner Machtpolitiker, dessen persönliche Interessen für ihn immer an erster Stelle stehen. Dörner (2016) unterscheidet idealtypisch drei Formen der Politik. Die Real-, Ideal-, oder eben jene Machtpolitik, die jegliches politisches Handeln aus dem Streben nach Macht ableitet und moralischen Werten keine Rolle beimisst. Im Gegensatz dazu versteht er unter Realpolitik „,ein Konzept, 
demzufolge Politik zwar, gute‘ Ziele erreichen möchte, dabei aber mitunter moralisch fragwürdige Handlungsweisen toleriert, um sie zu erreichen“ (Dörner 2016). Idealpolitik hingegen geht von einer idealtypischen politischen Bühne aus. Die Akteure handeln für hehre Ziele, weshalb ihnen vorranging das Gemeinwohl am Herzen liegt. In House of Cards spielen die Real- und IdealPolitiktypen allerdings eine untergeordnete Rolle. Zwar gibt es immer wieder Charaktere, die Anzeichen moralisch guter Politiker zeigen, wie beispielsweise Peter Russo nach seinen Alkohol- und Drogenexzessen oder wie der Reformführer Blythe, der mit aller Überzeugung für seinen liberalen Bildungsentwurf kämpft, um damit Verbesserungen für das amerikanische Schulsystem zu erreichen. Die Serie House of Cards wird jedoch durch den machthungrigen Protagonisten Frank Underwood dominiert, der seine Feinde Schachzug um Schachzug aus seinem perfiden Spiel nimmt. Weder Blythe noch Russo bleibt somit eine Chance, ihre idealpolitischen beziehungsweise realpolitischen Politikvorstellungen durchzusetzen. ${ }^{1}$

Da Frank Underwood im Mittelpunkt der Serie steht, kann er als Indikator bzw. Vertreter der amerikanischen Politik(er) gelten. Wie der Vergleich mit Machiavelli zeigt, hat die Politikdarstellung der Serie die größten Schnittmengen mit dem machtpolitischen Konzept nach Dörner. Jedoch dehnt Underwood Machiavellis Überzeugungen bis über alle moralischen Grenzen hinweg aus. Zwar ist Machiavelli der Meinung, Menschlichkeit müsse nicht zu jedem Zeitpunkt eingehalten werden (Machiavelli 2001: 88), von Mord spricht er dabei aber nicht. In Folge 11 der ersten Staffel geht Underwood trotzdem wortwörtlich über Leichen: Nachdem Peter Russo seine ihm zugedachte Rolle im Rachefeldzug erfüllt hat, bringt Underwood ihn um. Für die Zuschauer besteht spätestens dann kein Zweifel mehr daran, dass Underwood keine moralischen Grenzen kennt.

\section{Die Rolle der Medien: digitaler Medienwandel und Interdependenzmodell}

House of Cards, beziehungsweise vor allem Zoe Barnes' Handlungsstrang, stellt den digitalen Medienwandel anschaulich dar. Ihr erster Arbeitgeber, der strauchelnde Verlag des fiktiven Traditionsblatts Washington Herald, kämpft mit sinkenden Auflagen und einem mangelnden Online-Geschäftsmodell. Später wechselt Barnes zum Medien-Startup Slugline, das an real existierende Online-Seiten wie Buzzfeed und Huffington Post angelehnt ist (Carr/Parker 2013). Aufgegriffen

1 Im Gegensatz dazu dominiert in Borgen die Realpolitik, vgl. hierzu das Kapitel „Narrativ“ der entsprechenden Serienanalyse. 
wird diese Disruption in der sich schnell veränderten Verlagsbranche im Gespräch zwischen Barnes und ihrem Chef beim Washington Herald, Lucas Goodwin. Der Dialog verdeutlicht anschaulich, mit welchen marktwirtschaftlichen Problemen traditionelle Verlagshäuser im Online-Zeitalter zu kämpfen haben.

\section{$\underline{\text { Szenen-Beispiel }}$}

Barnes trifft in der Küche der Redaktion des Washington Herald auf Goodwin, ihren Vorgesetzten (S 1/F 1/6.40 - 7.27).

Barnes: Move me online. My own blog? First-person, subjective, 500 words.

Goodwin: Not going to happen.

Barnes: I go underground. Backrooms. The urinals. I'll win over staff members on the hill.

Goodwin: A gossip column?

Barnes: No! We lift the veil. What's really going on.

Goodwin: This is the Washington Herald, Zoe. It's not TMZ.

Barnes: Do you know how many people watch TMZ?

Goodwin: I couldn't care less.

Barnes: Which is why print journalism is dying.

Goodwin: Then it'll die with dignity, at least at this paper.

Barnes: You are stuck in the 20th century, Lucas. You lack imagination.

Bei ihrem neuen Arbeitgeber Slugline ist die redaktionelle Arbeitsweise durch einen schnelllebigen Sensationalismus getrieben und durch persönliche Zugänge und Infotainment geprägt. Der Medienforscher Schmidt befasst sich mit diesen neuen Kommunikationslogiken und hält dabei fest: „Themen und Informationen werden aufgrund persönlicher Relevanz ausgewählt und präsentiert, nicht auf der Grundlage institutionalisierter Nachrichtenfaktoren bzw. eines Anspruchs auf gesellschaftsweite Relevanz“ (Schmidt 2016: 272). Seine Schlussfolgerung lässt sich einwandfrei auf Slugline als Medienakteur anwenden.

Ebenso befasst sich die Serie mit der wechselseitigen Beziehung zwischen Medien und Politik. ${ }^{2}$ Eine meist „symbiotische Interaktion“ (Sarcinelli/Schatz 2002: 14) wird in diesem Kontext zwischen Underwood und Barnes deutlich, da - zumindest aus einer beruflichen Sicht - beide voneinander abhängen und jeder den jeweils anderen Akteur braucht, um seine eigene Agenda voranzubringen. Während Barnes dabei versucht, durch Insiderinformationen prestigeträchtige

2 Eine ausführliche Darstellung der Theoriemodelle in diesem Kontext ist im Kapitel „Spiegel“ der Analyse zur Serie Eichwald, MdB zu finden. 
Meldungen zu veröffentlichen, geht es für Underwood um das Agenda-Setting und die mediale Berichterstattung , seiner' Themen.

\section{Maklermacht: personelle Faktoren im Entscheidungsprozess politischer Akteure}

Jeden Morgen um kurz vor acht Uhr passiert eine Frau in einem dunkelfarbenen VW-Golf das Tor des Kanzleramts. Ihr Büro im siebten Stock der Berliner Regierungszentrale liegt nur drei Türen von dem der Bundeskanzlerin entfernt: Beate Baumann ist die wichtigste Mitarbeiterin von Angela Merkel. Doch kaum jemand kennt diese Frau, die wie die Kanzlerin den leisen Auftritt schätzt. (Delfs/Czuczka 2013)

So wird Beate Baumann, langjährige Merkel-Vertraute und Büroleiterin der Kanzlerin, beschrieben. Baumann ist ein Paradebeispiel für die sogenannten Machtmakler (Korte 2003), beratende Akteure, die, meist außerhalb der Öffentlichkeit, als langjährige Vertraute von politischen Spitzenakteuren ihre Vorgesetzten mit, gegebenenfalls informellen, Informationen versorgen und als Koordinatoren der personellen Netzwerke rund um den Politiker fungieren. Denn politische Entscheidungsvorbereitungen sind oft geprägt ,,vom Einfluss der personalen Faktoren. Für das Informationsmanagement der Spitzenakteure sind Personen mit Maklermacht (beratender Vermittlungsmacht) [...] unverzichtbar" (Korte 2003: 17). Doug Stamper verkörpert den Machtmakler in HoC. Er kennt die Absichten Underwoods wie kein anderer im Kapitol. Er ist an zentralen politischen Entscheidungen beteiligt und formt die externe sowie interne Kommunikation seines Chefs. Weiterhin hat er als Terminplaner des Politikers eine nicht zu überschätzende Machtposition inne, indem er Einfluss darauf nimmt, wer seinen Chef sehen darf und welche Informationen ihn erreichen (Oldopp 2005: 77). Er fungiert als Sparringspartner und verfügt über „abgeleitete Autorität“ (Korte 2003: 23), wenn er beispielsweise Underwood in den Verhandlungen zum Bildungsgesetz vertritt (S 1/F $3 / 5.13-5.49){ }^{3}$

\section{Der Revolving-Door-Effekt: die Drehtür zwischen Politik und Lobbying}

Der Revolving-Door-Effekt beschreibt den Wechsel eines politischen Amtsträgers beziehungsweise seines Angestellten zu einer anschließenden Tätigkeit bei

3 Die Rolle des Machtmaklers wird auch im Kapitel „Spiegel“ der Analyse zur Serie The Thick of It im Zusammenhang mit der Person Glenn Cullen besprochen. 
einer Interessenvertretung. Eine Definition hierfür kommt von Vidal et al. (2012), die diese Drehtür als „movement of federal public employees into the lobbying industry“ (Vidal et al. 2012: 3731) beschreiben. Jeffrey Berry ist dem Effekt empirisch nachgegangen und hat festgestellt, dass in den Vereinigten Staaten jeder zweite Lobbyist schon eine Art von staatlichem (Regierungs-) Angestelltenverhältnis hatte (Berry 1997).

Der ,Drehtür'-Lobbyist wird bei $\mathrm{HoC}$ durch Remy Danton, Partner bei der Lobbyvertretung Glendon Hill und ehemaliger Pressesprecher Underwoods, verkörpert. Danton nutzt seine alten Kontakte im Kongress, um für die Interessen seiner Klienten zu werben. Baumgartner et al. (2009) haben 1.200 Lobbyisten zu ihren Arbeitsmethoden befragt und herausgefunden, dass diese Taktik in der Realität ihre Anwendung findet: Sogenannte ,inside tactics of advocacy“, wie zum Beispiel persönliche Kontakte zu Abgeordneten und deren Mitarbeitern, zählen zu den beliebtesten Methoden der befragten Interessenvertreter: „Over three-quarters work through personal contacts with rank-and-file members of Congress and their staffs" (Baumgartner et al. 2009: 152).

\section{Bildungsgesetz als Fallbeispiel für das Arenen-Modell}

Der wichtigste Gesetzgebungsprozess der ersten Staffel ist das Bildungsgesetz, das Prestigeprojekt des neuen Präsidenten. Mit diesem Cliffhänger, ermöglicht durch den exklusiven Bericht über den Gesetzentwurf von Underwood an Barnes, endet die allererste Folge. Dieser legislative Prozess um die Bildungsgesetzgebung dient hervorragend, um das Arenenmodell des Politikmanagements (Korte/Fröhlich 2009) zu illustrieren. Dem Modell zufolge hängen politische Entscheidungsfindungen davon ab, in welchen Arenen sie stattfinden.

In der parlamentarischen Arena, die „,den Mehrheitsregeln des Parlamentarismus" (Korte/Fröhlich 2009: 224) folgt, befindet sich Underwood in seiner Funktion als majority whip des US-Repräsentantenhauses. Entscheidungen werden durch formale Beschlüsse nach Mehrheiten gefasst. Es gibt etliche Szenen, in denen Underwood mit seinem Machtmakler Stamper vor der weißen Tafel in dessen Büro steht und die einzelnen Stimmen der Abgeordneten zusammenzählt. Außerdem wird der rechtliche Gesetzgebungsprozess durch die Signatur des Bildungsgesetztes durch den Präsidenten veranschaulicht (S 1/F 7/5.00 - 6.15). Die Unterschrift stellt die institutionelle Komponente der parlamentarischen Arena dar; durch sie tritt das Gesetz formal in Kraft.

Die Logik der administrativen Arena ist geprägt durch nicht öffentliche Abstimmungen und die Koordination zwischen Partei- und Interessenvertretern. Die Prozesse folgen einer Verhandlungs- und Netzwerklogik. Veranschaulicht wird dies in der Serie auch durch die informellen Treffen zwischen Underwoods 
Team und den Vertretern der Lehrergewerkschaften - buchstäblich im Hinterzimmer von Underwoods Büro (S 1/F 3).

Im Gegensatz dazu werden in der öffentlichen Arena die Entscheidungsmuster durch Stimmungen und öffentliche Meinungen geprägt. Aufmerksamkeit dient als Währung im Zerren um die vermeintlich beste Darstellung der eigenen Politik. Korte und Fröhlich betonen, dass die politische Steuerung ,über die Beeinflussung und Aktivierung von Stimmungen, nicht über Hierarchie, Mehrheit oder Konsens“ (Korte/Fröhlich 2009: 225) erfolgt. Diese Regeln der politischen Kommunikationen werden im Falle des Bildungsgesetzes vor allem durch medial ausgeschlachtete Streiks und Demonstrationen der Lehrergewerkschaften (S 1/F 5 \& F6) sowie im Live-TV-Duell zwischen Underwood und Gewerkschaftsführer Martin Spinella (S 1/F 6/18.17 - 21.59) verdeutlicht. Außerdem gelingt es Underwood, mit dem Slogan „Disorganized Labor“ als Beschreibung des politischen Antagonisten einen politischen Kampfbegriff auf die mediale Agenda zu setzen, den die Medien für ihn aufnehmen und immerzu wiederholen (S 1/F 6).

\section{IN GOD WE TRUST: KULTURELLE WERTE UND NORMEN}

Das folgende Kapitel betrachtet die spezifischen US-Vorstellungen über Politik und die damit einhergehenden Normen und kulturellen Werte, die House of Cards aufgreift. Als Unterhaltungsserie spitzt $\mathrm{HoC}$ dabei selbstverständlich Themen und Rollen zu. Die Anlage der Szenarien sowie der handelnden Personen ist dabei angelehnt an real existierende politische Verfahren und zum Teil auch an Personen in öffentlichen Ämtern, an Medienvertreter und an Personengruppen aus der Wirtschaft. Es gilt, diese Bausteine zu erkennen, und herauszuarbeiten, welche Rückschlüsse aus der populärkulturellen Darstellung auf die politische Kultur und deren Wahrnehmung gezogen werden können. Oder anders: Wieviel Washington D.C. steckt in HoC und andersrum? Klar ist, dass eine umfassende Analyse der realen Begebenheiten in den USA, von den sozialen über die professionellen bis hin zu den systemischen Komplexen, aus dem Rahmen fallen würde. Daher beschränkt sich dieser Abschnitt darauf, die politische Kultur der USA zunächst in Ansätzen zu beschreiben und daran anschließend Elemente dieser Kultur aufzugreifen und Parallelen zur Anlage der Serie zu ziehen. 


\section{Politische Kultur in den USA - und in House of Cards?}

Zum Verständnis politischer Prozesse und öffentlicher Diskurse ist es stets notwendig, die politische Kultur einer Gesellschaft als kontextgebende Struktur mit einzubeziehen. Gleichwohl ist die Eingrenzung dessen, was unter politischer Kultur zu verstehen ist, naturgemäß schwer. Erstmals explizit eingeführt wird der Begriff der politischen Kultur von Gabriel Almond und Sidney Verba, die folgende Definition zugrunde legten:

„The political culture of a nation is the particular distribution of patterns of orientation toward political objects among the members of the nation." (Almond/Verba 1962: 14f.)

In ihrer Studie zur Entwicklungsbedingungen junger Demokratien nehmen die beiden Autoren an, dass eine gewisse Deckung der politischen Kultur in der Gesellschaft mit dem politischen System notwendig sei, um das Funktionieren von Demokratien zu sichern (Almond/Verba 1962: 14f). Darüber hinaus sind auch zunächst unpolitisch anmutende, zum Teil sehr persönliche Einstellungen wie religiöse Überzeugungen von hoher Relevanz. Insbesondere in den USA spielen diese, wie noch zu zeigen sein wird, eine zentrale Rolle. Anhand der Definition wird zudem auch deutlich, dass die politische Kultur einer Gesellschaft ein einendes Potenzial hat, allerdings auch in gleichem Maße spaltende Wirkung entfalten kann, sollten die Erwartungen der Öffentlichkeit als Ganzes oder die einzelner Gruppen in das politische System nachhaltig enttäuscht werden.

Zwischen den westeuropäischen Ausprägungen und der US-amerikanischen Kultur finden sich signifikante Unterschiede. Wesentliche Aspekte dieser Differenzen lassen sich historisch begründen. Die USA weisen eine äußerst heterogene ethnische Differenzierung auf. Neben der weißen Bevölkerung, der aktuell größten Gruppe, wächst der Anteil der schwarzen, hispanischen und asiatischen Bevölkerungsgruppen weiter an. Die ethnische Trennung impliziert eine Reihe weiterer segregierender Mechanismen, wie beispielweise sprachliche, soziokulturelle und nicht zuletzt auch ökonomische Unterschiede. In der Folge ist auch die Verfassung Ausdruck dieser „Segmentierung der Gesellschaft“ (Stüwe 2013: 58). Sie garantiert relativ unabhängige einzelne Bundesstaaten mit einem hohen Grad an politischer Autonomie und stellt somit einen Gegenentwurf zu zentralistischen politischen Systemen wie in Frankreich dar. Die Brücke zwischen den verschiedenen sozialen und politischen Gruppen schlägt dabei „eine gemeinsame, identitätsstiftende öffentliche Philosophie“ (Stüwe 2013: 58). Diese public philosophy fußt in der Verfassung und nimmt eine herausragende Stellung innerhalb der amerikanischen Öffentlichkeit ein. Sie zeichnet sich durch eine Rei- 
he fester belief systems, Symbole und Wertvorstellungen aus, die in weiten Teilen der Bevölkerung ,enthusiastische Verehrung“ (Stüwe 2013: 58) auslöst. Der Soziologe Robert N. Bellah prägte hierfür den Begriff der amerikanischen „Zivilreligion“ (Bellah 1967). In seinem bereits 1967 erschienenen Artikel Civil Religion in America analysiert er, ausgehend von der Amtsantrittsrede von John F. Kennedy am 20. Januar 1961, dieses spezifisch amerikanische Phänomen (Bellah 1967). Die starke Bezugnahme Kennedys auf Gott erscheint im höchsten politischen Amt eines säkularen Staates zunächst paradox, zumal Religion im Sinne einer strikten Trennung der politischen und religiösen Sphären etwas zutiefst Privates darstellt. Allerdings, so Bellah, gibt es in der heterogenen amerikanischen Gesellschaft gemeinsame religiöse Orientierungsmerkmale, die ein Großteil der Amerikanerinnen und Amerikaner teilen. Diese gemeinschaftlichen Elemente hatten zentralen Einfluss auf die Entwicklung der politischen und zivilgesellschaftlichen Institutionen, ohne dabei ihre religiösen Züge zu verlieren: "This public religious dimension is expressed in a set of beliefs, symbols, and rituals that I am calling the American civil religion." (Bellah 1967: 4)

Dabei unterscheidet sich die Zivilreligion von der Rousseau'schen Idee eines contrat social. Gleichwohl spielten vertragstheoretische Überlegungen im Sinne des französischen Philosophen eine Rolle, als die amerikanischen Gründerväter die Verfassung im Kontext ihrer Zeit erarbeiteten (Bellah 1967: 5-6). Weiter manifestierte sich die Zivilreligion während des Amerikanischen Bürgerkrieges von 1861 bis 1865. Die in dieser Zeit entstandenen National Cemeteries, allen voran in Gettysburg, schafften physische Orte, quasi Wallfahrtsstätten der Zivilreligion und mit dem ermordeten Präsidenten Lincoln zudem einen nationalen Märtyrer (Bellah 1967: 10-11). Auch in der politischen Gegenwart Bellahs erkennt der Soziologe die Lebendigkeit der Zivilreligion in Amerika wieder und konstatiert weiter: „It is certainly true that the relation between religion and politics in America has been singularly smooth" (Bellah 1967: 13) - eine Auffassung, die Nils Heisterhagen in seinem Beitrag auch für das 21. Jahrhundert teilt. Unter Bezug auf Bellah fasst er die Zivilreligion in Amerika als „Idee vom amerikanischen Israel“ zusammen, also eine Art Exodus-Erzählung nach biblischem Vorbild (Heisterhagen 2016). Dieses amerikanische Selbstverständnis als von Gott auserwähltes Volk wurde spätestens als Manifest Destiny auch (außen)politische Doktrin, die bisweilen in Imperialismus umschlug (Heisterberg 2016). Die christliche Komponente der Zivilreligion hat innenpolitisch vor allem zwei praktische Folgen: So sind amerikanische Politiker de facto stets zwei Souveränen verpflichtet, nämlich Gott und Volk (Heisterberg 2016). Zudem liebt die amerikanische Öffentlichkeit die mediale Aufbereitung der Erzählung vom reuigen Sünder. Für Letzteres hat die Medienlandschaft das griffige Schlagwort 
Comeback-Kid parat. Diese innenpolitische Dimension findet sich auch in der ersten Staffel von $\mathrm{HoC}$ wieder, wie folgend beschriebene Szene zeigt: Der von Frank Underwood instrumentalisierte Peter Russo geht mit dieser Phönix-ausder-Asche-Story auf Wahlkampftour: In einer Szene erhält der Abgeordnete Russo bei seinem ,Heimspiel' in Bristol im Wahlkampf um den Gouverneursposten in Pennsylvania Unterstützung durch den Vizepräsidenten Jim Matthews. Dieser führt in seinem Grußwort aus, dass er zunächst an Russo aufgrund seiner - teils öffentlichen - Verfehlungen als Politiker und Mensch gezweifelt hat, ihn jedoch jetzt vorbehaltlos unterstützen würde und in ihm den idealen Kandidaten für einen „fresh start“ - so das Wahlkampfmotto Russos - im leidgeprüften, wirtschaftlich in einer Talsohle weilenden Pennsylvania sieht (S 1/F 9/39.01 41.07). Laudatio und Hauptrede nutzen dabei beide das Element des gefallenen Individuums, welches mit hohem persönlichem Einsatz geläutert wurde. Der Funke springt auf die Menge über und so gelingt es Russo, seinem Wahlkampf massiv Auftrieb zu verleihen.

\section{,Klimawandel': $\mathrm{HoC}$ als Produkt einer veränderten politischen Kultur}

Weiter dient $\mathrm{HoC}$ auch als populäre Datengrundlage, um die veränderte öffentliche Rezeption des politischen Geschehens sichtbar zu machen. Die politische Kultur in den USA scheint sich in den letzten 15 Jahren merklich verändert zu haben. Dass die politische Kultur in den Vereinigten Staaten signifikant durch die DNA der Zivilreligion geprägt wurde, wurde eingangs dargestellt. Verändert sich das politische Klima, liegt ein analytischer Blick auf die zugrundeliegenden Parameter nahe: Woran krankt das Konzept der Zivilgesellschaft nach Bellah? Williams und Fuist argumentieren, dass sich die klassische civil religion, wie sie Bellah 1967 beschrieb, zu einer „tribal civil religion“ hin verändert hat (Williams/Fuist 2014: 1). Letztere unterscheidet sich in ihrem Effekt auf die amerikanische Gesellschaft fundamental von der Zivilreligion im herkömmlichen Sinne.

Die Reden von Matthews und Russo sind Ausdruck der politischen Kultur der USA, die vor dem Hintergrund der amerikanischen Zivilreligion zu betrachten ist. Aber: Im Verlauf der ersten Staffel wird vor allem in der Person von Russo deutlich, dass sich moralischer Anspruch und politische Realität in der Serie diametral entgegenstehen. Durch diesen Widerspruch zeichnet die Unterhaltungsserie $\mathrm{HoC}$ ein reales Bild der politischen Veränderungen in den Vereinigten Staaten. Angenommen, Bellahs civil religion-Ansatz trifft zu, so bedeutet dies im Umkehrschluss, dass die veränderte politische Kultur, die auch in $\mathrm{HoC}$ wie- 
derzufinden ist, Symptom einer tieferliegenden Veränderung ist: die Krise der Zivilreligion in den USA. Die Autoren argumentieren, dass civil religion, verstanden als einendes, prophetisch-soziales Fundament, erodiert (Williams/Fuist 2014: 932). An ihre Stelle ist die ,tribalist civil religion“ (Williams/Fuist 2014: 932) getreten: Diese Form der Zivilreligion betont ethno-religiöse Unterschiede in ihren geographischen, ökonomischen und ideellen Dimensionen, fokussiert also auf die sozialen Grenzen innerhalb einer Gesellschaft und wirkt schließlich segregierend (Williams/Fuist 2014: 932). Demnach gibt es, nach Phillip Gorski, drei Ausprägungen „transzendenter Legitimität“ (Gorski 2011), deren Ausrichtung sich in jeweils anderen politischen Programmen niederschlagen. Dabei ist die civil religion zwischen zwei Polen zu verorten, dem maximal inklusiven ,liberal secularism“ sowie dem exklusivem „religion nationalism“ (William/Fuist 2014: 932). Letzterer erstarkt etwa seit der Jahrtausendwende zunehmend. Begründet wird dieser Richtungswechsel unter anderem mit der Schwächung des Nationalstaates durch die ökonomische und politische Globalisierung (Williams/Fuist 2014: 933). Die Autoren resümieren:

„One could well argue that, civil religion" is in a crisis, as both as a concept and as a cultural phenomenon, because the legitimacy and what might be called the ,charisma" of the nation-state itself is in crisis." (Williams/Fuist 2014: 933)

Das gesellschaftliche Vertrauen in die politischen Institutionen eines Staates wird durch solche Verwerfungen nachhaltig gestört. Diese Vertrauenserosion wird in $\mathrm{HoC}$ aufgegriffen. In der Serie treten politische Inhalte zugunsten isolierter Karrieristen und deren Machtstreben vollkommen in den Hintergrund. Samuels sieht in $\mathrm{HoC}$ die Versinnbildlichung eines politischen Sozialdarwinismus, der keinen Platz für Ideale lässt (Samuels 2014). Gewichtige soziale Themen werden in den Händen von Frank Underwood ,reine Verhandlungsmasse“ (Klatt 2016) ohne feste Wertvorstellungen: „Cynicism here defined as the desire to succeed in a system in which one does not believe“ (Samuels 2014) - oder wie es Frank Underwood an sein Publikum gerichtet ausdrückt: „Democracy is so overrated“ (S2/F2/2.30). Greg Honan, Mitarbeiter des Institute of Politics (IOP), teilt diese Diagnose eines merklich abgekühlten politischen Klimas und untermauert diesen Negativtrend in seinem Artikel statistisch. Demnach ergab eine Umfrage von $C N N$ im August 2014, dass nur 13 Prozent der Bevölkerung der Regierung vertrauen. Diesem Tiefpunkt stehen immerhin 42 Prozent Vertrauen in die Clinton-Administration gegenüber. Weiter führt Hogan aus, dass der 113. 
Kongress $^{4}$ der ineffizienteste Kongress überhaupt war (Milbank 2014) und so die politischen Institutionen weiter zur Entfremdung von Volk und Staat beitragen. $\mathrm{HoC}$ ist in dieser Lesart Ausdruck einer Generation, die vom politischen Geschehen desillusioniert ist. Der Filmwissenschaftler Martin Lampprecht sieht für diese gesellschaftliche Bestandsaufnahme diverse Anhaltspunkte in House of Cards. Zugleich betont er, dass genau in dieser Negativität paradoxerweise der Erfolg von $\mathrm{HoC}$ begründet liegt, denn die Serie verweigert jegliche moralische Pointe:

„Wenn die Politik in der Tat, wie wir anzunehmen geneigt sind, von Grund auf verdorben ist, dann muss moralische Empörung als antiquierter Aktionismus erscheinen. Die einzige angemessene und stilsichere Reaktion kann hier sein, unseren eigenen Fatalismus vollauf anzunehmen und in der amüsierten Anerkennung unserer eigenen Machtlosigkeit den Trost eines überlegenen Wissens zu finden.“ (Lampprecht 2015: 20)

\section{ERZÄHLUNG: POLITIK ALS MACHT DES WORTES}

Im Folgenden sollen die narrativen Elemente der Serie näher beleuchtet werden. Das Ziel ist es, zu erläutern, wie die Serie Politik als Stoff zum Geschichtenerzählen nutzt. Im Fokus steht dabei die Hauptfigur Frank Underwood. Es wird gezeigt, wie Underwood Sprache und gewisse sprachliche Methoden (Manipulation, vierte Wand) einsetzt, um seine Ziele zu erreichen. Warum ist das so interessant? Schaut man die einzelnen Folgen der Serie, wird man eines schnell erkennen: Underwood dominiert nicht nur mit seiner Körpersprache beinahe jede Szene, sondern vor allem mit der Art und Weise, wie er mit seinen Gegenspielern kommuniziert. Sei es mit anderen Seriencharakteren oder den Zuschauern, Underwood kreiert oftmals eine düstere Gesprächsatmosphäre. Er schafft es, sein Gegenüber zu lenken und zu manipulieren. Dabei ist das TV-Publikum abwechselnd positiv überrascht oder möchte sich ob Underwoods niederträchtiger Art aus der beobachteten Situation entziehen.

In den beiden nun folgenden Unterkapiteln liegt der Fokus auf einer kurzen Ausführung über Sprache sowie Underwoods Sprachstil. Anschließend zeigen Beispiele, wie Underwood sprachliche Mittel zum Zwecke der Manipulation einsetzt.

4 Amtierend von 2013 - 2015, zeitlich parallel zu den ersten Staffeln der Serie. 


\section{House of Cards - oder: Wo ist die Politik geblieben?}

Man könnte vermuten, eine Polit-Serie wie House of Cards würde politischen Reden im parlamentarischen Umfeld sowie der politischen Sprache einen großen Stellenwert einräumen. Schließlich gilt „Sprache [als] Bedingung für Machtausübung und [ist] selbst eine Macht“ (Klein 2010). Ein Mittel also, das im politischen Betrieb durchaus einzusetzen ist. Wenn es um politische Inhalte geht, sind Plenarreden dann auch ein Mittel, um Argumente vorzutragen, Thesen Anderer $\mathrm{zu}$ widerlegen und Überzeugungsarbeit zu leisten. Allerdings ist House of Cards keine Serie, die diese Art der politischen Darstellung zeigen möchte. Die Serienmacher konzentrieren sich in ihrer Erzählung von Politik nicht auf das politische, sondern auf das persönliche Handeln und Kommunizieren Underwoods. Policies spielen dabei keine herausragende Rolle. Abgesehen von dem Bildungsgesetz thematisiert die erste Staffel von $\mathrm{HoC}$ keine konkreten politischen Inhalte tiefergehender. Zum anderen liegt es an Underwoods Verständnis seiner politischen Funktion. Als demokratischer Abgeordneter des Staates South Carolina sitzt er zwar im Abgeordnetenhaus, besonderes Interesse an der tagesaktuellen politischen Arbeit hat er jedoch nicht. Ihm geht es einzig und alleine darum, seinen persönlichen Rachefeldzug zu orchestrieren. Politische Reden vor Anhängern oder während eines Gottesdienstes nutzt er nicht politisch. Vielmehr setzt er sie gezielt im Rahmen seines Racheplans ein. Für Reden im Repräsentantenhaus bleibt einerseits keine Zeit, andererseits sieht Underwood darin kein Mittel, um seine Ziele zu erreichen. Das zeigt die folgende Szene beispielhaft. Underwood zieht es vor, nicht im parlamentarischen Kontext zu sprechen, sondern tritt direkt vor die Kameras. Er bezieht damit öffentlich Stellung zur politischen Reform des Bildungsgesetzes, obwohl er sich normalerweise nicht an die Öffentlichkeit wendet. Jetzt ist das notwendig, weil er gegenüber Spinella, dem Sprecher der streikenden Lehrergewerkschaft, Stärke zeigen muss. Seit einigen Tagen sind die Schulen aufgrund des Lehrerstreiks geschlossen, als ein Jugendlicher vor seinem Elternhaus erschossen wird. Underwood tritt wenig später zusammen mit der Mutter des Opfers vor die Kameras.

\section{Szenen-Beispiel: S 1/F 6/41.07-41.25}

Underwood: It's okay. If Tylers School had been in session, this senseless killing would have never taken place. We need to end this strike now and get all our children back in school where they belong before we risk another death like this. So, Mr. Spinella, I am headed to the Capitol right now, and I will remain there until you are ready to meet and talk. 
Underwood gelingt es dank dieses Statements, die Öffentlichkeit auf seine Seite ziehen, wodurch sein Gegner Spinella an Rückhalt verliert. Im Repräsentantenhaus wäre ihm das nicht gelungen, weil erstens die Zielgruppe (Öffentlichkeit) nicht direkt angesprochen worden wäre und zweitens Spinella nicht mit der gleichen Wucht direkt aus dem Weg hätte geräumt werden können. Um dieses Ziel zu erfüllen, schreckt er nicht davor zurück, den Mord an dem Jugendlichen für seine Zwecke zu instrumentalisieren. Er verwendet darüber hinaus auf eine sehr perfide Art das verbindende Element des Wortes „wir“, um deutlich zu machen, dass er doch derjenige sei, der gerne mit Spinella arbeiten und den Streik beenden würde. Somit weist er alle Schuld am Tod des Jugendlichen von sich, verleiht Spinella aber die Aura eines Mitschuldigen. Es wird deutlich, dass die Macher von $\mathrm{HoC}$ eines zeigen möchten: Politische Inhalte haben im Universum Underwoods keinen Platz. Wenn er sich öffentlich äußert, dann nur, um seinen Racheplan voranzubringen, nicht, um für politische Inhalte zu werben. Deshalb stellt sich bei $\mathrm{HoC}$ die Frage, wo denn die Politik im klassischen Sinne geblieben ist? In den nun folgenden Unterkapiteln soll gezeigt werden, dass Politik in der Serie als niederträchtige Politik erzählt wird.

\section{Die Erzählung niederträchtiger Politik: Manipulation und der Gebrauch der vierten Wand}

Sprache wirkt persuasiv. Idealerweise werden Zuhörer von der Meinung des Sprechers überzeugt, indem sie den besten Argumenten folgen. In der Realität spielen die besten Argumente oftmals keine entscheidende Rolle. Trotzdem wollen Politiker ihre eigenen Ziele durchsetzen. Damit dies gelingt, greifen sie auf bestimmte Strategien zurück. Neben der Argumentation wird oft die Manipulation genutzt. Die linguistische Manipulation ist ein sprachliches Instrument, das vor allem von Politikern eingesetzt wird. Typische Merkmale sind die indirekte Rede sowie Allusionen. Die Autorinnen Rozina und Karapetjana geben für dieses sprachliche Mittel folgende Definition: ,,[P]hraseological allusion is an implicit mental reference to the image of a phraseological unit which is represented in discourse by one or more explicit image-bearing components hinting at the image“ (Hervorhebung im Original, Rozina/Karapetjana 2009: 115).

\section{Underwood: der große Manipulator}

Um deutlich zu machen, wie Frank Underwood einen Gegenspieler manipuliert, wird nun auf eine Szene aus der ersten Folge der ersten Staffel von House of Cards eingegangen. Underwood empfängt Blythe in dessen Rolle als Reformführer für ein neues Bildungsgesetz in seinem Büro. Blythe hat einen neuen 
Entwurf für das Gesetz vorgelegt. Allerdings ist der Entwurf in Präsident Walkers Augen zu liberal. Underwood sieht seine Chance gekommen, sich mit einem eigenen Bildungsgesetz-Entwurf gegenüber Präsident Walker als besonders handlungsfähig und loyal zu beweisen. Jedoch steht ihm Blythe im Weg und er muss sich einen Plan überlegen, wie er ihn aus dem Spiel nehmen kann. Underwood kommt die Idee, den sehr liberalen, links zu verortenden Entwurf durch Zoe Barnes ohne das Wissen der Verantwortlichen an die Presse durchsickern zu lassen. Das Gespräch findet statt, als der Entwurf an die Öffentlichkeit gelangt und die Walker Administration verärgert ist.

\section{Szenen-Beispiel: S 1/F 2/09.22-09.50}

Underwood: Now look. I'm on your side. But Linda is furious.

Blythe: What is she saying?

Underwood: They want to point fingers.

Blythe: At me?

Underwood: I told her we cannot do that. I mean, you are vital to this process. I'm up to here with them, Donald. For lying, for turning their back on you. You know, I'm of a mind to say ,screw it'. I'll fall on this grenade myself. Just to piss them off. [Picks up the phone]. Give me John King at CNN.

Blythe: Wait, Frank. This is not your fault.

Underwood: No. We have to protect your reputation.

Blythe: No, but you are the man that needs to get the bill trough the house.

Underwood: I will. Hang on. I will assign it to one of my deputies and quietly manage it. And I will guide you trough the process.

Blythe: I'm not comfortable with this.

Underwood: So than what do you suggest we do?

[Blythe is thinking. Underwood breaks the fourth wall]

Underwood: What a martyr craves more than anything is a sword to fall on, so you share the blade, hold it just the right angle, and then 3-2-1,

Blythe: It should be me.

Hier zeigt Underwood sein wahres Gesicht. Es gelingt ihm, Blythe durch eine Manipulation zum Rücktritt zu bewegen. Besonderes Augenmerk muss auf die verwendeten sprachlichen Mittel gelegt werden. Zu Beginn umschmeichelt er Blythe („I'm on your side“, „,you are vital to this process.“). Indem er sich als seinen Freund darstellt, baut Underwood eine Verbindung zu ihm auf. Dann nutzt er jedoch die Kunst der Allusion. Das heißt, er deutet gewisse Handlungen nur an und überlässt seinem Gegenspieler deren Interpretation. Als Blythe wissen möchte, was Linda gesagt habe, antwortet Underwood, ohne konkret auf ihre 
Äußerungen einzugehen. Allerdings gelingt es ihm, gegenüber Blythe ein Bedrohungsszenario aufzubauen (,They want to point fingers.“). Hinzu kommt die Verwendung der Metapher ,I'll fall on this grenade myself“. So wächst in Blythe schnell der Verdacht, Frank Underwood könnte sich an seiner Stelle opfern. Underwood muss nicht laut aussprechen, dass er sich opfern könnte. Durch die Allusion drängt er Blythe diese Interpretation jedoch förmlich auf. Verstärkt wird dieser Effekt noch, als Underwood (vermeintlich) bei $C N N$ anruft. Nun ist sich Blythe sicher, dass sich Underwood jeden Moment selbst als den Schuldigen darstellen könnte. Zu diesem Zeitpunkt hat Underwood Blythe genau dort, wo er ihn haben möchte - nämlich mit dem Rücken zur Wand und mit einem schlechten Gewissen ihm gegenüber. Underwood drängt Blythe in die Rolle eines Märtyrers, der sich selbst für Höheres opfern möchte. Durch seinen Rücktritt schützt Blythe Underwood letztendlich und gibt ihm zu verstehen, welch hohen Stellenwert er ihm im Washingtoner Politikbetrieb zuschreibt. Während der gesamten Szene fällt ihm nicht auf, dass sein vermeintlicher Verbündeter derjenige ist, der ihm das Messer in den Rücken rammt.

Underwood erreicht seinen Sieg ohne Zwang, er war alleine mit Hilfe einer manipulativen Strategie erfolgreich. Einerseits hat er genau gewusst, wie er sprachliche Mittel überzeugend einsetzen musste. Andererseits spielen, wie bereits Aristoteles wusste, der Charakter (ethos) des Redners und seine Fähigkeit, für seine Zuhörer eine von ihm bestimmte Gefühlslage zu schaffen (pathos), eine Rolle (Jörke 2016: 160). Als Machtpolitiker im Sinne Macchiavellis ist Underwood ein einnehmender Charakter, der auch vor moralisch schlechten Handlungen nicht zurückschreckt. Für eine Manipulation sind das Voraussetzungen. Da Blythe in Frank Underwood einen Freund zu erkennen glaubt, kann er dessen Plan nicht durchschauen. Vielmehr vertraut er vollkommen auf das, was ihm sein Verbündeter sagt. All diese Faktoren helfen dabei, Blythe während des Rachefeldzuges zu opfern.

\section{Underwood durchbricht die ,vierte Wand‘}

Manipulation durch Sprache gelingt nicht nur durch Allusionen, sondern auch durch andere Kniffe oder sprachliche Stilmittel. Besonderes Augenmerk sollte auf die sogenannte vierte Wand gelegt werden. Hierbei handelt es sich um eine konzeptionelle Idee mit Ursprüngen im naturalistischen Theater. Damit den Schauspielern ein möglichst natürliches Spiel gelingen konnte, sollten sie eine imaginäre Wand zwischen sich und dem Publikum errichten. Theaterregisseure wollten hiermit Interaktionen zwischen Schauspielern und Publikum verhindern, denn nur so war ein möglichst ablenkungsfreies Spiel mit völliger Konzentration auf die Rollen möglich. Des Weiteren liegt dieser Idee das Ziel zugrunde, „den 
Zuschauer in einen traumhaften, passiven, mitfühlenden Zustand zu versetzen und ihm so eine vollständige Identifikation mit dem bürgerlichen Helden zu ermöglichen“ (Stiftung digitale Spielkultur 2013).

In House of Cards wäre dieses Ziel höchstwahrscheinlich nur unzureichend erfüllt worden. Warum? Weil während der kompletten ersten Staffel immer wieder das Stilmittel der , vierten Wand“ eingesetzt wird. Diese Art zu erzählen ist ein Alleinstellungsmerkmal von $H o C$ und der Originalserie der $B B C$. Aufgrund des besonderen, machthungrigen, gefährlichen Charakters Frank Underwoods, wäre den Zuschauern die vollständige Identifikation mit der Figur wohl schwergefallen. Sie hätten seine Beweggründe für den Rachefeldzug sowie seine illegalen Handlungen nicht nachvollziehen können. Aus diesem Grund haben bereits die Macher der $B B C$-Version auf das Stilmittel ,Durchbrechen der vierten Wand' zurückgegriffen. Immer, wenn der Schauspieler aus der Spielszene ausbricht und seine Worte direkt an das Publikum richtet, findet ein solches Durchbrechen statt. Underwood blickt den Zuschauern dann direkt in die Augen.

Bei den Zuschauern kann das Durchbrechen der vierten Wand positive Gefühle hervorrufen. Immerhin werden sie zu Verbündeten Underwoods, die es wert sind, in seine geheimen Rachepläne eingeweiht zu werden. Im Gegensatz zu seinen schwachen, unfähigen Gegenspielern sind die Zuschauer immer über seine nächsten Schritte informiert. Man kann deshalb so weit gehen, sie als Mitwisser und Komplizen zu bezeichnen. Neben der Tatsache, dass die TVZuschauer Underwoods Komplizen sind, zeichnet ihre Beziehung noch ein weiteres Merkmal aus: Baut er im Umgang mit seinen politischen Feinden auf ein Konstrukt aus Lügen sowie Betrug, äußert er seinen Mitwissern gegenüber immer die Wahrheit (Sorlin 2015: 134). Er schafft es, dass sich das TV-Publikum mit ihm identifizieren kann. Sogar seine Taten kann er bis zu einem gewissen Grad rechtfertigen. Immer wieder erwischen sich die Zuschauer auch dabei, Sympathien für Underwood zu empfinden, obwohl er in der ersten Staffel von House of Cards alles andere als moralisch richtig handelt. Ohne das Stilmittel ,Durchbrechen der vierten Wand' würde das Publikum weder einen Einblick in Underwoods Gedanken noch hinter die Kulissen Washingtons erhalten, wodurch eine Verurteilung des Politikers sehr viel wahrscheinlicher wäre. Darüber hinaus gibt es bis heute nicht viele Serien, welche dieses Stilmittel nutzen. Die Macher von House of Cards waren sich dessen bewusst. Im Endeffekt geht es deshalb nicht nur darum, einen Keil zwischen das TV-Publikum und Underwoods Feinde zu treiben (Sorlin 2015: 127), sondern auch schlicht um den Unterhaltungswert.

Abschließend soll eine Szene nochmals Einblick darin geben, wie Underwood die vierte Wand durchbricht. Diese Szene wurde ausgewählt, weil er keinen Gesprächspartner hat, wodurch die Wirkung des Durchbrechens stärker ist. 
Ziel ist es diesmal jedoch nicht, das Publikum zum Mitwisser zu machen, sondern es soll Underwood Halt geben und ihm zuhören. Einige Zeit nachdem Frank Underwood Peter Russo in dessen Auto umgebracht hat, besucht er alleine eine Kirche. Vor dem Altar, mit dem Rücken zum Kirchenschiff, spricht er folgenden Monolog:

Szenen-Beispiel: S 1/F 13/21.42-22.28

Underwood: Every time I've spoken to you, you've never spoken back. Although, given our mutual disdain, I can't blame you for the silent treatment. [Breaks the fourth wall] Perhaps I'm speaking to the wrong audience. Can you hear me? Are you even capable of language, or do you only understand depravity? [Door thuds] [Looks around] Peter, is that you? Stop hiding in my thoughts and come out. Have the courage in death that you never had in life. [Breaks the fourth wall again] Come out, look me in the eye and say what you need to say.

Selbst Frank Underwood kommt manchmal an einen Punkt, an dem er sich Sorgen macht und Rat braucht. Während seines Monologs durchbricht er gleich mehrmals die vierte Wand, wodurch die Zuschauer direkt angesprochen werden („Can you hear me?“). Indem er das theatralische, unpersönliche ,Du“ nutzt, meint er jeden Zuschauer von House of Cards. Eigentlich kann sich so niemand diesem Monolog entziehen. Deshalb scheint es, als würde er sich mit allen Zuschauern unterhalten und von ihnen Antworten auf seine Fragen verlangen (Sorlin 2015: 128). Dadurch entsteht erneut eine Verbindung zwischen ihm und dem Publikum. Allerdings weiht er hier niemanden in seine Pläne ein. Alleine in der Kirche, sucht Underwood vielmehr eine Bestätigung für seine Taten durch das ihm verbundene TV-Publikum. Ihm dämmert wohl, wie verstörend der Mord für seine Verbündeten gewesen sein muss („I can't blame you for the silent treatment.“). Im letzten Satz „come out, look me in the eye and say what you need to say“ könnte er zwar den Geist Russos meinen, aber eigentlich ist jedem Zuschauer klar, dass Underwood diese Frage direkt an ihn richtet. Er fordert das Publikum quasi heraus, den von ihm verübten Mord zu verurteilen. Underwood kann diese Frage so provokant stellen, weil ihm natürlich eines bewusst ist: Die Zuschauer werden ihm nicht antworten und er wird mit dem Mord davonkommen.

Gemeinsam haben alle Beispiele eines: Den Machern der Serie gelingt es, eine Geschichte über Macht zu erzählen. Es ist keine schöne Geschichte, in der Macht und das Erreichen politisch hehrer Ziele Hand in Hand gehen. Es ist eine Geschichte von Manipulation, Intrigen, Niedertracht. In House of Cards ist Poli- 
tik nichts anderes als ein Stoff, um eine düstere Geschichte von Macht zu erzählen. Sie ist Mittel zum Zweck.

\section{FAZIT: FRAGE NACH DER MORAL}

Der Washingtoner Politikbetrieb, wie er in House of Cards dargestellt wird, wirkt für die Zuschauer oftmals wie ein Treffpunkt für Menschen ohne Moral. Viele Akteure, allen voran Frank Underwood, verfolgen sehr persönliche Ziele. Um diese zu erreichen, nutzen sie die unterschiedlichsten, moralisch fragwürdigen Methoden. Einige von ihnen gehen über Leichen, werfen Freundschaften über Bord oder bringen mit ihrem Handeln einen ganzen Berufsstand in Verruf. House of Cards zeichnet deshalb das Bild einer machthungrigen, egoistischen politischen Elite, die sich nicht für das Gemeinwohl einsetzt, geschweige denn interessiert. Vermeintlich schwache Charaktere, wie Russo oder Blythe, haben in einer solchen Atmosphäre keine Chance, sich zu profilieren. Vielmehr zählen in der Serie vor allem Skrupellosigkeit, Machtbewusstsein und Prinzipienlosigkeit. Manipulationen scheinen ein legitimes Instrument $\mathrm{zu}$ sein, mehr politische Macht zu gewinnen.

Diese Art der (Macht-)Erzählung hat nach Meinung der House of CardsRegisseurin Folgen: „Heute, nach House of Cards, denken die Menschen, dass Washington und die Politiker zynischer Abschaum sind und dass sie ihnen weder vertrauen noch sie respektieren müssen“ (Szyszkowitz 2016). Dieses Zitat Szyszkowitz' fasst sehr gut zusammen, was Viele vermuten: Die fehlende Moral Frank Underwoods und seiner Verbündeten könnte zu einer sehr negativen Rezeption der Zuschauer hinsichtlich politischer Eliten geführt haben. Auch der vorliegende Aufsatz macht dies deutlich. Es wurde herausgearbeitet, dass House of Cards eine eindeutig negative Darstellung der politischen Akteure in Washington und des politischen Systems der USA zeigt. Frank Underwood manipuliert seine politischen Gegner, nutzt die Presse geschickt für seine Ziele und geht sogar über Leichen. Für sein Ziel, politische Macht zu erlangen, überschreitet er oftmals die Grenzen der Moral. Hieraus ergibt sich die Frage, wie sich das Bild einer manipulativen, niederträchtigen und machthungrigen amerikanischen Politik tatsächlich auf die Rezipienten der Serie auswirkt. Mit diesem Aspekt haben sich bereits einige Studien beschäftigt. Sie kommen zu dem Ergebnis, dass die Art und Weise, wie eine Serie Politik erzählt, auf die Bewertung der Zuschauerinnen und Zuschauer hinsichtlich der realen politischen Akteure und deren Handlungen großen Einfluss nimmt. Holbert et al. belegen in ihrer Studie, dass die Serie The West Wing sogar auf das Ansehen der oftmals kritisierten BushAdministration eine positive Wirkung hatte (Holbert et al. 2003). Eine neuere 
Studie von Weiner sieht in politischen TV-Serien sogar Katalysatoren für politisches Engagement. So können Serien die Zuschauer in ihrem eigenen Selbstbild festigen und sie in politischen, gesellschaftlichen oder sozialen Einstellungen bestärken (Weiner 2012). Wenn Serien wie HoC allerdings eine korrupte, unmoralische Politikdarstellung zeigen, dann wird auch das Publikum gegenüber der realen Politik misstrauischer (Mulligan/Habel 2013). Die Darstellung Frank Underwoods in House of Cards belegt, dass dessen manipulative Schachzüge auf eine Weise eingesetzt wurden, die die Zuschauer zu folgender Überzeugung verleitet haben: Politiker müssen manipulativ sein (Morris/Evans 2014).

\section{LITERATUR}

Almond, Gabriel/Verba Sidney (1963): The Civic Culture. Political Attitudes and Democracy in Five Nations, Boston/Toronto.

Baumgartner, Frank/Berry, Jeffrey/Kimball, David/Leech, Beth (2009): Lobbying and Policy Change, Chicago.

Bellah, Robert N. (1967): Civil Religion in America, in: Daedalus 1/1967, S. 121.

Berry, Jeffrey (1997): The Interest Group Society, 5. Aufl., Harlow.

Carr, David/Parker, Ashley (2013): Journalism in 8-Minute Chunks: A Backand-Forth on ,House of Cards ', in: https://mediadecoder.blogs.nytimes.com/ 2013/03/07/journalism-in-8-minute-chunks-a-back-and-forth-on-house-ofcards/?_r=0 (zugegriffen am 1.3.2017).

Committee on House Administration (2009): Overview of 13 Common Staff Positions at the United States House of Representatives, in: http://chadiversity.house.gov/position-descriptions.pdf (zugegriffen am 19.3.2017).

Delfs, Arne/Czuczka, Tony (2013): Beate Baumann - Merkels mächtiges Phantom im Bundeskanzleramt, in: https://www.welt.de/newsticker/bloomberg/ article118037076/Beate-Baumann-Merkels-maechtiges-Phantom-im-Bundes kanzleramt.html (zugegriffen am 1.3.2017).

Dörner, Andreas (2016): Politserien: Unterhaltsame Blicke auf die Hinterbühnen der Politik, in: Aus Politik und Zeitgeschichte 51/2016, S. 4-11.

Gorski, Philipp S. (2011): Barack Obama and Civil Religion, in: Political Power and Social Theory 22: S. 179-214.

Hammelehle, Sebastian (2014): Der Teufel trägt Nadelstreifen, in: Spiegel Online vom 27.3.2017, http://www.spiegel.de/kultur/gesellschaft/serie-houseof-cards-bbc-orginal-aus-grossbritannien-a-960911.html (zugegriffen am 27.3.2017). 
Hartmann, Jürgen (2011): Westliche Regierungssysteme: Parlamentarismus, präsidentielles und semi-präsidentielles Regierungssystem, 3. Aufl., Wiesbaden.

Heisterhagen, Nils (2016): Skurrile Kandidaten für das Weiße Haus - und ihr Bibelfokus, in: http://www.theeuropean.de/nils-heisterhagen--2/10768-politische-kul tur-in-den-usa (zugegriffen am 20.3.2017).

Helpman, Elhanan/Persson, Torsten (2001): Lobbying and Legislative Bargaining, in: Advances in Economic Analysis \& Policy 1/2012, S. 1-31.

Holbert, Lance/Pillion, Owen/Tschida, Davd A./Armfield, Greg G./Kinder, Kelly/Cherry, Kristin L./Daulton, Amy R. (2003): The West Wing as Endorsement of the U.S. Presidency: Expanding the Bounds of Priming in Political Communication, in: Journal of Communication 3/2003, S. 427-443.

Honan, Greg (2015): House of Cards, West Wing and the Millennial Generation, in: http://iop.harvard.edu/iop-now/house-cards-west-wing-and-millennial-generation (zugegriffen am 14.3.2017).

Jörke, Dirk (2010): Aristoteles' Rhetorik: Eine Anleitung zur Emotionspolitik, in: Austrian Journal of Political Science, 2/2010, S. 160.

Klatt, Jöran (2016): Ränkespiele zweier Serien (1): House of Cards, in: http://www.demokratie-goettingen.de/blog/house-of-cards\#_ftn4 (zugegriffen am 25.3.2017).

Klein, Josef (2010): Sprache und Macht, in: Aus Politik und Zeitgeschichte 8/2010, S. 7-13.

Korte, Karl-Rudolf (2003): Maklermacht: Der personelle Faktor im Entscheidungsprozess von Spitzenakteuren, in: Hirscher, Gerhard/Korte, Karl-Rudolf (Hrsg.): Information und Entscheidung: Kommunikationsmanagement der politischen Führung, Wiesbaden, S. 15-28.

Korte, Karl-Rudolf/Fröhlich, Manuel (2009): Politik und Regieren in Deutschland: Strukturen, Prozesse, Entscheidungen, 3.Aufl., Paderborn.

Lampprecht, Martin (2015): Serial Nation: Amerikanische TV-Politdramen als Visionen der Macht, Vortrag am 17.11.2015 an der Atlantischen Akademie/Technische Universität Kaiserslautern, in: http://www.academia.edu/2079 7739/Serial_Nation_Amerikanische_TV-Politdramen_als_Visionen_der_Macht (zugegriffen am 24.9.2017).

Machiavelli, Niccolò (2001): Der Fürst, 11. Aufl., Frankfurt a.M.

Milbank, Dana (2014): Good riddance to the worst Congress ever, in: Washington Post http://wapo.st/16A1qZF (zugegriffen am 23.3.2017).

Morris, Joseph M./Evans, Henry (2014): Our House of Cards? Political Fiction and Belief Change, Paper prepared for presentation at the Western Political Science Association Conference, Seattle 17.-19.4.2014, in: https://wpsa. research.pdx.edu/papers/docs/Our\%20House\%20of\%20Cards\%20-\%20Ficti 
onal\%20Narratives\%20and\%20Belief\%20Change.pdf (zugegriffen am 25.10.2017).

Mulligan, Ken/Habel Philip (2013): The Implications of Fictional Media for Political Beliefs, in: American Politics Research 1/2013, S. 122-146.

Murphy, Katharine (2013): How similar to real life politics is House of Cards?, in: https://www.theguardian.com/commentisfree/2013/jul/04/house-of-cardsreal-life-politics (zugegriffen am 1.3.2017).

Oldopp, Birgit (2013): Das politische System der USA, 2. Aufl., Wiesbaden.

Rozina, Gunta/Karapetjana, Indra (2009): The Use of Language in Political Rhetoric: Linguistic Manipulation, in: http://sdu.dergipark.gov.tr/download/ article-file/117981 (zugegriffen am 10.3.2017).

Samuels, Bob (2014): From The West Wing to House of Cards, in: Huffington Post vom 17.2.2014, http://www.huffingtonpost.com/bob-samuels/from-thewest-wing-to-hou_b_4803155.html (zugegriffen am 24.3.2017).

Sarcinelli, Ulrich/Schatz, Herbert (2002): Mediendemokratie im Medienland?, Opladen.

Schmidt, Jan-Hinrik (2016). Soziale Medien in Krisen und Katastrophenfällen, in: Zoche, Peter/Kaufmann, Stefan/Arnold, Harald (Hrsg.): Grenzenlose Sicherheit?, Münster, S. 271-288.

Schwarz, Hunter (2015): Bill Clinton said ,House of Cards “ is 99 percent realistic. Here are eight plot twists that prove he's wrong, in: Washington Post http://wapo.st/1xTnpr7 (zugegriffen am 1.3.2017).

Sorlin, Sandrine (2015): Breaking the forth wall. The pragmatic functions of the second person pronoun in House of cards, in: Gardelle, Laure/Sorlin, Sandrine: The Pragmatics of Personal Pronouns, Amsterdam/Philadelphia, S. 125-146.

Stephenson Jr, D. Grier/Bresler, Robert J./Friedrich, Robert J./Karlesky, Joseph J. (1988): American Government, New York.

Stiftung Digitale Spielekultur (2013): Der entfremdete Spieler, in: http://www. stiftung-digitale-spielekultur.de/der-entfremdete-spieler/ (zugegriffen am 25.3. 2017).

Stüwe, Klaus (2008): Das politische System der USA, in: Stüwe, Klaus/Rinke, Stefan (Hrsg.): Die politischen Systeme in Nord- und Lateinamerika: Eine Einführung, Wiesbaden, S. 540-582.

Szyszkowitz, Tessa (2016): „House of Cards“-Regisseurin Agnieszka Holland: „Eine gefährliche Schöpfung“, in: Profil vom 27.5.2016, https://www.profil. at/gesellschaft/house-cards-Agnieszka-holland-kevin-spacey-6383363 (zugegriffen am 10.1.2017). 
Vidal, Blanes/Jordi, Mirko Draca/Fons-Rosen, Christian (2012): Revolving Door Lobbyists, in: American Economic Review 7/2012, S. 3731-3748.

Weiner, Juli (2012): West Wing Babies, in: http://www.vanityfair.com/news/ 2012/04/aaron-sorkin-west-wing (zugegriffen am 10.1.2017).

Williams, Rhys H./Fuist, Todd Nicholas (2014): Civil Religion and National Politics in a Neoliberal Era, in: Sociology Compass 7/2014, S. 929-938.

Zimmer, Robert (2004): Das Philosophenportal: Ein Schlüssel zu klassischen Werken, München. 



\title{
Fernsehserien und die Wahrnehmung der US-Präsidentschaft
}

\author{
Einstellungen, politischer Zynismus und House of Cards
}

Zaira Batroff, Lorena Capuozzo, Kai Jardner, Klaus Kamps

\section{EINLEITUNG}

,FU2016.com` - unter dieser Netzadresse betrieb der Streaming-Dienst Netflix ab Dezember 2015 für einige Monate eine Marketingmaßnahme ganz eigener Art: einen rundum realistisch gestalteten, alle Facetten abdeckenden Netzwahlkampf für Frank Underwood, den virtuellen US-Präsidenten der Erfolgsserie House of Cards. Underwood musste passenderweise in der gerade anlaufenden und also zu bewerbenden vierten Staffel sich selbst der Wahl stellen. Konsequenterweise platzierte Netflix dann in einer Werbeunterbrechung der ersten realen - Fernsehdebatte im Vorwahlkampf der Republikaner einen gleichfalls professionell aufgemachten Wahlkampfspot („It's a new day in America“), zu dessen Ende der virtuelle Präsident (gespielt von Kevin Spacey) der Nation direkt in die Kamera versprach: „America, I'm only getting started“. Dass damit ein für die Erfolgsserie charakteristisches, machtpolitisches Verhalten des ansonsten wahlweise wenig skrupulösen Underwood gemeint war (mit dem er übrigens an eine traditionelle Elitenkritik des US-Wahlkampfrepertoires anknüpfte), mag eine Mottosammlung der ,Wahlkampf“-Seite unterstreichen: „Progress. Forward Momentum. Getting things done. These are my mottos. I have no patience for useless things - like political gridlock and stagnation. And neither should you. Join me. Let's roll up our sleeves together. Let's plow through the stubborn and smallminded and send Washington a message loud and clear." (FU 2106) Und so wie derartige crossmediale Marketingelemente selbst das Spiel zwischen Fiktion und Realität auf die Spitze treiben, so kann man der Serie gleichfalls bescheinigen, durch z.B. den dokumentarischen Schulterblick oder 
die direkte Ansprache der Zuschauer durch den Protagonisten Underwood, die Grenze zwischen, wirklicher' Politik und,Politik in einer Fernsehserie' auf eigene Weise zu beleuchten.

Freilich soll und wird hier nicht der Realitätsgehalt von House of Cards näher betrachtet, also die ,Wirklichkeit der Medien“ in Form eines spezifischen Erzählstils. Vielmehr bietet die Serie, ihr Zugang zur US-Politik, der politische Detaillierungsgrad und ihr Publikumserfolg, Anlass genug, eine klassische Frage anzuschließen: die nach den ,Folgen“ der Wahrnehmung, der Rezeption von Medieninhalten. Was, grob gefragt, bewirkt denn eine solche Serie mit ihrer Art der Politikdarstellung, mit ihrer Weise des unterhaltungsorientierten „Blicke[s] auf die Hinterbühne der Politik“ (Dörner 2016)? Ziehen bestimmte Formate ein Publikum an, das tendenziell extremere, gar explizit zynische Einstellungen pflegt, etwa gegenüber dem politischen System an sich? Derart wäre eine ganze Palette konkreter Bezugnahmen und Fragestellungen denkbar. Im Folgenden konzentriert sich die kleine Studie auf den Zusammenhang zwischen dem Konsum der Serie House of Cards und der Wahrnehmung von Kandidaten im USWahlkampf einerseits sowie der Einstellung gegenüber der US-Präsidentschaft andererseits.

\section{THEORETISCHE EINORDNUNG}

Vor knapp einem Jahrhundert formulierte der amerikanische Publizist Walter Lippmann eine später vielzitierte Metapher zur Frage der ,Wirklichkeit der Medien“. Damals noch mit Blick auf die Presse sprach er von den „Pictures in our heads“ (Lippmann 1922: 1), den „Bildern in unseren Köpfen“, die wir der Presse verdankten: einer vornehmlich (und notwendigerweise) verzerrenden medialen Realitätsdarstellung, die von der Leserschaft übernommen werde, zumal dann, wenn es sich um Personen, Dinge oder Vorgänge handele, die sich der direkten, eigenen Wahrnehmung überwiegend entziehen (z.B. die internationale Politik). Lippmann entwickelte daraus den durchaus medienkritisch verstandenen Begriff des ,Stereotyps ${ }^{\text {: }}$ eines in (weiten Teilen) der Gesellschaft angenommenen, vereinfachenden mentalen Bildes von Gruppen, Personen oder Ereignissen - kognitiver Konzepte, die von der Presse systematisch angeboten und von den Rezipienten übernommen würden, weil sie das Verständnis von komplizierten und distanzierten Dingen erleichtern oder gar erst ermöglichen. Eine Nähe zu gegenwärtigen Konzepten und Modellen im Kontext öffentlicher, politischer Kommunikation wie etwa ,Skripts', ,Narrativen “ oder gar ,Frames ' ist offenkundig: Ausgangspunkt ist eine (wie auch begründete) komplexitätsreduzierende, gleichfalls Einzelheiten betonende und darüber rezeptionssteuernde ,Abbil- 
dung ' und „Beschreibung ' von Vorgängen, von Personen, Gruppen, ja Ländern, Institutionen, Organisationen. Und dass sich dabei unter Relevanzgesichtspunkten die Sozialwissenschaften vornehmlich für eben solche Gegenstände interessieren, die über das individuelle, idiosynkratrische Erfahren hinausgehen, hat Niklas Luhmann in der schon programmatischen Eröffnung seiner Realität der Massenmedien so zusammengefasst: „Was wir über unsere Gesellschaft, ja über die Welt, in der wir leben, wissen, wissen wir durch die Medien.“ (Luhmann 1995: 5)

Eine derartige Globalperspektive umfasst ein komplexes, differenziertes Medienwirkungsverständnis mit unterschiedlichen Niveaus und Dimensionen der Medienzuwendung und der Rezeptionsfolgen: nicht allein Einstellungen und Verhalten einzelner Rezipienten aufgrund dieser oder jener medialer Stimuli (z.B. des Kaufs eines Produktes als Werbewirkung), sondern zudem solche, die sich als weithin geteilte Wahrnehmungsmuster gegenüber auch abstrakten Größen wie beispielsweise politischen Institutionen, Organisationen, Systemen, Kulturen oder gar Gesellschaften manifestieren (Schweiger 2013: 20). In der Kommunikationswissenschaft hat sich dabei mit der ,Kultivierungsforschung ' in den 1960er Jahren ein Ansatz früh etabliert, der eben diesen weiten Zugang pflegt (Gerbner/Gross 1976, Jäckel 2011, Rossmann 2013). Dabei stand anfangs die Frage nach den Folgen von Gewaltdarstellungen im seinerzeitigen Leitmedium Fernsehen im Mittelpunkt: ,Kultiviert‘ das Fernsehen als primäre, wichtigste mediale Sozialisationsinstanz bei seinen Zuschauern eine Weltsicht, die Kriminalität und Gewalt gegenüber der wirklichen Gewaltproblematik überschätzt, eben weil es diese Problematik z.B. aus Gründen der Aufmerksamkeitsökonomie sowohl in Informationsformaten (Negativismus in Nachrichten) als auch in Unterhaltungsangeboten (Spannung, Dramatik) übertrieben darstellt? Wer sich auf das Fernsehen verlässt, hält die Welt für krimineller - und verhält sich dann auch ängstlicher? Allgemeiner ausgedrückt besagt die Kultivierungsthese also, dass Weltbilder, Normen und Werte langfristig stabil durch systematisch inadäquate Medienberichterstattung beeinflusst werden können. ,Können“ - denn eine zentrale Variable wurde seinerzeit schon unmittelbar deutlich: Relevante Unterschiede in den Effektstärken zeigten sich vornehmlich im Vergleich der Extreme ,Vielseher" einerseits und Personen andererseits, die das Fernsehen nur sehr wenig nutzten. Bei weniger deutlichen Differenzen im TV-Konsum traten sofort moderatere Effekte auf.

Im Kontext der Ökonomisierung des Mediensystems (Kabel- und Satellitenfernsehen, private Rundfunkanbieter) und angesichts einer steigenden Parteienund Politikverdrossenheit wurde dann Anfang der 90er Jahre eine ähnlich gelagerte These der ,Videomalaise‘ aufgestellt (Holtz-Bacha 1990). Im Kern handelt 
es sich auch dabei um Fragen der Kultivierung: Führen eine - der Konkurrenz geschuldete - vermeintlich weniger seriöse, unterhaltungsoriertierte Politikberichterstattung im Fernsehen sowie neue Formate des ,Politainments (Dörner 2001) zu einer Ablenkung von der Politik? Steigert eine Fernsehkultur, in der alles Politische (Personen, Ereignisse, Positionen und Programmentwürfe) letztlich über den Modus der Unterhaltung rezipiert wird, den Vertrauensverlust weiter Bevölkerungsteile gegenüber der Politik, den Parteien oder anderen politischen Institutionen? Auch die Arbeiten zur ,Videomalaise" differenzierten den breiten Kultivierungsansatz weiter aus. Zum Beispiel scheint die ursprünglich für die USA formulierte These (Robinson 1976) schon deshalb nur schwer auf Deutschland übertragbar, weil hierzulande solche Personen, die das Fernsehen viel nutzen, deutlich politikinteressierter sind als ihre Pendants jenseits des Atlantiks. Und Jens Wolling (1999) arbeitet unter Rekurs auf einen differenzierteren Einstellungsbegriff heraus, dass die (unterhaltungsorientierte) Fernsehnutzung einen geringeren Faktor ausmacht im Vergleich etwa zur persönlichen Betroffenheit von Politikinhalten oder der Einschätzung der eigenen wirtschaftlichen Erfahrungen und wahrgenommenen Chancen. Zudem zeigten sich durchaus widersprüchliche Zusammenhänge: beispielsweise ein positiver Zusammenhang zwischen der Nutzung von Unterhaltungsangeboten und politischen Kompetenzund Legitimitätszuschreibungen sowie der Identifikation mit der politischen Kultur. Daneben gleichwohl steigert eine zugespitzte, ja aggressive politische Auseinandersetzung und Sprache - etwa in Talkshow-Formaten oder Fernsehdebatten (Mutz 2007) - zwar ein Unterhaltungserlebnis und das Interesse an politischen Themen, verringert aber das Vertrauen in die beteiligten Akteure und das politische System. Ähnliches ist inzwischen auch für politische OnlineDiskussionen beschrieben worden (z.B. Borah 2013).

Am elaboriertesten und explizit unter demokratietheoretischen Gesichtspunkten hat wohl Pippa Norris (u.a. 2000) solche oder ähnliche Fragen an die Rezeption von politischer Kommunikation in den vergangenen Jahren in der vergleichenden Systemforschung formuliert - und später auch im Kontext der Etablierung des Internets und der Ausdifferenzierung von Informationsrepertoires (Norris 2006). In einer breiten, internationalen Studie zu Nachrichtenformaten und -rezeption belegt sie eine konstant positive Beziehung zwischen einem aufmerksamen Nachrichtenpublikum und politischem Wissen, ja der Bereitschaft, sich politisch zu engagieren. Zunehmender Negativismus in Nachrichtenformaten und politische Inszenierungen führten dagegen dazu, dass einzelnen policies und politischen Positionen weniger vertraut wurden. Norris formulierte insbesondere einen virtuous circle, einen zweiseitigen Prozess: Zum einen werde auf längere Sicht durch wiederholte Nutzung von Informationsmedien, aber auch 
durch Wahlkampagnen oder alltägliche politische Kommunikation vorhandenes politisches Interesse weiter gestärkt - durch die Aneignung von Wissen und Kompetenzen (was wiederum politische Partizipation fördere). Zum anderen aber könnten Nachrichtenmedien politisch Desinteressierte nicht stärker für den politischen Prozess erwärmen, da sie nicht nur weniger aufmerksam rezipierten, sondern gegenüber der medialen Quelle darüber hinaus misstrauischer seien. In Folgestudien hat Norris im Übrigen gezeigt, dass diese ,zwei Seiten der Medail-

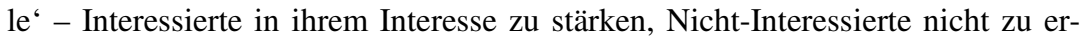
reichen bzw. nicht überzeugen zu können - auch für Netzangebote des Journalismus und für Parteien gelten (Norris 2006).

\section{FORSCHUNGSFRAGEN}

Allgemein gesprochen gehen Studien zur politischen Information und Unterhaltung - wie die eben exemplarisch vorgestellten Arbeiten - meist davon aus, auch Unterhaltungsformate oder unterhaltungsorientierte Informationsformate wie Talkshows hätten demokratietheoretisch wünschenswerte Effekte: ein durch ihren Konsum gesteigertes Interesse an Politik oder politischen Akteuren oder gar die Anregung zu anders gelagertem politischem Engagement (Baumgartner/ Morris 2008). Freilich verweisen solche Arbeiten dann meist auf diverse moderierende Variablen wie politisches Vorwissen, Betroffenheit in einer konkreten Situation, Informationsrepertoire der Nutzer allgemein und mehr. Daher werden für das eingangs dargelegte Interesse an House of Cards keine konkreten Thesen, sondern allgemeine Forschungsfragen formuliert. Dabei gilt insbesondere: Das Interesse der Studie liegt zentral auf dem Unterschied entsprechender Faktoren bei Zuschauern (,Vielsehern') und ,Nicht-“ bzw. ,Wenig-Sehern' der Serie.

Als erste, weit gefasste Frage: Inwiefern beeinflusst die Rezeption der Serie House of Cards die Einstellung gegenüber der US-Präsidentschaft? Diese Formulierung setzt zwar einen Einfluss voraus, lässt aber die Intensität offen und erfasst gegebenenfalls noch andere Zusammenhänge, etwa politische Einstellungen und Nutzung der Serie.

Um House of Cards sozusagen ,realweltlich“ zu verankern, wurde konkret Bezug genommen auf den US-Präsidentschafts-Wahlkampf 2016: Bewerten Zuschauerinnen und Zuschauer der Serie die Präsidentschaftskandidaten Hillary Clinton und Donald Trump oder den (seinerzeit) amtierenden US-Präsidenten Barack Obama jeweils besser oder schlechter - im Vergleich zu Personen, die die Serie nicht kennen respektive sie nicht gesehen haben?

Als wichtige moderierende Variable hat sich in der Kultivierungsforschung das Interesse am konkreten Gegenstand bzw. an Kenntnissen von ihm erwiesen; 
daher wird als dritte Forschungsfrage formuliert: Interessieren sich Zuschauer der Sendung auch allgemein mehr für die Politik in den USA? Dabei soll hier schon angemerkt werden, dass diese Studie in Deutschland realisiert wurde und entsprechend ein Interesse an einem fremden politischen System abgefragt wurde.

Explizit mit Blick auf House of Cards wurde, viertens, ein konkretes Einstellungskonstrukt näher betrachtet: politischer Zynismus. Es ist zumindest plausibel, dass Zynismus für die Rezeption der Serie eine besondere Rolle spielen mag - einer Serie, die gekennzeichnet ist durch den einerseits fast dokumentarischen Charakter im Verbund mit andererseits einem machtpolitisch skrupellosen und kriminellen Protagonisten wie Underwood. Daher wurde zuletzt formuliert: Zeichnet Zuschauer der Sendung ein im Vergleich zu Personen, die die Serie nicht kennen respektive sie nicht gesehen haben, größerer politischer Zynismus aus?

\section{FORSCHUNGSDESIGN}

Zur Beantwortung dieser Fragen wurde - auch aus forschungsökonomischen Gründen (es handelt sich um ein Lehrforschungsprojekt) - die Methode der vollstandardisierten Online-Umfrage gewählt; die Reihenfolge der Fragen variierte dabei nicht, und bis auf wenige persönliche Fragen zu den Probanden umfasste der Fragebogen ausschließlich geschlossene Fragen. Die wichtigsten Vorteile der Online-Befragung sind die rasche Realisierbarkeit und die vergleichsweise einfache Datenaufbereitung (Raab-Steiner/Bensch 2015: 49).

Die für die Studie herangezogenen Fragen und Skalen wurden weitgehend nicht selbst entwickelt, sondern wissenschaftlichen Publikationen entnommen; damit konnte sichergestellt werden, dass ein überwiegender Teil der verwendeten Items bereits in der Forschung validiert wurde. Der Fragebogen gliederte sich dabei in zwei Abschnitte:

Im ersten Teil fanden sich Skalen zu psychologischen Daten und Eigenschaften der Umfrageteilnehmer wie politische Einstellung oder Involvement. Etwa wurde hier das Konstrukt der Wahrnehmung der US-Präsidentschaft an eine Skala zu ,Spitzenkandidaten im Wahlkampf‘ angepasst und umfasste bspw. Vertrauen, Glaubwürdigkeit, Sympathie und wahrgenommene Macht (Klein et al. 2002). Eine andere Skala in diesem Abschnitt bezog sich auf das Involvement der Probanden in Bezug auf die US-Politik; hier wurde auf das von Zaichowsky (1994: 70) entwickelte und auf semantischen Differentialen beruhende Messmodell Personal Involvement Inventory zurückgegriffen. Eine weitere Item-Batterie befasste sich dann mit der politischen Einstellung, konkret mit dem politischen 
Zynismus, wobei hier eine elaborierte Skala von Hermann (2009) genutzt werden konnte.

Im zweiten Teil des Fragebogens standen konkrete Fragen zu House of Cards im Mittelpunkt. Zunächst wurden nach entsprechender Abfrage Teilnehmer als ,Nicht-Seher' der Serie klassifiziert, wenn sie weniger als mindestens drei Folgen einer Staffel gesehen hatten; nur House-of-Cards-Zuschauer füllten diesen zweiten Abschnitt des Fragebogens aus. Gefragt wurde hier u.a. nach einer Selbsteinschätzung: Wie sehr - etwa - habe die Serie die eigene Vorstellung vom Amt des US-Präsidenten geprägt. Erbeten wurde von den Probanden zudem eine subjektive Bewertung zum Realismus der Darstellung des Alltages des US-Präsidenten (also: Frank Underwood), z.B. in Bezug auf seinen Umgang mit Journalisten. Auch wurde hier eine Frage gestellt zur Sympathie gegenüber dem virtuellen Präsidenten - gleich jener Abfrage im ersten Teil zur Sympathie gegenüber den aktuellen, faktischen Kandidaten sowie Barack Obama. Abschließend folgten für alle Probanden die üblichen sozio-demografischen Fragen nach Alter, Geschlecht, Bildungsabschluss und beruflicher Tätigkeit.

\section{TEILNEHMER}

Für die Studie wurden Teilnehmer im Zuge eines convenience samples rekurriert, d.h. als Gelegenheitsstichprobe oder auch willkürliche Auswahl nach Verfügbarkeit (Brosius/Haas/Koschl 2016: 67, Frings 2010), weil die Probanden nach der „Prämisse ihrer Erreichbarkeit“ (Misoch 2015: 193) ausgewählt werden. Dabei wurden verschiedene Online-Verteiler und -Kanäle herangezogen: zunächst der studentische Verteiler einer größeren Hochschule in Süddeutschland; um aber nicht nur studentische Teilnehmer zu akquirieren, wurde der Fragebogen (respektive der entsprechende Link) noch über persönliche Profile sowie weitere Netzwerk-Gruppen verteilt. Selbstverständlich hat eine solche Gelegenheitsstichprobe erhebliche Limitationen, allen voran die fehlende Repräsentativität. Während conveniences samples für elaborierte Forschungsdesigns in den Sozialwissenschaften dann häufig abgelehnt werden, können solche Stichproben in bestimmten Zusammenhängen dennoch gerechtfertigt sein (Leiner 2016) „convenience samples enable research and explore ideas that may appear farfetched" (Meltzer et al. 2012: 252). So wird in dieser explorativen Studie eine Repräsentativität, wie beispielsweise in der Wahlforschung üblich und notwendig, nicht angestrebt. Und sie kann es auch nicht, weil die Merkmale der wichtigen Grundgesamtheiten ,Nutzer“ und ,Nicht-Nutzer der Serie“ (Alter, Geschlecht, Bildung u.Ä.) nicht bekannt sind bzw. entsprechende Zahlen u. W. nicht von Netflix veröffentlicht wurden. Zusätzlich muss die wichtige Einschränkung er- 
wähnt werden, dass die Ergebnisse möglicherweise durch die Nicht-Teilnahme bzw. durch den Abbruch des Fragebogens verzerrt wurden, weil angesichts des Themas ,Politik‘ Menschen, die sich nicht oder weniger für dieses interessieren oder gar als ,politikverdrossen“ gelten können, im Gegensatz zu politisch interessierteren Personen eher nicht teilgenommen haben dürften.

Letztlich den kompletten Fragebogen ausgefüllt haben 454 Teilnehmer; begonnen hatten ihn 663, so dass eine Nettoteilnehmerzahl von rund 68 Prozent erreicht wurde. (Da die Gesamtheit der angefragten Adressaten in den verschiedenen Verteilern nicht bekannt ist, kann keine Angabe zur Rücklaufquote gemacht werden.)

Gerundet 53 Prozent der Probanden $(n=243)$ waren Männer, 46 Prozent Frauen $(n=211)$. Hinsichtlich der Altersverteilung gibt es einen klaren Trend: Rund 79 Prozent ( $\mathrm{n}=360)$ waren zwischen 20 und 29 Jahren alt, nur rund 8 Prozent jünger, nur rund 13 Prozent älter. Das korrespondiert mit einem deutlichen Bias in der Tätigkeit: Rund 85 Prozent der Befragten waren Studenten ( $n=387)$; insofern ist eindeutig von einem weit überwiegenden studentischen Sample auszugehen, trotz der Hinzuziehung auch außer-akademischer Verteiler.

Eine zentrale Unterscheidung war noch die zwischen House-of-CardsZuschauern und entsprechenden ,Nicht-Zuschauern“. Das Verhältnis dieser beiden Gruppen ist in etwa $1 \mathrm{zu}$ 2. 34,14 Prozent der Probanden dürfen als $\mathrm{HoC}$ Zuschauer gelten $(n=155), 65,86$ Prozent als ,Nicht-HoC-Zuschauer' $(n=299)$. Dabei ändert sich die Zusammensetzung hinsichtlich der Tätigkeit und die hinsichtlich des Alters nur marginal. Auffällig ist hingegen und insbesondere, dass sich bei dieser Gruppierung dann die Verteilung beim Geschlecht deutlich verändert: Bei den $H o C$-Zuschauern sind immerhin rund 61 Prozent (n=95) Männer (gegenüber n=60 Frauen), während sich das Verhältnis bei den ,Nicht-HoCZuschauern' verkehrt: Hier finden sich noch rund 39 Prozent Männer ( $n=118)$, rund 60 Prozent Frauen $(\mathrm{n}=181)$.

\section{ERGEBNISSE}

Zentral hat sich die vorliegende Studie auf vier Fragekomplexe konzentriert: die Einstellung gegenüber der US-Präsidentschaft (1); die Bewertung der Präsidentschaftskandidaten und des seinerzeit realen Präsidenten Obama sowie des virtuellen Amtsinhabers Underwood (2); das Interesse an US-Politik (3) sowie schließlich das besondere Einstellungskonstrukt ,politischer Zynismus ‘ (4). Dabei werden insbesondere Unterschiede zwischen den Gruppen der House-ofCards-Zuschauer und ,Nicht-Zuschauer" der Serie berücksichtigt und Chi- 
Quadrat-Tests unterzogen; gemeldet werden hier entsprechende asymptotische, d.h. zweiseitige Signifikanzen.

Hinsichltich der Einstellung gegenüber der US-Präsidentschaft bzw. des Interesse an der US-Politik weisen die Gruppen Unterschiede auf dem 000.Signifikanz-Niveau auf, d.h. die beobachteten Unterschiede sind mit ausnehmend hoher Wahrscheinlichkeit nicht zufällig: HoC-Zuschauer weisen ein höheres Involvement und Interesse an US-Politik auf, verbunden mit einem stärkeren aktiven Informationsverhalten zur US-Politik. Diese Aussage ist korrelativ zu verstehen, also als ,Zusammenhang'. Eine Kausalität soll und kann aus methodologischen Gründen nicht angenommen werden. Hingegen nicht signifikant ist die Einschätzung der Macht des US-Präsidenten; die Serie verleitet also nicht zu der Annahme einer machtvolleren oder weniger machtvolleren Präsidentschaft was sicher auch auf die Dramaturgie zurückzuführen ist: Der virtuelle Präsident muss sich immer wieder schwierigen Hindernissen im Prozess der Mehrheitsbeschaffung im Kongress stellen. Ebenfalls nicht signifikant sind die Unterschiede zwischen den Nutzergruppen hinsichtlich ihrer Skalenwerte zum politischen Zynismus. Insofern hat die Serie in dieser Erhebung zumindest keinen messbaren ,Kultivierungseffekt' dergestalt, dass ,Vielseher' die (US-)Politik pauschal (im Vergleich zu ,Nicht-Sehern') ablehnen und ihr kritisch gegenüber eingestellt sind; vermutlich dürften hier die erwähnten höheren Informationsaktivitäten der HoC-Zuschauer moderierend wirken.

Die Bewertungen der US-Präsidenten bzw. der Präsidentschaftskandidaten, d.h. die in verschiedenen Dimensionen gemessenen Einschätzungen von Barack Obama, Hillary Clinton, Donald Trump und Frank Underwood, ergaben die aus unserer Sicht wohl interessantesten Ergebnisse. Sicher - einen virtuellen Präsidenten mit den Probanden als real bekannten, wenngleich distanzierten Spitzenpolitikern aus den USA vergleichen zu lassen, hat auch spielerischen Charakter. Gleichwohl mag, bei allen Einschränkungen, das einen explorativen Zugang zur realweltlichen Verankerung der Serie erlauben.

Dabei ist zweierlei vorab zu erwähnen. Erstens, über verschiedene Einschätzungsdimensionen hinweg gilt: Die Nutzung der Serie hat keinen signifikanten Effekt auf die personengebundenen Antworten zu Barack Obama; seine Einschätzung durch die Befragten hängt nicht von House of Cards ab. Hingegen gilt das genau umgekehrt (im Detail unten) für Hillary Clinton und Donald Trump: Deren Wahrnehmung ist in dieser Untersuchung sehr wohl mit der $\mathrm{HoC}$ Rezeption verbunden. Zweitens: Die Rangfolge der den Personen zugewiesenen politischen und persönlichen Qualitäten ist über die verschiedenen erhobenen Dimensionen hinweg bei den $\mathrm{HoC}$-Nutzern identisch mit der Rangfolge, die die ,Nicht-Seher' der Serie erstellten: Am positivsten wird der zum Zeitpunkt der 
Studie amtierende Präsident Obama beurteilt; danach folgen Hillary Clinton und Donald Trump. Interessanterweise ordnen die HoC-Zuschauer Frank Underwood meist zwischen Clinton und Trump ein. Zu lesen ist $\mathrm{u}$. E. diese recht konstante Einschätzung auch als liberaler Bias innerhalb des studentischen Samples.

Zunächst ein Vergleich der Mittelwerte der zugeschriebenen ,Politischen Qualitäten' (Tabelle 1); bei diesen Mittelwerten handelt es sich um einen Summenindex, der sich aus 9 Dimensionen einer fünfstufigen Likert-Skala ergibt (was ein positives Maximum von 45 ermöglicht). Die Items bezogen sich dabei beispielsweise auf Führungsqualitäten, Problemlösungskompetenz, Einsatz für die USA.

Tabelle 1: Mittelwertvergleich Politische Qualitäten

\begin{tabular}{|l|l|c|c|c|c|}
\hline \multicolumn{2}{|c|}{} & Obama & Trump & Clinton & Underwood \\
\hline \multirow{2}{*}{$\begin{array}{l}\text { mit } \\
\text { HoC }\end{array}$} & Mittelwert & 28,0645 & 12,355 & 19,3613 & 19,4452 \\
\cline { 2 - 6 } ohne & Mittelwert & 27,7291 & 155 & 155 & 155 \\
\cline { 2 - 6 } HoC & N & 299 & 299 & 299 & - \\
\hline \multicolumn{2}{|l}{ Signifikanz (2-seitig) } & 0,496 & 0,002 & 0,019 & - \\
\hline
\end{tabular}

Aus der Tabelle wird ersichtlich, dass die besten politischen Qualitäten Barack Obama zugeschrieben werden; auf den virtuellen Frank Underwood fallen sogar minimal bessere Werte als auf Hillary Clinton. Zuletzt folgt Donald Trump.

Bei den ,Persönlichen Qualitäten` zeigt sich ein ähnliches Bild (Tabelle 2; fünf Fragen einer 5er-Likert-Skala, maximal positiver Wert 25): Hier wird der fiktive Charakter Frank Underwood jedoch schlechter $(-1,90)$ bewertet als Hillary Clinton, allerdings auch deutlich besser als Donald Trump (+4,07). Die Items dieses Abschnittes umfassten Fragen etwa nach Sympathie, Vertrauenswürdigkeit, Solidität des Privatlebens.

Tabelle 2: Mittelwertvergleich Persönliche Qualitäten

\begin{tabular}{|l|l|c|c|c|c|}
\hline \multicolumn{2}{|c|}{} & Obama & Trump & Clinton & Underwood \\
\hline \multirow{2}{*}{$\begin{array}{l}\text { mit } \\
\text { HoC }\end{array}$} & Mittelwert & 15,2452 & 3,3419 & 9,3161 & 7,4129 \\
\cline { 2 - 6 } ohne & Mittelwert & 15,3545 & 155 & 155 & 155 \\
\cline { 2 - 6 } HoC & N & 299 & 299 & 299 & - \\
\hline \multicolumn{2}{|l}{ Signifikanz (2-seitig) } & 0,733 & 0,055 & 0,001 & - \\
\hline
\end{tabular}


Diesen personengebundenen Teil abschließen soll ein Blick auf die politische und die persönliche Vertrauenswürdigkeit (Tabelle 3), denen jeweils zwei Items zugeordnet werden können, so dass der maximal mögliche Wert 20 beträgt. Die Unterschiede zwischen den $\mathrm{HoC}$-Nutzern und den ,Nicht-Nutzern' sind auch hier (bis auf die Werte von Obama) signifikant. Im Vergleich liegt das Vertrauen in den fiktiven Charakter Frank Underwood unterhalb von Barack Obama und Hillary Clinton, jedoch über der Vertrauenswürdigkeit in den damaligen (Sommer 2016) Präsidentschaftskandidaten Donald Trump.

\section{Tabelle 3: Mittelwertvergleich Vertrauen in die Person}

\begin{tabular}{|l|l|c|c|c|c|}
\hline \multicolumn{2}{|c|}{} & Obama & Trump & Clinton & Underwood \\
\hline \multirow{2}{*}{$\begin{array}{l}\text { mit } \\
\text { HoC }\end{array}$} & Mittelwert & 11,7548 & 2,0774 & 7,0065 & 3,9419 \\
\cline { 2 - 6 } ohne & Mittelwert & 11,7993 & 1,5886 & 155 & 155 \\
\cline { 2 - 6 } HoC & N & 299 & 299 & 299 & - \\
\hline \multicolumn{2}{|l}{ Signifikanz (2-seitig) } & 0,887 & 0,024 & 0,003 & - \\
\hline
\end{tabular}

Die in diesen drei Tabellen gemeldeten Werte sind statistisch übrigens nicht durch den Faktor ,Geschlecht der Probanden“ beeinflusst.

Diese personengebundenen Vergleiche sind u. E. vor allem in zweierlei Hinsicht interessant. Erstens: Die so positive Einschätzung Barack Obamas belegt nicht allein dessen positive Wahrnehmung in Deutschland respektive im Sample; sie verweist auch auf den Faktor, Wissen', der für Probanden einer solchen Studie eine Rolle spielt: Über Obama ,weiß` man weit mehr, als man ,ahnt ‘ - und zwar in einer faktischen, nicht fiktionalen Dimension. Zweitens spiegeln die Werte natürlich eine Image-Hierarchie wider. Donald Trump hat - zumindest in dem überwiegend studentischen Sample im Frühsommer 2016 - ein deutliches Image-Defizit, als Politiker wie als Person. Darauf verweist die Konstanz dieser Einschätzung über die Probandengruppen hinweg. Interessanterweise wird er dabei von der Gruppe der House-of-Cards-Nutzern immer schlechter als Frank Underwood - ein virtueller Spitzenpolitiker, der auch bei einer gelegentlich sympathischen Darstellung seiner Person seine Machtinteressen durch Intrige, Lüge, Manipulation, Täuschung und auch Mord durchsetzt - eingeschätzt. Trump wird konstant schlechter eingeordnet. Aber, und das ist womöglich wiederum ein Effekt der Serie: Bei aller Negativität in der Einschätzung von Trump ist diese in den verschiedenen Dimensionen bei den Zuschauern von House of Cards immer leicht besser als bei denen, die Frank Underwood nicht kennen. Ohne dass das Untersuchungsdesign das valide belegen kann, drängt sich hier doch die These auf, dass die Kenntnis von House of Cards die überwiegend 
schlechte Wahrnehmung des Präsidentschaftskandidaten Trump geradezu positiv moderiert, jedenfalls bei weiter niedrigen Werten seine negative Wahrnehmung etwas abschwächt. Kurz: Frank Underwood als Referenzmodell lässt Donald Trump in etwas weniger üblem Licht erscheinen.

\section{DISKUSSION UND FAZIT}

Mit der Fernsehserie House of Cards hat der Streaming-Anbieter Netflix ein ausgesprochen erfolgreiches Politainment-Format gefunden, das sich durch eine Mischung aus dokumentarischen und klassisch-dramaturgischen Elementen kennzeichnet. Aus der Sicht der Kultivierungsforschung lässt sich fragen, ob der spezifische Blick, den diese Serie auf die US-Politik und ihre Akteure wirft, deren Wahrnehmung durch die Zuschauer wiederum ,kultiviert'. Es konnte gezeigt werden, dass die Nutzung der Serie mit einem höheren Involvement einhergeht, auch mit einer höheren Informationsaktivität. Eine klassische Kultivierungsfolge - ein erhöhter Zynismus - wie hier vermutet, wurde indes nicht vorgefunden. Die Studie konnte keinen Hinweis darauf identifizieren, dass ein höheres oder geringeres Maß an politischem Zynismus ein Merkmal der Zuschauer der Serie ist. Die interessanteren Zusammenhänge zeigten sich im personengebundenen Teil der Befragung, wobei u. E. diese Daten dann auch auf eine Kultivierungsfolge hinweisen: Der virtuelle Präsident Frank Underwood war für die eine Gruppe der Befragten dieser Studie als Referenzpunkt verfügbar, für die andere Gruppe nicht. Es scheint, als habe diese virtuelle Referenz bei der einen Gruppe einen Einfluss auf die Wahrnehmung der realen Bewerber Hillary Clinton und Donald Trump (zumindest in der konkreten Befragungssituation) - im Unterschied übrigens zu Barack Obama, bei dem die Studie keinen Unterschied in der Bewertung nachweisen konnte. Das legt die Vermutung nahe, dass die Qualität wie Quantität des Wissens über eine solche Person entscheidend dafür sein könnte, ob der fiktive Referenzpunkt tatsächlich Einfluss hat. Frappierend bleibt vor allem: Dem fiktiven Charakter Frank Underwood werden - obwohl er in der Serie mehrere Morde und auch sonst regelmäßig gegen Moral und andere Normen verstößt - konstant höhere politische wie persönliche Kompetenzen zugeschrieben als Donald Trump.

\section{LITERATUR}

Baumgartner, Jody C./Morris, Johnathan S. (Hrsg.) (2008): Laughing matters. Humor and american politics in the media age, New York. 
Borah, Porismita (2013): Interactions of news frames and incivility in the political blogosphere: Examing perceptual outcomes, in Political Communication, 30, S. 456-473.

Brosius, Hans-Bernd/Haas, Alexander/Koschel, Friederike (2016): Methoden der empirischen Kommunikationsforschung: Eine Einführung, Wiesbaden.

Dörner, Andreas (2001): Politainment: Politik in der medialen Erlebnisgesellschaft, Frankfurt a.M.

Döner, Andreas (2016): Politserien: Unterhaltsame Einblicke auf die Hinterbühne der Politik, in: Aus Politik und Zeitgeschichte (51), S. 4-11.

Frings, Cornelia (2010): Soziales Vertrauen: Eine Integration der soziologischen und der ökonomischen Vertrauenstheorie, Wiesbaden.

FU 2016 (2016): Frank Underwood 2016 - Anything for America. Online unter: https:/www.fu2016.com (zugegriffen am 18.08.2016).

Gerbner, George/Gross, Leonard (1976): The scary world of TV's heavy viewer, in: Psychology today, 89(4), S. 41-45.

Hermann, Michael C. (2009): Politische Weltbilder Jugendlicher in der Mediendemokratie: Eine interdisziplinäre Analyse am Beispiel von Wahlkampfkommunikation, Bad Heilbrunn.

Holtz-Bacha, Christina (1990): Ablenkung oder Abkehr von der Politik? Mediennutzung im Geflecht politischer Orientierung, Opladen.

Jäckel, Michael (2011): Medienwirkungen. Ein Studienbuch zur Einführung, 5. Aufl., Wiesbaden.

Klein, Markus/Ohr, Dieter/Heinrich, Stefanie (2002): Spitzenkandidaten im Wahlkampf. Die Veränderbarkeit von Kandidatenimages durch Wahlkampf und Medien, in: Publizistik 47 (4), S. 412-435.

Leiner, Dominik J. (2016): Our research's breadth lives on convinience samples. A case study of the online respondent pool „SoSci Panel“, in: Studies in Communication and Media, 5 (4), S. 367-396.

Lippmann, Walter (1922): Public Opinion, London.

Luhmann, Niklas (1995): Die Realität der Massenmedien, Wiesbaden.

Meltzer, Christine E./Naab, Thorsten/Daschmann, Gregor (2012): All student samples differ: On participant selection in communication science, in: Communication Methods and Measures, 6 (4), S. 251-262.

Misoch, Sabina (2015): Qualitative Interviews, Berlin.

Mutz, Diana (2007): Effects of „,in-your-face“-television discourse on perceptions of legitimate opposition, in: American Political Science Review 101, S. 621-635.

Norris, Pippa (2000): A Virtuous Circle. Political Communications in Postindustrial Societies, Cambridge/New York. 
Norris, Pippa (2006): Die Überzeugten überzeugen? Pluralismus, Partizipation und Parteien im Internet, in: Kamps, Klaus/Nieland, Jörg-Uwe (Hrsg.): Regieren und Kommunikation, Köln, S. 261-284.

Raab-Steiner, Elisabeth/Benesch, Michael (2015): Der Fragebogen: von der Forschungsidee zur SPSS-Auswertung, 4. Aufl., Wien.

Robinson, Michael J. (1976): Public Affairs Television and the Growth of Political Malaise: The Case of „The Selling of the Pentagon“, in: The American Political Science Review 70, S. 409-432.

Rossmann, Constanze (2013): Kultivierungsvorschung: Idee, Entwicklung und Integration, in: Schweiger, Wolfgang/Fahr, Andreas (Hrsg.): Handbuch Medienwirkungsforschung, Wiesbaden, S. 207-223.

Schweizer, Wolfgang (2013): Grundlagen: Was sind Medienwirkungen? Überblick und Systematik, in: Schweiger, Wolfgang/Fahr, Andreas (Hrsg.): Handbuch Medienwirkungsforschung, Wiesbaden, S. 15-37.

Wolling, Jens (1999): Politikverdrossenheit durch Massenmedien? Der Einfluss der Medien auf die Einstellung der Bürger zur Politik, Opladen/Wiesbaden.

Zaichowsky, Judy L. (1987): The emotional aspect of product involvement, in: NA - Advances in Consumer Research 14, S. 32-35. 


\section{Veep}

Das Amt des Vizepräsidenten der USA

als institutionalisierte Bedeutungslosigkeit?

\section{Alexander Stock}

\section{SATIRE ÜBER DEN WASHINGTONER POLITIKBETRIEB}

\section{Hintergrund: Eine VP im Alltagskampf}

In Veep - Die Vizepräsidentin übernimmt die ehrgeizige Ex-Senatorin Selina Meyer (Julia Louis-Dreyfus) den Posten der Vizepräsidentin der USA. Der vermeintlich mächtige Job im Zentrum der Macht erweist sich in der ersten Staffel als Scheinmacht ohne bedeutungsvolle Befugnisse. Veep steht für die umgangssprachliche Abkürzung von VP - Vice President. Die Polit-Satire und „Arbeitsplatz-Comedy“ (Rehfeld 2012a) schildert den bürokratischen Alltag voller Stolpersteine in den politischen Sphären Washingtons. Hierbei blickt der Zuschauer der Protagonistin in den ,Hinterzimmern der Macht' über die Schultern. Die Serie wählt die Form der Fokussierung und Personalisierung des politischen Akteurs der Vizepräsidentin, des Menschen Selina Meyer und ihres persönlichen Stabs. Politische Prozesse und Abläufe der politischen Figur der fiktiven Vizepräsidentin werden abgebildet sowie künstlerisch inszeniert. Durch Humor und böse Sprüche ist es eine ,irrwitzige Persiflage auf den politischen Betrieb in Washington“ (Schmieder 2016) und damit das witzige Gegenstück zum ernsten Politdrama House of Cards.

Veep steht in der Tradition von The Thick of it - der Erfinder Armando Iannucci ist auch der Produzent von Veep - und der britischen Politik-Sitcom Yes, Prime Minister. Iannucci bereitete sich intensiv auf das Thema vor und recherchierte beziehungsweise hospitierte beim ehemaligen Obama-Assistenten Reggie Love (Rehfeld 2012b). Mit Veep möchte Iannucci „einen Blick auf das dysfunk- 
tionale Washington werfen“ (Rehfeld 2012b). In bislang sechs Staffeln mit insgesamt 58 Folgen schildert er das Schicksal einer Vizepräsidentin und später Präsidentin, die „von inkompetenten Mitarbeitern umgeben und selbst einfach blöd ist" (Möller 2015). Eine weitere siebte Staffel wurde bereits angekündigt.

Die US-Polit-Serien konzentrieren sich in der großen Mehrheit auf die Fokussierung des Akteurs Präsident, der - wie narrative Unterhaltungs- und Stilelemente verdeutlichen - der mächtige Protagonist ist. In der Konzentrierung und völligen Verdichtung des Serienstoffes auf die Vizepräsidentin - und damit ,nur' auf die Nummer zwei des Staates - für drei volle Serienstaffeln ist Veep nahezu einzigartig. Dieser Artikel analysiert im Folgenden die erste Staffel hinsichtlich der Ausgestaltung der Rolle der Vizepräsidentin und dem Narrativ des Kampfes gegen die eigene Bedeutungslosigkeit. Ferner werden die kulturelle Symbolik sowie der Realitätsbezug der Serie untersucht.

\section{Synopsis zur ersten Staffel: Ein Ausnahmetalent für Fettnäpfchen}

In den acht Folgen der ersten Staffel von 2012 handelt es sich durchgehend um die Themen Macht, Einfluss und Interessendurchsetzung im Washingtoner Alltagswahnsinn. Einzelne zentrale Themenmotive und Handlungsstränge ziehen sich wie ein roter Faden durch alle acht Folgen, jede Folge kann darüber hinaus aber auch singulär mit ihren individuellen inhaltlichen Stories angeschaut werden.

Die $V P$ verfolgt im Kern der ersten Staffel die von ihr angestoßene Filibuster-Reform als auch ihre ,Grüne-Jobs-Kampagne' mit dem Kernstück der Einführung von kompostierbarem Besteck in allen Regierungsgebäuden. Dadurch verärgert sie allerdings die Plastikindustrie sowie die Öl-Lobby. Bei beiden Vorhaben ist sie Feuer und Flamme, jedoch zugleich abhängig von der Unterstützung anderer Akteure.

Die Filibuster-Reform bedarf der Unterstützung eines Senators und der Stimmen seiner Anhängerschaft im Senat. Für seine Zustimmung stellt er die Bedingung, Öl-Lobbyisten aus der ,Grüne-Jobs-Kommission“ herauszuhalten. Der Präsident begrüßt es hingegen, dass sich die $V P$ bei der Filibuster-Reform künftig zurückhalten solle, zugleich entzieht der besagte Senator seine Unterstützung, was die Stimmenmehrheit für die Reform verloren gehen lässt.

Die ,Grüne-Jobs-Kampagne' kommt ins Schleudern, da aufgrund der Konzentration auf einen eigenen präsidialen Gesetzentwurf zur verantwortungsvollen Finanzpolitik der Präsident andere Gesetzentwürfe fallen lässt - einer davon ist der zu den ,Grünen Jobs‘. Beim Stab der VP entsteht die Idee, den Hauptinhalt 
der ,Grünen Jobs‘ als Zusatz an den Gesetzentwurf des Präsidenten anzuhängen und ihn durch die Hintertür durch den befreundeten Senator Macauley einbringen zu lassen. Dadurch besteht die Hoffnung, den Kern der ,Grünen-Jobs-Idee“ zu erhalten, ohne dass die $V P$ in Verdacht gerät, dahinterzustecken. Als es zu einer Stimmengleichheit im Senat kommt, muss die VP schweren Herzens mit Nein stimmen, da der Präsident den Zusatz in keiner Form will. Am Ende der Staffel kocht der gescheiterte Macauley-Gesetzeszusatz wieder hoch. Der stellvertretene Vorsitzende der Kongressaufsichtskommission droht ein Ermittlungsverfahren gegen das Amt der VP und gegen ihren Mitarbeiter Dan Egan wegen des Zusatzes und der damit einhergehenden Korruption anzustrengen.

Um die kaltgestellte $V P$ nach der Abstimmungsniederlage $\mathrm{zu}$ besänftigen, überträgt der Präsident ihr die Leitung eines Programmes gegen Adipositas, das ihm sehr am Herzen liegt. Eine verärgerte $V P$, die sich mit dem neuen Thema Adipositas nicht anfreunden kann, startet nur widerwillig mit ihrer frisch übertragenen Gesundheitsinitiative unter dem Motto „Get Moving“.

Nachdem sich die VP über einen lächelnden Secret-Service-Agenten ärgert lässt sie ihn durch ihre Stabschefin Amy Brookheimer versetzen. Durch die von der Presse thematisierte Story zur hochgekochten „Lächel-Affäre“ stehen die VP und ihre Stabchefin mächtig unter Druck. Um ihren Job zu behalten, geht Brookheimer einen Deal ein, um so die VP aus der Schusslinie zu holen: sie schildert einem Magazin die Story, sie sei schwanger gewesen, habe eine Fehlgeburt gehabt und habe sich daher in einem emotionalen Trauma befunden, was ihren Geisteszustand erkläre, aufgrund dessen sie den Secret-Service-Agenten versetzt habe.

Die in den acht Folgen dargestellten Politiker sind

„keine Bösewichte, sondern eher arme Schweine, deren letzte Reste von Idealismus in den Kleinkriegen des Tagesgeschäftes aufgerieben werden. Moralisch verkommen ist vor allem ihre Entourage, jene, die sich von ihrer Nähe Vorteile erhoffen. Da nutzen Regierungsbeamte ihre Stellung für Sex aus [...], betteln um Aufmerksamkeit [...] oder versuchen sich mit Nonsens-Ideen zu profilieren“ (Brühl 2012).

Das zentrale Leitmotiv, das sich durch die gesamte Serie zieht, ist Schadensbegrenzung zu betreiben. Die VP und ihr Team sind ständig bemüht den ,,neuesten Fauxpas zu überspielen. Die Frau hat ein Ausnahmetalent für Fettnäpfchen“ (Rehfeld 2012b). 


\section{KONTEXT: DER VP ZWISCHEN THEORETISCHER ROLLE UND PRAKTISCHER AKTION}

\section{Verfassungswirklichkeit und Rolle im politischen System der USA}

Der Vizepräsident der Vereinigten Staaten ist einerseits nur „einen Herzschlag von der Macht entfernt“ (Sattar 2017) und zugleich ist seine Rolle ,undankbar“ (Oldopp 2013: 82). Diese Ambivalenz wird deutlich, wenn man sich die Verfassungswirklichkeit und die Rolle des VP im politischen System näher anschaut. Er ist als designierter Nachfolger sowohl ein Präsident ,in Wartestellung und zugleich ohne klare Ressortverantwortung absolut darauf angewiesen, „dass er vom Präsidenten ins politische Leben einbezogen wird“ (Oldopp 2013: 82). Der erste Vizepräsident der USA, John Adams, bezeichnete sein Amt im 18. Jahrhundert verdrossen als das bedeutungsloseste, ,das menschliche Erfindungskraft je geschaffen und menschliche Phantasie je vorzustellen vermocht haben“ (Adams zit. n. DER SPIEGEL 1963: 74).

Rechtlich ist der VP laut US-Verfassung Präsident des Senats, der im Falle der Stimmengleichheit mit seiner Stimme den Ausschlag gibt. Im zwölften Zusatzartikel ist die Verfassungsbestimmung hinterlegt, dass Präsident und VP aus unterschiedlichen Bundesstaaten stammen sollten. Seit 1804 wird der VP vorgeschlagen und jeder Kandidat muss auch zum Präsidenten wählbar sein: mindestens 35 Jahre alt, seit mindestens 14 Jahren einen festen Wohnsitz in den USA und gebürtiger US-Staatsbürger (,natural born citizen“). Im 25. Zusatzartikel von 1967 ist geregelt, dass der VP dem Präsidenten während der Amtszeit bei Tod, Rücktritt, Arbeitsunfähigkeit oder Amtsenthebung im Amt folgt - als ,Reservepräsident‘ (Oldopp 2013: 83-84).

Die Geschichte der bisher vom Präsidentschaftskandidaten ausgewählten $V P$ hat deutlich gemacht, dass die Auswahl der sogenannten running mates einem großen Satz an Kriterien und parteipolitischen, wahltaktischen und persönlichen Variablen unterliegt. Die Kandidaten zur Vizepräsidentschaft sind daher ,selten Wunschkandidaten“ (Oldopp 2013: 84) und eher Zünglein an der Waage im politischen Wettbewerb. Mal geht es ganz schlicht um die nüchterne Verbrüderung mit dem stärksten Vorwahlkampfrivalen, mal um religiöse Gründe und ein anderes Mal um regionale Aspekte, so zum Beispiel etwa, wenn der Kandidat durchseine Erfahrung und Verwurzelung in den entscheidenden Bundesstaaten dem Präsidenten zu einer besseren Zielgruppenansprache verhilft. In der Mehrzahl der Fälle wird der $V P$-Kandidat aber mit dem Motiv der Komplettierung des eigenen Profils ausgewählt. Er kann zum Beispiel eigene Schwächen durch ver- 
vollständigende Eigenschaften oder andere policies ausgleichen und damit eine signifikante Anhängerschaft an ihn binden, so z.B. ethnische Schlüsselgruppen wie die Hispanics oder Afroamerikaner im Wahlkampf mobilisieren. Gerade ein völlig gegensätzlicher Charakter als der des Präsidentschaftskandidaten kann jenen Wählern und Parteigruppen gefallen, die sich mit diesem schwertun.

Letztendlich angekommen im Amt des $V P$, werden wichtige Rollen und Funktionen wahrgenommen. Als Mitglied der Regierung mit einem eigenen Stab ist der VP zu Beginn der Amtszeit eine wichtige Stütze in der Transitionsphase. Er ist darüber hinaus als Präsident des Senats wertvoll für die Koordination mit Senat und US-Repräsentantenhaus und unterstützt den Präsidenten bei repräsentativen Aufgaben wie beispielweise Auslandsreisen und Benefizveranstaltungen. Er soll dem Präsidenten den Rücken stärken und stand in der Vergangenheit eher für unaufgeregte Sacharbeit während der Legislaturen. In dem Zusammenspiel mit dem Präsidenten ist vor allem das persönliche Vertrauen zueinander die wichtigste Grundlage - da er nicht selbstständig politische Entscheidungen treffen kann, hängen seine grundsätzliche Stellung, die ihm übertragenen Kompetenzen und mögliche Sonderbefugnisse ,im erheblichen Maße vom persönlichen Vertrauensverhältnis zum Präsidenten ab“(Oldopp 2013: 82).

\section{Vizepräsidenten in Aktion seit dem 20. Jahrhundert}

Die herausgearbeiteten Rollen des $V P$ sind in der Theorie ebenso differenziert wie die Praxisbeispiele der VP seit dem 20. Jahrhundert. Die Bandbreite der Bewertungen reicht da vom machtvollen Amt bis hin zur Bewertung des Amtes als „ein warmer Eimer Spucke“ (Brühl 2012). Bei den bislang 24 amtierenden VP seit 1900 lassen sich mehrere Klassifizierungen durchführen, wie die bisherigen $V P$ ins Amt gekommen sind (Hess 2002, Lutteroth 2008, Süddeutsche Online 2008, Oldopp 2013). Fünf VP rückten aus der zweiten Reihe durch die ,Hintertür ${ }^{6}$ - ohne stattgefundenen Wahlgang - zum Präsidenten auf: Theodore Roosevelt, Calvin Coolidge, Harry Truman, Lyndon B. Johnson und Gerald Ford. Zwei weitere VP wurden später durch das Wählervotum selbst Präsident: Richard Nixon und George Bush sen. Im Hinblick auf ihre Bewertung sind folgende drei Typen zu unterscheiden: die machtvollen $V P$, die stark im Team agierenden $V P$ sowie die bedeutungslosen $V P$.

Mächtige $V P$, bei verhältnismäßig schwachen Präsidenten, waren Nixon und Cheney. Nixon konnte als Vize beim kranken Präsidenten Dwight D. Eisenhower stark mitregieren und dem Amt neue Bedeutung geben. Bei Dick Cheney, VP von George W. Bush, habe es die Standardabfrage seitens des Präsidenten gegeben: „What does Dick think?“ (Milkis/Nelson zit. n. Oldopp 2013: 83). Cheney war der mächtige ,Strippenzieher' im Hintergrund, der aus der zweiten Reihe 
wahrlich regierte. Er war Architekt des Kriegs im Irak und der Antiterrorbekämpfung (Lutteroth 2008).

Starke politische Gespanne waren beispielsweise Truman/Barkley, Carter/Mondale oder Clinton/Gore. Alben Barkley hatte auch den Spitznamen , The Veep " und wurde von Truman zum Vorsitzenden des Nationalen Sicherheitsrates ernannt. Walter Mondale als wichtiger Berater von Carter „was consulted on all major decisions“ (Hess 2002: 141) und wertete das Amt des VP auf. Al Gore bildete mit Präsident Bill Clinton ebenfalls ein sichtbares Team. Gore hatte die Möglichkeit, Politik aktiv mitzugestalten, so in der Umweltpolitik.

Eine letzte Kategorie an $V P$ sind die Bedeutungslosen, zu nennen wären hier Truman, Spiro Agnew und Johnson. Truman hatte mit Roosevelt nahezu keinen Umgang, war nicht eingebunden und ,wusste nicht, dass die USA an der Atombombe forschten“ (Brühl 2012). Agnew als Vize von Nixon war ebenfalls nicht in den engeren Zirkel eingebunden. Johnson war zuvor mächtiger Fraktionsführer im Senat und als $V P$ in Gesetzesverfahren und außenpolitischen Themen von John F. Kennedy nicht einbezogen. Verstimmt betonte er einst: „Ich gebe legislativen Rat nur, wenn ich danach gefragt werde.“ (DER SPIEGEL 1963: 75) Meistens wurde er nicht gefragt, stattdessen mit vielen Auslandsreisen betraut. In einer Johnson-Biografie findet sich sein verbittertes Fazit seiner Vizepräsidentschaft: „Ich habe kein Budget und ich habe keinerlei Macht, ich habe nichts." (Caro 2012: 173).

\section{NAH AN DER MACHT, ABER DOCH MACHTLOS}

\section{Narrativ des Kampfes gegen die eigene Bedeutungslosigkeit}

Die VP kämpft die gesamte erste Staffel über mit der gefürchteten und empfundenen Bedeutungslosigkeit und Machtlosigkeit. „Ich bin die Veep, die ich nie sein wollte“ (S 1/F 6/1.01), äußert sie betrübt in der sechsten Folge. Denn sie will mehr Einfluss und Bedeutung innehaben. Sie hat die ständige Angst, ausgebootet und nicht mehr gebraucht zu werden. Sie ist selbst unsicher und spürt direkt die Sorge, beim Auftritt eines potentiellen Konkurrenten, dem Gouverneur von Minnesota Danny Chung, ein Kriegsheld asiatischer Herkunft, eventuell als Vize ersetzt zu werden (S 1/F 4). Bei der VP kommen erste Zweifel auf, ob der Präsident weiter auf sie als $V P$ setzt oder sie vielleicht gar durch so einen charismatischen Akteur ersetzen könnte. Sie ist so nervös, dass sie direkt eine lausige Hintergrundrecherche zu seiner Person anfertigen lässt. Die Angst bleibt bis zur letzten Folge, in der sie erfährt, dass Chung nun bereits mit dem Präsidenten Golf spielt. 
Sie fragt sich, ob sie weiterhin die uneingeschränkte Unterstützung des Präsidenten für ihren Gesetzesentwurf hat (S 1/F 5) und beklagt sich: „Der Präsident meidet mich" (S 1/F 5/5.26). Für diese Unsicherheit gibt es aus ihrer Sicht auch klare Argumente, die sich aus den erlebten Erfahrungen speisen. Sie wird bei wichtigen Entscheidungen nicht mitgedacht, das Briefing zur verantwortungsvollen Finanzpolitik, zum dem sie nicht eingeladen wurde (S 1/F 5), ist bloß ein Beispiel für das Außenvorbleiben der VP.

Sie kämpft dauernd um Anerkennung und unternimmt angestrengte Profilierungsversuche bei wenig bedeutsamen Terminen: ein Community-collegeTreffen mit Schülern (S 1/F 6), eine Rede beim Feuerwehrverband (S 1/F 5), ein reiner PR-Besuch in einem Frozen-yoghurt-Laden (S 1/F 2) oder die belanglose Stippvisite beim Interstate Police Breakfast (S 1/F 7). Keiner der Termine vermittelt dem Zuschauer politische Inhalte, was stellvertretend für die Belanglosigkeit steht.

Sie wünscht sich mehr Macht und weiß, dass sie diese derzeit nicht hat: „Ich halte mehr Macht in meinen Händen, wenn ich eine dieser schwachsinnigen Segway-Touristenführungen durch D.C. leiten würde“ (S 1/F 5/17.07 - 17.12). Die $V P$ befindet sich in einem permanenten und fortdauernden Krisenmanagement. Durch aufkommende Hektik, Krisenintervention, Handeln unter ständigem Zeitdruck mit Informationsdefiziten und Treffen von Ad-hoc-Entscheidungen ist immer nur Reaktion statt Aktion gefragt, unmittelbares statt weitsichtiges Handeln. Bei ausnahmslosem Löschen von politischen Brandherden gibt es keine langfristigen Visionen, keine Planungen, keine Strategie und man sieht, wie „weit der politische Betrieb von der bewussten Strategiebildung entfernt [ist], wie viel Dilettantismus und Tollpatschigkeit am Werk sind [...]“ (Möller 2015). Die VP behält nie die Deutungshoheit über ihre Themen und sieht sich immer wieder unerwarteten und unkalkulierbaren Situationen ausgesetzt. Durch die vielen Krisen und Skandale ist sie nicht nur fremdgesteuert, sondern man macht sich auch über sie lustig.

Neben den Vetospielern aus der Wirtschaft und aus den Lobbyverbänden ist der Präsident für die VP ein Vetospieler. Sie ist dem Präsidenten weisungsgebunden und alle Wünsche und Vorstellungen des Präsidenten haben immer Vorrang, eigene Zielvorstellungen müssen dahinter zurücktreten. Dies gibt den Rahmen vor für ihre empfundene Bedeutungslosigkeit. Das Amt des VP definiert „sich durch das Verhältnis zum Präsidenten. Wenn der dich nicht dabeihaben will, hängst du im Leeren“ (Iannucci zit. n. Rehfeld 2012a). Der Präsident führt keine direkte Kommunikation mit ihr, so dass sie imaginäre Anrufe des Präsidenten vortäuscht (S 1/F 3). Er entscheidet, ob sie ihr Herzensthema weiter fortführen darf oder abbrechen muss. In einer Szene lässt er das gesamte Redeskript 
so zusammenstreichen, dass nur noch die dümmliche Plattitüde „In der Politik geht es um die Menschen“ (S 1/F 1/14.33) übrigbleibt. Andererseits ist die VP für den Präsidenten keinesfalls unbedeutend. Sie ist ein Spielball für ihn und sie kann dadurch instrumentalisiert werden: ,Will er mich jetzt etwa per Fernbedienung steuern?“ (S 1/F 4/5.44 - 5.47). Er kann sich von ihr auf Benefizveranstaltungen vertreten lassen (S 1/F 1), er kann von ihren Ausarbeitungen und Kompetenzen profitieren (S 1/F 2) oder sich kraft seines Amtes Themen der VP mit neuem Labeling einverleiben. Das Narrativ der Bedeutungslosigkeit der $V P$ wird unterfüttert mit der absoluten Erfolglosigkeit ihrer Projekte. Es gibt nur einzelne Versuche, substantielle policies umzusetzen, die dann allesamt bitterlich scheitern.

Am Ende hat sie nur Niederlagen vorzuweisen, alle ihre Herzensprojekte schlagen fehl und andere Vorhaben misslingen: die Filibuster-Reform (S 1/F 4) und das ,Grüne-Jobs-Projekt‘ (S 1/F 5) scheitern aufgrund von fehlender Unterstützung durch den Präsidenten und Mehrheiten im Senat. Dass diese beiden Großprojekte scheitern steht stellvertretend für das Gesamtscheitern der $V P$ : „Haben Sie eigentlich eine Vorstellung davon, was das für mich für eine Niederlage ist?" (S 1/F 5/17.12 - 17.16). Die Suche nach einem ,Privat-Hund“ (S 1/F 3), um Sympathiepunkte in der Öffentlichkeit zu gewinnen, muss gecancelt werden, da auch die First Lady sich einen ,First Dog' anschaffen will und diese Aktion nicht überschattet werden darf. Die ersehnte Dienstreise nach Paris wird wegen einer Senatssitzung kurzfristig abgesagt (S 1/F 5) und der von der VP versetzte Secret-Service-Agent kehrt gegen ihren Willen zurück (S 1/F 8). Einziger unbedeutender Erfolg ist es, dass ein Hurrikan nicht ,Selina' genannt wird, der im kommenden Jahr als Hurrikan-Name vorgesehen war (S 1/F 3).

\section{Akteure in der Serie Veep}

Das Format der politischen Serie fußt auf Handlungsstrukturen und Akteurskonstellationen. Es stellt sich daher die Frage, wie Politik in Veep durch die Akteure dargestellt und bewertet wird. In Veep gibt es eine zentrale Hauptdarstellerin: VP Selina Meyer. Sie ist vom Präsidenten aufs Abstellgleis geschoben worden und nimmt in seiner Vertretung an symbolischen Akten, Fototerminen und Benefizveranstaltungen teil. Sie ist kein Polit-Neuling mehr, sondern bereits seit 20 Jahren in Washington tätig. Sie ist eine egozentrische $V P$, wankelmütig, dünnhäutig, narzisstisch und sehr öffentlichkeitsbezogen. All ihr Handeln ist auf ihr Auftreten in der Öffentlichkeit ausgerichtet, sie ärgert sich gerne über die negativen Pressemeldungen. Die VP hat eine unglaubliche Begabung, ein Lächeln vorzutäuschen und fröhlich zu wirken, wie sie es selbst ausdrückt, „die Sonne aus meinen Arsch scheinen [zu] lassen“ (S 1/F 6/1.10). 
Während der reale VP in der Realität einen Stab von circa 70 Mitarbeitern hat (Lutteroth 2008), besteht in der ersten Staffel ihr innerer Entscheidungszirkel aus fünf Protagonisten, die die Serie in den Fokus nimmt: der Stabschefin Amy Brookheimer, dem Kommunikationschef Mike McLintock, dem stellvertretenden Kommunikationschef Dan Egan, dem persönlichen Assistenten Gary Walsh und der Sekretärin Sue Wilson.

Amy Brookheimer ist eine Karrierefrau mit Organisationskompetenz und versteht sich als ,Problemlöserin rund um die Uhr‘. Sie organisiert den Entscheidungsprozess und ist immer sehr engagiert, eine Art 24-Stunden-Modus mit Überstunden nimmt sie in Kauf. Ihr Job ist es, das Image der $V P$ aufrechtzuerhalten. Selbst eine Schwangerschaft der $V P$ verkauft sie pressewirksam in der Öffentlichkeit als ihre eigene, um den potentiellen medialen Shitstorm präventiv abzuwehren (S 1/F 7).

Mike McLintock ist der schusselige Kommunikationschef, der sich persönlich als „Fußabtreter“ (S 1/F 3/3.21) sieht. Mit seinem Habitus wirkt er unprofessionell, zumal er selbst nicht immer zu 100 Prozent bei der Sache ist. Er vermeidet es, besonders viel Arbeit in seinen Job zu stecken, schläft während der Arbeitszeit und benutzt einen imaginären Labrador als Vorwand keine Überstunden machen zu können (S 1/F 1). Er beweist häufig mangelhafte strategische Kommunikationskompetenz.

Dan Egan ist für die VP der „Scheiß-Kerl-Alter-Ego“ (S 1/F 1/26.45). Er ist keinesfalls loyal und hat seinen Vorgängerjob bei einer Senatorin für diese reizvollere Position gekündigt und zuvor noch eine diskreditierende, kompromittierende Story an die Presse durchsickern lassen. Auch diesen Job sieht er als Sprungbrett. Er ist überheblich und versucht, andere auszunutzen. Für die VP ist er ein wichtiger strategischer Kopf mit guter Intuition und vielen Ideen, die in Krisensituationen zu Lösungsansätzen führen. Egan ist sehr ambitioniert, ehrgeizig und gerissen, er spekuliert auf den Abgang von McLintock oder schielt auf den Job des Stabschefs.

Gary Walsh ist als persönlicher Assistent loyal und fungiert als ,„duckmäuserische Zofe“ (Rehfeld 2012a). Sein Job ist es, die VP zu beschützen und glücklich zu halten. Er ist geduldig und nachgiebig, zu viel Stress und Nervosität kann er aber nicht vertragen. Er besorgt der $V P$ alles, was sie braucht, und übernimmt auch Zusatzaufgaben; so stellt er sein Privathaus zur Verfügung und macht im Auftrag und in Abwesenheit der VP mit ihrem Freund Schluss (S 1/F 7). Er flüstert ihr immer Infos zu Gesprächspartnern ins Ohr, als „,menschlicher Teleprompter für Smalltalk“ (S 1/F 3/17.12) ist er jederzeit an ihrer Seite. Er wirft sich für sie ins Feuer, sein Job ist sein Leben, die VP wäre ohne ihn verloren und hilflos. 
Für die Sekretärin Sue Wilson ist es „ein Job, alles zu wissen“ (S 1/F 4/13.00). Sie schirmt die $V P$ von unbequemen Gästen ab, managt den Terminkalender und nimmt im Vorzimmer die Anrufe entgegen. Sie ist direkt, kommt gleich zur Sache und streitet sich mit Walsh, wer der VP nähersteht (S 1/F 7).

Eine weitere wichtige Nebenrolle spielt der Verbindungsmann zum Weißen Haus Jonah Ryan. Er ist arrogant und betont dauernd, dass er im Weißen Haus arbeitet. Er ist geltungssüchtig, selbstgefällig, selbstzufrieden und herablassend und wird von niemandem gemocht. Der Begriff „Jonah“ ist bereits im gängigen Washington-Sprachgebrauch angekommen und inzwischen „eine Bezeichnung für aufgeblasene Aufschneider niederen Ranges am Capitol Hill“ (Rehfeld 2015).

Diese Akteure der Serie vermitteln keinen Teamspirit, sondern wirken wie die Zusammenballung von Individualisten. Zusagen werden gebrochen, man ist selbstsüchtig auf den eigenen Vorteil bedacht. In der Geisteshaltung der ,Fahrradfahrer-Mentalität“ werden Ideale verraten und,,angestrengt der nächste Rangniedere in Reichweite düpiert und die eigene Wichtigkeit ausgestellt" (Rehfeld 2015). Aber auch die VP spricht in ihrem Beraterkreis keinerlei Gemeinschafts- oder Gruppendenken an, das den gemeinsamen Teamgeist stärken könnte - es herrscht eher Ich-Bezogenheit vor. Vielmehr echauffiert sie sich über ihr Büro, „das, verdammte Scheiße, nicht richtig funktioniert“ (S 1/F 7/13.12). Ihr Stab soll aber auch oft Unmögliches möglich machen, so zum Beispiel dabei helfen, etwas Gesagtes „ungesagt zu machen“ (S 1/F 2/12.05). „Die Figuren sind Opfer der Umstände, ihr Karriereziel ist, sich über Wasser zu halten“ (Rehfeld 2012a). Das ,Küchenkabinett“ besteht aus politischen Arrangeuren und eher glücklosen Organisationstechnikern, deren oftmals scheiterndes Handeln sich abschließend fast komplett mit pejorativen Adjektiven charakterisieren lässt. Es ist ein dysfunktionales Team, changierend zwischen Hektik und Chaos. Die administrative Rationalität ihres Apparates ist damit begrenzt. VP Meyer bringt die Kompetenz ihres Stabs zusammenfassend selbst auf den Punkt: „Das Niveau der Inkompetenz in diesem Büro ist einfach erschütternd“ (S 1/F 1/21.04 - 21.07).

\section{Auswahl dargestellter policies}

Die erste Staffel erzählt von drei größeren Policy-Feldern, die in mehreren Episoden behandelt werden. Die Umweltpolitik steht an erster Stelle, mit den Widerständen und Einflusssphären der Öl-Lobby, Meyers ,Grüne-Jobs-Kampagne“ und der ,Maisstärkebesteck-Kampagne“ - es ist das Kernthema der VP, eine Form regulativer Politik mit den Instrumenten von Geboten, Verboten und Anreizprogrammen. Der Umweltschutz-Gesetzentwurf scheitert am Ende der fünften Episode. Als zweites inkludiert die Gesundheitspolitik mit dem Projekt 
gegen Fettleibigkeit die Themen Ernährung und Prävention. Das Politikproblem wird zwar erkannt, hat aber in seiner Darstellung ein negatives Policy-Image. Selbst die VP kann mit dem ihr vom Präsidenten übertragenen Thema nichts anfangen: „Und jetzt, was soll ich sagen? Ich bin die Vizepräsidentin der Vereinigten Staaten. Legen Sie den Muffin zurück!“ (S 1/F 5/25.05 - 25.10)

Und schließlich gibt es noch das innenpolitische Thema der FilibusterReform, die aufgrund der fehlenden Stimmenmehrheit in Folge 4 ihr Ende findet. Filibuster-Reden sind durchaus im Senat zur Verzögerung von Abstimmungen üblich. Der in der Serie nicht näher ausgeführte und definierte Reformansatz für eine Filibuster-Reform greift das durchaus reale US-Thema der Notwendigkeit einer Reform auf und deren thematische Aktualität auf. Noch jüngst gab es sogar im Repräsentantenhaus ein Filibuster der Sprecherin der demokratischen Minderheit, die über acht Stunden zum Thema Bleiberecht für Kinder von illegalen Einwanderern sprach. An weiteren Stellen werden die verantwortungsvolle Finanzpolitik mit einem verabschiedeten Gesetzesentwurf, die Außenhandelspolitik mit China als Handelspartner sowie die notwendige Einwanderungsreform als Plots eingestreut. Die Serie hat nicht den Anspruch, die Policy-Felder näher in den Vordergrund zu stellen und beispielsweise wie bei The West Wing durchzuexerzieren. Es sind immer nur kurze Handlungsstränge, die Politikfelder werden nur oberflächlich gestreift und inhaltlich nicht näher ausgeführt. Als Nebeneffekt können angeführte storylines und benutzte Narrative Themen auf die öffentliche Agenda setzen.

Probleme werden definiert, aber nicht gelöst, über das Agenda-Setting hinaus wird höchstens die Politikformulierung, aber nicht die Implementation erreicht. Die dargestellten policies sind nur das Vehikel zum Transport der Grundstory rund um den Kampf der $V P$ um Anerkennung ihrer eigenen Projekte, um die Interaktionen der Akteure untereinander sowie um die Darstellung der „Chronik aus Blamagen, Demütigungen und PR-Desastern“ (DER SPIEGEL 2016: 142) mit dem gewohnt bissig-trockenen Humor. Policies dienen somit als Hilfsmittel zur Darstellung der politics und polities.

\section{DIE POLITISCHE KULTUR IM FIKTIVEN WASHINGTON}

\section{„Hat der Präsident angerufen?“ - „Nein!“}

Die Korrelation zwischen den präsidialen Erfolgen und der im Zentrum stehenden Performanz des Präsidenten einerseits und dem daraus resultierenden Handlungskorridor und den Restriktionen der $V P$ andererseits erklärt die hohe Dependenz von VP Meyer in ihrer täglichen Amtsausübung. Das Kräfteverhältnis ist 
klar entschieden. Statt des Teamgedankens stehen die Konkurrenz und die deutliche Über- und Unterordnung im Fokus der Serie. Der Interaktionsprozess ist augenfällig geprägt durch ein hohes $\mathrm{Ma} \beta$ an fehlender Kommunikation. Alle Entscheidungen oder Botschaften des Präsidenten werden bloß mitgeteilt, nicht durch ihn persönlich, sondern hauptsächlich durch den Verbindungsmann Ryan. Der Präsident taucht in keiner einzigen Szene auf, der Präsident kontaktiert die $V P$ nie leibhaftig. Dies begründet auch den wiederkehrenden Running-Gag der gesamten ersten Staffel, bei dem die VP ihre Sekretärin jedes Mal fragt: „Sue, hat der Präsident angerufen?“ (beispielsweise S 1/F 1/08.05 - 08.08). Nein, der Präsident hat nie angerufen und bindet sie auch nicht mit in Entscheidungsprozesse ein, so wurde exemplarisch ein Treffen zum Verteidigungsetat ohne sie durchgeführt und auch ins Briefing zur verantwortungsvollen Finanzpolitik wird sie nicht miteinbezogen. Nach außen repräsentiert die $V P$ den Präsidenten und versucht, ihn mit mal besserer und mal schlechter Laune zu vertreten. Nach innen verflucht sie ihn mit Beschimpfungen: „Scheiße-im-Hirn-Präsidenten“ (S 1/F 2/16.38), „Dieser blöde Arsch“ (S 1/F 5/16.36), „Ich rufe den bescheuerten Präsidenten höchstpersönlich an. Ich sage ihm, er soll es sich in den Arsch stecken" (S 1/F 5/17.52 - 17.56). Sie hadert sehr mit ihrer derzeitigen, unbefriedigenden Situation, muss sich aber mit der gegebenen Lage arrangieren und ist deshalb ständig verärgert: „Ich muss dahin. Die können mich nicht ausschließen“ (S 1/F 5/3.50 - 3.51). Misstrauen belastet das Verhältnis zum Präsidenten, da die $V P$ dauernd erlebt, marginalisiert zu werden. $\mathrm{Zu}$ diesem Argwohn gehört auch, dass die $V P$ dem Präsidenten böse Absichten bei seinen Entscheidungen unterstellt: „Der Präsident weiß genau, wie unwohl ich mich in Gegenwart von fetten Menschen fühle“ (S 1/F 5/24.26-24.33).

\section{Kulturelle Symbolik - vermittelte Motive und Stereotypen}

Politische Kultur setzt sich zusammen aus Meinungen, Werten und Einstellungen und bezieht sich auf Bewusstseinslagen, Denk- und Handlungsweisen und Mentalitäten (Berg-Schlosser 2011). Veep fügt sich ein in ein amerikanisches Gesellschaftsklima, ,in dem der Kompromiss als Verrat und die Halsstarrigkeit als prinzipienfeste Tugend gilt“ (Ross 2013). Drei zur politischen Kultur zugehörige zentrale Motive und kulturelle Symbole lassen sich analytisch in Veep herauskristallisieren.

\section{Medien/Öffentlichkeit}

Ein durchgängiges Stilmittel politischer Kultur ist das Thema der Dominanz und Deutungsmacht der Medien. Das Team um die VP ist oft gesteuert durch mediale 
Ereignisse und versucht, den Imperativen der Mediendemokratie zu entsprechen. Gleichzeitig ist Meyer das Ansehen in der Öffentlichkeit sehr wichtig. Viele der Ereignisse sind reine öffentlichkeitsgetriebene Handlungen und PR-Aktionen und damit wissenschaftlich gesehen ,Pseudo-Ereignisse“: „Pseudo-Ereignisse sind Aktionen, die ausschließlich dazu dienen, die Aufmerksamkeit der Medien auf sich zu ziehen und die Berichterstattung zu stimulieren." (Pfetsch/SchmittBeck 1994: 238). Bei dem PR-Termin im Frozen-yoghurt-Laden (S 1/F 2), dem Gruppenfoto mit den Spielern der Baseball-Mannschaft der Baltimore Orioles (S 1/F 6) oder einem Krankenhaus-Besuchstermin (S 1/F 4), nachdem ein Kran auf ein Schiff gestürzt ist und zu Toten und Verletzten führt, geht es in Form von ,Placebo-Politik' einzig darum, sich medial gut zu inszenieren und positive Presse zu bekommen. Ähnliches gilt bei der Hurrikan-Namensdiskussion (S 1/F 3) oder der Frage der Anschaffung eines privaten Hundes zur Steigerung der Beliebtheit in der Öffentlichkeit, gerade da sie zu Letzterem ehrlich äußert: „Ich hasse diese Viecher" (S 1/F 3/15.34).

\section{Lobbyismus}

Öl- und Plastik-Lobbyisten erhalten in den ersten fünf Folgen eine äußerst wichtige Rollenzuschreibung und eine gewisse Vetospieler-Funktion, die bei politischen Entscheidungen mitgedacht werden müssen. Die Lobbyisten versuchen, politische Prozesse und Politikinhalte im Sinne ihrer Interessen zu beeinflussen und auch aktiv zu intervenieren. Eine Senatorin formuliert es mal so: „Wenn Du es Dir mit Plastik verscherzt, verscherzt Du es Dir mit Öl. Und mit Öl verscherzt man es sich nicht. Die Jungs sind nicht zum Scherzen aufgelegt" (S 1/F 1/06.08 - 06.13). Sie umgehen teils die Verfahren der parlamentarischen Demokratie und haben in Folge 1 einen direkten Zugang zum Präsidenten und damit anscheinend einen kurzen Draht ins Weiße Haus. Die Serie vermittelt eine starke Interdependenz zwischen politischen und wirtschaftlichen Subsystemen, die zu einer starken Stellung von Verbandsvertretern und Lobbyisten führt und damit auch zu einem möglichen Einfallstor für Korruption wird.

\section{Stereotypen/Klischees}

Politische Kultur umfasst Bewusstseinslagen und Mentalitäten, damit auch Stereotypen und Klischees. Veep erfüllt davon gleich mehrere und greift dessen Omnipräsenz in der Gesellschaft auf. Es wird mit der landläufigen Meinung und der Ironie gespielt. 
- Stereotyp 1: Politiker denken nur an sich und sind egoistisch: „Das ist ja echt großartig für mich“ - „Und für unser Land“ - „Ja, ja, das habe ich gemeint“ (S 1/F 2/1.05 - 1.10). Das Stereotyp nimmt das Klischee auf, dass Politiker nur nach persönlichem Aufstieg streben und Posten und Diäten im Blick haben.

- Stereotyp 2: Überheblichkeit und Allmacht der Politiker. Die VP ärgert sich über einen lächelnden Secret-Service-Agenten und versetzt ihn sofort (S 1/F 5). Bei Stereotyp 1 und 2 wird mit dem klischeehaften Bild gespielt Politiker könnten sich alles erlauben und hätten als Volksvertreter weniger den Bürger und Gesellschaft als vielmehr sich selbst im Sinn.

- Stereotyp 3: Das Polit-Establishment ist inkompetent. Die gesamte Entourage und die Politiker werden als Chaoten-Truppe dargestellt. Selbst die Schauspielerin Louis-Dreyfus unterstreicht, Meyer sei ,,,eine arme, lächerliche Frau“, die sie im wahren Leben nicht wählen würde“ (Louis-Dreyfus zit. in DER SPIEGEL 2016). Dieses Inkompetenz-Stereotyp ist ein Vorstellungsbild, das sich recht einfach verfängt und ohne die notwendige Kontextualisierung eine reine attributive Zuschreibung bleibt.

- Stereotyp 4: Politikerverdrossenheit. Dies wird in Folge 5 mit einer Vielzahl an albernen, aber auch zugleich verunglimpfenden Spitznamen der VP aufgegriffen. Die Stereotypen 1-4 stehen charakteristisch für eine gewisse Krise der Repräsentation und die Frustration über den aktuellen Politikbetrieb.

- Stereotyp 5: Politik ist unvereinbar mit der Familie. Meyer ist geschieden, sieht ihre Tochter Catherine zu selten und schafft es nicht, sich mit ihr normal zu unterhalten: „Du musst nur so tun, als würdest Du Dich ernsthaft mit mir unterhalten - wir tun nur so, als würden wir miteinander reden" (S 1/ F 3/15.54 - 15.57). Hier wird das Thema der Vereinbarkeit von Familienzusammenhalt, Kindererziehung und politischem Mandat kritisch aufgegriffen und auch das Geschlechter-Stereotyp miteinbezogen.

- Stereotyp 6: Politiker sind Schauspieler. Das Thema ,manipuliertes Weinen der $V P^{‘}$ sowie ,Fake-Tränen' (S 1/F 8) greift dieses Klischee einer inszenierten Medienfassade und fehlenden Authentizität auf und stellt die indirekte Frage, was in Zeiten von ,Fake-News ‘ noch geglaubt werden darf.

- Stereotyp 7: Reine Fokussierung auf Umfragewerte. Auch dieses Klischee geht auf das Thema der Glaubwürdigkeit von Politikern ein und vermittelt speziell in Folge 8 die Vorrangigkeit von öffentlicher Meinungsbildung und Performance statt politischer Inhalte, und das ständige Schielen auf demoskopische Umfragewerte von Meinungsforschungs- und Umfrageinstituten wie Gallup \& Co bei jedweder politischen Handlung. Aufgrund von miesen Umfragewerten der $V P$ bittet ein Gouverneurskandidat darum, dass die $V P$ sich nicht mehr öffentlich für ihn ausspricht - sie sei das ,totale Kassengift“ (S 1/F 
8/1.48) und „beschädigte Ware“ (S 1/F 8/7.11). Manipulierte emotionale Weinausbrüche in einem Fernsehinterview führen unterdessen zu fabelhaften Medienreaktionen und positiver Presse und zu verbesserten Umfragewerten.

- Stereotyp 8: Politik ist hart und rau. Politische Kultur inkludiert auch politischen Stil. Die Kultur in Veep ist rau und die Sprache schmutzig. Es wird obszön geflucht und es gibt drastische Ausdrucksweisen. Fast 250 „fucks“ werden in den acht Folgen gezählt (Rehfeld 2012a).

Diese exemplarischen acht Stereotypen sorgen dafür, dass Vorurteile damit bedient werden. Ein Problem entsteht, wenn das vermittelte Bild ebenfalls auf die Realität übertragen würde und die eigene selektive Wahrnehmung verengt bliebe und zugleich zu einer Manifestation der persönlichen Ansichten führt. Klischees werden pointiert und Politik dadurch entpolitisiert.

Diese Klischees stehen für eine Persiflage auf den politischen Betrieb, die kritisch mit dem politischen Pathos der US-Gesellschaft umgeht. Ebenfalls wird dadurch US-spezifisch auf die in der Realität stärkere Polarisierung in der Politik und die ,zerrüttete politische Kultur und das funktionsschwache politische System, bis hin zur Gefahr der Regierungsunfähigkeit.“ (Ross 2013) eingegangen, wie die bisweilen bevorstehende Shutdown-Situation beweist, bei der die Kluft zwischen den beiden Parteien offenbar wird und jedes Mal ein Zwangsstillstand droht.

\section{REALITÄTSBEZUG VERSUS UNTERHALTUNGSWERT}

Während in der Realität politische Entscheidungsprozesse strukturell immer komplexer werden, handelt es sich bei Veep um eine Reduktion von Komplexität, um dem Unterhaltsformat zu entsprechen. Die Serienmacher arbeiten mit einer Simplifizierung, um für das Narrativ der fiktionalen Vizepräsidentin zuschauerorientiert eine Unterhaltungs- und Erzählstruktur zu ebnen und so die Darstellung des US-Politikbetriebs zu ermöglichen. Politik als Serien-Fiktion ist immer nur reduzierte Wirklichkeit - und damit ist auch Veep konfrontiert. Serien müssen

„[...] verkürzen, verdichten, zuspitzen und beschleunigen. Alles passiert schneller, einfacher, eindeutiger als in Wirklichkeit, daher sind die Serien orientierungsfreundlich. Sie wollen mit dramaturgischen Mitteln unterhaltsame Spannungsbögen oder amüsante Situationskomik produzieren - und sie dürfen doch bei aller Zuspitzung die Verbindung mit dem, was wir als außermediale Wirklichkeit kennen, nicht völlig verlieren, sonst erschei- 
nen sie uns belanglos. Es geht um die richtige Balance zwischen Unterhaltungswert und Realitätsbezug“ (Dörner 2016: 7).

Während sich beispielsweise The West Wing gegen die Politikverdrossenheit wendet, spielt Veep mit vielen Klischees und Vorurteilen, gerade auch, um Witz und Satire der Serie zu unterstützen. „Die Als-ob-Welten sind kurzweiliger als der graue politische Alltag“ (Dörner 2016: 11). Prozesse und Interaktionen werden nicht so genau beschrieben, politische Parteien werden nicht genannt, Realitäten sind nicht operationalisierbar, im Gegensatz zu The West Wing gibt es wenig Zeit für politische Erzählungen, dennoch zeichnet Veep „,das realistischere Bild der Politik im politischen System“ (Möller 2015). Entscheidungen und Handlungsebenen im Tagesablauf der VP sind sehr einer symbolischen Politik in Form einer Darstellungs- statt Entscheidungspolitik (Sarcinelli 1987) unterworfen. Es gibt immer eine Wechselwirkung zwischen Realität und Fiktion, denn gerade die gewollte Rückkopplung und der mögliche Vergleich zwischen Realität und Fiktion „,begründe[n] die Relevanz der erfundenen Wirklichkeit einer Serie“ (Dörner 2016: 7). Vorherrschend bei Veep sind vereinzelt realistisches Agenda-Setting, der thematisierte Lobbyeinfluss und die VP als Spielball eines Präsidenten. Insgesamt weist Veep aber eher einen geringen Identifikations- und Orientierungsgrad hinsichtlich eines Rückschlusses auf reale Regierungswirklichkeiten auf.

Beim Studieren der ersten Staffel von Veep lässt sich herauslesen, dass die vorrangige Intention der Macher Iannucci/Blackwell nicht der realistische Blick in die amerikanische Regierungspraxis und das US-Polit-Geschäft gewesen sein kann. Teile der Episoden „,bereiten die untergründigen Machttechniken auf, die der Öffentlichkeit kaum zugänglich sind“ (Möller 2015), spitzen zu und simplifizieren. Gags und Pointen sind hier stärker gewichtet als Sachthemen, der kritische Diskurs fehlt und auf Idealismus wird völlig verzichtet.

Veep zeichnet eher ein negatives Bild der Politik. Politik wird als „Machtpolitik“ (Dörner 2016) gezeigt. Macht wird zwar legitim gebraucht, aber nicht für die Realisierung von policies und zur aktiven Gestaltung, sondern vielmehr als Selbstzweck und zur Steigerung von Anerkennung und Selbstwertgefühl. Aus extrinsischen Gründen wird Macht angestrebt, um der Macht willen. Veep gibt diese dargestellte Machtpolitik ,dem Gelächter des Publikums preis. Ihre Protagonisten [...] denunzieren das eigene Tun durch Unfähigkeit und übertriebene Eitelkeit“" (Dörner 2016: 10).

Die Serienmacher haben sich für einen fiktiven Präsidenten entschieden, der nie in Erscheinung tritt und alle für ihn unwichtigen zeremoniellen Verpflichtungen an seine $V P$ delegiert. Dies ist realistisch, da es bei einigen vergangenen 
Präsidenten so praktiziert wurde: „Some have viewed these duties as frivolous, as a waste of valuable presidential time, and have suggested that they be turned over to the vice president" (Hess 2002: 205).

Wenn die Wirklichkeit nunmehr die Fantasie überrollt, stellt der Journalist Jürgen Schmieder die provokant zugespitzte Frage: „Wer braucht so eine fiktive Satire mit bitterbösen Beleidigungen, wenn es in der wirklichen Welt Donald Trump gibt“" (Schmieder 2016).

Veep fügt sich US-spezifisch passend in die breite US-Satirelandschaft ein und entspricht dem Interesse an Satire-Serien wie Alpha House oder satirischen Berichterstattungen in Late Night Shows wie Last Week Tonight with John Oliver, The Colbert Report, The Daily Show oder den Trump Sketches von Alec Baldwin. Satire fungiert als Möglichkeit sich über Humor mit Politik auseinanderzusetzen - man kann einerseits über die dargestellte Politik lachen und andererseits versuchen für sich eine eigene Interpretation zu suchen.

\section{ZUSAMMENFASSUNG}

Eine politische Serie ist ,eine in Episoden und Staffeln unterteilte, fiktionale und audiovisuelle Erzählung über ,politische Wirklichkeiten“" (Dörner 2016: 6). Serien fungieren immer auch als „Seismographen des Politischen“ (Rahlf 2017: 337). Auch Veep macht sich Bezüge zu politischen Wirklichkeiten zunutze, verarbeitet Politik als Kulisse und zeigt eine egozentrische $V P$ mit Ellenbogen, die weg will vom institutionellen Abstellgleis, mit dem Ziel, mehr zu regieren, statt zu repräsentieren. Beim Politikmanagement geht dabei nahezu alles schief, was schiefgehen kann. Veep wird daher zu einer Farce.

„Kein Plan geht auf, ständig bekleckern sich alle den Pullover mit Kaffee, man kann gar nicht viel entscheiden, von Strategie ganz zu schweigen - und trotzdem funktioniert es am Ende irgendwie: muddling through.“ (Möller 2015)

Es ist ein Durchlavieren durch die Alltagsprobleme in Washington, ständige Schwierigkeiten treten auf, es handelt sich um Reaktion statt Aktion. Die meisten aktiven Politikvorhaben scheitern, Erfolge sind nicht zu verzeichnen.

Dargestellte Fallbeispiele der acht Episoden zeigen ein sehr satirisches Abbild der amerikanischen Politik und der Gesellschaft. Die mediale Konstruktion des Politischen ist charakterisiert durch eine moderne Darstellung von äußeren Einflüssen, der eigenen Wirkmächtigkeit und der zur Verfügung stehenden Instrumentenkästen. Entscheidungen werden in Veep immer taktisch, strategisch 
und zugleich sehr erratisch getroffen - mit großem Gespür für Machkonstellationen. Kurzfristigkeit und Netzwerkrelevanzen sind stets wichtig.

Das Drehbuch der Serie bietet einerseits keinen durchexerzierten Politikzyklus bei dargestellten policies, andererseits ermöglicht es kein Terrain für Idealisten, vielmehr sind machbewusste und zugleich inkompetente Akteure am Werk.

Als Fazit lässt sich konstatieren, dass die Macht des $V P$ nicht statisch ist und sich nicht allein an den formalen Kompetenzen ablesen lässt - es geht beim $V P$ nie bloß um die reine Addition der in der Verfassung normierten Kompetenzen. Die Durchsetzungsfähigkeit, der Prägefaktor sowie die zugestandene Rolle als Vizepräsident werden in nennenswertem Maße von der jeweiligen Beziehung zum Präsidenten bestimmt. Je nach Nähe zum Präsidenten und dem persönlichen Vertrauen zueinander wachsen der Einfluss, die ihm übertragene Kompetenz und damit die Bedeutung des Amtes des VP. Im Falle von Veep ist es eine ,Schaltstelle der Macht' ohne Schalthebel.

\section{LITERATUR}

Berg-Schlosser, Dirk (2011): Politische Kultur, in: Nohlen, Dirk/Grotz, Florian (Hrsg.): Kleines Lexikon der Politik, 5. Aufl. München, S. 467 - 468.

Brühl, Jannis (2012): US-Vizepräsidenten-Comedy „Veep“. Zweite Wahl, http://www.sueddeutsche.de/politik/us-vizepraesidenten-comedy-veep-zweitewahl-1.1506948. (zugegriffen am 28.12.2017).

Caro, Robert (2012): The Passage of Power: The Years of Lyndon Johnson, New York.

DER SPIEGEL (1963): USA/Johnson. Der 36., http://www.spiegel.de/spiegel/ print/d-46172996.html. (zugegriffen am: 30.12.2017).

DER SPIEGEL (2016): Hillarys Vorbild, in: DER SPIEGEL 17 vom 23. April 2016. S. 142.

Dörner, Andreas (2016): Politserien: Unterhaltsame Blicke auf die Hinterbühnen der Politik, in: Aus Politik und Zeitgeschichte 66 (51), S. 4-11.

Hess, Stephen (2002): Organizing the Presidency, 3. Aufl. Washington D.C.

Lutteroth, Johanna (2008): US-Vizepräsidenten. Regieren aus der zweiten Reihe, http://www.spiegel.de/einestages/us-vizepraesidenten-regieren-aus-der-zweitenreihe-a-947950.html. (zugegriffen am 28.12.2017).

Möller, Kolja (2015): House of Cards, Veep, Borgen. Was kann man von den neuen Polit-Serien lernen?, https:/www.prager-fruehling-magazin.de/de/ article/1209.house-of-cards-veep-borgen.html. (zugegriffen am 28.12.2017). 
Oldopp, Birgit (2013): Das politische System der USA. Eine Einführung, 2. Aufl. Wiesbaden.

Pfetsch, Barbara/Schmitt-Beck, Rüdiger (1994): Amerikanisierung von Wahlkämpfen? Kommunikationsstrategien und Massenmedien im politischen Mobilisierungsprozeß, in: Jäckel, Michael/Winterhoff-Spurk, Peter (Hrsg.): Politik und Medien. Analysen zur Entwicklung der politischen Kommunikation, Berlin, S. 231-252.

Rahlf, Katharina (2017): Von „House of Cards“ bis „Game of Thrones“, in: Bleckmann, Julia/Kallinich, Daniela/Lorenz, Robert/Mueller-Stahl, Robert/ Rahlf, Katharina (Hrsg.): Die neue Unordnung. Jahrbuch des Göttinger Instituts für Demokratieforschung, Stuttgart, S. 336-343.

Rehfeld, Nina (2012a): Amerikanische Polit-Serie „Veep“. Ihr Boss hat noch immer nicht angerufen, http://www.faz.net/aktuell/feuilleton/medien/ameri kanische-politsatire-veep-ihr-boss-hat-noch-immer-nicht-angerufen11787202.html. (zugegriffen am 28.12.2017).

Rehfeld, Nina (2012b): Neue Polit-Serien im TV. Frau Vize macht sich lächerlich, http://www.spiegel.de/kultur/tv/newsroom-veep-political-animals-polit-seriendominieren-us-tv-a-847723.html. (zugegriffen am 28.12.2017).

Rehfeld, Nina (2015): Viertel Staffel „Veep“. Alles ist jetzt ihre Schuld, http://www.spiegel.de/kultur/tv/veep-mit-julia-louis-dreyfus-vierte-staffelstartet-a-1027868.html. (zugegriffen am 28.12.2017).

Ross, Jan (2013): Fiskalklippe. Unter Feinden, http://www.zeit.de/2013/02/01USA-Haushaltsstreit/komplettansicht (zugegriffen am 08.02.2018).

Sarcinelli, Ulrich (1987): Symbolische Politik. Zur Bedeutung symbolischen Handelns in der Wahlkampfkommunikation der Bundesrepublik Deutschland, Wiesbaden.

Sattar, Majid (2017): Mike Pence. Amerikas Nummer zwei, http://www.faz.net /aktuell/politik/trumps-praesidentschaft/mike-pence-spielt-als-us-vizepraesidenteine-wichtige-rolle-14708057.html (zugegriffen am 30.12.2017).

Schmieder, Jürgen (2016): Bei so vielen Beleidigungen würde selbst Muhammad Ali neidisch, http://www.sueddeutsche.de/medien/us-serie-veep-houseof-cards-in-lustig-1.2873122 (zugegriffen am 28.12.2017).

Süddeutsche Online (2008): US-Vizepräsidenten. Die Nummer zwei, http:// www.sueddeutsche.de/politik/us-vizepraesidenten-die-nummer-zwei-1.576091 (zugegriffen am 04.01.2018). 



\section{„Whatever it takes“"}

\section{4 und die Normalisierung des Ausnahmezustandes}

Frank Gadinger

„America wants the war on terror fought by Jack Bauer. He's a patriot.“

Joel Surnow, zit. n. Mayer 2007

\section{EINLEITUNG ${ }^{1}:$ DIE ENTWICKLUNG EINER FILMPOLITOLOGIE UND DIE RELEVANZ VON 24}

In der jüngeren politikwissenschaftlichen Forschung lässt sich ein verstärktes Interesse für die Beschäftigung mit Filmen, Serien und der Visualisierung von Politik in der Populärkultur beobachten. Dies ist zunächst begrüßenswert und folgt der mit der Diskursforschung begonnenen methodologischen Öffnung zu anderen Orten der Bedeutungskonstruktion jenseits des ,offiziellen Gesprächs` der politischen Eliten. Allerdings fokussierte sich die Auseinandersetzung zunächst auf den Einsatz von Filmen und Serien in der Lehre (Spencer/Engert 2009, Hamenstädt/Hellmann 2015) und interessierte sich demnach primär für die Spiegelfunktion, die auch Switek in seinem einführenden Aufsatz zu diesem Band im Anschluss an den programmatischen Beitrag von Neumann und Nexon (2006) als eine mögliche Anwendung hervorgehoben hat. In dieser Lesart wird dann beispielsweise argumentiert, dass man durch Herr der Fliegen oder Game of Thrones die Denktradition des Realismus besser verstehe oder in Star Trek Feindbildkonstruktionen studieren könne. Aus meiner Sicht verschenkt diese Engführung das bislang wenig genutzte Potenzial von Filmen und Serien als

1 Für hilfreiche Anmerkungen und Kommentare danke ich Anna Gadinger, Elena Simon und dem Herausgeber Niko Switek. 
Untersuchungsobjekte, in denen zentrale Dimensionen des Politischen wie Legitimierungsprozesse, Herrschaftsverhältnisse und demokratischer Wandel analysiert werden können. Begreift man Filme und Serien als Daten, wie Switek dies als weitere mögliche Funktion betont, dann geht es weniger um Fragen der Didaktik, sondern um die methodologische Herausforderung, wie sich die performativen Effekte visueller Inszenierungen auf Alltagsdiskurse angemessen studieren lassen.

Eine solche Forschungsperspektive impliziert, dass Filme, Serien und andere visuelle Artefakte als Orte der alläglichen Bedeutungsproduktion und Sinnvermittlung analytisch aufgewertet werden und die diskreditierenden Konnotationen von Freizeit und Hobby im Begriff der Populärkultur verlieren. Dass Filmen der Stellenwert als ernstzunehmender Untersuchungsgegenstand häufig abgesprochen wird und dieser Zugang beispielsweise als bloßes Medium fiktiver Eindrücke oder persönlicher Erfahrungen abgetan wird, ist auch deshalb ignorant, wie Neumann und Nexon (2006: 7f.) argumentieren, da für die meisten Menschen populärkulturelle Vermittlungen (Repräsentationen zweiter Ordnung) als Wissensquellen weitaus bedeutsamer sind als die Redebeiträge politischer Eliten, die in der politikwissenschaftlichen Forschung als Repräsentationen erster Ordnung eindeutig bevorzugt werden. Auch der Einwand, dass Filme doch primär der Unterhaltung dienten, ist irreführend. Natürlich verfolgen Regisseure, Produzentinnen, Drehbuchautoren und Schauspielerinnen, wie Heck und Schlag (2016) argumentieren, das Ziel, ihr Publikum zu unterhalten, zu fesseln und zu begeistern. Jedoch verkennt diese landläufige Kritik die Performativität von populärkulturellen Artefakten gerade dort, wo gesellschaftlich und politisch hoch umstrittene Themen verhandelt werden; Filme und Serien produzieren in diesem Verständnis „mehr oder weniger autoritative Geschichten und Narrative über gender, Gewalt und Konflikte“ (Heck/Schlag 2016). Die Beschäftigung mit visuellen Artefakten in ihrem politischen Kontext ist demnach nicht zu verwechseln mit dem alltäglichen Gemurmel der Leute auf der Straße. Vielmehr bieten Filme und Serien einen nützlichen methodologischen Ansatzpunkt dafür, in Bildern, Mythen, Geschichten und Symbolen herauszufinden, wie sich Menschen ihr zwischenmenschliches Miteinander vorstellen, welche Regeln gelten sollen und welche normativen Erwartungen gestellt werden. Geteilte Bilder, Metaphern und Narrative geben demnach Aufschluss darüber, welche moralischen Vorstellungen sich verändern, welches politische Handeln als legitim gilt und was wir in unscharfer Form als Wirklichkeit auffassen (Horn 2014: 22). Switek bringt dies in der Einleitung dahingehend auf den Punkt, dass wir in der Lesart dieser Forschungsperspektive einen weit gefassten soziologischen Blick einnehmen und uns auf Spurensuche begeben, wie Bilder und Muster sich in einer bestimmten 
Werteordnung bewegen und wir dadurch letztlich mehr darüber erfahren, ,wie eine Gesellschaft sich selbst sieht".

Wenn nun Filmen oder Serien vorschnell ein Etikett angeheftet wird, dass dies doch ein klarer Propaganda- bzw. ein Anti-Kriegs-Film sei, dann ist dies in den seltensten Fällen eindeutig zu beantworten und bleibt stattdessen in der jeweiligen Einordung und Botschaft weitaus häufiger umstritten. Die kontroverse Debatte um den Film American Sniper (2014) in den USA, in dem die Geschichte des effektivsten Scharfschützen im Irakkrieg erzählt wird, der als gebrochener Held endet, verdeutlicht diese häufige Polarisierung zwischen ,Propaganda ' und ,Anti-Kriegs-Film‘ im politischen Diskurs. Heck und Schlag (2015: 141-143) greifen beispielsweise den Film Zero Dark Thirty (2012) auf, in dem es um die Suche und Ermordung von Osama Bin Laden geht, und zeigen, dass die Rechtfertigungspraxis von Folter keineswegs moralisch eindeutig transportiert wird, sondern genau die ambivalente Haltung in der US-Gesellschaft widerspiegelt, in der über die Effektivität dieser, verschärften Verhörmethoden“ kontrovers diskutiert wird. In einem eigenen Beitrag habe ich den Film The Hurt Locker (2008) analysiert (Gadinger 2016), in dem die alltägliche Arbeit eines Bombenentschärfungskommandos im Kriegseinsatz in Irak gezeigt wird. In einer Phase der gesellschaftlichen Kriegsmüdigkeit zu Barack Obamas Amtsantritt wird der Krieg gegen den Terror als ein von der US-Gesellschaft völlig abgekoppelter Einsatz von Spezialkräften gezeigt, für die der Krieg nicht nur zur Routine, sondern zur Alltagsdroge geworden ist. Das hier geformte Narrativ der Entmenschlichung und Technologisierung des Krieges kann als Kritik am heroischen und patriotischen Narrativ der Post-9/11-Phase interpretiert werden, jedoch auch als zynische Bestandsaufnahme, wie sich der Krieg gegen den Terror als technologisches Projekt mittlerweile öffentlichkeitswirksamer legitimieren lässt.

In einem programmatischen Beitrag haben Heck und Schlag (2015) nun jedoch zu Recht darauf hingewiesen, dass eine politikwissenschaftliche Analyse von Filmen und Serien dann besonders gewinnbringend ist, wenn nicht nur die genrespezifischen Konventionen und erzählerischen Techniken (Filmsprache) reflektiert, sondern auch filmtheoretische und filmanalytische Ansätze aufgegriffen werden. Hieraus könnte sich aus der Verbindung von Politikwissenschaft und Filmanalyse ein innovatives Forschungsfeld entwickeln, das sie als „Filmpolitologie“ beschreiben. In einer ersten Heuristik sehen sie die Analyse von „Film als Narration“ im Zentrum des Forschungsinteresses, was jedoch neben der Beschäftigung mit dem filmischen Narrativ auch weitere Dimensionen beinhaltet, etwa den Erzähler und die Kamera oder den Schnitt und die Produktionsbedingungen. Aus meiner Sicht liegt ein methodologischer Schlüssel für eine gewinnbringende Filmpolitologie darin, die alltägliche Kulturtechnik des 
Erzählens als grundlegenden Modus der Sinnvermittlung und Bedeutungskonstruktion aufzuwerten und daraus folgend wirkungsmächtige politische Narrative in Filmen und Serien zu identifizieren. Die Beschäftigung mit Filmen und Serien als zentraler Bestandteil des Alltagsdiskurses ist deshalb so elementar, weil sie uns vor Augen führt, dass wir uns zur Vermittlung einer Geschichte allgemeinverständlicher Formeln bedienen müssen, die in kulturellen Erzählformen verankert sind. In der politikwissenschaftlichen Narrativforschung (Gadinger et al. 2014, Gadinger/Yildiz 2017) wird gegenwärtig untersucht, welche spezifischen Merkmale in dominanten Erzählformen der Politik besonders relevant sind. Hierbei wird deutlich, dass die Überzeugungskraft in der politischen Sprache wesentlich von Metaphorisierungen, Rollenkonstruktionen und Plotstrukturen abhängt (Yildiz et al. 2015, 2018), da erst durch narrative Konfigurationen aktuelle Problembeschreibungen mit der Vergangenheit und der Zukunft verknüpft werden können und dadurch politische Handlungsoptionen entstehen.

So zeigt Eva Horn (2014) in ihrer Studie Zukunft als Katastrophe in einem breiten Spektrum von Filmen (I am Legend, Take Shelter, Minority Report), wie dominant in unseren gesellschaftspolitischen Diskursen Narrative des drohenden Untergangs, der Katastrophe, bis hin zur Apokalypse in der fiktiven Auseinandersetzung mit der Zukunft sind. Eine solche Analyse ist in hohem Maße politikwissenschaftlich relevant, da sie zeigt, wie sich der Glaube an die Prävention und das Paradigma der Sicherheit entwickelt hat, denn die ,Zukunft als Katastrophe schreit nach ihrer Verhinderung, nach einem präventiven Eingreifen" (Horn 2014: 25). Wie Horn argumentiert, spielen Fiktionen in diesen Inszenierungen eine besondere Rolle, nicht nur als Symptome, sondern ,als Agenten einer Formatierung von Zukunftserwartung" (Horn 2014: 23). Denn diese sind nicht einfach Abbildungen allgemeiner psychischer Verfassungen oder Medien ideologischer Indoktrination, sondern sollten als „Interventionen in dieses kollektive Imaginäre verstanden werden, Interventionen, in denen dieses überhaupt erst hergestellt, strukturiert und vor allem auch verhandelt wird" (Horn 2014: 23). Elisabeth Bronfen (2012: 11) weist in ihrer Analyse Hollywoods Kriege auf einen weiteren zentralen Aspekt hin, wonach durch Kriegsfilme eine „Rekodierung des tatsächlichen militärischen Konflikts" mit kinematographischen Stilmitteln stattfindet, die zweistimmig angelegt ist: „Sie nutzen das Genregedächtnis, um Erinnerungen an die Vergangenheit in die Gegenwart zu transportieren, sie reimaginieren und rekonzeptualisieren Geschichte aus der Position des zeitgenössischen Jetzt heraus.“ Das Kino als Denkraum ermöglicht damit einen „Prozess des Aufarbeitens", der in psychologischer und ästhetischer Weise eine „Form des Durcharbeitens“ ist, der in hohem Maße selbstreflexiv ist (Bronfen 2013: 11). 
Anhand der Serie 24 lassen sich diese von Horn und Bronfen betonten politischen und kulturellen Dimensionen visueller Narrative studieren. Die erste Staffel der Serie wurde 2001 ausgestrahlt, war in ihrer Resonanz beim Publikum extrem erfolgreich und bildete mit vielen weiteren Staffeln ein filmisches und meist widersprüchliches Narrativ zur gesellschaftlichen Kontroverse in den 2000er Jahren der USA, wie der Krieg gegen den Terror unter demokratischen Bedingungen wirkungsvoll und noch angemessen zu führen sei. Die Narration von 24 berührt dabei sowohl die kollektive Imagination und Intervention in die Zukunft (Horn) als auch die Selbstreflexion einer Nation (Bronfen), die sich zunehmend unsicherer wird, ob der eingeschlagene Weg der Terrorbekämpfung insbesondere die Legitimierung von Folter - mit der eigenen Werteordnung noch $\mathrm{zu}$ vereinbaren ist. In dieser Hinsicht bildete 24 einen gesellschaftlichen Imaginationsraum, in dem über die demokratischen Grenzen moralisch gestritten wurde, welche politischen Maßnahmen und Konsequenzen die vom damaligen USPräsidenten George W. Bush häufig benutzte Formulierung, whatever it takes beinhalten sollte.

Obwohl die öffentliche Empörung verständlich ist, nachdem durch journalistische Recherchen offenkundig wurde, dass der eigenwillige Stil der Terrorbekämpfung des Spezialagenten Jack Bauer (Kiefer Sutherland) jenseits aller rechtsstaatlichen Prinzipien sowohl gewöhnliche Militärangehörige als auch führende Juristen des Justizministeriums im Weißen Haus beeinflusst bzw. zumindest inspiriert hat (Lithwick 2008, Sands 2008: 73, Mayer 2007, Lowry 2014), ist die einfache Schuldzuweisung an die Adresse der Produktionsfirma Fox Television und an die der Macher der Serie 24 zwar entlastend, aber auch etwas scheinheilig. Vielmehr zeigt die Serie, dass sich dominante Erzählformen innerhalb des mächtigen War on Terror-Narrativs aus dem Wechselspiel zwischen Alltags- und Elitendiskursen ergeben und die Frage der Urheberschaft nicht in einem linearen Verständnis zu beantworten ist.

Die interessantere Frage ist, warum sich so viele Zuschauer - speziell in den USA - mit Jack Bauer identifizieren können und sein Handeln als legitim erachten. Inwiefern finden politische Narrative im Medium der Serie einen kulturellen Resonanzraum, der in anderen Kontexten (Deutschland) weniger funktioniert? Der Beitrag identifiziert drei dominante Erzählformen, die einerseits zur Legitimation einer schleichenden Normalisierung des Ausnahmezustandes genutzt wurden, andererseits aber auch eine Projektionsfläche anbieten zur Formulierung von Kritik: der patriotische Glaube an den aufrechten Amerikaner als zeitlosen Helden, die Metapher der tickenden Bombe und die Normalität des Krieges sowie die Logik des Verdachts und die Legitimierung von Folter. 
Der Beitrag geht in drei Schritten vor. Zunächst werde ich einige methodologische Überlegungen einer Filmpolitologie zusammenfassen (Heck/Schlag 2015, 2016, Heck 2017) und mich auch auf Klassiker der Filmanalyse (Mikos 2015, Bienk 2014) beziehen. Hieraus entwickle ich eine pragmatische Heuristik, die meine eigene Analyse der Serie 24 anleitet. Der dritte Abschnitt beinhaltet die eigentliche Analyse und die Herausarbeitung der drei Erzählformen. Im Fazit gehe ich auf die methodologischen Herausforderungen ein und schließe mit der These, dass eine kritische Filmpolitologie durch die Beschäftigung mit den Alltagsdiskursen und der Polyphonie auch als demokratisches Projekt interpretiert werden kann.

\section{ÜBERLEGUNGEN ZU EINER FILMPOLITOLOGIE UND DIE METHODIK DER FILMANALYSE}

In ihrem Vorschlag einer filmpolitologischen Heuristik benennen Heck und Schlag (2015: 133) fünf Dimensionen der Filmanalyse: das filmische Narrativ, den Erzähler und die Kamera, Schnitt und Montage, Genre und Konventionen sowie Produktion, Distribution und Rezeption. Sie beziehen sich hierbei unter anderem auch auf die Arbeit von Mikos (2015: 43), der in verwandter Form auch fünf Dimensionen der Filmanalyse vorgeschlagen hat: Inhalt und Repräsentation, Narration und Dramaturgie, Figuren und Akteure, Ästhetik und Gestaltung sowie den Kontext des Films. Was Mikos (2015: 45) unter dem Erkenntnisinteresse von Inhalt und Repräsentation diskutiert, berührt Überlegungen aus der Einleitung, wonach Filme als Repräsentationen mit gesellschaftlichen Strukturen korrespondieren und in ihrer ideologischen Komponente in einer fundamentalen Beziehung zu Macht und Herrschaftsverhältnissen stehen. Zugleich beziehen sich Filme, wie Mikos (2015: 45) weiter ausführt, auf den gesellschaftlichen Wissensvorrat, der die Positionierung des Individuums bestimmt und demnach für die Identität und Subjektivität des Zuschauers eine wichtige Rolle spielt.

In meiner eigenen Analyse, die besonders auf die Identifikation politisch relevanter Erzählformen abzielt, konzentriere ich mich auf drei Ebenen von Mikos (Narration und Dramaturgie, Figuren und Akteure, Ästhetik und Gestaltung), wobei dies die Hervorhebung des Erzählers und der Kamera sowie des Schnitts und der Montage bei Heck und Schlag miteinschließt. Auf die Produktions- und Kontextbedingungen der Serie gehe ich in kurzer Form in den einführenden Bemerkungen ein.

Die ,Narration und Dramaturgie' eines Films bzw. einer Serie hängt zwar mit dem Inhalt zusammen, jedoch geht es hier weniger darum, was gezeigt wird, 
sondern primär darum, wie es in audiovisueller Form gezeigt wird. Während die Narration vor allem aus der kausalen Verknüpfung von Situationen, Akteuren und Handlungen zu einer Geschichte besteht, ist die Dramaturgie die Art und Weise ,wie diese dem Medium entsprechend aufgebaut ist, um sie im Kopf und im Bauch der Zuschauer entstehen zu lassen“ (Mikos 2015: 43). Nach Heck und Schlag (2015: 133) ist das filmische Narrativ in einem doppelten Sinne perspektivisch:

„Zum einen werden durch die Bewegung der Kamera sowie durch Schnitt und Montage Akteure, Handlungen und Ereignisse zueinander in Beziehung gesetzt. Perspektivität zeigt sich zum anderen durch die vielfältigen Auslassungen dessen, was nicht gezeigt, nicht gesagt und damit nicht erzählt wird; Filme ordnen ihre Erzählung sequentiell, zeitlich und räumlich - auch wenn dies nicht zwangsläufig eine chronologische und kontinuierliche Abfolge beinhalten muss.“

Wie Mikos (2015: 47) erläutert, bedienen sich Erzählungen bestimmter Erzählstrategien, um das Publikum in die Geschichte miteinzubeziehen, wobei diese Strategien und Techniken immer mit Aktivitäten der Zuschauer verknüpft sind, die im Verlauf ihrer Mediensozialisation auch ein narratives Wissen erworben haben, etwa typische Handlungsstrukturen und -episoden, gängige Protagonistenrollen sowie typische Erzählkonventionen und Plots. Das Sehen eines Films bzw. einer Serie ist demnach kein rein passiver Prozess, sondern ist - ähnlich dem Betrachten eines Bilds - als aktiver und wechselseitiger Prozess einer sozialen Praxis zu verstehen. Filme und Serien orientieren sich zwar häufig an klassischen Plotstrukturen des Literaturgenres, wie der Tragödie oder der Komödie (Gadinger et al. 2014: 74), haben aber eigene Strukturen der Erzählung und Dramaturgie herausgebildet. Im Zentrum der Dramaturgie stehen Konflikte, die Figuren aktiv werden lassen und die Handlung vorantreiben (Mikos 2015: 48). Demnach hat die Dramaturgie die Aufgabe, ,die Kette von Ereignissen, in denen Personen handeln, so zu gestalten, dass bestimmte kognitive und emotionale Aktivitäten bei den Zuschauern angeregt werden“, so dass Wissen aufgebaut und Gefühle hervorgerufen werden (Mikos 2015: 48). Der Film oder die Serie werden dadurch performativ, da sie die Wirklichkeit durch ihr Medium hervorbringen (Heck/Schlag 2015: 133). Die Filmanalyse muss demnach herausarbeiten, mit welchen erzählerischen Strategien und Techniken es gelingt, diese emotionale Bindung und Identifikation des Zuschauers aufzubauen.

In der Film- und Serienanalyse geht es primär um ,Figuren“ als zentrale Handlungsträger (Akteure wie Moderatoren spielen im Fernsehen eine Rolle). So sind die auftretenden Personen als Handlungs- und Funktionsträger für die 
Dramaturgie und die narrative Struktur wichtig, da die Geschichte häufig aus der Perspektive einer der zentralen Figuren erzählt wird. Mit Hilfe von Filmfiguren verständigt sich die Gesellschaft über ihre Identitäts- und Rollenkonzepte, da diese eine wesentliche Funktion im Rahmen der Repräsentation für die Subjektpositionierung und Identitätsbildung beim Zuschauer einnehmen. Ein klassisches Rollenschema liefert die Gegenüberstellung von Helden und Schurken, deren moralische Zuweisungen sich aus der Filmgeschichte ergeben, beispielsweise aus der Figur des James Bond und den klassischen Bösewichten. Angelpunkt einer Romanze ist beispielsweise stets die Überschreitung der Erfahrungswelt durch den Helden, der nur so im Kampf gegen das Böse den drohenden Niedergang abwenden kann (Gadinger et al. 2014: 74). Figuren können auch lebensweltlicher angelegt sein, zu denken ist etwa an die fürsorgliche Großmutter, den patriarchalischen Stiefvater oder den abgehalfterten Kommissar, die für die Zuschauer aufgrund ihres lebensweltlichen Wissens besetzt und durch bestimmte Merkmale charakterisiert sind. Mit kulturellen Veränderungen wandeln sich auch typische Figuren, wenn beispielsweise die Figur des James Bond als Geheimagent mittlerweile verletzlicher und weniger machohaft gezeichnet wird. Für Mikos (2015: 51) steht das Personal in Filmen und Serien immer in Bezug zu den jeweiligen Vorstellungen von Selbst und Identität sowie zu dem Wissen über Personen- und Rollentypisierungen, das im Rahmen spezifischer kultureller Kontexte zirkuliert. Für die Analyse ist demnach ein entscheidendes Moment, dass die Inszenierung von Figuren nicht nur für Kognition und Verstehen von Bedeutung ist, sondern gerade auch für die emotionalen Prozesse in der Rezeption und Aneignung; vor allem über die Figuren wird das Verhältnis von Nähe und Distanz der Zuschauer zum Geschehen auf der Leinwand oder dem Bildschirm bestimmt.

Die , Ästhetik und Gestaltung` eines Films bzw. einer Serie operiert sowohl mit gewissen Regeln von Schnitt und Montage als auch mit Genres und Konventionen, so dass die Geschichte, die bei den Zuschauern in den Köpfen entsteht, eng mit dem vorhandenen narrativen Wissen verknüpft ist. Die klassische Schnitt-Montage-Idee zielt etwa darauf ab, dem Zuschauer Orientierung im Wechsel von Raum und Zeit zu bieten, wobei hier verschiedene Praktiken unterschieden werden können, die verschiedene narrative Anschlüsse ermöglichen, beispielsweise der unsichtbare Schnitt, der zwar mit einem Orts- und/oder Zeitwechsel verbunden ist, aber Kontinuität schafft (Heck/Schlag 2015: 134). Heck und Schlag (2015: 135) argumentieren zu Recht, dass die Kenntnis von Schnittund Montagetechniken wichtig ist, um die Narrativierung des Films zu verstehen, d.h. mit welchen technischen und ästhetischen Mitteln Sinn erzeugt wird. Mikos (2015: 51-52) geht davon aus, dass insbesondere die spezifischen Dar- 
stellungsmittel die Zuschauer während der Rezeption eines Films an das Geschehen binden, da sie dadurch emotional durch die Erzählung geführt werden, sie werden in bestimmte Stimmungen versetzt, ihre Aufmerksamkeit wird auf einzelne Aspekte im Filmbild gelenkt, ohne dass ihnen dies immer bewusst ist. Auf diese Weise werden sie in die Perspektiven der Erzählung und der Repräsentation eingebunden. In einer Filmanalyse gilt es nun, ,gerade diese Aspekte herauszuarbeiten und sie in Beziehung zum Wissen der Zuschauer über filmische Darstellungsweisen zu setzen, um zur Bewusstmachung dieses Prozesses beizutragen“ (Mikos 2015: 52). Die Gestaltungsweisen beruhen wiederum auf Genres und Konventionen der Darstellung, die sich durch häufigen Einsatz in der Film- und Seriengeschichte herausgebildet und Lernerfahrungen beim $\mathrm{Zu}$ schauer erzeugt haben. So kann der Zuschauer beim Schauen eines Westerns, Horrorfilms oder Science-Fiction-Films von gewissen Konventionen ausgehen, die von stilbildenden Filmen (Spiel mir das Lied vom Tod, Halloween, Blade Runner) vorgegeben wurden. Es ist beispielsweise davon auszugehen, dass die Spannung in der Dramaturgie steigt, wenn in einem Horrorfilm eine Person allein in einen Keller geht. Gleichzeitig werden Erzählregeln bis hin zu ganzen Genrekonventionen immer wieder offengelegt, gebrochen und verändert, beispielsweise durch Autoren-Filme (Heck/Schlag 2015: 135).

Bei der konkreten Methodik der Filmanalyse geht es vor allem um die Codierung und Interpretation, wobei dies einer rekonstruktiven Forschungslogik folgt. Die Filmanalyse lässt sich in eine Grob- und Feinanalyse differenzieren, wobei sich in der Grobanalyse strukturelle und formale Elemente bestimmen sowie Schlüsselszenen und -sequenzen selektieren lassen, während in der Feinanalyse ein Sequenzprotokoll mit Bildinhalten, Kameraaktivitäten, Handlungsbeschreibung, Dialogen, Musik und Geräuschen erstellt wird (Heck und Schlag 2015: 139-140, auch Bienk 2014). Allerdings sollte eine Film- bzw. Serienanalyse in ihrem Codierprozess nicht als rein technischer Schritt verstanden werden, worauf Heck und Schlag zu Recht hinweisen, da das Codieren der visuellen, auditiven und narrativen Elemente in ihren technischen und symbolischen Effekten immer eine kreative Interpretationsleistung erfordert. In meiner Analyse folge ich dem Beispiel Hecks (2017: 374), der in Anlehnung an David Bordwells narrative Filmanalyse das Dokudrama Eine mörderische Entscheidung untersucht und hierbei drei politische Narrative identifiziert hat, die wiederum den Wandel des deutschen gesellschaftspolitischen Diskurses zum Einsatz in Afghanistan demonstrieren. Im Fokus meiner Serienanalyse stehen die Staffeln 1 und 4, da die Erzählstruktur seit der ersten Staffel vergleichbar ist und diese stilbildend für die weiteren Staffeln wurde. In der vierten Staffel wird die Thematik der Folter als legitimer Verhörmethode in besonders drastischer Form dargestellt, wodurch 
die Kontroverse um 24 auf die Spitze getrieben wurde. Allerdings wird auch auf andere Szenen aus den weiteren Staffeln Bezug genommen, wenn dies notwendig ist.

\section{TICKENDE BOMBEN, EINSAME HELDEN UND DIE LOGIK DES VERDACHTS: ERZÄHLFORMEN IN 24}

Die erste Staffel der Serie 24 ging kurz nach den Anschlägen des 11. September 2001 auf Sendung und traf den Zeitgeist eines verwundeten Amerikas. Die Serie schien dem Entsetzen und der Wut der Amerikaner Ausdruck zu verleihen und präsentierte mit dem Agenten Jack Bauer (Kiefer Sutherland) einen patriotischen Helden, der zwar ständig jenseits der offiziellen Strukturen operiert, um immer extremere Terroranschläge zu verhindern, dem man aber vertraute und der alleine die Verantwortung für ein brüchiges Gemeinwesen trug (Rehfeld 2014). Die Serie brachte „den nationalen Ausnahmezustand in bisher nicht dagewesener Unmittelbarkeit ins amerikanische Wohnzimmer“ (Šíp 2014). Während sich die Serie in den USA mit streckenweise über 13 Millionen Zuschauern zu einem Erfolg für den Sender Fox entwickelte, blieb sie im deutschsprachigen Raum hinter den Erwartungen der ausstrahlenden Sender zurück. In jeder Staffel aufs Neue versuchen Terroristen, die USA mit Atomwaffen, Giftgas und anderen Mitteln zu Fall zu bringen; und Jack Bauer muss in einsamer Mission die Welt retten und kann niemanden trauen, da der Feind auch im Innern lauert und Verschwörungen an der Tagesordnung sind. In dieser Notlage des permanenten Ausnahmezustandes greift Jack Bauer zu allen erdenklichen Mitteln bis hin zu Folter, um die nationale Sicherheit zu bewahren. Die Serie wurde damit, wie Daniel Šíp (2014) treffend argumentiert, zum ,,primären Imaginationsraum für das Austesten demokratischer Grenzen“ im Anti-Terror-Kampf und „Bauer zum ambivalenten Helden, der den Zuschauer zwang, das eigene Wertesystem ständig zu hinterfragen“. Mit dem Helden Bauer, der unter ständigem Entscheidungsdruck steht, erlebte der Zuschauer demnach alle moralischen Dilemmata einer Terrorbekämpfung westlich-liberaler Demokratien. Den politischen und ideologischen Kampf in den USA, wie der Krieg gegen den Terror zu führen sei, spiegelt 24 in extremer Form wider. So wurde Jack Bauers Credo ,whatever it takes ${ }^{\text {' zu einer }}$ Metapher der neokonservativen Kriegstreiber und sowohl vom damaligen USPräsidenten George W. Bush als auch von seinem Verteidigungsminister Donald Rumsfeld als Rechtfertigungsformel benutzt.

Was zusätzlich zur Beliebtheit und Relevanz der Serie beitrug, die 20 Emmys und zwei Golden Globes erhielt, ist die verschwimmende Grenze zwischen Fakt 
und Fiktion (siehe hierzu den Beitrag von Yildiz in diesem Band). So wurden der Pilotfilm und die erste Staffel noch vor dem 11. September 2001 produziert und nahmen damit einen solchen Anschlag in seiner Unvorstellbarkeit vorweg; die Wahl des in der ersten Staffel bedrohten Senators und Präsidentschaftskandidaten David Palmer zum US-Präsidenten in der zweiten Staffel kann als geradezu prophetische Antizipation der späteren, realen Wahl von Barack Obama als erstem schwarzen Präsidenten gesehen werden. Gleichzeitig wurde die Serie mehrfach von der Realität eingeholt. Während in der ersten Staffel die Verhörtechniken meist noch legal sind, wird in der zweiten Staffel der Direktor der NSA mit der Legitimation des Präsidenten per Elektroschock gefoltert, um notwendige Informationen zur Vereitelung des Anschlags zu erhalten. Die Ausstrahlung dieser Staffel (2003) lief zu einem Zeitpunkt, zu dem in den USA noch keine öffentliche Diskussion zur Folter stattfand und der Skandal im irakischen Gefängnis von Abu Ghraib (2004) erst noch bevorstand. Wie Jane Mayer (2007) aufdecken konnte, lassen sich die Entwicklung der ,verschärften Verhörtechniken“ und die Legitimation von Folter als wechselseitige Konstitution von Fiktion und Fakt nachzeichnen. So versuchten Verhörspezialisten des US-Militärs die Macher der Serie davon abzubringen, immer unrealistischere Folterdarstellungen zu zeigen, die die Soldaten beeinflussten. Die Schreiber der Serie ließen sich jedoch auch umgekehrt inspirieren, etwa vom Verhör- und Folterhandbuch Kubark der CIA (Šíp 2014). Die Imagination betrifft auch die Vorstellung von Anschlagsszenarien, wenn etwa die fiktive Bedrohung eines Atomwaffenanschlags auf Los Angeles (zweite Staffel) als reale Bedrohung in die Seiten des National Commission on Terrorist Attacks Upon the United States (9/11 Commission) Report wandert (Van Veeren 2009: 373). Die wechselseitige Konstitution von Realität und Fiktion betrifft sogar architektonische Aspekte, wie den Bau einer Zentrale für Terrorismusbekämpfung, die der Counter Terrorist Unit $(C T U)$ in 24 nachempfunden ist, und verdeutlicht die Intertextualität des sicherheitspolitischen Post-9/11-Diskurses (Van Veeren 2009: 368).

24 kann zwar den Genres Action, Polit-Thriller und Drama zugeschrieben werden, der innovative Charakter der Serie entsteht jedoch durch das Erzählformat - eine Staffel bildet genau einen Tag ab und die Handlung läuft quasi in Echtzeit ab; d.h. jede Staffel hat 24 Folgen von jeweils einer Stunde (aufgrund der Werbeunterbrechungen dauert eine Folge jedoch nur 45 Minuten). Zu Beginn jeder Folge erklärt die Stimme Jack Bauers „Alles, was sie sehen, geschieht in Echtzeit“ („Events occur in real time“). Die häufige Einblendung einer digitalen Zeitanzeige, die einen Countdown herunterzählt, verbunden mit einem pochenden Bassgeräusch, lässt den Zuschauer die Hektik und den akuten Stress des Protagonisten nachempfinden und für ihn auch körperlich spürbar werden. 
Diese Countdown-Struktur bzw. der Wettlauf gegen die Zeit ist ein zentrales erzählerisches Mittel. So wird die Rettung einer Person oder die Gewinnung einer notwendigen Information stets erst in letzter Sekunde erreicht. Die häufige Nutzung schneller Schnitte, des Split-Screens als Montagetechnik, um die Gleichzeitigkeit verschiedener Handlungsstränge zu verdeutlichen, sowie die verwackelte Kamera suggerieren in der Erzählweise die Dringlichkeit einer tickenden Zeitbombe. $\mathrm{Zu}$ diesem Zeitpunkt noch unüblich und für die Sehgewohnheiten des Zuschauers herausfordernd sind die Vielzahl der Handlungsstränge (durchschnittlich sechs pro Episode) und die kurze Dauer der Szenen (zwischen einer und drei Minuten), wodurch die Erzählweise von einem hohen Maß an Tempo, Dichte und Dramatik geprägt ist. Diese Erzählweise sei der entscheidende Trick der Serie, wie einer ihrer Erfinder, Joel Surnow, erläutert. Der Plot einer tickenden Bombe ist zwar keineswegs realistisch (Mayer 2007), fesselt den Zuschauer jedoch durch den extremen Spannungsbogen an die Handlung. Zusätzliche Spannung entsteht durch die permanente Unsicherheit, welche Figuren auf der guten bzw. bösen Seite stehen: Enge Freunde werden als tödliche Gegner enthüllt, Figuren wechseln innerhalb der Handlung wiederholt die Seite und zentrale Personen werden plötzlich getötet. Spannung wird zudem dadurch erzeugt, dass Figuren doppeldeutig bzw. nicht die sind, die sie vorgeben. Der Topos des Maulwurfs in den eigenen Reihen ist zudem allgegenwärtig.

Die Serie 24 führte damit das serielle Erzählen ins Extreme und bot dem Zuschauer die Möglichkeit, eine Staffel in Echtzeit zu erleben (im binge viewing lässt sich dies in 24 Stunden tun) und sich mit der Rolle des Protagonisten zu identifizieren. Bis 2010 entstanden 192 Episoden in acht Staffeln und ein Fernsehfilm (24: Redemption). Fortgesetzt wurde die Serie 2014 mit der 12-teiligen Mini-Serie 24: Live Another Day, allerdings schwand die Beliebtheit der Serie während der Präsidentschaft Obamas und des damit einhergehenden, weniger öffentlichkeitswirksamen War on Terror. In der folgenden Darstellung der drei dominanten Erzählformen wird anhand von einzelnen Szenen eine Feinanalyse durchgeführt.

\section{Die Metapher der tickenden Bombe und der Plot der Verschwörung}

Jede Folge der Serie beginnt mit einem nervösen Piepen, knisternden Geräuschen und der visuellen Darstellung einer digitalen Uhr und der Zahl 24. Die tickende Uhr, die ständig eingeblendet wird, wird zu einem zentralen Motiv und zur Metapher des Ausnahmezustandes. Die Reihe von Terroranschlägen, die von Staffel zu Staffel in ihrem Ausmaß immer extremer werden, wird als ständige 
Bedrohung im Alltag präsentiert und suggeriert, dass wir uns als Zuschauer in einem mittlerweile zur Normalität gewordenen Ausnahmezustand befinden. Das Motiv der tickenden Bombe sorgt dafür, dass eine ständige Anspannung und Nervosität bei allen beteiligten Figuren die Regel ist und nie die Zeit gegeben ist, eine Entscheidung abzuwägen, sondern stets die schnelle Handlungsbereitschaft gefordert ist.

In der ersten Folge der ersten Staffel dringt die Nachricht durch, dass ein Anschlag auf den Präsidentschaftskandidaten David Palmer (Dennis Haysbert) geplant ist. Bauer wird von seiner Kollegin Nina Myers (Sarah Clarke) in die Zentrale der CTU gerufen, da ihr direkter Vorgesetzter Richard Walsh (Michael O`Neill) zu einem dringenden Briefing gebeten hat. Walsh erklärt dem Team, dass auf Senator Palmer ein Anschlag geplant ist. Nach dem offiziellen Gespräch wendet er sich Bauer zu:

\section{$\underline{\text { Szenen-Beispiel (S 1/F 1/ab 12.30) }}$}

Walsh: Jack!

Bauer: Ja [Walsh nickt ihm subtil zu und gibt ihm zu verstehen, zu bleiben] Ich komme gleich nach.

Walsh: Was ich Ihnen gleich sage, darf diesen Raum nicht verlassen.

Bauer: Klar [macht eine Geste des Schwurs und setzt sich].

Walsh: Es wäre möglich, dass vielleicht ein Agent der Agency in den Anschlag auf Palmer verwickelt ist.

Bauer: Was?

Walsh: Ich will, dass Sie in den nächsten 24 Stunden alles in Bewegung setzen, Sie werden mit allen Bezirken zusammenarbeiten.

Bauer: Ich bin der letzte Mensch auf diesem Planeten, dem Sie mit so was kommen sollten.

Walsh: Das sehe ich anders.

Bauer: Richard, ich habe gegen drei unserer Agenten wegen Bestechung ermittelt und die Brüder eingebuchtet. Und wenn bei der Agency einige ein Attentat auf Palmer planen, erfahre ich es mit Sicherheit nicht...

Walsh: Das müssen Sie aber, Jack, weil sie nämlich der einzige sind, dem ich vertrauen kann [setzt sich, sein Profil spiegelt sich in der schwarzen Tischplatte, Pause des bedrohlichen Schweigens]. Wird Palmer erschossen, der erste Afroamerikaner mit echten Aussichten auf die Präsidentschaft, wird dieses Land darüber zugrunde gehen [ernste Minen bei beiden Figuren, kurze Pause].

Bauer: Ich will versuchen, mein Bestes zu tun... 
Walsh: Gut, gut, George Mason wird vom Hauptquartier rüberkommen und Ihnen Informationen zu Palmer geben.

Bauer: Und ich soll George Mason vertrauen?

Walsh: Bis wir genaueres wissen, vertrauen Sie niemandem. Niemandem, verstehen Sie [beugt sich vor]? Nicht mal ihren Mitarbeitern. Wir müssen den Attentäter finden Jack, koste es, was es wolle!

[Die Szene endet im Split-Screen mit den beiden ernsten Mienen von Walsh und Bauer und einem eingeblendeten Flugzeug im Nachthimmel.]

Die Szene ist exemplarisch für die Erzählstruktur von 24, auch im Hinblick auf die weiteren Staffeln. Nach dem offiziellen Gespräch spricht Walsh im Vertrauen mit Bauer und vermittelt ihm den Auftrag, das Geheimwissen und die alleinige Verantwortung. Der Verdacht einer internen Verschwörung zwingt Bauer dazu, niemandem zu vertrauen. Gleichzeitig wird im Gespräch klar, dass Bauer durch seine Ermittlungen gegen korrupte Mitarbeiter unbestechlich ist. Der Auftrag der Vereitelung des Anschlags auf den Präsidenten hat zwar noch nicht das Zerstörungspotenzial der späteren Staffeln, ist aber in gleicher Weise dringend und funktioniert nach dem Plot der tickenden Bombe. Zwar geht es hier noch nicht um die Rettung der Welt oder um die drohende Explosion einer Atombombe (Staffel 2), aber das Schicksal der Nation steht auf dem Spiel, wenn Walsh gegenüber Bauer prophezeit, dass das Land bei einem Scheitern des Auftrags zugrunde gehen werde, wodurch er Bauer eine enorme Last der Verantwortung aufträgt. Der Abschluss des Gesprächs und die Mahnung und Aufforderung Walshs, dass Bauer niemandem vertrauen könne und alle erdenklichen Mittel einsetzen müsse, greift wiederum das grundlegende Motiv, whatever it takes auf, als pragmatische Notwendigkeit in einer Ausnahmesituation.

Bei einer späteren Szene (S 1/F 1/ ab 18.50), in der der von Walsh angekündigte George Mason (Xander Berkeley) in der CTU eintrifft und Bauer zusätzliche Informationen gibt, aber die Quelle nicht preisgeben will, wird die Erzählform auf die Spitze getrieben. Mason wirkt dubios und könnte der potentielle Verschwörer sein. Bauer bittet ihn, seinen Vorgesetzten anzurufen und sich autorisieren zu lassen. Mason geht zunächst scheinbar darauf ein. Er telefoniert und Bauer verlässt den Raum, um von einem zweiten Apparat das Gespräch Masons mithören zu können. Hierbei wird der Split-Screen eingesetzt und der Zuschauer kann verfolgen, dass Mason nur mit der Zeitansage telefoniert und Bauer sich mit einer Waffe aus einem Koffer präpariert. Zusätzlich setzt in der etwa zweiminütigen Szene das Musikstück Iguazu von Gustavo Santaolalla ein, das Jahre später auch in der Goldgräber-Serie Deadwood zu einem zentralen Motiv des Soundtracks wird. Es entsteht das Gefühl des bevorstehenden Showdowns und 
die technische Atmosphäre der $C T U$ vermischt sich mit der Western-Stimmung von Prärie und Duell. Bauer schießt Mason einen Betäubungspfeil ins Bein, verschafft sich Informationen darüber, dass dieser korrupt gehandelt hat, erpresst ihn damit und erfährt so die Quelle des verschlüsselten Datenpakets. Bauer erreicht sein Ziel, indem er gegen jede Regel agiert und sich nur auf seinen Instinkt verlässt.

Im gesamten Verlauf der ersten Staffel wird nie ganz klar, wer der Maulwurf in der $C T U$ ist. Bei der geplanten Ermordung Palmers wird zunehmend ersichtlich, dass sich eine Verschwörergruppe um Victor Drazen (Dennis Hopper) für einen früheren geheimen Einsatz von Bauer (unter Befehl von Palmer) auf dem Balkan rächen möchte. Bauers Tochter Kim (Elisha Cuthbert) und Frau Teri (Leslie Hope) geraten mehrfach in die Fänge der Verschwörer und Bauer wird erpresst, den Präsidentschaftskandidaten Palmer zu töten. In der vorletzten Folge der ersten Staffel geschieht die vollkommen überraschende Wendung (41.30), dass Nina Myers als engste Vertraute, Kollegin und frühere Geliebte Bauers der Maulwurf ist und für den Drazen-Clan arbeitet. Myers belügt Bauer hinsichtlich des angeblichen Tods seiner Tochter, um ihn in eine Falle zu locken. Nachdem Bauer diese Lüge aufdeckt und nur knapp daran gehindert werden kann, die Verräterin Nina Myers zu töten, findet er seine Tochter unversehrt in der CTU vor. Allerdings entdeckt er seine Frau Teri tot in einem Serverraum, wo sie offensichtlich durch Myers ermordet worden ist. Die Tragik erhöht sich dadurch, dass Teri sowohl ihrem Mann Jack (S 1/F 23/30.00) als auch Myers (S 1/F 23/39.40) von einer erneuten Schwangerschaft erzählt hat.

\section{Der patriotische Glaube an den aufrechten Amerikaner als zeitlosen Helden}

In der zweiten Szene der ersten Staffel (ab 03.18) wird Bauer im Kreise seiner Familie eingeführt. Er spielt mit seiner Tochter Kim Schach und beide reden vertraut miteinander, was auf ein enges Vater-Tochter-Verhältnis schließen lässt. Er schickt seine Tochter ins Bett und sagt ihr mit einem Gute-Nacht-Kuss, dass er sie liebe. Diese sagt ihm wiederum, dass sie sich freue, dass er wieder hier wohne. Auch im darauffolgenden Gespräch mit seiner Frau Teri wird das Bild des treusorgenden Familienvaters vermittelt. Da sowohl seine Tochter Kim als auch seine Frau Teri mehrfach entführt werden, muss Bauer fortlaufend an zwei Fronten kämpfen: Er muss die Nation und seine Familie retten. Dies wird in vielen Szenen deutlich, wenn Bauer trotz des Zeitdrucks in ausweglosen Situationen häufiger mit seiner Frau telefoniert (Split-Screen) oder wenn er alles tut, um seine verschwundene Tochter zu finden. Obwohl durch den Plot der Verschwörung 
der Zuschauer niemals sicher sein kann, wer von den zentralen Figuren Teil der Verschwörung sein könnte, kann man sich auf Bauer als integren Menschen verlassen, der für Patriotismus, Verantwortungsgefühl, Selbstlosigkeit, Familiensinn, Unbestechlichkeit und Loyalität steht. Diese Loyalität verbindet ihn mit Palmer, der in ähnlicher Form agiert, mit Intrigen zu kämpfen hat und sich sogar von seiner Frau trennt, nachdem er ihre karrieristischen Absichten und ihr brüchiges Wertegefüge durchschaut. Moralische Zuordnungen sind demnach zumindest bei den zentralen Figuren im Gegensatz von Gut und Böse eindeutig. Durch dieses Identifikationspotenzial verzeiht der Zuschauer auch leichter, dass Bauer häufig jenseits der formellen Regeln operiert, da er sich für höhere moralische Prinzipien des Gemeinwesens einsetzen muss. Der Schutz der Nation und der Familie wird damit in Entscheidungssituationen gegen die als lapidar wirkenden rechtsstaatlichen Prinzipien abgewogen; und für den Zuschauer ist das Handeln emotional nachvollziehbar. So will sich Bauer in einem Deal mit den Entführern seiner Tochter für diese eintauschen und unterstreicht damit seine Selbstlosigkeit. In jeder Szene wird die Entschlossenheit Bauers betont, der im Zweifel lieber handelt als zaudert und dies in der klassischen Rolle des ActionHelden von Hollywood-Filmen (Bruce Willis, Sylvester Stallone) in einem traditionellen Verständnis von Männlichkeit tut.

Emotionen werden der Figur Bauers primär zugestanden, wenn es um Familienangelegenheiten oder den drohenden Verlust von engsten Angehörigen geht. So wird Bauer von Tränen überwältigt, als ihm seine Frau Teri erzählt, dass sie ein Kind von ihm erwarte (S 1/F 23/ab 30.00). Im Gespräch wird klar, dass dadurch die Möglichkeit der zweiten Chance gegeben ist, nachdem die Ehe in eine Krise geraten war. Für den Zuschauer wirkt dies wie ein Moment der möglichen Erlösung für Bauer. Nachdem Bauer in der letzten Folge der ersten Staffel einen Anruf von Myers mit der Nachricht des angeblichen Todes seiner Tochter erhält (ab 14.00), sieht der Zuschauer bei sakralen Klängen für einen kurzen Zeitraum (eine Minute) den Zusammenbruch Bauers, der sich an einen Pfosten kauert und seinen Tränen freien Lauf lässt. Allerdings kippt die Emotion der Verletzlichkeit direkt in den finsteren Blick der Rache und Wut, der wiederum die zentrale Eigenschaft der Entschlossenheit erneut entfaltet. Bauer tötet die beiden Verschwörer und schießt, im Stile des vergeltenden Rächers, bei der Ermordung Victor Drazens sein gesamtes Magazin leer. In der letzten Szene der ersten Staffel wird die Tragik auf die Spitze getrieben (S 1/F 24/ab 39.30), wenn Bauer sich nach dem glücklichen Wiedersehen mit seiner Tochter auf die Suche nach seiner Frau Teri begibt (die Nachrichtensprecherin verkündet im Hintergrund, dass die Nation es Jack Bauer zu verdanken habe, dass Palmer noch lebt). Bauer findet seine leblose Frau im Serverraum und bricht, sie dabei auf den Armen haltend, 
zusammen. Im Split-Screen zeigen sich in Schwarz-Weiß frühere, glückliche Familienszenen, Bauer hält seine Frau in den Armen und sagt ihr, dass es ihm unendlich leidtue. Auch wenn die Zuschauer nicht mit Gewissheit davon ausgehen können, dass Teri stirbt, erzeugt es ein emotionales Mitgefühl, dass möglicherweise alle Anstrengungen vergeblich waren und Bauer als tragischer und gebrochener Held endet.

\section{Die Logik des Verdachts und die Legitimierung von Folter}

Eine fortlaufende Erzählform in 24 ist die Logik des Verdachts, die dazu führt, dass im Ausnahmezustand nicht nur ständiges Misstrauen gegenüber vertrauten Personen herrscht, sondern auch kaum noch Raum bleibt für rechtsstaatliche Maßnahmen gegenüber potentiellen Terrorverdächtigen. In der vierten Staffel bedroht der arabischstämmige Terrorist Habib Marwan (Arnold Vosloo) die Sicherheit der USA. Er kommandiert mehrere Terrorzellen und lässt den USVerteidigungsminister James Heller (William Devane) und dessen Tochter Audrey Raines (Kim Raver) entführen, um Heller in einem im Internet live übertragenen Schauprozess wegen angeblicher Verbrechen gegen die Menschlichkeit zum Tode zu verurteilen. Bauer ist mit Audrey liiert und arbeitet mittlerweile für den Verteidigungsminister, wechselt jedoch nach dessen Entführung wieder flieBend in die CTU. Nachdem in der ersten Folge ein Terroranschlag auf einen Personenzug stattfindet und durch Bauers Hilfe der Verdächtige Tomas Sherek festgenommen wird, geht es im Verhör darum, diesem Informationen zu entlocken, was jedoch nach formellen Regeln nicht gelingt. Die Leiterin der CTU, Erin Driscoll (Alberta Watson), und der Agent Curtis Manning (Roger Cross) diskutieren daraufhin über mögliche verschärftere Maßnahmen. Bauer überlegt in diesem Moment weniger und verschafft sich gewaltsam Zugang zum Verhörraum, wirft einen Tisch um und erklärt Sherek, dass nun nach anderen Regeln gespielt werde (S 4/F 1/ab 38.00). Ohne längere Vorankündigung schießt Bauer dem Verdächtigen ins Bein und droht ihm, dies zu wiederholen. Der Verdächtige Sherek schreit vor Schmerzen und erklärt direkt, dass die Entführung Hellers für acht Uhr geplant sei. Bauer kann zwar Audrey per Telefon noch von der Entführung unterrichten, diese lässt sich aber nicht mehr verhindern. Gegenüber der Leiterin der $C T U$, Driscoll, kann Bauer jedoch triumphieren, dass seine Methoden wirksamer sind.

Dieses Muster wiederholt sich ständig in einer Reihe von extrem gewaltsamen Terroranschlägen (bei einem der Anschläge wird eine Kernschmelze in einem Atomkraftwerk durchgeführt) und Verhinderungsversuchen. In diesem Handlungszeitraum sterben 225 Personen, allein 39 davon werden von Bauer getötet. Ist Folter in den ersten beiden Staffeln der Serie noch die absolute Aus- 
nahme, wird sie besonders in der vierten Staffel zur Normalität (in Staffeln 1-5 gibt es insgesamt 67 Folterszenen); die Methoden reichen von chemischen Injektionen, Elektroschocks bis hin zu Knochenbrüchen. So wird beispielsweise der widerspenstige, regierungskritische Sohn des Verteidigungsministers, Richard (Logan Marshall-Green), sogar gefoltert, nur wegen des Verdachts, er könne mehr über die Hintergründe der Entführung wissen; später muss er sich von seinem Vater dann in paternalistischem Ton erklären lassen, dass dies in der Situation notwendig war. War es im Genre des Action-Thrillers bis dahin üblich, dass die Schurken foltern und der Held sich aus solchen Situationen befreien muss, ändert sich dies grundlegend in 24. Die Legitimation der Folter wird dadurch beschworen, dass die Terrorverdächtigen niemals unschuldig sind, die relevanten Informationen schnell preisgeben und dadurch jeweils größerer Schaden abgewendet werden kann. Folter wirkt in dieser Erzählform nicht nur äußerst effektiv, sondern wird auch selbstverständlicher Bestandteil zwischenmenschlicher Beziehungen, etwa wenn Bauer mit Audreys Einverständnis ihren NochEhemann Paul Raines (James Frain) foltert und hierbei klar wird, dass er nicht Teil der Verschwörung ist. Später hingegen rettet Bauer Paul aus einer Foltersituation, während dieser bei einem Schusswechsel wiederum für Jack eine Kugel abfängt und später daran stirbt. Folter wird zunehmend zu einem alltäglichen Phänomen und produziert dadurch einen Gewöhnungseffekt beim Zuschauer.

Eine neue Dimension in der Logik des Verdachts entsteht in der vierten Staffel durch die Einführung einer arabischstämmigen Familie (Araz), die aus dem Ehepaar Navi (Nestor Serrano) und Dina (Shohreh Aghdashloo) sowie dem jugendlichen Sohn Behrooz (Jonathan Ahdout) besteht und Teil des Terrornetzwerks ist; seit fünf Jahren leben Navi, Dina und Behrooz als Schläfer in den USA. Der Sender Fox nutzte das Motiv der Familie Araz zur Ankündigung der Staffel mit dem Slogan „They could be living right next door“. Die Familie wird in islamophoben Stereotypen dargestellt, exemplarisch durch den autoritären Vater, der die Familie auf den Auftrag für eine höhere Sache einschwört und keinen Widerspruch duldet. Einzig Sohn Behrooz wird in menschlichen Zügen dargestellt, er hat sich in ein amerikanisches Mädchen verliebt (Debbie) und scheint von der Mission nicht überzeugt. Nachdem Debbie jedoch Behrooz an der Lagerhalle von Navi gesehen hat, wo Verteidigungsminister Heller und seine Tochter gefangen gehalten werden, stellt sie in den Augen von Navi und Dina ein Sicherheitsrisiko dar. Dina lädt Debbie in ihr Haus ein und täuscht ein herzliches Verhältnis vor. In einem unbemerkten Moment fordert sie, mit Verweis auf den Vater, ihren Sohn auf, Debbie mit einer Pistole zu ermorden, was dieser nur unwillig tun will (S 4/F 4/ab 26.00). Als Behrooz Debbie zur Flucht verhelfen will, bricht sie zusammen und stirbt. Dina erklärt daraufhin ihrem Sohn, dass sie 
Debbie vergiftet habe, da sie wusste, dass er nicht hart genug sei, um Debbie zu erschießen. Bevor Navi nach Hause kommt, schießt Dina mit der Waffe, um dem Vater vorzutäuschen, dass Behrooz die Tat begangen habe, der dies wiederum wohlwollend zur Kenntnis nimmt. Erst als im Verlauf der Serie sogar Behrooz vom Chef des Terrornetzwerks, Marwan, und dem eigenen Vater als Gefährdung gesehen wird und getötet werden soll, verhilft ihm Dina zur Flucht und erinnert sich an ihre Mutterrolle. Insgesamt werden Dina und insbesondere Navi als kaltblütige und hinterhältige Personen dargestellt, die ihren amerikanischen Nachbarn eine Fassade vorspielen, etwa wenn Debbies Mutter auf der vergeblichen Suche nach ihrer Tochter ist, die als Leiche im Hintergrund im Flur liegt. Die Strukturen des Terrornetzwerks und der Terrorzelle in Form der Familie Araz werden als absolute Hierarchie dargestellt, die zu unbedingtem Gehorsam verpflichtet, so dass der Vater Navi sogar den Befehl von Marwan akzeptiert, seinen Sohn zu opfern, da dieser durch seine westliche Entfremdung nicht mehr von ihrer Mission überzeugt sei (S 4/F 6/23.50). War die Logik des Verdachts in den ersten Staffeln von 24 noch auf potentielle Maulwürfe in den eigenen Reihen beschränkt und waren die Täter auch phänotypisch stets klar erkennbar, vermitteln die Mitglieder der Familie Araz als Schläfer einer Terrorzelle den Eindruck, dass hinter jeder nachbarschaftlichen Ecke die terroristische Gefahr lauern könnte. Der Terrorverdacht sickert in den gesellschaftlichen Alltag und provoziert bei den Zuschauern Ängste vor dem Fremdem, beispielhaft dargestellt in der jugendlichen Beziehung zwischen Debbie und Behrooz, die durch den heimtückischen Mord der Mutter dramatisch endet.

\section{FAZIT}

Die Entwicklung eines filmpolitologischen Forschungsprogramms steht erst am Anfang. Allerdings ist es für Politikwissenschaftler lohnenswert, sich dieser methodologischen Herausforderung zu stellen. Dies bedeutet nicht, dass eine solche Analyse auf jedes technische Detail einer Filmanalyse eingehen muss, jedoch ist die Bereitschaft notwendig, sich in einer kulturwissenschaftlichen Sensibilität auf die erzählerischen Mittel und Techniken der Filmsprache einzulassen, um zu verstehen, wie durch das Medium der Serie oder des Films performative Effekte auf die Konstruktion politischer Wirklichkeit erzeugt werden. Hierbei wird deutlich, dass die vermeintliche Trennung von Realität und Fiktion zunehmend verschwimmt. Die Serie 24 ist hierfür das beste Beispiel, da sich vielerlei Hinweise dafür finden, dass sich das filmische Narrativ und der reale War on Terror in einem sich wechselseitig konstituierenden Verhältnis befinden. Eine filmpolitologische Analyse kann diese Wechselwirkung deutlich und die enge Verschrän- 
kung von Eliten- und Alltagsdiskursen sichtbar machen. Die zentrale politikwissenschaftliche Relevanz von Film- und Serienanalysen ergibt sich daraus, dass die politische Selbstverständigung von Gesellschaften nicht allein durch ihre Eliten und Repräsentanten geschieht, etwa durch Debatten im Parlament, sondern vor allem durch die Reproduktion kultureller Repräsentationen und visueller Artefakte, an denen die Bürger gestalterisch mitwirken und in ihrer Praxis des Sehens aktiv beteiligt sind. Eine Filmpolitologie ist in ihrer methodologischen Öffnung demnach auch von einem demokratischen Impuls getrieben, die vermeintlichen Ränder des politischen Diskurses (Bilder, Filme, Serien, Literatur, Kunst) analytisch aufzuwerten.

\section{LITERATUR}

Bienck, Alice (2014): Filmsprache. Einführung in die interaktive Filmanalyse, Marburg.

Bronfen, Elisabeth (2012): Hollywoods Kriege. Geschichte einer Heimsuchung, München.

Engert, Stefan/Spencer, Alexander (2009): International Relations at the Movies: Teaching and Learning about International Politics through Film, in: Perspectives. Review of International Affairs 17, S. 83-103.

Gadinger, Frank (2016): Narrating War. Human Deformation in „The Hurt Locker", in: Gadinger, Frank/Kopf, Martina/Mert, Aysem/Smith, Christopher (Hrsg.): Political Storytelling. From Fact to Fiction, Duisburg, S. 70-80.

Gadinger, Frank/Jarzebski, Sebastian/Yildiz, Taylan (2014): Vom Diskurs zur Erzählung. Möglichkeiten einer politikwissenschaftlichen Narrativanalyse, in: Politische Vierteljahresschrift 55 (1), S. 67-93.

Gadinger, Frank/Yildiz, Taylan (2017): Politik, in: Martinez, Matias (Hrsg.): Erzählen. Ein interdisziplinäres Handbuch, Stuttgart, S. 158-165.

Hamenstädt, Ulrich/Hellmann, Jens H. (2015): „It’s the Movies, Stupid!“ Filme in der Didaktik der Internationalen Beziehungen, in: Zeitschrift für Internationale Beziehungen 22 (2), S. 149-167.

Heck, Axel/Schlag, Gabi (2015): „And... Cut!“ Theoretische und methodologische Überlegungen zur Analyse von Filmen in Lehre und Forschung, in: Zeitschrift für Internationale Beziehungen 22 (2), S. 125-148.

Heck, Axel/Schlag, Gabi (2016): Plädoyer für eine kritische Filmpolitologie, in: ZIB Blog, https://zib-online.org/2016/01/25/plaedoyer-fuer-eine-kritische-filmpolito logie/ (zugegriffen am 1.2.2018). 
Heck, Axel (2017): Analyzing Docudramas in International Relations: Narratives in the Film A Murderous Decision, in: International Studies Perspectives 18 (4), S. 365-390.

Horn, Eva (2014): Zukunft als Katastrophe, München.

Lithwick, Dahlia (2008): The Bauer of Suggestion, in: Slate vom 26.7., http://www.slate.com/articles/news_and_politics/jurisprudence/2008/07/the_ bauer_of_suggestion.html (zugegriffen am 1.2.2018).

Lowry, Brian (2014): The ,24‘ Effect: How ,Liberal Hollywood`Carried Water For Torture, in: Variety vom 14.12.

Mayer, Jane (2007): Whatever it takes. The politics of the man behind ,24', in: The New Yorker vom 19.2., https://www.newyorker.com/magazine/2007/02/ 19/whatever-it-takes (zugegriffen am 1.2.2018).

Neumann, Iver/Nexon, Daniel (2006): Harry Potter and the Study of World Politics, in: Nexon, Daniel/Neumann, Iver (Hrsg.): Harry Potter and International Relations, Lanham, S. 1-25.

Rehfeld, Nina (2014): Die Uhr tickt wieder, in: Frankfurter Allgemeine Zeitung vom 5.5.

Šíp, Daniel (2014): Gewissen ist Macht, in: Zeit Online, 6.5., http://www.zeit.de/ kultur/film/2014-05/24-jack-bauer-staffel-neun (zugegriffen am 1.2.2018)

Van Veeren, Elspeth (2009): Interrogating 24: Making Sense of US Counterterrorism in the Global War on Terrorism, in: New Political Science 31 (3), S. 361-384.

Yildiz, Taylan/Gadinger, Frank/Jarzebski, Sebastian (2015): Das narrative Element des Politischen. Überlegungen zu einer Poetologie des Wissens, in: Zeitschrift für Politikwissenschaft 25 (3), S. 421-431.

Yildiz, Taylan/Gadinger, Frank/Smith, Christopher (2018): Narrative Legitimierung. Exekutive, repräsentative und subversive Erzählstrategien in der Überwachungskontroverse, in: Leviathan - Berliner Zeitschrift für Sozialwissenschaft, i.E. 



\title{
人民的名义 (Im Namen des Volkes) versus House of Cards
}

\author{
Polit-Serien als Legitimierungsinstrument
}

aktueller chinesischer Reformpolitik

Ulrike Gansen, Martin Miller, Nele Noesselt, Jonas Seyferth

\section{EINLEITUNG}

Chinas Politik ist „,kein ,House of Cards““ (Renmin Ribao 2016) - mit dieser Formulierung trat der chinesische Staats- und Parteichef Xi Jinping indirekt Szenarien entgegen, welche eine Systemkrise des chinesischen Ein-Parteien-Staates prognostizieren. Korruption, Machtwillkür von lokalen Partei-Kadern sowie ein generelles Koordinationsversagen staatlicher Organe werden als Hauptfaktoren derart düsterer Untergangsszenarien identifiziert (Li 2012: 596, Pei 2012: 3637). Hatte sich jahrzehntelang in der politikwissenschaftlichen China-Analyse der Ansatz der Resilienz und Lernfähigkeit autoritärer Systeme als Erklärung für den Fortbestand und die relative Robustheit des chinesischen „sozialistischen“ Ein-Parteien-Systems behaupten können (Nathan 2003: 6), wird im Zuge der sichtbar gewordenen parteiinternen Faktionskämpfe und interelitärer Spannungen seit 2012 verstärkt über die Grenzen einer langfristigen Systemstabilisierung debattiert (Li 2012: 619).

Die seit 2012/2013 im Amt befindliche fünfte Führungsgeneration der Kommunistischen Partei Chinas (KPCh) hat auf diese in der internationalen China-Analyse zirkulierenden Transformationsmodelle und parallel zugleich auch auf Reformerwartungen innerchinesischer Akteursgruppen reagiert, indem sie offensiv ein großangelegtes Programm zur Bekämpfung von Fehlentwicklungen des Systems und zur Unterbindung von Korruption und Kaderwillkür vorgelegt hat. Allegorisch wird die seit 2012 unter Xi Jinping laufende AntiKorruptionskampagne gegen Partei-Kader auf allen Systemebenen - gegen so- 
genannte kleine ,Fliegen“ und mächtige ,Tiger" - als erfolgreiche Aktion zur Wiederherstellung der alten Ordnung präsentiert. Doch jede Adjustierung innerhalb des chinesischen Ein-Parteien-Systems birgt die Gefahr, dass sich Gegeneliten mit alternativen Ordnungsmodellen formieren und die etablierten Herrschaftsstrukturen in Frage stellen. Die politische Führung scheint dies bei der Präsentation ihrer Reformprogramme einkalkuliert zu haben. Denn die Veröffentlichung von Reformdokumenten wird begleitet von Polit-Serien, welche die aktuellen Maßnahmen und Kampagnen in eine übergeordnete Masternarrative einbetten und die Reformnotwendigkeit symbolisch untermauern. 2017 hat der chinesische Sender Hunan TV mit Im Namen des Volkes (Renmin de mingyi) eine 52-teilige Polit-Serie ausgestrahlt, welche den Kampf gegen die Korruption als Reformprojekt der Partei ausweist.

In westlichen Tageszeitungen wurde die Serie als chinesisches Pendant zu der Netflix-Produktion House of Cards rezipiert. Hinsichtlich der Popularität und auch hinsichtlich der Szenendramaturgie liegt dies auf den ersten Blick nahe. Doch Xi Jinpings Stellungnahme verdeutlicht, dass hier keine Parallelerzählung, sondern eine chinesische Gegenerzählung vorgelegt wird: Der in der NetflixSerie entworfenen Dystopie des US-amerikanischen demokratischen Präsidialsystems wird mit Im Namen des Volkes ein positiv besetztes ,Chinesisches Modell $^{11}$ der Ein-Parteien-Meritokratie entgegengesetzt.

Die folgende Analyse der Kopplung zwischen der Instrumentalisierung westlicher Polit-Serien und der symbolischen Systemlegitimierung über den Entwurf chinesischer ,Spiegel'-Serien erfolgt in drei Schritten. Zunächst soll kurz der Kontext der chinesischen House of Cards-Debatte skizziert werden. Der Fokus liegt auf den Jahren 2012 (Aufstieg der fünften Führungsgeneration, Beginn der Antikorruptionskampagne) bis 2017 (19. Parteitag). Welche Konzeptionen politischer Herrschaft und welche staatsphilosophischen Grundideen unterliegen der chinesischen Polit-Serie? Welche Reformentwicklungen werden in der Serie aufgegriffen? In einem zweiten Schritt soll zum einen die in House of Cards omnipräsente Kritik an Chinas Ein-Parteien-System und den korrupten Machenschaften von indirekten Systemvertretern am Beispiel der Figur Xander Feng nachgezeichnet werden. Zum anderen soll expliziert werden, wie die amerikanische Polit-Serie der chinesischen Staatspropaganda Anknüpfungsmöglichkeiten zur Demontage liberaler Demokratie und Abwertung westlicher Chinakritik verschafft. In einem dritten Schritt soll die chinesische Polit-Serie Im Namen des Volkes als indirekte Antwort und Gegenerzählung zu House of Cards entschlüsselt werden.

1 Zur innerchinesischen Konfiguration des, Chinesischen Modells‘ vgl. Zhao (2017). 


\section{KONTEXT: POLITISCHE SYSTEMREFORMEN DER VR CHINA IM 21. JAHRHUNDERT}

Als moderne Autokratie fällt die VR China in die Gruppe der lern- und adaptationsfähigen Ein-Parteien-Systeme, die sowohl die Erfahrungen anderer „,sozialistischer" Systeme reflektieren wie auch selektiv Governance-Elemente und Steuerungsinstrumente demokratisch-pluralistischer Systeme aufnehmen. Die politische Geschichte der VR China läßt sich einteilen in Phasen der Repression und Kontrolle und solche der partiellen Liberalisierung und Systemreformen. Eine Schlüsselfunktion kommt dem 3. Plenum von $1978 \mathrm{zu}$, auf welchem die Beschlüsse zu Reform und Öffnung verabschiedet wurden. Seitdem experimentiert die VR China mit einer eigenen Version des Kapitalismus. Das 3. Plenum von 2013 setzt das symbolische Erbe Deng Xiaopings, des Architekten der Wirtschaftsrestrukturierung in der post-maoistischen Ära, fort. Zudem aber werden negative Begleiterscheinungen des 1978 eingeleiteten Transformationsprozesses korrigiert. Im Folgenden sollen die jüngsten Kurskorrekturen unter der fünften Führungsgeneration (Xi Jinping/Li Keqiang) skizziert werden.

\section{Top-Down-Reformen statt Bottom-up-Revolution}

Das chinesische Ein-Parteien-System beruht im Unterschied $\mathrm{zu}$ westlichliberalen pluralistischen Parteiendemokratien auf dem Prinzip der zentralistischen Kontrolle und der Gewaltenverschränkung. Im Zuge der post-maoistischen Reform- und Öffnungspolitik hat die KPCh eine Wandlung von einer revolutionären Partei der Arbeiter, Bauern und Soldaten zu einer ,regierenden Partei“ (zhizheng dang) des gesamten Volkes vollzogen (Womack 2005: 23). Die grundlegenden Systemstrukturen des bürokratischen Partei- und Staatsapparats sind jedoch weitgehend unverändert fortgeschrieben worden. Allerdings wurde das Prinzip der charismatischen Führung Maos in der Reformära durch das Modell der kollektiven Führung substituiert, statt eines Parteivorsitzenden wurde ein Generalsekretär als princeps inter pares ${ }^{2}$ ernannt. Pragmatismus dominierte die Neuausrichtung der chinesischen Politik seit den frühen 1980er Jahren. Die KPCh vertritt seit 2002/2003 auch die Interessen der neuen Staatskapitalisten, ebenso wie jene der Intellektuellen - beide Gruppen waren während der MaoÄra als Feindes des Volkes gebrandmarkt und verfolgt worden.

2 Zum Konzept der kollektiven Führung und der hierzu seit 2012/2013 in China erneut aufflammenden kritischen Betrachtungen siehe Li (2016: 12-15). 
Seit dem Antritt der fünften Führungsgeneration zeichnet sich ein Kurswechsel ab. Formal sind die seit 1978 eingeführten Strukturen und Prinzipien weiterhin gültig. Jedoch wird ein neuer Personenkult um Xi Jinping aufgebaut, der Reminiszenzen an die Mao-Ära aufweist (Lam 2015: xi). Die Jahre des politischen Experimentierens ${ }^{3}$ und des „De-facto-Föderalismus“ (Zheng 2007: 30) scheinen passé: Die Parteispitze hat unmittelbar nach der Übernahme der Amtsgeschäfte (2013) ein „Top-Level-Design“ (dingceng sheji) der Systemsteuerung ausgerufen (Noesselt/Senghaas 2016: 566). Die vergangenen drei Dekaden waren von lokalen Reformexperimenten geprägt, die zu einer Erstarkung und zunehmenden Autonomie lokaler Verwaltungseinheiten geführt hatten. Diesen zentrifugalen Tendenzen und der Fragmentierung des Systems tritt die fünfte Führungsgeneration mit einem zentral koordinierten Reformpaket entgegen. Es reflektiert den seit vielen Jahren - nicht zuletzt im Rahmen der alljährlichen Arbeitsberichte der Regierung und der Konzeption der Fünf-Jahres-Pläne - bereits identifizierten Reformbedarf und die rezenten Reformforderungen systemrelevanter sozio-ökonomischer Akteursgruppen. Im Zentrum der mit den Beschlüssen des 3. Plenums des 18. Zentralkomitees 2013 angeschobenen Restrukturierungsmaßnahmen steht die Sicherstellung des chinesischen Wirtschaftswachstums. Der durch die Ein-Kind-Politik ausgelöste demographische Wandel hat unmittelbare Implikationen für das chinesische Wirtschaftsmodell, das bislang auf den Überschuss billiger Arbeitskräfte setzen konnte. Um der abzusehenden Überalterung der chinesischen Bevölkerung entgegenzuwirken, wurde 2013 der Übergang zu einer Zwei-Kind-Politik beschlossen. Um die Versorgung der urbanen Wirtschaftszentren mit Arbeitskräften zu gewährleisten, wurde zudem eine Legalisierung der Land-Stadt-Migration verabschiedet (Naughton 2014).

Herausgefordert wird das Ein-Parteien-System durch lokale Unruhen und Massenproteste, die sich zumeist gegen illegale Landnahme und Amtsmissbrauch von Partei-Kadern jenseits der Ebene der Zentrale richten (Tong/Lei 2014: 498). Mit der Antikorruptionskampagne von 2012 und begleitenden Maßnahmen zur Korrektur des Arbeitsstils der Partei-Kader hat die politische Führung symbolisch unterstrichen, dass diese Systemauswüchse nicht geduldet werden. ${ }^{4}$ Inwiefern im Zuge der Antikorruptionskampagne politische Gegner ausgeschaltet und strategische Machtnetze etabliert werden, wird sich erst in der histo-

3 Für einen Überblick über Chinas lokale Policy-Experimente vgl. Teets/Hurst (2015: $1-24)$.

4 Zur Konzeptualisierung von Korruption im Kontext der innerchinesischen Governance-Debatten und Herrschaftskonzeption siehe He (2000). 
rischen Rückblende eruieren lassen. Offiziell bekennt sich der chinesische Partei-Staat unter Xi Jinping zu einem Ausbau der „rechtsgestützten Herrschaft“ ( $y i$ fa zhi guo), zu verstehen als ,rule by law“ und nicht „rule of law“ in Verbindung mit Demokratisierung (Peerenboom 2002: 8-9). 2014 widmete sich die Partei mit einer Plenarsitzung erstmals ausschließlich der Frage des Zusammenspiels von Politik und Recht. ${ }^{5}$ Die damit erfolgende Formalisierung der chinesischen Politik impliziert, dass informelle Mechanismen, die sich bislang auf lokaler Ebene bewährt hatten, nunmehr nicht länger zulässig sind und möglicherweise Gegenstand einer zentral koordinierten Korruptionsuntersuchung werden könnten.

Die Ära Xi Jinping ist damit geprägt von zwei partiell widersprüchlichen Tendenzen: dem Aufbau eines neuen charismatischen Personenkults und, parallel hierzu, der Stärkung der legal-rationalen Herrschaft. Auf der einen Seite wird der neoliberale Wirtschaftskurs fortgesetzt, auf der anderen Seite aber die ,sozialistische' Systemidentität und Wirtschaftsplanung betont. 2014 proklamierte die chinesische Staatsführung offiziell den Eintritt in die Phase der „Neuen Normalität“: Nachhaltigkeit, Innovation, Stärkung des Binnenmarktes und Diversifizierung der Handelskontakte sind $\mathrm{zu}$ den neuen Zielvorgaben erhoben worden (Xinhua 2014). Die Ausrichtung auf ein jährliches Wirtschaftswachstum von sechs bis sieben Prozent wird als gezielte Umsteuerung in Richtung eines umwelt-und sozialverträglichen Entwicklungsweges ausgegeben. Zusätzlich proklamierte Xi Jinping auf dem 19. Parteitag 2017 den „Sozialismus mit chinesischen Charakteristika in der neuen Ära“. ${ }^{6}$ Mit dieser Formel erhebt der chinesische Ein-Parteien-Staat den Anspruch, einen partikularen Entwicklungsweg zu verfolgen, der sich nicht an den historischen Szenarien anderer (sozialistischer) Systeme messen lässt.

\section{Legalismus und Han Fei}

Der staatstheoretische Referenztext, auf den sich die Rückbesinnung auf eine starke Staatsführung und die Verrechtlichung der chinesischen Politik bezieht, sind die Schriften des chinesischen Legalisten Han Fei (280 bis 233 v. Chr.). Dieser identifiziert drei Grundelemente politischer Herrschaft: Autorität (shi), Führungstechniken $(s h u)$ und Gesetze $(f a)$. In der Kompilation der wichtigsten Zitate Xi Jinpings aus der vormodernen chinesischen Staatsphilosophie finden

5 Die englische Version der Resolution findet sich unter: http://news.xinhuanet.com/ politics/2014-10/23/c_1112953884.htm.

6 Für eine Zusammenfassung der wichtigsten Punkte des 19. Parteitags: Xinhua (2017). 
sich in dem Kapitel zur rechtsgestützten Herrschaft gleich zwei Schlüsselsätze aus den Schriften Han Feis. Abstrakt zusammengefasst unterstreichen diese, dass Gesetze die Grundlage für die Stärke und Stabilität eines Herrschaftssystems darstellen, da hiermit eine hierarchische Ordnung und ein verbindlicher Verhaltenskodex festgeschrieben werden, welche Chaos unterbinden und das Herrschaftsregime stabilisieren (Renmin Ribao Pinglunbu 2015: 267, 271). Die Rückbesinnung auf einen autoritären Zentralismus wird symbolisch als Rückbesinnung auf die Prinzipien des chinesischen Leg(al)ismus präsentiert (Seppänen 2016: 30), nicht als Übernahme, westlicher' Vorstellungen von Rechtsstaatlichkeit und Transparenz. Wie die Kompilation der Zitate Xi Jinpings aus den Klassikern der vormodernen chinesischen Staatsphilosophie verdeutlicht, werden zur Konzeption eines modernen ,chinesischen' Rechtsmodells konkurrierende Elemente des Konfuzianismus und Leg(al)ismus in einer neuen Synthese zusammengeführt. Die Parteiführung entwirft für sich die Rolle des aufgeklärten wohlwollenden Koordinators und regiert paternalistisch ,für' (nicht , durch') das Volk. Die Antikorruptionskampagne und auch die Korrektur des Führungsstils der Partei erfolgen unter Rückgriff auf legalistische Ideen, die Führung des Volkes durch die Partei aber wird von konfuzianischen Vorstellungen eines hierarchischen Ordnungssystems der wechselseitigen Pflichten und Verpflichtungen geleitet.

\section{HOUSE OF CARDS IM CHINESISCHEN POLIT-DISKURS}

Dass House of Cards auch in China legal über das Internetportal Sohu abrufbar ist, vermag in Anbetracht des in der Serie vermittelten negativen, stereotypen Chinabildes nur auf den ersten Blick zu überraschen. Gerade in der zweiten Staffel der Serie werden rezente Konfliktfelder wie der Inselkonflikt zwischen China und Japan, der Vorwurf der Währungsmanipulation gegenüber Peking, Bestechungsversuche des Weißen Hauses durch einen zwielichtigen chinesischen Geschäftsmann oder auch die China zugeschriebenen Hackerangriffe auf USamerikanische Server in den Plot eingebaut. Doch letztendlich zeigt sich hier eine strategische Weiterentwicklung und pragmatische Adaption in der chinesischen Medienpolitik: Wenn Wege existieren, über Proxyserver auch auf gesperrte Medienangebote zuzugreifen, und dies in Anbetracht der Popularität von Serien wie House of Cards auch zu erwarten wäre, ist es strategisch gesehen zielführender, diese Serie offiziell freizugeben und in den eigenen Polit-Diskurs zu integrieren. So wird Wang Qishan, der die 2012 durch Xi Jinping angestoßene Anti-Korruptionskampagne bis 2017 koordinierte, zugeschrieben, die Partei- 
Kader aktiv angewiesen zu haben, House of Cards als warnendes Beispiel anzuschauen und kritisch zu studieren. ${ }^{7}$

Es ist sicherlich kein Zufall, dass zur Diskreditierung, westlicher' Systemwerte nicht irgendeine der zahlreichen Polit-Serien der vergangenen Jahre (wie Scandal, Borgen, The West Wing) herangezogen wurde, sondern gerade House of Cards: Kaum eine Polit-Serie überwindet so meisterhaft die Grenzen zwischen Fiktion und politischer Gegenwart. Nicht zuletzt durch den Clou, dass der ,Wahlkampf' des Serien-Protagonisten mit den realen Präsidentschaftswahlen in den USA 2016 gekoppelt wurde - konzipiert als Teaser für die angekündigte Folgestaffel der Serie (Dörner 2016). Diese so geschaffene ,Als-ob-Welt‘ suggeriert dem Betrachter sehr sublim, dass die Serie einen Blick hinter die Kulissen, in die Abgründe des realen politischen Tagesgeschäfts erlaubt. Der Zuschauer wird über die Monologe der Charaktere in ihre (niederen) Beweggründe und korrupten Machtergreifungsstrategien eingebunden, wird eingeweiht in die Kalkulationen und Triebkräfte der innersten Machtzirkel (siehe hierzu die beiden Kapitel zur Serie House of Cards in diesem Band). Die US-amerikanische Serie spielt zudem gezielt mit der Faszination, die von der Entschlüsselung geheimer, interner Informationen ausgeht, setzt auf einen fiktionalen investigativen Reportagestil. Der gekonnten Verbindung von Politthriller, Krimi und TV-Drama kommt eine hohe Zugkraft zu - die professionelle Hochglanzproduktion schlägt den Betrachter in ihren Bann, spricht diesen durch ein ausgeklügeltes Zusammenspiel von Ton und formelhafter Symbolsprache nicht zuletzt auf einer höchst emotionalen Ebene an.

Die zweite Staffel von House of Cards zeichnet insgesamt ein ambivalentes Bild von der Volksrepublik China, auf dessen Basis die chinesische Staatspropaganda eine Erzählung von der Demontage westlich-liberaler Demokratie und der Entwertung westlicher Chinakritik entwirft. Die Korrumpierung des westlichen Regierungssystems durch den Aufstieg Frank Underwoods zum US-Präsidenten und die Korruption des chinesischen Milliardärs Xander Feng offerieren eine dankbare Vorlage, mit der westliche Ordnungsvorstellungen delegitimiert und das chinesische Herrschaftssystem aufgewertet werden kann. Die Figur des

7 Neben House of Cards soll Wang Qishan den Führungskadern der Partei zudem Tocquevilles Abhandlung Der alte Staat und die Revolution und die chinesische Übersetzung der von Michael Sandel herausgegebenen Textsammlung Justice zum Selbststudium empfohlen haben. Diese drei Vorlagen dienen als Ausgangspunkt der kritischen Bestimmung und Diskussion des gegenwärtigen Zustands des chinesischen EinParteien-Systems, wenngleich offiziell hierbei ausdrücklich nicht-chinesische Regime thematisiert werden (Noesselt 2014: 1). 
Xander Feng, der sich in der Serie als inoffizieller Gesprächskontakt zur chinesischen Regierung ausgibt, bestimmt in diesem Zusammenhang maßgeblich das in der Serie vermittelte Bild des modernen Chinas, mit dem sich die US-Regierung auseinanderzusetzen hat. Underwood postuliert hierzu: „Mao is dead, and so is his China“" (S 2/F 5/41.10). Feng übernimmt das Repräsentationsmonopol eines modernen Chinas, dem der Kaderkapitalismus neuen Wohlstand und Reichtum beschert hat. Sein Repräsentationsmonopol ergibt sich daraus, dass seine Figur der einzige Vertreter Chinas ist, der im Staffelverlauf persönlich auftritt. Zwar verweisen die Seriencharaktere auf verschiedene weitere chinesische Staatsvertreter (u.a. Präsident Qian), diese betreten jedoch nicht selbst die Bühne, sondern werden lediglich mittelbar zitiert und so in das Geschehen eingebunden, weshalb sie kaum an Kontur gewinnen. In diesem Sinne manifestiert sich die Präsenz des chinesischen Parteistaats im Serienkontext maßgeblich über die Absenz einer offiziellen Repräsentation.

In Fengs Habitus offenbart sich dem Serienzuschauer eine Neigung zu Kriminalität, Lasterhaftigkeit und Materialismus: So wurde er in der Vergangenheit bereits zweifach wegen Korruption in China angeklagt, jedoch nicht verurteilt, was eine unterschwellige Duldung von Korruption in China suggeriert. Fengs Figur wird zudem mit einer Szene eingeführt, die ihn beim erotischen Fetischspiel mit zwei Prostituierten zeigt. Ferner offenbart er eine Vorliebe für kostspielige alkoholische Getränke, auf deren Wert er explizit verweist. Zu den Praktiken, die ihm seinen persönlichen Aufstieg ermöglicht haben, bekennt er im vertraulichen Gespräch:

$\underline{\text { Szenen-Beispiel: }}$

Feng im Gespräch mit dem Stabschef Underwoods, Doug Stamper (S 2/F 7/38.40)

Feng: Everyone in China who works on this level pays who they need to pay. And they kill who they need to kill. People disappear all the time, Mr. Stamper.

Scheint diese Darstellung zunächst ein negatives Chinabild zu vermitteln, so zeigen sich bei einer Kontextualisierung mit aktuellen Reformbestrebungen unter der fünften Führungsgeneration Chinas und mit den zugehörigen Legitimationsstrategien Komplementaritäten: Die Figur des Xander Feng verkörpert in ihrer Korruption, Kriminalität und Dekadenz Eigenschaften, die die Dringlichkeit von Maßnahmen zu Korruptionsbekämpfung und ideologischer Rektifikation, wie sie in der Antikorruptionskampagne unter Xi Jinping realen Ausdruck gefunden haben, illustrieren.

Jenseits der Figur des Xander Feng porträtiert die zweite Staffel House of Cards die Volksrepublik als eine selbstbewusste Großmacht, die in vielen Be- 
langen zwar auf Augenhöhe mit den Amerikanern agiert, dabei aber auf konventionelle Instrumente destruktiver und am Eigennutz orientierter Machtpolitik zurückgreift. China ist im Serienkontext damit im Konzert der Großmächte eine unter Gleichen. Diese Darstellung steht dem chinesischen Ansinnen entgegen, international als „responsible power“ wahrgenommen zu werden, was unter der Ägide von Xi Jinping noch einmal an Bedeutung gewonnen hat, wie es auch in der Betonung des Selbstbildes als „responsible country“ durch Li Keqiang in seinem Arbeitsbericht auf dem Nationalen Volkskongress vom März 2017 zum Ausdruck kam (Heberer 2014: 121, Wang 2008: 268, Li 2017). Vizepräsident Frank Underwood hebt in seinen Äußerungen wiederholt die Bedeutung Chinas hervor, betont aber zugleich, dass die Chinesen nur eine Sprache der Stärke verstünden.

\section{Szenen-Beispiel:}

Underwood gegenüber Präsident Walker (S 2/F 5/42.40)

Underwood: And I can tell you firsthand that we are dealing with a regime that is not being forthright and will seize upon the faintest whiff of trepidation.

Underwoods Vorwürfe bauen auf dem Verhalten chinesischer Staatsvertreter im Umgang mit ihren amerikanischen Pendants auf, das ein robustes Selbstbewusstsein erkennen lässt. So weigert sich eine chinesische Delegation in Handelsgesprächen mit den Amerikanern, das Ultimatum der US-Seite zu akzeptieren, dass Cyberangriffe auf US-Unternehmen zum Bestandteil der Gespräche werden müssten. Stattdessen führt sie den Abbruch der Gespräche herbei. Im Staffelverlauf ignoriert der chinesische Staatspräsident zudem Versuche der Kontaktaufnahme von US-Präsident Walker. Der chinesische Botschafter in Washington lässt übermitteln, dass seine Regierung nicht mit einem Präsidenten verhandeln werde, der sich hinter Pekings Rücken mit korrupten Unternehmern abspricht. Xander Feng offenbart einen vergleichbaren Habitus, als er nach seinem ersten Treffen mit Vizepräsident Underwood mit dessen Stabschef Douglas Stamper zusammenkommt. Trotz eines formalen Hierarchiegefälles zwischen dem Vizepräsidenten der USA und einem inoffiziellen Gesandten der Volksrepublik erhält Feng direkten Zugang zu Underwood. Dass er dies auch erwartet, verdeutlicht sein herablassender Umgang mit Stamper.

\section{Szenen-Beispiel:}

Feng im Gespräche Doug Stamper (S 2/F 5/24.55)

Feng: Don't inflate your status, Mr. Stamper. You're nothing but an errand boy. 
Besonders deutlich tritt das Motiv eines illegitimen, aggressiven Gebarens Chinas in Vorwürfen der Cyberspionage hervor, die Mitglieder der US-Regierung gegen China erheben. Die Rolle des Aggressors wird der chinesischen Seite ebenso in einer Krise mit Japan zugeschrieben, als zwei chinesische Zerstörer in die Hoheitsgewässer um eine japanische Insel vordringen. US-Präsident Garrett Walker stellt mit dem Verweis auf den Konflikt um die Senkaku-Inseln den Bezug zu realen Vorfällen her:

Szenen-Beispiel:

Das US-Kabinett berät über den japanisch-chinesischen Inselkonflikt (S2/F10/3.49 3.58).

Walker: Now this is different than the standoff over the Senkaku Islands.

Durant: It could have to do with shipping routes.

Underwood: Or the trade war. They can't pick on the big kid, so they're picking on the little one.

China wirkt im Serienkontext zudem im Zusammenhang mit der Affäre um illegale Parteispenden als manipulative Kraft im Hintergrund: So fließen chinesische Gelder über verschlungene Wege an die amerikanischen Parteien. Die Geldwäsche erfolgt in amerikanischen Casinos, von wo aus sie an super PACs der Demokraten und nachfolgend der Republikaner weitergereicht werden. Mit diesem illegalen Konstrukt ausländischer Einflussnahme nimmt China in Grundzügen eine ähnliche Rolle ein, wie sie in Bezug auf Russland bei der Präsidentschaftskampagne Donald Trumps diskutiert wird (siehe hierzu die Übersicht in New York Times 2018).

In der politischen Identitätskonstruktion des liberalen Westens hat China als autoritär verfasster Staat immer wieder als Gegen- und Zerrbild fungiert (Zhang 2016: 797). Einige Elemente dieses imaginierten System-Antagonismus scheinen auch im Serienkontext durch. Das chinesische Regierungssystem präsentiert sich in diesem Zusammenhang weitgehend als Blackbox, dessen Undurchsichtigkeit in markantem Kontrast zur Einsicht steht, die der Serienzuschauer in das US-System erhält, wenn die Figur Underwoods zum stilisierten Durchbrechen der , vierten Wand ' ansetzt und sich direkt an den Serienzuschauer wendet ${ }^{8}$. Im weiteren Verlauf bleibt auch die tatsächliche Rolle des Xander Feng unklar, der für sich selbst in Anspruch nimmt, Kontakte zur Parteispitze der KPCh bis hinein in das Politbüro zu unterhalten und als inoffizieller Sprecher der chinesi-

8 Näheres hierzu findet sich im Kapitel „Erzählung“ der Analyse zur Serie House of Cards. 
schen Regierung aufzutreten. Andere Serienfiguren ziehen diesen Status jedoch in Zweifel, ohne dass es letztlich zu einer Auflösung kommt. Vizepräsident Underwood und sein Stab behandeln Feng zwar als quasioffiziellen Emissär Pekings, gleichzeitig gibt Underwood jedoch zu erkennen, dass Feng seiner Auffassung nach allenfalls für einen Teil der chinesischen Staatsführung spricht und seine eigentliche Loyalität den eigenen Geschäftsinteressen gilt. Die spärlichen Einblicke in die Blackbox des chinesischen Regierungssystems, die House of Cards offeriert, suggerieren die Existenz von Spaltungen und Konfliktlinien in den Machtzirkeln um den Ständigen Ausschuss des Politbüros herum. In Bezug auf die Wiederaufnahme einer WTO-Klage der USA zur Währungsmanipulation durch China erklärt Feng zu seinen Möglichkeiten der Einflussnahme auf den chinesischen Entscheidungsprozess:

\section{Szenen-Beispiel:}

Feng im Gespräch mit Doug Stamper (S 2/F 5/25.54)

Feng: When you do, there will be those on the Standing Committee who protest, but I'll manage them. The majority want reform.

Weniger markant fällt der Kontrast der Systeme hingegen bei der Anfälligkeit für Korruption und Verbrechen aus: In der Person von Xander Feng treten die Auswüchse des chinesischen Systems zutage, wohingegen mit der Machtusurpation durch Frank Underwood die Schattenseiten der US-Demokratie ausgesprochen scharf konturiert werden. Diese Hyperrealität in der Darstellung des USSystems als Beute des Frank Underwood, der sich das System mittels Mord, Verrat und Verbrechen untertan gemacht hat, ist, so die offiziöse Mitteilung an die chinesische Bevölkerung, eine Geschichte des Abstiegs westlicher Demokratien hinsichtlich ihrer hehren Werte der Demokratie und Rechtsstaatlichkeit. Sie bezieht ihre besondere Qualität daraus, dass die Dekonstruktion der liberalen Demokratie aus dem Zentrum des Systems heraus erfolgt. Underwood macht sich gegenüber dem Serienzuschauer selbst zum ersten Fürsprecher dieser Lesart:

\section{Szenen-Beispiel:}

Underwood wird als Vizepräsident vereidigt. Währenddessen wendet er sich an den Serienzuschauer (S 2/F 2/2.30).

Underwood: One heartbeat away from the presidency, and not a single vote cast in my name. Democracy is so overrated. 
Diese Hyperrealität tritt zugleich in eine erweiterte Rückkopplung mit der Wirklichkeit, stellt sie doch zugleich eine Erzählung aus dem Amerika des Donald Trump dar, wenn dieser propagiert: „We have a rigged system“ (CNN 2017). Von einem solch dekadenten System an China geübte Kritik ist, folgt man dieser offiziellen Leseanleitung, nicht weiter ernst zu nehmen, da sie sich schlussendlich selbst delegitimiert. Ungewollt übernimmt House of Cards somit die Funktion eines das chinesische Ein-Parteien-System stabilisierenden Narrativs. Dieses wird durch eine positive Gegenerzählungen der Systemmodernisierung und Korruptionsbekämpfung unter der von Xi Jinping geführten fünften Führungsgeneration (seit 2012/2013) flankiert.

\section{IM NAMEN DES VOLKES ALS CHINESISCHE GEGENERZÄHLUNG ZU HOUSE OF CARDS}

Anknüpfend an den Erfolg von House of Cards erfolgte unter der fünften Führungsgeneration im März 2017 mit Im Namen des Volkes die Ausstrahlung einer chinesischen Polit-Serie, über welche Xi Jinpings Anti-Korruptionskampagne als notwendiger Eingriff im Interesse und ,im Namen“ des chinesischen Volkes ausgewiesen wird. Präsentiert als Fiktion, greift die chinesische Serie - darin westlichen Polit-Serien vergleichbar - gezielt Ereignisse der jüngsten politischen Geschichte und ausgewählte aufgedeckte Korruptionsfälle hochrangiger Führungskader der Kommunistischen Partei auf. Die Serie basiert auf einem Polit-Thriller des chinesischen Autors Zhou Meisen (2017), der, legitimiert durch die Oberste Volksstaatsanwaltschaft der VR China, auch die Drehbuchvorlage lieferte.

Die erste offizielle Ausstrahlung von Im Namen des Volkes begann Ende März 2017 - und somit nach den ,zwei Sitzungen“, den Jahrestagungen des Nationalen Volkskongresses und der Politischen Konsultativkonferenz des chinesischen Volkes. Damit wurde vorausschauend vermieden, dass es ausgehend von der Serie zu Debatten über die Themen und Inhalte der „zwei Sitzungen“ kommen würde - was wiederum verdeutlicht, wie vorsichtig die Serie ausgetestet wird.

Hochkarätig besetzt und mit einem Trailer beworben, der den gegenwärtigen ,westlichen' Polit-Serien in keinerlei Hinsicht nachsteht, erreichte die Serie sofortigen Kultstatus - und wurde auch von im Ausland lebenden Chinesen mitverfolgt. Die Serie präsentiert als Spielort das moderne urbane China, das in Trailern mittels Kamerafahrten zwischen Hochhäusern und Häuserschluchten in Szene gesetzt wird und generell inszenatorisch in vielerlei Hinsicht die Ästhetik westlicher Film- und Serienblockbuster nachempfindet. Im Unterschied zu 
House of Cards wird mit dieser Serie keine Zukunftsdystopie des erfolgreichen Spiels von Intrigen zur Erringung politischer Macht und der Übernahme der Staatsführung entworfen. Vielmehr werden reale, in den letzten Jahren über die Staatsmedien dokumentierte Einzelfälle von Macht- und Amtsmissbrauch hochrangiger Führungskader aufgegriffen.

Held der Serie ist ein unermüdlich gegen die Korruption zu Felde ziehender junger Sonderermittler (Hou Liangping, gespielt von Lu Yi), der in einer imaginären chinesischen Provinz - nicht auf der Ebene der Zentrale - den Netzwerken korrupter Partei-Kader entgegentritt. Er ist ausgestattet mit einem offiziellen Mandat, agiert mithin im Auftrag der obersten Führungsebene und nicht als selbsternannter Rächer der Opfer lokaler Machtwillkür oder oppositioneller Systemreformer. Das Alter der Protagonisten ist insofern ein relevanter Faktor, als der Kampf gegen die Korruption auch als Konflikt der Generationen präsentiert wird. Eine junge Riege von Sonderermittlern tritt an, die Seilschaften der alten Kader zu zerschlagen. Das chinesische Staatssystem ist somit in dieser Darstellung dazu fähig, aus sich selbst heraus Regenerationskräfte zu mobilisieren, um sich gegen die Ausbreitung von Korruption zu immunisieren und den eigenen Fortbestand zu sichern. Aus chinesischer Sicht illustriert die fiktionalisierte politische „Realität“ der USA in House of Cards das Endspiel der westlichen Demokratie. Checks-and-Balances und die moralisch-ethischen Grundlagen des Systems werden durch Frank Underwood systematisch ausgehebelt. Die Instrumente des westlichen Rechtsstaats versagen. Das chinesische System hingegen wehrt, so die mit Im Namen des Volkes gesetzte Leitnarrative, die von Korruption und Machtmißbrauch ausgehende Gefahr effektiv ab. Dies zeigt sich nicht zuletzt allegorisch in den martialisch in Szene gesetzten Einsätzen von schwer bewaffneten Spezialkräften des Anti-Korruptionskorps, deren resolutes Vorgehen an Kommandomissionen westlicher Anti-Terror-Einheiten erinnert.

Augenfällig ist, dass Korruption als primär auf die Provinzebene begrenzt dargestellt wird, die oberste Staats- und Parteiführung folglich als immun gegenüber entsprechenden Versuchungen dargestellt wird, was anderslautenden Berichten über fragwürdige Verflechtungen und Aktivitäten der vierten Führungsgeneration und ihrer Familienangehörigen, veröffentlicht von der New York Times (2012), entgegensteht. Dass die Konstruktion von Realitätsbezügen für das chinesische Publikum darüber hinaus von Bedeutung ist und auch teilweise selbst hergestellt wird, verdeutlichen Debatten, die kurz nach Ausstrahlung der Pilotfolge entbrannten und darum kreisten, wo der Handlungsort der (fiktiven) 
Stadt Jingzhou, gelegen in der Provinz Handong, ${ }^{9}$ realiter zu verorten sei (Sohu 2017). ${ }^{10}$ Eine weitere doppelte Ebene der Serienkonzeption ergibt sich aus der Art und Weise, wie die Erzählfigur des geständigen Sünders eingesetzt wird. Bei Razzien werden Korruptionsverdächtige gezeigt, die in geduckter Haltung vor den Ermittlern fliehen, bisweilen auch kriechend, bevor sie unter der Last der kraftvoll vorgetragenen Anklage scheinbar kollabieren. Derartige Vorführungen sind dem chinesischen Fernsehzuschauer bereits aus der Realität bekannt, in der das Staatsfernsehen vermeintliche Geständnisse von Verdächtigen in realen Strafsachen dokumentiert. ${ }^{11}$ Dem realen TV-Pranger wird so ein fiktionaler beiseitegestellt, wobei der Serienkontext keinen Zweifel an der Schuld der Angeklagten lässt. Dieses Wechselspiel von Fiktion und Realität ist somit geeignet, die Glaubwürdigkeit der im Staatsfernsehen ausgestrahlten Geständnisse von Partei-Kadern, die der Korruption beschuldigt werden, für den Zuschauer sublim zu untermauern.

\section{CONCLUSIO}

Mit der Übernahme der Amtsgeschäfte durch die fünfte Führungsgeneration um Xi Jinping sind in China Reformprozesse in Gang gesetzt worden, aus denen der politischen Führung signifikanter Legitimierungsbedarf erwachsen ist. Beim Werben um strategische Mehrheiten für diesen partiell durchaus radikalen Reformkurs setzt die Parteiführung neben den altetablierten Kanälen - Printmedien, Staatsfernsehen, Online-Portalen - auf ein sehr sublimes Medium der Perzeptions- und Emotionssteuerung: Polit-Serien. Dabei wird die Begeisterung der chinesischen Massen für Blockbuster wie die US-amerikanische Serie House of

9 In der Romanvorlage als Jingzhou in der Provinz „H“ (im Original mit dem lateinischen Buchstaben abgekürzt) angegeben (Zhou 2017: 1). Durch den lateinischen Buchstaben in einem chinesischen Text springt dem Leser die Anonymisierung und Verfremdung von Handlungsort und Personen direkt mit dem Eröffnungssatz ins Auge. Suggeriert wird damit eine investigative Abhandlung der politischen Gegenwart, die aus politischen Gründen abstrahiert wird.

10 Ein Eintrag auf Baidu (chinesisches Pendant zu Wikipedia) notiert, dass sich hinter Handong die Provinz Jiangsu verbergen könnte. Andere Kommentare sehen hier eine Verdichtung von Korruptionsfällen, die sich in verschiedenen Provinzen ereignet haben, und interpretieren die Provinz Handong daher als abstrakten Platzhalter.

11 Beispiele für diese Praxis und Tendenzen in der öffentlichen Rezeption finden sich bei BBC (2016). 
Cards gezielt einkalkuliert, um die diffuse Folgebereitschaft und Sympathie für das chinesische Ein-Parteien-System zu erhöhen.

Dabei ist das Phänomen House of Cards in China weit mehr als ein in Kreisen der urbanen Mittelschicht konsumiertes Unterhaltungsprogramm. Gerade in autoritären Systemen erfolgt in Literatur und Film ein Spiel mit Allegorien und Parabeln immer dann, wenn es darum geht, unter Umgehung der Zensur und Sanktionierung eine kritische Aufarbeitung historischer Entwicklungen und politischer Systemstrukturen vorzunehmen. Die chinesische Staatspropaganda instrumentalisiert diese Rezeptionsprozesse, indem der offizielle Politdiskurs der VR China so gelenkt wird, dass die in House of Cards skizzierten Schattenseiten des politischen Machtkampfs als Beleg für die generelle Dekadenz und Dysfunktionalität der westlichen Demokratie fungieren. Das chinesische System hingegen wird über Im Namen des Volkes in Abgrenzung von demokratischpluralistischen Systemen zur erfolgreichen Meritokratie stilisiert. Über die chinesische Polit-Serie werden Deutungsnarrativen angeboten, wird eine offizielle Leseanleitung der Antikorruptionskampagne und der Reformpolitik der fünften Führungsgeneration vorgelegt. Diese gemeinsamen Referenznarrative dienen der Generierung einer gemeinsamen ,chinesischen“ Identität und der Stärkung des Legitimitätsglaubens der Bevölkerung. Über Polit-Serien lässt sich dies sehr viel effektiver und nachhaltiger erzielen als über das klassische Format der staatlichen Printmedien.

\section{LITERATUR}

BBC (2016): How seriously do Chinese take ,confession“ videos?, in: BBC Online vom 2.8., http://www.bbc.com/news/world-asia-china-36882956 (zugegriffen am 25.1.2018).

CNN (2017): Trump: We have a rigged system, in: CNN Online vom 9.12., http://edition.cnn.com/videos/politics/2017/12/09/trump-rally-pensacola-riggedsystem-sot.cnn/video/playlists/president-donald-trump/ (zugegriffen am 22.1. 2018).

Dörner, Andreas (2016): Politserien: Unterhaltsame Blicke auf die Hinterbühnen der Politik, in: Aus Politik und Zeitgeschichte (51), S. 4-11.

He, Zengke (2000): Corruption and Anti-Corruption in Reform China, in: Communist and Post-Communist Studies 33 (2), S. 243-270.

Heberer, Thomas (2014): China in 2013: The Chinese Dream's Domestic and Foreign Policy Shifts, in: Asian Survey 54 (1), S. 113-128.

Lam, Willy Wo-Lap (2015): Chinese Politics in the Era of Xi Jinping, London/New York. 
Li, Cheng (2012): The End of the CCP's Resilient Authoritarianism? A Tripartite Assessment of Shifting Power in China, in: The China Quarterly 211, S. $595-623$.

Li, Cheng (2016): Chinese Politics in the Xi Jinping Era: Reassessing Collective Leadership, Washington.

Li, Keqiang (2017): Report on the Work of the government. Delivered at the Fifth Session of the 12th National People's Congress of the People's Republic of China on March 5, 2017, in: http://en.people.cn/n3/2017/0306/c900009186379.html (zugegriffen am 17.1.2018).

Nathan, Andrew (2003): Authoritarian Resilience, in: Journal of Democracy 14 (1), S. 6-17.

Naughton, Barry (2014): After the Third Plenum: Economic Reform Revival Moves toward Implementation, in: China Leadership Monitor 43, https://www.hoover.org/research/after-third-plenum-economic-reformrevival-moves-toward-implementation (zugegriffen am 18.1.2018).

New York Times (2012): Billions in Hidden Riches for Family of Chinese Leader, in: New York Times Online vom 25.10., http://www.nytimes.com/2012/10/26/ business/global/family-of-wen-jiabao-holds-a-hidden-fortune-in-china.html (zugegriffen am 15.1.2018).

New York Times (2018): Russian Hacking and Influence in the U. S. Election, in: New York Times Online vom 25.1., https://www.nytimes.com/newsevent/russian-election-hacking (zugegriffen am 25.1.2018).

Noesselt, Nele (2014): Alexis de Tocqueville in China: Spiegeldebatten über Reformbedarf und Revolutionsgefahr, in: Leviathan 42 (3), S. 346-362.

Noesselt, Nele/Senghaas, Dieter (2016): Neue Normalität (xin changtai) und ReKonfuzianisierung des chinesischen Marxismus: Die VR China auf der Suche nach einem neuen Konzept politischer Herrschaft, in: Leviathan 44 (4), S. $558-577$

Peerenboom, Randall (2002): China's Long March toward Rule of Law, New York.

Pei, Minxin (2012): Is CCP Rule Fragile or Resilient?, in: Journal of Democracy 23 (1), S. 27-41.

Renmin Ribao Pinglunbu (2015): Xi Jinping Yongdian (Kompilation der Zitate Xi Jinpings aus den chinesischen Klassikern), Beijing.

Renmin Ribao (2016): Xi Jinping Zhongjiwei jianghua: Fan fu bu shi „Zhipai wu“ (Rede des Generalsekretärs Xi Jinping: Der Kampf gegen die Korruption (in China) ist kein „House of Cards“), in: People's Daily Online vom 12.1., http://news.sina.com.cn/c/nd/2016-05-03/doc-ifxrtztc3157086.shtml (zugegriffen am 18.1.2018). 
Seppänen, Samuli (2016): Ideological Conflict and the Rule of Law in Contemporary China: Useful Paradoxes, Cambridge.

Sohu (2017): Renmin de mingyi li Handong sheng Jingzhou shi shi nali? (Wo liegt die Stadt Jingzhou der Provinz Handong aus Im Namen des Volkes?), in: http://www.sohu.com/a/133135560_248541 (zugegriffen am 20.1.2018).

Teets, Jessica C./Hurst, William J. (2015): Introduction: The Politics and Patterns of Policy Diffusion in China, in: Teets, Jessica/Hurst, William (Hrsg.): Local Governance Innovation in China: Experimentation, Diffusion, and Defiance, London/New York, S. 1-24.

Tong, Yanqi/Lei, Shaohua (2014): Social Protest in Contemporary China: 20032010, London/New York.

Wang, Yiwei (2008): Public Diplomacy and the Rise of Chinese Soft Power, in: The Annals of the American Academy of Political and Social Science 616 (2), S. 257-273.

Womack, Brantly (2005): Democracy and the Governing Party: A Theoretical Perspective, in: Journal of Chinese Political Science 10 (1), S. 23-42.

Xinhua (2014), Xi Jinping shouci xitong chanshu xin changtai (Xi Jinpings erste systematische Darlegung zur „Neuen Normalität“), in: Xinhua Online vom 9.11., http://news.xinhuanet.com/world/2014-11/09/c_1113175964.htm (zugegriffen am 10.12.2017).

Xinhua (2017): Highlights of Xi's report to 19th CPC National Congress, in: Xinhua Online vom 17.10., http://news.xinhuanet.com/english/2017-10/18/c_136688994. htm (zugegriffen am 18.1.2018).

Zhang, Yongjin (2016): China and liberal hierarchies in global international society: power and negotiation for normative change, in: International Affairs 92 (4), S. 795-816.

Zhao, Suisheng (2017): Whither the China Model: Revisiting the Debate, in: Journal of Contemporary China 26 (103), S. 1-17.

Zheng, Yongnian (2007): De Facto Federalism in China, Singapore.

Zhou, Meisen (2017): Renmin de Mingyi (In the Name of the People), Beijing. 



\title{
Sind Drachen, Zombies und Aliens politisch?!
}

\author{
Das Politische in der Phantastik \\ am Beispiel der SF-Serie The Expanse
}

\section{Arne Sönnichsen}

\section{EINLEITUNG}

In den vergangenen Jahren sind Fernsehserien vom verschrienen Massenmedium zum intellektuellen Qualitätsmedium avanciert, welches insbesondere mit seinem gritty realism (engl. schmutzigen Realismus) zum Einschalten animiert (Dellwing/Harbusch 2015: 8-11). Mit Politserien wie House of Cards oder The West Wing hat sich ein Serienkosmos etabliert, bei dem durch Fernsehserien das Innenleben von Politik durchleuchtet und auf unterhaltsame Weise dargestellt wird. Die Serien sind keine Abbildung der Realität, sondern Fiktionen, doch sind sie zugleich realistisch, das heißt so oder so ähnlich könnten die Erzählungen in der realen Welt stattfinden. Während eine Vielzahl von Beiträgen in diesem Band solche (Polit-)Serien behandelt, widmet sich dieser Beitrag der Beleuchtung von Serien, welche auf den ersten Blick wenig mit alltäglicher Politik zu tun haben, die Rede ist von ,phantastischen' Serien.

Phantastische Formate sind vielfältig und es ist nicht verwunderlich, dass weder eine einheitliche Definition noch eine Übereinstimmung hinsichtlich einer Nomenklatur existiert. ,Fantastik', ,Phantastik“ oder ,speculative fiction" - es lässt sich fast alles subsummieren, was nur den Anstrich des Unmöglichen trägt. ${ }^{1}$ Für die Science-Fiction (SF) behauptet Jutta Weldes: „,W]e all know SF when we see it, there exists no standard, generally accepted definition of it" (2003: 8). Neben ihrer inhärenten Fiktionalität lässt sich Phantastik - so der Schluss - intuitiv durch die Identifikation des Unrealistischen, durch das, was diese phantas-

1 Siehe auch Brittnacher/May (2013). 
tische Welt in der Realität unmöglich macht, erkennen. So ist die heute betriebene Raumfahrt weit davon entfernt, andere Sterne zu bereisen, bisher gab es (noch?) keinen Kontakt mit Aliens und feuerspeiende Drachen oder Zombies sind - zum Glück - Produkte der Fantasie. Taugen phantastische Unterhaltungsformate trotz oder gar wegen ihrer Unmöglichkeit zur Diskussion von Politik und dem Politischen?

Der Beitrag untersucht, wie Politik und das Politische in phantastischen Fernsehserien verhandelt werden. Die These des Beitrags ist, dass phantastische Serien durch einen radikalen Weltenbau die Realität so stark verfremden, dass sie die Möglichkeit gewinnen, allegorisch und weitreichend politische Grundfragen zu verhandeln. Anhand der Science-Fiction-Serie The Expanse (eine erstmals 2015 auf Syfy ausgestrahlte US-amerikanische Serie nach der Idee von Mark Fergus und Hawk Ostby) möchte der Beitrag anregen, ausgewählte phantastische Serien als politische Serien zu verstehen.

\section{FILMPOLITOLOGIE UND POPULÄRKULTUR}

Populärkulturelle Erzeugnisse wie Filme sind bereits seit einiger Zeit auf der Agenda von Forschung und Lehre der politikwissenschaftlichen Teildisziplin der Internationalen Beziehungen. In der angelsächsischen Debatte wird dies unter dem Begriff Popular Culture and World Politics (PCWP) zusammengefasst ${ }^{2}$, die deutsche Debatte bezeichnet diese Auseinandersetzungen als Filmpolitologie. ${ }^{3}$

Eine Grundannahme ist jeweils, dass politische Texte und populärkulturelle Texte vereinbar sind. Zwar folgen beide Textarten unterschiedlichen Regeln der Textproduktion, doch beschreiben beide die Realität, nur auf eine jeweils andere Art und Weise. In einem poststrukturalistischen Sinne können sie als kommunikative Handlungen, als „Intertext“ (Weldes 2003: 13-16), verstanden werden, wobei politische Texte eine „first-order representation“, die Populärkultur eine „second-order representation“ der Realität ist (Neumann 2001: 603-604). Populärkultur lässt sich als „Spiegel“, als pädagogisches Tool zur Veranschaulichung politikwissenschaftlicher Konzepte (Carpenter 2016: 54-55, Neumann/Nexon 2006: 13-15), als „Data“ und Beleg für die Existenz bestimmter Diskurse in der Gesellschaft (Carpenter 2016: 55, Neumann/Nexon 2006: 13-14) und als erklä-

2 Für einen Überblick siehe den Sammelband von Caso/Hamilton (2015).

3 Für eine Übersicht über die filmpolitologische Debatte siehe den Beitrag Göler/Zech (2017). 
rende Variable zum Verständnis sozialer und politischer Phänomene untersuchen (Carpenter 2016: 54-55, Neumann/Nexon 2006: 13-15).

Kulturbegrifflich lehnt sich die Debatte an die cultural studies an: „Culture is [...] composed of potentially contested codes and representations; it designates a field on which battles over meaning are fought" (Weldes 2003: 6). Anders formuliert bezeichnet Kultur die von Individuen gedeutete, politische Realität, in der manche Dinge sagbar und in der Folge machbar sind und andere nicht (Dörner/Vogt 2012: 14). Medienkulturelle Artefakte und Prozesse sind deshalb relevant, weil sie - egal ob kritisch oder unkritisch - auf das Wirken von Macht hindeuten (Weldes 2003: 6-7). Die (politische) Kultur kann verstanden werden als „Software“, die innerhalb einer polit-institutionellen „Hardware“ operiert (Dörner/Vogt 2012: 14-15). Weil die politische Kultur instrumentell zur Erfüllung politischer Ziele genutzt werden kann und weil Machtbeziehungen verdeckte Wirkungen entfalten, sind kulturelle Artefakte und Praktiken relevant, um einen Zugang zum Denken über Politik zu erlangen: [D]as „Nachdenken über Politik [...] muss offen [sein] für das [...], was von wem auch immer zum Verständnis von Politik einer Zeit beigetragen wird“"(Ottmann 2001: V).

\section{THEORIE: POLITIK UND PHANTASTIK}

\section{Politik und das Politische}

Die Politikwissenschaft hat eine kaum zu überblickende Zahl von Definitionsversuchen für den Begriff ,Politik‘ hervorgebracht. Eine von zwei bekannte Definitionen stammt von David Easton, nach welcher Politik auf die Verteilung von Werten innerhalb einer (unhinterfragten) Ordnung reduziert wird: „the authoritative allocation of values for the society“ (Easton 1953: 128). Eine Definition von Harold Lasswell fokussiert auf den Einfluss von Akteuren: „The study of politics is the study of influence and the influential“ (Lasswell 1936: 295). Diese Grundannahme diente in abgewandelter Form auch als Titel seines Buches: „Politics is the study of who gets what, when, and how." Damit geht es nicht nur um Ordnung und Werteverteilung, sondern auch um Machtbeziehungen im Sinne Max Webers (Weber 1980 [1922]: 28). Beide Definitionen sind jedoch technisch, grundsätzliche Fragen, wie die Ordnung einer Gesellschaft überhaupt aussehen soll, bleiben ausgeklammert.

Seit den 1980er Jahren wird eine lebhafte Debatte um das Andere von Politik, um das Politische, geführt, wobei die Wurzeln der Debatte noch weiter zurückreichen (Bedorf 2010: 13-16). Bedorf bezeichnet das Politische auch als „politische Differenz“ und ordnet die Debatten nach fünf Großbegriffen, die das 
Politische von der Politik abgrenzen: ,Norm‘ beschreibt nach Hannah Arendt den Maßstab, an dem Politik gemessen wird. ,Hegemonie“ baut auf dem Antagonismus-Konzept von Carl Schmitt und Chantal Mouffe auf und versteht das Politische als Prozess der Identifikation und Abgrenzung eines ,Wir' und eines ,Sie‘. ,Unterbrechung' ist in Anlehnung an Jacques Rancière und Alain Badiou das Hervortreten von Kontingenzerfahrungen, von Momenten, die den Status quo von Politik unterbrechen. ,Stiftung' ist der Prozess der Wahrnehmungsordnung und Inszenierung nach Claude Lefort. ,Sozialität‘ findet sich bei Philippe Lacoue-Labarthe und Jean-Luc Nancy wieder und ist bezogen auf das Mit-Sein mit Anderen einer Gesellschaft (Bedorf 2010: 16-32). Gemein ist diesen Debatten, dass sie sich einer sektoralen Zuordnung, etwa zur Ökonomie oder zur Wissenschaft, entziehen. Damit geht jedoch keine Beliebigkeit des Politischen gemäß der Devise , alles ist politisch“ einher. Das Politische tritt stattdessen dann hervor, wenn der Status quo von Politik - die Beziehungen zwischen dem ,Wir` und dem ,Sie " und die Kontingenz von Politik - hinterfragt und neu ausgehandelt wird. Dieser Moment der Kontingenz kann dabei im Großen, in revolutionären Umwälzungen wie der Französischen Revolution stattfinden. Rancière sieht die Kontingenz von Politik und dem Politischen aber gerade auch im Kleinen wirken, oder, wie Bedorf schreibt: „Politisches [existiert] genau dann, wenn die Ordnung der Herrschaft und der Verteilungsprozesse unterbrochen und die zugrundeliegende Kontingenz dieser Ordnung und ihrer Verteilungen offengelegt wird" (Bedorf 2010: 25).

Zusammenfassend ist das Politische nicht nur die normative Bewertung von Politik, sie akzentuiert auch eine Unterscheidung von ,Wir' und ,Sie', leistet Sinnstiftung und Sozialität und hebt die Kontingenz von Politik - im Großen und im Kleinen - hervor und macht diese erfahrbar. Sie schafft einen Möglichkeitsraum, politische Grundfragen erneut zu stellen und die Organisation von Gesellschaft, die Politik und damit die Verteilung von Gütern und das Wirken von Macht, zu aktualisieren.

Zurzeit populäre Polit-Serien wie House of Cards oder The West Wing - also Serien, die primär das Wirken eines oder mehrerer Politiker zeigen - stellen oftmals nicht Fragen des Politischen, sondern stellen das Prozessuale der Politik in den Mittelpunkt (siehe den Beitrag von Henrik Schillinger in diesem Band sowie Dörner (2016: 6-7)). Die Inhalte sind fast egal - der Schauwert entsteht dadurch, dass dem Politiker über die Schulter geschaut wird, wie er sein oder ihr Ziel auf eine idealpolitische, eine realpolitische oder eine machtpolitische Weise erreicht oder daran scheitert (Dörner 2016: 7-10). Wenn die Polit-Serien also schon nicht politisch sind, wie kann die Phantastik politisch sein? 


\section{Phantastik und Kontingenz}

Nach dem Literaturtheoretiker Darko Suvin (1979) unterscheidet sich phantastische Literatur von realistischer Literatur dadurch, dass die empirisch erfahrbare Realität und Umwelt des Produzierenden - die „Nullwelt“ (Suvin 1979: 31) durch ein für die Handlung relevantes, zeithistorisch phantastisches „Novum“ verfremdet wird (Suvin 1979: 93, Steinmüller 1995: 16, Schröder 1998: 17). Fantasy, Märchen und Mythen sind „metaphysisch“ (Suvin 1979: 31) - das Novum ist hier fast ausschließlich unwissenschaftlich, magisch oder unmöglich begründet und ignoriert die physikalischen Gesetze der ,Nullwelt‘. Drachen, Orks, Monster und Magie können Teil der Handlung sein, etwa wie bei Herr der Ringe oder Harry Potter, sind in der ,Nullwelt" aber schlicht unmöglich. Im Horror zieht ebenfalls etwas Unmögliches ein, etwa ein Monster, auf das die Figuren mit Verunsicherung, Angst oder Grauen reagieren. In der Science-Fiction ist das Novum naturwissenschaftlich begründet und steht nicht immer im Einklang, aber zumindest nicht in offenem Widerspruch zu physikalischen Gesetzen der ,Nullwelt‘. Durch die Galaxis zu reisen wie das Raumschiff Enterprise in Star Trek ist heute unmöglich, doch ist ein ,Warpantrieb“ theoretisch ebenso denkbar wie auch die dargestellte liberale Utopie oder der Kontakt mit Außerirdischen.

Die vielen Spielarten der Phantastik wie Utopien, Dystopien, kontrafaktische Geschichten, (Post-)Apokalypse, Superhelden und etliche Grenzgänger zwischen Science-Fiction, Horror und Fantasy offenbaren, dass eine spezifische Genrebeschreibung der Phantastik scheitern muss. Anders als bspw. Krimis zeichnet sich das Phantastische weniger durch typische Handlungsmuster als vielmehr durch Topoi aus, die auch Weldes meint, wenn sie attestiert, dass Rezipienten intuitiv das Phantastische erkennen. Beispielsweise finden sich in der Anthologie-Serie Black Mirror die Figuren in Realitäten vor, in denen technische Trends von Digitalisierung und Social Media in bester Science-Fiction-Manier in die Zukunft extrapoliert werden. Zunehmend stehen die Figuren der Technik hilflos gegenüber, die Handlung wird dann von Elementen der Dystopie, der Satire, des Horrors und des Thrillers dominiert. Star Wars mit seiner Welt voller Raumschiffe, Roboter und Aliens sieht aus wie Science-Fiction, der ikonische Lauftext „Es war einmal vor langer Zeit in einer weit, weit entfernten Galaxis..." ist dem Märchen entlehnt, und angelegt ist es als Heldenepos mit der Macht als QuasiMagie. Das verdeutlicht, dass eine spezifische Genrebeschreibung der Phantastik scheitern muss.

Der Umfang des Novums und damit die Verfremdung der ,Nullwelt' variieren stark und ermöglichen einen Zugriff auf politische Kontingenz. Möglich werden ganze Weltenentwürfe mit eigenen Mythologien, Kreaturen, Gesell- 
schaftsordnungen und Gimmicks - etwa wie in Star Wars oder in Herr der Ringe - oder auch nur subtile Veränderungen. So unterscheidet sich die Welt in der schwedischen Serie Äkta Människör (schwed. für ,Echte Menschen', Real Humans) von der Realität lediglich dadurch, dass Androiden, genannt Hubots, existieren. Gerade Alternativwelt-Geschichten bringen politische Kontingenz auf die Spitze, wenn die ,Nullwelt“ als ihrerseits kontingent entlarvt wird. In den Serien SS-GB und The Man in the High Castle haben das nationalsozialistische Deutschland bzw. die Achsenmächte gewonnen. Beide Handlungen befassen sich daraufhin mit der Frage, wie Widerstand und Kollaboration gegen ein totalitär-faschistisches Regime möglich sind. In der Erfindung einer neuen Welt macht die Phantastik Kontingenz plastisch. Eine Revolution kann bereits stattgefunden haben, oder sie ist im Begriff, sich zu entfalten. Handlungstreibend ist dann meistens der Umstand, dass die Figuren gezwungen werden, ihre Kontingenz zu nutzen.

\section{Phantastik und die ,fantastisch Anderen‘}

Der Kontakt des ,Wir' mit den ,(fantastisch) Anderen“ ist der wohl ursprünglichste Topos phantastischer Erzählungen. Regelmäßig treffen Protagonisten auf ,Andere", die sich mehr als einmal als Monster im Sinne einer Verletzung natürlicher oder gesellschaftlicher Gesetze (Parr/Foucault 2013: 3) entlarven lassen. Schmitz-Emans argumentiert, dass in Monstren und dem Monströsen verschiedene Menschenentwürfe abgebildet werden, die aber letztliche Abgrenzungsversuche verhindern, denn in der Spiegelung des Monsters zeigt sich der monströse Facettenreichtum des Menschen selbst (Schmitz-Emans 2013: 17). Die Protagonisten treffen also nicht auf wirklich, Andere', sondern auf Menschen, die nur auf den ersten Blick wie Monster aussehen. Diese ,Anderen' sind sozialwissenschaftlich interessant, denn durch sie wird ein politischer Antagonismus verhandelt; sie ermöglichen das Zur-Sprache-Bringen von Themen, die sonst unsagbar sind:

„[...] fantastische Konstruktionen lösen die vermeintliche Unausweichbarkeit einer bedrängenden Realität nicht nur auf und nehmen ihr damit die Gefährlichkeit. Der Deckmantel $[\ldots]$ besteht aus einer offenen Darstellung des Anderen, gegen das sich abgegrenzt wird, als fantastisch und erfunden. Das wiederum erlaubt es, diesen Anderen in einem Licht darzustellen, der risikoloser und deutlicher sein kann, als eine direktere Darstellung eines Anderen das sein könnte, der von Zuschauerinnen als ,real existierender Anderer ‘ dargestellt wird.“ (Dellwing/Harbusch 2015: 14-15) 
An einem Serienbeispiel verdeutlicht, lassen sich die Hubots in Real Humans sowohl als Auseinandersetzung lesen, wie eine Gesellschaft auf eine neue Technik reagiert und diese - wenn sie menschenähnlich ist - behandelt. Die Hubots sind intentional aber auch so angelegt, dass sie als ,Andere“ im Sinne von Immigranten gelesen werden können und abbilden, wie eine Gesellschaft auf das ihr Fremde reagiert und mit dem möglichen Verlust tradierter Werte und Identitäten umgeht (Hamrell 2017: 99).

Phantastische Formate umgehen damit die Fallstricke des politisch Sagbaren und erzeugen eine ,ideologische Elastizität“ (Saunders 2015a: 152), die Aussagen über Personengruppen, Kulturen oder Länder ermöglicht, ohne Stereotype und Ressentiments zu schüren. Die Anwesenheit phantastischer Elemente betont zusätzlich die Fiktionalität einer Serie und verschiebt die Handlung in eine hypothetisches ,what-if*, welches die Gefahr der Verwechslung mit der Realität verhindert (Besand 2016: 6.30-8.30). Realistische Unterhaltungsformate riefen in der Vergangenheit nicht nur harsche Kritik hervor, etwa die stereotype Darstellung des Nahen Ostens in den Serien The West Wing (Dellwing/Harbusch 2015: 15) und Homeland (Saunders 2015b). Untersuchungen zeigen auch, dass populärkulturelle Erzählungen und Erzeugnisse durchaus einen Einfluss auf politische Einstellungen von Rezipienten nehmen (Furman/Musgrave 2017).

Auch andere Serien nutzen Phantastik und die ,fantastisch Anderen', um über verschiedene gesellschaftliche Probleme nachzudenken. Beispielsweise reflektierte Star Trek oft gesellschaftliche Probleme (Sönnichsen 2016) oder nutzte den Serienkontext als philosophischen Diskursraum, als „casuistry“ (Lewis 2016). In der Zombieserie The Walking Dead lassen sich die Staffeln als Auseinandersetzung um die Mechanismen und die Legitimation unterschiedlicher, politischer Ordnungsmuster lesen (Besand 2016, Loyola 2015). In der SF-Serie Battlestar Galactica werden bewusst Diskurse, die nach 9/11 in den USA geführt wurden, reflektiert (Neumann/Kiersey 2013). Diese Beispiele werden auch immer wieder als Beleg für die sozialkritische Aussagekraft der SF herangezogen (Weldes 2003: 14, Grayson et al. 2009: 158, Carpenter 2016). Dass die Intention, Sozialkritik zu äußern, aber kein entscheidendes Merkmal ist, beweist hingegen die Serie Game of Thrones, die in einer mittelalterlichen Fantasywelt den Krieg adeliger Häuser um den Königsthron erzählt. Die Serie wurde als Allegorie für den Klimawandel gelesen, obwohl dies von dem Autor der Buchreihe, George R. R. Martin, nie intendiert war:

„Like Tolkien I do not write allegory, at least not intentionally. Obviously you live in the world and you're affected by the world around you, so some things sink in on some level, 
but, if I really wanted to write about climate change in the 21 st century I'd write a novel about climate change in the 21st century.“ (Martin, zit. nach Hughes 2013)

Zusammenfassend werden in der Phantastik neue Welten geschaffen, die anders sind als die reale Welt, die ,Nullwelt", die jedoch naturwissenschaftlich oder metaphysisch rückgebunden bleibt. Durch die Einführung eines Novums wird die Welt gestört und die Figuren werden gezwungen, Stellung zu beziehen. In dieser Reaktion ergibt sich die Option - intentional oder nicht -, Fragen des Politischen, die Kontingenz des Politischen und das Verhältnis eines ,Wir ‘ und eines ,Sie ${ }^{\text {- }}$ (neu) zu verhandeln. Durch die Verfremdung der Welt wirkt die Phantastik als kontrafaktisches ,what-if*, als Sozialkritik, ohne Ressentiments zu schüren oder Rezipienten ein verfälschtes Bild der Wirklichkeit einzuimpfen. Die Phantastik avanciert, in der Tradition der politischen Utopie (Schölderle 2011), zur „social fiction“ (Schröder 1998: 60-73) .

\section{FALLBEISPIEL: THE EXPANSE}

Die Serie The Expanse, die auf der erfolgreichen Buchreihe gleichen Namens des Autorenduos Daniel Abraham und Ty Franck (unter dem Pseudonym James S.A. Corey) basiert, spielt in einem von der Menschheit besiedelten Sonnensystem der Zukunft. Der Mars, verschiedene Kleinstplaneten, Asteroiden, Monde und Raumstationen sind besiedelt. Die Erde ist unter Führung der UN die vorherrschende Großmacht. Der Mars hat sich als Martian Congressional Republic unabhängig erklärt und ein Militär aufgebaut, welches die Vormachtstellung der Erde herausfordert. Die Menschen im und jenseits des Asteroidengürtels, der einfach nur Belt und dessen Bewohner Belter genannt werden, werden von Erde und Mars ausgebeutet. Innerhalb der Handlung werden drei Themenkomplexe angesprochen, die überdeutlich mit gegenwärtigen politischen Themen der internationalen Politik korrelieren, weshalb der Politikprofessor Daniel Drezner The Expanse eine der brisantesten Serien über internationale Politik nannte (Drezner 2016).

Das erste Thema der Serie betrifft die Beziehungen zwischen der Erde und deren Herausforderer, dem Mars. Diese sind geprägt von Feindseligkeiten und dem Streben, die jeweils andere Partei auszustechen. Erde und Mars sind auch bereit, zu militärischen Mitteln zu greifen um ihre Interessen durchzusetzen. $\mathrm{Zu}$

4 Schon Isaac Asimov nannte seine Geschichten „social science fiction“ (1979) und auch Franke nennt Science Fiction „social fiction“ (1984), während einige Autoren ihre Werke als „speculative fiction“ (Nicholls/Langford 2015) bezeichnen. 
Beginn der zweiten Staffel halten das Erd-Kriegsschiff Nathan Hale und das Mars-Kriegsschiff Scirocco auf den Asteroiden Phoebe zu. Auf der Scirocco unterhält sich der Kommandant Lieutenant Sutton mit der Marinerin Bobbie Draper, die ihre Unzufriedenheit über den Kalten Krieg zwischen Erde und Mars ausdrückt:

Szenen-Beispiel: S 2/F 1/17.21 - 17.59

Sutton: [...] I was a private during the Vesta blockade. Half the Earth's fleet was headed to Mars, to annihilate its former colony. If they couldn't have what we built, they were going to pound it back into dust. We went to bed every night thinking that we would wake in nuclear fire. Luckily for everyone, cooler heads prevailed.

Draper: We all grew up with that story.

Sutton: Because it was a warning. We've managed to avoid a shooting war with Earth up till now. That's our job. To make sure that war never happens.

Draper: And because of Vesta we pushed back the terraforming project. Fifty years and fifty more. All those resources to the military. Now, none of us will live to see an atmosphere over Mars.

Das Setting scheint einem neorealistischen Lehrbuch der Internationalen Beziehungen entsprungen zu sein. ${ }^{5}$ Beide Fraktionen sind relativ gleichstark. Ein verhältnismäßig stabiles geostrategisches System hat sich herausgebildet. Weil keine von beiden Fraktionen antizipieren kann, was die jeweils andere vorhat, neigen beide zur Aufrüstung, die ihr Überleben und die Durchsetzung ihrer Interessen sicherstellen soll. Dadurch entsteht jedoch ein typisches Sicherheitsdilemma, welches zu einer Rüstungsspirale und zu höchst volatilen Beziehungen führt. Neutrale und ausgleichende Institutionen oder Organisationen, die das Wettrennen eindämmen könnten, gibt es nicht, der Weltraum ist archetypisch anarchisch. Lohnenswerte Projekte, wie das ,Terraformingprojekt‘ des Mars, sind auf Eis gelegt, und auch wenn beide Fraktionen im Rahmen verschiedener Projekte kooperieren, wird die Volatilität der Beziehungen überdeutlich, als es mehrfach zu militärischen Zusammenstößen kommt. Als die zuvor genannte Scirocco Torpedos abfeuert, um den Asteroiden Phoebe zu zerstören, vermuten UN-Politiker zunächst einen Erstschlag (S 2/F 1). Die Provokation der Marsbewohner beantwortet die UN mit der Zerstörung des Marsmondes Deimos (S 2/F 3). Über dem Jupitermond Ganymede entfaltet sich kurz darauf ein militärischer Schlagabtausch zwischen Erde und Mars (S 2/F 6). Ein systemweiter Krieg wird nur

5 Zur Einführung siehe Masala (2017). 
durch einen äußerst unterkühlten Friedensgipfel verhindert, der abermals die schlechten Beziehungen hervorhebt (S 2/F 6 \& F 7). Die Auswirkungen des Konflikts - die schweren Schäden auf Ganymede und die Flüchtlingswellen werden nicht ausgespart. The Expanse entwirft damit ein Setting, welches überdeutlich an den Kalten Krieg des 20. Jahrhunderts erinnert und die ganzen Widersprüche - Krieg als Mittel zur Friedenssicherung und zugleich als Auslöser von Konflikten - widerspiegelt.

Vor dieser Hintergrundfolie wird ein weiterer Konflikt entfaltet, nämlich das Verhältnis zwischen den inneren Planeten Erde und Mars und den Beltern, die auf Zwergplaneten wie Ceres, Asteroiden wie Eros, Monden wie dem Jupitermond Ganymede und auf verschiedenen Raumstationen leben. Der Belt wird von Erde und Mars gemeinsam verwaltet und als Ressourcengarant und Sammelbecken für billige Arbeitskräfte ausgenutzt. Zugleich ist der Belt fast vollständig von den inneren Planeten abhängig. Durch die Darstellung der Menschen im Belt als in ihrem Selbstbestimmungsrecht marginalisiert und imperial ausgebeutet wird eine Allegorie für ein koloniales Setting geschaffen, welches in sehr ähnlicher Weise während der weltpolitischen Phase des europäischen Kolonialismus vorherrschte (Conrad 2012). Ganz wie im Vorbild ist die imperiale Herrschaft von Willkür und Gewalt geprägt. In einer Episode fängt ein Patrouillenschiff vom Mars ein kleines Prospektorenschiff ab und konfisziert wegen eines veralteten Transponders und trotz Flehens des Piloten die Ladung. In seiner Verzweiflung versucht der Pilot, das Mars-Schiff durch einen Asteroiden zu zerstören (S $1 / \mathrm{F} 6)$.

Im Belt versucht eine Organisation, die Outer Planet Alliance (kurz OPA), einen Prozess der Dekolonisierung, anzustoßen. In der $O P A$ gibt es radikale terroristische Zellen, aber auch einen gemäßigten Zweig unter der Führung von Fred Johnson, der nach politischer Anerkennung des Belt strebt. Ein wieder anderer Teil der $O P A$, angeführt von Anderson Dawes, sieht keine Möglichkeit für einen Ausgleich zwischen den Inneren und der $O P A$, weist aber auch Mittel des Terrorismus zurück (S 1/F 5). Er sieht nur eine umfassende Revolution als Option, um die strukturell angelegte Ungleichheit und Gewaltordnung zu durchbrechen. Fred Johnson und Anderson Dawes verkörpern die nicht triviale Frage, wer für die $O P A$ und den Belt sprechen darf und welche Richtung Dekolonisationsprozesse nehmen sollten. Diese Debatte nimmt auch Anleihen an reale Dekolonisierungsprozesse (Jansen/Osterhammel 2014: 107-116), die in der Fachdisziplin der Internationalen Beziehungen gespiegelt werden (Castro Varela/Dhawan 2017). Fred Johnson und Anderson Dawes wünschen sich beide die Unabhängigkeit von der imperialistischen Herrschaft der inneren Planeten und Anerkennung des Belt als souveränen Akteurs. Dawes und Johnson stimmen überein, dass thermonukleare Raketen, die 
Fred Johnson der Erde gestohlen hat, in dem volatilen geostrategischen Szenario als Waffen nutzlos, als politische Währung jedoch überaus wertvoll sind (S 2/F 6). Ihre Strategien unterschieden sich jedoch fundamental.

In der zweiten Staffel versucht Fred Johnson die $O P A$ von einer gemeinsamen Strategie zu überzeugen. Weil er von der Erde stammt, wird ihm abgesprochen, die Belange der Belter vertreten zu können. Auch er selbst ist überzeugt, dass nur ein Belter für den Belt sprechen kann. Anderson Dawes misstraut Johnsons Motiven:

\section{Szenen-Beispiel: S 2/F 7/14.56 - 16.31}

Dawes: [...] It's a beautiful dream he has, huh? Earth and Mars at peace. And the belt, equal partners in that peace. But is it too much an Earther's dream? I do not doubt his heart. Fred Johnson has proven himself to be a true friend to the belt. But as much as I fear war between Earth and Mars, I fear the peace more. For that is when they will turn their sights back on all of us. The inners are not like us. Earthers cannot look upon a thing but wonder who it belongs to, huh? To make it their possession. ,Possession is ninetenths of the law", they say. But, that is not the way of the belt. We say, ,the more you share, the more your bowl will be plentiful. ' [...] Earthers, Martians, they see us as their possessions. Animals to test their new weapons on. And, make no mistake, they will do it again. We must protect ourselves against these weapons.

Damit öffnet sich ein drittes großes Thema: Rassismus. Der grundsätzliche Konflikt zwischen den Fraktionen verläuft nicht zwischen Hautfarben oder Geschlechtern. Die Serie bricht mit der indischen UN-Untersekretärin Chrisjen Avasarala, der farbigen Ingenieurin Naomi Nagata und der polynesischen Marinerin Bobbie Draper die üblichen Genderklischees sogar auf (Robinson 2016). Die Konfliktlinien sind stattdessen von stereotypem Rassismus geprägt, von Fraktionszugehörigkeit, Philosophie, Sprache, physiologischen Eigenschaften und Herkunft. Besonders im Belt sind die Ressentiments gegenüber Erde und Mars, abfällig Duster genannt (von engl. dust = Staub in Anspielung auf die Wüstenlandschaft des Mars) stark. Die Abneigung drückt sich durch die Beleidigung welwala aus (das Kunstwort setzt sich aus dem englischen (gravity-)well und lover zusammen), mit der jemand belegt wird, der sich übermäßig mit den inneren Planeten identifiziert oder dem Belt gegenüber illoyal verhält. Obwohl sich Fred Johnson als Belter identifiziert, spricht er doch nicht deren Sprache, das Belterkreol, und weist auch nicht die physiologischen Veränderungen auf, die Beltern das Überleben auf der Erde unmöglich machen und auf der Erde als 
Folterinstrument genutzt werden (S 1/F 1). Auch ikonografisch wird die Unterscheidung hervorgehoben. Terraner kleiden sich ,normal', Marsianer treten fast ausschließlich in Militäruniformen auf. Belter tragen oft ausgefallene Frisuren und Tattoos - beispielsweise das Logo der $O P A$, die Buchstaben $\mathrm{O}$ und $\mathrm{P}$, gefolgt von dem für eine anarchistische Symbolik stilisierten A in einem Kreis was gut zu deren Ablehnung zentraler Autoritäten passt.

Auch bezüglich der inneren Haltung zeigen sich offenkundige Unterschiede. Dawes zufolge dominieren in der Belter-Kultur Pragmatismus und eine Philosophie des Teilens. Marsianer zeichnen sich durch eine kommunitarische Grundhaltung und Zielfokussierung - ,Terraformung' des Mars und Militärdienst aus. Sie blicken auf die Terraner herab, die scheinbar lethargisch den Planeten verschmutzen und auf , basic ' leben - einem bedingungslosen Grundeinkommen, welches nötig ist, weil die UN den 30 Milliarden Menschen keine sinnvollen Jobs vermitteln können (S 2/F 9). UN-Untersekretärin Chrisjen Avasarala ist in dem Dilemma gefangen, einerseits einen systemweiten Krieg verhindern zu wollen, andererseits auf die populistische Lösung „Earth first!“ festgenagelt zu sein. Einer von Bobbies Marinern, Richard Travis, der mit fünf Jahren von der Erde zum Mars migrierte, wird immer wieder angefeindet, weil er von der Erde stammt, und ultimativ als Bauernopfer in den Friedensverhandlungen präsentiert, um einen Krieg zu verhindern (S 2/F 9).

Alle drei Themen lassen die Ambitionen der Menschheit, den Weltraum zu kolonisieren, in einem äußerst pessimistischen Licht erscheinen. Hier schafft sich die Menschheit kein liberales Utopia wie in Star Trek, hier unterdrückt eine Fraktion die andere und ist notfalls auch bereit, einen Krieg zu riskieren, wenn es ihren Interessen dient. Die Serie schreibt somit die Gewaltherrschaften der Menschheit fort und extrapoliert diese in die Zukunft. So fasst eine der Protagonistinnen, die Belterin Naomi Nagata, das Szenario zusammen:

\section{Szenen-Beispiel: S 2/F 13/38.19-38.57:}

Nagata: [...] I don't ever remember a time when Earth, Mars and the belt weren't fighting. The sides change sometimes. What we think we're fighting for. Who we tell ourselves are the good people. But, it just seems we can't ever stop fighting war after war after war. It's part of being human. An ugly part but, I don't think it will ever change. Technology certainly hasn't changed it. Guns. Railguns. Nuclear bombs. No weapon ever brings peace.

Diese grimmige Botschaft hinterlässt eine gewisse Ernüchterung. Doch positiv gewendet kann sie als starke Botschaft dahingehend gelten, dass sich solche $\mathrm{Zu}$ - 
stände nicht wiederholen dürfen und dass reflektiert werden muss, wie stark die heutige Welt von diesen Problemen geprägt ist.

The Expanse ist eine sowohl politische wie auch aktuelle Serie, die gegenwärtige weltpolitische Herausforderungen, vor denen die internationale Gemeinschaft steht, abbildet. Spannungen entstehen hier wie dort nicht nur zwischen Nationen und deren unterschiedlichen Kulturen, sondern auch zwischen jeweils unterschiedlichen Interessen und Fähigkeiten sowie Unfähigkeiten, diese Interessen durchzusetzen. Der Konflikt zwischen den Inneren und dem Belt lässt sich dabei als Allegorie für Dekolonisierungsdiskurse zwischen dem Globalen Norden und dem Globalen Süden interpretieren.

\section{FAZIT}

Auf den ersten Blick scheinen phantastische Serien wenig mit realer Politik zu tun zu haben. Zwar entwerfen auch sie Institutionen, Verfahren und Regeln der Politik, doch wird das Prozessuale nur bedingt in den Mittelpunkt der Handlung gerückt. Stattdessen schaffen phantastische Serien Welten, die allegorisch - ob beabsichtigt oder unbeabsichtigt - Diskussionen über politische Grundfragen anstoßen, etwa was die normative Bewertung von Politik betrifft, das Verhältnis zwischen dem ,Wir' und den ,Anderen', und sie machen politische Kontingenz erfahrbar. Diese ,what-if ${ }^{*}$-Szenarien ermöglichen das Nachdenken über unterschiedliche Themen, vermeiden Stereotype und Ressentiments und unterstreichen die Fiktionalität des Gezeigten. So ermöglichen die Verfremdung der ,Nullwelt', die Einführung eines Novums und die ,fantastisch Anderen' bei einem gleichzeitigen Rückbezug an unsere Welt eine Auseinandersetzung mit sensiblen oder unbekannten Themen auf unterhaltsame Art und Weise. Damit eignen sich diese Serien auch zur Dekonstruktion problematischer Themen.

Durch die Mittel der Phantastik - den Weltenbau und die Kontrastierung verschiedener Fraktionen mittels des ,fantastisch Anderen' - entwirft The Expanse ein glaubwürdiges Szenario politischer Zustände der Zukunft und behandelt dabei relevante Debatten und Positionen, die für die gegenwärtige internationale Politik relevant sind. Am prominentesten vertreten sind das dysfunktionale geostrategische Setting, das ausbeuterische, imperiale Gehabe der inneren Planeten und der Rassismus zwischen den Fraktionen. Damit verschafft die Serie dem Zuschauer einen Zugang zu diesen Diskursen, wobei die konkurrierenden Positionen in einem Ressentiment-enthobenen Setting dargestellt und zur Disposition gestellt werden. The Expanse ist nur ein Beispiel für das Spektrum der Phantastik und der vielen Szenarien, mit Hilfe derer politische Prozesse durchgespielt und politische Fragen gestellt werden. Es wird höchste Zeit, ausgewählte phan- 
tastische Erzählungen und Serien als seriöse politik- und sozialwissenschaftlich relevante Werke zu betrachten, als, social science fiction'.

\section{LITERATUR}

Asimov, Isaac (1979): Social Science Fiction, in: Bretnor, Reginald/Campbell, John W. (Hrsg.) Modern Science Fiction, its Meaning and its Future, 2. Aufl. New York, NY, S. 157-197.

Bedorf, Thomas (2010): Das Politische und die Politik. Konturen einer Differenz, in: Bedorf, Thomas/Röttgers, Kurt (Hrsg.): Das Politische und die Politik, Frankfurt, S. 13-37.

Besand, Anja (2016): Was wir von ZOMBIES lernen können oder: Über politische Grundfragen in der Serie The Walking Dead, https://www.bpb.de/veranstal tungen/dokumentation/238562/politische-grundfragen-in-der-serie-the-walkingdead (zugegriffen am 25.01.2018).

Brittnacher, Hans Richard/May, Markus (Hrsg.) (2013): Phantastik. Ein interdisziplinäres Handbuch, Stuttgart.

Carpenter, Charli (2016): Rethinking the Political/-Science-/Fiction Nexus: Global Policy Making and the Campaign to Stop Killer Robots, in: Perspectives on Politics 14 (1), S. 53-69.

Caso, Frederica/Hamilton, Caitlin (Hrsg.) (2015): Popular Culture and World Politics. Theories, Methods, Pedagogies, Bristol, UK.

Castro Varela, María do Mar/Dhawan, Nikita (2017): Postkoloniale Studien in den IB: Die IB dekolonisieren, in: Sauer, Frank/Masala, Carlo (Hrsg.): Handbuch Internationale Beziehungen, Berlin, S. 233-256.

Conrad, Sebastian (2012): Kolonialismus und Postkolonialismus: Schlüsselbegriffe der aktuellen Debatte, in: APuZ, 62 (44-45). S. 3-9.

Dellwing, Michael/Harbusch, Martin (2015): Vergemeinschaftung in Zeiten der Distinktion: Fantastische Andere und transgressives Fernsehen, in: Dellwing, Michael/Harbusch, Martin (Hrsg.): Vergemeinschaftung in Zeiten der Zombie-Apokalypse. Gesellschaftskonstruktion am fantastisch Anderen, Wiesbaden, S. 7-20.

Dörner, Andreas (2016): Politserien: Unterhaltsame Blicke auf die Hinterbühne der Politik, in: Aus Politik und Zeitgeschichte, 66 (51). S. 4-11.

Dörner, Andreas/Vogt, Ludgera (2012): Unterhaltungskultur als politische Kultur: Politikvermittlung in der Gegenwartsgesellschaft, in: Dörner, Andreas/Vogt, Ludgera (Hrsg.): Unterhaltungsrepublik Deutschland. Medien, Politik und Entertainment, Bonn, S. 11-21. 
Drezner, Daniel W. (2016): The Best Show About International Relations on Television Right Now Is on - Wait for It - Syfy, in: Washington Post http://wapo.st/2obqLBY (zugegriffen am 22.11.2017).

Easton, David (1953): The Political System: An Inquiry into the State of Political Science, New York.

Franke, Herbert, W. (1984): Science Fiction - Denken in Modellen, in: Aus Politik und Zeitgeschichte (B1), S. 29-38.

Furman, Daniel/Musgrave, Paul (2017): Synthetic Experiences: How Popular Culture Matters for Images of International Relations, in: International Studies Quarterly, 61 (1), S. 503-516.

Göler, Daniel/Zech, Lukas (2017): „Füße still“ und „,keine Beunruhigung Zuhause“. Eine filmpolitologische Analyse zur strategischen Kultur Deutschlands, in: Zeitschrift internationale Beziehungen (ZiB), 24 (2), S. 6-35.

Grayson, Kyle/Davies, Matt/Philpott, Simon (2009): Pop Goes IR?, in: Politics 29 (3), S. 155-163.

Hamrell, Harald (2017): Echte Menschen? - Über die Entstehung der Fernsehserie Äkta Människör - Real Humans, in: Jaspers, Kristina/Warnecke, Nils/ Waz, Gerlinde/Zill, Rüdiger (Hrsg.): Future Worlds. Science-Fiction-Film, Berlin, S. 97-107.

Hughes, C. S. (2013): George R. R. Martin - On the End of Game of Thrones, http://nerdalicious.com.au/books/george-r-r-martin-on-the-end-of-thrones/ (zugegriffen am 29.11.2017).

Jansen, Jan C./Osterhammel, Jürgen (2014): Dekolonisation. Das Ende der Imperien, Bonn.

Lasswell, Harold Dwight (1936): Politics: Who Gets What, When, and How, Cleveland

Lewis, Courtland (2016): Casuistry in the Final Frontier, in: Decker, Kevin S./Eberl, Jason T. (Hrsg.) The Ultimate Star Trek and Philosophy. The Search for Socrates, Chichester, S. 138-147.

Loyola, Mario (2015): The Walking Dead's Political Philosophy, http://www. nationalreview.com/article/428043/walking-deads-political-philosophy-mario-lo yola (zugegriffen am: 22.11.2017).

Masala, Carlo (2017): Realismus in den Internationalen Beziehungen, in: Sauer, Frank/Masala, Carlo (Hrsg.): Handbuch Internationale Beziehungen, Berlin, S. 141-175.

Neumann, Iver B. (2001): ,Grab a Phaser, Ambassador': Diplomacy in Star Trek, in: Millennium: Journal of International Studies, 30 (3), S. 603-624.

Neumann, Iver B./Kiersey, Nicholas J. (2013): Battlestar Galactica and International Relations, New York, NY. 
Neumann, Iver B./Nexon, Daniel H. (2006): Introduction: Harry Potter and the Study of World Politics, in: Nexon, Daniel H./Neumann, Iver B. (Hrsg.): Harry Potter and International Relations, Lanham, MD, S. 1-23.

Nicholls, Peter/Langford, David (2015): Speculative Fiction (zugegriffen am 25.01.2018).

Ottmann, Henning (2001): Geschichte des politischen Denkens, Stuttgart u.a.

Parr, Rolf/Foucault, Michel (2013): Die Anormalen, in: Aus Politik und Zeitgeschichte 63 (52), S. 3-10.

Robinson, Tasha (2016): How SyFy's The Expanse Cast its Multiracial Future. Genre TV is Relying on Increasingly Gender-Balanced, Multicultural Casts to Populate Its Far-Flung Worlds, https://www.theverge.com/2016/2/25/1110 3434/syfy-the-expanse-series-diverse-cast (zugegriffen am 29.11.2017).

Saunders, Robert A. (2015a): Imperial Imaginaries: Employing Science Fiction to Talk About Geopolitics, in: Caso, Frederica/Hamilton, Caitlin (Hrsg.): Popular Culture and World Politics.Theories, Methods, Pedagogies, Bristol, UK, S. 149-159.

Saunders, Robert A. (2015b): Homeland's Popular Geopolitics Gets Punked, http://www.e-ir.info/2015/11/24/homelands-popular-geopolitics-getspunked/ (zugegriffen am 24.11.2017).

Schmitz-Emans, Monika (2013): Monster: Eine Einführung, in: Aus Politik und Zeitgeschichte 63 (52), S. 11-17.

Schölderle, Thomas (2011): Utopia und Utopie, Baden-Baden.

Schröder, Torben (1998): Science Fiction als Social Fiction, Münster.

Sönnichsen, Arne (2016): Interstellar Relations? Star Trek als Spiegel USamerikanischer Außenpolitik, in: Zeitschrift für Fantastikforschung 5 (2), S. $22-41$.

Steinmüller, Karlheinz (1995): Gestaltbare Zukünfte. Zukunftsforschung und Science Fiction, Gelsenkirchen.

Suvin, Darko (1979): Poetik der Science Fiction. Zur Theorie und Geschichte einer literarischen Gattung, Frankfurt am Main.

Weber, Max (1980 [1922]): Wirtschaft und Gesellschaft, 5 Aufl. Tübingen.

Weldes, Jutta (2003): Popular Culture, Science Fiction, and World Politics: Exploring Intertextual Relations, in: Weldes, Jutta (Hrsg.): To Seek Out New Worlds.Science Fiction and World Politics, New York, S. 1-30. 
Reflektion \& didaktischer Einsatz 



\title{
Warum eigentlich nicht?
}

\author{
Über die Unmöglichkeit, deutsches Politikmanagement \\ im Fernsehen abzubilden
}

Karl-Rudolf Korte

Man wundert sich. Die Dänen schaffen das - die US-Amerikaner auch. Mit Borgen präsentieren die Dänen ein vorzeigbares Modell einer parlamentarischen Demokratie. Mit House of Cards produzieren die Amerikaner ein versuchtes Abbild ihrer Präsidialdemokratie. Unterschiedliche Regierungssysteme finden nicht nur hohe Resonanz in den TV-Serien. Die Filme bieten auch facettenreiche Empirie des tatsächlichen Politikmanagements. Das gilt natürlich mit großen Einschränkungen. Denn je mehr die Serien über Resonanzmessungen die Konfigurationen, die Themenzuspitzungen und die Dramaturgie an die Wünsche der Zuschauer anpassten, desto mehr verlor auf der Strecke der weiteren Staffeln die politikwissenschaftliche Empirie deutlich an Substanz.

Die wenigen deutschen Versuche, das politische System adäquat im Unterhaltungsformat abzubilden, scheiterten. Das kann man auf die Resonanz der Einschaltquoten ebenso beziehen wie auf die Substanz der Darstellung des politischen Gegenstands. Vielleicht liegt es schlicht an den im Vergleich zum angelsächsischen Markt geringen Honoraren für Drehbuchautoren? Vielleicht fehlen deutsche Schauspieler, die sich eignen oder die sich für solche schwierigen politischen Rollen zur Verfügung stellen? Vielleicht fürchten Schauspieler Karriereoder Reputationsnachteile, wenn sie parteipolitisch im Film zugeordnet werden? Antworten kann ich als Politikwissenschaftler darauf nicht verlässlich geben. Das verbleibt im Bereich der Spekulation. Am Ende sind es sicher viele verschiedene Gründe, warum es schwer erscheint, Berliner Politik im Film zu spielen.

Mein Argumentationsstrang orientiert sich an der spezifischen Komplexität des deutschen Politikmanagements, das deshalb nachfolgend kurz skizziert wird. 
Meine Überlegungen gehen davon aus, dass der Stoff der Politik, das konkrete Entscheidungsmanagement und die Erwartungen der Bürger an ihre Politiker das politische Serien-Genre in Deutschland extrem erschweren. Jede pointierte Vereinfachung, die in den Polit-Serien Einschaltquoten sichert, macht die Geschichten so fiktional, dass die zentralen Mechanismen der Mehrheitsfindung und die Logik der politischen Führung in einer Parteiendemokratie banalisiert werden. Zum Hintergrund deshalb ein paar einführende Erklärungen über die Komplexität des Gegenstandes.

\section{DIE RATIONALITÄT DES POLITISCHEN ENTSCHEIDENS: KOMPLEXES ENTSCHEIDEN}

Wieso entscheidet Politik so, wie sie entscheidet? Warum wird aus der besten Expertise nicht auch ein Gesetz? Politische Rationalität bedeutet immer Sachund Machtfragen zusammenzubringen. Die Nicht-Entscheidung ist der Alltag des Politikers, nicht die machtvolle Entscheidung. Das kann auch kein FilmRegisseur ändern, wenn er an der Authentizität der deutschen Politik interessiert ist. Politiker sind als Tagesintegrationsweltmeister unterwegs. Ihre Macht ist extrem stimmungsflüchtig. Insofern ist es rational, auch Entscheidungen nicht zu treffen. Denn jede Entscheidung polarisiert, was man in einem Integrationsamt verhindern sollte oder zumindest hinausschieben müsste. Dilatorisch zu handeln ist insofern höchst rational, da der Handlungsrahmen immer auf die Legislaturperiode begrenzt bleibt. Unabhängig von diesen Zugängen und Einordnungen ist politisches Entscheiden mit vollkommen neuen Rahmenaspekten konfrontiert, die ein Drehbuch berücksichtigen müsste.

Politisches Entscheiden tritt zudem in eine neue formative Phase. Das hängt mit einer Modernisierung von Instrumenten, Techniken und Stilen des Entscheidens in digitalen Demokratien zusammen. Online-Kontexte verschieben die ZeitDimension des Entscheidens. Noch wirkungsmächtiger auf den Modus des demokratischen Entscheidens sind allerdings die veränderten Zeitläufe, die eine enorme Ereignisdichte mit sich bringen und seit einigen Jahren das Risiko zum Regelfall der Politik machen. Für die politischen Spitzenakteure kommen in Folge dieser Veränderungen immer mehr Entscheidungen als purer ,Stresstest ‘ daher. Ohne Risikokompetenz droht den Akteuren das politische Aus. Die Dramaturgie des Films belohnt den Risikoentscheider, der sich als Power-Entscheider durchsetzt und für seine Idee vom Wähler belohnt oder abgestraft wird. Sowas lässt sich mit besonderen Phasen des Richtungsentscheidens, wie beispielsweise die Agenda-Politik der Schröder-Fischer-Regierung, exemplarisch zeigen. Aber 
es ist die absolute Ausnahme in der deutschen Kanzlerdemokratie. Eher ist das pragmatische ,Auf-Sicht-Fahren“ absichernd und abwägend der politische Alltag in Berlin, was Langeweile für filmische Dramaturgie bedeutet.

Die politischen Spitzenakteure einer Regierung sind in eine Regierungsformation eingefügt. Als Kollektivakteur ist die Formation extrem fragil und fluid. Handlungsfähig sind die Spitzenakteure, die wiederum ihr Politikmanagement unter den Bedingungen von politischer Komplexität und Unsicherheit organisieren. Wer die Handlungskorridore des Regierens erweitern möchte, kann sich eines mittlerweile etablierten Repertoires an Instrumenten bedienen, um mehrheitsfähig zu bleiben. Dazu sind auch die drei Arenen des Politikmanagements (parlamentarisch, administrativ, öffentlich) klug miteinander zu verzahnen. Das setzt eine Strategiefähigkeit der Akteure voraus: Strategiefähigkeit bedeutet zumeist eine Nichtwissensbasiertheit. Mit dem nicht-gewussten Wissen sollte ein Spitzenakteur umgehen können. Strategiefähigkeit bemisst sich daher an der Fähigkeit zur Antizipation von Erwartungsunsicherheit. Strategiefähigkeit beruht folglich auf der Verfügbarkeit von Orientierungswissen für offene Problemsituationen, immer im Bewusstsein extrem stimmungsflüchtiger Machtgrundlagen. Wie stellt man sowas filmisch überzeugend dar?

Die aktuelle Kaskade von Krisen stellt jede Regierung vor besondere Probleme. Denn im Zentrum steht dabei nicht nur die Bewältigung der Probleme im Sinne einer Problemlösung in Zeiten entfesselter Dynamiken. Vielmehr zeigt sich im Politikmanagement ein Kampf um das Primat der Politik, um den Ort und die Verteilung politischer Entscheidungsmacht. Wo fallen heute die Entscheidungen: in der Regel nicht mehr über Telefongespräche über Festnetzapparate an einem bestimmten Ort und auch nicht in den dafür vorgesehenen Gebäuden der Verfassungsorgane.

Parallel zu wachsenden Risiken entwickelt sich exponentiell politische Komplexität mit überraschenden Rückkopplungseffekten. Immer mehr Akteure in immer stärker globalisierten Verhandlungsformaten ohne hierarchische Formen der Handlungskoordination arbeiten an Lösungen von konzeptionell nicht linearen Problemen. Unter dem Druck der Ereignisdichte scheint sich ein neuer Rhythmus der Politik zu entwickeln. Wichtiger und bindender als Verträge werden für die politischen Akteure auf vielen Ebenen die Krisenreaktionskräfte. Wenn es serienmäßig zum Triumph des Unwahrscheinlichen über das Wahrscheinliche kommt, muss Politik stets das Überaschende erwarten. Wie passt das in Serien-Formate? 


\section{DIE VERHANDELNDE WETTBEWERBSDEMOKRATIE: VERWISCHUNG VON ZUSTÄNDIGKEITEN}

Das politische System der Bundesrepublik Deutschland kann als Mischform charakterisiert werden: Es handelt sich um eine Kombination von parlamentarischen Strukturen und Verhandlungssystemen. Als Unterscheidungsmerkmal dient dabei der Typus der Konfliktregelung. In den parlamentarischen Strukturen einer Wettbewerbsdemokratie wird über den Parteienwettbewerb und das Mehrheitsprinzip entschieden. Die politische Macht ist in repräsentativer Form bei der Parlamentsmehrheit und ihrer Regierung konzentriert, was eine weitgehende Machtausübung ohne besondere Rücksichtnahme auf die Opposition erlaubt. Doch eine Konfliktregelung nach dem Muster der Wettbewerbsdemokratie im strikten Sinne einer Mehrheits- und Konkurrenzdemokratie - entsprechend dem ,Westminster-Modell ‘ - charakterisiert keinesfalls den klassischen Machtkreislauf der Berliner Republik.

Aber trifft das Gegenbild einer Verhandlungsdemokratie zu? Hierbei entfalten Parteienwettbewerb und Mehrheitsprinzip bei Wahlen und parlamentarischen Prozessen in nur eingeschränktem Maße ihre Wirkung. Denn die Konfliktregelung erfolgt strikt durch Aushandlungsprozesse. Das gütliche, freiwillige, konsensuale, kompromissgeprägte und informelle Einvernehmen der beteiligten Akteure führt zur Konfliktlösung weitgehend ohne die Transparenz der Willensund Entscheidungsbildungsprozesse. Dennoch lässt sich eine Vielzahl von konkreten politischen Entscheidungen anführen, in denen die Politik die Einbindung aller möglichen Interessengruppen (Korporatismus) zwar zuließ, sich dennoch, der Mehrheitsregel verpflichtet, am Ende des Prozesses gegen die Wünsche und Zielsetzungen machtvoller gesellschaftlicher Gruppen entschied. Die Bundesrepublik Deutschland ist somit weder eine reine Wettbewerbsdemokratie noch eine reine Verhandlungsdemokratie. Mehrheitsdemokratische und konsensuale Entscheidungsmodi haben nebeneinander Gültigkeit, so dass sich das Regierungshandeln in der Bundesrepublik primär am Modell der verhandelnden Wettbewerbsdemokratie orientiert. In diesem institutionellen Rahmen erfolgen Konfliktregelungen und Problemlösungen in gegenseitiger Abhängigkeit, die Verhandlungszwänge entstehen lassen.

Die Überlappungen in den Ebenen Wettbewerb (Mehrheitsregel im Parlament), Hierarchie (Verwaltungshandeln bei der Ausführung und dem Vollzug von Gesetzen, Verordnungen etc.) und Verhandlung (Runde Tische für korporatistische Interessenartikulation) lassen die Schlussfolgerung zu, dass der sogenannte, verhandelnde Staat' des Einsatzes von Steuerungsinstrumenten bedarf, die über den traditionellen politischen Prozess zwischen den Verfassungsorga- 
nen weit hinausweisen. Gleichgültig von welcher konkreten Akteurskonstellation Deutschland regiert wird, die Kennzeichen der verhandelnden Wettbewerbsdemokratie gelten für alle. Das politische Entscheiden scheint sich danach an immerwährenden Runden Tischen abzuspielen. Ein Gesetz benötigt deshalb auch in Deutschland im Durchschnitt 264 Tage. Wo liegt die besondere Pointe, sowas filmisch umzusetzen, wenn der langwierig zu legitimierende Kompromiss Weg und Ergebnis darstellt?

Welche konkreten ,Nebenregierungen“ (,Veto-Spieler') können nun aus systematischen Gesichtspunkten heraus benannt werden, welche eine politische Entscheidung für jede Bundesregierung komplexer und zeitintensiver machen? Wie gestaltet sich der institutionelle Pluralismus? Die nachfolgend dargestellten Nebenregierungen sind nicht alle im Verlauf der letzten Jahrzehnte linear stärker geworden; die Bundesregierung ist nicht kontinuierlich schwächer geworden. Der politischen Realität kommt eher ein Kurvenverlauf nahe. $\mathrm{Zu}$ benennen sind folgende Aspekte:

Der Einigungszwang in der Koalitionsdemokratie: Stärke und Schwäche des Koalitionspartners hängen nicht nur von den Mehrheitsverhältnissen im Bundestag ab, sondern auch von persönlichen Prägungen und dem politischen Gewicht der verhandelnden Hauptakteure. Die Macht der Ländervertretung, die Politikverflechtung zwischen Bund und Ländern und die gegenläufige Machtverteilung zwischen den Parteien in Bund und Ländern.

Mitwirkung organisierter Interessen: Neue Verhandlungsarrangements ergeben sich auch dadurch, dass Verbände, Gewerkschaften und andere organisierte Interessen an der Formulierung und Umsetzung bindender Entscheidungen mitwirken. Regieren bedeutet in dieser Hinsicht, auch auf neokorporatistische Arrangements einzugehen.

Die Verfassungsgerichtsbarkeit: Trotz zunehmender Verrechtlichung der politischen Entscheidungen und der zum Teil bewussten Entscheidungsverlagerung nach Karlsruhe, dem Sitz des Bundesverfassungsgerichts, sind gerade Beispiele auf dem Gebiet der Außenpolitik anzuführen, die eine parlamentarische Mitsteuerung - und damit den zentralen Ort der Gesetzgebung - wieder gestärkt haben.

Die autonome Bundesbank: Sie konnte bis zur Einführung der Europäischen Zentralbank (EZB) als weitere Nebenregierung aufgelistet werden.

Das europäische Mehrebenensystem: Im Zentrum der Vernetzung steht das europäische Mehrebenensystem. Kaum ein Politikfeld mehr kann autonom in Berlin entschieden werden. Das deutsche Regierungssystem ist ein penetriertes politisches System, in dem die europäische Einmischung vertraglich verankert 
und legitimiert ist. Regierungshandeln verläuft in vielen Politikfeldern in zunehmendem Maß analog zur europäischen Entscheidungsfindung.

Äußerer Souveränitätsverlust: Seit einigen Jahren wird in der Literatur der Befund der ,Nebenregierungen“ durch den Aspekt des ,äußeren Souveränitätsverlustes` ergänzt. In dem Maße, wie die Bundesrepublik seit 1990 auch völkerrechtlich souverän wurde, hat sie - vergleichbar mit den zentralen Phasen des außenpolitischen Prozesses der Bonner Republik - durch weitere Integration formal Souveränität abgegeben. Heute spricht man von ,governance without government“ (James Rosenau), „Regieren jenseits der Staatlichkeit“ (Markus Jachtenfuchs), „government without statehood“(William Wallace), „postmodern state“ (John Ruggie).

Die hier skizzierten Faktoren und Nebenregierungen der verhandelnden Wettbewerbsdemokratie sind typische Merkmale des Regierens in Deutschland, die nachfolgend weiter ergänzt werden sollen.

Die Komplexität hat somit auch strukturelle Ursachen: Eine internationale Problemstruktur, europäische Entscheidungsstrukturen und nationale Legitimationsstrukturen markieren eine Diskrepanz, die man nur durch unterschiedliche Spielorte filmisch auflösen könnte.

\section{KANZLER UND KANZLERAMT ALS FÜHRUNGSZENTRALE: DER PREIS DER MEDIENDEMOKRATIE}

Das Politikmanagement verleiht einer Regierungszentrale ihre Steuerungsfähigkeit. Dies gilt für die Koordination der wichtigen politischen Akteure (Kanzler, Bundesminister, Fraktion etc.) ebenso wie für die Steuerbarkeit des politischen Systems (Gang der Gesetzgebung, Koordination der politischen Institutionen mit Veto-Macht etc.). Immer vermischen sich sachliche Überlegungen mit machtpolitischen Absichten und persönlichen Profilierungssüchten.

An der Spitze der Informationspyramide soll idealtypisch der Kanzler stehen. Die Informationen sind gefiltert und selektiert durch die hausinternen Prozesse. Rund zwei Drittel der Nachrichten, die den Bundeskanzler täglich erreichen, sind öffentlicher Natur. Die Exklusivität solcher Informationen liegt nicht im Inhalt der Nachricht, sondern in der Frühzeitigkeit und Schnelligkeit ihres Zugangs. Wenn die Informationen als Grundlage einer politischen Lageeinschätzung so wichtig sind, dann stellt sich die Frage nach dem Webmuster einer Lageanalyse und Problemdeutung. Nach welchen Kriterien erstellt ein politischer Spitzenakteur eine Risikoeinschätzung? 
Das Bundeskanzleramt ist das Drehkreuz des Kanzler-, Ressort-, Kollegial-, Partei- und Koalitionsprinzips. Unter dem Aspekt des operativen Regierens bilden der Regierungschef und das Bundeskanzleramt das Zentrum der core executive. Von hier aus erfolgt die Koordination der Exekutive, die immerhin rund 25.000 Beamte und Angestellte in den Bundesministerien und Bundesbehörden umfasst. Das Kanzleramt ist ein Sekretariat der Bundesregierung. Gleichzeitig stellt es gegenüber den Ressorts kein ,Überministerium‘ dar. Es hat kein Weisungsrecht gegenüber den Fachministerien. Diejenigen Chefs des Kanzleramtes, die nicht beamtete Staatssekretäre waren, sondern der Bundesregierung angehörten, titulierten sich stets als „Bundesminister für besondere Aufgaben“, ohne Portefeuille. Im Verhältnis zum Bundestag und zum Bundesrat hat das Kanzleramt die gleichberechtigte Kompetenz der Einbringung von Gesetzentwürfen gemäß Art. 76 GG. Für die Zuleitung dieser Gesetzentwürfe der Bundesregierung ist das Kanzleramt zuständig.

Medial sichtbar sind in der Regel nur die Kanzler. Nicht grundlos gilt die Bundesrepublik als Kanzlerdemokratie. Kanzler agieren oft präsidial, oft parteiund koalitionsübergreifend. Sie stehen im Meinungsbild der Bürger in der Verantwortung der Politikergebnisse. Nicht Regierungen werden gewählt, sondern Kanzlerkandidaten, die über die jeweilige Parteiliste zur Auswahl stehen. Mit ihnen lassen sich Filmabende sicher gestalten. Was sie entscheiden, hat maßgeblich auch Folgen. Doch diese extrem verkürzte Sicht auf den Chefentscheider im Kanzleramt gehört ins Reich des Fiktionalen. Denn die aufgeführte Entscheidungskomplexität macht aus dem Kanzler einen Interdependenzmanager auf Zeit, der nicht grundlos täglich auf Sicht fährt. Wer den Kanzler medial ins Zentrum rückt, muss zeitgleich auch andere Strukturmerkmale des Regierens in Bilder und Personen übersetzen. Alles andere banalisiert die Kanzlerdemokratie.

\section{ES BLEIBT SCHWIERIG}

Deutsche Zuschauer sind politisch anspruchsvoll. Denn die politische Informiertheit und politische Interessiertheit ist im europäischen und US-amerikanischen Vergleich hoch - zugegebenermaßen auf einem insgesamt sehr geringen Ausgangsniveau. Deutsche Wähler gehen auch davon aus, dass keine Partei ihr Wahlprogramm vollständig umsetzt. Denn die Tradition der Koalitionsregierungen führt automatisch zum Verständnis für eine Kompromiss-Suche bei der Umsetzung von Wahlversprechen. Noch immer agieren Volksparteien in Deutschland, die mit einem breiten Themenangebot Mitglieder binden. Wer sich zu einer Partei bekennt, wird hier in Mithaftung genommen für die gesamte Programmatik, unabhängig von wechselndem Personal. Auch das schreckt viele Bürger ab, sich zu 
binden. Trotz zunehmender Personalisierung und Präsidentialisierung bleibt die deutsche Demokratie eine Parteiendemokratie. So ein Befund führt zu vielfältigen Problemen bei der Umsetzung in ein Genre für Polit-Serien, die in der Regel mit dem Machtkampf der Spitzenpolitiker Quote machen. Wer insofern die Komplexität des politischen Systems und Entscheidens angemessen filmisch umsetzen möchte, steht vor großen Herausforderungen. Fiktionale Spielräume sind unendlich. Sie wurden auch durchaus bislang genutzt. Nur endeten diese Serienversuche meist im Klamauk oder in der Seifenoper, weil die kluge filmisch heruntergebrochene Reduktion von Komplexität großer Meister des Narrativen bedarf.

\section{LITERATUR}

Benz, Arthur/Dose, Nicolai (2010): Governance. Modebegriff oder nützliches sozialwissenschaftliches Konzept, in dies. (Hrsg.): Governance. Regieren in komplexen Regelsystemen, Wiesbaden, S. 13-36.

Bröchler, Stephan/Timo Grunden (Hrsg.) (2014): Informelle Politik. Konzepte, Akteure und Prozesse, Wiesbaden.

Busse, Volker/Hofmann, Hans (2010): Bundeskanzleramt und Bundesregierung. Aufgaben - Organisation - Arbeitsweise, 5. Aufl., Heidelberg u.a.

Florack, Martin (2013): Transformation der Kernexekutive. Eine neoinstitutionalistische Analyse der Regierungsorganisation in NRW 2005-2010, Wiesbaden.

Florack, Martin/Grunden, Timo (Hrsg.) (2011): Regierungszentralen. Organisation, Steuerung und Politikformulierung zwischen Formalität und Informalität, Wiesbaden.

Florack, Martin/Grunden, Timo/Korte, Karl-Rudolf (2011): Kein Governance ohne Government, in: Bröchler, Stephan/Blumenthal, Julia von (Hrsg.): Regierungskanzleien im politischen Prozess, Wiesbaden.

Grasselt, Nico/Korte, Karl-Rudolf (2007): Führung in Politik und Wirtschaft. Instrumente, Stile, Techniken. Wiesbaden.

Hirscher, Gerhard/Korte, Karl-Rudolf Korte (Hrsg.) (2001): Aufstieg und Fall von Regierungen. Machterwerb und Machterosion in westlichen Demokratien, München.

König, Klaus (2015): Operative Regierung, Tübingen.

Korte, Karl-Rudolf (2008): Die Praxis regierungsförmiger Steuerung, in: Holtmann, Everhard/Patzelt, Werner J. (Hrsg.): Führen Regierungen tatsächlich? Zur Praxis gouvernementalen Handelns, Wiesabden, S. 59-71. 
Korte, Karl-Rudolf (2012): Besondere Herausforderungen der angewandten Regierungsforschung, in: Glaab, Manuela/Korte, Karl-Rudolf (Hrsg.): Angewandte Politikforschung, Wiesbaden, S. 91-98.

Korte, Karl-Rudolf (2010): Präsidentielles Zaudern. Der Regierungsstil von Angela Merkel in der Großen Koalition, in: Bukow, Sebastian/Seemann, Wenke (Hrsg.): Die Große Koalition, Wiesbaden.

Korte, Karl-Rudolf (2011): Risiko als Regelfall: Über Entscheidungszumutungen in der Politik, in: Zeitschrift für Politikwissenschaft (3), S. 465-477.

Korte, Karl-Rudolf/Fröhlich, Manuel (2009): Politik und Regieren in Deutschland, 3. Aufl., Paderborn u.a.

Korte, Karl-Rudolf/Michels, Dennis/Schoofs, Jan/Switek, Niko/Weissenbach, Kristina (2018): Parteiendemokratie in Bewegung. Organisations- und Entscheidungsmuster der deutschen Parteien im Vergleich, Baden-Baden.

Korte, Karl-Rudolf/Grunden, Timo (Hrsg.) (2013): Handbuch Regierungsforschung, Wiesbaden.

Niclauß, Karlheinz (2015): Kanzlerdemokratie. Regierungsführung von Konrad Adenauer bis Angela Merkel, 3. Aufl., Wiesbaden.

Pannes, Anna-Tina (2015): Informalität. Theoretische und empirische Dimensionen informeller Entscheidungsprozesse in Regierungsformationen. Ein Analyseansatz, Wiesbaden.

Schimank, Uwe (2005): Die Entscheidungsgesellschaft. Komplexität und Rationalität der Moderne, Wiesbaden. 



\title{
Polit-Serien im Fachunterricht
}

\author{
Empirische Befunde zum Medieneinsatz
}

aus Schüler- und Lehrerperspektive

Sabine Manzel

Zu guter Bildung im 21. Jahrhundert gehören IT-

Kenntnisse und der souveräne Umgang mit der Technik und den Risiken digitaler Kommunikation ebenso wie das Lernen mittels der vielen neuen Möglichkeiten digitaler Medien. Deutschland muss diese Chancen viel stärker nutzen als bisher.

Bundesministerin Johanna Wanka, BMBF 2016

\section{EINLEITUNG}

Mit dem DigitalPakt\#D hat das Bundesministeriums für Bildung und Forschung (BMBF) Ende 2016 eine Bildungsoffensive für die digitale Wissensgesellschaft gestartet. Nicht nur das Zitat der ehemaligen Bundesbildungsministerin Johanna Wanka zeigt die Bedeutsamkeit, die diese Offensive für eine zukunftsfähige Gesellschaft einnimmt. Auch die Fördersumme von rund 5 Milliarden Euro, die das BMBF über einen Zeitraum von fünf Jahren für die rund 40.000 Grund- bis weiterführenden Schulen in Deutschland anbietet, um die digitale Ausstattung mit Breitbandanbindung, W-LAN und Geräten voranzutreiben, spricht für die Relevanz. Im Gegenzug sind die Länder aufgefordert, entsprechende pädagogische und didaktische Konzepte zu entwickeln, die Aus- und Fortbildung von Lehrern ${ }^{1}$

1 Aus Gründen der Lesbarkeit werden männliche Substantive verwendet, diese schlieBen die weibliche Form der Begriffe jedoch selbstverständlich mit ein. 
zu sichern sowie technische Standards umzusetzen. Den gemeinsamen Auftakt der Länder bildet die Strategie Bildung in der digitalen Welt, beschlossen im Dezember 2016 von der Kultusministerkonferenz. Als Handlungsfelder für Schulund Hochschulentwicklung werden unter anderem Bildungspläne und Unterrichtsentwicklung, Aus- und Weiterbildung von Erziehenden und Lehrenden, aber auch Infrastruktur und Ausstattung genannt (KMK 2016).

Im internationalen Vergleich der ICIL-Studie sind deutsche Schüler unterdurchschnittlich im Umgang mit digitalen Medien ausgebildet (Bos et al. 2014). Und das, obwohl nahezu alle Jugendliche ein Mobiltelefon, einen Computer und Internetzugang besitzen (MPFS 2016: 8). Internet-Recherchen (70 Prozent), WhatsApp-Nachrichten (58 Prozent) und YouTube-Videos (50 Prozent) fallen unter die regelmäßige Tätigkeit von Kindern und Jugendlichen (MPFS 2017: 41). Besonders YouTube-Videos werden aufgrund der humorvollen und ironischen Darstellungsweise häufig angeklickt. Auf der Beliebtheitsskala rangieren nach Musikvideos (55 Prozent) „lustige Clips“ (40 Prozent) und „Comedy/lustige Videos von YouTubern“ (32 Prozent). Gleichzeitig sehen Jugendliche aber auch Kommentarvideos (22 Prozent) von bekannten YouTubern, wie z.B. LeFloid, Tilo Jung, Ben Bode oder MrWissen2go. Aktuelle Nachrichten und politische Sachverhalte werden hier locker, visuell ansprechend und verständlich präsentiert und bieten einen Kontrast zu herkömmlichen Nachrichtenformaten.

\section{Abbildung 1: Nutzungshäufigkeit von Serien im Fernsehen nach Alter}

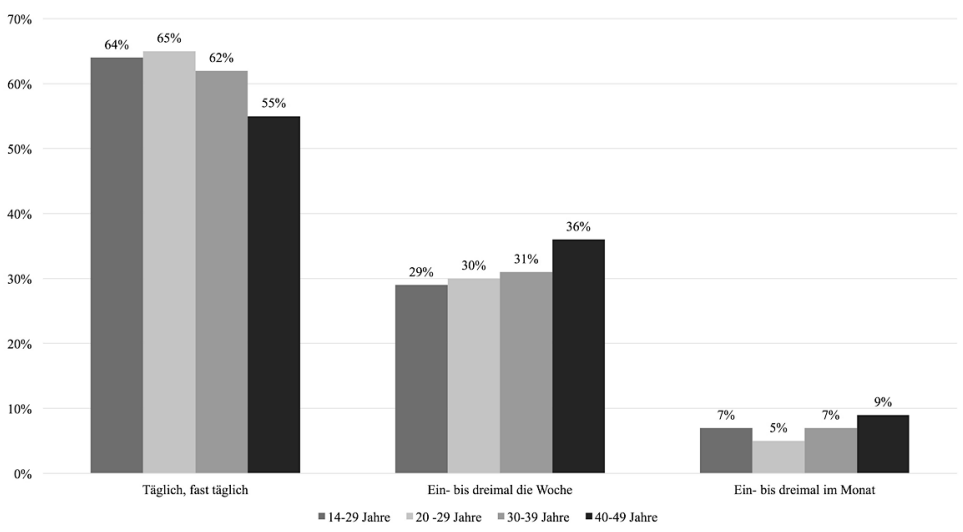

Quelle: Statista-Umfrage 2017, 1.011 Befragte; 14-49 Jahre; Personen, die mindestens einmal im Monat Serien schauen. 
Zum Alltagsrepertoire von Jugendlichen gehört auch der Film- oder Serienkonsum bei Streaming-Anbietern wie Netflix (MPFS 2016: 36). Gerade Netflix ist auch durch seine Polit-Serie House of Cards bekannt geworden.

Diese Rahmenbedingungen und Voraussetzungen nimmt der folgende Beitrag zum Anlass, über Politische Bildung mit medialen Formaten nachzudenken. Es stellt sich die Frage, ob der Einsatz von Polit-Serien im Politik- und sozialwissenschaftlichen Unterricht beim Aufbau politischer Kompetenzen hilfreich sein kann. Besand (2016) sieht Serien, die politische oder gesellschaftliche Themen adressieren, als gewinnbringend für den Politikunterricht an. Sie eröffnen Einblicke auf die Hinterbühnen der Politik und ermöglichen die kritische Auseinandersetzung mit Fiktion und Realität (Dörner 2016: 4f.). Bislang sind die Potentiale für den Erwerb von Medien- und Politikkompetenz theoretisch beleuchtet, aber nicht empirisch belegt. Hier werden erstmals Befunde zu PolitSerien im Fachunterricht aus Schüler- und Lehrerperspektive vorgestellt.

\section{THEORETISCHE ÜBERLEGUNGEN}

Für die Konzeption eines Untersuchungsinstrumentes für Schüler und Lehrkräfte bedarf es einiger theoretischer Vorüberlegungen hinsichtlich Medieneinsatz und Politikunterricht. „Medien dienen der politischen Informationsvermittlung und sind daher im Politikunterricht einerseits Quelle inhaltlicher Themen, andererseits verlangen sie entsprechendes Wissen über Produktions- und Rezeptionsbedingungen sowie Medien- und domänenspezifische Kompetenzen“ (Manzel 2007: 44). Polit-Serien zählen zu den Unterrichtsmedien des Politikunterrichts und können politische Inhalte in den Klassenraum transportieren (Breit/Weißeno 2004: 84). Sie besitzen hohes Potenzial, Wissen und Informationen zu vermitteln, besonders durch das Ansprechen zweier Wahrnehmungskanäle (Simon 2007: 4). Der Einsatz von Medien wirkt sich positiv auf die Lernmotivation aus und fördert das intrinsische Interesse am Lerninhalt durch differenziertere Einblicke in den Lerngegenstand (Kerres 2003: 3f.). Es wird von einem dauerhaft motivationssteigernden Effekt für leistungsstarke und -schwache Schüler durch Medien ausgegangen (Eickelmann/Gerick/Schulz-Zander 2008) und nicht nur von einem „Neuartigkeitseffekt“ bei der Verknüpfung von kognitiven Prozessen der Auseinandersetzung mit den Inhalten (Kerres 2000: 29). Medien unterstützen die kognitive und emotionale Aktivierung der Schüler (Kerres/ Preussler/Schiefner-Rohs 2013: 585, Benkert 2001: 65). Zur vertieften Theorie bezüglich Medienkompetenz und Medienerziehung in der Schule sei auf einschlägige Literatur von Herzig (2014), Tulodziecki (2009), Spanhel (2006) und den aktuellen Band zur Medienkompetenz der Bundeszentrale für Politische Bildung von 
Gapski/Oberle/Staufer (2017) verwiesen. Für die Politikdidaktik bieten Formate der alltäglichen Mediennutzung von Jugendlichen die Chance, politische Problemfelder und Inhalte aus dem politischen Alltag zu thematisieren (Manzel 2017). Schüler sprechen außerhalb der Schule über Polit-Serien und tauschen sich über diese aus. Ein Medieneinsatz, der an die Lebensgewohnheiten und Bedürfnisse der Schüler anknüpft, zielt auf mehr ab als die Befähigung zur kompetenten Zeitungslektüre zur Herausbildung des Bürgerideals von interessierten und kritischen Zeitungslesern. Wichtig ist an dieser Stelle die Verzahnung des Mediums Polit-Serien mit den vier Kompetenzdimensionen Fachwissen, politische Handlungsfähigkeit, Einstellung/Motivation aus dem Politikkompetenzmodell von Detjen et al. (2012) und der politischen Urteilsfähigkeit, ausdifferenziert in Teilfähigkeiten nach Manzel/Weißeno (2017).

\section{Abbildung 2: Kompetenzdimensionen im Politikkompetenzmodell}

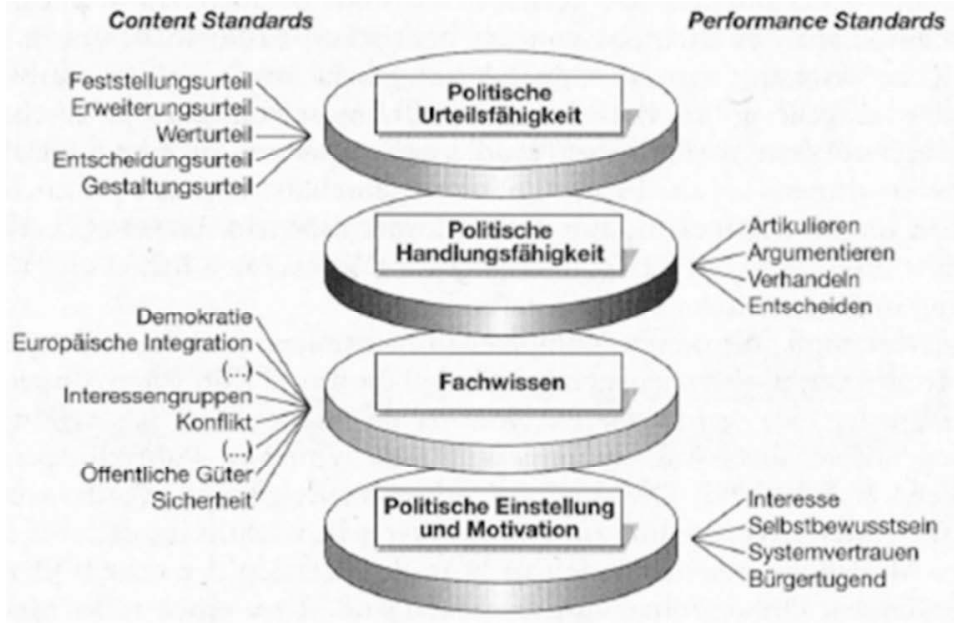

Quelle: Detjen et al. 2012: 172.

Die Kompetenzdimension Fachwissen mit den dazugehörigen Fachkonzepten wie Parteien, Wahlen, Macht oder Konflikt spielen in Polit-Serien wie House of Cards eine zentrale Rolle. Der Aufbau von konzeptuellem Wissen ist möglich anhand der Konfrontation fiktionaler und realer Politik wie zum Beispiel der Vorbereitung von Abstimmungen und Gesetzen oder der Organisation von Mehrheiten. In der Polit-Serie House of Cards erklärt und begründet der Präsident der Vereinigten Staaten, Francis Underwood, seine Gedanken, Vorgehensweisen und Strategien durch eine direkte Zuwendung an das Publikum (Besand 2016: 197). Dadurch ergibt sich nicht nur die Gelegenheit, den politischen Ak- 
teuren bei ihrer politischen Arbeit über die Schulter zu schauen, sondern der $\mathrm{Zu}$ schauer wird zum Mitwisser geheimer Strategien (Dörner 2016: 7). Genau an dieser Stelle ist die Lehrkraft gefragt, das ,Insiderwissen der Betrachter ' einem Realitätscheck zu unterziehen und mögliche Stereotype und Fehlkonzepte aufzubrechen, um konzeptuelles Fachwissen zu vertiefen. Durch einen Einsatz neuer Medien im Unterricht kann träges und erfahrungsleeres Wissen in anwendungsfähiges Wissen umgewandelt werden (Manzel 2008: 46).

Massenmedien selbst sind ein Fachkonzept, das Schüler im Politikunterricht im Rahmen des Fachwissens erwerben und zusammen mit anderen Fachkonzepten vernetzen können (Detjen et al. 2012). Medien haben als vierte Gewalt Einfluss auf die Politik und öffentliche Meinungsbildung (Geyer \& Kölbl 2015). Zu den zentralen Aufgaben von Massenmedien in einer demokratischen Gesellschaft zählen Meinungsbildung, Kontrolle, Unterhaltung, Information und Bildung. Gerade in Polit-Serien lässt sich ein direkter Bezug zum Zusammenhang von Medien und Politik herstellen. So nehmen Medien in der Polit-Serie House of Cards eine zentrale Rolle ein und zeigen ihren Einfluss auf das politische Alltagsgeschäft. Medienvertretern wie Journalisten und Reportern kommt in dieser Serie eine entscheidende Funktion zu. Die Serie zeigt einerseits, wie die Medien ihrer Informationsfunktion sowie Kritik- und Kontrollfunktion Rechnung tragen, indem sie die Handlungen und Aussagen der politischen Akteure durch ihre Berichterstattung kritisch hinterfragen und beleuchten. Gleichzeitig werden andererseits Einblicke ermöglicht, die zeigen, wie politische Akteure die Medien bewusst (aus)nutzen, um ihre Interessen und Vorhaben möglichst öffentlichkeitswirksam zur Geltung zu bringen, was wiederum gewinnbringend für den journalistischen Erfolg von Medienakteuren scheint. Polit-Serien zeigen, ,welche Kooperationen und Konfrontationen sich zwischen Politik und Medien herausbilden können“ (Dörner 2016: 7) und tragen nicht nur zur Kompetenz Fachwissen, sondern auch zur Urteilsbildung bei.

Die Kompetenzdimension Urteilsfähigkeit basiert auf Fachwissen und der Fähigkeit, sich mit Hilfe von Argumenten für oder gegen einen Sachverhalt unter Einbezug von Werten wie Freiheit, Gerechtigkeit begründet Position zu beziehen. Nach dem Social-Cognition-Ansatz spielt die Informationsverarbeitung und die Interaktion von neuen Informationsreizen und vorherigem Wissen eine entscheidende Rolle beim Urteilen (Betsch et al. 2010: 17f.). Durch den Vergleich zwischen dem realen politischen Alltag und der Fiktionalität in PolitSerien ergeben sich Rückbezüge zwischen diesen beiden Wirklichkeitsebenen, wodurch diese kritisch in Beziehung zueinander gesetzt werden können (Dörner 2016: 11). Diese Fiktionalität einer Polit-Serie gibt Lehrkräften die Möglichkeit, Gestaltungsurteile anzubahnen, die verschiedene Auswirkungen von politischen 
Entscheidungen thematisieren und szenisch durchspielen (Detjen et al. 2012: 57 und 63, Besand 2016: 211). Allerdings kann es auch zu einer Vermischung von Realität und fiktionalen Erfahrungen kommen, wodurch sich das Bild und die Wahrnehmung der Politik und einzelner Politiker verschieben können (Steppat 2014). So zeigt eine amerikanische Studie, dass die Politserie The West Wing zu höheren Sympathie- und Kompetenzwerten des US-Präsidenten führen und damit das Bild von realen Politikern beeinflussen kann (Zegart 2000). Die Ergebnisse einer amerikanischen Telefonumfrage bestätigen diesen Befund, dass $\mathrm{Zu}$ schauer von Serien wie Homeland den Geheimdiensten mehr Befugnisse zugestehen (Zegart 2000).

Polit-Serien stehen somit in einem engen Zusammenhang zu den individuellen Einstellungen, womit die dritte Kompetenzdimension der politischen Einstellung und Motivation angesprochen ist. Politische Einstellungen umfassen kognitive, affektiv/evaluative und konative Orientierungen gegenüber politischen Sachverhalten, Institutionen und Personen, und beeinflussen das politische Handeln, politische Wissen und politische Urteilen (Detjen et al. 2012: 89f.). Sie haben folglich einen Einfluss auf die Wahrnehmung von Politikern (Niedermayer 2005: 16).

Politische Handlungsfähigkeit, unterteilt in kommunikative und partizipative Handlungsfähigkeit (Detjen et al. 2012: 68), kommt insbesondere in der kommunikativen Form zum Tragen. Das Sprechen über Polit-Serien und ihre Inhalte ermöglicht die politische Meinungsbildung und den Austausch von Argumenten (Straßner 2013: 13ff.). Wird z.B. ein Schwerpunkt auf Interaktion gelegt, bieten sich Rollenspiele wie Pro-Kontra-Diskussionen oder Talkshows an. Lehrkräfte können Schüler auch dazu ermuntern, eigene Youtube-Videos zu erstellen, um politische Zusammenhänge darzustellen. Politische Medienkompetenz, die auf fachlicher Ebene Wissen, kritische Reflexivität in der Bewertung der fachlichen Informationen, Handlungsfähigkeit und kommunikative Interaktion im Sinne politischer Argumentation umfasst, wird zu einer wichtigen Aufgabe auch für die politische Bildung und die politische Urteilsbildung sowie zum Zugangsticket für Partizipation. Die Aufgabe von Lehrkräften ist es, diese Politische Medienkompetenz zu fördern. Dafür bedarf es gemäß der Modelle professioneller Handlungskompetenz wie bei der COACTIV- oder PKP-Modellierung (Kunter et al. 2011, Weschenfelder 2014: 55f.) seitens der Lehrkräfte auch Professionswissen, d.h. Fachwissen, fachdidaktisches und pädagogisches Wissen, aber auch beliefs, z.B. Überzeugungen zum Lehren und Lernen mit Medien. Diese theoretischen Annahmen sind aber in der Politikdidaktik zum Einsatz von Polit-Serien noch nicht empirisch überprüft, wenngleich die Beschäftigung in der Politikdi- 
daktik mit (digitalen) Medien bis 2000 zurückreicht (Harth 2001, Hauk 2016, Motyka/Lipowsky 2017).

\section{STUDIENDESIGN}

Das Forschungsvorhaben gliedert sich in zwei Perspektiven: die von Lehrkräften im Fach Politik und die von Schülern. Ziel beider Studien ist es zu untersuchen, ob und mit welcher Intention Politiklehrkräfte im Fachunterricht Polit-Serien einsetzen und wie sich das Medienverhalten von Schülern bezüglich Politik gestaltet. Damit soll das bisherige Forschungsdesiderat aufgebrochen und ein Beitrag zum Beschreibungswissen mit ersten empirischen Daten geleistet werden.

\section{Forschungsfragen und Hypothesen}

Um dem Serienverhalten von Schülern nachzugehen, wird als erstes überprüft, ob Schüler überhaupt Polit-Serien schauen, und wenn ja, welche. Die weiteren Forschungsfragen beziehen sich auf den Einsatz von Polit-Serien im Unterricht und die Analyse von Unterschieden hinsichtlich Schulform, Jahrgangsstufe, Gender und Migrationserfahrung. Folgende Hypothesen werden mittels t-Tests und Korrelationen geprüft:

(H1) Es gibt einen Unterschied zwischen dem Medien- und Serienverhalten von Schülern der Gesamtschule und des Gymnasiums.

(H2) Es gibt Unterschiede im Nutzungsverhalten von Polit-Serien im Hinblick

a) auf das Geschlecht,

b) die Jahrgangsstufe und

c) die Migrationserfahrung.

(H3) Zudem interessiert das Bild, das sich Schüler von Politikern machen. Auch hier wird vermutet, dass es Unterschiede in der Wahrnehmung von Politikern im Hinblick
a) auf das Geschlecht,
b) die Jahrgangsstufe und
c) die Migrationserfahrung gibt.

Die Lehrerinterviews dienen primär einer Hypothesengenerierung zur Bedeutung, Nutzung und Herausforderung von Polit-Serien im Politikunterricht. 


\section{Stichprobe}

Sowohl die Lehrer- als auch die Schülerstichprobe sind reine Gelegenheitsstichproben in Nordrhein-Westfalen (NRW), was die Aussagekraft der Ergebnisse einschränkt. Bei der Auswahl der Lehrkräfte (Positivselektion von Lehrern, die sich für die Interviews bereit erklärt hatten) ist auf eine gleichmäßige Merkmalsausprägung hinsichtlich Gender, Alter, Dienstjahre Schulform, Stadt-LandSchule geachtet worden (Bortz/Döring 2016: 303). Von den elf Befragten sind fünf Frauen und sechs Männer im Alter von 27 bis 56 Jahren $(M=38.8)$, die an den Schulformen Gesamtschule, Gymnasium und Berufskolleg das Fach Politik/Sozialwissenschaften unterrichten. Fünf Personen unterrichten an einer Stadtschule (250.000 bis 500.000 Einwohner), 6 Personen an einer Landschule (13.000 bis 50.000 Einwohner). Die Dienstjahre variieren zwischen einem und 23 Jahren $(M=10.5)$. In Bezug auf die Schülerstichprobe gibt es mit den befragten Lehrkräften keine Überschneidungen, diese sind unabhängig voneinander ausgewählt worden. Es wurden insgesamt $N=276$ Schüler in der 8. und 11. Klasse befragt, davon 167 Schüler der Schulform Gymnasium und 109 der Schulform Gesamtschule. Knapp 70 Prozent der Befragten sind Achtklässler, 30 Prozent besuchen die Klasse 11. Das Alter der Befragten liegt zwischen 13 und 19 Jahren: $\mathrm{M}=14.65$, $(S D=1.44)$. 33 Prozent der Schüler weisen Migrationserfahrung auf. Die Politiknote ist im Schnitt der Probanden gut bis mittelmäßig: M $=2.65(S D=0.90)$.

\section{Methoden}

Zwei methodische Verfahren wurden entsprechend der Forschungsfragen ausgewählt, um die unterschiedlichen Sichtweisen zum Einsatz von Polit-Serien im Fachunterricht von Schülern und Lehrern zu erfassen. Dabei ist die Perspektive der Schüler quantitativ über standardisierte Fragebögen erhoben worden, die Perspektive von Lehrkräften mittels leitfadengestützter Interviews.

Perspektive der Lehrkräfte: Die halbstandardisierten Interviews dauerten zwischen 30 und 45 Minuten, wurden auf Tonband aufgezeichnet und inhaltlich vollständig wortwörtlich in MAXQDA (Kuckartz 2005) transkribiert ${ }^{2}$. In Anlehnung an Gropengießer (2005) wurden diese Originaltranskripte redigiert, um eine bessere Lesbarkeit durch eine grammatikalische Glättung herzustellen. Auf linguistische Besonderheiten und kommunikative Merkmale (Pause, Wörter wie

2 An dieser Stelle sei Annika Overdick gedankt, die diese Daten in ihrer Masterarbeit erhoben hat. 
ähm, Lachen etc.) wurde angesichts des Fokus auf die Sinngehalte verzichtet (Bortz/Döring 2016: 367). Der Leitfaden umfasst vier Themenkomplexe: allgemeine Einstiegsfragen zur Mediennutzung an der Schule, Einsatz von PolitSerien im Fachunterricht (fachdidaktisches Wissen), Interesse (Überzeugungen zum Lehren und Lernen) sowie abschließend personenbezogene Daten. Die Interviews wurden mit Hilfe eines Kategoriensystems gemäß der qualitativen Inhaltsanalyse nach Mayring (2010) ausgewertet. Diese Methode verbindet ein induktives und deduktives Vorgehen. Im ersten Schritt werden die Analyseeinheiten bestimmt (Kodier-, Kontext- und Auswertungseinheit), danach erfolgen Analyseschritte mittels eines erstellten Kategoriensystems mit Ankerbeispielen, das wiederholt an Theorie und am Material überprüft wird und ggfls. zu einem erneuten Materialdurchlauf führen kann. Vier Interpretationsschritte helfen bei der Reduktion zur Vergleichbarkeit des Materials: Paraphrasierung, Generalisierung auf das Abstraktionsniveau, erste und zweite Reduktion (Mayring 2010: 70). Fünf Oberkategorien mit Unterfragen wurden gebildet: Ausstattung und Vorgaben der Schule, Erfahrungen zum Medieneinsatz, fachdidaktische Aufbereitung von Polit-Serien im Fachunterricht, Chancen, technische Probleme und inhaltliche Risiken sowie Schülerinteressen und Einstellungen. Die InterraterReliabilität beträgt bei zwei Ratern Cohens Kappa $=.75$ und wird als akzeptabel bei hoch-inferenten Ratings eingeschätzt.

Perspektive von Schülern: Die Erhebung mit Hilfe standardisierter Fragebögen $^{3}$ erfolgte im Querschnittdesign. Die Wissensfragen zum Politischen System Deutschlands wurden als geschlossene Single-Choice-Items gestellt, bei denen eine Antwortmöglichkeit richtig war. Das Interesse der Schüler an Serien und am Fach Politik wurde über eine vierstufige Likert-Skala erhoben. Neben geschlossenen Fragen (z.B. Erfahrung mit Polit-Serien in der Freizeit und im Unterricht, Kommunikation über Polit-Serien, Vertrauen in die mediale Berichterstattung) wurden auch offene Fragen (Politische Partizipation, Wahrnehmung Politiker) gestellt. Verzerrungen durch Selbsteinschätzungen und soziale Erwünschtheit sind bei der Auswertung zu berücksichtigen.

Beide Erhebungsmethoden wurden vorab im Forschungskolloquium und mit einer Seminargruppe im Sommersemester 2017 an der Universität DuisburgEssen pilotiert.

3 An dieser Stelle sei Alexander Brefort gedankt, der diese Daten in seiner Bachelorarbeit erhoben hat. 


\section{ERGEBNISSE}

In diesem Teil werden zuerst die Ergebnisse der Schülerbefragung und danach die Ergebnisse der Lehrerinterviews berichtet. 97 Prozent der befragten Schüler haben generelle Serienerfahrung (z.B. Breaking Bad, Die Simpsons), 42 Prozent geben an, dass sie „häufig“ mit ihren Freunden über Serien sprechen. 35,1 Prozent haben bereits eine Polit-Serie gesehen und 10,5 Prozent sogar im Unterricht. Hier unterscheiden sich die Erfahrungen jedoch nach Schulform. Während 21 Prozent der Gesamtschüler bereits eine Polit-Serie im Unterricht gesehen haben, sind es nur 3,6 Prozent der Gymnasialschüler. Trotz der höheren Erfahrung der Schüler der Gesamtschule mit Polit-Serien lässt sich feststellen, dass Schüler des Gymnasiums über eine höhere Kenntnis von Polit-Serien wie House of Cards oder Breaking Bad verfügen, wie es Abbildung 3 veranschaulicht. Rund 48 Prozent der Gymnasiasten geben an, dass sie Polit-Serien kennen, wohingegen dies lediglich 25,7 Prozent der Gesamtschüler angeben. Weiterhin zeigt sich, dass 75,4 Prozent der Gymnasiasten glauben, dass Serien politische Sachverhalte ansprechen und im Vergleich dazu 50,5 Prozent der Schüler der Gesamtschule an eine Thematisierung politischer Sachverhalte in Serien glauben. Hypothese 1 kann für die befragte Stichprobe zugestimmt werden.

\section{Abbildung 3: Vergleich Polit-Serien in den Schulformen Gesamtschule} und Gymnasium

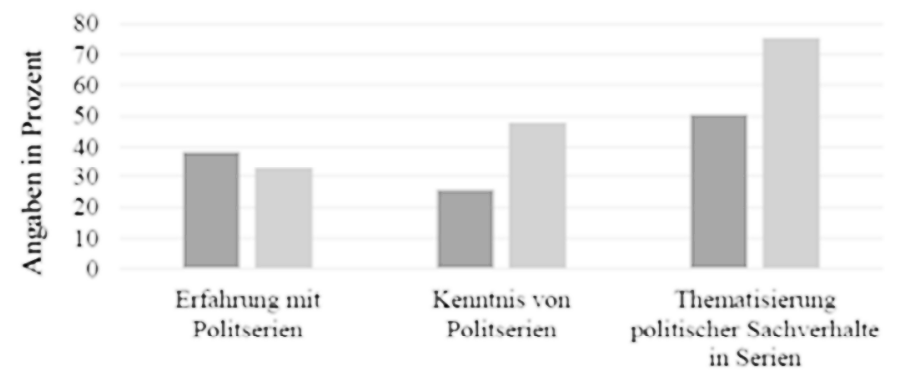

Fragestellung

Eesamtschule Eymnasium

Quelle: Eigene Darstellung.

Hinsichtlich Genderunterschieden kennen Mädchen signifikant weniger PolitSerien als Jungen $\mathrm{t}(268)=2.434, \mathrm{p}<.05$, obwohl sie mehr über Serien anderer Themenformate kommunizieren $\mathrm{t}(268)=3.667, \mathrm{p}<.001$. Möglicherweise er- 
klärt sich dies zum Teil durch das Serieninteresse. Bei der befragten Strichprobe lassen sich Hinweise auf eine positive Korrelation zwischen dem weiblichen Geschlecht und dem Interesse an Serien $\left(r=0.13^{*}\right)$ finden. Gleichzeitig gibt es einen signifikanten Genderunterschied bei der Frage, wie realistisch die Darstellung von Politik in Serien (4-stufige Likertskala) eingeschätzt wird. Jungen sind hier etwas kritischer als Mädchen $\mathrm{t}(167)=2.248, \mathrm{p}<.05$. Darüber hinaus besteht eine positive Korrelation zwischen dem weiblichen Geschlecht und dem Politikunterricht als Verständnishilfe zum Verstehen von politischen Nachrichten ( $\mathrm{r}=$ $\left..15^{* *}\right)$. Hypothese $2 \mathrm{a}$ ist somit für diese Stichprobe bestätigt. Zu Hypothese $3 \mathrm{a}$ hingegen finden sich keine signifikanten Unterschiede, so dass die Hypothese verworfen werden muss. Hypothese $2 b$ bezüglich Jahrgangsunterschieden trifft zu, denn Schüler der 11. Jahrgangsstufe haben signifikant mehr Erfahrung mit Polit-Serien im Unterricht mit $\mathrm{t}(268)=2.945, \mathrm{p}<.01$ und kennen mehr politische Serien $\mathrm{t}(271)=3.638, \mathrm{p}<.001$ als Schüler der 8 . Jahrgangsstufe. Jahrgangsältere Schüler sehen in Polit-Serien signifikant häufiger eine Thematisierung politischer Inhalte als jüngere Schüler $\mathrm{t}(269)=3.378, \mathrm{p}<.01$. Zudem schreiben Schüler der 11. Klasse Politikern eine stärkere Machtorientierung zu als Schüler der 8. Klasse $\mathrm{t}(220)=2.331, \mathrm{p}<.05$. Dies bestätigt gleichzeitig Hypothese $3 b$.

Tabelle 1: Mittelwerte, Standardabweichung und Korrelationen zu Jahrgangsunterschieden zwischen Klasse 8 und 11

\begin{tabular}{|l|c|c|c|c|}
\hline & $\begin{array}{c}\text { Erfahrung } \\
\text { mit Polit- } \\
\text { Serien: MW } \\
\text { (SD) }\end{array}$ & $\begin{array}{c}\text { Kenntnis } \\
\text { Polit-Serien }\end{array}$ & $\begin{array}{c}\text { Thematisie- } \\
\text { rung von } \\
\text { Politik in Po- } \\
\text { lit-Serien }\end{array}$ & $\begin{array}{c}\text { Machtorien- } \\
\text { tierung von } \\
\text { Politiker/- } \\
\text { innen }\end{array}$ \\
\hline $\begin{array}{l}\text { 8. Jahr- } \\
\text { gangsstufe }\end{array}$ & $.30(.46)$ & $.32(.47)$ & $.62(.79)$ & $.52(.50)$ \\
\hline $\begin{array}{l}\text { 11. Jahr- } \\
\text { gangsstufe }\end{array}$ & $.48(.50)$ & $.55(.50)$ & $.85(.36)$ & $.68(.47)$ \\
\hline $\begin{array}{l}\text { Korrelation } \\
\text { nach Spearman }\end{array}$ & $.17 * *$ & $.22^{* *}$ & $.16^{*}$ & $.15^{* *}$ \\
\hline
\end{tabular}

MW = Mittelwert, SD = Standardabweichung, Quelle: Eigene Darstellung.

Bei Schülern mit Migrationserfahrung finden sich Hinweise auf eine negative Korrelation zur Kenntnis politischer Serien $\left(r=-0.13^{*}\right)$. In der Wahrnehmung von Politikern im Hinblick auf eine Machtorientierung und der Erfahrung mit Polit-Serien lassen sich keine signifikanten Unterschiede feststellen. 
Tabelle 2: Zustimmungswerte zu Thesen zur Wahrnehmung von Politikern

\begin{tabular}{|c|c|c|c|c|}
\hline & $\begin{array}{c}\text { N mit } \\
\text { Migrations- } \\
\text { erfahrung Eltern }\end{array}$ & $\begin{array}{l}\text { MW } \\
\text { (SD) }\end{array}$ & $\begin{array}{c}\text { N keine } \\
\text { Migrations- } \\
\text { erfahrung }\end{array}$ & $\begin{array}{l}\text { MW } \\
\text { (SD) }\end{array}$ \\
\hline $\begin{array}{l}\text { Politiker handeln stets für } \\
\text { mein persönliches Wohl- } \\
\text { ergehen. }\end{array}$ & 80 & $\begin{array}{l}.50 \\
(.50)\end{array}$ & 156 & $\begin{array}{l}.32 \\
(.47)\end{array}$ \\
\hline $\begin{array}{l}\text { Politiker arbeiten dafür, } \\
\text { dass es den Menschen in } \\
\text { Deutschland besser geht. }\end{array}$ & 81 & $\begin{array}{l}.74 \\
(.44)\end{array}$ & 165 & $\begin{array}{l}.76 \\
(.43)\end{array}$ \\
\hline $\begin{array}{l}\text { Politiker sind macht- } \\
\text { orientiert. }\end{array}$ & 72 & $\begin{array}{l}.58 \\
(.50)\end{array}$ & 149 & $\begin{array}{l}.57 \\
(.50)\end{array}$ \\
\hline $\begin{array}{l}\text { Politiker handeln stets zu } \\
\text { ihrem eigenen Vorteil. }\end{array}$ & 77 & $\begin{array}{l}.44 \\
(.50)\end{array}$ & 155 & $\begin{array}{l}.37 \\
(.48)\end{array}$ \\
\hline
\end{tabular}

Quelle: Eigene Darstellung.

Jedoch zeigt sich, dass Schüler mit Migrationserfahrung signifikant mehr daran glauben, dass Politiker für das Wohlergehen des Einzelnen handeln $\mathrm{t}(234)=$ $2.718, \mathrm{p}<.01$. Hypothese $2 \mathrm{c}$ kann somit zugestimmt werden, Hypothese $3 \mathrm{c}$ nur eingeschränkt.

Mit vier Wissensitems zum Politischen System Deutschlands ist ein Teil des Fachwissens kontrolliert. Von den maximal 4 erreichbaren Punkten erzielten die Schüler im Mittelwert MW = 2,35 (SD = 1,0). Ihr Wissen hängt signifikant mit ihrer Politiknote zusammen: $\mathrm{t}(93)=2.519, \mathrm{p}<.05$, und hoch signifikant mit einem Tageszeitungsabonnement im Haushalt: $\mathrm{t}(245)=3.939, \mathrm{p}<.001$. Der Einfluss des Bildungshintergrundes auf den Wissenserwerb wurde in ICCS 2016 (Abs/Laudenberg 2017) für NRW erneut bestätigt. Es lässt sich in der Stichprobe kein Genderunterschied nachweisen. Zum Einflussfaktor Geschlecht liegen in der Fachunterrichtsforschung disparate Befunde vor (u.a. Oberle 2012, Weißeno/Eck 2013). Hinweise gibt es auf den Zusammenhang mit Migrationserfahrung. Insgesamt schneiden Schüler mit Migrationserfahrung signifikant schlechter im Wissenstest ab als Schüler ohne Migrationserfahrung $\mathrm{t}(259)=2.638, \mathrm{p}<$ .01. Es zeigt sich ebenfalls eine negative Korrelation zwischen Schülern mit elterlicher Migrationserfahrung und Fachwissen $\left(r=-0.17^{* *}\right)$. Diese Befunde de- 
cken sich mit bisherigen Studien (Landwehr 2017, Goll et al. 2010). Ebenfalls im Einklang mit den Befunden anderer Studien (u.a. Kohring 2006, Matthes/Kohring 2006) sind die Hinweise auf einen Zusammenhang zwischen Wissen und Medienvertrauen $(\mathrm{r}=0.29 * *)$. Je höher das Fachwissen bei Schülern ist, desto ausgeprägter ist ihr Medienvertrauen, verstanden auch als Systemvertrauen (Weißeno/Weschenfelder/Landwehr 2015).

Die leitfadengestützten Lehrerinterviews werden in diesem Beitrag nur auszugsweise vorgestellt. Sie dienen einer möglichen Hypothesengenerierung. Bezüglich des Einsatzes von Medien im Unterricht geben 8 der 10 befragten Lehrkräfte an, regelmäßig Beamer und PC zu nutzen. Allerdings gab nur ein Lehrer an, dass er Filme regelmäßig als Medium im Unterricht einsetzt (LM 2, Abs. 6). Obwohl 9 von 11 der Befragten der Meinung sind, dass Serien oder Filme im Spielfilmformat Unterrichtsinhalte anschaulicher aufbereiten können, nutzen nur 5 dieses Medium zur Vermittlung von Inhalten (primär über Dokumentationen oder Erklärvideos), da die Spielfilmhandlung zu sehr im Vordergrund steht: „Was mich immer stört bei guten Filmen ist, dass meistens doch sehr viel Hollywood drin ist" (LM 6, Abs. 10). Als Auswahlkriterium für einen potentiellen Serieneinsatz gilt zuvorderst die Vermittlung von politischen oder gesellschaftlichen Inhalten. Interessant ist, dass alle Lehrer auch das eigene Interesse bei der Auswahl angegeben haben, während dies hingegen nur eine Frau zugibt. Andererseits geben alle Lehrerinnen an, dass sie Serien danach auswählen würden, ihre Unterrichtsziele zu erreichen, während dies wiederum nur die Hälfte der Männer angibt. Als Ziele lassen sich in den Interviews sechs Dimensionen erkennen: Kompetenzentwicklung, Verdeutlichung von politischen Zusammenhängen und Prozessen, Visualisierung, Schüleraktivierung, Aufzeigen von Perspektiven und Kontroversen, Abwechslung. 5 von den 11 befragten Lehrkräften glauben, dass die Kompetenzdimension Fachwissen durch den Serieneinsatz nicht besser gefördert werden könne als eine thematische Erarbeitung ohne den Einsatz von Medien, während 6 dies umgekehrt sehen. Noch deutlicher bejahen mit 8 der 11 Interviewten die Förderung der politischen Urteilsfähigkeit durch Serien: „Vor allem die Diskussion nach dem Unterricht ist stärker vorhanden“ (LW 1, Abs. 68). Bei der Frage nach der didaktischen Einbettung im Unterrichtsverlauf steht bei allen Befragten der Einsatz in der Einstiegsphase auf Platz 1 (10 Personen sehen Serien als motivationsfördernd), nur 3 der Befragten können sich eine Serie in der Sicherungsphase vorstellen. In der Lehramtsausbildung konnten mit 91 Prozent fast alle interviewten Personen keine Erfahrung mit dem Einsatz von Polit-Serien sammeln. Nur ein Lehrer konnte während des Referendariats eine Fortbildung zu dem Thema besuchen (LM 6, Abs. 30). Hier schlieBen sich auch die Probleme an, die von den Lehrkräften benannt werden. Tech- 
nische Schwierigkeiten (technische Ausstattung der Schule, Urheberrechte, Download) sehen 7 Lehrer als hinderlich für den Einsatz von Serien, die Mehrheit steht jedoch vor inhaltlichen oder didaktischen Herausforderungen: „Die Dimension, die so ein Serienausschnitt hat, kann auf ganz verschiedene Art und Weise falsch verstanden werden und das kann dann im Unterricht nicht abgedeckt werden und so was bleibt dann hängen“ (LM 4, Abs. 68). Hier ist eine didaktische Aufarbeitung notwendig, ,damit die auch wirklich wissen, was Realität und was Fiktion ist“" (LW 2, Abs. 64). Obgleich nur 4 der 11 befragten Lehrkräfte glauben, dass die Trennung von Fiktion und Realität ihren Schülern Probleme bereitet, sind 10 der Befragten der Ansicht, dass ihre Schüler durch den Konsum von Polit-Serien in ihren Vorurteilen bestärkt werden könnten, ,,weil es oft überspitzt und übertrieben dargestellt wird und deswegen glaube ich schon, dass sich dadurch Vorurteile schüren" (LW 5, Abs. 69).

Insgesamt zeigen die Lehrerinterviews, dass hinsichtlich medialer Kompetenzen noch Nachholbedarf besteht und Fortbildungsangebote ausgebaut werden müssen, um auch Polit-Serien für den Unterricht zu nutzen (Fröhlich 2004: 75f.). Das Bewusstsein, dass Polit-Serien fachdidaktisch aufzubereiten sind, ist den Befragten sehr deutlich. Daraus lässt sich hinsichtlich des Professionswissens jedoch nicht ableiten, dass die befragten Lehrkräfte über fachdidaktisches Wissen und Können verfügen, um dies in Unterrichtshandeln umzusetzen (siehe Einsatz von Politserien von nur einer Person der Befragten und Rückgriff auf aufbereitete Lehrfilme). Die befragten Lehrkräfte äußern zudem Ängste hinsichtlich einer Überforderung mit fiktiven Inhalten oder dem Ausbau von Vorurteilen bei Schülern, was ihre fachdidaktische beliefs zum Lehr-Lern-Prozess offenbaren kann. Hier sind vertiefte Analysen notwendig.

\section{FAZIT UND AUSBLICK}

Serien nehmen einen zentralen Platz im Medienverhalten der befragten Schüler ein, mehr als ein Drittel hat bereits eine Polit-Serie gesehen. Die Schülerbefragung zeigt Unterschiede zwischen Schulformen, Gender und Jugendlichen mit Migrationserfahrung. Gerade in Verbindung mit Wissen lassen sich die üblichen Effekte beobachten. In den Interviews betonen Lehrkräfte die Bedeutung von Medienkompetenz bei ihren Schülern, dennoch fühlen sie sich beim Einsatz von Polit-Serien eher verunsichert oder nicht gut vorbereitet, weshalb dieser Medieneinsatz eher weniger im alltäglichen Unterrichtsgeschehen zum Tragen kommt.

Sowohl auf Schüler- als auch auf Lehrerseite besteht weiterer Forschungsbedarf in der Fachdidaktik zum Einsatz von multimedialen Formaten als Inhalt und Medium im politik- und sozialwissenschaftlichen Unterricht. Dass dies nicht nur 
angesichts der allseits geforderten digitalen Medienkompetenz gewinnbringend ist, zeigen diese ersten empirischen Untersuchungen. Spannend wäre, in Interventionsstudien mit Follow-Up-Design zu analysieren, ob die Urteilskompetenz von Schülern durch die Auseinandersetzung mit Polit-Serien im Vergleich zu Textanalysen steigt, ob sich das Bild von Politikern dadurch beeinflussen lässt und ob das Interesse am Fach durch diese Art des Medieneinsatzes steigt und anhält. Zugleich wäre es für die Qualitätssteigerung in der Lehramtsausbildung und den wissenschaftlichen Praxistransfer bedeutsam, mehr gesicherte empirische Daten zur Professionskompetenz von Politiklehrern im Hinblick auf PolitSerien zu gewinnen, um evidenzbasiert passende Angebote und Fortbildungen zu konzipieren.

\section{LITERATUR}

Abs, Hermann J./Hahn-Laudenberg, Katrin (Hrsg.) (2017): Das politische Mindset von 14-Jährigen. Ergebnisse der International Civic and Citizenship Education Study 2016, Münster/New York.

Besand, Anja (2016): Zweite Wahl? - Von House of Cards bis Breaking Bad. Didaktische Reflexionen über die Chancen und Grenzen der Vermittlung politischer Konzepte durch Fernsehserien, in: Sanders, Olaf/Besand, Anja/Arenhövel, Marc (Hrsg.): Ambivalenzwucherungen. Breaking Bad aus bildungs-, kultur- und sozialwissenschaftlichen Blickwinkeln, Köln, S. 195213.

Betsch, Tilmann/Funke, Joachim/Plessner, Henning (2010): Denken - Urteilen, Entscheiden, Problemlösen, Wiesbaden.

BMBF (2016): Sprung nach vorn in der digitalen Bildung. Pressemitteilung: 117/2016 https://www.bmbf.de/de/sprung-nach-vorn-in-der-digitalen-bildung3430.html (zugegriffen am 27.10.2017).

Bortz, Jürgen/Döring, Nicola (2016): Forschungsmethoden und Evaluation in den Sozial- und Humanwissenschaften. Wiesbaden.

Bos, Wilfried/Eickelmann, Birgit/Gerick, Julia/Goldhammer, Frank/Schaumburg, Heike/Schwippert, Knut/Senkbeil, Martin/Schulz-Zander, Renate/Wendt, Heike (Hrsg.) (2014): ICILS 2013: Computer- und informationsbezogene Kompetenzen von Schülerinnen und Schülern in der 8. Jahrgangsstufe im internationalen Vergleich, Münster.

Breit, Gottfried/Weißeno, Georg (2004): Planung des Politikunterrichts. Eine Einführung, Schwalbach am Taunus.

Detjen, Joachim/Massing, Peter/Richter, Dagmar/Weißeno, Georg (2012): Politikkompetenz - Ein Modell, Wiesbaden. 
Dörner, Andreas (2016): Politserien: Unterhaltsame Blicke auf die Hinterbühnen der Politik, in: Aus und Politik und Zeitgeschichte (51), S. 4-11.

Eickelmann, Birgit/Gerick, Julia/Schulz-Zander, Renate (2008): Lernen mit digitalen Medien: auf Dauer motivationsfördernd? Analysen zur Lernmotivation aus Lehrer- und Schülersicht, https://kw.uni-paderborn.de/fileadmin/fakultaet/ Institute/erziehungswissenschaft/Schulpaedagogik/PDF/Poster_Kiel_final.pdf (zugegriffen am 16.07.2017).

Fröhlich, Arnold (2004): Medienkompetenz in der Schule, in: Bonfadelli, Heinz/Bucher, Priska/Paus-Hasebrink, Ingrid/Süss, Daniel (Hrsg.): Medienkompetenz und Medienleistungen in der Informationsgesellschaft, Zürich, S. 72-87.

Gapski, Harald/Oberle, Monika/Staufer, Walter (Hrsg.) (2017): Medienkompetenz. Herausforderung für Politik, politische Bildung und Medienbildung, Bonn.

Geyer, Robby/Kölbl, Claudia (2015): Medien - die „vierte Gewalt“?, in: Bundeszentrale für politische Bildung (Hrsg.): Themenblätter im Unterricht 107, Bonn.

Goll, Thomas/Richter, Dagmar/Weißeno, Georg/Eck, Valentin (2010): Politisches Wissen zur Demokratie von Schüler/-innen mit und ohne Migrationshintergrund (POWIS-Studie), in: Weißeno, Georg (Hrsg.): Bürgerrolle heute, Bonn, S. 21-48.

Gropengießer, Harald (2005): Qualitative Inhaltsanalyse in der fachdidaktischen Lehr-Lernforschung, in: Mayring, Peter/Gläser-Zikuda, Michaela (Hrsg.): Die Praxis der qualitativen Inhaltsanalyse, Weinheim, S.172-190.

Harth, Thilo (2001): Medien und Demokratie. Der Einfluss des Internet und die Folgen für Politik und politische Bildung, in: Meier-Walser, Reinhard/Harth, Thilo (Hrsg.): Politikwelt Internet. Neue demokratische Beteiligungschancen mit dem Internet?, München, S. 23-48.

Haug, Dennis (2016): Digitale Medien in der Politischen Bildung, Wiesbaden.

Herzig, Bardo (2014): Wie wirksam sind digitale Medien im Unterricht?, Gütersloh.

Kerres, Michael/Preussler, Annabell/Schiefner-Rohs, Mandy (2013): Lernen mit Medien, in: Kuhlen, Rainer/Semar, Wolfgang/Strauch Dietmar (Hrsg.): Grundlagen der praktischen Information und Dokumentation. Handbuch zur Einführung in die Informationswissenschaft und -Praxis, Berlin, S. 583-594.

Kerres, Michael (2003): Wirkungen und Wirksamkeit neuer Medien in der Bildung, in: Learning Lab Universität Duisburg-Essen, https://learninglab.unidue.de/sites/default/files/eq-wirkungen-kerres_1.pdf - Stand: o.A. 2003, (zugegriffen am 16.07.2017). 
Kerres, Michael (2000): Medienentscheidungen in der Unterrichtsplanung. $\mathrm{Zu}$ Wirkungsargumenten und Begründungen des didaktischen Einsatzes digitaler Medien, in: Bildung und Erziehung 52, S. 19-39.

Kohring, Matthias (2006): Zum Verhältnis von Wissen und Vertrauen. Eine Typologie am Beispiel öffentlicher Kommunikation, in: Pühringer, Karin/Zielmann, Sarah (Hrsg.): Vom Wissen und Nicht-Wissen einer Wissenschaft. Kommunikationswissenschaftliche Domänen, Darstellungen und Defizite, Berlin, S. 121-134.

KMK (2016): Strategie der Kultusministerkonferenz „Bildung in der digitalen Welt“. Beschluss der Kultusministerkonferenz vom 08.12., https://www.kmk.org/ fileadmin/Dateien/pdf/PresseUndAktuelles/2016/Bildung_digitale_Welt_Web version.pdf (zugegriffen am 27.10.2017)

Kuckartz, Udo (2005): Einführung in die computergestützte Analyse qualitativer Daten, Wiesbaden.

Kunter, Mareike/Baumert, Jürgen/Blum, Werner/Klusmann, Uta/Krauss, Stefan/Neubrand, Michael (Hrsg.) (2011): Professionelle Kompetenz von Lehrkräften. Ergebnisse des Forschungsprogramms COACTIV, Münster.

Landwehr, Barbara (2017): Partizipation, Wissen und Motivation im Politikunterricht, Wiesbaden.

Manzel, Sabine (2017): Medienkompetenz als eine Schlüsselkompetenz für politische Urteils- und Handlungsfähigkeit, in: Gapski, Harald/Oberle, Monika/Staufer, Walter (Hrsg.): Medienkompetenz. Herausforderung für Politik, politische Bildung und Medienbildung, Bonn, S. 207-217.

Manzel, Sabine/Weißeno, Georg (2017): Modell der Politischen Urteilsfähigkeit - eine Dimension der Politikkompetenz, in: Oberle, Monika/Weißeno, Georg (Hrsg.): Politikwissenschaft und Politikdidaktik. Theorie und Empirie, Wiesbaden, S. 59-86.

Manzel, Sabine (2008). Wissensvermittlung und Problemorientierung im Politikunterricht: Lehr-Lern-Forschung; eine anwendungsorientierte Einführung, Schwalbach/Ts.

Manzel, Sabine (2007). Kompetenzzuwachs im Politikunterricht: Ergebnisse einer Interventionsstudie zum Kernkonzept Europa, Münster/New York.

Matthes, Jörg/Kohring, Matthias (2006): Seeing is believing? Zum vermeintlichen Glaubwürdigkeitsvorsprung des Fernsehens im Vergleich zu Tageszeitung, Zeitschrift und Radio, in: Weischenberg, Siegfried/Loosen, Wiebke/Beuthner, Michael (Hrsg.): Medien-Qualitäten. Öffentliche Kommunikation zwischen ökonomischem Kalkül und Sozialverantwortung, Konstanz, S. 367-382. 
Mayring, Philipp (2010): Qualitative Inhaltsanalyse. Grundlagen und Techniken, Weinheim/Basel.

Medienpädagogischer Forschungsverbund Südwest (MPFS) (2017): JIM_Studie 2017, Jugend, Information, (Multi-) Media. Basisstudie zum Medienumgang 12- bis 19-Jähriger in Deutschland, Stuttgart, https://www.mpfs.de/file admin/files/Studien/JIM/2016/JIM_Studie_2016.pdf （zugegriffen am 08.12.2017).

Medienpädagogischer Forschungsverbund Südwest (MPFS) (2016): JIM_Studie 2016, Jugend, Information, (Multi-) Media. Basisstudie zum Medienumgang 12- bis 19-Jähriger in Deutschland, Stuttgart, https://www.mpfs.de/file admin/files/Studien/JIM/2017/JIM_2017.pdf (zugegriffen am 31.12.2017).

Motyka, Marc/Lipowsky, Frank (2017): Mögen die Spiele beginnen. Eine empirische Forschungsagenda für das Lernen mit Computerspielen im Politikunterricht, in: Manzel, Sabine/Schelle, Carla (Hrsg.): Empirische Forschung zur schulischen Politischen Bildung, Wiesbaden, S. 171-179.

Niedermayer, Oskar (2005): Bürger und Politik. Politische Orientierungen und Verhaltensweisen der Deutschen, Wiesbaden.

Oberle, Monika (2012): Politisches Wissen über die Europäische Union. Subjektive und objektive Politikkenntnisse von Jugendlichen, Wiesbaden.

Simon, Erik (2007): Medienwirkung. Wissenschaftliche Theorien und Ergebnisse, WDR http://www1.wdr.de/unternehmen/der-wdr/medienundbildung/ kapitel_X_medienwirkung102.pdf, (zugegriffen am 16.07.2017).

Spanhel, Dieter (2006): Handbuch Medienpädagogik. Medienerziehung, Band 3, Stuttgart.

Statista (2017): Anzahl der Streaming-Abonnenten von Netflix weltweit vom 3. Quartal 2011 bis zum 1. Quartal 2017 (in Millionen), in: Statista - Das Statistik-Portal, https://de.statista.com/statistik/daten/studie/196642/umfrage/ abonnenten-von-netflix-quartalszahlen/ (zugegriffen am 16.07.2017).

Steppat, Timo (2014): Von „House of Cards“ bis „Homeland“. Wie uns Fernsehserien manipulieren, in: Handelsblatt, http://www.handelsblatt.com/pano rama/kultur-kunstmarkt/von-house-of-cards-bis-homeland-wie-uns-fernseh serien-manipulieren/9484368.html (zugegriffen am 27.09.2017)

Straßner, Veit (2013) (Hrsg.): Filme im Politikunterricht. Wie man Filme professionell aufbereitet, das filmanalytische Potenzial entdeckt und Lernprozesse anregt - mit zehn Beispielen für die Sekundarstufe II, Schwalbach/Ts.

Tulodziecki, Gerhard (2009): Funktionen von Medien im Unterricht, in: Arnold, Karl-Heinz/Sandfuchs, Uwe/Wiechmann, Jürgen (Hrsg.): Handbuch Unterricht, 2. Aufl., Bad Heilbrunn, S. 291-297. 
Weißeno, Georg/Weschenfelder, Eva/Landwehr, Barbara (2015): Motivation, Systemvertrauen und Leistungsfähigkeit von Schülerinnen und Schülern im Politikunterricht, in: Weißeno, Georg/Schelle, Carla (Hrsg.): Empirische Forschung in gesellschaftswissenschaftlichen Fachdidaktiken, Wiesbaden, S. 53-66.

Weißeno, Georg/Eck, Valentin (2013): Wissen, Selbstkonzept und Fachinteresse - Ergebnisse einer Interventionsstudie zur Politikkompetenz, Münster.

Weschenfelder, Eva (2014): Professionelle Kompetenzen von Politiklehrkräften, Wiesbaden.

Zegart, Amy B. (2000): Flawed by Design: The Evolution of the CIA, JCS, and NSC, Stanford. 

Anhang 



\section{Autorinnen- und Autorenverzeichnis}

Zaira Batroff M.A., Unternehmenskommunikation, Hochschule der Medien, Stuttgart (zaira.batroff@gmx.net)

Jonathan Beierl B.A., Masterprogramm Politikmanagement, Public Policy und öffentliche Verwaltung, NRW School of Governance, Institut für Politikwissenschaft, Universität Duisburg-Essen (jonathan.beierl@stud.uni-due.de)

Ann-Kathrin Binot B.A., Masterprogramm Politikmanagement, Public Policy und öffentliche Verwaltung, NRW School of Governance, Institut für Politikwissenschaft, Universität Duisburg-Essen (ann-kathrin.binot@stud.unidue.de)

Johannes Bongardt B.A., Masterprogramm Politikmanagement, Public Policy und öffentliche Verwaltung, NRW School of Governance, Institut für Politikwissenschaft, Universität Duisburg-Essen (johannes.bongardt@gmx.net)

Florian Breitweg B.A., Masterprogramm Politikmanagement, Public Policy und öffentliche Verwaltung, NRW School of Governance, Institut für Politikwissenschaft, Universität Duisburg-Essen (florian.breitweg@stud.uni-due.de)

Lisa Brose M.A., Persönliche Referentin Wahlkreisbüro Mahmut Özdemir, MdB (brose_lisa@web.de)

Lorena Capuozzo M.A., Unternehmenskommunikation, Hochschule der Medien, Stuttgart (lorenacapuozzo@web.de)

Yannick Chougrani B.A., Masterprogramm Politikmanagement, Public Policy und öffentliche Verwaltung, NRW School of Governance, Institut für Poli- 
tikwissenschaft, Universität Duisburg-Essen (yannick.chougrani@stud.unidue.de)

Prof. Dr. Andreas Dörner, Institut für Medienwissenschaft, Universität Marburg (andreas.doerner@staff.uni-marburg.de)

Dr. Frank Gadinger, Center for Global Cooperation Research, Universität Duisburg-Essen (frank.gadinger@gcr21.uni-due.de)

Ulrike Gansen B.A., Masterprogramm Politikmanagement, Public Policy und öffentliche Verwaltung, NRW School of Governance, Institut für Politikwissenschaft, Universität Duisburg-Essen (ug@gaha.de)

Rieke Gießelmann B.A., Masterprogramm Politikmanagement, Public Policy und öffentliche Verwaltung, NRW School of Governance, Institut für Politikwissenschaft, Universität Duisburg-Essen (rieke.giesselmann@stud.unidue.de)

Florian Gilberg B.A., Masterprogramm Politikmanagement, Public Policy und öffentliche Verwaltung, NRW School of Governance, Institut für Politikwissenschaft, Universität Duisburg-Essen (florian.gilberg@ stud.uni-due.de)

Jakob Hager B.A., Masterprogramm Politikmanagement, Public Policy und öffentliche Verwaltung, NRW School of Governance, Institut für Politikwissenschaft, Universität Duisburg-Essen (jakob.hager@ stud.uni-due.de)

Kai Jardner M.A., Unternehmenskommunikation, Hochschule der Medien, Stuttgart (kaijardner@gmail.com)

Matthias Jüschke B.A., Masterprogramm Politikmanagement, Public Policy und öffentliche Verwaltung, NRW School of Governance, Institut für Politikwissenschaft, Universität Duisburg-Essen (matthias.b@jueschke.de)

Prof. Dr. Klaus Kamps, Hochschule der Medien, Stuttgart (kamps@hdmstuttgart.de)

Anatoli Kolembach B.A., Masterprogramm Politikmanagement, Public Policy und öffentliche Verwaltung, NRW School of Governance, Institut für Poli- 
tikwissenschaft, Universität Duisburg-Essen (anatoli.kolembach@stud.unidue.de)

Prof. Dr. Karl-Rudolf Korte, NRW School of Governance, Institut für Politikwissenschaft, Universität Duisburg-Essen (karl-rudolf.korte@uni-due.de)

Julia Linn B.A., Masterprogramm Politikmanagement, Public Policy und öffentliche Verwaltung, NRW School of Governance, Institut für Politikwissenschaft, Universität Duisburg-Essen (julia.linn@stud.uni-due.de)

Michelle Magaletta B.A./B.Sc., wissenschaftliche Hilfskraft TalentKolleg Ruhr und Masterprogramm Politikmanagement, Public Policy und öffentliche Verwaltung, NRW School of Governance, Institut für Politikwissenschaft, Universität Duisburg-Essen (michelle.magaletta@uni-due.de)

apl. Prof. Dr. Manfred Mai, Staatskanzlei Nordrhein-Westfalen und Institut für Politikwissenschaft, Universität Duisburg-Essen (manfred.mai@stk.nrw.de)

Prof. Dr. Sabine Manzel, CIVES School of Civic Education, Institut für Politikwissenschaft, Universität Duisburg-Essen (sabine.manzel@uni-due.de)

Martin Miller B.A., Masterprogramm Politikmanagement, Public Policy und öffentliche Verwaltung, NRW School of Governance, Institut für Politikwissenschaft, Universität Duisburg-Essen (martin.miller@stud.uni-due.de)

Vanessa Molter B.A., Masterprogramm Politikmanagement, Public Policy und öffentliche Verwaltung, NRW School of Governance, Institut für Politikwissenschaft, Universität Duisburg-Essen (vanessa.molter@stud.uni-due.de)

Raphael David Moser B. A., wissenschaftliche Hilfskraft am Wuppertal Institut für Klima, Umwelt, Energie und Masterprogramm Politikmanagement, Public Policy und öffentliche Verwaltung, NRW School of Governance, Institut für Politikwissenschaft, Universität Duisburg-Essen (raphael.moser@alumni. uni-due.de)

Prof. Dr. Nele Noesselt, Institut für Politikwissenschaft, Universität DuisburgEssen (nele.noesselt@uni-due.de) 
Jan Pfeifer M.A., Masterprogramm Politikmanagement, Public Policy und öffentliche Verwaltung, NRW School of Governance, Institut für Politikwissenschaft, Universität Duisburg-Essen (jan.pfeifer@alumni.uni-due.de)

Ilka Rasch B.A., Masterprogramm Politikmanagement, Public Policy und öffentliche Verwaltung, NRW School of Governance, Institut für Politikwissenschaft, Universität Duisburg-Essen (ilka.rasch@ stud.uni-due.de)

Said Rezek B.A., Masterprogramm Politikmanagement, Public Policy und öffentliche Verwaltung, NRW School of Governance, Institut für Politikwissenschaft, Universität Duisburg-Essen (rezeksaid@gmail.com)

Henrik Schillinger M.A., Institut für Politikwissenschaft, Universität DuisburgEssen (henrik.schillinger@uni-due.de)

Christina-Johanne Schröder B.A., Masterprogramm Politikmanagement, Public Policy und öffentliche Verwaltung, NRW School of Governance, Institut für Politikwissenschaft, Universität Duisburg-Essen (info@cjschroeder.de)

Steffen Schulze B.A., Masterprogramm Politikmanagement, Public Policy und öffentliche Verwaltung, NRW School of Governance, Institut für Politikwissenschaft, Universität Duisburg-Essen (steffen.schulze@ stud.uni-due.de)

Jonas Seyferth B.A., Masterprogramm Politikmanagement, Public Policy und öffentliche Verwaltung, NRW School of Governance, Institut für Politikwissenschaft, Universität Duisburg-Essen (jonas.seyferth@stud.uni-due.de)

Stefan Heinrich Simond M.A., Institut für Medienwissenschaft, Universität Marburg (stefan.simond@staff.uni-marburg.de)

Arne Sönnichsen M.A., Institut für Politikwissenschaft, Universität DuisburgEssen (arne.soennichsen@uni-due.de)

Dipl. Soz.-Wiss. Alexander Stock, Abteilungsleiter Sozialmanagement GAG Immobilien AG (alexstock81@gmx.de)

Dr. Niko Switek, NRW School of Governance, Institut für Politikwissenschaft, Universität Duisburg-Essen (niko.switek@uni-due.de) 
Cornelius Witt M.A., Manager Public Policy, SCOPE Europe (cwitt@scopeeurope.eu)

Sören Witt B.A., Masterprogramm Politikmanagement, Public Policy und öffentliche Verwaltung, NRW School of Governance, Institut für Politikwissenschaft, Universität Duisburg-Essen (soeren.witt@stud.uni-due.de)

Dr. Taylan Yildiz, NRW School of Governance, Institut für Politikwissenschaft, Universität Duisburg-Essen (taylan.yildiz@uni-due.de)

Almut Zimmer M.A., Masterprogramm Politikmanagement, Public Policy und öffentliche Verwaltung, NRW School of Governance, Institut für Politikwissenschaft, Universität Duisburg-Essen (zimmer.almut@gmail.com) 


\section{Politikwissenschaft}

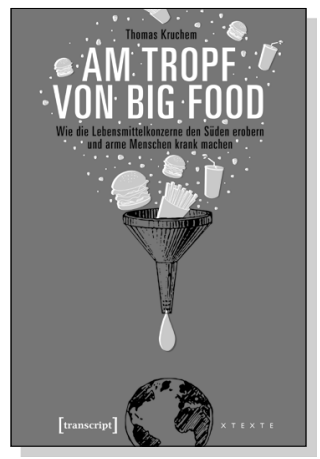

Thomas Kruchem

Am Tropf von Big Food

Wie die Lebensmittelkonzerne den Süden erobern und arme Menschen krank machen

Juni 2017, 214 S., kart., zahlr. Abb.

$19,99 €(D E), 978-3-8376-3965-0$

E-Book

PDF: $16,99 €(D E)$, ISBN 978-3-8394-3965-4

EPUB: $16,99 €(D E)$, ISBN 978-3-7328-3965-0

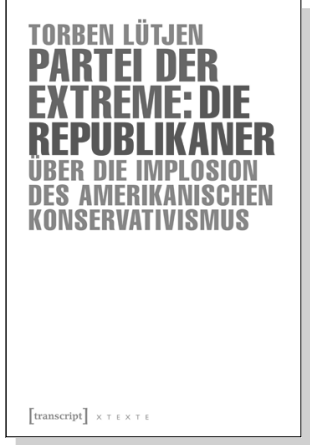

Torben Lütjen

Partei der Extreme: Die Republikaner

Über die Implosion des amerikanischen Konservativismus

2016, 148 S., kart.

$14,99 €(D E), 978-3-8376-3609-3$

E-Book

PDF: $12,99 €(D E)$, ISBN 978-3-8394-3609-7

EPUB: $12,99 €(D E)$, ISBN 978-3-7328-3609-3

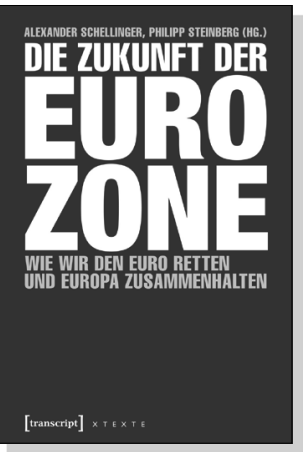

Alexander Schellinger, Philipp Steinberg (Hg.)

\section{Die Zukunft der Eurozone}

Wie wir den Euro retten und Europa zusammenhalten

2016, 222 S., kart.

$19,99 €(D E), 978-3-8376-3636-9$

E-Book

PDF: $17,99 €(D E)$, ISBN 978-3-8394-3636-3

EPUB: $17,99 €(D E)$, ISBN 978-3-7328-3636-9 


\section{Politikwissenschaft}

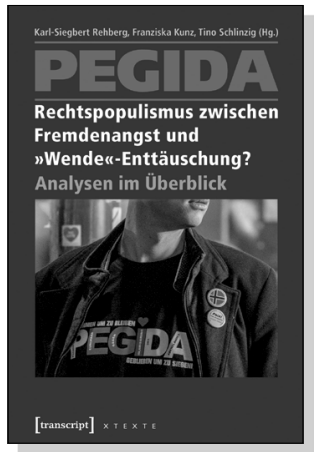

Karl-Siegbert Rehberg, Franziska Kunz, Tino Schlinzig (Hg.)

PEGIDA - Rechtspopulismus zwischen

Fremdenangst und "Wende "-Enttäuschung?

Analysen im Überblick

2016, 384 S., kart.

29,99€ (DE), 978-3-8376-3658-1

E-Book

PDF: $26,99 €$ (DE), ISBN 978-3-8394-3658-5

EPUB: $26,99 €(D E)$, ISBN 978-3-7328-3658-1

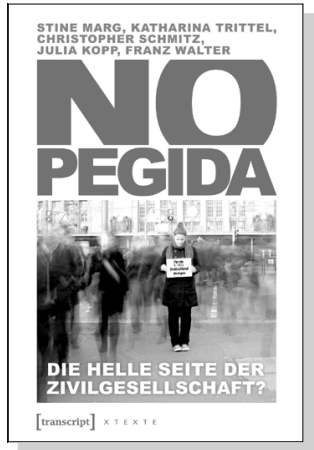

Stine Marg, Katharina Trittel,

Christopher Schmitz, Julia Kopp, Franz Walter

\section{NoPegida}

Die helle Seite der Zivilgesellschaft?

2016, 168 S., kart.

$19,99 €(D E), 978-3-8376-3506-5$

E-Book

PDF: $17,99 €$ (DE), ISBN 978-3-8394-3506-9

EPUB: $17,99 €(D E)$, ISBN 978-3-7328-3506-5

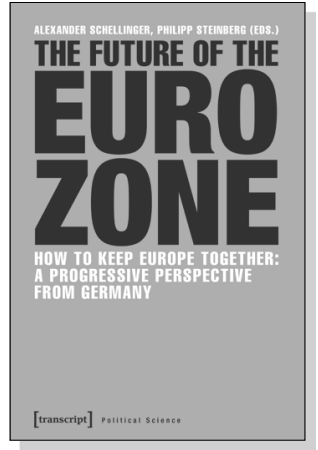

Alexander Schellinger, Philipp Steinberg (eds.)

The Future of the Eurozone

How to Keep Europe Together:

A Progressive Perspective from Germany

October 2017, 202 p., pb.

$29,99 €(D E), 978-3-8376-4081-6$

E-Book

PDF: $26,99 €$ (DE), ISBN 978-3-8394-4081-0

EPUB: $26,99 €(D E)$, ISBN 978-3-7328-4081-6 
